
Dr. iur. Dominik Elser

\section{Die privatisierte Erfüllung staatlicher Aufgaben}

Verfassungsrechtliche Probleme und Spielräume

Dissertation 
Inauguraldissertation zur Erlangung der Würde eines Doctor iuris der Rechtswissenschaftlichen Fakultät der Universität Bern.

Die Fakultät hat diese Arbeit am 21. Februar 2019 auf Antrag der beiden Gutachter, Prof. em. Dr. Pierre Tschannen und Prof. Dr. Markus Müller, als

Dissertation angenommen und mit dem Prädikat «summa cum laude» versehen.

Für die Publikation wurden Literatur und Rechtsprechung bis zum 31. Dezember 2019 berücksichtigt. 


\section{Vorwort}

Eine abgeschlossene Dissertation ist der Triumph des Wahnsinns über die Vernunft.

Nur schon der Anspruch, einer derart überbearbeiteten Disziplin wie der Rechtswissenschaft neue Erkenntnisse abzuringen, ist reichlich kühn. Diesen Anspruch dann nicht aufzugeben, bedingt sodann die vollständige Verdrängung der Vernunft. Jetzt, wo es vollbracht ist und das Manuskript alsbald der Druckmaschine - und der E-Book-Maschine - übergeben wird, kann ich erleichtert feststellen: gerade nochmal gutgegangen! Ich wünschte, es hätte nicht mehr als eine halbe Dekade gedauert, möchte aber keinen der kleinen Fortschritte, keine der langen Durststrecken und keinen der seltenen Einfälle der letzten Jahre missen. Ich hätte diese Zeit bestimmt zielführender und sinnvoller zubringen können. Doch niemand wird jetzt behaupten, es hätte sich nicht gelohnt - auch darin liegt der Wahnsinn einer Dissertation.

Dieses Werk ist nur dank der Unterstützung folgender Personen entstanden: Pierre Tschannen, Doktorvater und Chef, dessen Vorbild und Anleitung mich ermutigten, es zu versuchen und abzuschliessen. Er hat mir gezeigt, wie gutes akademisches Schreiben gehen und was man damit erreichen kann. Und weshalb sich die gewissenhafte Beschäftigung mit dem Staats- und Verwaltungsrecht lohnt; mehr noch, weshalb jede gewissenhafte Juristin und jeder gewissenhafte Jurist dazu verpflichtet ist.

Markus Müller, dessen Pausengespräche und dessen Ermutigung zur provokativen Aussage mich weiter darin bestärkten, dass ich etwas beisteuern kann.

Ein besonderer Dank gebührt dem Bernischen Juristenverein, dessen grosszügige finanzielle Unterstützung den Abschluss dieser Dissertation ermöglicht hat. Namentlich verdankt seien Dr. Christoph Zimmerli, Prof. Dr. Sibylle Hofer, und Dr. Thomas Müller.

Die Kolleginnen und Kollegen am Institut für öffentliches Recht der Universität Bern, die das wahnsinnige Unterfangen durchwegs freudig und unterhaltsam gestaltet haben.

Peter Bieri, Anja Eugster, Nula Frei, Fabian Mösching, Charlotte SieberGasser und Karl-Marc Wyss, die jeweils einen Teil des ersten Entwurfs gelesen und mit ihren aufmerksamen Hinweisen massgeblich verbessert haben.

Das Organisationsteam der 54. Assistententagung Öffentliches Recht an der Universität Graz, die mich erste Erkenntnisse dieser Arbeit haben vortragen 
lassen, und die Kolleginnen und Kollegen der Wirtschaftsuniversität Wien, die mich das Vorgetragene in einer genüsslichen Woche haben aufschreiben lassen.

Daniel Hürlimann, Marc Thommen, Wendelin Hess und Beat Müller, die mir die Chance geben, als erster Autor ein Buch im suigeneris Verlag zu publizieren.

Dieses Buch sei gewidmet: meinem Vater, der jetzt sehr stolz gewesen wäre; meiner Mutter, die ohnehin stolz ist; und Mirjam, deren unbändiges Vertrauen in meine Fähigkeiten ich streckenweise teilte.

Bern, im Februar 2020

Dominik Elser 


\section{Übersicht}

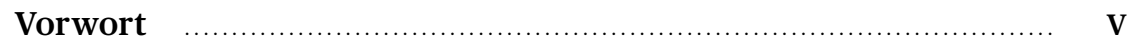

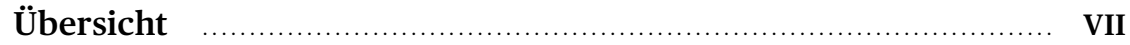

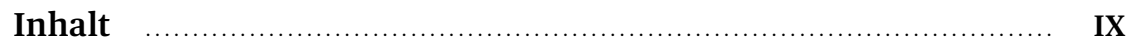

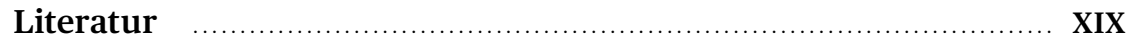

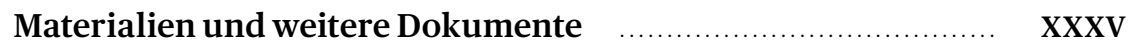

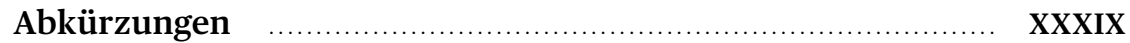

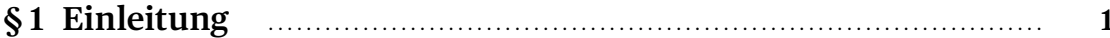

\$2 Begriffsklärung _............................................................

\$3 Welchen verfassungsrechtlichen Anforderungen unterliegt die Erfüllung staatlicher Aufgaben?

\$4 In welcher Hinsicht kann die Erfüllung staatlicher Aufgaben privatisiert werden?

\$5 Welche Probleme schaffen die verfassungsrechtlichen Anforderungen für die Erfüllungsprivatisierung?

\$6 Welche Spielräume belassen die verfassungsrechtlichen Anforderungen der Erfüllungsprivatisierung? 



\section{Inhalt}

Vorwort

Übersicht

VII

Inhalt

IX

Literatur XIX

Materialien und weitere Dokumente XXXV Abkürzungen XXXIX

I. Ausgangspunkt: Die Erwartung von weniger Staat

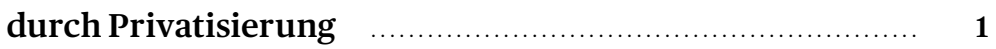

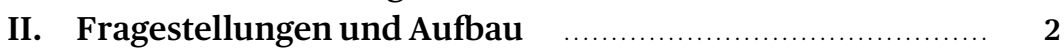

III. Verfassungsdogmatischer Zugang ……................... 3

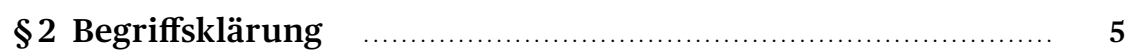

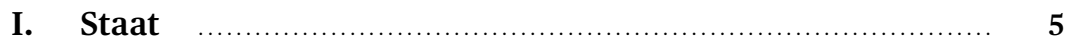

1. Staatlichkeit als Begriff der Bundesverfassung $\ldots \ldots \ldots \ldots . .5$

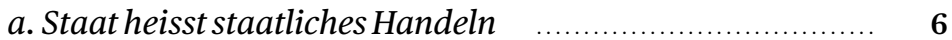

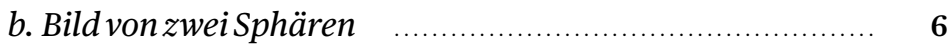

$\begin{array}{llll}\text { c. Staatstätigkeit als Erfüllung staatlicher Aufgaben } & \text {........ } \quad 6\end{array}$

2. Staatsaufgaben ……..........................................

a. Positivrechtliche Zuweisung _................................. 8

b. Pflicht zur Erfüllung _....................................... 9

c. Verwaltungsaufgaben und andere Staatsaufgaben $\quad \ldots . . . . \quad 11$

d. Verhältnis zu öffentlichen Aufgaben $\quad$.......................... 11

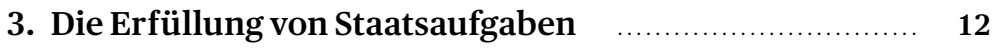

4. Veranschaulichung anhand konkreter Staatsaufgaben 13

5. Einschub: Zuordnung verschiedener Stufen staatlicher Verantwortung zur Staatsdefinition $\ldots \ldots \ldots \ldots \ldots \ldots \ldots \ldots \ldots \ldots$

6. Zwischenfazit: Der funktionelle Staatsbegriff des Verfassungsrechts ….................................... 15

II. Privatisierung ….................................................. 16

1. Ein facettenreicher Prozess ohne scharfe Konturen? ... 16

2. Verhältnis von Privatisierung und Entstaatlichung $\quad \ldots \quad 16$

3. Das Private als Begriff der Bundesverfassung $\quad \ldots \ldots \ldots \ldots \ldots . .17$ 
a. Zweiteilung in private und andere Akteure ................... 18

b. Lebensbereich Privater an sich ................................. 19

c. Privatwirtschaft .............................................. 20

d. Zwischenfazit: Keine Begriffsklarheit aus der Verfassung $\quad \ldots \quad 21$

4. Zweiteiliger Privatisierungsbegriff für einen zweiteiligen Staatsbegriff ….................................................. 21

a. Aufgabenprivatisierung ….................................... 22

b. Erfüllungsprivatisierung ........................................ 22

c. Einschub: Schwierige Grenzziehung zwischen privatisierter und nichtprivatisierter Erfüllung ............................. 23

5. Unterschiedliche Privatisierungsbegriffe in

Lehre und Praxis ............................................... 25

a. Echte und unechte, materielle und formelle Privatisierung $\quad \ldots \quad 25$

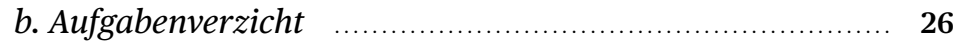

c. Teilprivatisierung …............................................ 28

d. Organisationsprivatisierung …............................... 28

e. Finanzierungsprivatisierung ............................... 29

$f$. Vermögensprivatisierung ….................................. 30

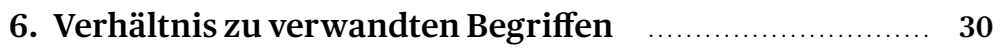

a. Dezentralisierung …….................................... 30

b. Auslagerung …....................................................... 31

c. Deregulierung ….................................................. 31

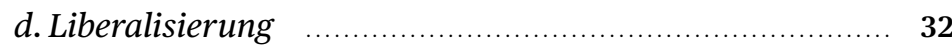

e. New Public Management .................................... 33

7. Zwischenfazit: Privatisierung heisst staatliche Aufgaben oder deren Erfüllung vom Staat zu entfernen $\quad \ldots \ldots \ldots \ldots \ldots . \quad 35$

\$3 Welchen verfassungsrechtlichen Anforderungen unterliegt die Erfüllung staatlicher Aufgaben?

I. Begriff und Bedeutung der verfassungsrechtlichen Anforderungen

1. Anforderungen an staatliches Handeln als Kehrseite des funktionellen Staatsbegriffs

2. Legitimationspflicht des Staats als Geltungsgrund und Funktion der Anforderungen

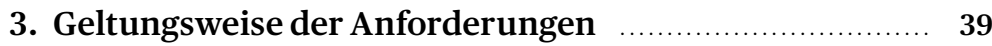

II. Gehalt der verfassungsrechtlichen Anforderungen f...... 41

1. Übersicht und Quellen der Anforderungen $\quad \ldots \ldots \ldots \ldots \ldots \ldots . . . . . .41$ 
2. Gesetzmässigkeit

a. Das Erfordernis einer genügend bestimmten Norm (Normdichte)

b. Das Erfordernis einer Norm genügender Erlassstufe (Normstufe) 46

c. Sonderstellung gegenüber anderen Anforderungen $\quad \ldots \ldots . \quad 46$

d. Geltungsweise

e. Gerichtliche Durchsetzbarkeit

3. Öffentliches Interesse

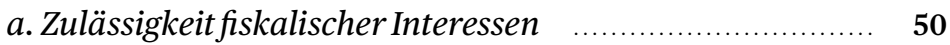

b. Konkretisierung im politischen Prozess $\quad \ldots \ldots \ldots \ldots \ldots \ldots \ldots . . \ldots 51$

c. Geltungsweise ..................................................... 51

d. Gerichtliche Durchsetzbarkeit _............................... 52

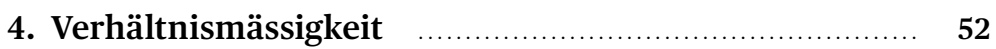

a. Einzelne Postulate …........................................ 53

b. Geltungsweise .................................................... 5

c. Gerichtliche Durchsetzbarkeit ................................. 55

5. Teilgehalte des Grundsatzes von Treu und Glauben $\quad \ldots \quad \mathbf{5 5}$

a. Anforderung an staatliches Handeln trotz Geltung im gesamten Rechtsverkehr? ................................. $\quad 56$

b. Einzelne Teilgehalte, welche den Staat verpflichten $\quad \ldots \ldots . .56$

c. Gerichtliche Durchsetzbarkeit .............................. 58

6. Grundrechtsbindung …................................... 59

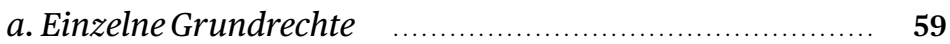

b. Geltungsweise: Grundrechtsbindung bei der Wahrnehmung

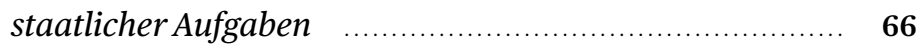

c. Gerichtliche Durchsetzbarkeit ................................ 76

d. Folgen von Grundrechtsverletzungen $\quad \ldots \ldots \ldots \ldots \ldots \ldots \ldots \ldots \ldots$

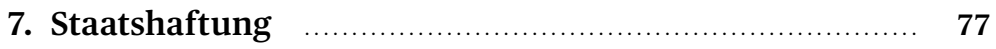

8. Planung und Koordination staatlicher Tätigkeit $\quad \ldots \ldots \ldots . . \quad 78$

III. Was sind keine Anforderungen? ….......................... $\quad 80$

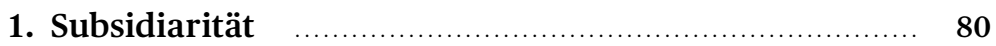

2. Bedarfsgerechtigkeit und Wirtschaftlichkeit $\ldots \ldots \ldots \ldots . . . \quad 81$

3. Grundversorgung …......................................... 82

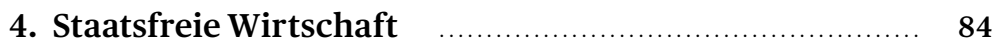

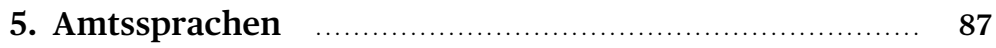

IV. Zwischenfazit: Jede Erfüllung staatlicher Aufgaben ist gleich rechtfertigungspflichtig 
\$4 In welcher Hinsicht kann die Erfüllung staatlicher

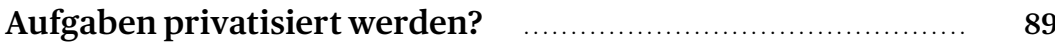

I. Ansatzpunkt: Aspekte der Aufgabenerfüllung …......... 90

1. Unmittelbare Aufgabenerfüllung …................... 90

2. "Nebenaktivitäten»-eine neue Kategorie des Bundesgerichts? .......................................... 93

3. Aufgabenerfüllung aus Sicht der betroffenen Privaten 94

4. Einschub: Abgrenzung von Reformen der wirkungsorientierten Verwaltungsführung $\quad \ldots \ldots \ldots \ldots . . . \quad 95$

II. Privatisierung der Organisationsformen f................. 100

1. Begriff und Bedeutung der Organisationsformen $\quad \ldots . .100$

2. Beschränkte Nützlichkeit von Organisationsmodellen …............................................... 101

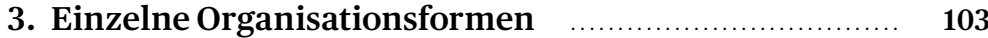

a.Zentralverwaltung _........................................... 103

b. Weisungsfreie Einheiten der Bundesverwaltung $\quad \ldots \ldots \ldots . .104$

c. Öffentlichrechtliche Anstalten _.............................. 104

d. Öffentlichrechtliche Körperschaften ........................ 105

e. Öffentlichrechtliche Genossenschaften $\quad$.................... 105

f. ÖffentlichrechtlicheStiftungen ............................. 106

g. Öffentlichrechtliche Aktiengesellschaften $\quad$................. 106

h. Privatrechtliche Gesellschaften $\quad \ldots \ldots \ldots \ldots \ldots \ldots \ldots \ldots \ldots \ldots \ldots$

4. Kennzeichen der Erfüllungsprivatisierung in

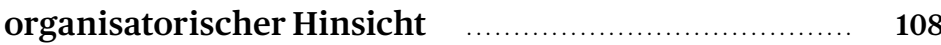

a. Vorab: Die privatisierten Organisationsformen $\quad \ldots \ldots \ldots . .108$

b. Grundlegend: Einzelfallregelung der Rechtsfolgen $\quad . . . . . \quad 109$

c. Eingeschränkte Aufsichtsmittel _........................... 110

d. Periodische Steuerung statt ständiger Leitung $\quad \ldots \ldots \ldots \ldots . . .112$

5. Zwischenfazit: Grössere Spielräume und nur

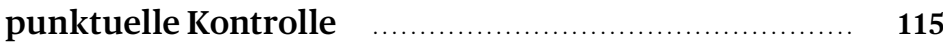

III. Privatisierung der Handlungsformen $\ldots \ldots \ldots \ldots \ldots \ldots \ldots \ldots \ldots$

1. Begriff und Bedeutung der Handlungsformen $\quad \ldots \ldots \ldots \ldots . \quad 116$

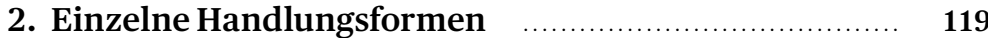

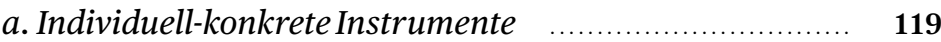

b. Generell-abstrakte Instrumente …......................... 128

c. Andere sachgesetzliche Instrumente, insbesondere Pläne 134 
3. Kennzeichen der Erfüllungsprivatisierung in instrumenteller Hinsicht

a. Vorab: Die privatisierten Handlungsformen $\quad$............. 137

b. Gestaltungsfreiheit im Einzelfall ............................. 137

c. Vermeintliche Aufgabe von Machtvorsprung $\quad$............ 139

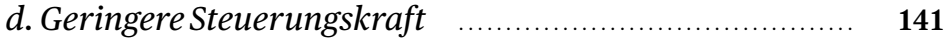

e. Gesteigerte Wirkungserwartung durch höhere Akzeptanz und freiwillige Befolgung …................................. 142

f. Inkaufnahme von Interessenkonflikten …............... 143

g. Einbindung von externem Fachwissen $\quad . . \ldots \ldots \ldots \ldots \ldots \ldots \ldots . . . . . . .144$

h. Erschwerte Abgrenzung zwischen staatlicher Aufgabenerfüllung und privater Tätigkeit $\quad$................ $\quad 145$

i. Ziviles Verfahrensrecht bei zivilrechtlichen Verträgen $\quad \ldots \quad \mathbf{1 4 5}$

4. Zwischenfazit: Die Verwaltung verzichtet auf hoheitliche Vorrechte und hofft auf dankbare Verwaltungsadressaten

IV. Privatisierung der Finanzierungsformen $\ldots \ldots \ldots \ldots \ldots \ldots \ldots . \ldots \ldots$

1. Begriff und Bedeutung der Finanzierungsformen $\quad \ldots \quad 147$

2. Das System der öffentlichen Abgaben $\quad . . \ldots \ldots \ldots \ldots \ldots \ldots \ldots \ldots . . \ldots 149$

3. Einzelne Finanzierungsformen …....................... 152

a. Verwaltungsgebühren und Benutzungsgebühren $\quad \ldots \ldots . . .152$

b. Abgaben zur Kostenanlastung zwischen Steuern und

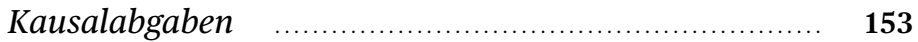

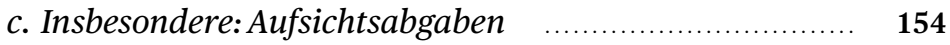

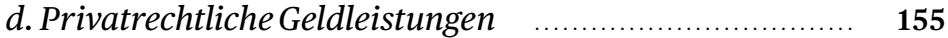

e. Quersubventionierung aus privatwirtschaftlicher Tätigkeit

4. Kennzeichen der Erfüllungsprivatisierung in

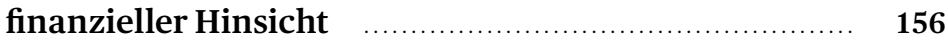

a. Vorab:Die privatisierten Finanzierungsformen $\quad \ldots \ldots \ldots . .157$

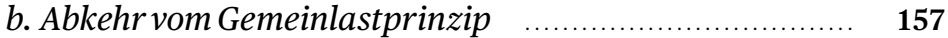

c. Aufweichung des Verursacherbegriffs ..................... 158

d. GelockerteSteuerung durch das Parlament $\quad$............... 159

e. Anreize gegen die Verursachung staatlicher Tätigkeit $\quad \ldots \quad 159$

f. Anreiz zur Monetarisierungjedes Bestandteils des

Verwaltungshandelns ................................... $\quad 160$

g. Interessenkonflikte aus gegenseitiger Abhängigkeit $\quad \ldots . . \quad 160$

5. Zwischenfazit: Staatsaufgaben werden zur wirtschaftlichen Verhandlungsmasse 
V. Zwischenfazit: Erfüllungsprivatisierung gibt staatlichem Handeln den Anstrich privater Flexibilität

\$5 Welche Probleme schaffen die verfassungsrechtlichen Anforderungen für die Erfüllungsprivatisierung?

I. Probleme aus der Gesetzmässigkeit 165

1. Insbesondere bei den privatisierten Organisationsformen 166

2. Insbesondere bei den privatisierten Handlungsformen 167

a. Dogmatisch ungefestigte Sui-generis-Instrumente 167

b. Verminderte Steuerungskraft trifft auf zwingendes Recht 167

c. Insbesondere: Eingeschränkte Anpassbarkeit an veränderte Rechtslage 168

d. Problem aus Normdichte bei ausgelagerter Normsetzung

3. Insbesondere bei den privatisierten Finanzierungsformen

II. Probleme aus dem öffentlichen Interesse

1. Insbesondere bei den privatisierten Organisationsformen

a. Spannung aus Zweckbindung wegen eingeschränkter Aufsicht und weniger enger Kontrolle

b. Spannung aus Renditezielen 173

c. Kein grundsätzliches Problem aus der «Kohärenz des staatlichen Handelns»

2. Insbesondere bei den privatisierten Handlungsformen

a. Grundsätzliche Spannung zwischen Gestaltungsfreiheit und Zweckbindung

b. Einbezug von Fachexperten kann Sondergruppeninteressen über öffentliche Interessen stellen

c. Nurvermeintliche Spannung zwischen privatem Auftreten und Gemeinwohl

3. Insbesondere bei den privatisierten

Finanzierungsformen

a. Kein grundsätzliches Problem aus Abkehrvon Gemeinlastprinzip

b. Spannung aus verschobener Optik von Verwaltungsadressaten zu Kunden 
III. Probleme aus der Verhältnismässigkeit

1. Keine besonderen Probleme bei der Privatisierung der organisatorischen Erfüllungsaspekte

2. Insbesondere bei den privatisierten Handlungsformen

a. Quasiprivates Auftreten kommt tendenziell privaten Interessen zugute

b. Problem aus neuen Formen staatlicher Beeinflussung

3. Insbesondere bei den privatisierten

Finanzierungsformen

IV. Probleme aus den Teilgehalten des Grundsatzes von Treu und Glauben

1. Keine besonderen Probleme bei der Privatisierung der organisatorischen Erfüllungsaspekte

2. Insbesondere bei den privatisierten Handlungsformen

a. Potentielle Probleme aus erschwerter Erkennbarkeit staatlicher Instrumente

b. Probleme aus dem stärkeren Vertrauen in Beständigkeit von Verträgen

3. Insbesondere bei der Privatisierung der finanziellen Erfüllungsapekte

V. Probleme aus der Grundrechtsbindung

1. Insbesondere bei den privatisierten

Organisationsformen

a. Strapazierte Dogmatik zur Grundrechtsbindung bei Organisationen mit gemischten Aufgaben

b. Spannungen aus Renditezielen und Quersubventionierungen

c. Probleme bezüglich Durchsetzung der Grundrechtsbindung wegen gelockerter Aufsicht

2. Insbesondere bei den privatisierten Handlungsformen

3. Insbesondere bei den privatisierten

Finanzierungsformen

a. Gefährdete Rechtsgleichheit bei der Kostenanlastung nach aufgeweichter Verursacherfinanzierung

b. Abkehrvom Gemeinlastprinzip kann Grundrechtsansprüchevereiteln 
VI. Querschnittsprobleme bei der Einhaltung der verfassungsrechtlichen Anforderungen

1. Erschwerte Erkennbarkeit des Staats gefährdet die Anrufung der verfassungsrechtlichen Anforderungen $\quad 197$

2. Rechtsschutzdefizite des zivilen Verfahrensrechts

3. Die Einhaltung der verfassungsrechtlichen Anforderungen bedingt zusätzlichen Aufsichts- und Kontrollaufwand

VII. Zwischenfazit: Erfüllungsprivatisierung stellt Freiräume über gesetzliche Leistungspflichten und Zweckbindung

\$6 Welche Spielräume belassen die verfassungsrechtlichen Anforderungen der Erfüllungsprivatisierung? 205

I. Allgemein: Spielräume ausserhalb der verfassungsrechtlichen Anforderungen 206

II. Spielräume aus der Gesetzmässigkeit 208

1. Insbesondere bei den privatisierten Organisationsformen 209

a. Zulässigkeit von Einzelfallregelungen als Spielraum $\quad \ldots . .209$

b. Eingeschränkte Aufsicht als Spielraum .................... 209

c. Unbestimmte Aufgabenübertragungen dank Spezialitätsprinzip

2. Insbesondere bei den privatisierten Handlungsformen

3. Insbesondere bei den privatisierten

Finanzierungsformen

a. Das aufgeweichte Verursacherprinzip weitet die Spielräume des Äquivalenzprinzips aus

b. Marktpreise ersetzen gesetzliche Bemessungsgrundsätze

III. Spielräume aus dem öffentlichen Interesse 215

1. Insbesondere bei den privatisierten Organisationsformen

2. Insbesondere bei den privatisierten Handlungsformen 216

3. Insbesondere bei den privatisierten Finanzierungsformen 
IV. Spielräume aus der Verhältnismässigkeit

1. Allgemein: Zurückhaltende Ausübung der gerichtlichen Kognition

2. Insbesondere bei den privatisierten Handlungsformen

a. Einbezug von Fachwissen garantiert die Berücksichtigung privater Interessen

b. Einvernehmliche Handlungsformen garantieren Zumutbarkeit

3. Insbesondere bei den privatisierten

Finanzierungsformen

a. Äquivalenzprinzip als Kompensat für mangelnde Normdichte schafft Spielraum

b. Höhergewichtung privater Interessen dank finanzieller Abhängigkeit des Aufgabenträgers

V. Spielräume aus den Teilgehalten des Grundsatzes von Treu und Glauben

VI. Spielräume aus der Grundrechtsbindung

1. Allgemein: Eingeschränkte Grundrechtsgeltung bei privatisierter Aufgabenerfüllung?

a. Lehre hält Beschränkung aufrechtsstaatliche Garantien für grundsätzlich zulässig

b. Doch Beschränkung auf Art. 8 und 9 BV reflektiert bloss typische Gefährdungslage

c. Ergebnis: Eingeschränkte Geltung nur auf ausdrückliche Anordnung des Gesetzgebers hin

2. Insbesondere bei den privatisierten

Organisationsformen

a. Herabgesetzte Forderung einer "protection juridique minimum»

b. Unterschiedliche Aufsicht über privatisierte Aufgabenträger als Spielraum bei der Rechtsgleichheit

c. Grundrechtsträgerschaft ausserhalb des staatlichen Aufgabenbereichs beigemischten Organisationen

3. Insbesondere bei den privatisierten Handlungsformen

VII. Zwischenfazit: Erfüllungsprivatisierung nutzt Einzelfallregelungen und Freiwilligkeit 
I. Die Erfüllungsprivatisierung im Kontext eines funktionellen Staatsbegriffs

II. Aufgabenfremde Ziele und Anreize der Erfüllungsprivatisierung

III. Einzelfallregelungen als Mehraufwand und Garant für Freiräume zugleich

IV. Einzelne Probleme bei der privatisierten Erfüllung staatlicher Aufgaben

V. Einzelne Spielräume bei der privatisierten Erfüllung staatlicher Aufgaben

VI. Empfehlungen

1. Probleme und Spannungen als Checkliste für Privatisierungsvorhaben 237

2. Detaillierte Aufgabenzuweisung im Gesetz 239

3. Insbesondere bezüglich der Organisationsformen 239

4. Insbesondere bezüglich der Handlungsformen 240

5. Insbesondere bezüglich der Finanzierungsformen $\quad \ldots \quad 240$

VII. Schlusswort 


\section{Literatur}

Das Verzeichnis nennt alle im Text zitierten Werke. Sie werden jeweils mit Nachnamen und Kurztitel zitiert.

ABEGG ANDREAS, Der Verwaltungsvertrag zwischen Staatsverwaltung und Privaten. Grundzüge einer historisch und theoretisch angeleiteten Dogmatik öffentlichrechtlicher und privatrechtlicher Verwaltungsverträge, Zürich 2009 (zit. Verwaltungsvertrag).

ABEGG ANDREAS/FREI MARCO, Können sich öffentliche Unternehmen auf die Wirtschaftsfreiheit berufen?, in: recht 2017, S. 290-299 (zit. Wirtschaftsfreiheit).

ACHERMANN URS PHILIPP, Privatisierung im öffentlichen Verkehr. Voraussetzungen der Privatisierung und Steuerung des privatisierten Vollzugs am Beispiel des öffentlichen Verkehrs, Zürich/Basel/Genf 2008 (zit. Privatisierung).

ADLER DENIS OLIVER/TÖNZ STEFAN, Die Verwendung von Verwaltungsvermögen im Rahmen einer Privatisierung. Unter besonderer Berücksichtigung der Wasserversorgungsanlagen im Kanton Zürich, in: ZBl 2017, S. 355-376 (zit. Privatisierung).

AMSTUTZ MARC/ABEGG ANDREAS/KARAVAS VAIOS (Hrsg.), Soziales Vertragsrecht. Eine rechtsevolutorische Studie, Basel 2006 (zit. Soziales Vertragsrecht).

ARNET RUTH, Bemerkungen zu BGE 129 III 35, in: AJP 2003, S. 593-597 (zit. Bemerkungen zum Post-Urteil).

AUBERT JEAN-FRANÇOIS/MAHON PASCAL (Hrsg.), Petit commentaire de la Constitution fédérale de la Confédération suisse, Zürich/Basel/Genf 2003 (zit. Petit commentaire).

BAUER HARTMUT, Privatisierung von Verwaltungsaufgaben, in: VVDStRL 1995, S. 243-286 (zit. Privatisierung).

\section{BIAGGINI GIOVANNI}

- Rechtsstaatliche Anforderungen an die Auslagerung und an den ausgelagerten Vollzug staatlicher Aufgaben sowie Rechtsschutz, in: René Schaffhauser et al. (Hrsg.), Auslagerung und Privatisierung von staatlichen und 
kommunalen Einheiten: Rechtsformen und ihre Folgen, Referate der Tagung vom 14. November 2001 in Luzern, St. Gallen 2002, S. 143-175 (zit. Rechtsstaatliche Anforderungen).

- Entwicklungen und Spannungen im Verfassungsrecht. Versuch einer Standortbestimmung zehn Jahre nach Inkrafttreten der Bundesverfassung vom 18. April 1999, in: ZBl 2010, S.1-41 (zit. Entwicklungen im Verfassungsrecht).

- Bemerkungen zu BGE 138 I 378, in: ZBl 2012, S. 665-674 (zit. Bemerkungen zum Glarnersach-Urteil).

- Die polizeiliche Generalklausel: ein verkanntes Rechtsinstitut. Bemerkungen aus Anlass des Urteils des Bundesgerichts vom 15. Juli 2011 in Sachen FINMA/UBS (2C_127/2010), in: ZBl 2012, S.35-45 (zit. Polizeiliche Generalklausel).

- Bemerkungen zu BGE 138 I 274, in: ZBl 2013, S. 176-180 (zit. Bemerkungen zum SBB-Plakatfall).

- Art.187, in: Bernhard Ehrenzeller et al. (Hrsg.), Die schweizerische Bundesverfassung, Zürich/St. Gallen 2014, S. 2994-3006 (zit. St. Galler Kommentar).

- Art. 43a, in: Bernhard Waldmann et al. (Hrsg.), Bundesverfassung, Basel 2015, S. 846-857 (zit. Basler Kommentar).

- Art. 5a, in: Bernhard Waldmann et al. (Hrsg.), Bundesverfassung, Basel 2015, S. 133-143 (zit. Basler Kommentar).

- Was die Verfassung leisten kann und soll (und was nicht) - am Beispiel «Grundversorgung», in: ZBl 2015, S. 113-114 (zit. Grundversorgung).

- BV Kommentar. Bundesverfassung der Schweizerischen Eidgenossenschaft, 2. Aufl., Zürich 2017 (zit. Kommentar BV).

BIGLER-EGGENBERGER MARGRITH/KÄGI-DIENER REGULA, Art. 8, in: Bernhard Ehrenzeller et al. (Hrsg.), Die schweizerische Bundesverfassung, Zürich/St. Gallen 2014, S. 236-256 (zit. St. Galler Kommentar).

BÖCKENFÖRDE ERNST-WOLFGANG, Die politische Funktion wirtschaftlichsozialer Verbände und Interessenträger in der sozialstaatlichen Demokratie, in: Wilhelm Hennis et al. (Hrsg.), Regierbarkeit, Stuttgart 1977 (zit. Sozialstaatliche Demokratie).

BOGUMIL JÖRG/JANN WERNER, Verwaltung und Verwaltungswissenschaft in Deutschland. Einführung in die Verwaltungswissenschaft, 2. Aufl., Wiesbaden 2009 (zit. Verwaltungswissenschaft). 
BOVET CHRISTIAN/HÉRITIER LACHAT ANNE, Intermédiares financiers, in: Christian Bovet (Hrsg.), Finanzmarktaufsicht, Basel 2016, S. 53-285 (zit. Intermédiares financiers).

BÜHLER CHRISTOPH B., Eigenheiten der Corporate Governance von Aktiengesellschaften mit staatlicher Beteiligung, in: SJZ 2011, S. 513-521 (zit. Corporate Governance).

BUNDESAMT FÜR JUSTIZ (BJ), Erfordernis der gesetzlichen Grundlage im Bereich der Leistungsverwaltung. Bedarfsverwaltung, administrative Hilfstätigkeit und «schlichtes Verwaltungshandeln», in: VPB 1996, Nr.1 (zit. Leistungsverwaltung).

BÜREN LUCIE VON, Akkreditierte Zertifizierung im gesetzlich geregelten Bereich. Systeme, Einordnung und Rechtsschutz, Bern 2013 (zit. Akkreditierte Zertifizierung).

DAENIKER DANIEL, Überführung staatlicher Aufgaben auf verwaltungsexterne Rechtsträger, in: Tobias Jaag (Hrsg.), Dezentralisierung und Privatisierung öffentlicher Aufgaben, Zürich 2000, S. 49-66 (zit. Überführung staatlicher Aufgaben).

DOLDE KLAUS-PETER, Verwaltungsverfahren und Deregulierung, in: NVwZ 2006, S. 857-865 (zit. Verwaltungsverfahren und Deregulierung).

DUBEY JACQUES/ZUFFEREY JEAN-BAPTISTE, Droit administratif général, Basel 2014 (zit. Droit administratif général).

EGLI PATRICIA, Mehr Staat durch Beleihung Privater?, in: Eva Maria Belser et al. (Hrsg.), Mehr oder weniger Staat?, Bern 2015, S. 193-205 (zit. Beleihung Privater).

EHRENZELLER BERNHARD, Art.180, in: Bernhard Ehrenzeller et al. (Hrsg.), Die schweizerische Bundesverfassung, Zürich/St. Gallen 2014, S. 2899-2906 (zit. St. Galler Kommentar).

EHRENZELLER BERNHARD/SCHINDLER BENJAMIN/SCHWEIZER RAINER J./ VALLENDER KLAUS A. (Hrsg.), Die schweizerische Bundesverfassung, 3. Aufl., Zürich/St. Gallen 2014 (zit. St. Galler Kommentar).

EICHENBERGER KURT

- Der geforderte Staat: Zur Problematik der Staatsaufgaben, in: Wilhelm Hennis et al. (Hrsg.), Regierbarkeit, Stuttgart 1977, S.103-117 (zit. Staatsaufgaben). 
- Leistungsstaat und Demokratie, in: Verfassungsrat und Regierungsrat des Kantons Aargau (Hrsg.), Der Staat der Gegenwart, Basel 1980, S. 56-72 (zit. Leistungsstaat).

ELSER DOMINIK, Der funktionelle Staatsbegriff und das verkannte Merkmal staatlichen Handelns, in: Lisa Heschl et al. (Hrsg.), L'État, c'est quoi? Staatsgewalt im Wandel, Baden-Baden 2015, S. 59-78 (zit. Funktioneller Staatsbegriff).

EPINEY ASTRID, Art. 5, in: Bernhard Waldmann et al. (Hrsg.), Bundesverfassung, Basel 2015, S. 85-133 (zit. Basler Kommentar).

ERRASS CHRISTOPH, Technikregulierungen zur Gewährleistung von Sicherheit, in: S\&R 2016, S. 63-89 (zit. Technikregulierungen).

FLÜCKIGER ALEXANDRE, Régulation, dérégulation, autorégulation: l'émergence des actes étatiques non obligatoires, in: ZSR 2004/II, S. 159-303 (zit. Actes non obligatoires).

FREUDIGER PATRICK, Anstalt oder Aktiengesellschaft? Zur Bedeutung der Rechtsform bei Ausgliederungen, Bern 2016 (zit. Anstalt oder Aktiengesellschaft?).

FREY RENÉ L.

- Die drei Stossrichtungen der Privatisierung, in: Hans Schmid et al. (Hrsg.), Finanz- und Wirtschaftspolitik in Theorie und Praxis, Bern 1997, S. 339-359 (zit. Drei Stossrichtungen).

- Privatisierung und Liberalisierung sind weiterhin nötig. Marktbefürworter haben nach der Krise einen schweren Stand gegen Verheissungen von besserer Regulierung und Service public, in: NZZ vom 18. August 2010, S. 25 (zit. Privatisierung und Liberalisierung).

FRICK MARTIN, Das Verursacherprinzip in Verfassung und Gesetz, Bern 2004 (zit. Verursacherprinzip).

GÄCHTER THOMAS, Schnell, schwammig, schlecht. Zugespitztes zu Art. 43a und Art. 43b ATSG, in: HAVE 2018, S. 216-221 (zit. Schnell, schwammig, schlecht).

GÄCHTER THOMAS/WERDER GREGORI, Art. 41, in: Bernhard Waldmann et al. (Hrsg.), Bundesverfassung, Basel 2015, S. 807-826 (zit. Basler Kommentar).

GAMMA MARCO, Die Kontrolle des ruhenden Verkehrs durch Private, in: AJP 1999, S. 1233-1240 (zit. Kontrolle). 
GAUCH PETER, Ein Vorwort zum «Sozialen Vertragsrecht» von Peter Gauch, in: Marc Amstutz et al. (Hrsg.), Soziales Vertragsrecht, Basel 2006, S. 1-11 (zit. Soziales Vertragsrecht).

GILI NATASSIA, Staatlich gesteuerte Selbstregulierung. Am Beispiel der medizinisch-ethischen Richtlinien der Schweizerischen Akademie der Medizinischen Wissenschaften (SAMW), Zürich/Basel/Genf 2017 (zit. Selbstregulierung).

HAEFLIGER ARTHUR, Alle Schweizer sind vor dem Gesetze gleich. Zur Tragweite des Artikels 4 der Bundesverfassung, Bern 1985 (zit. Alle Schweizer).

HÄFELIN ULRICH/HALLER WALTER/KELLER HELEN/THURNHERR DANIELA, Schweizerisches Bundesstaatsrecht, 9. Aufl., Zürich/Basel/Genf 2016 (zit. Schweizerisches Bundesstaatsrecht).

HÄFELIN ULRICH/MÜLLER GEORG/UHLMANN FELIX, Allgemeines Verwaltungsrecht, 7. Aufl., Zürich/St. Gallen 2016 (zit. Allgemeines Verwaltungsrecht).

HALLER WALTER/KÖLZ ALFRED/GÄCHTER THOMAS, Allgemeines Staatsrecht. Eine juristische Einführung in die Allgemeine Staatslehre, 5. Aufl., Zürich/ Basel/Genf 2013 (zit. Allgemeines Staatsrecht).

HANDSCHIN LUKAS/SIEGENTHALER THOMAS, Privatisierung öffentlicher Aufgaben, in: SJZ 2000, S. 405-414 (zit. Privatisierung).

HÄNER ISABELLE

- Privatisierung staatlicher Ausgaben (Finanzierungsprivatisierung) unter verfassungsrechtlichen Aspekten, in: ZBl 2001, S. 423-443 (zit. Privatisierung).

- Die Einwilligung der betroffenen Person als Surrogat der gesetzlichen Grundlage bei individuell-konkreten Staatshandlungen, in: ZBl 2002, S. 57-76 (zit. Einwilligung).

- Grundrechtsgeltung bei der Wahrnehmung staatlicher Aufgaben durch Private, in: AJP 2002, S.1144-1153 (zit. Grundrechtsgeltung).

- Art. 25a, in: Bernhard Waldmann et al. (Hrsg.), Praxiskommentar Verwaltungsverfahrensgesetz (VwVG), Zürich 2016, S. 521-540 (zit. Praxiskommentar VwVG).

HANGARTNER YVO

- Verwaltung durch Private, in: Verwaltungs-Praxis 1982, S. 4-6 (zit. Verwaltung durch Private). 
- Öffentlich-rechtliche Bindungen privatrechtlicher Tätigkeit des Gemeinwesens, in: Ernst Brem et al. (Hrsg.), Festschrift zum 65. Geburtstag von Mario M. Pedrazzini, Bern 1990, S. 129-158 (zit. Öffentlich-rechtliche Bindungen).

- Grundrechtsbindung öffentlicher Unternehmen. Bemerkungen aus Anlass der Auseinandersetzung zwischen einer Bürgerbewegung und der Post, in: AJP 2000, S. 515-520 (zit. Grundrechtsbindung).

- Bemerkungen zu BGE 129 III 35, in: AJP 2003, S. 690-693 (zit. Bemerkungen zum Post-Urteil).

HÄSLER PHILIPP, Geltung der Grundrechte für öffentliche Unternehmen, Bern 2005 (zit. Geltung der Grundrechte).

HEINEMANN ANDREAS, Recht, Ökonomie und Realität, in: Robert Waldburger et al. (Hrsg.), Law \& Economics, Bern 2015, S. 21-41 (zit. Recht, Ökonomie und Realität).

HERMES GEORG, Staatliche Infrastrukturverantwortung. Rechtliche Grundstrukturen netzgebundener Transport- und Übertragungssysteme zwischen Daseinsvorsorge und Wettbewerbsregulierung am Beispiel der leitungsgebundenen Energieversorgung in Europa, Tübingen 1998 (zit. Staatliche Infrastrukturverantwortung).

HETTICH PETER

- Bemerkungen zu BGE 138 I 378, in: AJP 2012, S. 1467-1471 (zit. Bemerkungen zu BGE 138 I 378).

- Kooperative Risikovorsorge. Regulierte Selbstregulierung im Recht der operationellen und technischen Risiken, Zürich, Basel, Genf 2014 (zit. Kooperative Risikovorsorge).

HOFSTETTER DAVID, Das Verhältnismässigkeitsprinzip als Grundsatz rechtsstaatlichen Handelns (Art. 5 Abs. 2 BV). Ausgewählte Aspekte, Zürich/ Basel/Genf 2014 (zit. Verhältnismässigkeitsprinzip).

HUSMANN MARKUS

- Demokratiefeindliche Polizeikostenüberwälzung. Grenzen und Gefahren der Haftbarkeit des Störers, in: S\&R 2015, S.143-156 (zit. Demokratiefeindliche Polizeikostenüberwälzung).

- Überwälzung von Polizeikosten bei Demonstrationen. Ein Update nach BGE 143 I 147 unter besonderer Berücksichtigung der Situation im Kanton Luzern, in: S\&R 2018, S. 72-82 (zit. Update Polizeikosten). 
IMBODEN MAX, Der verwaltungsrechtliche Vertrag, in: ZSR 1958/II, S. 1a-218a (zit. Verwaltungsrechtlicher Vertrag).

\section{JAAG TOBIAS}

- Privatisierung von Verwaltungsaufgaben. Länderbericht Schweiz, in: VVDStRL 1995, S. 287-300 (zit. Privatisierung Länderbericht Schweiz).

- Dezentralisierung und Privatisierung öffentlicher Aufgaben: Formen, Voraussetzungen und Rahmenbedingungen, in: TobiasJaag(Hrsg.), Dezentralisierung und Privatisierung öffentlicher Aufgaben. Referate einer Tagung vom 29. September 1999, Zürich 2000, S. 23-48 (zit. Dezentralisierung und Privatisierung).

- Staats- und Beamtenhaftung. Organisationsrecht, Bd. I/3, 3. Aufl., Basel 2017 (zit. Staats- und Beamtenhaftung).

JAAG TOBIAS/HÄNNI JULIA, Art.146, in: Bernhard Ehrenzeller et al. (Hrsg.), Die schweizerische Bundesverfassung, Zürich/St. Gallen 2014, S. 2573-2583 (zit. St. Galler Kommentar).

KÄGI-DIENER REGULA, Art. 70, in: Bernhard Ehrenzeller et al. (Hrsg.), Die schweizerische Bundesverfassung, Zürich/St. Gallen 2014, S.1442-1460 (zit.St. Galler Kommentar).

KÄLIN WALTER, Die staatsrechtliche Rechtsprechung des Bundesgerichts in den Jahren 2003 und 2004, in: ZBJV 2004, S. 633-696 (zit. Staatsrechtliche Rechtsprechung 2003/2004).

KÄLIN WALTER/LIENHARD ANDREAS/WYTTENBACH JUDITH, Auslagerung von sicherheitspolizeilichen Aufgaben, Basel 2007 (zit. Auslagerung).

KIENER REGINA/KÄLIN WALTER/WYTTENBACH JUDITH, Grundrechte, 3. Aufl., Bern 2018 (zit. Grundrechte).

KÖLZ ALFRED/HÄNER ISABELLE/BERTSCHI MARTIN, Verwaltungsverfahren und Verwaltungsrechtspflege des Bundes, 3. Aufl., Zürich 2013 (zit. Verwaltungsverfahren und Verwaltungsrechtspflege des Bundes).

KRAEMER RAPHAEL/STÖCKLI ANDREAS, Grenzenlose Staatswirtschaft? Bemerkungen zum Bundesgerichtsurteil «Glarnersach» vom 3. Juli 2012, in: recht 2013, S. 28-45 (zit. Grenzenlose Staatswirtschaft?).

KRÄHENMANN BEAT, Privatwirtschaftliche Tätigkeit des Gemeinwesens, Basel 1987 (zit. Privatwirtschaftliche Tätigkeit). 
KRUCK ANDREAS, Privatisierung von Regieren und die Dynamiken und Grenzen der rechtlichen Kontrolle privater Autorität(en), in: Kritische Justiz 2016, S. 439-452 (zit. Privatisierung).

KÜHNI OLIVIA, Weder staatlicher Fisch noch Vogel AG, in: Republik vom 17. April 2018 (zit. Weder staatlicher Fisch noch Vogel AG).

KUSTER MATTHIAS, Zur Rechtsnatur der Sanktionsentscheide von Selbstregulierungsorganisationen und der Schweizer Börse, in: AJP 2005, S.1502-1508 (zit. Selbstregulierungsorganisationen).

\section{LIENHARD ANDREAS}

- Deregulierung - Leitmotiv im Wirtschaftsverwaltungsrecht? Mit aktuellen Tendenzen und Potentialen im Bund und im Kanton Bern, Bern 1995 (zit. Deregulierung).

- Organisation und Steuerung der ausgelagerten Aufgabenerfüllung, in: AJP 2002, S. 1163-1174 (zit. Ausgelagerte Aufgabenerfüllung).

- Staats- und verwaltungsrechtliche Grundlagen für das New Public Management in der Schweiz. Analysen - Anforderungen - Impulse, Bern 2005 (zit. Grundlagen NPM).

- Grundlagen der Public Corporate Governance, in: Schweizerische Vereinigung für Verwaltungsorganisationsrecht SVVOR, Association suisse du droit public de l'organisation (Hrsg.), Verwaltungsorganisationsrecht Staatshaftungsrecht - öffentliches Dienstrecht, Bern 2009, S. $43-77$ (zit. Corporate Governance).

- Das Neue Führungsmodell für die Bundesverwaltung (NFB), in: Eva Maria Belser et al. (Hrsg.), Mehr oder weniger Staat?, Bern 2015, S. 247-261 (zit. Neues Führungsmodell für die Bundesverwaltung).

\section{MÄCHLER AUGUST}

- Rechtsfragen um die Finanzierung privater Träger öffentlicher Aufgaben, in: AJP 2002, S. 1175-1185 (zit. Finanzierung privater Träger).

- Bemerkungen zu BGE129 III 35, in: ZBl 2003, S.365-376 (zit. Bemerkungen zum Post-Urteil).

- Vertrag und Verwaltungsrechtspflege. Ausgewählte Fragen zum vertraglichen Handeln der Verwaltung und zum Einsatz des Vertrages in der Verwaltungsrechtspflege, Zürich 2005 (zit. Vertrag und Verwaltungsrechtspflege).

MAHON PASCAL, Remarques liminaires Art. 7, in: Jean-François Aubert et al. (Hrsg.), Petit commentaire de la Constitution fédérale de la Confédération suisse, Zürich/Basel/Genf 2003, S. 59-66 (zit. Petit commentaire). 
MARTENET VINCENT, La protection contre les discriminations émanant de particuliers, in: ZSR 2006/I, S. 419-458 (zit. Discriminations émanant de particuliers).

MARTI ARNOLD

- Aufgabenteilung zwischen Staat und Privaten auf dem Gebiet der Rechtsetzung - Ende des staatlichen Rechtsetzungsmonopols?, in: AJP 2002, S.1154-1162 (zit. Aufgabenteilung).

- Die Kosten im heutigen Zivilprozess. Was bleibt vom Grundsatz der wohlfeilen Rechtspflege, in: Anwaltsrevue 2018, S. 116-123 (zit. Kosten im Zivilprozess).

MASTRONARDI PHILIPPE, Gewaltenteilung unter NPM. Zum Verhältnis von Parlament und Regierung, in: ZBl 1999, S. 449-464 (zit. Gewaltenteilung unter NPM).

MAURER HARTMUT/WALDHOFF CHRISTIAN, Allgemeines Verwaltungsrecht, 19. Aufl., München 2017 (zit. Allgemeines Verwaltungsrecht).

MOOR PIERRE/BELLANGER FRANÇOIS/TANQUEREL THIERRY, Droit administratif. Volume III : L'organisation des activités administratives. Les biens de l'Etat, 2. Aufl., Bern 2018 (zit. Droit administratif III).

MOOR PIERRE/FLÜCKIGER ALEXANDRE/MARTENET VINCENT, Droit administratif. Volume I: Les fondements, 3. Aufl., Bern 2012 (zit. Droit administratifI).

MORELL RETO/VALLENDER KLAUS A., Art. 74, in: Bernhard Ehrenzeller et al. (Hrsg.), Die schweizerische Bundesverfassung, Zürich/St. Gallen 2014, S. 1503-1517 (zit. St. Galler Kommentar).

MOSIMANN HANS-JAKOB, New Public Management zwischen theoretischem Programm und praktischer Umsetzung, in: ZBl 2000, S. 337-355 (zit. New Public Management).

MÜLLER ANDREAS/JENNI VITTORIO, Rahmenbedingungen bei der Privatisierung kommunaler Aufgaben - eine aktuelle Übersicht, in: AJP (1999), S. 1071-1082 (zit. Privatisierung).

MÜLLER GEORG

- Art. 4, in: Kommentar zur Bundesverfassung der Schweizerischen Eidgenossenschaft, Basel/Zürich/Bern 1997 ff., S. 1-80 (zit. Kommentar aBV).

- Wie wird ein Staat schlank?, in: Solothurnischer Juristenverein (Hrsg.), Solothurner Festgabe zum Schweizerischen Juristentag 1998, Solothurn 1998, S. 159-173 (zit. Schlank). 
- Zulässigkeit des Vertrages und zulässige Vertragsinhalte, in: Isabelle Häner (Hrsg.), Der verwaltungsrechtliche Vertrag in der Praxis, Zürich/Basel/ Genf 2007, S. 25-37 (zit. Zulässigkeit des Vertrags).

MÜLLER JÖRG PAUL

- \$39 Allgemeine Bemerkungen zu den Grundrechten, in: Daniel Thürer et al. (Hrsg.), Verfassungsrecht der Schweiz, Zürich 2001, S. 621-645 (zit. Allgemeine Bemerkungen).

- \$202 Geschichtliche Grundlagen, Zielsetzung und Funktionen der Grundrechte, in: Detlef Merten et al. (Hrsg.), Grundrechte in der Schweiz und in Liechtenstein, Heidelberg, Zürich/St. Gallen 2007, S. 3-30 (zit. Grundlagen der Grundrechte).

\section{MÜLLER MARKUS}

- Rechtsschutz im Bereich des informalen Staatshandelns. Überlegungen am Beispiel der staatlichen Empfehlungen, in: ZBl 1995, S. 533-556 (zit. Informales Staatshandeln).

- Die Rechtsweggarantie - Chancen und Risiken. Ein Plädoyer für mehr Vertrauen in die öffentliche Verwaltung, in: ZBJV 2004, S. 161-197 (zit. Rechtsweggarantie).

- Verwaltungsrecht. Eigenheit und Herkunft, Bern 2006 (zit. Verwaltungsrecht).

- Art. 5, in: Christoph Auer et al. (Hrsg.), Kommentar zum Bundesgesetz über das Verwaltungsverfahren (VwVG), Zürich/St. Gallen 2008, S. 63-100 (zit. Kommentar VwVG).

- Verhältnismässigkeit. Gedanken zu einem Zauberwürfel, Bern 2013 (zit. Verhältnismässigkeit).

- Art. 187, in: Bernhard Waldmann et al. (Hrsg.), Bundesverfassung, Basel 2015, S. 2721-2732 (zit. Basler Kommentar).

MÜLLER-GRAF THOMAS, Entrechtlichung durch Informalisierung? Ein Beitrag zur Handlungsformen- und zur Rechtsverhältnislehre im Verwaltungsrecht, Basel 2001 (zit. Informalisierung).

OSTERLOH LERKE, Privatisierung von Verwaltungsaufgaben, in: VVDStRL 1995, S. 204-242 (zit. Privatisierung).

PETERS B. GUY, The Failure of Managerial Reform in a Managerial Society: Public Sector Reform in the United States, in: Joachim Jens Hesse et al. (Hrsg.), Paradoxes in Public Sector Reform, Berlin 2003, S. 25-52 (zit. Failure of Managerial Reform). 
PFISTER LOÏC, Approche constitutionnelle de l'obligation de contracter, in: Daniel Dédeyan (Hrsg.), Vertrauen, Vertrag, Verantwortung, Zürich 2007, S.127-144 (zit. Obligation de contracter).

PIRKER BENEDIKT, Behördliche Interventionen in Abstimmungskämpfe, in: AJP 2017, S. 1366-1381 (zit. Behördliche Intervention).

POLEDNA TOMAS, Öffentliche Aufgaben und Kriterien für die Wahl der Organisationsform, in: René Schaffhauser et al. (Hrsg.), Auslagerung und Privatisierung von staatlichen und kommunalen Einheiten: Rechtsformen und ihre Folgen, Referate der Tagung vom 14. November 2001 in Luzern, St. Gallen 2002, S. 9-25 (zit. Öffentliche Aufgaben).

POLTIER ETIENNE, Les gentlemen's agreements à participation publique, in: ZSR 1987/I, S. 367-402 (zit. Gentlemen's agreements).

REICH JOHANNES, Grundsatz der Wirtschaftsfreiheit. Evolution und Dogmatik von Art. 94 Abs. 1 und 4 der Bundesverfassung der Schweizerischen Eidgenossenschaft vom 18. April 1999, Zürich 2011 (zit. Grundsatz der Wirtschaftsfreiheit).

RHINOW RENÉ, Verfügung, Verwaltungsvertrag und privatrechtlicher Vertrag, in: Juristische Fakultät der Universität Basel (Hrsg.), Privatrecht, öffentliches Recht, Strafrecht, Basel 1985, S. 295-322 (zit. Verfügung, Verwaltungsvertrag und privatrechtlicher Vertrag).

RHINOW RENÉ, Die Bundesverfassung 2000. Eine Einführung, Basel 2000 (zit. Bundesverfassung 2000).

RHINOW RENÉ/BIAGGINI GIOVANNI/SCHMID GERHARD/UHLMANN FELIX, Öffentliches Wirtschaftsrecht, 2. Aufl., Basel 2011 (zit. Öffentliches Wirtschaftsrecht).

RHINOW RENÉ/SCHEFER MARKUS/UEBERSAX PETER, Schweizerisches Verfassungsrecht, 3. Aufl., Basel 2016 (zit. Schweizerisches Verfassungsrecht).

RICHLI PAUL, Interdisziplinäre Daumenregeln für eine faire Rechtsetzung. Ein Beitrag zur Rechtsetzungslehre im liberalen sozial und ökologisch orientierten Rechtsstaat, Basel 2000 (zit. Rechtsetzung).

RUCH ALEXANDER

- Informalisierung und Deregulierung im schweizerischen Verfassungsrecht, in: ZBl 2002, S. 505-528 (zit. Informalisierung).

- Regulierungsfragen der Gentechnologie und des Internet, in: ZSR 2004/ II, S. 373-475 (zit. Regulierungsfragen). 
XXX

RÜTSCHE BERNHARD

- Was sind öffentliche Aufgaben?, in: recht 2013, S. 153-162 (zit. Öffentliche Aufgaben).

- Staatliche Leistungsaufträge und Rechtsschutz, in: ZBJV 2016, S. 71-113 (zit. Leistungsaufträge).

SÄGESSER THOMAS

- Regierungs- und Verwaltungsorganisationsgesetz (RVOG) vom 21. März 1997, Bern 2007 (zit. Kommentar RVOG).

- 3. Teil / $3^{\text {ème }}$ partie: Die Entwicklung der Gesetzgebung im Bereich des Verwaltungsorganisationsrechts, in: Schweizerische Vereinigung für Verwaltungsorganisationsrecht SVVOR, Association suisse du droit public de l'organisation (Hrsg.), Verwaltungsorganisationsrecht - Staatshaftungsrecht - öffentliches Dienstrecht/Droit public de l'organisation responsabilité des collectivités publiques - fonction publique, Bern 2016, S. 445-500 (zit. Verwaltungsorganisationsrecht).

SALADIN PETER, Grundrechtsprobleme, in: Die Kunst der Verfassungserneuerung, Basel 1998, S. 211-232 (zit. Grundrechtsprobleme).

SCHAFFHAUSER RENÉ/POLEDNA TOMAS (Hrsg.), Auslagerung und Privatisierung von staatlichen und kommunalen Einheiten: Rechtsformen und ihre Folgen, St. Gallen 2002 (zit. Auslagerung und Privatisierung).

SCHINDLER BENJAMIN

- Zur Rollenverteilung zwischen Justiz und Verwaltung, in: ZBl 2013, S. 359-373 (zit. Rollenverteilung).

- Art. 5, in: Bernhard Ehrenzeller et al. (Hrsg.), Die schweizerische Bundesverfassung, Zürich, St. Gallen 2014, S. 100-142 (zit. St. Galler Kommentar).

SCHNIDER MICHÈLE, Schutz des Arbeitnehmers vor psychischem Druck, Bern 2017 (zit. Schutz des Arbeitnehmers).

\section{SCHWEIZER RAINER J.}

- Art. 35, in: Bernhard Ehrenzeller et al.(Hrsg.), Die schweizerische Bundesverfassung, Zürich/St. Gallen 2014, S. 793-821 (zit. St. Galler Kommentar).

- Art. 42, in: Bernhard Ehrenzeller et al. (Hrsg.), Die schweizerische Bundesverfassung, Zürich/St. Gallen 2014, S. 941-945 (zit. St. Galler Kommentar).

- Art. 8, in: Bernhard Ehrenzeller et al. (Hrsg.), Die schweizerische Bundesverfassung, Zürich/St. Gallen 2014, S. 206-236 (zit. St. Galler Kommentar).

- Vorbemerkungen zu Art. 7-36, in: Bernhard Ehrenzeller et al. (Hrsg.), Die schweizerische Bundesverfassung, Zürich/St. Gallen 2014, S. 165-186 (zit. St. Galler Kommentar). 
SCHWEIZER RAINER J./MÜLLER LUCIEN

- Art. 43a, in: Bernhard Ehrenzeller et al. (Hrsg.), Die schweizerische Bundesverfassung, Zürich/St. Gallen 2014, S. 949-959 (zit. St. Galler Kommentar).

- Art. 5a, in: Bernhard Ehrenzeller et al. (Hrsg.), Die schweizerische Bundesverfassung, Zürich/St. Gallen 2014, S. 143-154 (zit. St. Galler Kommentar).

SPANNOWSKY WILLY, Die Verantwortung der öffentlichen Hand für die Erfüllung öffentlicher Aufgaben und die Reichweite ihrer Einwirkungspflicht auf Beteiligungsunternehmen, in: DVBl 1992, S.1072-1079 (zit. Verantwortung der öffentlichen Hand).

SPRECHER FRANZISKA, Datenschutz und Big Data im Allgemeinen und im Gesundheitsrecht im Besonderen, in: ZBJV 2018, S. 482-552 (zit. Datenschutz und Big Data).

STEINER RETO/FINGER MATTHIAS/HUBER ETIENNE/REIST PASCAL, Praxis des Bundes bei der Steuerung von Post, SBB und Swisscom, Bern 2012 (zit. Praxis des Bundes bei der Steuerung).

STEINER THOMAS, Besprechung BGE 139 I 306. Urteil des Bundesgerichts vom 16. November 2012 (2C_1032/2012), in: Medialex 2014, S. 29-30 (zit. Besprechung SRG-Urteil).

STEINMANN GEROLD, Art.34, in: Bernhard Ehrenzeller et al. (Hrsg.), Die schweizerische Bundesverfassung, Zürich/St. Gallen 2014, S. 776-792 (zit. St. Galler Kommentar).

STÖCKLI ANDREAS/PIOLINO MARINA, Religiöse Privatschulen im Spannungsfeld zwischen Privatschulfreiheit und Recht auf ausreichenden Grundschulunterricht, in: AJP 2018, S. 42-55 (zit. Privatschulen).

TANQUEREL THIERRY, Manuel de droit administratif, 2. Aufl., Genf/Zürich/ Basel 2018 (zit. Droit administratif).

THALER RICHARD H./SUNSTEIN CASS R., Nudge. Improving decisions about health, wealth and happiness, New Haven 2008 (zit. Nudge).

THOMA ANSELM CHRISTIAN, Regulierte Selbstregulierung im Ordnungsverwaltungsrecht, Berlin 2008 (zit. Selbstregulierung).

TSCHANNEN PIERRE

- Der Richtplan und die Abstimmung raumwirksamer Aufgaben, Bern 1986 (zit. Richtplan).

- Bundesstaatliche Aufgabenteilung und politisches System - Grenzen der Verfassungsästhetik, in: ZSR (1995/I), S. 143-169 (zit. Aufgabenteilung). 
- Privatisierung: Ende der Verfügung?, in: Wolfgang Wiegand (Hrsg.), Rechtliche Probleme der Privatisierung, Bern 1998, S. 209-240 (zit. Privatisierung).

- Systeme des allgemeinen Verwaltungsrechts, Bern 2008 (zit. Systeme).

- Zentral, dezentral, ausserhalb - oder: Wie zeichne ich das Organigramm der Bundesverwaltung?, in: Markus Rüssli et al. (Hrsg.), Staats- und Verwaltungsrecht auf vier Ebenen, Zürich/Basel/Genf 2012, S. 517-531 (zit. Organigramm).

- Staatsrecht der schweizerischen Eidgenossenschaft, 4. Aufl., Bern 2016 (zit. Staatsrecht).

TSCHANNEN PIERRE/LOCHER THOMAS, Aufsicht über das Versicherungsgeschäft kantonaler Gebäudeversicherungen ausserhalb des Monopols, in: Verwaltungsorganisationsrecht - Staatshaftungsrecht - öffentliches Dienstrecht/Droit public del'organisation - responsabilité des collectivités publiques - fonction publique, Bern 2014, S. 63-97 (zit. Aufsicht Gebäudeversicherungen).

TSCHANNEN PIERRE/ZIMMERLI ULRICH/MÜLLER MARKUS

- Allgemeines Verwaltungsrecht, 3. Aufl., Bern 2009 (zit. Allgemeines Verwaltungsrecht 3. Aufl.).

- Allgemeines Verwaltungsrecht, 4. Aufl., Bern 2014 (zit. Allgemeines Verwaltungsrecht).

TSCHENTSCHER AXEL/LIENHARD ANDREAS, Öffentliches Recht. Ein Grundriss, Zürich/St. Gallen 2011 (zit. Öffentliches Recht).

UEBERSAX PETER, Privatisierung der Verwaltung, in: ZBl 2001, S. 393-422 (zit. Privatisierung).

UHLMANN FELIX, Gewinnorientiertes Staatshandeln. Möglichkeiten und Zulässigkeit gewinnorientierter staatlicher Eigenbetätigung aus wirtschaftsverfassungsrechtlicher Sicht, Basel 1997 (zit. Gewinnorientiertes Staatshandeln).

VALLENDER KLAUS A., Der ordnungspolitische Grundentscheid der schweizerischen Bundesverfassung, in: Max-Emanuel Geis et al. (Hrsg.), Staat Kirche - Verwaltung, München 2001, S. 1033-1051 (zit. Ordnungspolitischer Grundentscheid). 


\section{VOGEL STEFAN}

- Der Staat als Marktteilnehmer. Voraussetzungen der Zulässigkeit wirtschaftlicher Tätigkeit des Gemeinwesens in Konkurrenz zu Privaten, Zürich 2000 (zit. Staat als Marktteilnehmer).

- Einheit der Verwaltung - Verwaltungseinheiten. Grundprobleme der Verwaltungsorganisation - Rechtliche Rahmenbedingungen, Konzepte, Strukturen und Formen für die Organisation von Aufgabenträgern der öffentlichen Verwaltung, Zürich 2008 (zit. Einheit der Verwaltung).

VOSSKUHLE ANDREAS

- Gesetzgeberische Regelungsstrategien der Verantwortungsteilung zwischen öffentlichem und privatem Sektor, in: Gunnar Folke Schuppert (Hrsg.), Jenseits von Privatisierung und «schlankem» Staat, Baden-Baden 1999, S. 47-90 (zit. Verantwortungsteilung).

- Beteiligung Privater an der Wahrnehmung öffentlicher Aufgaben und staatliche Verantwortung, in: VVDStRL 2003, S. 266-335 (zit. Beteiligung Privater).

\section{WALDMANN BERNHARD}

- Der verwaltungsrechtliche Vertrag - Eine Einführung, in: Isabelle Häner (Hrsg.), Der verwaltungsrechtliche Vertrag in der Praxis, Zürich/Basel/ Genf 2007, S.1-23 (zit. Verwaltungsrechtlicher Vertrag).

- Art.35, in: Bernhard Waldmann et al. (Hrsg.), Bundesverfassung, Basel 2015, S. 723-745 (zit. Basler Kommentar).

- Art. 8, in: Bernhard Waldmann et al. (Hrsg.), Bundesverfassung, Basel 2015, S. 174-223 (zit. Basler Kommentar).

WALDMANN BERNHARD/BELSER EVA MARIA/EPINEY ASTRID (Hrsg.), Bundesverfassung, Basel 2015 (zit. Basler Kommentar).

WALTHER SIMONE, Kooperative Steuerungsinstrumente im schweizerischen Stromversorgungsrecht, Zürich 2014 (zit. Kooperative Steuerungsinstrumente).

WEBER-DÜRLER BEATRICE, Vertrauensschutz im öffentlichen Recht, Basel 1983 (zit. Vertrauensschutz).

WIEDERKEHR RENÉ

- Kausalabgaben, Bern 2015 (zit. Kausalabgaben).

- Sonderabgaben, in: recht 2017, S. 43-59 (zit. Sonderabgaben).

- Das Legalitätsprinzip im Kausalabgaberecht. Eine Übersicht über die neuere Rechtsprechung und Doktrin, in: recht 2018, S. 40-50 (zit. Legalitätsprinzip im Kausalabgaberecht). 
WIEDERKEHR RENÉ/RICHLI PAUL, Praxis des allgemeinen Verwaltungsrechts. Eine systematische Analyse der Rechtsprechung; Band II, Bern 2014 (zit. Praxis des allgemeinen Verwaltungsrechts).

WIEGAND MARC ANDRÉ, Die Beleihung Privater im Kernbereich hoheitlicher Aufgabenwahrnehmung. Zugleich eine Anmerkung zur Entscheidung des BVerfG vom 18.01.2012 (2 BvR 133/10), in: DVBl 2012, S. 1134-1140 (zit. Beleihung Privater).

WIEGAND WOLFGANG (Hrsg.), Rechtliche Probleme der Privatisierung. Tagung vom 15./16. Oktober 1997 an der Universität Bern, Bern 1998 (zit. BTJP 1997).

WYSS DANIELA, Kausalabgaben. Begriff, Bemessung, Gesetzmässigkeit, Basel 2009 (zit. Kausalabgaben).

WYSS MARTIN PHILIPP, Öffentliche Interessen - Interessen der Öffentlichkeit? Das öffentliche Interesse im schweizerischen Staats- und Verwaltungsrecht, Bern 2001 (zit. Öffentliche Interessen).

ZYSSET PASCAL, Selbstregulierung im Finanzmarktrecht. Grundlagen, verwaltungsrechtliche Qualifikationen und rechtsstaatlicher Rahmen, Zürich 2017 (zit. Selbstregulierung). 


\section{Materialien \\ und weitere Dokumente}

Das Verzeichnis nennt alle im Text zitierten Materialien und Dokumente. Sie werden jeweils mit Kurztitel zitiert.

Bericht Bundesfinanzen 2016 - FINANZDELEGATION, Bericht der Finanzdelegation an die Finanzkommissionen des Nationalrates und des Ständerates betreffend die Oberaufsicht über die Bundesfinanzen im Jahre 2016 vom 31. März 2017, BBl 2017 5245-5334.

Bericht FLAG BUNDESRAT, Bericht über das Führen mit Leistungsauftrag und Globalbudget - Evaluation und weiteres Vorgehen (Evaluationsbericht FLAG) vom 19. Dezember 2001, BBl 2002 3535-3567.

Bericht ParlG SPK-N, Parlamentarische Initiative. Parlamentsgesetz (PG). Bericht der Staatspolitischen Kommission des Nationalrates vom 1. März 2001, BBl 2001 3467-3620.

Bericht Staat und Wettbewerb - BUNDESRAT, Staat und Wettbewerb. Auswirkungen staatlich beherrschter Unternehmen auf die Wettbewerbsmärkte. Bericht des Bundesrates in Erfüllung der Postulate 12.4172 FDP-Liberale Fraktion vom 13.12.2012 und 15.3880 Schilliger vom 22.09.2015, Bern 8. Dezember 2017.

Bericht strategische Ziele SIFEM - EFK, Prüfung der Aufsicht über die Einhaltung der strategischen Ziele 2014-2017 des Bundesrates durch die SIFEM AG, 12.Juli 2017, nicht amtlich publiziert, abrufbar unter www.efk.admin.ch/de/publikationen/ wirtschaft-verwaltung/wirtschaft-und-landwirtschaft.

Botschaft Bahnreform - BUNDESR AT, Botschaft zur Bahnreform vom 13. November 1996, BBl 1997 I 909-995.

Botschaft BV

BUNDESRAT, Botschaft über eine neue Bundesverfassung vom 20. November 1996, BBl 1997 I 1-642. 
XXXVI

Botschaft FINMAG BUNDESRAT, Botschaft zum Bundesgesetz über die Eidgenössische Finanzmarktaufsicht (Finanzmarktaufsichtsgesetz; FINMAG) vom 1. Februar 2006, BBl 2006 2829-2916.

Botschaft GwG BUNDESRAT, Botschaft zum Bundesgesetz zur Bekämpfung der Geldwäscherei im Finanzsektor (Geldwäschereigesetz, GwG) vom 17.Juni 1996, BBl 1996 III 1101-1177.

Botschaft HMG BUNDESRAT, Botschaft zu einem Bundesgesetz über Arzneimittel und Medizinprodukte (Heilmittelgesetz, HMG) vom 1. März 1999, BBl 1999 3453-3618.

Botschaft Initiative «Gleiche Rechte» - BUNDESRAT, Botschaft über die Volksinitiative «Gleiche Rechte für Mann und Frau» vom 14. November 1979, BBl 1980 I 69-149.

Botschaft NFA BUNDESRAT, Botschaft zur Neugestaltung des Finanzausgleichs und der Aufgaben zwischen Bund und Kantonen (NFA) vom 14. November 2001, BBl 2002 2291-2559.

Botschaft NFB BUNDESRAT, Botschaft über die Weiterentwicklung der ziel- und ergebnisorientierten Verwaltungsführung Neues Führungsmodell für die Bundesverwaltung (NFB) vom 20. November 2013, BBl 2014 767-882.

Botschaft POG BUNDESRAT, Botschaft zum Bundesgesetz über die Organisation der Schweizerischen Post (Postorganisationsgesetz, POG) vom 20. Mai 2009, BBl 2009 5265-5308.

Botschaft Umwandlung PTT - BUNDESRAT, Botschaft zu einem Postorganisationsgesetz und zu einem Telekommunikationsunternehmungsgesetz vom 10.Juni 1996, BBl 1996 III 1306-1383.

Corporate-Governance-Bericht - BUNDESRAT, Bericht zur Auslagerung und Steuerung von Bundesaufgaben (Corporate-GovernanceBericht) vom 13. September 2006, BBl 2006 8233-8304.

Jahresrechnung Swissmedic - SwISSMEDIC, Jahresrechnung Swissmedic 2017, 11. April 2018, nicht amtlich publiziert, abrufbar unter www.swissmedic.ch/swissmedic/de/home/ueber-uns/ publikationen/aktueller-geschaeftsbericht.html.

Legislaturplanung 1987-1991 - BUNDESRAT, Bericht über die Legislaturplanung 1987-1991 vom 18. Januar 1988, BBl 1988 I 395-568. 
Legislaturplanung 1991-1995 - BUNDESRAT, Bericht über die Legislaturplanung 1991-1995 vom 25. März 1992, BBl 1992 III 1-200.

Legislaturplanung 1995-1999 - BUNDESRAT, Bericht über die Legislaturplanung 1995-1999 vom 18. März 1996, BBl 1996 II 293-369.

Legislaturplanung 1999-2003 - BUNDESRAT, Bericht über die Legislaturplanung 1999-2003 vom 1. März 2000, BBl 2000 2276-2356.

Legislaturplanung 2003-2007 - BUNDESRAT, Bericht über die Legislaturplanung 2003-2007 vom 25. Februar 2004, BBl 2004 11491252.

Legislaturplanung 2007-2011-BUNDESRAT, Botschaft zur Legislaturplanung 2007-2011 vom 23.Januar 2008, BBl 2008 753-832.

Leistungsauftrag Swissmedic - BUNDESRAT, Leistungsauftrag Swissmedic 2017-2018 (LA), 17. Oktober 2017, nicht amtlich publiziert, abrufbar unter www.swissmedic.ch/swissmedic/de/ home/ueber-uns/swissmedic--schweizerisches-heilmit telinstitut/strategische-vorgaben.html.

Strategische Ziele Post - BUNDESRAT, Strategische Ziele des Bundesrates für die Schweizerische Post AG 2017-2020 vom 9. Dezember 2016, BBl 2017 125-128.

Strategische Ziele RUAG - BUNDESRAT, Strategische Ziele des Bundesrates für die RUAG Holding AG 2016-2019, Ziff. 5; nicht amtlich publiziert, abrufbar unter www.vbs.admin.ch/de/vbs/ bundesnahe-betriebe/ruag.html.

Strategische Ziele SIFEM - BUNDESRAT, Strategische Ziele 2014-2017 des Bundesrates für die SIFEM AG vom 29. November 2013, BBl 2013 9409-9414.

Strategische Ziele Swisscom - BUNDESRAT, Strategische Ziele des Bundesrates für die Swisscom AG 2018-2021 vom 22. November 2017, BBl 2017 7867-7870.

Strategische Ziele Swissmedic - SwISSMEDIC, Strategische Ziele 2019 bis 2022 vom 24. Oktober 2018. 



\section{Abkürzungen}

$\mathrm{AB}$

Abs.

AG

AJP

ARE

Art.

AS

AtraG

Aufl.

BAFU

BAG

BAKOM

BBG

$\mathrm{BBl}$

Bd.

$\mathrm{BE}$

BehiG

BGBM

BGE

BGG

BGÖ

BGRB

BJ

BLV
Amtliches Bulletin

Absatz

Aktiengesellschaft

Aktuelle Juristische Praxis

Bundesamt für Raumentwicklung

Artikel

Amtliche Sammlung des Bundesrechts

Bundesgesetz über den Bau der schweizerischen Eisenbahn-Alpentransversale vom 4. Oktober 1991 (Alpentransit-Gesetz, SR 742.104)

Auflage

Bundesamt für Umwelt

Bundesamt für Gesundheit

Bundesamt für Kommunikation

Bundesgesetz über die Berufsbildung vom 13. Dezember 2002 (Berufsbildungsgesetz, SR 412.10)

Bundesblatt

Band

Kanton Bern

Bundesgesetz über die Beseitigung von Benachteiligungen von Menschen mit Behinderungen vom 13. Dezember 2002 (SR 151.3).

Bundesgesetz über den Binnenmarkt vom 6. Oktober 1995 (SR 943.02)

Entscheidungen des Schweizerischen Bundesgerichts (Amtliche Sammlung)

Bundesgesetz über das Bundesgericht vom 17.Juni 2005 (Bundesgerichtsgesetz, SR 173.110)

Bundesgesetz über das Öffentlichkeitsprinzip der Verwaltung vom 17. Dezember 2004 (Öffentlichkeitsgesetz, SR 152.3)

Bundesgesetz über die Rüstungsunternehmen des Bundes vom 10. Oktober 1997 (SR 934.21)

Bundesamt für Justiz

Bundesamt für Lebensmittelsicherheit und Veterinärwesen 
BöB

BSG

Bst.

BV

BVG

BWIS

bzw.

CENELEC

Diss.

DSG

DVBl

E.

EBG

EFK

EleG

EPDG

EPDV

ETH-Gesetz

f., ff.

FHG

FINMA

FINMAG

FKG

FKV

FLAG

FMG
Bundesgesetz über das öffentliche Beschaffungswesen vom 16. Dezember 1994 (SR 172.056.1)

Bernische Systematische Gesetzessammlung Buchstabe

Bundesverfassung der Schweizerischen Eidgenossenschaft vom 18. April 1999 (SR 101)

Bundesgesetz über die berufliche Alters-, Hinterlassenenund Invalidenvorsorge vom 25.Juni 1982 (SR 831.40)

Bundesgesetz über Massnahmen zur Wahrung der inneren Sicherheit vom 21. März 1997 (BWIS, SR 120)

beziehungsweise

European Committee for Eletrotechnical Standardization Dissertation

Bundesgesetz über den Datenschutz vom 19.Juni 1992 (SR 235.1)

Deutsches Verwaltungsblatt

Erwägung

Eisenbahngesetz vom 20. Dezember 1957 (SR 742.101)

Eidgenössische Finanzkontrolle

Bundesgesetz betreffend die elektrischen Schwach- und Starkstromanlagen vom 24.Juni 1902 (SR 734.0)

Bundesgesetz über das elektronische Patientendossier vom 19.Juni 2015 (SR 816.1)

Verordnung über das elektronische Patientendossier vom 22. März 2017 (SR 816.11)

Bundesgesetz über die Eidgenössischen Technischen Hochschulen vom 4. Oktober 1991 (SR 414.110)

folgende, fortfolgende

Bundesgesetz über den eidgenössischen Finanzhaushalt vom 7. Oktober 2005 (Finanzhaushaltgesetz, SR 611.0)

Eidgenössische Finanzmarktaufsicht

Bundesgesetz über die Eidgenössische Finanzmarktaufsicht vom 22.Juni 2007 (Finanzmarktaufsichtsgesetz, SR 956.1)

Bundesgesetz über die Eidgenössische Finanzkontrolle vom 28. Juni 1967 (Finanzkontrollgesetz, SR 614.0)

Verordnung über Frequenzmanagement und Funkkonzessionen vom 9. März 2007 (SR 784.102.1)

Führen mit Leistungsauftrag und Globalbudget

Fernmeldegesetz vom 30. April 1997 (SR 784.10) 
Fn.

Fr.

GlG

GwG

HAVE

HFKG

HGVAnG

HMG

Hrsg.

i.d.R.

IEC

IGW

insb.

i.S.v.

i.V.m.

KG

KVG

LSV

LwG

MedBG

MSG

$\mathrm{N}$

NEF
Fussnote

Schweizer Franken

Bundesgesetz über die Gleichstellung von Frau und Mann vom 24. März 1995 (SR 151.1)

Bundesgesetz über die Bekämpfung der Geldwäscherei und der Terrorismusfinanzierung vom 10. Oktober 1997 (Geldwäschereigesetz, SR 955.0)

Haftung und Versicherung

Bundesgesetz über die Förderung der Hochschulen und die Koordination im schweizerischen Hochschulbereich vom 30. September 2011 (Hochschulförderungs- und -koordinationsgesetz, SR 414.20)

Bundesgesetz über den Anschluss der Ost- und der Westschweiz an das europäische Eisenbahn-Hochleistungsnetz vom 18. März 2005 (HGV-Anschluss-Gesetz, SR 742.140.3)

Bundesgesetz über Arzneimittel und Medizinprodukte vom 15. Dezember 2000 (Heilmittelgesetz, SR 812.21)

Herausgeber

in der Regel

International Electrotechnical Commission

Immissionsgrenzwert

insbesondere

im Sinne von

in Verbindung mit

Bundesgesetz über Kartelle und andere Wettbewerbsbeschränkungen vom 6. Oktober 1995 (SR 251)

Bundesgesetz über die Krankenversicherung vom 18. März 1994 (SR 832.10)

Lärmschutz-Verordnung vom 15. Dezember 1986

(SR 814.41)

Bundesgesetz über die Landwirtschaft vom 29. April 1998 (Landwirtschaftsgesetz, SR 910.1)

Bundesgesetz über die universitären Medizinalberufe vom 23. Juni 2006 (Medizinalberufegesetz, SR 811.11)

Bundesgesetz über die Museen und Sammlungen des Bundes vom 12. Juni 2009 (Museums- und Sammlungsgesetz, SR 432.30)

Nationalrat

Neue Verwaltungsführung [im Kanton Bern] 
NFA

NFB

NHG

NPM

Nr.

NSG

NVwZ

NZZ

OR

OV-WBF

ParlG

PG

POG

PTT

recht

RPV

RVOG

RVOV

Rz.

$\mathrm{S}$

S.

SBBG

SIFEM

SJZ

sog.
Neugestaltung des Finanzausgleichs und der Aufgaben zwischen Bund und Kantonen

Neues Führungsmodell für die Bundesverwaltung

Bundesgesetz über den Natur- und Heimatschutz vom 1.Juli 1966 (SR 451)

New Public Management

Nummer

Bundesgesetz über die Nationalstrassen vom 8. März 1960 (SR 725.11)

Neue Zeitschrift für Verwaltungsrecht

Neue Zürcher Zeitung

Bundesgesetz betreffend die Ergänzung des Schweizerischen Zivilgesetzbuches (Fünfter Teil: Obligationenrecht) vom 30. März 1911 (SR 220)

Organisationsverordnung für das Eidgenössische Departement für Wirtschaft, Bildung und Forschung vom 14.Juni 1999 (SR 172.216.1)

Bundesgesetz über die Bundesversammlung vom 13. Dezember 2002 (Parlamentsgesetz, SR 171.10)

Postgesetz vom 17. Dezember 2010 (SR 783.0)

Bundesgesetz über die Organisation der Schweizerischen Post vom 17. Dezember 2010 (Postorganisationsgesetz, SR 783.1)

Post-, Telefon- und Telegrafenbetriebe

Zeitschrift für juristische Ausbildung und Praxis

Raumplanungsverordnung vom 28.Juni 2000 (SR 700.1)

Regierungs- und Verwaltungsorganisationsgesetz vom 21. März 1997 (SR 172.010)

Regierungs- und Verwaltungsorganisationsverordnung vom 25. November 1998 (SR 172.010.1)

Randziffer(n)

Ständerat

Seite(n)

Bundesgesetz über die Schweizerischen Bundesbahnen vom 20. März 1998 (SR 742.31)

Swiss Investment Fund for Emerging Markets

Schweizerische Juristen-Zeitschrift

sogenannt(e) 
SpG

SpV

SR

SRG

StromVG

SuG

S\&R

THG

TUG

u.a.

USG

UVEK

UVG

UWG

VG

VGG

vgl.

VID

VPB

VPG

VPOG
Bundesgesetz über die Landessprachen und die Verständigung zwischen den Sprachgemeinschaften vom 5. Oktober 2007 (Sprachengesetz, SR 441.1)

Verordnung über die Landessprachen und die Verständigung zwischen den Sprachgemeinschaften vom 4.Juni 2010 (Sprachenverordnung, SR 441.11)

Systematische Sammlung des Bundesrechts

Schweizerische Radio- und Fernsehgesellschaft

Bundesgesetz über die Stromversorgung vom 23. März 2007 (Stromversorgungsgesetz, SR 734.7)

Bundesgesetz über Finanzhilfen und Abgeltungen vom 5. Oktober 1990 (Subventionsgesetz, SR 616.1)

Sicherheit \& Recht

Bundesgesetz über die technischen Handelshemmnisse vom 6. Oktober 1995 (SR 946.51)

Bundesgesetz über die Organisation der Telekommunikationsunternehmung des Bundes vom 30. April 1997 (Telekommunikationsunternehmungsgesetz, SR 784.11)

unter anderem

Bundesgesetz über den Umweltschutz vom 7. Oktober 1983 (SR 814.01)

Eidgenössisches Departement für Umwelt, Verkehr, Energie und Kommunikation

Bundesgesetz über die Unfallversicherung vom 20. März 1981 (SR 832.20)

Bundesgesetz gegen den unlauteren Wettbewerb vom 19. Dezember 1986 (SR 241)

Bundesgesetz über die Verantwortlichkeit des Bundes sowie seiner Behördemitglieder und Beamten vom 14. März 1958 (SR 170.32).

Bundesgesetz über das Bundesverwaltungsgericht vom 17.Juni 2005 (Verwaltungsgerichtsgesetz, SR 173.32) vergleiche

Verordnung über Internet-Domains vom 5. November 2014 (SR 784.104.2)

Verwaltungspraxis der Bundesbehörden

Postverordnung vom 29. August 2012 (SR 783.01)

Postorganisationsverordnung vom 24. Oktober 2012 (SR 783.11) 
XLIV

VRPG-BE Gesetz [des Kantons Bern] über die Verwaltungsrechtspflege vom 23. Mai 1989 (BSG 155.21)

VS. versus

VVDStRL Veröffentlichungen der Vereinigung der Deutschen Staatsrechtslehrer

VwVG

Bundesgesetz über das Verwaltungsverfahren vom 20. Dezember 1968 (Verwaltungsverfahrensgesetz, SR 172.021)

z.B. zum Beispiel

ZBJV

Zeitschrift des Bernischen Juristenvereins

$\mathrm{ZBl}$ Schweizerisches Zentralblatt für Staats- und Verwaltungsrecht

Ziff. $\quad$ Ziffer(n)

zit.

ZPO zitiert

Schweizerische Zivilprozessordnung vom 19. Dezember 2008 (Zivilprozessordnung, SR 272)

ZSR Zeitschrift für Schweizerisches Recht

ZWG Bundesgesetz über Zweitwohnungen vom 20. März 2015 (Zweitwohnungsgesetz, SR 702)

ZWV Zweitwohnungsverordnung vom 4. Dezember 2015 (SR 702.1) 




\section{\$1 Einleitung}

\section{Ausgangspunkt: Die Erwartung von weniger Staat durch Privatisierung}

Der Staat ist zu gross, zu langsam, zu teuer und zu weit von seinen Bürgerinnen und Bürgern entfernt. Diesen Eindruck vermitteln die Motive hinter Privatisierungsvorhaben, die den Staat reformieren möchten. Privatisierung führe zu einem schlankeren, günstigeren Staat, der geringeren Aufwand betreiben müsse. ${ }^{1}$ Die vielfältigen Ziele der Privatisierung lassen diese als Universalwaffe gegen den ausufernden Staat erscheinen: Sie soll Kundenund Bürgerorientierung fördern, indem die Aufgaben auf die Bedürfnisse ausgerichtet werden; die Kosten sollen gesenkt, die Qualität aber verbessert werden; und dies alles diene der Senkung von defizitären Staatshaushalten. ${ }^{2}$

Privatisierungen sind auch von der Überzeugung getrieben, Private würden «besser, beweglicher, billiger» handeln als der Staat. ${ }^{3}$ Man erwartet, «dass die Privaten kostengünstiger wirtschaften als der Staat. In der Regel dürfte dies auch zutreffen, ist der Kostendruck bei den Privaten doch höher und sind die betriebswirtschaftliche Organisation sowie die unternehmerische Führung flexibler.»4 Der Staat soll «die Verhaltensmuster des wirtschaftlichen Wettbewerbs» annehmen und dadurch eine «Steigerung der Aufgabeneffizienz» erreichen. 5 «Der ökonomische Gedanke soll mithin auch im Staat vermehrt Eingang finden. Mittels der verschiedenen Privatisierungsmöglichkeiten soll mehr Flexibilität und Effizienz erreicht werden.»6 Insgesamt strebe

1 FRICK, Verursacherprinzip, S. 48; FREY, Drei Stossrichtungen, S. 345 f.; HANDSCHIN/ SIEGENTHALER, Privatisierung, S. 408; MOOR/BELLANGER/TANQUEREL, Droit administratif III, S. 119; TSCHANNEN/ZIMMERLI/MÜLLER, Allgemeines Verwaltungsrecht, \$11 Rz.3. Vgl. aus der deutschen Lehre auch BAUER, Privatisierung, S. 256f.; BOGUMIL/JANN, Verwaltungswissenschaft, S. 49; OSTERLOH, Privatisierung, S. 213.

2 FREY, Drei Stossrichtungen, S.345 f.

3 TSCHANNEN, Privatisierung, S. 216.

4 UEBERSAX, Privatisierung, S. 402.

5 MÜLLER/JENNI, Privatisierung, S. 1072; gleichlautend TSCHANNEN/ZIMMERLI/MÜLLER, Allgemeines Verwaltungsrecht, §11 Rz.3. Ähnlich auch HÄNER, Privatisierung, S. 423; LIENHARD, Ausgelagerte Aufgabenerfüllung, S.1166.

6 HÄNER, Privatisierung, S. 423. 
die Privatisierung mehr Flexibilität und Autonomie der Verwaltungsträger an. ${ }^{7}$ Damit gingen auch «ordnungspolitische Bereinigungen im Grenzbereich zwischen Staat und Markt» 8 einher, die wirtschaftliche Tätigkeiten vom Staat befreien sollen. «Ein Hauptgrund für Privatisierungen besteht darin, durch marktwirtschaftlichen Effizienzdruck den nachfrageoptimierten Ressourceneinsatz sicherzustellen.» ${ }^{9}$ Die Losung scheint somit klar: Wäre der Staat nur weniger Staat, käme alles besser.

Womöglich müssen einige dieser hehren Hoffnungen enttäuscht werden. Doch bedeutet dies, dass stattdessen düstere Warnungen zutreffen müssen? Durch die Zurückdrängung hoheitlicher Handlungsformen würden demnach rechtsstaatliche und demokratische Verpflichtungen abgebaut. ${ }^{10}$ Es werde versucht, «durch die zivilrechtliche Einkleidung» des Staats «die - vor allem in wirtschaftlicher Hinsicht - hemmenden Aspekte der Staatlichkeit (z.B. Grundrechte, Legalitätsprinzip, staatliche Verantwortlichkeit etc.) teilweise abzustreifen»11. Es gehe um eine «Loslösung von den demokratischen Entscheidungsprozessen», weil diese «unflexibel und innovationshemmend» seien. ${ }^{12}$

\section{Fragestellungen und Aufbau}

Diese Arbeit untersucht, ob Privatisierungen die Erwartung von weniger Staat einlösen können, oder ob die geäusserten Befürchtungen eintreffen, wonach Privatisierungen die rechtsstaatlichen und demokratischen Vorgaben zurückdrängen. Um diesen Anspruch einzulösen, beantwortet die Arbeit der Reihe nach vier Fragen:

- Welchen verfassungsrechtlichen Anforderungen unterliegt die Erfüllung staatlicher Aufgaben? (\$3)

- In welcher Hinsicht kann die Erfüllung staatlicher Aufgaben privatisiert werden? (\$4) Diese Frage legt den Fokus auf die Erfüllungsprivatisierung und spart die Aufgabenprivatisierung bewusst aus.

7 HANDSCHIN/SIEGENTHALER, Privatisierung, S. 408; LIENHARD, Ausgelagerte Aufgabenerfüllung, S. 1166 («Flexibilität/unternehmerischer Handlungsfreiraum») MÜLLER/ JENNI, Privatisierung, S. 1073; UEBERSAX, Privatisierung, S. 402 («unternehmerische[...] Flexibilisierung»).

8 TSCHANNEN/ZIMMERLI/MÜLLER, Allgemeines Verwaltungsrecht, \$11 Rz.3.

9 HANDSCHIN/SIEGENTHALER, Privatisierung, S. 412.

10 TSCHANNEN, Privatisierung, S. 233-239.

11 MÜLLER, Verwaltungsrecht, S. 88.

12 HANDSCHIN/SIEGENTHALER, Privatisierung, S. 408. 
- Welche Probleme schaffen die verfassungsrechtlichen Anforderungen für die Erfüllungsprivatisierung? (\$5)

- Welche Spielräume belassen die verfassungsrechtlichen Anforderungen der Erfüllungsprivatisierung? (\$6)

Diese vier Fragen ergeben eine rasterartige Prüfung. Im Zentrum des Erkenntnisinteresses stehen die Probleme und Spielräume der Erfüllungsprivatisierung. Den Prüfmassstab bilden die Anforderungen der Verfassung an staatliches Handeln. Die verschiedenen Erscheinungsformen der privatisierten Aufgabenerfüllung sind der Prüfgegenstand. Eingangs erfolgt eine Begriffsklärung (\$2). Ein Résumé der Ergebnisse (\$7) schliesst die Arbeit ab.

Diese Arbeit strebt nicht an, den rechtlichen Vorgang der Privatisierung neu zu erfinden oder zu beschreiben - Lehre und Praxis haben die Rechtsfragen in mehreren Entwicklungsschritten ausgiebig erörtert. ${ }^{13}$ Zahlreiche Studien haben untersucht, ob und unter welchen Voraussetzungen bestimmte Aufgabenbereiche privatisiert werden können.Jedes konkrete Privatisierungsprojekt könnte in eine eigene Dogmatik gehüllt werden, um seine typischen Ausprägungen zu erfassen. Diese Arbeit hat ein anderes Ziel: Sie will vereinfachen und ordnen. Sie unterscheidet Privatisierungen ganz grundlegend danach, ob sie die Aufgaben- oder die Erfüllungsseite der staatlichen Tätigkeit betreffen. Und sie legt die Anforderungen der Verfassung in allgemeingültiger Weise an Privatisierungen an, ohne die Besonderheiten eines einzelnen Aufgabenbereichs mit dogmatischen Sonderkategorien zu erfassen.

\section{Verfassungsdogmatischer Zugang}

Diese Arbeit verfolgt einen strikt rechtswissenschaftlichen Zugang. Volks- und betriebswirtschaftliche Vorstellungen von Effizienz werden ebenso ausgespart wie politikwissenschaftliche und staatsphilosophische Überlegungen über die Rolle des Staats.

Innerhalb der Rechtswissenschaft wird die Optik weiter auf das Verfassungsrecht verengt. Welche Vorgaben macht die Verfassung an das staatliche Handeln? Soweit es für die Erörterung der Fragestellungen notwendig ist, wird zudem das Verwaltungsorganisationsrecht aufgegriffen.

13 Stellvertretend für die Dichte der Privatisierungsdiskussion um die Jahrtausendwende stehen drei Tagungsbände: WIEGAND, BTJP 1997; JAAG, Dezentralisierung und Privatisierung; SCHAFFHAUSER/POLEDNA, Auslagerung und Privatisierung. Siehe für eine umfassendere Übersicht über die Literatur HÄFELIN/MÜLLER/UHLMANN, Allgemeines Verwaltungsrecht, §26. 
Die Arbeit bleibt bewusst auf abstrakter Flughöhe. Sie will nicht untersuchen, ob einzelne Privatisierungsprojekte ihre Erwartungen erfüllen konnten oder wie die optimale Verwaltungsorganisation zur Erfüllung eines bestimmten Aufgabenbereichs, etwa des Postwesens, aussehen müsste. Die verschiedenen Aufgabengebiete des besonderen Verwaltungsrechts dienen vielmehr der punktuellen Veranschaulichung. Der rote Faden dieser Arbeit bleibt aber das Verfassungsrecht: Was ist aus Sicht der Verfassung Staatstätigkeit, und was bewirken die Vorgaben der Verfassung, wenn diese Tätigkeit privatisiert wird?

Und nicht zuletzt: Der Staat, der hier untersucht wird, ist die Schweizerische Eidgenossenschaft. Wenn immer diese Arbeit von der Verfassung spricht, meint sie die Bundesverfassung ${ }^{14}$. Die gliedstaatliche Ebene (Kantonsverfassungen) wird ebenso ausgeblendet wie die überstaatliche (Völkerrecht).

14 Bundesverfassung der Schweizerischen Eidgenossenschaft vom 18. April 1999 (BV, SR101). 


\section{§2 Begriffsklärung}

\section{Staat}

\section{Staatlichkeit als Begriff der Bundesverfassung}

Weshalb muss überhaupt definiert werden, was der Staat ist?15 Weil das Verfassungsrecht den Staat besonderen Anforderungen unterwirft, die für privates Handeln nicht gelten (siehe dazu hinten \$3/I.1).

Im Folgenden beschränkt sich diese Untersuchung auf das aktuell geltende Verfassungsrecht des Bundes. Kantonales und kommunales Verfassungsrecht werden ebenso ausgespart wie verfassungsgeschichtliche Erwägungen. Die Bundesverfassung erwähnt den Staat in insgesamt fünfzehn Artikeln ausdrücklich. Zwei dieser Erwähnungen betreffen konkrete Aufgabenbereiche ${ }^{16}$, drei weitere Artikel erwähnen die Staatsrechnung17. Die übrigen zehn Artikel erwähnen den Staat in grundsätzlicher Weise und lassen Rückschlüsse auf das Staatsverständnis der Bundesverfassung zu:

- Art. 5 BV: Grundsätze rechtsstaatlichen Handelns;

- Art. 5 a und 43a BV: Subsidiarität und andere Grundsätze bei der Zuweisung und Erfüllung staatlicher Aufgaben;

- Art. 6 BV: individuelle und gesellschaftliche Verantwortung bei der Bewältigung der Aufgaben in Staat und Gesellschaft;

- Art. 9 BV: willkürfreie Behandlung durch staatliche Organe;

- Art. 35 Abs. 2 BV: Grundrechtsbindung bei der Wahrnehmung staatlicher Aufgaben;

- Art. 41 Abs. 4 BV: Ansprüche auf staatliche Leistungen;

- Art.146 BV: Staatshaftung bei der Ausübung amtlicher Tätigkeiten;

- Art. 173 Abs. 1 Bst. g und Art. 180 BV: Planung und Koordinierung der Staatstätigkeit sowie öffentliche Information darüber.

15 Dieser Abschnitt lehnt sich teilweise an eine frühere Publikation an: ELSER, Funktioneller Staatsbegriff, S. 62-66.

16 Art. 62 BV zur Grundschule und Art. 72 BV zum Verhältnis von Kirche und Staat.

17 Art.126 Abs.4, Art. 167 und 183 BV. 
Nebenbei: Diese Liste führt nur die ausdrücklichen Erwähnungen von Staatlichkeit in der Bundesverfassung auf. Die Verfassung in ihrer Gesamtheit betrifft den Staat. Hier wird aber untersucht, wie das Verfassungsrecht den Staat konzipiert und in welchen Kontexten es ihn erwähnt.

\section{a. Staat heisst staatliches Handeln}

Diesen Bestimmungen ist gemein, dass ihre Rechtsfolgen jeweils an eine Tätigkeit staatlicher Natur knüpfen. Die aufgelisteten Artikel sprechen von staatlichem Handeln (Art. 5 BV); Erfüllung, Wahrnehmung bzw. Bewältigung staatlicher Aufgaben (Art. 5a, 6, 35 Abs. 2, Art. 43a BV); staatlicher Behandlung von Personen (Art. 9 BV); staatlichen Leistungen (Art. 41 Abs. 4 BV) sowie staatlicher Tätigkeit (Art. 173 Abs. 1 Bst. g und Art.180 BV). Die Begriffe sind vielfältig, das verfassungsrechtliche Verständnis von Staatlichkeit hingegen eindeutig: Die Verfassung unterwirft das staatliche Handeln bestimmten Anforderungen, nicht staatliche Einrichtungen oder Personen an sich.

\section{b. Bildvon zwei Sphären}

Weiter offenbaren diese Bestimmungen ein Verständnis zweier getrennter Sphären mit ihren je eigenen Regeln: ${ }^{18}$ Das staatliche Handeln auf der einen Seite und das private Handeln auf der anderen. Aus verfassungsdogmatischer Sicht erübrigt es sich, von transformierter Staatlichkeit oder neuen Zwischenformen zu sprechen. ${ }^{19}$ Das Bild der zwei Sphären mag zwar irreführend sein, da die staatlichen und privaten Bereiche aufeinander einwirken, ${ }^{20}$ verfassungsrechtlich zutreffend ist es dennoch. Generell droht die verfassungsmässige Zweiteilung in staatliche und private Tätigkeiten vergessen zu gehen, wenn man Transformation und Dynamik der Staatlichkeit zu prägenden Prinzipien erhebt. ${ }^{21}$

\section{c. Staatstätigkeit als Erfüllung staatlicher Aufgaben}

Die Frage danach, was der Staat ist, verdichtet sich also zur Frage, wann eine Tätigkeit staatlich ist. Die Verfassung gibt darauf eine klare Antwort: Staatlich ist eine Tätigkeit dann, wenn sie die Erfüllung staatlicher Aufgaben bezweckt. Dies ergibt sich zum Teil direkt aus dem Wortlaut, ${ }^{22}$ andernorts zeigt dies die

\footnotetext{
18 HALLER/KÖLZ/GÄCHTER, Allgemeines Staatsrecht, \$3.

19 In diese Richtung etwa BÖCKENFÖRDE, Sozialstaatliche Demokratie, S. 225.

20 So TSCHANNEN, Staatsrecht, \$1 Rz. 21.

21 EICHENBERGER, Leistungsstaat, S. 58, der Verbindlichkeit und Geltung bedroht sieht, wenn die stabilisierende Kraft des Staats einem «Kult der Dynamik» geopfert wird.

22 Art. 5a, 6 und 35 Abs. 2 BV.
} 
Auslegung des Geltungsbereichs einer Bestimmung. ${ }^{23}$ Dabei ist jeweils die Staatlichkeit der Aufgabe ausschlaggebend, nicht die staatliche Erfüllungsweise.

Die Grundsätze von Art. 5 BV richten sich an alle Staatsorgane, auch an den privatrechtlich handelnden Staat sowie an Private, sofern ihnen Staatsaufgaben übertragen sind. ${ }^{24}$ Die Grundrechte gelten ebenfalls unabhängig von den Erscheinungsformen der staatlichen Tätigkeit.25 Auch bei der Staatshaftung hängt alles an der Aufgabe. ${ }^{26}$ Staatlicher Verantwortlichkeit unterstehen auch Private, «insoweit sie unmittelbar mit öffentlich-rechtlichen Aufgaben des Bundes betraut sind ${ }^{27}$. Die schädigende Handlung erfolgt «in Ausübung amtlicher Tätigkeiten» gemäss Art. 146 BV, wenn sie «in einer sachlichen Beziehung zum Aufgabenbereich der fehlbaren Person stehen»28.

Im Versuch, Ordnung zu schaffen, konstatiert RÜTSCHE ganz allgemein:

«Die Sphären des Staates auf der einen und der Gesellschaft sowie Wirtschaft auf der anderen Seite sind im Recht funktional getrennt: Wer im Auftrag des Gesetzgebers öffentliche Aufgaben erfüllt, gehört zum Staat, alles andere Handeln ist privates Handeln.»29

\section{Staatsaufgaben}

Die Staatsdefinition verlagert sich somit auf eine Bestimmung der staatlichen Aufgaben. Wie im Folgenden zu zeigen ist, zeichnen sich Staatsaufgaben durch die Inpflichtnahme eines Akteurs auf Wegen des positiven Rechts aus. Mit anderen Worten liegt eine Staatsaufgabe immer dann vor, wenn eine Tätigkeit einer Person oder Organisation per Verfassung oder Gesetz derart übertragen wird, dass eine Pflicht zur Ausübung dieser Tätigkeit entsteht.

23 In diesem Sinn etwa bei der Gesetzmässigkeit SCHINDLER, St. Galler Kommentar, Art. 5 Rz. 28; ebenso bei der StaatshaftungJAAG/HÄNNI, St. Galler Kommentar, Art.146 Rz. $20,23$.

24 BIAGGINI, Kommentar BV, Art. 5 Rz. 6; EPINEY, Basler Kommentar, Art. 5 Rz. 33; SCHINDLER, St. Galler Kommentar, Art. 5 Rz. 28.

25 BIAGGINI, Kommentar BV, Art.35 Rz.9, 13; HANGARTNER, Grundrechtsbindung, S. 517; KÄLIN/LIENHARD/WYTTENBACH, Auslagerung, S.63. So auch das Bundesgericht in ständiger Rechtsprechung BGE 139 I 306 E.3.2.2 S.311; 138 I 289 E.2.3 S. 292; 138 I 274 E. 2.2.1 S. 281. Zur Grundrechtsbindung eingehender hinten \$3/II.6.b.

26 BGE 107 Ib 5E.1S.6.

27 Art.1 Abs.1Bst.f VG.

28 BIAGGINI, Kommentar BV, Art.146 Rz.7.

29 RÜTSCHE, Öffentliche Aufgaben, S. 162. Ferner auch RUCH, Informalisierung, S. 506. 


\section{a. Positivrechtliche Zuweisung}

Staatsaufgaben sind «Tätigkeitsfelder, die kraft Verfassung und Gesetz dem Staat zugewiesen sind».30 Entscheidend ist also erstens die positiv-rechtliche Zuweisung. ${ }^{31}$

Hier wird deutlich, dass die Verfassung das staatliche Handeln grundlegend anderen Regeln unterstellt als das private. Der Staat braucht zunächst eine rechtliche Ermächtigung, bevor er tätig werden darf, während Private grundsätzlich alles tun dürfen, was ihnen nicht ausdrücklich verboten ist. ${ }^{32}$ Dies ist Ausfluss des Gesetzmässigkeitsprinzips, wonach es «für das Gemeinwesen keinen rechtsfreien Raum»33 gibt.

Wofür der Staat zuständig ist, bestimmt sich also nicht ein für alle Mal abstrakt, sondern aus je einzelnen Aufgabenzuweisungen und deren Konkretisierung im positiven Recht. Kein anderer Massstab bestimmt, welche Aufgaben dem Staat zufallen. Entscheidend ist alleine die «rechtliche Überantwortung an den Staat» ${ }^{34}$. Insbesondere gibt es kein ordnendes Kriterium, das bestimmt, welche Aufgabe dem Staat zugewiesen werden kann und welche nicht. Es liegt in der Macht der Staats zu entscheiden, wofür er zuständig sein soll und wofür nicht. 35

Es ist auch nicht zielführend, Staatsaufgaben über ihre Anbindung an Staatszwecke, Staatsziele oder öffentliche Interessen zu definieren. Die Aufgaben, die dem Staat tatsächlich zugewiesen sind, können zwar als Konkretisierung der Staatsziele aufgefasst werden, die wiederum die Staatszwecke konkretisieren. ${ }^{36}$ Allerdings bleibt für die Bestimmung der Staatsaufgaben alleine entscheidend, ob sie positivrechtlich dem Staat zugewiesen werden, und nicht, ob sie aufgrund dieses oder jenen Staatszwecks geboten sind. ${ }^{37}$

30 TSCHANNEN/ZIMMERLI/MÜLLER, Allgemeines Verwaltungsrecht, §1 Rz.14; TSCHANNEN, Staatsrecht, §7 Rz. 31. Ähnlich auch DAENIKER, Überführung staatlicher Aufgaben, S. 52; KIENER/KÄLIN/WYTTENBACH, Grundrechte, §4 Rz. 54.

31 RÜTSCHE, Öffentliche Aufgaben, S. 158; TANQUEREL, Droit administratif, Rz.4; UHLMANN, Gewinnorientiertes Staatshandeln, S. $230 \mathrm{f}$.

32 EPINEY, Basler Kommentar, Art. 5 Rz. 41.

33 TSCHANNEN/ZIMMERLI/MÜLLER, Allgemeines Verwaltungsrecht, \$19 Rz.1.

34 TSCHANNEN/ZIMMERLI/MÜLLER, Allgemeines Verwaltungsrecht, §1 Rz.14.

35 EICHENBERGER, Staatsaufgaben, S. 103; TSCHANNEN, Privatisierung, S. 239.

36 So etwa DAENIKER, Überführung staatlicher Aufgaben, S. 51, der den hier vertreten Ansatz «positivistisch» nennt und wegen der möglichen Uferlosigkeit staatlicher Aufgaben ablehnt.

37 Vgl. RUCH, Informalisierung, S. 509-512; und UHLMANN, Gewinnorientiertes Staatshandeln, S. 228-231, die Staatsaufgaben aus Zwecken, Zielen und öffentlichen Interessen ableiten, aber schlussendlich nicht um das entscheidende Kriterium der rechtlichen Zuweisung herumkommen. Anders RICHLI, Rechtsetzung, Rz.4, der viel auf das «Verhältnis fortschreitender Konkretisierung» dieser Begriffe gibt und eine deutliche Unterscheidung unterlässt, vgl. auch Rz.39. 
Eine beschränkende Bestimmung staatlicher Aufgaben ist auf diesem Weg jedenfalls nicht zu erreichen.

Schliesslich grenzt die positivrechtliche Aufgabenzuweisung die Erfüllung von Staatsaufgaben gegenüber der Verwaltungshilfe ab. Bei Letzterer leistet jemand «lediglich Teilbeiträge an die Erfüllung einer fremden Aufgabe»38. Diese Abgrenzung ist vor allem dort notwendig, wo Private mit der Erfüllung von Staatsaufgaben betraut werden. In der Form der Beleihung werden Private Träger eigener staatlicher Aufgaben. ${ }^{39}$ Wer aber bloss «nach Art einer Hilfskraft zur technischen Ausführung bestimmter Verwaltungsaufgaben eingespannt» ${ }^{40}$ wird, ist Verwaltungshelfer und nicht selbst Aufgabenträger.

Ein Beispiel zu dieser Abgrenzung: Gestützt auf seine aussenpolitischen Befugnisse trifft der Bund Massnahmen der internationalen Entwicklungszusammenarbeit und humanitären Hilfe. ${ }^{41}$ Die Entwicklungszusammenarbeit kann gemäss einschlägigem Bundesgesetz unter anderem in der Form der «Förderung des Einsatzes privatwirtschaftlicher Mittel» ${ }^{42}$ erfolgen. Gemäss der dazugehörigen Verordnung schafft der Bund «zur Förderung des Einsatzes privatwirtschaftlicher Mittel, namentlich von Investitionen in Entwicklungsländern, eine Gesellschaft in der Form einer privatrechtlichen Aktiengesellschaft.» ${ }^{43}$ Diese Gesellschaft, die SIFEM AG ${ }^{44}$, ist Trägerin dieser Aufgabe. Diese AG besorgt ihre Geschäfte allerdings nicht selbst; seit 2011 übernimmt die OBVIAM DFI AG mit Zustimmung des Bundesrats die Geschäftsführung der SIFEM AG. ${ }^{45}$ Diese zweite Aktiengesellschaft ist nicht selbst Trägerin der staatlichen Aufgabe, sie ist blosse Verwaltungshelferin.

\section{b. Pflicht zur Erfüllung}

Durch die Zuweisung einer Tätigkeit an den Staat wird diese noch nicht zur Staatsaufgabe. Zusätzlich ist erforderlich, dass die positivrechtlich überant-

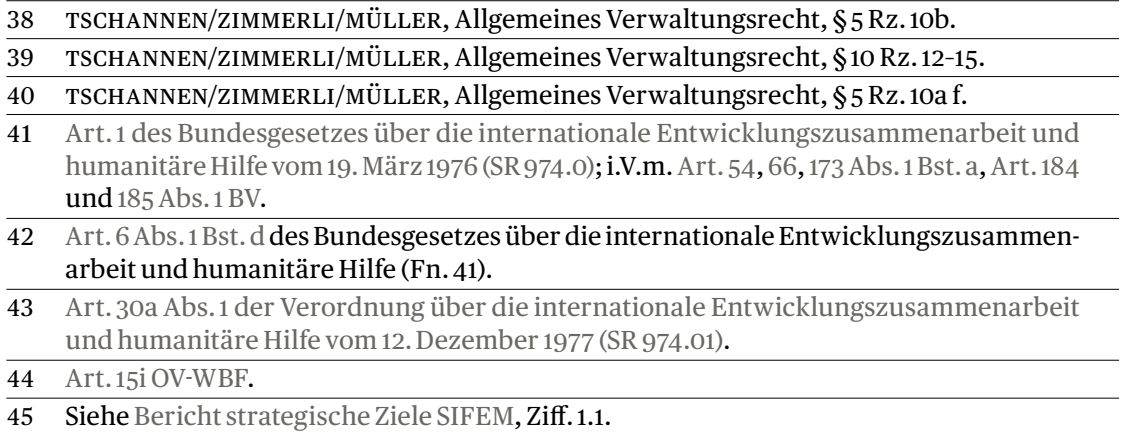


wortete Tätigkeit zwingend erfüllt werden muss; zur gesetzlichen Übertragung muss also eine gesetzliche Erfüllungspflicht treten. ${ }^{46}$

Dies ergibt sich schon aus der zwingenden Natur desöffentlichen Rechts. ${ }^{47}$ Für den Bund hält Art. 42 Abs. 1 BV zudem fest, dass ihm verfassungsrechtlich zugewiesene Aufgaben erfüllt werden müssen. ${ }^{48}$ Auch das Bundesgericht verdeutlicht, dass Staatsaufgabe nur ist, was verpflichtend zu erfüllen ist: Die Tätigkeiten der Post, welche (nach damaligem Postrecht) Wettbewerbsdienste sind, können keine «staatliche Aufgabe» im Sinne von Art. 35 Abs. 2 BV sein, weil «die Post in diesem Bereich - im Gegensatz zum Universaldienst - nicht verpflichtet ist, die entsprechenden Dienstleistungen zu erbringen.» ${ }^{49}$

Dass die Erfüllungspflicht entscheidend für das Vorliegen einer Staatsaufgabe ist, zeigt sich insbesondere bei der Beleihung, bei der Privaten die Erfüllung staatlicher Aufgaben übertragen wird. ${ }^{50}$ Das verpflichtende Element der Aufgabenzuweisung unterscheidet Staatsaufgaben von Konzessionen und Finanzhilfen.

Privaten kann mittels Konzession erlaubt werden, eine im staatlichen Monopolbereich liegende Tätigkeit auszuüben. ${ }^{51}$ Dadurch werden sie allerdings nicht zum Träger staatlicher Aufgaben. ${ }^{52}$ Die Konzession überträgt ihnen die Befugnis, tätig zu werden, ohne sie allerdings in die Pflicht zu nehmen. Anders verhält es sich mit den sogenannten Konzessionen des öffentlichen Dienstes: Hierbei ist die Konzession das Mittel, um Private zu verpflichten, eine staatliche Aufgabe zu erfüllen. Es handelt sich dann um eine Beleihung durch Konzession; doch eine Konzession alleine ist keine Inpflichtnahme.

Wie bei der Konzession fehlt die Erfüllungspflicht auch bei der Entrichtung von Finanzhilfen nach dem Subventionsgesetz (SuG). ${ }^{53}$ Diese Abgrenzung ist erforderlich, weil Subventionen mit Staatsaufgaben einhergehen können, aber vom einen nicht zwingend auf das andere zu schliessen ist. Finanzhilfen

46 ABEGG/FREI, Wirtschaftsfreiheit, S. 292; ADLER/TÖNZ, Privatisierung, S. 358; DUBEY/ ZUFFEREY, Droit administratif général, Rz.16; BAUMANN, Wettbewerbsverzerrungen, Rz.60; KIENER/KÄLIN/WYTTENBACH, Grundrechte, § 4 Rz. 54; RÜTSCHE, Öffentliche Aufgaben, S.159 f. Vgl. zudem JAAG, Staats- und Beamtenhaftung, Rz. 226, wonach nur die Aufgabenübertragung mit Erfüllungsauftrag die Staatshaftung begründet.

47 TSCHANNEN/ZIMMERLI/MÜLLER, Allgemeines Verwaltungsrecht, §30 Rz. 26.

48 SCHWEIZER, St. Galler Kommentar, Art. 42 Rz. 9.

49 BGE 129 III 35 E. 5.2 und 5.3 S.40 f.

50 EGLI, Beleihung Privater, S.195-200; TSCHANNEN/ZIMMERLI/MÜLLER, Allgemeines Verwaltungsrecht, §10 Rz.12-15.

51 TSCHANNEN/ZIMMERLI/MÜLLER, Allgemeines Verwaltungsrecht, §10 Rz.14, auch zum Folgenden.

52 BIAGGINI, Kommentar BV, Art.35 Rz.13.

53 Näher dazu RÜTSCHE, Öffentliche Aufgaben, S.159. 
i.S.v. Art. 3 Abs. 1 SuG sind nämlich geldwerte Vorteile für selbstgewählte Aufgaben - im Unterschied zu Abgeltungen nach Abs. 2 derselben Bestimmung, welche Lasten mildern oder ausgleichen, die aus der Erfüllung von verpflichtend übertragenen Aufgaben folgen.

\section{c. Verwaltungsaufgaben und andere Staatsaufgaben}

Staatsaufgaben werden bisweilen mit Verwaltungsaufgaben gleichgesetzt. ${ }^{54}$ Dies entspricht der negativen Definition der Verwaltung als diejenige Staatstätigkeit, die übrig bleibt, wenn Rechtsetzung und Rechtsprechung ausgespart werden. ${ }^{55}$ Eine strikte Trennung in Verwaltungs- und andere Aufgaben des Staats ist nicht erforderlich, um die Fragestellungen dieser Arbeit zu beantworten. Untersucht wird die Privatisierung jeder Tätigkeit, die dem Staat verpflichtend zur Erfüllung übertragen ist, unabhängig davon, welcher Staatsfunktion sie zufällt. Hauptsächlich wird die Privatisierung die Verwaltungstätigkeit betreffen, ${ }^{56}$ allerdings können damit insbesondere auch Rechtsetzungsaufgaben verbunden sein.

\section{d. Verhältnis zu öffentlichen Aufgaben}

Ein Teil der Lehre verwendet die Begriffe Staatsaufgabe und öffentliche Aufgabe synonymisch. ${ }^{57}$ Und auch das Bundesgericht scheint die Begriffe für austauschbar zu halten. 58

Gemäss einer anderen Auffassung besteht aber eine inhaltliche Differenz zwischen staatlichen und öffentlichen Aufgaben: Demnach seien Staatsaufgaben diejenigen öffentlichen Aufgaben, die der Staat selber ausführe. Öffentliche Aufgaben, die «von ausserstaatlichen Stellen aufgrund von Aufgabenübertragungen erfüllt» 59 werden, seien keine Staatsaufgaben.

Es ist nicht sinnvoll, diesen Unterschied mit abweichenden Aufgabenbegriffen nachzuzeichnen. Ob eine Aufgabe verwaltungsintern oder durch ausgelagerte Aufgabenträger erfüllt wird, ist keine Frage der Aufgabe selbst,

54 Ohne weiteren Kommentar etwa RÜTSCHE, Öffentliche Aufgaben, S.153. Diese Einschränkung macht auch die Verfassung bezüglich Aufgabenübertragungen in Art. 178 Abs. 3 BV.

55 HÄFELIN/MÜLLER/UHLMANN, Allgemeines Verwaltungsrecht, Rz.13 f.; TSCHANNEN/ ZIMMERLI/MÜLLER, Allgemeines Verwaltungsrecht, §1 Rz.9.

56 Ähnlich auch TSCHANNEN, Privatisierung, S. 211. Die Privatisierung ganz auf Verwaltungsaufgaben beziehend JAAG, Dezentralisierung und Privatisierung, S. $25 \mathrm{f}$.

57 RÜTSCHE, Öffentliche Aufgaben, S. 153; UHLMANN, Gewinnorientiertes Staatshandeln, S. 230. BAUMANN begründet die synonyme Verwendung ausdrücklich, Wettbewerbsverzerrungen, Rz. 63 .

$58 \quad$ BGE 140 I 338 E.6 S.343 und E.7 S.346.

59 RICHLI, Rechtsetzung, Rz. 8. Ähnlich auch HÄNER, Grundrechtsgeltung, S.1150; UEBERSAX, Privatisierung, S. 395. 
sondern ihrer Erfüllung (siehe zu den Organisationsformen hinten \$4/II). Deshalb vermeidet diese Arbeit den Begriff der öffentlichen Aufgabe, um den Eindruck zu vermeiden, damit sei etwas anderes als eine Staatsaufgabe bezeichnet. ${ }^{60}$ Ausserdem ist «staatliche Aufgabe» ein Begriff der Verfassung, ${ }^{61}$ während «öffentliche Aufgabe» darin keine Erwähnung findet.

\section{Die Erfüllung von Staatsaufgaben}

Staatstätigkeit ist definiert als die Erfüllung staatlicher Aufgaben. Die Aufgaben geben vor, was zu tun ist - welche Leistungen erbracht werden müssen und in welcher Qualität. Diesbezüglich gilt die verfassungsrechtliche Dichotomie zweier abgetrennter Sphären: Tätigkeiten, die dem Staat positivrechtlich und verpflichtend zur Erfüllung übertragen sind, sind staatlich, alles andere ist privates Handeln.

Die Modalitäten der Erfüllung bestimmen, wie die Aufgabe tatsächlich erfüllt wird. Es handelt sich quasi um die Bürgerfront der Aufgabenerfüllung: Wie werden die Leistungen den Bürgern gegenüber erbracht, wie treten die Verwaltungsträger auf, um ihre Aufgaben zu erfüllen? Was im Einzelnen zur Aufgabenerfüllung dazugehört, wird hinten in \$ 4/I herausgearbeitet; dem besseren Verständnis halber folgt schon hier eine kurze Übersicht:

- Es stellt sich zunächst die organisatorische Frage, was für eine Organisationseinheit für die Aufgabe zuständig ist. Soll es eine weisungsgebundene Einheit der Zentralverwaltung sein oder etwa eine autonome Körperschaft mit eigener Rechtspersönlichkeit?

- Die als Aufgabe definierten Leistungspflichten können mit verschiedenartigen Instrumenten erbracht werden. Es können Verfügungen erlassen oder Verträge geschlossen werden. Verwaltungseinheiten können mit echten Privaten Kooperationen eingehen oder sie am Markt mit ihren Leistungen konkurrenzieren.

- Sodann stehen verschiedene Möglichkeiten zur Finanzierung der Staatstätigkeit offen. Decken allgemeine Steuermittel die Kosten der Aufgabenerfüllung? Oder werden diese gänzlich auf die Nutzer oder Verursacher überwälzt?

Auf der Erfüllungsseite passt das Bild der zwei voneinander abgegrenzten Sphären nicht, es ist vielmehr ein gradueller Übergang entlang eines Spek-

60 Vom Begriff der öffentlichen Aufgabe abratend auch HÄFELIN/MÜLLER/UHLMANN, Allgemeines Verwaltungsrecht, Rz. 25; TSCHANNEN/ZIMMERLI/MÜLLER, Allgemeines Verwaltungsrecht, \$1 Rz. 14.

$61 \quad$ Art. 5a, 35 Abs. 2 und Art. 43a BV. 
trums. ${ }^{62}$ Für jeden Aspekt mag es zwar klassisch staatliche Modalitäten geben. Auch wenn davon abgewichen wird, bleibt die fragliche Tätigkeit staatlich, solange staatliche Aufgaben erfüllt werden. ${ }^{63}$

\section{Veranschaulichung anhand konkreter Staatsaufgaben}

Staatsaufgaben sind diejenigen Tätigkeiten, die einer Person oder Organisation positivrechtlich und verpflichtend zur Erfüllung übertragen sind. Diese Definition erlaubt es, Staatsaufgaben konkret zu benennen. Dies sollen die folgenden drei Beispiele veranschaulichen.

- Im Bereich des Postwesens beauftragt Art. 92 Abs. 2 Satz 1 BV den Bund, «für eine ausreichende und preiswerte Grundversorgung mit Post- und Fernmeldediensten in allen Landesgegenden» zu sorgen. Diesen breit gefassten Aufgabenbereich konkretisieren dann Gesetz und Verordnung weiter, sodass sich einzelne zu erbringende Dienstleistungen ergeben. Das Gesetz hält etwa fest, welche Arten von Sendungen zum Grundversorgungsauftrag gehören, ${ }^{64}$ in welcher Häufigkeit die Zustellung erfolgen muss ${ }^{65}$ und welche Abdeckung mit Poststellen und öffentlichen Briefeinwürfen notwendig ist. ${ }^{6}$ Per Verordnung werden die einzelnen Dienstleistungen dann spezifiziert und etwa festgehalten, dass die Post Briefe bis ein Kilogramm und Pakete bis 20 Kilogramm ins In- und Ausland befördern muss. ${ }^{67}$

- Im Bereich der Eisenbahnen weist die Verfassung dem Bund zunächst nur die Gesetzgebung auf diesem Gebiet zu. ${ }^{68}$ Das Gesetz über die Schweizerischen Bundesbahnen schafft sodann einzelne Aufgaben, indem es die SBB etwa zur Bereitstellung der notwendigen Infrastruktur verpflichtet. ${ }^{69}$ Den Umfang dieser Infrastruktur beschreibt das Eisenbahngesetz

62 So auch JAAG, Privatisierung Länderbericht Schweiz, S. 293 f. Bezogen auf organisatorische Aspekte der Aufgabenerfüllung DUBEY/ZUFFEREY, Droit administratif général, Rz.1406. Bezogen auf verschiedene «Regulierungstypen» zwischen staatlicher Rechtsetzung und privater Selbstregulierung auch HETTICH, Kooperative Risikovorsorge, Rz.512.

63 Anders RHINOW, Bundesverfassung 2000, S.153, der staatlichen Aufgaben ihren «Staatscharakter» abspricht, wenn sie "gestützt auf eine gesetzliche Ermächtigung [...] an Private delegiert werden".

\begin{tabular}{ll}
\hline 64 & Art.14 Abs.1 PG. \\
\hline 65 & Art.14 Abs.3 PG. \\
\hline 66 & Art.14 Abs. PG. \\
\hline 67 & Art.29 Abs.2 Bst. a VPG. \\
\hline 68 & Art. 87 BV. \\
\hline 69 & Art. 3 Abs.1 SBBG.
\end{tabular}


dann im Einzelnen und überantwortet etwa auch Betrieb und Unterhalt der Publikumsanlagen dem Staat. ${ }^{70}$ Daraus ergibt sich dann etwa, dass auch die Bewirtschaftung von Bahnhofswänden, einschliesslich ihrer Vermietung zu Werbezwecken, eine staatliche Aufgabe ist. ${ }^{71}$

- Aufgabenzuweisungen müssen nicht zwingend so systematisch erfolgen wie in diesen zwei Beispielen, wo zunächst ein Aufgabengebiet ausgeschieden, dieses in einzelne Teile gegliedert und sodann zu einzelnen Leistungen konkretisiert wird. Gerade über direktdemokratische Interventionen können detaillierte Einzelaufgaben dem Staat übertragen werden. So sieht etwa Art. 75b BV sehr spezifisch vor, dass der Bund Zweitwohnungen nach einer bestimmten Berechnungsart auf einen festgesetzten Anteil beschränken soll.

\section{Einschub: Zuordnung verschiedener Stufen staatlicher Verantwortung zur Staatsdefinition}

Staatliche Aufgaben werden oft in Verbindung mit staatlicher Verantwortung gebracht. Staatsaufgaben seien «dem Staat zurechenbare Verantwortung» 72 .

Die in dieser Arbeit verwendeten Definitionen von Staatlichkeit und Staatsaufgaben kommen ohne Verantwortungsbegriffe aus. Dieser Einschub soll immerhin einen Überblick über die gängigsten Varianten staatlicher Verantwortung liefern und diese Begriffe in die verwendeten Definitionen einpassen.

Die verschiedenen Verantwortungsarten unterscheiden sich danach, ob eine staatliche Aufgabe vorliegt und wer diese zu erfüllen hat. Liegt die Erfüllungsverantwortung beim Staat, so ist der Staat selbst verantwortlich, dass eine Staatsaufgabe tatsächlich erfüllt wird. ${ }^{73}$ Überträgt der Staat jedoch die Aufgabenerfüllung auf Private, so wird die Erfüllungs- zur Gewährleistungsverantwortung. ${ }^{74}$ Beide Verantwortungstypen beziehen sich also auf staatliche Aufgaben, sie unterscheiden sich lediglich in den Modalitäten der Aufgabenerfüllung. ${ }^{75}$ Erfüllungs- und Gewährleistungsverantwortung können

\footnotetext{
$70 \quad$ Art. 62 Abs.1 Bst. d und Art. 63 EBG.

71 Diese Aufgabe war Gegenstand des BGE 138 I 274, insb. E.1.4 S. 278-280.

72 TSCHANNEN, Aufgabenteilung, S.150. Ähnlich für das deutsche Recht auch THOMA, Selbstregulierung, S. 58.

73 HÄNER, Grundrechtsgeltung, S.1149; RÜTSCHE, Leistungsaufträge, S. 74; TSCHANNEN, Privatisierung, S. 216.

74 KÄLIN/LIENHARD/WYTTENBACH, Auslagerung, S.62 f.; TSCHANNEN, Privatisierung, S. 215 .

75 MÜLLER, Schlank, S. 165. Anders HÄNER, Grundrechtsgeltung, S. 1149, die von Gewährleistungspflicht spricht, wenn keine Pflicht zur Aufgabenerfüllung besteht und der Staat lediglich die selbstgewählte Tätigkeit mit Anforderungen belegt (z.B. mit Bewilligungen).
} 
in den Oberbegriff Aufgabenverantwortung eingeordnet werden, wobei diesem Begriff wenig dogmatische Aussagekraft zukommt: Er sagt nur aus, dass eine Staatsaufgabe vorliegt, nicht aber, ob deren Erfüllung dem Staat selbst oder anderen Aufgabenträgern obliegt.

Vor allem die deutsche Lehre hat darüber hinaus Verantwortungen geschaffen, die den Staat treffen, auch wenn keine Staatsaufgaben vorliegen. Regulierungs-, Kontroll-, Überwachungs- und Steuerungsverantwortung bezeichnen Fälle, in denen der Staat gesetzliche Rahmenbedingungen für private Tätigkeiten setzt. ${ }^{76}$ Dazu kommt die Auffangverantwortung, womit die Möglichkeit des Staats bezeichnet wird, eine private Tätigkeit an sich zu ziehen und zur Staatsaufgabe zu machen. ${ }^{77}$

\section{Zwischenfazit: Der funktionelle Staatsbegriff des Verfassungsrechts}

Die Bundesverfassung definiert den Staat über seine Tätigkeit: die Erfüllung von Staatsaufgaben. Staatsaufgaben liegen immer dann vor, wenn einer Person oder Organisation eine Tätigkeit positivrechtlich und verpflichtend zur Erfüllung übertragen wird. Diese Staatsdefinition erlaubt eine präzise Festlegung des Staatsumfangs und eine Abgrenzung gegenüber privaten Tätigkeiten. Der Staat ist die Gesamtheit der Tätigkeiten, die der Erfüllung staatlicher Aufgaben dienen. Ein Rückgriff auf Staatszwecke und -ziele sowie öffentliche Interessen ist nicht nötig, um den Staat zu definieren. Ebenso wenig sind Verantwortungsbegriffe dazu geeignet, den Staat einzugrenzen; sie können beschreibend hinzugenommen werden, leisten aber keinen eigenen Beitrag zur Staatsdefinition.

76 Siehe dazu HERMES, Staatliche Infrastrukturverantwortung, S. 152-156; THOMA, Selbstregulierung, S. 58, 472; vosSKuHLE, Verantwortungsteilung, S. 48, 74-76; vosSKUHLE, Beteiligung Privater, S. 285. Zur Kontrollverantwortung auch in der Schweizer Lehre HÄNER, Grundrechtsgeltung, S.1149.

77 THOMA, Selbstregulierung, S. 57-62 


\section{Privatisierung}

\section{Ein facettenreicher Prozess ohne scharfe Konturen?}

Wenn sich der Staat klar definieren lässt, sollte dies auch für die Privatisierung möglich sein. Doch die rechtswissenschaftliche Literatur lässt die erforderliche Klarheit oft vermissen. Es wird hingegen resigniert festgehalten, die Privatisierung sei ein «Begriff von geradezu lästiger Konturlosigkeit» ${ }^{78}$, oder darauf verzichtet, «auf die verschiedenen Arten und Qualifikationen des schillernden Begriffes der Privatisierung» 79 einzugehen. Bisweilen bedient sich die Literatur Allgemeinplätzen wie etwa, dass die Privatisierung ein «vielschichtiger und vielgestaltiger Prozess der Umverteilung vom Staat zur Gesellschaft» ${ }^{80}$ sei. Die Privatisierung sei ein kaum fassbares Phänomen, was dogmatische Definitionen erübrige. 81

Müssen wir uns also damit begnügen, die Privatisierung als einen facettenreichen Prozess mit unscharfen Konturen zu beschreiben, dessen dogmatische Einordnung müssig ist? Wie im Folgenden zu zeigen ist, lässt sich die Privatisierung zumindest dann scharf umreissen, wenn sie den Bestand der staatlichen Aufgaben reduziert (siehe zur Aufgabenprivatisierung hinten 4.a). Die Konturen lassen sich aber nicht vollends schärfen, soweit lediglich die Erfüllungsseite privatisiert wird (siehe hinten 4.b und 4.c).

\section{Verhältnis von Privatisierung und Entstaatlichung}

Eine erste Annäherung an die Privatisierung ist zu erreichen, wenn man sich ihrem naheliegenden Begriffspartner, der Entstaatlichung, annimmt. So ist zunächst davon auszugehen, «die Privatisierung bezweck[e] die Entstaatlichung gesellschaftlicher Aufgaben» 82 bzw. bedeute sie «die Entstaatlichung bisheriger Verwaltungsverantwortungen» 83 .

\footnotetext{
78 TSCHANNEN, Privatisierung, S. 210, ohne die Hervorhebung im Original.

79 POLEDNA, Öffentliche Aufgaben, S.11.

80 MÜLLER/JENNI, Privatisierung, S. 1082. Ähnlich auch TSCHANNEN, Privatisierung, S. 211. Vgl. aus der deutschen Verwaltungsrechtslehre auch MAURER/WALDHOFF, Allgemeines Verwaltungsrecht, §23 Rz. 67.

81 So etwa MÜLLER/JENNI, Privatisierung, S.1071f.

82 HÄFELIN/MÜLLER/UHLMANN, Allgemeines Verwaltungsrecht, Rz.1867, ohne die Hervorhebung im Original.

83 TSCHANNEN/ZIMMERLI/MÜLLER, Allgemeines Verwaltungsrecht, \$11 Rz.1, ohne die Hervorhebungen im Original; früher noch als «Entstaatlichung bisheriger Verwaltungsaufgaben» bezeichnet, TSCHANNEN, Privatisierung, S. 211.
} 
Rein begrifflich sollten sich Privatisierung und Entstaatlichung entsprechen. Mehr «Privates» sollte weniger «Staatliches» bedeuten. Ausgehend vom hier verwendeten Staatsbegriff muss dies aber relativiert werden. Die Staatsqualität einer Tätigkeit hängt daran, ob sie in Erfüllung einer staatlichen Aufgabe ergeht. Als Privatisierung werden auch Prozesse beschrieben, die den Aufgabenbestand des Staats unberührt lassen (siehe zu den verschiedenen Arten hinten 5). So kann zum Beispiel ein Verwaltungsträger in eine privatrechtliche Form gegossen werden, ohne dass sich etwas an der Staatlichkeit der Aufgabe ändert, die er erfüllt. Oder das Handlungsinstrumentarium eines Verwaltungsträgers kann von hoheitlichen Mitteln auf konsensuale, privatrechtliche Verträge umgestellt werden. Entstaatlichung beschreibt also nicht nur ein Weniger an Staat im verfassungsrechtlichen Sinn, sprich die Verringerung des Aufgabenbestands, sondern auch die Erfüllung staatlicher Aufgaben in weniger staatstypischer Weise (siehe dazu hinten 4.c). Entstaatlichung - wie auch Privatisierung - deckt Phänomene ab, die den Aufgabenbestand des Staats betreffen, sowie solche, die nur bei der Erfüllung dieser Aufgaben ansetzen.

Dass Privatisierung nicht zwingend Entstaatlichung bedeutet, hängt daran, dass das Bild zweier abgetrennter Sphären, einer staatlichen und einer privaten, nur auf der Aufgaben-, nicht aber auf der Erfüllungsseite zutrifft. Die Art und Weise, wie Staatsaufgaben erfüllt werden können, entspricht einem Spektrum mit vielen Zwischenstufen. Hingegen gibt es eine klare Trennlinie zwischen dem Staat und der privaten Sphäre: das Vorliegen staatlicher Aufgaben.

\section{Das Private als Begriff der Bundesverfassung}

Da dem vermeintlichen Gegenbegriff Entstaatlichung keine klaren Konturen für die Privatisierung zu entnehmen sind, stellt sich die Frage, ob Begriffsklarheit aus dem Verfassungsverständnis der privaten Sphäre zu gewinnen ist.

Privatisierung an sich ist kein Begriff der Verfassungssprache, das Private allerdings wird in vierzehn Artikeln erwähnt. Diese Normstellen sollen im Folgenden daraufhin untersucht werden, ob sie Rückschlüsse auf den Prozess der Verschiebung von der staatlichen in die private Sphäre zulassen. Im Folgenden werden die verschiedenen Erwähnungen des Privaten zu drei Konstellationen gegliedert. 


\section{a. Zweiteilung in private und andere Akteure}

An vier Stellen unterscheidet die Verfassung private und andere Akteure:

- Art. 5 Abs. 3 BV: Private müssen ebenso wie staatliche Organe den Grundsatz von Treu und Glauben beachten;

- Art. 41 Abs.1 BV: Die Sozialziele, für die Bund und Kantone verantwortlich sind, ergänzen die persönliche Verantwortung und die private Initiative;

- Art. 83 Abs. 2 BV: Bau, Betrieb und Unterhalt von Nationalstrassen kann vom Bund an öffentliche, private oder gemischte Trägerschaften übertragen werden;

- Art. 196 Ziff. 3 Abs. 2 Bst. f: Der Bundesrat kann für Eisenbahngrossprojekte eine ergänzende Finanzierung durch Private sowie internationale Organisationen vornehmen.

An zwei weiteren Stellen teilt die Verfassung die Akteure nach ihrer Rechtsgrundlage in zwei Gruppen:

- Art. 96 Abs. 2 Bst. a BV: Die wettbewerbspolitischen Massnahmen des Bundes treffen Unternehmen und Organisationen des privaten wie des öffentlichen Rechts gleichermassen;

- Art. 178 Abs. 3: Verwaltungsaufgaben können an Organisationen und Personen des öffentlichen wie des privaten Rechts übertragen werden.

Diese sechs Stellen lassen einige Schlussfolgerungen zu. Erstens wird darin das Bild der zwei Sphären bestätigt: Die Verfassung kennt nur staatliche oder private Akteure und unterscheidet keine weiteren Kategorien. Die Trennlinie zwischen den Sphären bleibt aber funktionell, also an der Aufgabe hängend; diesen Stellen ist nicht zu entnehmen, die Verfassung gehe von einem personellen Begriffsverständnis des Staats aus.

Art. 41 Abs. 1 BV führt neben dem Privaten auch das Persönliche auf. Dies lässt darauf schliessen, dass «privat» einen überindividuellen, gesellschaftlichen Aspekt hat, wobei das Persönliche nur eine einzelne Person betrifft. 84 An den anderen Stellen scheint das Private sowohl individuelle wie überindividuelle Aspekte aufzuweisen. Daher erübrigt es sich, neben dem Staatlichen und dem Privaten eine dritte Kategorie aus der Verfassung abzuleiten.

Diese Textstellen belegen zweitens, dass für staatliche Akteure andere Regeln gelten als für private. Auch Art. 5 Abs. 3 BV ändert nichts daran, obwohl er «[s]taatliche Organe und Private» in einem Atemzug nennt (siehe dazu hinten \$3/II.5.a). Private werden durch diese Bestimmung «nicht [...] gleicher-

84 In diese Richtung auch GÄCHTER/WERDER, Basler Kommentar, Art. 41 Rz. 15 
massen wie der Staat auf ein widerspruchsloses Handeln verpflichtet»85, vielmehr ist die Bindung an den Vertrauensgrundsatz asymmetrisch. Dies geht darauf zurück, dass im nichtstaatlichen Bereich die Privatautonomie gilt. ${ }^{86}$

Und schliesslich wird die Zweiteilung in eine staatliche und eine private Sphäre aufgeweicht, wenn es um die Erfüllung staatlicher Aufgaben geht. Kooperationen, Mischformen und die Einbeziehung Privater in die Erfüllung staatlicher Aufgaben sind von vornherein vorgesehen. Wie vorne schon festgestellt (I.3), gilt das Zwei-Sphären-Bild für die Aufgabenseite. Was die Erfüllung betrifft, ist vielmehr von einem Spektrum auszugehen.

\section{b. Lebensbereich Privater an sich}

An fünf Stellen erwähnt die Verfassung den Lebensbereich Privater, um diesen besonders zu schützen oder besonders zu fördern:

- Art.13 BV: Der grundrechtliche Schutz der Privatsphäre und des Privatlebens;

- Art.35 Abs. 3 BV: Grundrechte sollen auch unter Privaten wirksam werden;

- Art. 95 Abs. 3 BV: Die Bestimmungen über Vergütungen in Aktiengesellschaften bezwecken u.a. den Schutz von Privateigentum;

- Art.108 Abs.1BV: Eigenbedarf Privater bei der Förderung des Wohnungsbaus und des Erwerbs von Wohnungs- und Hauseigentum;

- Art. 180 Abs. 2 BV: Private Interessen, die der öffentlichen Information über die Tätigkeiten des Bundesrats entgegenstehen können.

Diese fünf Artikel zeigen erstens, dass es Bereiche des Privaten gibt, die dem Staat nur unter besonderen Bedingungen zugänglich sind - die Freiheitsrechte des Individuums. In einer anderen Hinsicht scheidet die Verfassung Bereiche des Privaten aus, denen staatlicher Schutz oder staatliche Förderung zukommen soll.

Ausserdem ist diesen Artikeln wiederum das Zwei-Sphären-Bild zu entnehmen, indem das Staatliche als (zum Teil nur impliziertes) Gegenstück zum Privaten auftaucht: Der Staat muss Eingriffe in die Privatsphäre oder das Privatleben rechtfertigen. Die staatlichen Behörden sind dafür verantwortlich, dass Grundrechte auch unter Privaten wirksam werden. Der Staat erlässt besondere Vorschriften zum Schutz des privaten Eigentums, die in dieser

85 SCHINDLER, Rollenverteilung, Rz. 55. Ferner BIAGGINI, Kommentar BV, Art. 5 Rz. 24, der «zulasten Privater direkt sanktionierbare Handlungspflichten» aus Art. 5 Abs. 3 BV verneint.

86 Vgl. SCHINDLER, Rollenverteilung, Rz. 55, der dies in der «individuellen Freiheit» begründet sieht. 
Form das Staatseigentum nicht betreffen. Der Staat wird nicht gefördert, wenn er Wohnungsraum bauen oder erwerben will. Und der Staat muss auf private Interessen Rücksicht nehmen, wenn er die Öffentlichkeit über seine Tätigkeiten informiert.

Diese Bestimmungen lassen das Private also als einen vom Staat abgegrenzten Bereich erscheinen, der anderen Regeln unterliegt.

\section{c. Privatwirtschaft}

Das Private taucht schliesslich als Teil des verfassungsrechtlichen Wirtschaftsverständnisses auf. Vier Bestimmungen regeln Freiheit und Rahmenbedingungen der privaten Wirtschaft:

— Art. 27 Abs. 2 BV schützt den freien Zugang zu einer privatwirtschaftlichen Erwerbstätigkeit;

- Art. 95 Abs. 1 BV gibt dem Bund sodann das Recht, über diese Erwerbstätigkeit Vorschriften zu erlassen;

- Art. 94 Abs. 2 BV hält Bund und Kantone an, zusammen mit der Privatwirtschaft für Wohlfahrt und wirtschaftliche Sicherheit der Bevölkerung zu sorgen;

- Art. 94 Abs. 3 BV fordert den Staat auf, der privaten Wirtschaft günstige Rahmenbedingungen zu setzen.

Art. 98 Abs. 3 BV erwähnt zudem die Privatversicherungen als Unterkategorie der Privatwirtschaft.

Diese Normen suggerieren, dass staatliche Einmischung in die Wirtschaft von vornherein vorgesehen ist. Der viel beschworene Grundsatz der staatsfreien Wirtschaft erscheint in diesem Zusammenhang als ideologisch aufgeladene Wunschvorstellung (siehe dazu hinten \$ 3/III.4). Wirtschaftliche Betätigung ist nicht der privaten Sphäre allein vorbehalten.

Die Bestimmungen über die Privatwirtschaft dienen insgesamt nicht dazu, den Privatisierungsbegriff fassbarer zu machen. Ebenso wenig helfen sie, staatliche Tätigkeit e contrario aus der privatwirtschaftlichen Tätigkeit zu definieren. Dieser letztere Begriff ist als sachlicher Schutzbereich eines Grundrechts bedeutsam (Art. 27 Abs. 2 BV). So werden etwa Tätigkeiten in Ausübung einer Monopolkonzession von der privatwirtschaftlichen Tätigkeit ausgespart, 87 ohne dadurch zur Staatstätigkeit zu werden (es sei denn, die Konzession sei mit einer Erfüllungspflicht verbunden, siehe dazu vorne I.2.b). 


\section{d. Zwischenfazit: Keine Begriffsklarheit aus der Verfassung}

Vorab ist festzuhalten, dass die Verfassung keine eigentliche Definition des Privaten enthält. Dies zu erwarten, wäre auch verfehlt, regelt die Verfassung doch in erster Linie die Grundlagen des Staats. Folglich prägt die Verfassung auch kein eigenes Verständnis der Privatisierung. Sie verdeutlicht allerdings, dass das Bild zweier abgetrennter Sphären nuanciert gesehen werden muss. Eine klare Trennlinie zwischen der staatlichen und der privaten Sphäre wird nur bezüglich der Aufgaben gezogen, deren Erfüllung eine Tätigkeit dient. Diese trennscharfe Definition geht auf den funktionellen verfassungsrechtlichen Staatsbegriff zurück (siehe vorne I). Was die Aufgabenerfüllung betrifft, ist von einem Spektrum auszugehen mit typisch staatlichen Erfüllungsformen auf der einen und typisch privaten auf der anderen Seite.

\section{Zweiteiliger Privatisierungsbegriff für einen zweiteiligen Staatsbegriff}

Weder die Entstaatlichung als vermeintlicher Gegenbegriff noch das Verfassungsverständnis des Privaten tragen dazu bei, die Privatisierung begrifflich einzugrenzen. Die Privatisierung ist begrifflich schwer zu fassen; insoweit ist das vorne bei 1 angeführte Lamento der rechtswissenschaftlichen Literatur über die Konturenlosigkeit dieses Begriffs verständlich.

Ansatzweise kehren die Konturen in den Begriff zurück, wenn man ihn passend zum Staatsbegriff zweiteilt und dadurch leicht ausweitet. Staatstätigkeit ist die Erfüllung staatlicher Aufgaben. Dementsprechend können Privatisierungen danach eingeteilt werden, ob sie den Aufgabenbestand oder die Erfüllungsseite betreffen.

Vor dem Hintergrund des verfassungsrechtlichen Staatsbegriffs sind also lediglich zwei Privatisierungsarten zu unterscheiden: die Aufgabenprivatisierung und die Erfüllungsprivatisierung. Privatisierungen verringern entweder die Aufgaben, die der Staat erfüllen muss, oder sie setzen bei der Art und Weise der Erfüllung dieser Aufgaben an.

Diese Zweiteilung möglicher Privatisierungsprozesse ist der rechtswissenschaftlichen Literatur nicht unbekannt. Sie taucht dort im Begriffspaar echte/unechte, seltener auch formelle/materielle Privatisierung auf ( $v g l$. hinten 5.a). Die Lehre verwendet diese Zweiteilung allerdings nicht dazu, um die Privatisierung grundlegend zu strukturieren. ${ }^{88}$

88 Vgl. etwa BIAGGINI, Rechtsstaatliche Anforderungen, S.144-148. Ähnlich auch JAAG, Dezentralisierung und Privatisierung, S.24, der «den Umfang und die Art der Aufgabenerfüllung durch den Staat» unterscheidet, aber diese Zweiteilung nicht wieder aufgreift. 
Um die Privatisierung als verfassungsrechtliches Phänomen besser zu verstehen, wird der zweigeteilte Privatisierungsbegriff im Folgenden näher umschrieben.

\section{a. Aufgabenprivatisierung}

Eine Aufgabenprivatisierung liegt vor, wenn der Staat weder eine Erfüllungsnoch eine Gewährleistungsverantwortung für die Erfüllung der betreffenden Aufgabe trägt (siehe zu den Verantwortungsbegriffen vorne I.5). Erst wenn der Staat weder darauf verpflichtet ist, eine Aufgabe selbst zu erfüllen, noch zumindest bei Schlechterfüllung der Aufgabe einzugreifen, verliert die Aufgabe ihre staatliche Qualität. ${ }^{89}$ Die Aufgabenprivatisierung erlaubt damit eine klare Grenzziehung: Eine Aufgabe ist entweder staatlich oder nicht - Zwischenstufen, was die Verantwortungszuweisung für eine Aufgabe betrifft, gibt es nicht.

Die Aufgabenprivatisierung ist ein wohlausgebildeter Begriff der rechtswissenschaftlichen Literatur. Sie bedeute einen Verzicht auf die Erfüllung einer bisherigen Aufgabe, 90 einen Wechsel von «Staatsaufgaben auf wirkliche Private»91. Anders gewendet werde eine Aufgabe privatisiert, wenn der Staat sie «den Privaten überlässt und sich aus der Verantwortung zurückzieht»92.

Der Begriff der Aufgabenprivatisierung wird teilweise unpräzise verwendet. Mitunter wird die Aufgabenprivatisierung unpassend ausgedehnt auf die Auslagerung oder Abtretung der Aufgabenerfüllung, ohne dass die Verantwortung für die Aufgabe an sich abgegeben würde. ${ }^{93}$

Zur Wiederholung: Diese Arbeit spricht nur dann von Aufgabenprivatisierung, wenn sie den Bestand staatlicher Aufgaben an sich betrifft.

\section{b. Erfüllungsprivatisierung}

Eine Erfüllungsprivatisierung ist jede Privatisierung, die den Aufgabenbestand des Staats unberührt lässt. Anders gewendet liegt eine Erfüllungsprivatisierung immer dann vor, wenn nur die Art und Weise der Aufgabenerfüllung privatisiert wird. Bei der Aufgabenprivatisierung geht es um die

89 Anders HÄNER, Privatisierung, S. 424 f., die dem Staat auch bei der Aufgabenprivatisierung «die Gewährleistung, dass die Privaten die Aufgabe in einer bestimmten Art und Weise wahrnehmen", zuschreibt.

90 HÄFELIN/MÜLLER/UHLMANN, Allgemeines Verwaltungsrecht, Rz.1869.

91 TSCHANNEN, Privatisierung, S. 213.

92 HÄSLER, Geltung der Grundrechte, S. 46. Fast gleichlautend auch HÄNER, Privatisierung, S. 424.

93 TSCHANNEN/ZIMMERLI/MÜLLER, Allgemeines Verwaltungsrecht, §11 Rz.4. 
Substanz: Ist eine Tätigkeit staatlich oder privat? Die Erfüllungsprivatisierung beschlägt das Erscheinungsbild: Ähnelt staatliches Handeln privatem Handeln?94

Die Erfüllung staatlicher Aufgaben lässt sich in drei Aspekte auffächern: organisatorische, instrumentelle und finanzielle. Inwiefern diese Aspekte der Aufgabenerfüllung privatisiert werden können, wird hinten in \$4/II-IV detailliert behandelt. Um diese Begriffe hier schon zugänglich zu machen, folgen einige Beispiele:

- Erfüllungsprivatisierung in organisatorischer Hinsicht: Die Grundversorgung mit Postdiensten (eine staatliche Aufgabe nach Art.14 ff. PG; siehevorne I.4) obliegt einer Aktiengesellschaft, nicht länger einer öffentlichrechtlichen Anstalt.

- Erfüllungsprivatisierung in instrumenteller Hinsicht: Finanzintermediäre schliessen sich Selbstregulierungsorganisationen an, um ihre Sorgfaltspflichten nach dem Geldwäschereigesetz in selbst erlassenen Reglementen zu regeln (Art.24 f. GwG).

- Erfüllungsprivatisierung in finanzieller Hinsicht: Das Schweizerische Heilmittelinstitut Swissmedic erhebt Gebühren für die Überwachung des Arzneimittelverkehrs, um ihre Leistungen im öffentlichen Interesse zu finanzieren (Art. 65 HMG).

Der Begriff der Erfüllungsprivatisierung ist der Lehre nicht unbekannt, ${ }^{95}$ wenn er auch selten verwendet wird und fast durchgängig verkannt wird, welches Potential zur Vereinfachung der Diskussion um verschiedene Privatisierungsphänomene ihm innewohnt.

\section{c. Einschub: Schwierige Grenzziehung zwischen privatisierter und nichtprivatisierter Erfüllung}

Diese Arbeit untersucht die verfassungsrechtlichen Probleme und Spielräume bei der privatisierten Erfüllung staatlicher Aufgaben (siehe hinten \$\$ 5und 6). Damit der Untersuchungsgegenstand greifbar bleibt, muss also eine Grenze gezogen werden zwischen privatisierter und nichtprivatisierter Aufgaben-

94 Vgl. zu diesem Aspekt der Äusserlichkeit TSCHANNEN, Organigramm, S. 528, bezüglich Organisationsformen, die «für Bürger und Politik als Staat erkennbar» sind.

95 Siehe etwa Gamma, Kontrolle, passim, insb. S. 1236; LIENHARD, Ausgelagerte Aufgabenerfüllung, S.1166; STÖCKLI/PIOLINO, Privatschulen, S. 44; ZYSSET, Selbstregulierung, Rz.347. In der deutschen Lehre MAURER/WALDHOFF, Allgemeines Verwaltungsrecht, §23 Rz.69. Anderes Begriffsverständnis bei LIENHARD, Grundlagen NPM, S. 56, der Erfüllungsprivatisierung als die Betrauung Privater mit der Erfüllung von öffentlichen Aufgaben bezeichnet, was in der Terminologie dieser Arbeit einer Organisationsprivatisierung entspricht. 
erfüllung. Es soll nicht verheimlicht werden, dass diese Grenzziehung zu einem gewissen Grad willkürlich ist: Der funktionelle Staatsbegriff der Verfassung bedeutet, dass nur die Aufgaben entweder staatlich oder privat sind; bei der Erfüllung ist diese Grenze immer künstlich und auf dem freien Feld zu ziehen. Auch die gefestigten Privatisierungsarten (sogleich 5) beschreiben nicht, ob eine Verwaltungstätigkeit staatlich oder privat ist, sondern dienen bloss der didaktischen Veranschaulichung. ${ }^{96}$

Die Zweiteilung in Aufgaben- und Erfüllungsprivatisierung (soeben a und b) vereinfacht die Privatisierungsdiskussion - zugegebenermassen - nur vordergründig. Die unscharfen Konturen verschieben sich einfach auf den Begriff der Erfüllungsprivatisierung. Die Aufgabenprivatisierung lässt sich zwar scharf umreissen, für die Erfüllungsprivatisierung gilt dies aber nicht.

Wie also umgrenzt diese Arbeit die Erfüllungsprivatisierung? Sie sieht jede Verwaltungstätigkeit als privatisiert an, die privater Tätigkeit ähnelt. Die Abgrenzung muss sich an Äusserlichkeiten orientieren, weil es der Substanz nach keine private oder staatliche Aufgabenerfüllung gibt. ${ }^{97}$

Dieser Zugang bedeutet, dass ein enger Kreis um typisch staatliche Formen gezogen wird. Die exakte Grenzziehung folgt in \$ 4 jeweils nach der Erörterung der verschiedenen Organisations-, Handlungs- und Finanzierungsformen. Dort werden die Kennzeichen der Erfüllungsprivatisierung ausdifferenziert und mithin dem phänotypischen Abgrenzungskriterium der Privat-Ähnlichkeit etwas mehr Substanz verliehen. Diese Arbeit bezeichnet alle Aufgabenträger ausserhalb der Zentralverwaltung als privatisiert (hinten \$4/II.4.a). Auch bei den Handlungsformen ist der nichtprivatisierte Bereich eng umgrenzt: Nur gerade Verfügungen und Rechtsverordnungen sowie von den Realakten die Vollstreckungs- und Vollzugshandlungen gehören dazu (hinn ten \$ 4/III.3.a). Und schliesslich sind diejenigen Finanzierungsformen als privatisiert anzusehen, die den Bestand eines Aufgabenträgers mit anderen als allgemeinen Steuermitteln sichern (hinten \$ 4/IV.4.a).

Die Erfüllungsprivatisierung bringt zwar eine Vereinfachung in die Begrifflichkeiten der Privatisierungsdiskussion, sie weitet den Privatisierungsbegriff aber auch aus. Dezentralisierte Aufgabenträger sind der Verwaltung ebenso altbekannt wie Verwaltungsverträge und hauptsächliche Gebührenfinanzierung. Das weite Verständnis der privatisierten Erfüllung, das dieser Arbeit zugrunde liegt, erlaubt es aber, die Gemeinsamkeiten von sehr unter-

96 Angelehnt an MOOR/BELLANGER/TANQUEREL, Droit administratif III, S. 108, die allen Privatisierungskategorien ausserhalb der Aufgabenprivatisierungjede «conséquence juridique» absprechen und höchstens pädagogischen Wert beimessen.

97 Ähnlich auch DUBEY/ZUFFEREY, Droit administratif général, Rz.159; MÜLLER, Basler Kommentar, Art. 178 Rz. 39. 
schiedlichen Vorgängen zu untersuchen. Hier sollen nicht nur neue und explizit als Privatisierung bezeichnete Phänomene untersucht werden, sondern jede Aufgabenerfüllung, die ihrem Äusseren nach ebenso gut im privatautonomen, wettbewerbswirtschaftlichen Bereich angesiedelt sein könnte. Was die verfassungsrechtlichen Konsequenzen sind, wenn staatliche Aufgaben mit solchen Formen erfüllt werden, bildet ja gerade das Erkenntnisinteresse dieser Arbeit.

\section{Unterschiedliche Privatisierungsbegriffe in Lehre und Praxis}

Die Lehre unterscheidet zahlreiche Privatisierungsarten, um alle Erscheinungsformen der Privatisierung zu erfassen. Die soeben skizzierte Zweiteilung in Aufgabenprivatisierung und Erfüllungsprivatisierung soll die Privatisierungsdiskussion nicht zusätzlich verkomplizieren, sondern die rechtliche Bewertung der verschiedenen Vorgänge vereinfachen. Die bestehenden Begriffe müssen sich also in diese Zweiteilung einordnen lassen.

\section{a. Echte und unechte, materielle und formelle Privatisierung}

Die Zweiteilung in Aufgabenprivatisierung und Erfüllungsprivatisierung taucht in der rechtswissenschaftlichen Lehre unter anderen Chiffren auf: Regelmässig wird von echter und unechter bzw. von materieller und formeller Privatisierung gesprochen. Echt und materiell werden dabei gleichbedeutend verwendet, ebenso unecht und formell auf der anderen Seite.

Eine echte oder materielle Privatisierung liege etwa vor, wenn der Staat sich seiner Verantwortung für eine bestimmte Aufgabe entledige. ${ }^{98}$ Dagegen handle es sich um eine rein formelle oder unechte Privatisierung, wenn lediglich die Erfüllung einer staatlichen Aufgabe übertragen wird, der Staat aber die Verantwortung dafür behält. 99

98 BÜREN, Akkreditierte Zertifizierung, S. 145; TSCHANNEN, Privatisierung, S. 213. Ähnlich auch HÄFELIN/MÜLLER/UHLMANN, Allgemeines Verwaltungsrecht, Rz.1869; TSCHANNEN/ZIMMERLI/MÜLLER, Allgemeines Verwaltungsrecht, \$11 Rz. 4. Etwas ungenau hingegen DUBEY/ZUFFEREY, Droit administratif général, Rz.43, die eine materielle Privatisierung (oder "privatisation proprement dite») daran festmachen, ob der ausgelagerte Aufgabenträger einen «rapport organique» zum Staat habe.

99 Vgl. DUBEY/ZUFFEREY, Droit administratif général, Rz. 43 («improprement dite»); JAAG, Dezentralisierung und Privatisierung, S.30; TSCHANNEN/ZIMMERLI/MÜLLER, Allgemeines Verwaltungsrecht, §11 Rz.4. Ähnlich auch HÄFELIN/MÜLLER/UHLMANN, Allgemeines Verwaltungsrecht, Rz.1868. Etwas ungenau TANQUEREL, Droit administratif, Rz.108, der die unechte Privatisierung mit der Dezentralisierung vermischt. 
Diese Begriffspaare dienen aber selten dazu, die vielgestaltigen Privatisierungsarten zu ordnen, sondern sind meist zusätzliche Beschreibungen für bestimmte Privatisierungsarten: unecht/formell etwa für die Organisationsprivatisierung ${ }^{100}$ und echt/materiell für die Aufgabenprivatisierung101.

Während die echte bzw. materielle Privatisierung dem hier verwendeten Begriff der Aufgabenprivatisierung entspricht, kann die unechte bzw. formelle Privatisierung nicht ohne Weiteres mit der Erfüllungsprivatisierung gleichgesetzt werden. In der Lehre wird damit ausschliesslich die Organisationsprivatisierung bezeichnet, während die Erfüllungsprivatisierung - wie hier verwendet - weitere Phänomene abdeckt.

\section{b. Aufgabenverzicht}

Beim Aufgabenverzicht gibt der Staat seine Verantwortung für eine bestimmte Aufgabe ab. ${ }^{102}$ Der Staat ist also weder für das Ob noch das Wie der Aufgabenerfüllung verantwortlich. Beispiele für diesen Vorgang finden sich etwa auf kantonaler Ebene: Die Kantone Solothurn und Appenzell Ausserrhoden haben darauf verzichtet, eigene Banken zu betreiben, und der Kanton Zürich hat seine Staatskellerei veräussert. ${ }^{103}$

Nach dem Begriffsverständnis dieser Arbeit ist der Aufgabenverzicht gleichbedeutend mit der Aufgabenprivatisierung. Die Literatur nimmt zwei Unterscheidungen vor, die diese Arbeit ausklammert. Erstens beschlage der Aufgabenverzicht die dahinterliegenden Motive, was bei der Aufgabenprivatisierung nicht der Fall ist. Ein Aufgabenverzicht liege vor, wenn das öffentliche Interesse an einer Aufgabe entfalle ${ }^{104}$ oder eine staatliche Aufsicht nicht mehr nötig sei ${ }^{105}$. Diese Zusätze sind für die Begriffsdefinition irrelevant; jeder Privatisierungsvorgang ist irgendwie motiviert. Entscheidend bleiben

100 So bei HÄFELIN/MÜLLER/UHLMANN, Allgemeines Verwaltungsrecht, Rz.1868; HANDSCHIN/SIEGENTHALER, Privatisierung, S. 405; JAAG, Dezentralisierung und Privatisierung, S. 30; TSCHANNEN/ZIMMERLI/MÜLLER, Allgemeines Verwaltungsrecht, §11 Rz. 4 .

101 So bei BÜREN, Akkreditierte Zertifizierung, S.145, HÄFELIN/MÜLLER/UHLMANN, Allgemeines Verwaltungsrecht, Rz.1869; TSCHANNEN, Privatisierung, S. 213; TSCHANNEN/ ZIMMERLI/MÜLLER, Allgemeines Verwaltungsrecht, §11 Rz. 4.

102 HANDSCHIN/SIEGENTHALER, Privatisierung, S. 406; JAAG, Dezentralisierung und Privatisierung, S.33; TSCHANNEN/ZIMMERLI/MÜLLER, Allgemeines Verwaltungsrecht, \$11 Rz. 6; TSCHANNEN, Privatisierung, S. 215; UEBERSAX, Privatisierung, S. 398.

103 Beispiele nach JAAG, Dezentralisierung und Privatisierung, S. 33, wobei im Fall der Staatskellerei überprüft werden müsste, ob der Kellereibetrieb überhaupt im Bereich staatlicher Aufgaben lag oder vielleicht lediglich der Mittelbeschaffung diente.

104 So ACHERMANN, Privatisierung, S. 28, der fälschlicherweise gar das Vorliegen einer staatlichen Aufgabe am öffentlichen Interesse festmacht; sowie JAAG, Dezentralisierung und Privatisierung, S.33; und TSCHANNEN, Privatisierung, S. 215.

105 HANDSCHIN/SIEGENTHALER, Privatisierung, S. 406. 
sein Ansatzpunkt und seine Wirkung: Betrifft er die Aufgabenverantwortung an sich oder lediglich die Modalitäten der Aufgabenerfüllung? Wird staatliche Verantwortung abgetreten oder nicht?

Eine zweite Unterscheidung nimmt insbesondere TSCHANNEN vor, der den Aufgabenverzicht im Zusammenhang mit verschiedenen Privatisierungsgraden oder -intensitäten behandelt. ${ }^{106}$ Er bezeichnet den Aufgabenverzicht als Vollprivatisierung im Bereich der Aufgabenprivatisierung. Dagegen gebe es bloss teilweise Aufgabenprivatisierungen, wenn der Staat eine Kontroll- oder Gewährleistungsverantwortung für die privatisierte Aufgabe behalte. ${ }^{107}$ Im Begriffsverständnis dieser Arbeit ist diese Unterscheidung unpräzis. Aufgabenprivatisierung heisst, dass eine bestimmte Leistung aus dem Bestand staatlicher Aufgaben entlassen wird. So gefasst gibt es keine vollständige oder teilweise Aufgabenprivatisierung. Möglich ist einzig, dass die Aufgabenprivatisierung nur eine bestimmte Leistung aus einem grösseren Aufgabenbereich umfasst (wenn etwa der Staat gewisse Postdienstleistungen nicht mehr erbringen muss, aber für andere weiterhin verantwortlich bleibt; siehe sogleich c). Wenn hingegen der Staat für gewisse Aufgaben weiterhin eine wie auch immer geartete Verantwortung behält, sollte nicht von einer Aufgabenprivatisierung gesprochen werden.

Der Aufgabenverzicht ist rechtlich einfach zu beurteilen: Eine bestimmte Tätigkeit, zu deren Erbringung der Staat vorher verpflichtet war, wird Privaten überlassen. In diesem Zusammenhang stellen sich vorab politische Fragen: Wofür soll der Staat verantwortlich sein? Soll er gewisse Fälle definieren, in denen er die Verantwortung für die privatisierte Aufgabe wieder an sich nimmt? Die Antworten darauf sind nicht im Verfassungsrecht zu finden.

Verfassungsrechtlich irrelevant ist auch die Frage, ob es einen bestimmbaren Bereich unverzichtbarer Aufgaben gibt - sogenannte Kernaufgaben. ${ }^{108}$ Die Verfassung definiert den Aufgabenbestand ${ }^{109}$ des Staats, und ihre Revision unterliegt keinen diesbezüglichen materiellen Grenzen.110 Ob der Staat

106 Vgl. TSCHANNEN/ZIMMERLI/MÜLLER, Allgemeines Verwaltungsrecht, §11 Rz. 5 f.; TSCHANNEN, Privatisierung, S. 215.

107 TSCHANNEN/ZIMMERLI/MÜLLER, Allgemeines Verwaltungsrecht, \$11 Rz.7.

108 Siehe für verschiedene Ansätze in dieser Diskussion: EICHENBERGER, Staatsaufgaben, S.113f. (Unterscheidung zwischen unerlässlichen, gebotenen, wünschenswerten und entbehrlichen Aufgaben); RICHLI, Rechtsetzung, Rz. 18 (unverzichtbare Aufgaben); UEBERSAX, Privatisierung, S. 395 f. (zwingende bzw. unausweichliche und fakultative Aufgaben); WIEGAND, Beleihung Privater, S. 1135 f. (notwendige und nicht primär hoheitliche Aufgaben).

109 Für die Schweizerische Eidgenossenschaft insbesondere im Kapitel über die Zuständigkeiten der Bundesversammlung (Art. 54-135 BV).

110 Einzige materielle Schranke der Verfassungsrevision ist das zwingende Völkerrecht (Art.193 Abs. 4 BV für die Totalrevision und Art.194 Abs. 2 BV für die Teilrevision). 
gewisse Aufgaben zwingend erbringen muss, ist wiederum eine politische und rechtsphilosophische Frage, deren Beantwortung zugegebenermassen durch die Verfassung selbst vorgezeichnet ist: Ein Staat, der «die Unabhängigkeit und die Sicherheit des Landes» $\mathbf{1 1 1}$ schützt, muss Landesverteidigung betreiben.EinStaat, der «dieStärke des Volkes [...] am Wohlder Schwachen»112 misst, wird Sozialversicherungen bestellen müssen.

\section{c. Teilprivatisierung}

Teilprivatisierungen lösen Teile aus einem grösseren Aufgabenbereich heraus. Wenn etwa die Pflicht der Post, Briefsendungen zuzustellen, auf Briefe bis zu einem Kilogramm Gewicht eingeschränkt wird. ${ }^{113}$ Insoweit entfällt die Erfüllungspflicht, und die Tätigkeit «Zustellung von Briefen über 1 Kilogramm» verliert ihre Qualität als staatliche Aufgabe.

Der Aufgabenbegriff dieser Arbeit knüpft bei einzelnen Tätigkeiten an, die dem Staat verpflichtend zur Erfüllung übertragen sind (siehe vorne I.2). Daher wird im Folgenden darauf verzichtet, Teilaufgaben oder Teilprivatisierungen auszuscheiden.

Die Lehre bezeichnet mitunter auch andere Privatisierungserscheinungen als Teilprivatisierung, um zu kennzeichnen, dass nur ein Teil der staatlichen Verantwortung abgegeben wird.114 Für diese Fälle ist der Begriff aber unnötig; es bestehen präzisere Begriffe für die verschiedenen Phänomene. Oft handelt es sich um eine Erfüllungsprivatisierung in organisatorischer oder finanzieller Hinsicht. Die Teilprivatisierung füllt also keine dogmatische Lücke; der Begriff überlagert vielmehr andere Kategorien und verwirrt mehr, als er klärt.

\section{d. Organisationsprivatisierung}

Die Organisationsprivatisierung betrifft die Trägerschaft von Staatsaufgaben, ohne die Staatlichkeit der Aufgabe an sich anzutasten. Entscheidend ist dabei die Rechtsform des Aufgabenträgers: Von einer Organisationsprivatisierung ist auszugehen, wenn eine staatliche Aufgabe auf ein Privatrechts-

111 Art.2 Abs.1 BV.

112 Präambel BV.

113 So geschehen mit dem Postgesetz von 2010: Art.14 PG i.V.m. Art. 29 Abs. 1 VPG. Vgl. die gewichtsmässig nicht beschränkte Zustellpflicht in Art. 3 Abs. 1 des früheren Postgesetzes vom 30. April 1997 (AS 1997 2452).

114 HANDSCHIN/SIEGENTHALER, Privatisierung, S. 405, verstehen unter Teilprivatisierung die Möglichkeit Privater, sich als Minderheitsaktionäre an öffentlichen Unternehmen zu beteiligen; und auch UEBERSAX, Privatisierung, S. 398 («mit Mehr- oder Minderheitsbeteiligung des Staates»). 
subjekt übertragen wird.115 TSCHANNEN spezifiziert überdies, dass es sich dabei um ein staatlich beherrschtes Privatrechtssubjekt handeln muss. ${ }^{116}$

Diese Art der Privatisierung wird in dieser Arbeit als Erfüllungsprivatisierung in organisatorischer Hinsicht behandelt, also als Privatisierung der Organisationsformen, wobei dieser Begriff weiter ist und jegliche Situation umfasst, in der eine Staatsaufgabe ausserhalb klassisch staatlicher Organisationsformen erfüllt wird (siehe hinten \$ 4/II).

Der Begriff der Organisationsprivatisierung kann dazu verleiten, die staatliche Aufgabe als entscheidendes Kriterium für die Abgrenzung zwischen staatlicher und privater Sphäre aus dem Blick zu verlieren. BIAGGINI etwa unterscheidet zwischen staatlicher und privatisierter Aufgabenerfüllung je nach Rechtsnatur des Aufgabenträgers. ${ }^{117}$

Die Organisationsprivatisierung überschneidet sich teilweise mit der Dezentralisierung. ${ }^{118}$ Zur Abgrenzung der Dezentralisierung von der Privatisierung im Allgemeinen siehe hinten 6.a.

\section{e. Finanzierungsprivatisierung}

Die Finanzierungsprivatisierung meint ganz allgemein die Abkehr vom Gemeinlastprinzip bei der Erbringung staatlicher Aufgaben. Gewöhnlich kommt der mit Steuereinnahmen geäufnete Staatshaushalt für die Kosten der Staatsaufgaben auf. Konkreter ausgedrückt handelt es sich um eine Finanzierungsprivatisierung, wenn die Kosten einer staatlichen Leistung von den Leistungsbezügern getragen werden. ${ }^{119}$ Da die Finanzierungsprivatisierung Leistungserbringung und Leistungsbezahlung in einen direkten $\mathrm{Zu}$ sammenhang setzt, wird sie auch als Äquivalenzfinanzierung bezeichnet. 120

Die Finanzierungsprivatisierung betrifft das Ob der staatlichen Aufgabenerfüllung in keiner Weise, sondern setzt bei einem bestimmten Aspekt des Wie an: Wie sollen die Kosten gedeckt werden, die durch die Erfüllung staat-

115 HÄFELIN/MÜLLER/UHLMANN, Allgemeines Verwaltungsrecht, Rz.1868; HÄNER, Privatisierung, S. 424; HÄSLER, Geltung der Grundrechte, S. 46; TSCHANNEN/ZIMMERLI/MÜLLER, Allgemeines Verwaltungsrecht, §11 Rz. 4. Auch als rechtlich-organisatorische Privatisierung bezeichnet, MÜLLER/JENNI, Privatisierung, S.1072.

116 TSCHANNEN, Privatisierung, S. 212; TSCHANNEN/ZIMMERLI/MÜLLER, Allgemeines Verwaltungsrecht, §11 Rz.4.

117 BIAGGINI, Rechtsstaatliche Anforderungen, S. 144-148, insb. Schaubild auf S. 146.

118 TSCHANNEN/ZIMMERLI/MÜLLER, Allgemeines Verwaltungsrecht, §11 Rz. 4.

119 DUBEY/ZUFFEREY, Droit administratifgénéral, Rz. 43; FREY, Drei Stossrichtungen, S. 343f., HÄFELIN/MÜLLER/UHLMANN, Allgemeines Verwaltungsrecht, Rz.1871; HÄNER, Privatisierung, S. 425; HÄSLER, Geltung der Grundrechte, S. 47; TSCHANNEN/ZIMMERLI/MÜLLER, Allgemeines Verwaltungsrecht, \$11 Rz. 4.

120 TSCHANNEN, Privatisierung, S. 213, m.w.H. 
licher Aufgaben entstehen? ${ }^{121}$ In der hier verwendeten Terminologie handelt es sich um Erfüllungsprivatisierung in finanzieller Hinsicht, also eine Privatisierung der Finanzierungsformen (siehe hinten \$ 4/IV).

\section{f. Vermögensprivatisierung}

Vermögensprivatisierung bedeutet den Verkauf staatlichen Eigentums an Private. ${ }^{122}$ Diese Form der Privatisierung wird auch als Eigentumsprivatisierung123 bezeichnet; in der französischsprachigen Schweiz ist sie als "privatisation patrimoniale»124 bekannt.

Übliche Beispiele sind der Verkauf von Liegenschaften oder Unternehmensbeteiligungen. Die Vermögensprivatisierung erfolgt aus rein finanziellen Motiven und berührt weder den staatlichen Aufgabenbestand noch die Aufgabenerfüllung. Sachenrechtlich gesprochen handelt es sich bei vermögensprivatisierten Objekten zwingend um Finanzvermögen, da mit der Vermögensprivatisierung keine Sachen veräussert werden können, die der Erfüllung einer Staatsaufgabe dienen und somit Verwaltungsvermögen darstellen. ${ }^{\mathbf{2 5}}$

Folglich lässt sich die Vermögensprivatisierung weder als Aufgabennoch als Erfüllungsprivatisierung einordnen. Streng genommen ist es unangebracht, sie überhaupt als Privatisierung zu bezeichnen, da sie nichts mit Staatstätigkeit zu tun hat. Konsequenterweise sollte die Vermögensprivatisierung einfach als Vermögensveräusserung bezeichnet werden.

\section{Verhältnis zu verwandten Begriffen}

Neben der Privatisierung betreffen auch andere Phänomene die Abgrenzung der staatlichen von der privaten Sphäre. Die verschiedenen Begriffe werden oft in einem Atemzug genannt, ${ }^{126}$ was deren begriffliche Eingrenzung nicht gerade erleichtert.

\section{a. Dezentralisierung}

Die Dezentralisierung meint den verwaltungsorganisatorischen Vorgang, Verwaltungsaufgaben auf Aufgabenträger ausserhalb der Zentralverwaltung

121 Ähnlich auch MOOR/BELLANGER/TANQUEREL, Droit administratif III, S. 105.

122 HÄFELIN/MÜLLER/UHLMANN, Allgemeines Verwaltungsrecht, Rz.1871; HÄNER, Privatisierung, S. 424; HÄSLER, Geltung der Grundrechte, S. 46 f.; TSCHANNEN, Privatisierung, S. 212; TSCHANNEN/ZIMMERLI/MÜLLER, Allgemeines Verwaltungsrecht, §11 Rz. 4.

123 BÜREN, Akkreditierte Zertifizierung, S. 145; TSCHANNEN, Privatisierung, S. 212.

124 DUBEY/ZUFFEREY, Droit administratif général, Rz. 43.

125 Für eine Übersicht über die Arten von öffentlichen Sachen siehe anstelle vieler TSCHANNEN/ ZIMMERLI/MÜLLER, Allgemeines Verwaltungsrecht, \$48 Rz.11-18.

126 So etwa JAAG, Dezentralisierung und Privatisierung, S.24. 
zu übertragen. ${ }^{127}$ Die Lehre unterscheidet weiter zwischen sachlicher und örtlicher Dezentralisierung, wobei Letztere die Aufgabenteilung zwischen mehreren föderalen Staatsebenen meint. ${ }^{128}$ Dieses Phänomen kann für die Zwecke dieser Arbeit ausgeblendet werden: Vorliegend soll nur untersucht werden, wie der Staat seine Aufgabenerfüllung innerhalb der höchsten Staatsebene organisiert.

Die sachliche Dezentralisierung hingegen überschneidet sich teilweise mit einer Kategorie, die noch vertieft zu analysieren sein wird: der Erfüllungsprivatisierung in organisatorischer Hinsicht (siehe dazu hinten \$4/II).

\section{b. Auslagerung}

Die Auslagerung nimmt in der Privatisierungsdiskussion die Stellung eines diffusen Oberbegriffs ein; sie bezeichnet verschiedenartige Vorgänge wie etwa Dezentralisierungen und Aufgabenübertragungen. ${ }^{129}$ BIAGGINI unterscheidet die Auslagerung vom Aufgabenverzicht und von der Vermögensprivatisierung. ${ }^{130}$ Der Auslagerung wird überdies dem englischsprachigen Sammelbegriff Outsourcing gleichgesetzt. ${ }^{131}$ Auslagerung bedeutet, eine staatliche Aufgabe «durch Beizug vorgefundener Privatrechtssubjekte» ${ }^{132}$ zu erfüllen, was sie abgrenzt von Verschiebungen der Aufgabenträgerschaft innerhalb der Verwaltung und von Neugründungen privater Aufgabenträger.

Die Auslagerung kommt - in die Begriffe dieser Arbeit gefasst - einer Privatisierung der Organisationsformen gleich. Sie bezeichnet die Betrauung nichtstaatlicher Akteure mit der Erfüllung staatlicher Aufgaben.

\section{c. Deregulierung}

Die Deregulierung bezeichnet Bestrebungen, den Einfluss staatlicher Normen auf privatwirtschaftliche Tätigkeit zu verringern. ${ }^{133}$ Das kann eine Reduktion von Normen bedeuten (Aufhebung von Erlassen oder Artikeln) oder eine

127 HÄFELIN/MÜLLER/UHLMANN, Allgemeines Verwaltungsrecht, Rz.1595;JAAG, Dezentralisierung und Privatisierung, S.24; TSCHANNEN, Systeme, Rz.61.

128 HÄFELIN/MÜLLER/UHLMANN, Allgemeines Verwaltungsrecht, Rz.1598. Dieselbe Unterscheidung mit den Begriffen administrativ und politisch bei TSCHANNEN/ZIMMERLI/ MÜLLER, Allgemeines Verwaltungsrecht, §5 Rz.15-21.

129 Ersteres bei BIAGGINI, Rechtsstaatliche Anforderungen, S.151; Letzteres beiJAAG, Dezentralisierung und Privatisierung, S. 26; beides bei BIAGGINI, Kommentar BV, Art. 178 Rz. 20.

130 BIAGGINI, Rechtsstaatliche Anforderungen, S.144-148.

131 JAAG, Dezentralisierung und Privatisierung, S. 26.

132 TSCHANNEN/ZIMMERLI/MÜLLER, Allgemeines Verwaltungsrecht, §5 Rz. 21c.

133 JAAG, Dezentralisierung und Privatisierung, S. 25; LIENHARD, Deregulierung, S. 109 f.; TSCHANNEN, Privatisierung, S. 213 f.; TSCHANNEN/ZIMMERLI/MÜLLER, Allgemeines Verwaltungsrecht, §11 Rz. 2; UEBERSAX, Privatisierung, S. 398. 
Flexibilisierung der staatlichen Vorschriften.134 Der Begriff Deregulierung drückt vor allem die Grundeinstellung aus, dass weniger staatliche Vorschriften mehr wirtschaftliche Entfaltung bedeuten; ${ }^{135}$ genaue Instrumente sind damit noch nicht bezeichnet, was sich auch an den unbestimmten Begriffen «Normenverbesserung» oder «Gesetzesverlagerung» zeigt.136

Die unter Deregulierung gefassten Vorgänge teilen die Zielrichtung von Privatisierungen: eine Verlagerung vom Staatlichen hin zum Privaten. Die Deregulierung setzt bei der Norm als Mittel staatlicher Einflussnahme an. ${ }^{137}$ Sie lässt sich nicht als eine Unterart der Privatisierung einteilen. Ein Abbau von Normen kann sowohl eine Aufgabenprivatisierung bedeuten (ein Gesetz wird aufgehoben, weil der Staat seine Verantwortung für einen Aufgabenbereich abgibt) oder auch irgendeine Form der Erfüllungsprivatisierung (die Art und Weise der staatlichen Aufgabenerfüllung wird weniger detailliert geregelt).

\section{d. Liberalisierung}

Die Liberalisierung ist ein originär volkswirtschaftlicher Begriff, der zwar mit rechtlichen Phänomenen zusammenhängt, diese aber aus einem anderen Blickwinkel betrachtet: Er befasst sich mit der Öffnung von Märkten. ${ }^{138}$ Welche rechtlichen Veränderungen dazu notwendig sind, ist damit nicht vorgezeichnet. Liberalisierung heisst typischerweise Monopolabbau 139 - dies kann über Konzessionsvergabe, Reduktion der Regelungsdichte, Rechtsformumwandlung der staatlichen Leistungserbringer oder sonst wie erfolgen.

Dies zeigt sich exemplarisch in der bundesrätlichen Legislaturplanung: Ab den späten 1980er-Jahren wird die Liberalisierung von Märkten als Teil der internationalen Rahmenbedingungen thematisiert. ${ }^{140}$ Mitte der 1990er sodann erhebt der Bundesrat Liberalisierungen verschiedener Märkte zu politischen Zielen. ${ }^{141}$ Diese Legislaturplanungen kommen allerdings gänzlich ohne die Begriffe Privatisierung, Auslagerung oder Deregulierung aus.

134 Vgl. RUCH, Informalisierung, S. 506f., der davon ausgeht, dass sich die Regulierung lediglich auf private Regelsetzung «verschiebt» und sich der Staat auf Rahmensetzung beschränkt.

135 Aus ökonomischer Sicht betrachtet spricht FREY, Privatisierung und Liberalisierung, S. 25, von einer «Erhöhung der Wettbewerbsintensität».

136 So bei LIENHARD, Deregulierung, S.110.

137 TSCHANNEN/ZIMMERLI/MÜLLER, Allgemeines Verwaltungsrecht, \$11 Rz. 2, die in Abgrenzung davon die Privatisierung als «eher subjektbezogen» beschreiben.

138 BAUMANN, Wettbewerbsverzerrungen, Rz.90.

139 Vgl. aus der deutschen Lehre, THOMA, Selbstregulierung, S. 53.

140 Legislaturplanung 1987-1991, 429; Legislaturplanung 1991-1995, 7, 66.

141 Legislaturplanung 1995-1999, 312 (Telekommunikation), 314 (Getreide), 325 (Energie). Die Legislaturplanungen des Zeitraums 1999 bis 2011 erwähnen die Liberalisierung dann nur noch im internationalen Kontext, Legislaturplanung 1999-2003, 2286; Legislaturplanung 2003-2007, 1183; Legislaturplanung 2007-2011, 784 . 
Sofern die rechtswissenschaftliche Literatur Liberalisierung überhaupt im Kontext rechtlicher Konzepte thematisiert, tut sie dies entweder in einer losen Aufzählung ohne weitere Strukturierung, ${ }^{142}$ oder indem sie die Liberalisierung als Generalisierung über andere Kategorien setzt. ${ }^{143}$

\section{e. New Public Management}

New Public Management (NPM) ist ein betriebswirtschaftlich orientiertes Modell144 für eine Verwaltungsführung, die stärker an messbaren Wirkungen orientiert ist denn an rechtlichen Normen und finanziellen Vorgaben. ${ }^{145}$ NPM kann verstanden werden als Sammelbegriff für vielgestaltige Vorschläge zur Verbesserung der staatlichen Aufgabenerfüllung. Die mit NPM verbundene Vorstellung einer besseren Verwaltung 146 fusst in der Überzeugung, dass die Funktionsweise der Privatwirtschaft derjenigen der staatlichen Verwaltung überlegen ist und ihr als Vorbild dienen soll. ${ }^{147}$ MASTRONARDI beschreibt den Kern des NPM-Ansatzes so:

«Die Verwaltung wird politisch neutralisiert und kann sich auf rein zweckrationales Management konzentrieren, weil sie von der Politik einen klaren Leistungsauftrag erhält. Die Politik ist verantwortlich für den Auftrag, die Verwaltung für dessen effiziente Erfüllung. Das Parlament delegiert alle operativen Entscheide an die Verwaltung.»148

Diese «betriebswirtschaftliche Verkürzung» ${ }^{149}$ verdeutlicht, dass NPM die Verwaltungstätigkeit mit einer originär nichtstaatsrechtlichen Sichtweise betrachtet. ${ }^{150}$ Es geht um Zweckrationalität, Management und Effizienz. Privatisierungsbestrebungen treten aus einer anderen Warte an die Verwaltungstätigkeit heran: Sie betreffen die Staatlichkeit von Aufgaben und die Art und Weise der Aufgabenerfüllung. DieZwecktauglichkeit einer Verwaltungshandlung kann

142 Bei JAAG, Dezentralisierung und Privatisierung, S. 24, neben Deregulierung, Dezentralisierung, Privatisierung und New Public Management; beim Bundesrat neben Deregulierung, Legislaturplanung 1991-1995, $7 \mathrm{f}$.

143 So etwa FREY, Privatisierung und Liberalisierung, S. 25.

144 LIENHARD, Grundlagen NPM, S. 113.

145 HÄFELIN/MÜLLER/UHLMANN, Allgemeines Verwaltungsrecht, Rz.1578; TSCHANNEN/ ZIMMERLI/MÜLLER, Allgemeines Verwaltungsrecht, §5 Rz. 36 .

146 TSCHANNEN/ZIMMERLI/MÜLLER, Allgemeines Verwaltungsrecht, §11 Rz. 2.

147 Vgl. LIENHARD, Grundlagen NPM, S. 23; MASTRONARDI, Gewaltenteilung unter NPM, S. 462; TSCHANNEN, Privatisierung, S. 214.

148 MASTRONARDI, Gewaltenteilung unter NPM, S. 450.

149 MASTRONARDI, Gewaltenteilung unter NPM, S. 450; ferner LIENHARD, Grundlagen NPM, S. 23-53, wo die Charakteristiken des NPM als «betriebswirtschaftliches Modell» abgehandelt werden.

150 Gemäss LIENHARD, Grundlagen NPM, S. 56, «zielt [NPM] grundsätzlich in eine andere Richtung als Privatisierungsvorhaben» wegen seiner «Ausprägung als Binnenmodernisierung». 
zwar optimiert werden, die Nützlichkeit (im Sinne eines Beitrags zur Förderung des Gemeinwohls) ist allerdings mit der gesetzlichen Zuweisung abschliessend beurteilt worden. Aus staatsrechtlicher Warte definieren Verfassung und Gesetz den Rahmen, in welchem die Aufgabenträger operieren können. ${ }^{151}$ Dem Verfassungsgeber und dem Gesetzgeber ist es freigestellt, wie stark er nicht nur das Was, sondern auch das Wie der Verwaltungstätigkeit vorgibt.152

NPM soll hier nicht weiter bewertet, ${ }^{153}$ sondern lediglich gegen die Privatisierung abgegrenzt werden. Beide Phänomene können ähnliche Änderungen an der Einrichtung und Funktionsweise der staatlichen Verwaltung bedeuten. ${ }^{154}$ Doch sie treten mit unterschiedlicher Optik - und teilweise mit unterschiedlichem Vorverständnis - an diese Änderungsvorschläge heran. ${ }^{155}$ NPM soll die staatlichen Leistungen verbessern, indem die Verwaltung ihre Aufgaben effizienter erbringt. ${ }^{156}$ Der Bestand staatlicher Aufgaben ist dadurch von vornherein nicht betroffen. NPM setzt also bei der Erfüllungsseite an; allerdings nicht unter dem Vorzeichen der Entstaatlichung, sondern der vermehrten Wirkungsorientierung. Mit anderen Worten ist die Motivation und Logik hinter NPM eine andere als bei der Erfüllungsprivatisierung, sie kann allerdings deckungsgleiche Formen annehmen. Die neuesten Erscheinungen der Verwaltungsreformen im Bund werden hinten in \$ 4/I.4 in die Aspekte der Aufgabenerfüllung eingeordnet.

Im Folgenden wird die privatisierungsspezifische Betrachtungsweise eingenommen, da diese Arbeit einen verfassungsdogmatischen Ansatz verwendet, welcher mit den Grundannahmen von New Public Management in einem gewissen Spannungsverhältnis steht. Kurz gesagt: Das funktionelle Staatsverständnis, das die Verfassung ausdrückt, verträgt sich schlecht mit dem auf Privatwirtschaft und Effizienz fokussierten Verständnis von NPM. ${ }^{157}$

151 HALLER/KÖLZ/GÄCHTER, Allgemeines Staatsrecht, Rz.916.

152 Dass Parlamente auf einen Teil ihrer Macht verzichten müssten, um die Trennung von Politik und Betrieb aufrechtzuerhalten, führt MASTRONARDI, Gewaltenteilung unter NPM, S. 454 f. schlüssig aus.

153 Für eine Bewertung siehe die eingehende Würdigung bei TSCHANNEN/ZIMMERLI/MÜLLER, Allgemeines Verwaltungsrecht, §5 Rz.41-43 (insb. die in Rz. 43 zitierte Kritik, wie sie der Regierungsrat des Kantons Bern in der Evaluation der bernischen NPM-Variante NEF äusserte); sowie die gesammelte Kritik bei LIENHARD, Grundlagen NPM, S.113-118.

154 LIENHARD, Grundlagen NPM, S. 56.

155 Zur unterschiedlichen «Denkweise» von Betriebswirtschaft und Staatspolitik auch MASTRONARDI, Gewaltenteilung unter NPM, S. $454 \mathrm{f}$.

156 MÜLLER, Schlank, S. 163. Ähnlich bei TSCHANNEN/ZIMMERLI/MÜLLER, Allgemeines Verwaltungsrecht, §5Rz.36, 41.

157 Diese Abgrenzung ist nicht als die typisch gewordene Kritikgemeint, dass NPM die «staatsund verwaltungsrechtlichen Errungenschaften» gefährde oder «in einem potentiellen Widerspruch zu den staatstragenden Grundwerten der Schweiz» stehe; LIENHARD, Grundlagen NPM, S.113, mit ausführlicher Auseinandersetzung mit dieser Kritik, S.118-240. 


\section{Zwischenfazit: Privatisierung heisst, staatliche Aufgaben oder deren Erfüllung vom Staat zu entfernen}

Privatisierung bedeutet, staatliche Aufgabenerfüllung in Richtung der nichtstaatlichen, privaten Sphäre zu bewegen. In Form der Aufgabenprivatisierung geschieht dies, indem der Staat aus der Verantwortung für eine bestimmte Aufgabe entlassen wird. Als Erfüllungsprivatisierung hingegen bedeutet diese Bewegung, dass der Staat weiterhin für eine Aufgabe verantwortlich bleibt, deren Erfüllung aber privater Tätigkeit ähnelt. Bei der Erfüllungsprivatisierung bleibt die Tätigkeit staatlich, sie entfernt sich lediglich in ihrer Erscheinungsweise vom Staat.

Privatisierung schliesst also zwei Phänomene ein: die Reduktion staatlicher Substanz (Bestand von Staatsaufgaben nimmt ab) und die Zunahme staatlicher Tätigkeit, die privatem Handeln ähnelt (quasiprivate Erfüllungsformen). 



\section{\$3 Welchen verfassungs- rechtlichen Anforderungen unterliegt die Erfüllung staatlicher Aufgaben?}

Diese Arbeit untersucht, welche Probleme und Spielräume die verfassungsrechtlichen Anforderungen an staatliches Handeln entstehen lassen, wenn die Erfüllung staatlicher Aufgaben privatisiert wird. Im vorliegenden Paragrafen wird zunächst die Geltungsweise der Anforderungen an sich erörtert (I), um sie sodann überblickweise darzustellen (II). Anschliessend werden einige Rechtsfiguren erörtert, die gerade nicht zu diesen Anforderungen gehören (III).

\section{Begriff und Bedeutung der verfassungs- rechtlichen Anforderungen}

\section{Anforderungen an staatliches Handeln als Kehrseite des funktionellen Staatsbegriffs}

Überall dort, wo die Verfassung den Staat an besondere Regeln bindet, die in der privaten Sphäre nicht gelten, hängt dies von der Erfüllung staatlicher Aufgaben ab. Seien es die rechtsstaatlichen Grundsätze nach Art. 5 BV, die Grundrechtsbindung nach Art. 35 Abs. 2 oder auch die Staatshaftung nach Art.146 - Lehre und Rechtsprechung sind sich einig, dass es auf die Staatlichkeit der Aufgabe ankommt und nicht auf die Rechtsnatur der Handlungsoder Organisationsformen. ${ }^{158}$ 
Unbestritten ist, dass sämtliche Tätigkeiten, die der Erfüllung staatlicher Aufgaben dienen, den verfassungsrechtlichen Anforderungen an staatliches Handeln zu unterwerfen sind. Im Gegenzug ist umstritten, ob sämtliches Handeln staatlicher Organe - unabhängig vom Bezug zu einer Staatsaufgabe Staatstätigkeit darstellt. Mit anderen Worten gibt es einen Lehrstreit zwischen einem personellen und einem funktionellen Ansatz: Sollen staatliche Akteure auch dann den verfassungsrechtlichen Anforderungen unterliegen, wenn sie eine Tätigkeit ausüben, die ausserhalb ihrer staatlichen Aufgaben liegt? Dieser Lehrstreit spielt sich im Rahmen der Dogmatik zur Grundrechtsbindung $\mathrm{ab}^{159}$ und wird in dieser Arbeit auch in jenem Zusammenhang vertieft (siehe hinten II.6.a). So viel aber schon vorweg: Es ist unbestritten, dass privatrechtliche Akteure an die Verfassungsvorgaben für staatliches Handeln gebunden sind, soweit sie staatliche Aufgaben erfüllen. Umstritten ist lediglich, ob staatliche Akteure über ihren Aufgabenbereich hinaus gebunden sind.

Es mag zirkulär erscheinen, die Anforderungen an staatliches Handeln am funktionellen Staatsbegriff festzumachen. Diese Arbeit leitet die Notwendigkeit der Staatsdefinition ja gerade davon ab, dass staatliches Handeln verfassungsrechtlichen Anforderungen unterliegt, die für privates Handeln nicht gelten (siehe vorne \$2/I.1). Doch dieser Zusammenhang ist nur folgerichtig: Weil die Verfassung staatliches Handeln besonderen Anforderungen unterstellt, muss erkennbar sein, was Staatstätigkeit ist und wo sie endet. Gehalt und Geltungsweise der verfassungsrechtlichen Anforderungen sind damit noch nicht bestimmt.

Bevor nun die Anforderungen inhaltlich umgrenzt werden können (hinten II und III) muss in allgemeiner Weise geklärt werden, worin ihre Geltung begründet liegt (2) und wie ihre Geltung strukturiert ist (3).

\section{Legitimationspflicht des Staats als Geltungsgrund und Funktion der Anforderungen}

Der Staat unterliegt besonderen Verfassungsbindungen, weil er sich für den Zwang rechtfertigen muss, den er gegenüber seinen Bürgern anwenden darf. ${ }^{60}$ Diese Rechtfertigung verschafft seinem Handeln Legitimität. Auf eine kurze Formel gebracht: Er geniesst besondere Rechte und muss deshalb besondere Pflichten tragen. ${ }^{161}$

159 Vgl. zur Übersicht SCHWEIZER, St. Galler Kommentar, Art. 35 Rz. 45.

160 Vgl. TSCHANNEN, Privatisierung, S. 234.

161 Diesen Gedanken mit dem Begriff «puissance publique» ausdrückend TANQUEREL, Droit administratif, Rz. 79, 83 . 
Diese Rechtfertigung richtet das staatliche Handeln auf das Gemeinwohl aus. Dieses ist zugleich «staatspolitische Rechtfertigung und staatsrechtliche Voraussetzung allen staatlichen Handelns» ${ }^{\mathbf{1 6 2}}$.

Bei der Anwendung der verfassungsrechtlichen Anforderungen ist also zu bedenken, dass sie das Staatshandeln grundlegend legitimieren und deshalb gelten, weil der Staat in besonderer Weise die Freiheit von Individuen beschränken kann. Mit anderen Worten verliert der eingreifende, lenkende, umverteilende, planende und sorgende Staat seine Legitimation, wenn er sich seiner verfassungsrechtlichen Pflichten zu entledigen versucht: Sie sind der Preis für seine besondere Macht.

\section{Geltungsweise der Anforderungen}

Es stellen sich drei Fragen bezüglich Geltungsweise der Anforderungen: Gelten alle Anforderungen bei sämtlichem Staatshandeln, oder gibt es Handlungsfelder, die von gewissen oder gar allen Anforderungen befreit sind? Gelten die Anforderungen immer gesamthaft, oder gibt es verschiedene, abgestufte Intensitäten ihrer Geltung? Und hängen allfällige Abstufungen von den verschiedenen Modalitäten der Aufgabenerfüllung, wie Akteur und Handlungsinstrument, $a b$ ?

Die Literatur stellt für die Grundsätze nach Art. 5 BV fest, dass sie immer und uneingeschränkt anwendbar sind. Art. 5 BV gilt für sämtliches Staatshandeln. ${ }^{163}$ Nicht nur die Eingriffsverwaltung, bei der Rechtspositionen von Bürgern durch die Verwaltung beschränkt werden, sondern auch die Leistungsverwaltung unterliegt diesen verfassungsrechtlichen Bindungen.

Die Verfassung selbst sieht auch bei den anderen Anforderungen nicht vor, dass sie selektiv gälten oder je nach Art und Weise des staatlichen Handelns abgestuft wären. Wenn immer die Verfassung vom Staat spricht, auferlegt sie ihm ihre Rechtsfolgen pauschal: Die rechtsstaatlichen Grundsätze von Art. 5 BV gelten für «staatliches Handeln»; «staatliche Organe» behandeln «jede Person» willkürfrei und nach Treu und Glauben (Art. 9); «wer staatliche Aufgaben wahrnimmt», muss «die Grundrechte» beachten (Art. 35 Abs. 2); Staatshaftung kommt zum Zug bei Schäden, die «in Ausübung amtlicher Tätigkeiten» entstehen (Art.146). Die Verfassung unterscheidet keine verschiedenen Handlungs-, Erfüllungs-, Wahrnehmungs-, Tätigkeits- oder Organtypen. Sämtliches Staatshandeln unterliegt diesen Anforderungen und es unterliegt ihnen in gleicher Weise.

162 HANGARTNER, Öffentlich-rechtliche Bindungen, S.140.

163 AUBERT/MAHON, Petit commentaire, Art. 5 Rz. 4; BIAGgINI, Kommentar BV, Art. 5 Rz. 6;

EPINEY, Basler Kommentar, Art. 5 Rz. 33. 
Wie schon bei der Staatsdefinition dargelegt, stellt die Verfassung eine Dichotomie zwei abgetrennter Sphären auf: auf der einen Seite das staatliche Handeln, auf der anderen das private Handeln (siehe vorne \$2/I.3). Alles staatliche Handeln unterliegt grundsätzlich denselben verfassungsrechtlichen Anforderungen.

Nach diesen Vorbemerkungen allgemeiner Art sind nun die Anforderungen inhaltlich zu konkretisieren, welche die Verfassung an staatliches Handeln stellt. 


\section{Gehalt der verfassungsrechtlichen Anforderungen}

\section{1. Übersicht und Quellen der Anforderungen}

Diese Arbeit nimmt eine rasterartige Prüfung vor, wobei der Prüfgegenstand die verschiedenen Aspekte staatlicher Aufgabenerfüllung sind, die privatisiert werden können (\$4). Der Prüfmassstab, der auf diesen Gegenstand angewendet wird, sind die verfassungsrechtlichen Anforderungen an staatliches Handeln. Damit diese Prüfung zu konkreten Ergebnissen führt, ist eine möglichst präzise Beschreibung dieses Massstabs erforderlich. «Verfassungsrechtliche Anforderungen an staatliches Handeln» ist kein gefestigter Begriff;164 diese Arbeit möchte eine vereinfachende Systematik für bekannte Rechtsfiguren schaffen und keine querliegenden Kategorien einführen.

Das funktionelle Staatsverständnis erlaubt es, die verfassungsrechtlichen Anforderungen zuverlässig einzugrenzen: Immer wenn die Verfassung eine Rechtsfolge an die Erfüllung einer staatlichen Aufgabe knüpft, liegt eine verfassungsrechtliche Anforderung an das staatliche Handeln vor. Die in der Bundesverfassung ausdrücklich festgeschriebenen Bindungen, denen das Staatshandeln genügen muss, sind die folgenden:

— Gesetzmässigkeit (Art. 5 Abs.1BV);

- öffentliches Interesse (Art. 5 Abs. 2 BV);

— Verhältnismässigkeit (Art. 5 Abs. 2 BV);

- Teilgehalte des Grundsatzes von Treu und Glauben (Art. 5 Abs.3, Art. 9 BV);

— Beachtung des Völkerrechts (Art. 5 Abs. 4 BV);

- Grundrechtsbindung (Art. 35 Abs. 2 i.V.m. Art.7-34 BV);

- Haftung für widerrechtlich verursachte Schäden in Ausübung amtlicher Tätigkeiten (Staatshaftung; Art.146 BV);

- Planung und Koordination staatlicher Tätigkeiten durch den Bundesrat (Art.180 Abs.1Satz 2 BV).

Da sich diese Arbeit im Bundesstaatsrecht verordnet und einen verfassungsdogmatischen Zugang verfolgt, werden die völkerrechtlichen Bindungen (nach Art. 5 Abs. 4 BV) nicht weiter behandelt. Inwiefern sich Privatisierungen mit völkerrechtlichen Pflichten vertragen, wäre Gegenstand einer gesonderten Abhandlung. 
Allerdings ist die Suche nach möglichen verfassungsrechtlichen Anforderungen in zwei Richtungen zu öffnen. Auch das ungeschriebene Verfassungsrecht und die Verfassung im materiellen Sinn ${ }^{165}$ sind mögliche Quellen für verfassungsrechtliche Anforderungen. Die verfassungsrechtlichen Anforderungen an staatliches Handeln werden hier als Ausdruck von Grundentscheidungen der Staatsordnung verstanden, wobei der materielle Gehalt höher zu gewichten ist als die ausdrückliche Verankerung im Verfassungsdokument. Eine unkommentierte Beschränkung auf geschriebenes Verfassungsrecht im formellen Sinn wäre somit ungerechtfertigt.

Seit Inkrafttreten der nachgeführten Bundesverfassung von 1999 scheint die Lehre davon auszugehen, dass kein ungeschriebenes Verfassungsrecht besteht; eine systematische Darstellung von Rechtssätzen, die als ungeschriebenes Verfassungsrecht anerkennt wären, fehlt jedenfalls. ${ }^{166} \mathrm{Im} \mathrm{Zu-}$ sammenhang mit Art. 5 BV wird darauf hingewiesen, dass diese Bestimmung die Grundlagen des Rechtsstaats nicht abschliessend nenne und insbesondere das Rückwirkungsverbot sowie das Prinzip der Gewaltenteilung dazuzuzählen seien. ${ }^{167}$ Diese Ergänzungen sind allerdings nicht als eigenständige verfassungsrechtliche Anforderungen im Sinne dieser Arbeit anzusehen. Das Rückwirkungsverbot ist ein Teilgehalt des Gesetzmässigkeitsprinzips (siehe sogleich 2), und die Gewaltenteilung ist vielmehr ein Prinzip der Behördenorganisation ${ }^{168}$ als eine Vorgabe an die staatliche Aufgabenerfüllung. Dieses Prinzip geht überdies zu einem grossen Teil im Gesetzmässigkeitsprinzip auf: Es schlägt sich dort in Voraussetzungen für die Delegation von Rechtsetzungsbefugnissen nieder. ${ }^{169}$ Ausserdem ist das Gewaltenteilungsprinzip kein absolutes Gebot: Der Gesetzgeber hat es beispielsweise in der Hand, demselben Aufgabenträger zugleich exekutive und judikative Funktionen zuzuweisen. $\mathbf{1 7 0}^{170}$

Rechtssätze, die von ihrem Gewicht her eigentlich Teil der Verfassung bilden könnten, aber darin nicht vorkommen, werden als Verfassungsrecht

165 Siehe dazu HÄFELIN/HALLER/KELLER/THURNHERR, Schweizerisches Bundesstaatsrecht, Rz.17-23; TSCHANNEN, Staatsrecht, §3 Rz.11-16.

166 RHINOW/SCHEFER/UEBERSAX, Schweizerisches Verfassungsrecht, Rz.13-20; TSCHANNEN, Staatsrecht, \$3 Rz.19 a.E. In anderen Lehrbüchern fehlt die Unterscheidung zwischen geschriebenem und ungeschriebenem Verfassungsrecht komplett: BIAGGINI, §§7 und 8 in: Staatsrecht; HÄFELIN/HALLER/KELLER/THURNHERR, Schweizerisches Bundesstaatsrecht, §1.

167 BIAGGINI, Kommentar BV, Art. 5 Rz. 5.

168 Vgl. die Kapitelüberschrift in TSCHANNEN, Staatsrecht, \$27.

169 TSCHANNEN, Staatsrecht, \$27 Rz.27-34.

170 Vgl. ausführlich zu den Relativierungen der Gewaltenteilung TSCHANNEN, Staatsrecht, §27 Rz. 8-11. 
im materiellen Sinn bezeichnet. ${ }^{171}$ Dazu gehören die Strukturprinzipien, die ungeschriebenen Grundrechte sowie der Rechtssatzbegriff. Dieser Letzte gehört wiederum zum Gesetzmässigkeitsprinzip und entfaltet keine eigenständige Bindungswirkung auf das Staatshandeln. Ungeschriebene Grundrechte wären wohl eher als ungeschriebenes Verfassungsrecht denn als materielles Verfassungsrecht zu thematisieren; so oder anders: Zurzeit werden keine ungeschriebenen Grundrechte anerkannt; die nachgeführte Bundesverfassung von 1999 hat den Bestand geltender Grundrechte verbindlich festgelegt. ${ }^{172}$

Die Strukturprinzipien - Demokratie, Rechtsstaat, Sozialstaat, Bundesstaat - prägen das staatliche Handeln in grundlegender Weise, aber auch sie dienen nicht als eigenständige verfassungsrechtliche Anforderungen. Dies, weil die Prinzipien nur soweit unumstritten bestehen, als sie konkrete Ausprägungen im Verfassungstext gefunden haben:173 Die direktdemokratischen Instrumente geben dem Demokratieprinzip Gestalt; Art. 5 BV definiert den Rechtsstaat; der Sozialstaat wird u.a. mit den Zielen von Art. 41 und den Grundrechten von Art. 11, 12, 19 und Art. 29 Abs. 3 BV konkretisiert; und die Kompetenzordnung von Art.3, 42-43a BV konkretisiert das Bundesstaatsprinzip. Was SCHINDLER für den Rechtsstaat festhält, kann als Maxime für alle Strukturprinzipien genommen werden: Es sei «Aufgabe des Verfassungsund Gesetzgebers, mittels Normen dem Rechtsstaat schärfere Konturen zu verleihen» $\mathbf{1 7 4}$.

Für die Zwecke dieser Arbeit kann sich die Liste verfassungsrechtlicher Anforderungen also auf die ausdrücklich erwähnten Bindungen der Bundesverfassung beschränken.

Auch einfaches Gesetzesrecht und Verordnungen können Regeln aufstellen, die nur den Staat binden. ${ }^{175}$ So müssen Träger öffentlicher Aufgaben etwa freien Zugang zum Markt im Sinne des Binnenmarktgesetzes sicherstellen (Art. 2 BGBM) und grössere Aufträge in Übereinstimmung mit dem öffentlichen Beschaffungsrecht ausschreiben und vergeben (Art. 2, 6 BöB). Diese Arbeit untersucht jedoch nur die Anforderungen vertieft, die in der Verfassung selbst vorgesehen sind. Die verschiedenen Erscheinungsformen privatisierter Aufgabenerfüllung sollen an den Grundentscheidungen der Verfassung gemessen werden.

171 TSCHANNEN, Staatsrecht, §3 Rz.11, Rz.15 zum Folgenden.

172 TSCHANNEN, Staatsrecht, \$3 Rz. 20.

173 Siehe TSCHANNEN, Staatsrecht, §6Rz.19f., 28-31, 37-39, 43-46.

174 SCHINDLER, St. Galler Kommentar, Art. 5 Rz. 8.

175 Für eine Übersicht siehe RÜTSCHE, Öffentliche Aufgaben, S.153 f. 


\section{Gesetzmässigkeit}

Die erste und bedeutsamste verfassungsrechtliche Anforderung ist das Erfordernis einer genügenden gesetzlichen Grundlage. Die Verfassung verankert sie in Art. 5 Abs. 1 BV in grundlegender Weise für sämtliches staatliches Handeln und doppelt in Art. 36 Abs. 1 bezogen auf Grundrechtseinschränkungen nach. Ausserdem führt die Verfassung das Erfordernis in bestimmten Sachbereichen noch aus: Art.127 Abs. 1 bestimmt, welche Grundsätze für Besteuerungen zwingend gesetzlich zu verankern sind; Art. 178 Abs. 3 sieht für Aufgabenübertragungen ein Gesetzeserfordernis vor. Und in den Art. 163-165 werden Leitlinien für den Gesetzgeber aufgestellt, insbesondere erwähnt Art. 164 Abs.1, welche Regelungsgegenstände zwingend in einem formellen Gesetz unterzubringen sind. ${ }^{176}$

Der Gesetzmässigkeitsgrundsatz ist gleichzeitig legitimierende Grundlage wie begrenzende Schranke für staatliches Handeln - wie es der Wortlaut von Art. 5 Abs. 1 BV auf eine schöne Formel bringt. Der Staat muss sich auf eine gesetzliche Grundlage stützen können, um überhaupt tätig zu werden, und darf nur so weit gehen, wie es diese Grundlage erlaubt. ${ }^{177}$

Die Gesetzmässigkeit lässt sich - leicht verkürzend - in drei Teile gliedern:178 (1) Es muss eine gesetzliche Grundlage bestehen, die generelle Regeln aufstellt. (2) Dieser Rechtssatz muss richtig erlassen worden sein; im richtigen Verfahren und konform mit übergeordnetem Recht. (3) Der Rechtssatz muss geeignet sein, um darauf gestütztes Handeln zu legitimieren.

Die ersten zwei Teile der Gesetzmässigkeit sind gedankliche Vorstufen, die in der Praxis kaum Probleme schaffen. Anders liegt es beim dritten und entscheidenden Punkt: Eine gesetzliche Grundlage muss nach ihrer Stufe und nach ihrem Inhalt geeignet sein, das staatliche Handeln zu begründen und zu begrenzen. Mit anderen Worten müssen Normdichte und Normstufe genügend sein, worauf im Folgenden näher einzugehen ist.

176 Vgl. zum Teil AUBERT/MAHON, Petit commentaire, Art. 5 Rz. 6.

177 Auch bezeichnet als Vorbehalt des Gesetzes und Vorrang des Gesetzes, BIAGGINI, Kommentar BV, Art. 5 Rz. 7; HÄFELIN/MÜLLER/UHLMANN, Allgemeines Verwaltungsrecht, Rz.325.

178 Vgl. TSCHANNEN/ZIMMERLI/MÜLLER, Allgemeines Verwaltungsrecht, §19 Rz. 2 f., wo dieselben Elemente in fünf einzelne Postulate gegliedert werden. Leicht verkürzt auch BGE 130 I 1 E. 3.1 S. 5. Zweiteilige Gliederung in Erfordernis des Rechtssatzes und Erfordernis der Gesetzesform bei HÄFELIN/MÜLLER/UHLMANN, Allgemeines Verwaltungsrecht, Rz. $338 \mathrm{ff} ., 350 \mathrm{ff}$. 


\section{a. Das Erfordernis einer genügend bestimmten Norm (Normdichte)}

Das Gebot der genügenden Normdichte179 bedeutet gemäss dem Bundesgericht, dass Rechtssätze «so präzise formuliert sein [müssen], dass die Rechtsunterworfenen ihr Verhalten danach einrichten und die Folgen eines bestimmten Verhaltens mit einem den Umständen entsprechenden Grad an Gewissheit erkennen können» $\mathbf{1 8 0}$.

Dieser Teilgehalt des Gesetzmässigkeitsprinzips soll Rechtssicherheit gewährleisten, darf die Einzelfallgerechtigkeit aber nicht vereiteln. ${ }^{181}$ Rechtssätze sollen so gehaltvoll sein, dass darauf gestützte Entscheidungen von Behörden erwartbar werden, dass also deren Auslegung nicht völlig offen ist. ${ }^{182}$ Zugleich müssen generell-abstrakte Normen ja gerade auf eine unbestimmte Anzahl Fälle angewendet werden können, weshalb sie genügend Spielraum für Lösungen belassen müssen, die im Einzelfall gerecht sind. ${ }^{183}$ Oder in den Worten des Bundesgerichts:

«Der Grad der erforderlichen Bestimmtheit lässt sich nicht abstrakt festlegen. Er hängt unter anderem von der Vielfalt der zu ordnenden Sachverhalte, von der Komplexität und der Vorhersehbarkeit der im Einzelfall erforderlichen Entscheidungen, von den Normadressaten, von der Schwere des Eingriffs in die Verfassungsrechte sowie von der erst bei der Konkretisierung im Einzelfall möglichen sachgerechten Entscheidung ab»184 .

Das Erfordernis der genügenden Normdichte ist damit zwar selber wenig bestimmt, gibt aber immerhin einen handhabbaren Prüfmassstab vor: Kann sich eine Staatshandlung auf einen Rechtssatz stützen, der diese genügend vorgezeichnet hat, sodass sie nicht unbegründet erscheint? Und je stärker das staatliche Handeln die Rechtsstellung Privater betrifft, desto deutlicher vorgezeichnet muss es sein. 185

179 Alternative Begriffe Bestimmtheitsgebot und Normbestimmtheit bei BIAGGINI, Kommentar BV, Art. 5 Rz.10, Art. 36 Rz.12; TSCHANNEN/ZIMMERLI/MÜLLER, Allgemeines Verwaltungsrecht, \$19 Rz.19.

180 BGE143 I 310 E. 3.3.1 S. 314; 139 I 280 E. 5.1 S. 284; ähnlich schon BGE 109 Ia 273 E. 4d S. 283.

181 TSCHANNEN/ZIMMERLI/MÜLLER, Allgemeines Verwaltungsrecht, \$19 Rz.19. Ähnlich auch BIAGGINI, Kommentar BV, Art. 36 Rz.11.

182 HÄFELIN/MÜLLER/UHLMANN, Allgemeines Verwaltungsrecht, Rz.342.

183 HÄFELIN/MÜLLER/UHLMANN, Allgemeines Verwaltungsrecht, Rz.344.

184 BGE 143 II 162 E. 3.2.1 S. 169; fast gleichlautend auch BGE 139 II 243 E.10 S. 252; 136 I 87 E. 3.1 S. 90; grundlegend dazu BGE 128 I 327 E. 4.2 S. 339 f.

185 BIAGGINI, Kommentar BV, Art.36 Rz.12. 


\section{b. Das Erfordernis einer Norm von genügender Erlassstufe (Normstufe)}

Rechtssätze müssen nicht nur inhaltlich bestimmt genug sein, sie müssen auch in einem Erlass von genügender Stufe enthalten sein. Dieses Erfordernis bestimmt, ob eine Verordnungsnorm als Grundlage für eine Staatshandlung reicht oder ob ein Gesetz im formellen Sinn verlangt wird. ${ }^{186}$ In diesem Zusammenhang stellen sich zwei Fragen: Welches sind die «wichtigen» Regelungsgegenstände, die kraft Art. 164 Abs. 1 BV zwingend in das Gesetz selbst gehören? Und unter welchen Voraussetzungen ist eine Delegation der Rechtsetzungsbefugnis an den Verordnungsgeber zulässig?

Beide dieser Themen sind in der Lehre eingehend bearbeitet worden und müssen an dieser Stelle nicht wiederholt werden. Gesagt sei immerhin so viel: Was wichtig oder wesentlich ist, entspringt einer rechtspolitischen Wertung und lässt sich nicht verbindlich umreissen. ${ }^{187}$ Es lassen sich nur Anhaltspunkte und Indizien beiziehen wie etwa die Anzahl der Betroffenen, die Intensität des Eingriffs, finanzielle Auswirkungen oder die öffentliche Akzeptanz. ${ }^{188}$

Die Voraussetzungen der Gesetzesdelegation bilden gefestigte herrschende Lehre; sie wurden vor allem anhand von Grundrechtseingriffen erarbeitet. Hier zu resümieren ist nur Folgendes: Auch Staatshandlungen, die die Rechtsstellung des Einzelnen schwer betreffen, können sich auf Verordnungsnormen stützen, sofern das Gesetz selbst die «Grundzüge der delegierten Materie» regelt. ${ }^{189}$

Das Erfordernis einer genügenden Erlassstufe gründet in der legitimierenden Wirkung der Gesetzesform:190 Erlasse des Parlaments verfügen über die höchste demokratische Legitimation und können damit Staatshandlungen eher rechtfertigen, die einschneidend, umstritten und folgenschwer sind.

\section{c. Sonderstellung gegenüber anderen Anforderungen}

Die Gesetzmässigkeit nimmt eine besondere Rolle unter den verfassungsrechtlichen Anforderungen ein; sie ist das Öhr, durch welches die Einhaltung anderer Anforderungen beurteilt wird. So verzichtete etwa das Bundesgericht schon auf diegesonderte Prüfung des öffentlichen Interesses, weil diegesetzliche Grund-

186 HÄFELIN/MÜLLER/UHLMANN, Allgemeines Verwaltungsrecht, Rz.350-352; TSCHANNEN/ ZIMMERLI/MÜLLER, Allgemeines Verwaltungsrecht, §19 Rz.16 f.

187 TSCHANNEN/ZIMMERLI/MÜLLER, Allgemeines Verwaltungsrecht, §19 Rz.17.

188 HÄFELIN/MÜLLER/UHLMANN, Allgemeines Verwaltungsrecht, Rz.354; TSCHANNEN/ ZIMMERLI/MÜLLER, Allgemeines Verwaltungsrecht, §19 Rz. 5.

189 HÄFELIN/MÜLLER/UHLMANN, Allgemeines Verwaltungsrecht, Rz.368; fast gleichlautend auch TSCHANNEN/ZIMMERLI/MÜLLER, Allgemeines Verwaltungsrecht, §19 Rz. 38.

190 Vgl. «erhöhter Legitimationsbedarf» bei TSCHANNEN/ZIMMERLI/MÜLLER, Allgemeines Verwaltungsrecht, \$19 Rz. 6 . 
lage ja als Abschluss eines demokratischen Erörterungsprozesses anzusehen sei, der das Vorliegen eines öffentlichen Interesses bezeuge. ${ }^{191}$ Anders gewendet: Wenn der Gesetzgeber eine gewisse Tätigkeit gesetzlich erlaubt, drückt er damit aus, dass aus seiner Sicht ein öffentliches Interesse dafür besteht.

\section{d. Geltungsweise}

Grundsätzlich gilt das Gesetzmässigkeitsprinzip für jedes Staatshandeln 192 aber eben nur grundsätzlich. Es gibt zwei Arten von Ausnahmen: In gewissen Fällen ist das Staatshandeln befreit vom Gesetzmässigkeitsprinzip, in anderen wird dieses gelockert.

In zwei Fällen kann die gesetzliche Grundlage substituiert werden: durch die polizeiliche Generalklausel und die Sachherrschaft über öffentliche Sachen. ${ }^{193}$

- Die polizeiliche Generalklausel kann eine gesetzliche Grundlage ersetzen, wenn eine ernste und unmittelbar drohende Gefahr für hochstehende Rechtsgüter nicht anders abgewendet werden kann. ${ }^{194}$ Die polizeiliche Generalklausel darf aber nur subsidiär zum Einsatz kommen; sie kann also nur ein fehlendes Gesetz ersetzen, nicht von einem unpassenden abweichen. ${ }^{195}$

- Der Staat darf überdies aufgrund seiner Sachherrschaft den gesteigerten Gemeingebrauch einer öffentlichen Sache auch ohne ausdrückliche Grundlage für bewilligungspflichtig erklären. ${ }^{196}$ Die Verfügungsgewalt des Gemeinwesens über eine öffentliche Sache schliesst somit das Recht ein, eine Bewilligungspflicht für deren Benutzung einzuführen. Noch besteht diese Gerichtspraxis; nach deutlicher Kritik hat das Bundesgericht zuletzt 2009 offengelassen, ob es künftig an dieser Praxis festhalten wolle. ${ }^{197}$

191 BGE 138 I 378 E. 8.3 S.394.

192 BGE 130 I 1 E. 3.1 S. 5; EPINEY, Basler Kommentar, Art. 5 Rz. 33 unter Bezug auf Art. 5 BV insgesamt.

193 TSCHANNEN/ZIMMERLI/MÜLLER, Allgemeines Verwaltungsrecht, §19 Rz. 22a, spricht von Vorbehalten und Durchbrechungen.

194 Art. 36 Abs. 1 Satz 3 BV (für Grundrechtseingriffe); HÄFELIN/MÜLLER/UHLMANN, Allgemeines Verwaltungsrecht, Rz. 2580; TSCHANNEN/ZIMMERLI/MüLLER, Allgemeines Verwaltungsrecht, §19 Rz. 22a, §56 Rz.4-10.

195 BGE 137 II 431 E.3.3.1 S.444; TSCHANNEN/ZIMMERLI/MÜLLER, Allgemeines Verwaltungsrecht, §56 Rz. 6.

196 TSCHANNEN/ZIMMERLI/MÜLLER, Allgemeines Verwaltungsrecht, \$19 Rz. 22a, §51 Rz.13. A.M. HÄFELIN/MÜLLER/UHLMANN, Allgemeines Verwaltungsrecht, Rz.384, wonach die BV 1999 diese Praxis nicht mehr zulasse.

197 BGE 135 I 302 E. 3.2 S.307 f. In einem späteren Entscheid hat das Bundesgericht die Frage einer allfälligen Praxisänderung allerdings nicht mehr angesprochen, BGE 138 I 274 E. 3.3. S. 286; vgl. TSCHANNEN/ZIMMERLI/MÜLLER, Allgemeines Verwaltungsrecht, §51 Rz.15. 
In drei Bereichen des Verwaltungshandelns gelten geringere Anforderungen an die Normdichte:

- Leistungsverwaltung. In der Leistungsverwaltung muss zwar eine gesetzliche Grundlage vorliegen, 198 allerdings sind die Anforderungen an deren Bestimmtheit geringer. ${ }^{199}$ Besonders für die Bemessung von Kausalabgaben als Entgelt für staatliche Leistungen reicht eine weniger detaillierte gesetzliche Grundlage aus. ${ }^{200}$ Allerdings gilt auch bei der Leistungsverwaltung wieder die übliche Anforderungsintensität, wenn mit der Leistung Pflichten verbunden sind, die staatliche Leistung Vorbedingung für die Ausübung von Grundrechten ist oder wenn die Leistung Rechtssicherheits- und Rechtsgleichheitsfragen aufwirft.201

- Wirtschaftendes Staatshandeln. Als zweiten Fall herabgesetzter Anforderungen an die Bestimmtheit der gesetzlichen Grundlage identifiziert das Bundesgericht die staatlichen Tätigkeiten, «die nach marktwirtschaftlichen Prinzipien geregelt werden»202. Das Bundesgericht hat diese Lockerung aus dem Glarnersach-Entscheid noch nicht als allgemeine Praxis verstetigt. ${ }^{203}$ Doch hat es damit eine Richtung aufgezeigt: Das Gericht drängt die demokratische und die rechtsstaatliche Funktion des Gesetzmässigkeitsprinzips zurück, «um die unternehmerische Tätigkeit nicht zu behindern» ${ }^{204}$ (siehe auch hinten \$6/II.1.c).

- Besondere Rechtsverhältnisse. Gefestigte Praxis sind hingegen die deutlich herabgesetzten Anforderungen an Normstufe und -dichte im besonderen Rechtsverhältnis. ${ }^{205}$ Ein solches liegt vor, wenn «Bürgerinnen und Bürger in einer besonders nahen und engen Beziehung zum Staat stehen» 206 , etwa als Insassen einer staatlichen Anstalt oder als Nutzer einer öffentlichen Einrichtung. Nach aktueller Praxis darf sich der Staat für

198 BGE 130 I 1 E.3.1 S. 5; HÄFELIN/MÜLLER/UHLMANN, Allgemeines Verwaltungsrecht, Rz.39, 379.

199 BGE138 I 378 E. 7.2 S.392; HÄFELIN/MÜLLER/UHLMANN, Allgemeines Verwaltungsrecht, Rz.381.

200 BGE 125 I 182 E. 4a S. 193; 121 I 230 E. 3g/aa S. 238. Ferner WIEDERKEHR, Legalitätsprinzip im Kausalabgaberecht, S. 45.

201 TSCHANNEN, Systeme, Rz.26.

202 BGE 138 I 378 E.7.2 S.392.

203 Eine allgemeine Praxis scheinbar annehmend BAUMANN, Wettbewerbsverzerrungen, Rz.303.

204 BGE 138 I378 E.7.2 S.392.

205 TSCHANNEN/ZIMMERLI/MÜLLER, Allgemeines Verwaltungsrecht, §43 Rz.31.

206 HÄFELIN/MÜLLER/UHLMANN, Allgemeines Verwaltungsrecht, Rz. 450, dort als «Sonderstatusverhältnis» bezeichnet; TSCHANNEN/ZIMMERLI/MÜLLER, Allgemeines Verwaltungsrecht, \$43 Rz. 22. 
leichte Eingriffe auf weniger detaillierte Normen tieferer Stufe stützen (herabgesetzte Normdichte und Normstufe). ${ }^{207}$

\section{e. Gerichtliche Durchsetzbarkeit}

Die Gesetzmässigkeit kann in zwei Gebieten als verfassungsmässiges Recht eigenständig gerügt werden: im Strafrecht und im Abgaberecht. ${ }^{208}$ Eigenständig anrufbar ist das Prinzip überdies in der Beschwerde in öffentlichrechtlichen Angelegenheiten im Rahmen von Verletzungen von Bundesrecht.209 Soweit kantonale Erlasse oder auf kantonales Recht gestützte Entscheide angefochten werden, muss eine Verletzung des Gesetzmässigkeitsprinzips in Verbindung mit einem anderen Recht gerügt werden: der Verletzung eines Freiheitsrechts, des Willkürverbots oder im Rahmen der Gewaltenteilungsbeschwerde. 210

\section{3. Öffentliches Interesse}

Die Vorgabe von Art. 5 Abs. 2 BV, Staatstätigkeit müsse «im öffentlichen Interesse liegen», dient der «Begründung und Begrenzung staatlicher Handlungsmotive» 211. Staatliches Handeln muss aufdas Gemeinwohlausgerichtetsein,212 womit Anliegen gemeint sind, «welche die Öffentlichkeit (verstanden als Allgemeinheit, Bevölkerung, Publikum) für erstrebens- oder erhaltenswert hält.»213 Anders gewendet darf Staatstätigkeit nicht durch private Interessen oder Sondergruppeninteressen motiviert sein. ${ }^{214}$

Welche Interessen hingegen öffentlich sind, bestimmt die Verfassung selber nicht. ${ }^{215}$ Ziel- und Aufgabennormen können dafür ein Anhaltspunkt sein; jedenfalls nennt die Verfassung zulässige Interessen nicht abschlies-

207 HÄFELIN/MÜLLER/UHLMANN, Allgemeines Verwaltungsrecht, Rz.453 f.; TSCHANNEN/ ZIMMERLI/MÜLLER, Allgemeines Verwaltungsrecht, §43 Rz.31. Nach früherer Praxis war der Staat gänzlich vom Erfordernis einer gesetzlichen Grundlage befreit; MÄCHLER, Vertrag und Verwaltungsrechtspflege, \$2 Rz.15, m.w.H.

208 BGE 134 I 322 E. 2.1 S. 326; 129 I 161 E. 2.2 S.163; auch zum Folgenden.

209 Art. 89 ff., insb. 95 Bst. a BGG; TSCHANNEN/ZIMMERLI/MÜLLER, Allgemeines Verwaltungsrecht, \$19 Rz. 45 .

210 TSCHANNEN/ZIMMERLI/MÜLLER, Allgemeines Verwaltungsrecht, \$19 Rz. $46 \mathrm{f}$.

211 TSCHANNEN/ZIMMERLI/MÜLLER, Allgemeines Verwaltungsrecht, \$20 Rz.1, ohne die Hervorhebung im Original.

212 BIAGGINI, Kommentar BV, Art. 5 Rz. 15; HÄFELIN/MÜLLER/UHLMANN, Allgemeines Verwaltungsrecht, Rz.461.

213 TSCHANNEN/ZIMMERLI/MÜLLER, Allgemeines Verwaltungsrecht, §20 Rz. 2.

214 BIAGGINI, Kommentar BV, Art. 5 Rz.15.

215 BIAGGINI, Kommentar BV, Art. 5 Rz.16; TSCHANNEN/ZIMMERLI/MÜLLER, Allgemeines Verwaltungsrecht, $\$ 20$ Rz.3. 
send und stellt keine Hierarchie verschiedener Interessen auf. Für jedes Staatshandeln muss nach seinen konkreten Umständen erörtert werden, ob ein öffentliches Interesse vorliegt und ob es mit Blick auf das eingeschränkte Rechtsgut zulässig ist (Interessenqualifikation und -selektion). ${ }^{216}$

Um sodann zu beurteilen, ob die so bestimmten Interessen ein staatliches Handeln zu rechtfertigen vermögen, müssen sie gegen die Interessen der betroffenen Person(en) abgewogen werden, was unter dem Titel der Verhältnismässigkeit geschieht.

Im Folgenden ist die Sonderfrage abzuhandeln, ob auch monetäre Ziele staatliches Handeln begründen und legitimieren können.

\section{a. Zulässigkeit fiskalischer Interessen}

Bei den öffentlichen Interessen wird immer wieder diskutiert, ob auch rein fiskalische Interessen für ein Tätigwerden des Staats ausreichen oder ob der Staat zwingend auch über andere als finanzielle Motive verfügen muss. ${ }^{217}$

Dass fiskalische Interessen - neben anderen - ein legitimes Motiv bilden können, ist unbestritten. ${ }^{218}$ Dies wäre nur anders, wenn die Verfassung Steuern als ausschliessliche Einnahmequelle bestimmen würde. Das ist nicht der Fall: Die Auflistung der Steuerarten in den Art.128-132 BV lässt keine Ausschliesslichkeit erkennen und die Verfassung gibt dem Staat auch andernorts keinerlei Richtlinien bezüglich Mittelbeschaffung vor. Ausserdem werden Verwaltungsträger gerade mit der Begründung organisatorisch ausgelagert, dass sie dadurch an unternehmerischer Freiheit gewännen. Betriebswirtschaftliche Ziele, und damit letztlich fiskalische Interessen, werden dadurch vom Gesetzgeber zu legitimen öffentlichen Interessen erhoben. ${ }^{219}$

Ob unternehmerisches Staatshandeln alleine mit fiskalischen Interessen gerechtfertigt werden kann, hat das Bundesgericht bislang offengelassen.220 Die neuere Praxis legt diesen Schluss aber nahe. Im vieldiskutierten Glarnersach-Urteil wurde die wettbewerbliche Tätigkeit einer staatlichen Versicherungsanstalt «aus betriebswirtschaftlichen Überlegungen»221 für zulässig erklärt. Das Gericht umging es aber, die Frage abschliessend zu beurteilen.

216 TSCHANNEN/ZIMMERLI/MÜLLER, Allgemeines Verwaltungsrecht, §20 Rz.13; auch zum Folgenden.

217 Dieser Abschnitt ist teilweise entnommen aus einer früheren Publikation: ELSER, Funktioneller Staatsbegriff, S. $72 \mathrm{f}$.

218 BIAGGINI, Kommentar BV, Art. 5 Rz.16; HÄFELIN/MÜLLER/UHLMANN, Allgemeines Verwaltungsrecht, Rz. 483; TSCHANNEN/ZIMMERLI/MÜLLER, Allgemeines Verwaltungsrecht, §20 Rz.9.

219 HÄNER, Grundrechtsgeltung, S.1151 f.

220 BGE 138 I 378 E. 8.6.2 S.397; 120 II 321 E.2d S. 326.

221 BGE 138I 378 E. 8.6.2 S.397. 
Ebenfalls in diese Richtung weist allerdings die Wertung des Bundesgerichts, die Gewinnerzielung mit Werbeeinnahmen sei eine (zumindest mittelbare) Staatsaufgabe, da die Aufgabenerfüllung entsprechende Mittel voraussetze (siehe auch hinten \$ 4/I.2).222 Damit ist das Gericht kurz davor stehen geblieben, finanziellen Profit anderen Motiven für staatliches Handeln gleichzustellen. Es wäre dann nur noch konsequent, fiskalische Interessen auch alleine als zulässiges Motiv anzusehen.

\section{b. Konkretisierung im politischen Prozess}

Was Anliegen der Öffentlichkeit sind, bestimmt nicht die Verfassung abschliessend, sondern oft erst das Gesetz. ${ }^{223}$ Dort wird festgelegt, welche Zwecke und Ziele förderungswürdig und schützenswert sind und dadurch zu zulässigen Motiven für staatliches Handeln werden.

Die zulässigen Interessen sind der Rechtsordnung nicht vorgegeben, sondern durch diese selbst erst bestimmt und sind somit Ausdruck einer politischen Wertung im demokratischen Prozess. ${ }^{224}$ Öffentliche Interessen sind somit auch wandelbar, neue können entstehen und alte untergehen.225 Es liesse sich also beklagen, öffentliche Interessen würden dadurch beliebig und entfalteten «keine Steuerungs- oder Begrenzungsfunktion» 226 .

Allerdings muss man sich vor Augen führen, dass das öffentliche Interesse kein inhaltlicher, sondern ein prozessualer Begriff ist.227 Erstens muss ein solches überhaupt aufgeführt werden, und zweitens ist abschliessend vorgegeben, in welchem Verfahren neue Anliegen zu öffentlichen Interessen erhoben werden können.

\section{c. Geltungsweise}

Der Grundsatz desöffentlichen Interessesgiltfür sämtlichesStaatshandeln;228 Befreiungen davon gibt es nicht - anders als bei der Gesetzmässigkeit. Die Frage der abgestuften Anforderungen stellt sich hier aus zwei Gründen nicht:

222 BGE 139 I 306 E. 3.2.2 S.311; 138 I 274 E.1.4 S. 278 ff.

223 Vgl. BIAGGINI, Kommentar BV, Art.36 Rz. 18; HÄFELIN/MÜLLER/UHLMANN, Allgemeines Verwaltungsrecht, Rz.462.

224 BGE 142 I 49 E. 8.1 S. 66; 138 I 378 E. 8.3 S. 393; TSCHANNEN/ZIMMERLI/MÜLLER, Allgemeines Verwaltungsrecht, §20 Rz.3; TSCHANNEN/LOCHER, Aufsicht Gebäudeversicherungen, S. 85 .

225 HÄFELIN/MÜLLER/UHLMANN, Allgemeines Verwaltungsrecht, Rz.465; TSCHANNEN/ ZIMMERLI/MÜLLER, Allgemeines Verwaltungsrecht, §20 Rz.10.

226 UHLMANN, Gewinnorientiertes Staatshandeln, S. $239 \mathrm{f}$.

227 TSCHANNEN/LOCHER, Aufsicht Gebäudeversicherungen, S. 85.

228 HÄFELIN/MÜLLER/UHLMANN, Allgemeines Verwaltungsrecht, Rz.490-492; TSCHANNEN/ZIMMERLI/MÜLLER, Allgemeines Verwaltungsrecht, \$20 Rz.11. Allgemein für die rechtsstaatlichen Anforderungen von Art. 5 BV EPINEY, Basler Kommentar, Art. 5 Rz. 33. 
Erstens ist es von der konkreten Konstellation abhängig, welche Motive legitim sind, und nicht von einem absoluten Massstab. Zweitens entscheidet erst die Verhältnismässigkeitsprüfung, ob ein als legitim anerkanntes Interesse auch gewichtig genug ist, um die konkrete Handlung zu rechtfertigen.

Die vertiefte Praxis zu Art. 36 Abs. 2 BV bei der Prüfung von Grundrechtseingriffen bestätigt diese Relativierung: Es gibt keine Hierarchie zulässiger Interessen, keine bereichsweise Abstufung verschieden hoher Hürden. Für Eingriffe in dieselben Schutzbereiche mögen jeweils dieselben Interessen beigezogen werden, ${ }^{229}$ es bleibt aber bei einer einzelfallweisen Feststellung und Bewertung.

\section{d. Gerichtliche Durchsetzbarkeit}

Sofern Entscheide angefochten werden, die sich auf Bundesrecht stützen, kann die fehlerhafte Beachtung öffentlicher Interessen im Verfahren der öffentlichrechtlichen Beschwerde eigenständig gerügt werden. ${ }^{230}$ Allerdings ist der Grundsatz des öffentlichen Interesses kein verfassungsmässiges Recht und kann in allen anderen Fällen nur mittelbar gerügt werden: in Verbindung mit der Verletzung von Freiheitsrechten oder des Willkürverbots. ${ }^{231}$

Das Bundesgericht prüft das Vorliegen eines öffentlichen Interesses grundsätzlich frei, hält sich aber zurück, «soweit die Beurteilung von einer Würdigung der örtlichen Verhältnisse abhängt, welche die kantonalen Behörden besser überblicken»232. Diese Zurückhaltung legt sich das Gericht besonders in planungs- und baurechtlichen Fragen auf. ${ }^{233}$

\section{Verhältnismässigkeit}

Direkt anknüpfend an das öffentliche Interesse fordert Art. 5 Abs. 2 BV weiter, dass staatliches Handeln «verhältnismässig sein» muss. Eine staatliche Handlung muss nicht nur durch ein legitimes Anliegen motiviert sein, es muss dieses Motiv auch fördern, und dies möglichst schonend. Ausserdem ist das Motiv einer Handlung an dessen Wirkungen zu messen.234 Aus diesem Gedan-

229 HÄFELIN/MÜLLER/UHLMANN, Allgemeines Verwaltungsrecht, Rz. 494; TSCHANNEN/ ZIMMERLI/MÜLLER, Allgemeines Verwaltungsrecht, \$2O Rz.12.

230 Art. 89 ff., insb. 95 Bst. a BGG; TSCHANNEN/ZIMMERLI/MÜLLER, Allgemeines Verwaltungsrecht, §20 Rz.15.

231 HÄFELIN/MÜLLER/UHLMANN, Allgemeines Verwaltungsrecht, Rz. 494; TSCHANNEN/ ZIMMERLI/MÜLLER, Allgemeines Verwaltungsrecht, \$20 Rz.14-16.

232 BGE 142 I 162 E.3.2.2 S.165.

233 BGE 132 II 408 E. 4.3 S. 416; 129 I 337 E. 4.1 S. 344 (das Bundesgericht sei keine «autorità superiore di pianificazione»); 126 I 219 E. 2c S. 222, betreffend Denkmalschutz.

234 TSCHANNEN/ZIMMERLI/MÜLLER, Allgemeines Verwaltungsrecht, §21 Rz.1. 
ken haben Lehre und Rechtsprechung die drei bekannten Teilforderungen der Eignung, Erforderlichkeit und Zumutbarkeit abgeleitet. ${ }^{235}$

\section{a. Einzelne Postulate}

An dieser Stelle muss die Dogmatik nicht in allem Detail wiedergegeben werden, einige Hinweise auf einzelne Postulate sollen im Folgenden genügen.

Zwecktauglichkeit. Der erste Teilgehalt des Verhältnismässigkeitsprinzips, die Eignung, stellt sicher, dass staatliche Handlungen ein sinnvolles Ziel anvisieren und dieses auch erreichen.236 Darin liegt ein Kern rechtsstaatlicher Rechtfertigung von Staatstätigkeit: Interessen der Öffentlichkeit müssen nicht nur als Leitidee vorliegen, sie müssen auch tatkräftig befördert werden. Diesen Grundgedanken drückt auch Art. 178 Abs. 1 BV aus, der im Zusammenhang mit Organisationsvorgaben für die Bundesverwaltung eine «zielgerichtete Erfüllung der Aufgaben» fordert.

Zielgenauigkeit. Zur Zielgerichtetheit muss eine gewisse Präzision hinzukommen, denn die Zwecktauglichkeit legitimiert das Staatshandeln zwar, sie begrenzt es aber nicht. Der Staat könnte mit übermässigem Aufwand und übermässig einschränkendem Vorgehen seine Ziele erfüllen. ${ }^{237}$ Dieses Anliegen wird durch die Erforderlichkeit eingelöst. Staatshandeln darf die Rechtsstellung Privater nur soweit einschränken, als dies für die Zielerreichung notwendig ist - und zwar in sachlicher, räumlicher, zeitlicher und persönlicher Hinsicht.238

Interessenabwägung. Das Verhältnismässigkeitsprinzip dient nicht nur dazu, das staatliche Handeln möglichst genau an die verfolgten öffentlichen Interessen zu binden, es dient auch der Einzelfallgerechtigkeit, indem es die Sichtweise und Interessen der betroffenen Privatpersonen berücksichtigt. Eine staatliche Handlung mag noch so zielgenau legitime Motive verfolgen, sie bleibt illegitim, wenn dieprivaten Interessen dieöffentlichenimEinzelfallüberwiegen. ${ }^{239}$ Diese Interessenabwägung darf nicht als freihändiges, ergebnisorientiertes Herumhantieren missverstanden werden. Sie erfolgt gemäss TSCHANNEN in

235 Dazu ausführlich HÄFELIN/MÜLLER/UHLMANN, Allgemeines Verwaltungsrecht, Rz. 521-564; TSCHANNEN/ZIMMERLI/MÜLLER, Allgemeines Verwaltungsrecht, §21 Rz. 2-17.

236 HÄFELIN/MÜLLER/UHLMANN, Allgemeines Verwaltungsrecht, Rz. 522; TSCHANNEN/ZIMMERLI/MÜLLER, Allgemeines Verwaltungsrecht, §21 Rz. 4.

237 Genau unzutreffend HÄFELIN/MÜLLER/UHLMANN, Allgemeines Verwaltungsrecht, Rz. 522, wonach die «Geeignetheit» der Präzision staatlichen Handelns dienen soll.

238 HÄFELIN/MÜLLER/UHLMANN, Allgemeines Verwaltungsrecht, Rz. 530-554; TSCHANNEN/ZIMMERLI/MÜLLER, Allgemeines Verwaltungsrecht, \$21 Rz. 8-15.

239 Vgl. zur Zumutbarkeit ausführlich HÄFELIN/MÜLLER/UHLMANN, Allgemeines Verwaltungsrecht, Rz. 555-558; TSCHANNEN/ZIMMERLI/MÜLLER, Allgemeines Verwaltungsrecht, §21 Rz.16. 
drei gedanklichen Schritten:240 Die rechtlich bedeutsamen Interessen sind zunächst zu ermitteln und dann «mithilfe rechtlich ausgewiesener Massstäbe und mit Blick auf die möglichen Auswirkungen» zu beurteilen. Im dritten und entscheidenden Schritt sind die Interessen zu optimieren; es soll also diejenige Lösung gefunden werden, bei der die ermittelten und beurteilten Interessen gemäss ihrem Gewicht «möglichst umfassend zur Geltung kommen».

\section{b. Geltungsweise}

Das Verhältnismässigkeitsprinzip gilt in der gesamten Rechtsordnung241 und für sämtliches Staatshandeln, auch wenn dieses in privatrechtlichen Handlungsformen erbracht wird.242 In einzelnen Rechtsgebieten ist das Prinzip aber besonders bedeutsam oder erfährt besondere Ausprägungen.

Besonderes Gewicht hat der Grundsatz der Verhältnismässigkeit etwa im Polizeirecht, auch weil dieses die Ausübung des staatlichen Gewaltmonopols regelt.243 Die polizeirechtlichen Erlasse beschränken sich typischerweise darauf, eine Auswahl zulässiger Instrumente bereitzustellen, über deren Einsatz im Einzelfall anhand des Verhältnismässigkeitsprinzips zu entscheiden ist. ${ }^{244}$ Das Polizeirecht kennt ausserdem eine besondere Ausprägung der Erforderlichkeit in persönlicher Hinsicht: ${ }^{245}$ das Störerprinzip, wonach sich "polizeiliches Handeln gegen diejenigen Personen zu richten [hat], die den polizeiwidrigen Zustand unmittelbar zu verantworten haben» 246 .

Im Recht der öffentlichen Abgaben nimmt das Verhältnismässigkeitsprinzip die besondere Gestalt des Äquivalenzprinzips an. Dieses «bestimmt, dass eine Gebühr nicht in einem offensichtlichen Missverhältnis zum objektiven Wert der Leistung stehen darf und sich in vernünftigen Grenzen halten muss»247 (siehe dazu hinten \$ 4/IV.2). Diese besondere Ausgestaltung gibt ins-

240 TSCHANNEN/ZIMMERLI/MÜLLER, Allgemeines Verwaltungsrecht, \$26 Rz.37; einschliesslich der folgenden wörtlichen Zitate. Positivrechtliche Verankerung dieses Dreischritts in Art. 3 RPV.

241 Insbesondere «in allen Gebieten des öffentlichen Rechts», BGE 94 I 392 E. 3 S. 397.

242 EPINEY, Basler Kommentar, Art. 5 Rz. 33; TSCHANNEN/ZIMMERLI/MÜLLER, Allgemeines Verwaltungsrecht, §21 Rz.18f., §56 Rz.16.

243 BGE 140 I 353 E. 8.7 S.373.

244 TSCHANNEN/ZIMMERLI/MÜLLER, Allgemeines Verwaltungsrecht, §56 Rz.17.

245 HÄFELIN/MÜLLER/UHLMANN, Allgemeines Verwaltungsrecht, Rz. 554; TSCHANNEN/ ZIMMERLI/MÜLLER, Allgemeines Verwaltungsrecht, §56 Rz.29.

246 TSCHANNEN/ZIMMERLI/MÜLLER, Allgemeines Verwaltungsrecht, \$56 Rz. 28, ohne die Hervorhebung im Original. Vgl. dazu auch HÄFELIN/MÜLLER/UHLMANN, Allgemeines Verwaltungsrecht, Rz.2608.

247 BGE141 V 509 E. 7.1.2 S. 517; grundlegend BGE126I180 E.3a/bb S.188. Näher dazu: HÄFELIN/ MÜLLER/UHLMANN, Allgemeines Verwaltungsrecht, Rz.2786; TSCHANNEN/ZIMMERLI/ MÜLLER, Allgemeines Verwaltungsrecht, §58 Rz.19. 
besondere Vorgaben an die Interessenabwägung, indem die relevanten Beurteilungspunkte für die Bemessung des Leistungswerts vorgegeben werden. ${ }^{248}$

Ausserdem kennen Spezialgesetze mitunter besondere Verankerungen des Verhältnismässigkeitsprinzips. So etwa gilt im Datenschutzrecht die Besonderheit, dass auch Private verhältnismässig mit erhobenen Daten umgehen müssen. ${ }^{249}$

\section{c. Gerichtliche Durchsetzbarkeit}

Der Verhältnismässigkeitsgrundsatz ist - wie schon das Erfordernis des öffentlichen Interesses - nie selbständig anrufbares verfassungsmässiges Recht. Er kann eigenständig gerügt werden als Verletzung von Bundesrecht (Art. 95 Bst. a BGG) im Verfahren der öffentlichrechtlichen Beschwerde. ${ }^{250}$ In anderen Fällen muss mangelhafte Verhältnismässigkeit in Verbindung mit der Verletzung von Freiheitsrechten oder des Willkürverbots gerügt werden. 251

Das Bundesgericht prüft die Einhaltung der Verhältnismässigkeit grundsätzlich frei. Es legt sich aber dieselbe Zurückhaltung auf wie beim öffentlichen Interesse, wenn die Beurteilung von einer Kenntnis der örtlichen Verhältnisse abhängt. 252

\section{Teilgehalte des Grundsatzes von Treu und Glauben}

Treu und Glauben ist in der Verfassung doppelt verankert: als rechtsstaatlicher Grundsatz in Art. 5 Abs. 3 BV sowie als verfassungsmässiges Individualrecht in Art. 9.253 Die erste Verankerung nimmt Staat und Private gleichermassen in die Pflicht, während der als Grundrecht ausgestaltete Art. 9 nur den Staat verpflichtet. Treu und Glauben unterscheidet sich also von den übrigen Grundsätzen rechtsstaatlichen Handelns nach Art. 5. Vorab ist daher zu klären, ob Art. 5 Abs. 3 überhaupt eine Anforderung an staatliches Handeln ist, obgleich auch Private daran gebunden sind.

248 Nutzen für den Leistungspflichtigen oder Kostenaufwand der konkreten Leistungsnutzung, BGE126 I 180 E.3a/bb S.188. Näher dazu HÄFELIN/MÜLLER/UHLMANN, Allgemeines Verwaltungsrecht, Rz. 2788; TSCHANNEN/ZIMMERLI/MÜLLER, Allgemeines Verwaltungsrecht, \$58Rz. 20.

249 Art. 4 Abs. 2 DSG. Vgl. dazu BGE 138 II 346 E. 9.2 S.362.

250 BGE 140 I 257 E. 6.3.1 S. 267; 134 I 153 E. 4.1 S.156f.

251 TSCHANNEN/ZIMMERLI/MÜLLER, Allgemeines Verwaltungsrecht, \$21 Rz. 20-22. Im Abgaberecht kann das Äquivalenzprinzip eigenständig gerügt werden, Rz. 21.

252 BGE142 I162 E. 3.2.2 S. 165; 132 II 408 E.4.3 S. 416; 129 I 337 E. 4.1 S. 344; 126 I 219 E. 2c S. 222.

253 BIAGGINI, Kommentar BV, Art. 5 Rz. 22; HÄFELIN/MÜLLER/UHLMANN, Allgemeines Verwaltungsrecht, Rz.622; SCHINDLER, St. Galler Kommentar, Art. 5 Rz. 53; TSCHANNEN/ ZIMMERLI/MÜLLER, Allgemeines Verwaltungsrecht, §21 Rz.1. 


\section{a. Anforderung an staatliches Handeln trotz Geltung im gesamten Rechtsverkehr?}

Verfassungsrechtliche Anforderungen an staatliches Handeln markieren den Unterschied zwischen der staatlichen und der privaten Sphäre, wie sie die Verfassung eingerichtet hat. Es handelt sich um Vorgaben, die nur für diejenigen Tätigkeiten gelten, die der Erfüllung einer staatlichen Aufgabe dienen. Sie binden den (funktionellen) Staat, nicht aber (funktionell) Private. Der Grundsatz von Treu und Glauben liegt somit quer; er ist ein «allgemeiner Rechtsgrundsatz», dem "umfassende Geltung» zukommt. ${ }^{254}$ Gemeint ist damit, dass gewisse aus dem Grundsatz ableitbare Ansprüche auch unter Privaten und unter Gemeinwesen 255 durchsetzbar sind und dass auch Private gegenüber dem Staat darauf verpflichtet werden können. ${ }^{256}$

Die umfassende Geltung des Grundsatzes von Treu und Glauben bedeutet noch nicht, dass Private gleich weit und gleich eng an seine Teilgehalte gebunden sind. Die Bindung ist vielmehr «asymmetrisch»257: Private dürfen aufgrund ihrer individuellen Freiheit von getätigten Aussagen und eingenommenen Standpunkten freier abweichen als der Staat, welcher wiederum «regelmässig weitergehende Möglichkeiten [hat], die Richtigkeit von Zusicherungen oder Erklärungen Einzelner zu überprüfen»258.

Darin zeigt sich, dass Treu und Glauben die verfassungsrechtlich scharfe Trennlinie entlang der staatlichen Aufgabe entgegen dem ersten Eindruck gerade nicht aufweicht: Der Staat verfügt über grössere Macht und muss deshalb stärker gebunden sein, während Private originär über grössere Freiheiten verfügen, welche stärker geschützt werden müssen.

\section{b. Einzelne Teilgehalte, welche den Staat verpflichten}

Vertrauensschutz. Dieser Teilgehalt gibt die Voraussetzungen vor, unter welchen das Vertrauen von Privaten in staatliche Handlungen geschützt wird, die «unrichtig waren oder gar nicht hätten getätigt werden dürfen.» ${ }^{259} \mathrm{Die}$ Rechtsfolgen dieses Schutzes unterscheiden sich je nach Konstellation: Sie reichen von der Bindung des Staats an die getätigte Handlung, dem Ersatz des

\footnotetext{
254 EPINEY, Basler Kommentar, Art. 5 Rz.72.

255 Dazu insbesondere BIAGGINI, Kommentar BV, Art. 5 Rz. 25.

256 SCHINDLER, St. Galler Kommentar, Art. 5 Rz. 53; TSCHANNEN/ZIMMERLI/MÜLLER, Allgemeines Verwaltungsrecht, §22 Rz.1. Grundlegend BGE 121 I 181 E. 2a S. 183 f.; der von SCHINDLER zitierte BGE 131 I 166 E. 6.1 S.177 wiederholt allerdings nur den Wortlaut von Art. 5 Abs. 3 BV, SCHINDLER, St. Galler Kommentar, Art. 5 Rz. 17.

257 SCHINDLER, St. Galler Kommentar, Art. 5 Rz. 55.

258 SCHINDLER, St. Galler Kommentar, Art. 5 Rz. 55.

259 TSCHANNEN/ZIMMERLI/MÜLLER, Allgemeines Verwaltungsrecht, §22 Rz.3.
} 
Vertrauensschadens, der Erschaffung von Übergangsregeln bis zur Wiederherstellung verpasster Fristen. ${ }^{260}$ Die Figur des Vertrauensschutzes «schützt das individuelle Vertrauen des Bürgers in eine konkretisierte Rechtslage» ${ }^{261}$. Damit dieses Vertrauen als berechtigt gilt, müssen folgende Voraussetzungen erfüllt sein:262 Es muss eine ausreichende Vertrauensgrundlage vorliegen, gestützt auf welche das Vertrauen in Form einer nicht ohne Nachteil rückgängig machbaren Disposition betätigt worden ist - und zwar kausal. Weiter muss eine Abwägung im Einzelfall ergeben, dass die privaten Interessen die öffentlichen Interessen überwiegen. ${ }^{263}$ Praktisch bedeutsam ist vor allem der Vertrauensschutz bei amtlichen Zusicherungen und Auskünften, ${ }^{264}$ wozu die Dogmatik ein verfeinertes Schema entwickelt hat, das allerdings auf diesen Elementen basiert. 265 Andere Rechtsvorgänge, bei denen der Vertrauensschutz eine Rolle spielt, sind Praxisänderungen, Normänderungen, Widerruf, Änderung oder Aufhebung von Rechtsanwendungsakten sowie der Entzug wohlerworbener Rechte. ${ }^{266}$

Verbot widersprüchlichen Verhaltens. Der Grundsatz von Treu und Glauben gebietet ganz allgemein loyales Verhalten, woraus sich ein Verbot von folgewidrigem Handeln ableitet. ${ }^{267}$ Dieser Teilgehalt verbietet dem Staat, «plötzliche, sachlich unbegründete Kurswechsel» ${ }^{268}$ während eines konkreten Verfahrens.

Verbot des täuschenden Verhaltens. Über bloss widersprüchliches und folgewidriges Verhalten hinausgehend verbietet das Bundesgericht dem Staat

260 HÄFELIN/MÜLLER/UHLMANN, Allgemeines Verwaltungsrecht, Rz.700-711; TSCHANNEN/ ZIMMERLI/MÜLLER, Allgemeines Verwaltungsrecht, §22 Rz.3 (dort keine Erwähnung von Übergangsregeln).

261 TSCHANNEN/ZIMMERLI/MÜLLER, Allgemeines Verwaltungsrecht, \$22 Rz. 6, ohne die Hervorhebung im Original. Ähnlich auch HÄFELIN/MÜLLER/UHLMANN, Allgemeines Verwaltungsrecht, Rz.625.

262 BIAGGINI, Kommentar BV, Art. 9 Rz. 15; HÄFELIN/MÜLLER/UHLMANN, Allgemeines Verwaltungsrecht, Rz.627-666; TSCHANNEN/ZIMMERLI/MÜLLER, Allgemeines Verwaltungsrecht, §22 Rz.10-13. Grundlegend dazu WEBER-DÜRLER, Vertrauensschutz, $\$ \S 9-13$. Die Voraussetzungen werden unterschiedlich gruppiert und nummeriert, sind inhaltlich aber konsistent.

263 Diese Interessenabwägung spiegelt das Spannungsverhältnis zwischen der Gesetzmässigkeit und dem Vertrauensschutz, der grundsätzlich die Beständigkeit von Rechtsakten bevorzugt; TSCHANNEN/ZIMMERLI/MÜLLER, Allgemeines Verwaltungsrecht, §22 Rz. 8f.

264 BIAGGINI, Kommentar BV, Art. 5 Rz. 23.

265 TSCHANNEN/ZIMMERLI/MÜLLER, Allgemeines Verwaltungsrecht, \$22 Rz.16. Zu den einzelnen Voraussetzungen ebd., Rz.15-20; und HÄFELIN/MÜLLER/UHLMANN, Allgemeines Verwaltungsrecht, Rz.667-699.

266 TSCHANNEN/ZIMMERLI/MÜLLER, Allgemeines Verwaltungsrecht, §22 Rz. 4.

267 TSCHANNEN/ZIMMERLI/MÜLLER, Allgemeines Verwaltungsrecht, §22 Rz.16.

268 TSCHANNEN/ZIMMERLI/MÜLLER, Allgemeines Verwaltungsrecht, \$22 Rz. 22.

Ferner auch HÄFELIN/MÜLLER/UHLMANN, Allgemeines Verwaltungsrecht, Rz. $712 \mathrm{f}$. 
auch täuschendes Verhalten ausdrücklich: «En particulier, l'administration doit s'abstenir de tout comportement propre à tromper l'administré»269. Täuschendes Verhalten dürfte zugleich immer auch willkürliches Verhalten sein und ist somit doppelt von Art. 9 BV abgedeckt.

Verbot des Rechtsmissbrauchs. Die zweckwidrige Verwirklichung eines Rechtsinstituts verbindet gewissermassen folgewidriges und täuschendes Verhalten. ${ }^{270}$ Dies wird als Rechtsmissbrauch bezeichnet und ist in der gesamten Rechtsordnung untersagt. ${ }^{271}$ Über die typische Anwendung im Zivilrechtsverkehr (gestützt auf Art. 2 Abs. 2ZGB) hinaus verbietet das verfassungsrechtliche Gebot von Treu und Glauben dem Staat etwa, Rechte Privater durch Verzögerungen bewusst zu vereiteln. ${ }^{272}$

Den verschiedenen - und teilweise ungenau abgrenzbaren - Teilgehalten des Grundsatzes von Treu und Glauben gemein ist ihre Stossrichtung: Sie verpflichten den Staat auf verlässliches Handeln und schützen den Glauben der Machtbefohlenen darin. Verlässlichkeit umfasst dabei erwartungsgemässes, folgerichtiges, konsistentes und zweckmässiges Handeln. ${ }^{273}$ In etwas eindringlicheren Worten spricht das Bundesgericht von einem Verbot jeder «missbräuchliche[n] Machenschaft (ttoute machination abusive))» ${ }^{274}$. Der Grundsatz von Treu und Glauben bezweckt also zweierlei: Einerseits verpflichtet er den Staat, sich im Rechtsverkehr loyal zu verhalten, 275 andererseits schützt er Private, falls der Staat dies nicht tut.

\section{c. Gerichtliche Durchsetzbarkeit}

Die soeben behandelten Teilgehalte des Gebots von Treu und Glauben sind kraft Art. 9 BV verfassungsmässige Individualrechte. Sie können somit selbständig angerufen werden. ${ }^{276}$

269 BGE 121 I 181 E. 2a S. 183.

270 Formulierung eng angelehnt an BGE 131 I 166 E. 6.1 S.177.

271 TSCHANNEN/ZIMMERLI/MÜLLER, Allgemeines Verwaltungsrecht, §22 Rz. 26.

272 Vgl. die Beispiele bei TSCHANNEN/ZIMMERLI/MÜLLER, Allgemeines Verwaltungsrecht, $\S 22$ Rz.27.

273 Zusammenführung von TSCHANNEN/ZIMMERLI/MÜLLER, Allgemeines Verwaltungsrecht, §22 Rz. 3, 21, 26, wo die jeweiligen Zwecke des Vertrauensschutzes, des Verbots widersprüchlichen Verhaltens und des Rechtsmissbrauchsverbots diskutiert werden.

274 BGE 133 I 234 E. 2.5.1 S.239.

275 Diese Wortwahl insbesondere bei BIAGGINI, Kommentar BV, Art. 5 Rz. 23; EPINEY, Basler Kommentar, Art. 5 Rz. 73.

276 HÄFELIN/MÜLLER/UHLMANN, Allgemeines Verwaltungsrecht, Rz. $622 \mathrm{f}$; TSCHANNEN/ZIMMERLI/MÜLLER, Allgemeines Verwaltungsrecht, §23 Rz. 28. 


\section{Grundrechtsbindung}

Neben den rechtsstaatlichen Grundsätzen von Art. 5 BV drückt die Grundrechtsbindung die staatlichen Sonderpflichten am deutlichsten aus. Der Staat darf gegenüber Individuen Macht ausüben und gewaltweise durchsetzen, im Gegenzug muss er deren Rechte und Freiheiten schützen.

Dieser Abschnitt behandelt die Grundrechte in aller möglichen Kürze. Zunächst erfolgt eine Übersicht über die einzelnen grundrechtlichen Ansprüche (a). Die Geltungsweise der Grundrechte wirft eine umstrittene Frage auf: Wer ist bei welchen Handlungen an die Grundrechte gebunden $(b)$ ? Mit Blick auf die prozessuale Verwirklichung des Grundrechtsschutzes schliessen Bemerkungen zur gerichtlichen Durchsetzung $(c)$ und zu den Folgen von Grundrechtsverletzungen $(d)$ diesen Abschnitt ab.

\section{a. Einzelne Grundrechte}

Grundrechte vermitteln verschiedenartige Rechtsansprüche:277 Sie können vom Staat ein Tun oder ein Unterlassen erfordern und entsprechend in Leistungsrechte, Schutzpflichten und Abwehrrechte unterteilt werden oder dem Einzelnen als Teilhaberechte die Partizipation an bestimmten Prozessen garantieren. Doch ist dies keine ordnende Einteilung. Die Anforderungen an staatliches Handeln ergeben sich aus den jeweiligen Schutzbereichen der einzelnen Grundrechte. Überhaupt sind die Grundrechte kein geschlossener Block, kein einheitliches System aufeinander abgestimmter Rechte - ihr Schutz ist punktuell. ${ }^{278}$ Die Grundrechte vermitteln vielmehr in verschiedenen Sachverhalten Rechtspositionen ${ }^{279}$, in die nur mit besonderer Rechtfertigung eingegriffen ${ }^{280}$ werden kann oder die gar absolut geschützt ${ }^{281}$ sind. Grundrechte sind also Rechte des Einzelnen, die sich spiegeln als verschiedenartige Ansprüche an staatliches Handeln: gewisse Handlungen zu unterlassen, gewisse Handlungen strenger zu begründen oder gewisse private Tätigkeiten zu ermöglichen.

277 Ausführlich dazu KIENER/KÄLIN/WYTTENBACH, Grundrechte, §4 Rz.7-19. Ferner RHINOW/ SCHEFER/UEBERSAX, Schweizerisches Verfassungsrecht, Rz. 982-984; SCHWEIZER, St. Galler Kommentar, Vorbemerkungen zu Art. 7-36 Rz.35; TSCHANNEN, Staatsrecht, \$7 Rz.7f., 81.

278 RHINOW/SCHEFER/UEBERSAX, Schweizerisches Verfassungsrecht, Rz. 956.

279 Angelehnt an die Beschreibung des Schutzbereichs bei TSCHANNEN, Staatsrecht, §7 Rz. 82.

280 Siehe generell die Rechtfertigung von Grundrechtseingriffen nach Art. 36 BV; vgl. KIENER/ KÄLIN/WYTTENBACH, Grundrechte, \$9 Rz.1, 7 .

281 Die Willkür- und Diskriminierungsverbote etwa gelten absolut und kennen keine Rechtfertigungsmöglichkeit, KIENER/KÄLIN/WYTTENBACH, Grundrechte, §9 Rz.9, §33 Rz.31, §36Rz.14; TSCHANNEN/ZIMMERLI/MÜLLER, Allgemeines Verwaltungsrecht, §23 Rz. 21-27 e contrario; wie auch staatliche Vorzensur unter keinen Umständen gerechtfertigt ist, KIENER/KäLIN/WYTTENBACH, Grundrechte, §21 Rz. 45. 
Grundrechte im hier verwendeten Sinn meinen die in der Bundesverfassung unter diesem Titel garantierten Rechtsansprüche, also die Art. 7-34 BV. Die im internationalen Recht garantierten Menschenrechte mögen zwar punktuell weitergehende Ansprüche begründen, sind aber strukturell als Mindestgarantien angelegt. ${ }^{282}$ Diese Arbeit untersucht verfassungsrechtliche Anforderungen an das Staatshandeln auf Bundesebene; allenfalls weitergehende Grundrechte des kantonalen Verfassungsrechts 283 fallen ausser Betracht. Vereinzelt werden Rechtsansprüche auch ausserhalb des Grundrechtskatalogs der Verfassung als Grundrechte qualifiziert, so etwa Art. 118 Abs. 2 Bst. a BV,284 was im Folgenden auch nicht systematisch dargestellt werden kann. Zurzeit ist ausserdem anzunehmen, dass keine nennenswerten ungeschriebenen Grundrechte bestehen (siehe vorne 1). 285

Die einzelnen Schutzbereiche sind Gegenstand umfangreicher Spezialliteratur 286; im Folgenden soll immerhin ein gruppierter Überblick gegeben werden. In der einschlägigen Literatur finden sich zahlreiche Typisierungen, darunter keine zwei übereinstimmenden. ${ }^{287}$ Auch erscheinen die Anknüpfungspunkte der verschiedenen Kategorisierungen teilweise unklar. Die folgende Darstellung ordnet die Grundrechte nach ihrem Schutzgegenstand, das heisst nach der folgenden Frage: Welchen Lebensbereich von Individuen oder welchen Aspekt des gesellschaftlichen Zusammenlebens will das Grundrecht besonders schützen?288

282 KIENER/KÄLIN/WYTTENBACH, Grundrechte, §2 Rz.29 f.; RHINOW/SCHEFER/UEBERSAX, Schweizerisches Verfassungsrecht, Rz.1044. Vgl. scHWEIzER, St. Galler Kommentar, Vorbemerkungen zu Art. 7-36, Rz. 26, 31, der die Bedeutung völkerrechtlicher Garantien über blosse Mindeststandards hinaus betont.

283 Siehe dazu KIENER/KÄLIN/WYTTENBACH, Grundrechte, §2 Rz. 2-4; RHINOW/SCHEFER/ UEBERSAX, Schweizerisches Verfassungsrecht, Rz.1037 f.

284 Als Beispiel für «weitere spezielle Freiheitsrechte» aufgeführt bei scHWEIZER, St. Galler Kommentar, Vorbemerkungen zu Art. 7-36 Rz.5.

285 KIENER/KÄLIN/WYTTENBACH, Grundrechte, §3 Rz. 9; TSCHANNEN, Staatsrecht, §7 Rz.16f. Implizit auch RHINOW/SCHEFER/UEBERSAX, Schweizerisches Verfassungsrecht, Rz.1026f.

286 HÄFELIN/HALLER/KELLER/THURNHERR, Schweizerisches Bundesstaatsrecht, \$§10-26, 28-31; KIENER/KÄLIN/WYTTENBACH, Grundrechte, \$\$10-45; RHINOW/SCHEFER/UEBERSAX, Schweizerisches Verfassungsrecht, \$\$14-20. Und die Anmerkungen zu Art. 7-34 in den Kommentaren zur Bundesverfassung: AUBERT/MAHON, Petit commentaire; BIAGGINI, Kommentar BV; EHRENZELLER, St. Galler Kommentar; WALDMANN, Basler Kommentar.

287 Die beschränkte dogmatische Kraft solcher Typisierungen betonend auch SCHWEIZER, St. Galler Kommentar, Vorbemerkungen zu Art. 7-36 Rz. 5.

288 Zusammengestellt aus KIENER/KÄLIN/WYTTENBACH, Grundrechte, §3 Rz.11-21; MAHON, Petit commentaire, Remarques liminaires Art. 7 Rz.3; MÜLLER, Allgemeine Bemerkungen, Rz. 7; SCHWEIZER, St. Galler Kommentar, Vorbemerkungen zu Art. 7-36 Rz. 5. Vgl. insbesondere Schutzzwecke als Unterscheidungsmerkmal bei TSCHANNEN, Staatsrecht, §7 Rz.10-15. 


\section{aa. Menschenwürde (Art. 7 BV)}

Der Inhalt der Menschenwürde lässt sich nicht auf bestimmte Sachverhalte oder einen Lebensbereich festmachen, vielmehr zielt sie auf die «Subjektqualität des Menschen» 289 an sich und schützt die «Rechtsfähigkeit eines Menschen [als] zwingende Voraussetzung dafür, Träger von Rechten und Pflichten zu sein» 290 .

Die Garantie der Menschenwürde ist programmatisch für den Grundrechtsschutz insgesamt, indem sie die Individualität des einzelnen Menschen an den Anfang des Grundrechtskatalogs stellt. ${ }^{291}$ Sie ist in grundlegender Weise «ausgerichtet auf Anerkennung des Einzelnen in seiner eigenen Werthaftigkeit und individuellen Einzig- und allfälligen Andersartigkeit»292 .

\section{bb. Rechtsstaatliche Garantien (Art. 8, 9, 25, 29-32 BV)}

Diese Grundrechte betreffen das gesellschaftliche Zusammenleben, indem sie den Staat auf bestimmte Verhaltensweisen gegenüber Individuen verpflichten. Beim Setzen und Anwenden von Recht sind alle Menschen gleich und gerecht zu behandeln (Art. 8 und 9 BV).293 Der Schutz vor Ausweisung, Auslieferung und Ausschaffung gemäss Art. $25 \mathrm{BV}$ ist ebenfalls als rechtsstaatliche Garantie anzusehen,294 im Sinne einer internationalen und ausländerrechtlichen Verfahrensgarantie. Im Übrigen sind die eigentlichen Verfahrensgarantien von Art. 29-32 Garanten für eine auf Recht basierende Gesellschaftsordnung, in der alle Menschen gleich und gerecht behandelt werden - nicht nur in der Zuteilung von Rechten und Pflichten, sonderngerade auch in deren Durchsetzung. ${ }^{295}$

Die Gebote und Verbote von Art. 8 und 9 BV beschlagen jeweils Rechtsetzung und -anwendung gleichermassen ${ }^{296}$. Das allgemeine ${ }^{297}$ Gleichbehand-

\footnotetext{
289 KIENER/KÄLIN/WYTTENBACH, Grundrechte, §10 Rz. 8.

290 KIENER/KÄLIN/WYTTENBACH, Grundrechte, \$10 Rz.14.

291 Vgl. MÜLLER, Grundlagen der Grundrechte, Rz.13; RHINOW/SCHEFER/UEBERSAX, Schweizerisches Verfassungsrecht, Rz.1015; SCHWEIZER, St. Galler Kommentar, Vorbemerkungen zu Art. 7-36 Rz.33.

292 BGE132 I 49 E. 5.1 S. 55.

293 Vgl. die Überschrift «Gleiche und gerechte Behandlung» für Art. 8 und 9 BV bei KIENER/ KÄLIN/WYTTENBACH, Grundrechte, §§33-36.

294 Eigenständige Einordnung. Art. 25 BV wird in den zitierten Typisierungen nicht gesondert erwähnt; bei SCHWEIzER, St. Galler Kommentar, Vorbemerkungen zu Art. 7-36 Rz. 5, kommentarlos (und wenig überzeugend) als Freiheitsrecht eingestuft.

295 Anstelle vieler KIENER/KÄLIN/WYTTENBACH, Grundrechte, §3 Rz. 20.

296 BIAGGINI, Kommentar BV, Art. 8Rz. 9; KIENER/KÄLIN/WYTTENBACH, Grundrechte, \$35Rz.3 und 8, §36 Rz.32; TSCHANNEN/ZIMMERLI/MÜLLER, Allgemeines Verwaltungsrecht, §23 Rz. 4.

297 Im Unterschied dazu werden Art. 8 Abs. 2 und 3, Art. 27 BV als «besondere Gleichbehandlungsgebote» bezeichnet, TSCHANNEN/ZIMMERLI/MÜLLER, Allgemeines Verwaltungsrecht, §23 Rz. 2; ausserhalb des Grundrechtskatalogs auch Art. 37 Abs. 2 BV, siehe dazu HÄFELIN/ HALLER/KELLER/THURNHERR, Schweizerisches Bundesstaatsrecht, Rz. 797-803.
} 
lungsgebot von Art. 8 Abs. 1 BV fordert sachgerechte Differenzierung, je nachdem, ob rechtserhebliche Unterschiede tatsächlich bestehen oder nicht. ${ }^{298}$ Der Anspruch auf Gleichbehandlung richtet sich - anders als bei anderen Grundrechten - nicht an den Staat in seiner Gesamtheit, sondern ist territorial und verwaltungsorganisatorisch begrenzt: Rechtsgleichheit in der Rechtsetzung gilt jeweils nur innerhalb derselben Gebietskörperschaft (Bund, Kanton oder Gemeinde). ${ }^{299}$ Der Anspruch auf Gleichbehandlung in der Rechtsanwendung richtet sich laut Bundesgericht sogar nur an «ein und dieselbe Behörde» ${ }^{300}$. Was in diesem Zusammenhang noch dieselbe Behörde ist, ergibt sich aus den Umständen des Einzelfalls. So gelten etwa auch zwei verschiedene Behörden als gleich in diesem Sinn, wenn sie «ont toutes deux statué sur la même base et avec la même cognition» 301 oder eine Behörde sich in ähnlicher Lage befand, «s'il avait pris les deux décisions lui-même»302. Umstritten ist, ob dies generell anzunehmen ist bei Behörden, die derselben Aufsicht unterstehen. ${ }^{303}$ Welche Spielräume für organisatorisch ausgelagerte Aufgabenträger dadurch entstehen, wird hinten in \$6/VI.2.b behandelt.

Das Diskriminierungsverbot von Art. 8 Abs. 2 BV kann als Kerngehalt der allgemeinen Rechtsgleichheit angesehen werden. ${ }^{304}$ Das besondere Unrecht einer Diskriminierung liegt darin, dass die Schlechterstellung «an Unterscheidungsmerkmalen anknüpft, die einen wesentlichen und nicht oder nur schwer aufgebbaren Bestandteil der Identität der betroffenen Personen ausmachen» ${ }^{305}$.

298 KIENER/KÄLIN/WYTTENBACH, Grundrechte, §35Rz.9; TSCHANNEN/ZIMMERLI/MÜLLER, Allgemeines Verwaltungsrecht, §23Rz.3, 5, 11; WALDMANN, Basler Kommentar, Art. 8Rz. 26.

299 HÄFELIN/MÜLLER/UHLMANN, Allgemeines Verwaltungsrecht, Rz.581; SCHWEIZER, St. Galler Kommentar, Art. 8 Rz. 23; TSCHANNEN/ZIMMERLI/MÜLLER, Allgemeines Verwaltungsrecht, §23 Rz. 7. Kritisch dazu BIAGGINI, Kommentar BV, Art. 8 Rz. 15.

300 BGE 90 I 1 E. 2 S. 8. Fast gleichlautend (die gleiche, die nämliche Behörde) in BGE 121 I 49 E. 3c S. 51; 115 Ia 81 E. 3c S. 85; 103 Ia 115 E. 4c S. 119; 102 Ia 38 E. 2c S. 42. Auf diese Praxis verweisen auch BIAGGINI, Kommentar BV, Art. 8 Rz.15; HÄFELIN/MÜLLER/UHLMANN, Allgemeines Verwaltungsrecht, Rz. 588; TSCHANNEN/ZIMMERLI/MÜLLER, Allgemeines Verwaltungsrecht, §23 Rz.12. Grundlegend dazu schon HAEFLIGER, Alle Schweizer, S.71f.

301 BGE 102 Ia 81 E.3 S. 87.

302 BGE 91 I 169 E.1 S. 172. Auf Deutsch wiederholt in Urteil 2P.283/2001 vom 25. Februar 2002, E. 5.1.1. Vgl. dazu ausführlich MÜLLER, Kommentar aBV, Art. 4 Rz. 39; HAEFLIGER, Alle Schweizer, S. 72; und ferner HÄFELIN/MÜLLER/UHLMANN, Allgemeines Verwaltungsrecht, Rz. 588.

303 Vgl. Darstellung so lautender Lehrmeinungen bei MÜLLER, Kommentar aBV, Art. 4 Rz.39. Frage offengelassen in Urteil 2P.283/2001 vom 25. Februar 2002, E. 5.1.1; tendenziell wohl auch BGE138 I 321 E. 5.3.7 S. 330. Vielleicht auch HÄFELIN/MÜLLER/UHLMANN, Allgemeines Verwaltungsrecht, Rz.588, dort aber ohne weitere Erklärung.

304 So BIAGGINI, Kommentar BV, Art. 8 Rz.16 a.E.

305 Anstelle vieler BGE141 I 241 E. 4.3.2. Siehe ausführlich KIENER/KÄLIN/WYTTENBACH, Grundrechte, §36 Rz.15-24. Ferner auch BIAGGINI, Kommentar BV, Art. 8 Rz.19 m.w.H. auf die bundesgerichtliche Formel und Rz.24 zu den einzelnen Merkmalen. Auch zum Folgenden. 
Art. 8 Abs. 3 und 4 BV enthalten sodann spezifische Gleichbehandlungsgebote und Rechtsetzungsaufträge für die Gleichstellung unter den Geschlechtern und von Menschen mit Behinderung. ${ }^{306}$ Im letzten Satz von Abs. 3 wird zudem ein Anspruch auf gleichen Lohn für gleiche Arbeit statuiert, der im Sinne direkter Horizontalwirkung auch gegenüber privaten Arbeitgebern durchgesetzt werden kann ${ }^{307}$ - wie beim allgemeinen Gleichheitsgebot allerdings nur jeweils gegenüber demselben Arbeitgeber. ${ }^{308}$

Das Willkürverbot von Art. 9 BV übt eine Auffangfunktion ${ }^{309}$ aus, indem es Rechtsetzungs- und Rechtsanwendungsakte für verfassungswidrig erklärt, die «elementare Gerechtigkeitserwartungen verletz[en]»310. Das Willkürverbot erfasst erst qualifizierte, offenkundige Rechtsfehler, etwa bei sinnund zwecklosen oder in sich widersprüchlichen Anordnungen, die dann als Schikane erscheinen. ${ }^{311}$

Von den vielfältigen Verfahrensgarantien durchzieht insbesondere die Rechtsweggarantie von Art. 29a BV das Staatshandeln in grundlegender Weise. Dieses muss bei einer Instanz überprüft ${ }^{312}$ werden können, die nicht hierarchisch mit dem handelnden Aufgabenträger verbandelt ist - einer unabhängigen Justizbehörde. ${ }^{313}$

306 Vgl. allgemein zu Abs. 3: BGE 140 I 305 E. 4 S. 310; BGE 108 Ia 22 E. 5a S. 29; gestützt auf Botschaft Initiative «Gleiche Rechte», 130 f., 141. BIAGGINI, Kommentar BV, Art. 8 Rz. 27-35; HÄFELIN/HALLER/KELLER/THURNHERR, Schweizerisches Bundesstaatsrecht, Rz. 777-794; KIENER/KÄLIN/WYTTENBACH, Grundrechte, \$36 Rz. 82-110. Ferner BGE 137 I 305 E. 3.1 S.317; BIGLER-EGGENBERGER/KÄGI-DIENER, St. Galler Kommentar, Art. 8 Rz.112. Vgl. allgemein zu Abs. 4: BIAGGINI, Kommentar BV, Art. 8 Rz. 36 f.; HÄFELIN/HALLER/KELLER/ THURNHERR, Schweizerisches Bundesstaatsrecht, 795 f.; KIENER/KÄLIN/WYTTENBACH, Grundrechte, §36 Rz.111-114.

307 BIAGGINI, Kommentar BV, Art. 8 Rz. 33; BIGLER-EGGENBERGER/KäGI-DIENER, St. Galler Kommentar, Art. 8 Rz. 99, 122; HÄFELIN/HALLER/KELLER/THURNHERR, Schweizerisches Bundesstaatsrecht, Rz.793.

308 WALDMANN, Basler Kommentar, Art. 8 Rz.115.

309 BIAGGINI, Kommentar BV, Art. 9 Rz. 5; TSCHANNEN/ZIMMERLI/MÜLLER, Allgemeines Verwaltungsrecht, §23 Rz.23.

310 TSCHANNEN/ZIMMERLI/MÜLLER, Allgemeines Verwaltungsrecht, §23 Rz. 21. Geschützt wird ein «Mindestmass an Gerechtigkeit», BIAGGINI, Kommentar BV, Art. 9 Rz.3; KIENER/ KÄLIN/WYTTENBACH, Grundrechte, §33 Rz. 4.

311 HÄFELIN/MÜLLER/UHLMANN, Allgemeines Verwaltungsrecht, Rz. 610; KIENER/KÄLIN/ WYTTENBACH, Grundrechte, \$33 Rz. 9; TSCHANNEN/ZIMMERLI/MÜLLER, Allgemeines Verwaltungsrecht, \$23 Rz.24.

312 Die Verfassung spricht von «Beurteilung», womit volle Kognition über Rechts- wie Sachverhaltsfragen gemeint ist, nicht aber eine Angemessenheitskontrolle, MÜLLER, Rechtsweggarantie, S.176; ferner BIAGGINI, Kommentar BV, Art. 29a Rz. 8.

313 MÜLLER, Rechtsweggarantie, S.166f. Verlangt ist «mindestens eine» richterliche Behörde; HÄFELIN/HALLER/KELLER/THURNHERR, Schweizerisches Bundesstaatsrecht, Rz. 845b. Ähnlich auch BIAGGINI, Kommentar BV, Art. 29a Rz.3. 


\section{cc. Freiheits- und Sozialrechte (Art.10-24 BV)}

Freiheitsrechte stellen sachlich eingegrenzte Bereiche des menschlichen Lebens unter besonderen Schutz, indem staatliche Eingriffe darin nur in den Grenzen von Art. 36 BV möglich sind.314 Die so ausgeschiedenen «Bereiche menschlicher Existenz» ${ }^{315}$ oder «Manifestationen desmenschlichenLebens» ${ }^{316}$ sind aber kein Block absolut geschützter Freiheitsräume. Vielmehr ergeben sich die Schutzbereiche der einzelnen Freiheitsrechte aus der Summe unzulässiger staatlicher Eingriffe. Nur durch die Feststellung von Verletzungen klärt sich, welche Ansprüche ein bestimmtes Grundrecht garantiert. ${ }^{317}$

Die Wissenschaftsfreiheit (Art. 20 BV) etwa schützt nicht «die Wissenschaft» an sich, sondern gibt Lehrpersonen grundsätzlich das Recht, über Inhalt und Methoden ihres Unterrichts zu bestimmen ${ }^{318}$ - während sich die Tragweite dieses Rechts wiederum an den im Einzelfall zulässigen Einschränkungen am Massstab von Art. 36 BV bemisst. Ein weiteres Beispiel: Ob Hundehaltung, Rauchen und Nacktwandern durch die persönliche Freiheit von Art. 10 Abs. 2 BV geschützte Aktivitäten sind, lässt sich nur anhand konkreter staatlicher Beschränkungen erörtern. ${ }^{319}$

Üblicherweise werden Sozialrechte ${ }^{320}$ von den Freiheitsrechten unterschieden. Sie sollen «gezielt den sozialen Status eines Menschen verbessern» ${ }^{321}$ und zeichnen sich insbesondere dadurch aus, dass sie einklagbare Ansprüche auf staatliche Leistungen garantieren. ${ }^{322}$

314 KIENER/KÄLIN/WYTTENBACH, Grundrechte, §9 Rz.7; MÜLLER, Allgemeine Bemerkungen, Rz. 49; TSCHANNEN, Staatsrecht, §7 Rz.11.

315 MÜLLER, Allgemeine Bemerkungen, Rz. 49.

316 TSCHANNEN, Staatsrecht, \$7 Rz.11.

317 Vgl. die Schutzbereichsdefinition von TSCHANNEN, Staatsrecht, §7 Rz. 82.

318 KIENER/KäLIN/WYTTENBACH, Grundrechte, \$24 Rz. 6 und 10. Ferner auch BIAGGINI, Kommentar BV, Art. 20 Rz.9.

319 HÄFELIN/HALLER/KELLER/THURNHERR, Schweizerisches Bundesstaatsrecht, Rz.364, insbesondere mit Verweis auf die Tendenz des Bundesgerichts, einer Festlegung auszuweichen.

320 SCHWEIZER, St. Galler Kommentar, Vorbemerkungen zu Art. 7-36 Rz. 5, rät stattdessen zum Begriff soziale Grundrechte, der gesetzlich verankerte Sozialrechte ausklammert. Die übrige Literatur verwendet die beiden Begriffe synonymisch, KIENER/KäLIN/WYTTENBACH, Grundrechte, §3 Rz.16; oder spricht nur von Sozialrechten, TSCHANNEN, Staatsrecht, §7 Rz.12; MÜLLER, Allgemeine Bemerkungen, Rz. 20.

321 SCHWEIZER, St. Galler Kommentar, Vorbemerkungen zu Art.7-36 Rz. 5.

322 HÄFELIN/HALLER/KELLER/THURNHERR, Schweizerisches Bundesstaatsrecht, Rz. 907; MÜLLER, Allgemeine Bemerkungen, Rz. 20; RHINOW/SCHEFER/UEBERSAX, Schweizerisches Verfassungsrecht, Rz.1001. Sie setzen somit Justiziabilität voraus, siehe TSCHANNEN, Staatsrecht, §7 Rz.12. 


\section{dd. Garantien der Wirtschaftsordnung (Art. 26-28 BV)}

Allen Grundrechten kommt ein Gehalt zu, der über punktuelle, individualrechtliche Ansprüche hinausreicht. ${ }^{23}$ Dies gilt besonders für die Garantien der Wirtschaftsordnung: die Eigentumsgarantie, die Wirtschafts- und die Koalitionsfreiheit. ${ }^{324}$ Diese Grundrechte sind von den in der Menschenwürde begründeten, auf die Person bezogenen Freiheitsrechten etwas weiter entfernt. ${ }^{325}$ Sie schreiben ein marktorientiertes, auf Privateigentum und kollektiver Interessenwahrung aufbauendes Wirtschaftssystem fest.

\section{ee. Rechte zur politischen Partizipation (Art. 33 und 34 BV)}

Die politischen Grundrechte gewähren dem Einzelnen Anspruch, am politischen Prozess der Willensbildung und Entscheidfindung teilhaben zu können. ${ }^{326}$ Der Teilgehalt der Abstimmungsfreiheit «gewährleistet die für den demokratischen Prozess und die Legitimität direktdemokratischer Entscheidungen erforderliche Offenheit der Auseinandersetzung»327. Daraus folgen insbesondere verschiedene Anforderungen an staatliche Organe bezüglich ihres Verhaltens vor Abstimmungen.328

Diese Anforderungen gelten - dank bundesgerichtlicher Klarstellung in gleicher Weise für alle Träger staatlicher Aufgaben. ${ }^{329}$ Das Bundesgericht hat die frühere Unterscheidung aufgegeben, dass öffentliche Unternehmen sich gerade dann nicht äussern dürfen, wenn eine Abstimmung die «demokratische Legitimierung ihrer Existenz» betrifft. 330

323 Positivrechtlich festgemacht an Art. 35 Abs. 1 BV. Vgl. dazu grundlegend MÜLLER, Allgemeine Bemerkungen, Rz. 29-32; ferner BIAGGINI, Kommentar BV, Art. 35 Rz. 6; KIENER/ KäLIN/WYTTENBACH, Grundrechte, §4 Rz. 39-41; WALDMANN, Basler Kommentar, Art. 35 Rz.9, 11.

324 Als eigenständige Gruppe von Grundrechten bei BAUMANN, Wettbewerbsverzerrungen, Rz.16; MAHON PASCAL, Petit commentaire, Remarques liminaires Art. 7 Rz. 3 a.E.; MÜLLER, Allgemeine Bemerkungen, Rz.7, 17-19. Ferner BIAGGINI, Kommentar BV, zu den einzelnen Grundrechten: Art. 26 Rz. 5, Art. 27 Rz.4, Art. 28 Rz.3.

325 Angelehnt an MÜLLER, Allgemeine Bemerkungen, Rz.17.

326 KIENER/KÄLIN/WYTTENBACH, Grundrechte, S. 31; RHINOW/SCHEFER/UEBERSAX, Schweizerisches Verfassungsrecht, Rz. 999; TSCHANNEN, Staatsrecht, \$7 Rz.13. Ferner MÜLLER, Allgemeine Bemerkungen, Rz.15.

327 BGE140 I338 E.5 S.342.

328 Auch zum Folgenden: Grundlegend und ausführlich TSCHANNEN, Staatsrecht, §52 Rz.1-30. Ferner BIAGGINI, Kommentar BV, Art. 34 Rz.17 f., 26; KIENER/KÄLIN/WYTTENBACH, Grundrechte, \$27 Rz.34-43; STEINMANN, St. Galler Kommentar, Art. 34 Rz. 25.

329 BGE 140 I 338 E. 7 S. 346-348.

330 Schilderung dieser Lehrmeinungen in BGE 140 I 338 E. 6.3 S.345, Ablehnung e contrario E. 7.1 S.347. Vgl. dazu PIRKER, Behördliche Intervention, S. 1378 f. 
Das Petitionsrecht von Art. 33 BV ergänzt die politischen Rechte. Es gewährt zwar keinen justiziablen Anspruch auf Mitwirkung, aber gibt Privaten immerhin das Recht, «ungehindert Bitten, Vorschläge, Kritiken oder Beschwerden an die Behörden zu richten, ohne deswegen Belästigungen oder Rechtsnachteile irgendwelcher Art befürchten zu müssen»331 .

\section{b. Geltungsweise: Grundrechtsbindung bei der Wahrnehmung staatlicher Aufgaben}

Nur wer an die Grundrechte gebunden ist, kann diese verletzen. Unabhängig vom Gehalt der grundrechtlichen Ansprüche ist zu klären, welche Tätigkeiten überhaupt an die Grundrechte gebunden sind. Die verfassungsrechtliche Regelung der Grundrechtsbindung in Art. 35 Abs. 2 BV gibt Anlass zu unterschiedlichen Lehrmeinungen, die im Folgenden dargelegt werden.

\section{aa. Zwei Auslegungen von Art. 35 Abs. 2 BV}

In aller Kürze hält Art. 35 Abs. 2 BV fest: «Wer staatliche Aufgaben wahrnimmt, ist an die Grundrechte gebunden». ${ }^{332}$ Das knackige Postulat ist in einem entscheidenden Punkt undeutlich: Ist, «wer staatliche Aufgaben wahrnimmt», bei jeder Tätigkeit an die Grundrechte gebunden oder nur soweit tatsächlich staatliche Aufgaben erfüllt werden?333 Entlang dieser zwei Lesarten unterscheidet sich ein personeller von einem funktionellen Ansatz der Grundrechtsbindung.

Die beiden Ansätze sind zwar weitgehend deckungsgleich. Jede Handlung, die der Erfüllung einer Staatsaufgabe dient, ist an die Grundrechte gebunden. ${ }^{334}$ Die beiden Ansätze trennen sich jedoch in der Frage, ob Akteure, die staatliche Aufgaben erfüllen, in all ihren Tätigkeiten an die Grundrechte gebunden sind (so der personelle Ansatz) - oder nur in denjenigen Tätigkeiten, die zum Bereich der staatlichen Aufgabe gehören (so der funktionelle Ansatz). Entfällt also die Grundrechtsbindung, wenn ein Aufgabenträger ausserhalb seines Aufgabenbereichs handelt? Nur darin liegt der Unterschied.

331 BGE 119 Ia 53 E. 3 S. 55. Siehe dazu KIENER/KäLIN/WYTTENBACH, Grundrechte, § 36 Rz.14-16.

332 Der zweite Satzteil - «und ist verpflichtet, zu ihrer Verwirklichung beizutragen» - wiederholt den «allgemeinen Grundrechtsverwirklichungsauftrag des Abs.1» (BIAGGINI, Kommentar BV, Art. 35 Rz.14) und ist nicht weiter entscheidend für die Frage, welche Tätigkeiten grundrechtsgebunden sind. Siehe dazu auch BGE 139 I 114 E. 3 S. 116 f.

333 HÄSLER stellt dieselbe Frage als «immer oder nur soweit», Geltung der Grundrechte, S.119-121.

334 Oder anders: «[J]ede Wahrnehmung staatlicher Aufgaben [unterliegt] einer Grundrechtsbindung», HÄFELIN/HALLER/KELLER/THURNHERR, Schweizerisches Bundesstaatsrecht, Rz. 265. 
Dass ein privater Akteur an die Grundrechte gebunden ist, wenn er ihm übertragene Staatsaufgaben erfüllt, ist unbestritten. ${ }^{335}$

Der Hintergrund des Meinungsstreits ist ein staatstheoretischer, der einer politischen Wertung unterliegt: Sollen staatliche Akteure jemals ihre Verpflichtung auf die Grundrechte (und andere verfassungsrechtliche Anforderungen an ihr Handeln) zurücklassen können? Gehen die «gleich langen Spiesse» also in beide Richtungen: Soll auch staatlichen Akteuren dieselbe Rechtsstellung wie Private zukommen, wenn sie nichtstaatliche Tätigkeiten ausüben? Der Slogan «Flucht ins Privatrecht», oft als Vorwurfbenutzt, weist zu diesem Streithintergrund. Wird ein Träger staatlicher Aufgaben zum nichtstaatlichen Akteur, wenn er andere Aufgaben wahrnimmt? Oder gilt «einmal Staat, immer Staat»?

\section{bb. Das Bundesgericht verfolgt (noch) einen funktionellen Ansatz}

Umstritten ist, ob ein Akteur, der vormals rein staatliche Aufgaben wahrnahm, in all seinen Tätigkeiten an die Grundrechte gebunden bleiben muss oder ob die staatliche Sonderpflicht des Grundrechtsschutzes entfällt, soweit er Leistungen im freien Wettbewerb erbringt, zu deren Erbringung er nicht verpflichtet ist. ${ }^{336}$ Vertreter des personellen Ansatzes postulieren, der Staat dürfe sich nicht aus der Grundrechtsverantwortung ziehen, indem er sich in ein privatrechtliches Kleid hüllt. «Einmal Staat, immer Staat» ist die Maxime, nach der gerade ehemalige Monopolbetriebe auch dann grundrechtsgebunden bleiben sollen, wenn sie als Marktteilnehmer unter Privaten auftreten. Die Frage stellt sich nicht nur bei ehemaligen Monopolisten, dort aber besonders deutlich, weil der «Gesetzgeber in jüngerer Zeit wiederholt staatliche Monopole aufgebrochen und dem Markt geöffnet hat, ohne den bisherigen

335 BAUMANN, Wettbewerbsverzerrungen, Rz. 119; BIAGGINI, Kommentar BV, Art. 35 Rz.13; HÄFELIN/HALLER/KELLER/THURNHERR, Schweizerisches Bundesstaatsrecht, Rz. 277; KIENER/KÄLIN/WYTTENBACH, Grundrechte, §4 Rz.67; RÜTSCHE, Leistungsaufträge, S. 84 f.; TSCHANNEN, Staatsrecht, \$7 Rz. 46 und 53; TSCHANNEN/ZIMMERLI/MÜLLER, Allgemeines Verwaltungsrecht, \$10 Rz. 22; WALDMANN, Basler Kommentar, Art. 35 Rz. 25. Aus der Rechtsprechung zu privaten Trägern staatlicher Aufgaben BGE 143 I 37 E. 6.3 S. 42 (vom Kanton beauftragter Betreiber eines Flughafens); 141 V 557 E. 5.2 S. 566 f. (private Versicherungsunternehmen, die Leistungen der obligatorischen Krankenpflegeversicherung nach KVG anbieten); 140 I 2 E. 10.2.2 S. 30 (private Sicherheitskräfte, die polizeiliche Aufgaben übertragen erhalten); 138 I 289 E. 2.3 S. 292 (vorbestehende privatrechtliche Stiftung SWITCH, die das Register von .ch-Domainnamen im Auftrag des Bundes betreibt); 133 I 49 E.3.2 S. 55 f. (vom Kanton errichtete privatrechtliche Stiftung FAREAS, die im Auftrag des Kantons die Aufnahme von Flüchtlingen vollzieht); 103 Ia 544 E. 5c S. 551 (private Organisation, die im Auftrag des Kantons über die Zulassung zum Bergführerkurs entscheidet).

336 Siehe Darstellung des Lehrstreits bei BAUMANN, Wettbewerbsverzerrungen, Rz.120 f.; WALDMANN, Basler Kommentar, Art.35 Rz. 25 f. 
Staatsmonopolisten aus dem Verkehr zu ziehen»337. Der Lehrstreit entzündete sich just an dieser Frage im Post-Urteil von 2002 (BGE 129 III 35), wo sich das Bundesgericht unmissverständlich für den funktionellen Ansatz der Grundrechtsbindung ausgesprochen hatte:

«Gemäss Art. 35 Abs. 2 BV ist an die Grundrechte gebunden, wer staatliche Aufgaben wahrnimmt. Danach besteht bei der Erfüllung von staatlichen Aufgaben eine Grundrechtsbindung, und zwar unabhängig davon, ob diese Aufgaben durch den Staat oder durch privatrechtliche Organisationen erfüllt werden [m.w.H.]. Da die Post im hier relevanten Bereich der Wettbewerbsdienste keine sstaatlichen Aufgaben` wahrnimmt, sondern vielmehr Dienstleistungen erbringt, die von jedem anderen Privaten auch erbracht werden könnten, fällt eine Grundrechtsbindung der Post gestützt auf Art. 35 Abs. 2 BV ausser Betracht. Nur im Bereich der Universaldienste kann von der Erfüllung einer staatlichen Aufgabe (Art. 92 Abs. 2 BV) und dementsprechend auch von einer Grundrechtsbindung des jeweiligen Dienstleisters ausgegangen werden.»338

Die Post ist zweifellos Trägerin staatlicher Aufgaben;339 sie besitzt sogar ein Teilmonopol ${ }^{340}$. Doch wenn sie Tätigkeiten ausübt, die auch «von jedem anderen Privaten» erbracht werden könnten, ist die Post aus verfassungsrechtlicher Sicht nicht mehr Staat und daher auch nicht an die Grundrechte gebunden. Dieses Urteil wurde in der Lehre überwiegend kritisch aufgenommen. ${ }^{341}$ BIAGGINI etwa kommentiert, es stehe «nicht in der Macht des Gesetzgebers, die Post von der Beachtung der Grundrechte zu dispensieren", und wertet das Urteil als einen misslungenen «Befreiungsversuch».342 HANGARTNER hält die bundesgerichtliche Argumentation für «widersprüchlich und in ihren Kon-

337 TSCHANNEN, Staatsrecht, \$7 Rz. 58.

338 BGE 129 III 35 E. 5.2 S. 40. Nebenbemerkung zum System der Postdienstleistungen: Im neuen Postrecht, das seit 2012 in Kraft steht, haben sich die Begriffe geändert, das System bleibt allerdings gleich: Die Post ist verpflichtet, die Leistungen der Grundversorgung zu erbringen (Art. 1 Abs. 1 Bst. b, Art. 13 Abs.1, Art.14-17 PG); die Post ist frei, darüber hinaus weitere «Postdienste» zu erbringen, wie dies auch privaten Anbietern freisteht, die lediglich einer Meldepflicht unterstehen (Art.1 Abs.1 Bst. a, Art. 2 Bst. a, Art. 4 Abs. 1 PG).

339 Zum Zeitpunkt des Urteils war die Post eine selbständige Anstalt des öffentlichen Rechts mit Rechtspersönlichkeit (gemäss aPOG vom 30. April 1997; vgl. TSCHANNEN/ZIMMERLI/ MÜLLER, Allgemeines Verwaltungsrecht 3. Aufl., §7 Rz. 5, 9, 19). Seit 1. Oktober 2012 ist «Die Schweizerische Post AG» als spezialgesetzliche AG konstituiert (Art. 2 Abs. 1 POG). Die neue Rechtsform beschlägt die Gültigkeit der bundesgerichtlichen Argumentation nicht, da die Aufgabenlage in der Substanz unverändert ist.

340 Das Monopol umfasst noch «das ausschliessliche Recht, Briefe bis 50 Gramm zu befördern (reservierter Dienst)» (Art.18 Abs.1PG).

341 Vgl. die Hinweise auf diese Kritik bei TSCHANNEN, Staatsrecht, §7 Rz. 60 a.E.

342 BIAGGINI, Kommentar BV, Art. 35 Rz.11. 
sequenzen undurchdacht»343 und kritisiert den verwendeten Aufgabenbegriff. Seiner Auffassung nach seien der Staat und «seine Einrichtungen immer, in allen Funktionen, unmittelbar, also gestützt auf Art. 35 Abs. 2 BV, an die Grundrechte gebunden». Die Bindung bestehe also nicht nur bei Tätigkeiten, die entweder dem Staat vorbehalten sind, einem Verfassungsauftrag entspringen oder zumindest «rechtlich genau vorgeschrieben sind». Nebenbei: HANGARTNER missversteht die Aufgabendefinition des Bundesgerichts; das entscheidende Merkmal, die Erfüllungspflicht, erwähnt er nicht (siehe dazu vorne \$2/I.2.b). KäLIN hält das Urteil für eine «Diskrepanz der Rechtsprechung, [die] nicht nur dem Ansehen des Bundesgerichts, sondern auch der Sache des Grundrechtsschutzes» ${ }^{344}$ schadet. Es sei unzulässig, die Wettbewerbsdienste aus der Staatsaufgabe Postwesen (Art. 92 BV) herauszulösen, gerade «in Zeiten von New Public Management und anderen Reformen, welche auf die Erhöhung der Effizienz und Handlungsfähigkeit des Staates zielen»345. Auch MÄCHLER kritisiert den funktionellen Ansatz fundamental. Er zweifelt an, ob die Grundrechtsbindung entlang der Erfüllung staatlicher Aufgaben entschieden werden kann, der Aufgabenbegriff sei dafür zu weit: «Formal betrachtet sind alle Aufgaben staatlich, die der Staat erfüllt.» ${ }^{346}$ Wie HANGARTNER übergeht auch MÄCHLER die rechtliche Verpflichtung zur Aufgabenerfüllung als entscheidendes Merkmal; staatliche Aufgabe sei doch «all das, was der Staat aufgrund von Verfassung und Gesetz selbst bzw. durch seine Anstalten anpackt»347. Er bestätigt allerdings das grundsätzliche Entfallen der Grundrechtsbindung, indem er anfügt, die Post könne im Wettbewerbsbereich «selbst frei entscheiden, ob sie eine Dienstleistung überhaupt oder weiterhin anbieten will. [...] Werden sodann Aspekte einer Grundrechtsverwirklichung in eine Interessenabwägung eingebracht, so darf diesen ein wirtschaftliches oder finanzielles Interesse der Post gegenübergestellt werden.»

Das Bundesgericht hat seither an dieser Rechtsprechungslinie festgehalten und die funktionsbezogene Grundrechtsbindung bestätigt. ${ }^{348}$ Die Schweizerischen Bundesbahnen (SBB) seien bei der Bewirtschaftung von Werbeflächen in ihren Bahnhöfen an die Grundrechte gebunden, weil «die Verwaltung

343 HANGARTNER, Bemerkungen zum Post-Urteil, S. 691, auch für die folgenden wörtlichen Zitate.

344 KÄLIN, Staatsrechtliche Rechtsprechung 2003/2004, S. 646. Er mutmasst, die Zivilabteilung müsse wohl «so überlastet» sein, «dass sie nicht mehr dazu kommt, die Hefte der Reihe I zu lesen».

345 KÄLIN, Staatsrechtliche Rechtsprechung 2003/2004, S. 645.

346 MÄCHLER, Bemerkungen zum Post-Urteil, S. 375.

347 MÄCHLER, Bemerkungen zum Post-Urteil, S. 376; auch das folgende Zitat.

348 Die bundesgerichtliche Linie verteidigend auch KIENER/KäLIN/WYTTENBACH, Grundrechte, §4 Rz.73; TSCHANNEN, Staatsrecht, §7 Rz. 58-60. 
von öffentlichen Sachen i.e.S. Wahrnehmung einer Staatsaufgabe [und] deshalb grundrechtsgebunden»349 ist. Die Werbetätigkeit der SBB ist gerade deshalb an die Grundrechte gebunden, weil sie aufgrund der gesetzlichen Bestimmungen - im Unterschied zur Post - als staatliche Aufgabe anzusehen ist. ${ }^{350}$ Dem personellen Ansatz zufolge hätte es ausgereicht, auf die staatliche Beherrschung der SBB zu verweisen, um eine umfassende Grundrechtsbindung zu begründen. 351

\section{cc. Aufgeweichte Trennlinie zwischen den Ansätzen}

In einem darauffolgenden Urteil hielt das Bundesgericht an der Aufgabenerfüllung als Geltungsgrund für die Grundrechtsbindung fest, weichte allerdings die Grenze zum personellen Ansatz auf. Die Schweizerische Radio- und Fernsehgesellschaft (SRG), bzw. deren Tochterfirma publisuisse SA, ist beim Abschluss von Werbeverträgen nach Art. 35 Abs. 2 BV an die Grundrechte gebunden, weil der privatrechtlich bewirtschaftete Werbebereich «als Nebenaktivität zur Finanzierung ihrer Programme dient» ${ }^{352}$. Das Gericht verwies dabei auf den soeben beschriebenen SBB-Plakatfall, unterliess aber einen gewichtigen Hinweis: Der Plakataushang in Bahnhöfen war Teil des eigentlichen Aufgabenbereichs der $\mathrm{SBB}^{353}$ und ist damit eine unmittelbare Erfüllung einer staatlichen Aufgabe. Dagegen ist die SRG bei der «Akquisition und Ausstrahlung der Werbung nicht unmittelbar im Rahmen ihres Programmauftrags tätig [...]. Sie kann ihre Programme unter Einhaltung der öffentlich-rechtlichen Vorgaben mittels Werbung finanzieren, ist hierzu jedoch nicht verpflichtet.»354

Das Bundesgericht knüpft die Grundrechtsbindung also weiterhin an die Erfüllung staatlicher Aufgaben, schliesst darin aber auch mittelbare Aufgaben mit ein. ${ }^{355}$ Es wird sich zeigen, ob das Gericht den Unterschied zum perso-

349 BGE 138 I 274 E. 2.2.1 S. 281. Ähnliche Konstellation bei BGE 143 I 37 E. 6.2 S. 40 ff., wo der kantonale Gesetzgeber einen Flughafenbetreiber zur Bewirtschaftung von Parkplätzen verpflichtet.

350 BIAGGINI missversteht dieses Urteil als «deutlichen Kontrapunkt» und «klare Absage» an die Rechtsprechungslinie des Post-Urteils, weil er den Aufgabenbegriff in Art 35 Abs. 2 $\mathrm{BV}$ zu eng auf den «gesetzlichen Grundversorgungsauftrag» beschränkt; BIAGGINI, Bemerkungen zum SBB-Plakatfall, S.177 f.

351 In Verkennung der bundesgerichtlichen Herleitung einer «i.e.S.» staatlichen Aufgabe kritisiert BIAGGINI: «Entscheidend sollte im Falle der SBB doch eigentlich sein, dass es sich um ein Unternehmen des Bundes handelt», BIAGGINI, Bemerkungen zum SBBPlakatfall, S. 177 .

352 BGE 139 I306 E.3.2.2 S.311.

353 BGE 138 I 274 E.1.4 S.278-280.

354 BGE 139 I 306 E.3.2.1 S.310.

355 So auch BAUMANN, Wettbewerbsverzerrungen, Rz.123. Medienrechtliche Kritik an dieser Ausweitung bei STEINER, Besprechung SRG-Urteil, S. 30. 
nellen Ansatz vollständig auflöst, indem künftig jede wettbewerbliche Tätigkeit als unterstützend für die Erfüllung der staatlichen Aufgaben eingestuft wird. Alternativ könnte das Bundesgericht verdeutlichen, dass die Werbetätigkeit der SRG in einer besonders engen Beziehung zum staatlichen Pflichtbereich stehe, die bei anderen gemischtwirtschaftlichen Trägern staatlicher Aufgaben loser ist.

In diese Richtung deutet das Urteil betreffend die Stiftung SWITCH (vierzehn Monate vor dem diskutierten SRG-Urteil entschieden ${ }^{356}$ ), welche als Registerbetreiberin der .ch-Domain eine staatliche Aufgabe wahrnimmt und grundrechtsgebunden ist. Wenn diese Stiftung nun aber Werbung schaltet für eine Tochterfirma, die rein privatwirtschaftliche Leistungen erbringt, kann sie sich «wie jede andere privatwirtschaftlich tätige Person auf die Wirtschaftsfreiheit» berufen und ist grundrechtsberechtigt, nicht -verpflichtet. ${ }^{357} \mathrm{Im}$ Unterschied zum später entschiedenen SRG-Urteil wurde hier also der Werbebereich nicht zur mittelbaren Staatsaufgabe erklärt. Der Fall entspringt aber einer anderen Dynamik, da SWITCH ein originär privatrechtlicher Akteur ist.

Nach weiterhin geltender Rechtsprechung entfällt die Grundrechtsbindung von staatlichen Aufgabenträgern, wenn sie ausserhalb ihres Aufgabenbereichs tätig sind. ${ }^{358}$ Es mag befremden, einem staatlichen Akteur Privatautonomie zukommen zu lassen. Doch die heftige Kritik, die sich am Post-Urteil von 2002 entzündete, geht daneben: Es geht hier nicht darum, den Staat durch ein anderes Rechtskleid von seiner doch so lästigen Grundrechtsbindung zu befreien. ${ }^{359}$ Wenn ein staatlicher Akteur Dinge tut, die nicht mit seinen staatlichen Aufgaben zusammenhängen, ist er nicht Staat im Sinn der Verfassung. Das Befremdliche verschiebt sich vielmehr auf den Umstand, dass der Gesetzgeber Gebilde schafft, die gleichzeitig staatlich und privat sind. Die theoretische Möglichkeit, dass derselbe Schalterangestellte mit dem einen Handgriff staatlich handelt und mit dem anderen privat, mutet absurd an, kann aber gesetzgeberisch gewollt sein (siehe dazu hinten \$5/V.1.b). Dieses Nebeneinander staatlicher und nichtstaatlicher Aufgaben erschwert

356 Das Bundesgericht hat diese Frage seit dem SRG-Urteil vom 16. November 2013 (BGE 139 I306) nicht nochmals entschieden (Stand: 31. Dezember 2019). Die Linie des SRG-Urteils wurde immerhin bestätigt in BVGE B-6872/2017 E. 2.3.5 und 2.3.6.

357 BGE 138 I 289 E. 2.8.1 S. 294. Zustimmend auch BAUMANN, Wettbewerbsverzerrungen, Rz.126, 128.

358 So auch HÄSLER, Geltung der Grundrechte, S.120 f.; KIENER/KÄLIN/WYTTENBACH, Grundrechte, \$4 Rz.73; TSCHANNEN/ZIMMERLI/MÜLLER, Allgemeines Verwaltungsrecht, \$11 Rz.17; WALDMANN, Basler Kommentar, Art. 35Rz. 25. Insbesondere TSCHANNEN, Staatsrecht, \$7 Rz. 58, mit ausdrücklicher Verteidigung des Post-Urteils in Rz.6o.

359 So das ursprüngliche Postulat SALADINS, der Staat bleibe Staat, unbeachtlich seiner privatrechtlichen Kleider; SALADIN, Grundrechtsprobleme, S. 265. 
die Durchsetzung der verfassungsrechtlichen Anforderungen, die sich speziell und ausschliesslich an den Staat richten. Die Probleme, die daraus entstehen, sind Gegenstand des $\$ 5$.

\section{dd. Ergebnis: Zurückdrängung staatlicher Sonderpflichten ist gesetzgeberische Entscheidung}

Der funktionelle Ansatz ist richtig und unerlässlich: Wer von grundsätzlicher Privatautonomie ausgeht, die von einem mächtigen, aber begrenzten Staat eingeschränkt wird, darf die staatstypischen Pflichten nicht auf privates Handeln ausweiten. Oder wie es TSCHANNEN etwas schärfer ausdrückt: «Es ist nicht Sache der Grundrechtslehre, verfassungsrechtlich zulässige Rückzüge des Gemeinwesens durch dogmatische Tricks zu unterlaufen»360. Wird der staatliche Bereich durch gesetzgeberische Entscheidungen verkleinert, ist dies mit allen Konsequenzen zu akzeptieren, auch wenn der Gesetzgeber staatliche und private Aufgaben in demselben organisatorischen Gebilde vermischt. Das hält das Bundesgericht unmissverständlich fest: «Diese Schwierigkeiten sind aber durch die rechtliche Konstruktion und die damit verbundene Doppelstellung der Beschwerdeführerin bedingt und somit zwangsläufig vorhanden.» ${ }^{361}$ Die Grundrechtsbindung des Staats auf nichtstaatliches Handeln durch dogmatische Kniffe ${ }^{362}$ auszudehnen, macht diese gesetzgeberischen Entscheide nicht rückgängig.

Ob hingegen Grundrechte unter Privaten gelten sollen, ist eine separate Diskussion unter dem Stichwort Horizontalwirkung, ${ }^{363}$ die in Art. 35 Abs. 3 BV vorgezeichnet, aber nicht entschieden ist. ${ }^{364}$ Justiziable, verfassungsunmittelbare Grundrechtsansprüche im privaten Rechtsverkehr werden nur sehr restriktiv anerkannt. Dafür wird eine ausdrückliche Verankerung in der Verfassung verlangt, was einhellig beim Lohngleichheitsanspruch von Art. 8 Abs. 3 Satz 3 BV angenommen wird, bei anderen Rechten wie etwa der Koalitionsfreiheit und dem Streikrecht aber umstritten ist. 365 Grundrechtsverpflich-

360 TSCHANNEN, Staatsrecht, \$7 Rz. 59 .

361 So ausdrücklich in BGE 138 I 289 E. 2.8.2 S. 295. Ähnlich auch 129 III 35 E. 5.3 S. 41.

362 Vgl. die Darstellung bei WALDMANN, Basler Kommentar, Art.35 Rz.26, wonach der personelle Ansatz bedeutet, dass das «in Art. 35 Abs. 2 BV genannte funktionelle Kriterium der Aufgabenerfüllung [...] bloss einen Anwendungsfall der die Grundrechtsverpflichtung auslösenden Zurechenbarkeit zum Staat» darstelle.

363 Zum Folgenden und eingehender siehe HÄFELIN/HALLER/KELLER/THURNHERR, Schweizerisches Bundesstaatsrecht, Rz. 278-288; KIENER/KÄLIN/wYTTENBACH, Grundrechte, §4 Rz. 75-91; TSCHANNEN, Staatsrecht, §7 Rz.61-69.

364 BIAGGINI, Kommentar BV, Art. 35 Rz. 21.

365 BIAGGINI, Kommentar BV, Art. 35 Rz. 21; TSCHANNEN, Staatsrecht, \$7 Rz.63. Ferner SCHWEIZER, St. Galler Kommentar, Vorbemerkungen zu Art. 7-36 Rz. 9 a.E. 
tungen unter Privaten können ausserdem einfachgesetzlich verankert werden, ${ }^{366}$ wie etwa im Behindertengleichstellungsgesetz ${ }^{367}$. Darüber hinaus entfalten die Grundrechte indirekte Wirkungen in der gesamten Rechtsordnung, insbesondere in Form der verfassungskonformen Auslegung unbestimmter Normen. 368

Es darf aber nicht vergessen werden, dass nicht nur die Grundrechte Individualrechte schützen. Die Bedrohung und Verletzung privater Rechtsgüter zu ahnden, ist die grundlegende Aufgabe des Strafrechts. ${ }^{369}$ Daneben zeigt gerade die Kasuistik zu Art. 35 Abs. 2 BV, dass zivilrechtliche Instrumente wegfallenden Grundrechtsschutz kompensieren können; siehe dazu den folgenden Nachtrag.

\section{ee. Nachtrag: Zivilrechtliche Kompensation wegfallender Grundrechtsbindung}

Aus Sicht einer Privatperson, die sich von einem Dienstleistungsanbieter in ihren Rechten beschnitten fühlt, mag es unbefriedigend sein, dass ihr Grundrechtsschutz von der Art der Aufgabe abhängt. Auch im privaten Rechtsverkehr kann ein Machtgefälle bestehen, das Individualrechte systematisch bedroht. Doch hierfür bestehen zivilrechtliche Abhilfen, die diesen Dynamiken entgegenwirken können. Diese stehen zwar unter einem anderen Vorzeichen: Sie sind ausnahmsweise Einschränkungen der Privatautonomie, während verfassungsrechtliche Bindungen die generelle Rechtfertigungspflicht des Staats ausdrücken (vgl. vorne I.2). Im Folgenden sollen einige Rechtsfiguren kurz skizziert werden, die vom Bundesgericht als Kompensation für wegfallende Grundrechtsbindungen eingesetzt werden.

Kontrahierungspflicht. Ein Anbieter kann nicht nur gestützt auf das Gleichbehandlungsgebot oder das Diskriminierungsverbot zur Erbringung gewisser Leistungen verpflichtet werden; das Privatrecht kennt eigene Rechtsverkehrsregeln, die mitunter zwingend sein können. ${ }^{370}$ Als direkte Kompensation für das Diskriminierungsverbot, welches bei fehlender Grundrechtsbindung nicht greift, kann die privatrechtliche Kontrahierungspflicht angesehen werden. Eine solche Pflicht bricht die Vertragsfreiheit, welche zentraler Ausdruck

\footnotetext{
366 Ausführlich dazu MARTENET, Discriminations émanant de particuliers, S. 424-426, 429-434, 450-454.

367 Art. 3, 6, 7 Abs.1, Art. 8 Abs. 3 BehiG.

368 BIAGGINI, Kommentar BV, Art. 35Rz.18; HÄFELIN/HALLER/KELLER/THURNHERR, Schweizerisches Bundesstaatsrecht, Rz. 282; TSCHANNEN, Staatsrecht, \$7 Rz. 67.

369 TSCHANNEN, Staatsrecht, \$7 Rz.66 a.E.

370 KIENER/KäLIN/WYTTENBACH, Grundrechte, §4 Rz. 83; TSCHANNEN, Staatsrecht, $\S 7$ Rz. 59 .
} 
der Privatautonomie ist, und kann daher nur in engen Grenzen zur Anwendung kommen. ${ }^{371}$ Im Post-Urteil etablierte das Bundesgericht vier Voraussetzungen, aufgrund deren «sich eine Kontrahierungspflicht auch aus allgemeinen Prinzipien des Privatrechtes wie dem Verbot sittenwidrigen Verhaltens ergeben» ${ }^{372}$ kann; also keine ausdrückliche gesetzliche Verankerung dafür notwendig ist. 373 Die Verweigerung eines Vertragsschlusses ist unter anderem nur dann unzulässig, wenn «dem Interessenten aufgrund der starken Machtstellung des Anbieters zumutbare Ausweichmöglichkeiten» fehlen und wenn der Anbieter «keine sachlich gerechtfertigten Gründe» nennen kann. ${ }^{374}$ Diese Voraussetzungen verdeutlichen, dass der Anbieter in einer monopolistischen Rolle sein muss, um überhaupt die Rechte des Interessenten in einer Weise verletzen zu können, die einer Korrektur bedarf. Die zivilrechtliche Lehre bewertete diese Ausweitung der KontrahierungspflichtDogmatik als «Paradigmenwechsel» und «Neuausrichtung des Vertragsrechts ${ }^{375}$ und verschrie sie teils als justizielle Zentralisierung der Verteilung von Gütern und Dienstleistung, die der Wirtschaftsordnung unangemessen sei. ${ }^{376} \mathrm{Zu}$ anderen Teilen begrüsste sie die dahinter stehende «Offenheit [des Bundesgerichts] auf dogmatisch schwierigem Terrain» ${ }^{377}$ oder sieht darin gar die «Fragmente einer aus dem Vertragsrecht fliessenden Zivilverfassung» ${ }^{378}$, eine «kraftvolle Vision eines sozialen Vertragsrechts» 379 .

Wettbewerbsrechtliche Mittel. Bietet ein Träger staatlicher Aufgaben daneben auch wettbewerbliche Leistungen an, ist er als wirtschaftlicher Akteur denselben Regeln unterstellt, die auch für rein private Unternehmen gelten - keine Sonderpflichten (wie eben die Grundrechtsbindung), im Gegenzug aber auch keine Privilegierung. Dieses Umschalten von besonderen staatlichen Bindungen auf die allgemeine Rechtsordnung des wirtschaftlichen Wettbewerbs wird besonders deutlich im SWITCH-Fall von 2012:

«In Bezug auf ihre unter Wettbewerbsbedingungen erbrachte Tätigkeit gilt jedoch nicht die Grundrechtsbindung, sondern ist die Beschwerdeführerin

371 ARNET, Bemerkungen zum Post-Urteil, S. 596.

372 BGE 129 III 35 E. 6.3 S. 45; auch zum Folgenden.

$373 \mathrm{Zu}$ ausdrücklich vorgesehenen Kontrahierungspflichten hingegen siehe HANDSCHIN/ SIEGENTHALER, Privatisierung, S. $412 \mathrm{f}$.

374 BGE 129 III 35 E.6.3 S. 45 f.

375 GAUCH, Soziales Vertragsrecht, S. 2.

376 PFISTER, Obligation de contracter, S.134 f.

377 ARNET, Bemerkungen zum Post-Urteil, S. 597.

378 AMSTUTZ/ABEGG/KARAVAS, Soziales Vertragsrecht, S.11 f.

379 AMSTUTZ/ABEGG/KARAVAS, Soziales Vertragsrecht, S.13. 
den gleichen Regeln unterstellt wie ihre Konkurrentinnen; [...] Im Wettbewerbsbereich haben die Konkurrentinnen der Beschwerdeführerin keinen grundrechtlichen Anspruch auf Gleichbehandlung (BGE 129 III 35 E. 5.2; [m.w.H.]). Allfälligen Wettbewerbsverzerrungen, die sich daraus ergeben könnten, dass die Beschwerdeführerin daneben eine öffentliche Aufgabe erfüllt, ist mit einer Trennung der Geschäftsbereiche und wettbewerbsrechtlichen Mitteln zu begegnen».380

Das Bundesgericht verweist sodann gesamthaft auf die Massnahmen des Kartellgesetzes (KG) sowie des Bundesgesetzes gegen den unlauteren Wettbewerb (UWG). ${ }^{381}$ Die Rechtspositionen von allenfalls bedrängten Konkurrenten sollen also - in Abwesenheit des Grundrechtsschutzes - durch Verbote gewisser Wettbewerbsabreden (Art. 5 KG), Verbote des Missbrauchs einer marktbeherrschenden Stellung (Art. 7 KG) und die Widerrechtlichkeit täuschender und treuwidriger Geschäftsmethoden (Art. 2 ff. UWG) kompensiert werden. Diese zivilrechtlichen Vorschriften mögen gewisse Ungleichbehandlungen und Diskriminierungen unterbinden (insb. Art. 7 Abs. 2 Bst. b KG). Anbieter, die aufgrund ihrer Grösse möglichen Kunden wenig Ausweichmöglichkeiten belassen, 382 können im Interesse eines funktionierenden Wirtschaftswettbewerbs zurückgebunden werden. Diese Bindung hängt alleine an ihrer Marktmacht und ist nicht etwa stärker, wenn es sich um ehemalige Staatsmonopolisten handelt. Überhaupt gilt das Wettbewerbsrecht in gleicher Weise für privat- wie öffentlichrechtliche Unternehmen. ${ }^{383}$

Trennung der Geschäftsbereiche. Die gesetzgeberische Vermengung staatlicher Aufgaben und wettbewerblicher Leistungen in demselben Unternehmen mag zwar zu Wettbewerbsverzerrungen und tieferem Individualrechtsschutz führen, doch kann dieser Effekt begrenzt werden durch das Gebot, den staatlichen vom Wettbewerbsbereich kalkulatorisch zu trennen und eine systematische Quersubventionierung in diese Richtung zu unterlassen. ${ }^{384}$ Das

\footnotetext{
380 BGE 138 I 289 E. 2.8.1 S.294.

381 Ähnlich auch BGE 138 I 378 E. 9.4 S.401. Auch der Bundesrat betont die Anwendbarkeit der kartellrechtlichen Regelungen, Bericht Staat und Wettbewerb, S.12, 15.

382 Vgl. im Zusammenhang mit der allgemeinen Kontrahierungspflicht BGE 129 III 35 E. 6.2.2 und 6.3 S. $44 \mathrm{f}$. Allfällige Ausweichmöglichkeiten sind überdies auch bei grundrechtlichen Diskriminierungsverboten zu berücksichtigen, wonach «desto höhere Anforderungen [...] an die sachliche Begründetheit von potentiell diskriminierenden Zulassungsschranken zu stellen» sind, je mehr jemand auf die angebotene Leistung angewiesen ist, BGE 127 I 84 E. 4c S. 90 f.

383 Art. 2 Abs. 1 KG; vgl. BGE 138 I 378 E. 6.3.3 S.390; 137 II 199 E. 3.1 S. 205 f., zum Anwendungsbereich des KG generell und auf die Swisscom AG speziell.

384 BGE 138 I 378 E. 9.1 S.398;138 I 289 E.2.8.1 S.294.
} 
Bundesgericht wendet diese Beschränkungen allerdings zurückhaltend an: Diese «finanzielle Trennung» sei auch «innerhalb ein und derselben juristischen Person möglich» $\mathbf{3 8 5}$ und die «bloss theoretische Möglichkeit einer Quersubventionierung» ${ }^{386}$ sei noch kein Verstoss. Abschliessend sei die «Gefahr einer gewissen Wettbewerbsverzerrung» hinzunehmen, solange zumindest keine «rechtliche Bevorzugung» des (teil-)staatlichen Unternehmens im Wettbewerb bestehe. ${ }^{387}$ Das Bundesgericht scheint hier Anforderungen aufzustellen, die es aus Rücksicht auf die gesetzgeberisch gewollte Aufgabenvermischung nicht allzu streng handzuhaben wagt. ${ }^{388}$ Das Verbot der Quersubventionierung kann auch aus anderer Optik betrachtet werden: Sein Gegenstück ist die Ausweitung des für die Grundrechtsbindung relevanten Aufgabenbegriffs auf Nebentätigkeiten, die der Finanzierung der eigentlichen Staatsaufgabe dienen (siehe soeben cc). 389

Dass ausserhalb der staatlichen Aufgabenerfüllung weniger strenge Bindungen bestehen, ist aber gerade keine lamentable Schutzlücke, sondern bloss Konsequenz des verfassungsrechtlichen Entweder-oders der Staatlichkeit. Entweder ist eine Tätigkeit den besonderen Anforderungen an staatliches Handeln unterworfen oder eben nicht. Das unbefriedigende Ergebnis zweier Anforderungsniveaus an dieselbe Organisation ist allerdings nicht der verfassungsrechtlichen Ordnung anzulasten, sondern der gesetzgeberischen Entscheidung, diese zu durchbrechen.

\section{c. Gerichtliche Durchsetzbarkeit}

Grundrechte zeichnen sich gerade dadurch aus, dass sie individualrechtliche, von Einzelnen anrufbare Ansprüche verleihen. ${ }^{390}$ Staatliche Handlungen können direkt gestützt auf einen Grundrechtsartikel gerichtlich gerügt werden, wenn sie in den Schutzbereich eines Grundrechts eingreifen, sprich einen justiziablen Anspruch verkürzen. 391

385 BGE 138 I 378 E. 9.2 S. 399; es reiche vielmehr, die «Vermögens-, Finanz- und Ertragslage getrennt nach den Aufgabengebieten» darzulegen bzw. die mit der Wettbewerbstätigkeit «verbundenen Kosten sachgerecht in einer getrennten Kostenrechnung» auszuweisen, BGE138 I 289 E. 2.2 S. 291 f. Kritisch dazu BAUMANN, Wettbewerbsverzerrungen, Rz.352.

386 BGE 138I I78 E. 9.3.3 S.401.

387 BGE 138I 378 E.9.4 S.401.

388 Ähnlich auch BIAGGINI, Bemerkungen zum Glarnersach-Urteil, S. 673; HETTICH, Bemerkungen zu BGE 138 I 378, S. 1471.

389 Vgl. BGE 139 I 306 E.3.2.2 S.311.

390 HÄFELIN/HALLER/KELLER/THURNHERR, Schweizerisches Bundesstaatsrecht, Rz. 220; TSCHANNEN, Staatsrecht, \$7 Rz. 5, 18.

391 TSCHANNEN, Staatsrecht, §7 Rz.18, 82. Ferner KIENER/KÄLIN/WYTTENBACH, Grundrechte, §8 Rz.3; RHINOW/SCHEFER/UEBERSAX, Schweizerisches Verfassungsrecht, Rz.1009. 


\section{d. Folgen von Grundrechtsverletzungen}

Der Grundrechtsschutz erstreckt sich nicht schon darin, Eingriffe überprüfen und allfällige Verletzungen feststellen zu lassen. Die Folgen festgestellter Grundrechtsverletzungen sind vom Gericht im Einzelfall anzusetzen:392 Vorrang hat die Wiederherstellung des verfassungsmässigen Zustands; wenn immer möglich ist die Rechtsverletzung rückgängig zu machen. Dies dürfte zuvorderst zutreffen, wenn die Verletzung durch einen Rechtsakt erfolgt, der aufgehoben oder durch eine richterliche Ersatzregelung angepasst werden kann. Bei Realakten hingegen dürfte die Verletzung i.d.R. irreversibel sein. Dann liegt der Fokus auf Kompensation und Prävention, sprich der zumindest annähernden Wiedergutmachung in Form von Schadenersatz sowie der Verhinderung künftiger Verletzungen.

\section{Staatshaftung}

Eine weitere Anforderung an das staatliche Handeln sind die besonderen Verantwortlichkeiten oder Haftungsregeln, denen es unterliegt. Diese Regeln überlagern sich nach thematischen Bereichen und föderalen Staatsebenen. ${ }^{393}$ Die Verfassung äussert sich nur zur vermögensrechtlichen Haftung, indem sie eine ausschliessliche Staatshaftung des Bundes vorsieht für Schäden, die Personen oder Organisation in Erfüllung ihrer staatlichen Aufgaben widerrechtlich verursachen. ${ }^{394}$ Ausschliesslich heisst dabei, dass gegenüber dem Geschädigten nicht die handelnde Person, sondern das Gemeinwesen haftet. 395 Die einzelnen Haftungsvoraussetzungen richten sich nach dem Verantwortlichkeitsgesetz (VG) und müssen hier nicht vertieft werden. ${ }^{396}$

Die spezialgesetzlichen Grundlagen staatlicher Haftung können hier nicht ausgeführt werden. ${ }^{397}$ Zur strafrechtlichen Verantwortlichkeit staatlicher

392 Zum Folgenden ausführlich KIENER/KäLIN/WYTTENBACH, Grundrechte, §8 Rz. 25-38. Knapp auch HÄFELIN/HALLER/KELLER/THURNHERR, Schweizerisches Bundesstaatsrecht, Rz.271a.

393 JAAG/HÄNNI, St. Galler Kommentar, Art.146 Rz. 51; HÄFELIN/MÜLLER/UHLMANN, Allgemeines Verwaltungsrecht, Rz. 2095; TSCHANNEN/ZIMMERLI/MÜLLER, Allgemeines Verwaltungsrecht, §61 Rz. 2 .

394 BIAGGINI, Kommentar BV, Art.146 Rz.2;JAAG/HÄNNI, St. Galler Kommentar, Art.146 Rz.17, 50.

395 JAAG/HÄNNI, St. Galler Kommentar, Art.146 Rz.13; TSCHANNEN/ZIMMERLI/MÜLLER, Allgemeines Verwaltungsrecht, \$61 Rz.4, §62 Rz. 4.

396 Siehe dazu BIAGGINI, Kommentar BV, Art.146 Rz. 5-8;JAAG/HÄNNI, St. Galler Kommentar, Art.146 Rz.18-38; TSCHANNEN/ZIMMERLI/MÜLLER, Allgemeines Verwaltungsrecht, §62 Rz. 8-44.

397 Ausführlich dazu JAAG, Staats- und Beamtenhaftung, Rz. 22-36. Ferner HÄFELIN/MÜLLER/ UHLMANN, Allgemeines Verwaltungsrecht, Rz. 2145-2149; TSCHANNEN/ZIMMERLI/MÜLLER, Allgemeines Verwaltungsrecht, §62 Rz. 21. 
Aufgabenträger nur so viel: Sie zeichnet sich vor allem dadurch aus, dass die Strafverfolgung die vorgängige Ermächtigung einer staatlichen Stelle voraussetzt. 398

Die Staatshaftung knüpft an ein funktionelles Staatsverständnis an. Organ (i.S.v. Art. 146 BV) bzw. Beamter (i.S.v. Art. 3 VG) ist jede Person, die eine staatliche Aufgabe wahrnimmt. ${ }^{399}$ Und die Haftung ist umgekehrt beschränkt auf solche Handlungen, die in Erfüllung dieser staatlichen Aufgabe ergehen. 400 Der funktionelle Zugang des Staatshaftungsrechts gilt unbeachtlich der Rechtsform des Aufgabenträgers. Art. 19 VG sieht lediglich vor, dass ausserhalb der Zentralverwaltung stehende Aufgabenträger selber Anspruchsgegner der Staatshaftung sind und der Bund nur eine Ausfallhaftung trägt. ${ }^{401}$

Die Staatshaftung unterscheidet sich von den anderen Anforderungen: Art.146 BV greift nämlich immer dann, wenn bei der staatlichen Aufgabenerfüllung eine rechtliche Vorgabe missachtet wurde. Die Staatshaftung ist also vielmehr eine Konsequenz verletzter Anforderungen denn eine Anforderung selbst. Wenn im weiteren Verlauf dieser Arbeit die Probleme und Spielräume geprüft werden, die bei der Erfüllungsprivatisierung entstehen ( $\$ \$ 5$ und 6), wird daher die Staatshaftung ausgespart. Privatisierte Organisations-, Handlungs- und Finanzierungsformen führen zu keinen Besonderheiten. Mit Art. 19 VG ist bereits alles gesagt: Auch die ausgelagerte Aufgabenerfüllung kann eine staatliche Haftung begründen; einzig der Anspruchsgegner ist ein anderer.

\section{Planung und Koordination staatlicher Tätigkeit}

An einer weiteren Stelle knüpft die Verfassung ausdrücklich an staatliche Tätigkeit, also die Erfüllung staatlicher Aufgaben an: Der Bundesrat wird angehalten, die staatliche Tätigkeit zu planen und zu koordinieren (Art.180 Abs. 1 Satz 2 BV). Diese Bestimmung zwingt das staatliche Handeln nicht in

\footnotetext{
398 Art.13-16 VG.

399 Vgl. die Auffangbestimmung in Art.1 Abs. 1 Bst. f VG. Dazu ausführlich BIAGGINI, Kommentar BV, Art. 146 Rz. 6; JAAG/HÄNNI, St. Galler Kommentar, Art. 146 Rz. 21; HÄFELIN/ MÜLLER/UHLMANN, Allgemeines Verwaltungsrecht, Rz. 2102; TSCHANNEN/ZIMMERLI/ MÜLLER, Allgemeines Verwaltungsrecht, \$62 Rz.18 f.

400 «Amtliche Tätigkeit» nach Art.146 BV ist damit gleichbedeutend. BIAGGINI, Kommentar BV, Art. 146 Rz. 7; FREUDIGER, Anstalt oder Aktiengesellschaft?, S. 394 f.; JAAG/HÄNNI, St. Galler Kommentar, Art. 146 Rz. 24; HÄFELIN/MÜLLER/UHLMANN, Allgemeines Verwaltungsrecht, Rz.2108.

401 BIAGGINI, Kommentar BV, Art.146 Rz. 6; JAAG, Staats- und Beamtenhaftung, Rz. 202 f.; JAAG/HÄNNI, St. Galler Kommentar, Art.146 Rz.14; TSCHANNEN/ZIMMERLI/MÜLLER, Allgemeines Verwaltungsrecht, $\$ 62$ Rz. 20.
} 
bestimmte Formen. Im Sinne einer Organzuständigkeit erklärt sie lediglich den Bundesrat dafür zuständig, die verschiedenen Tätigkeiten aller Aufgabenträger des Bundes zu überblicken und aufeinander abzustimmen. ${ }^{402}$ Der Bundesrat muss die Staatstätigkeit umfassend planen, über die Bundesverwaltung hinaus. ${ }^{403}$ Die Funktion dieser Anforderung liegt darin, das staatliche Handeln ungeachtet seiner Organisations- oder Handlungsform an gemeinsame Ziele zu binden. Indem die Verfassung auch an dieser Stelle nicht an das Erscheinungsbild des Aufgabenträgers anknüpft, sondern an die staatliche Tätigkeit, wird verdeutlicht, dass jede Erfüllung staatlicher Aufgaben aus verfassungsrechtlicher Sicht gleich bedeutsam ist.

Während diese Bestimmung eine verfassungsrechtliche Anforderung im Sinn dieser Arbeit darstellt, ergeben sich aus ihr keine Bindungen, die auf verschiedene Erfüllungsformen des staatlichen Handelns angewendet werden können. Die Anforderung bindet alleine den Bundesrat. Entsprechend ist Art.180 BV nicht Teil des Prüfmassstabs bei den $\$ \$ 5$ und 6 .

402 BIAGGINI, Kommentar BV, Art. 180 Rz. 5; EHRENZELLER, St. Galler Kommentar, Art. 180 Rz.20.

403 EHRENZELLER, St. Galler Kommentar, Art.180 Rz.19. 


\section{Was sind keine Anforderungen?}

Vorne in II.1 wurden die verfassungsrechtlichen Anforderungen an das staatliche Handeln abschliessend aufgelistet. Im Folgenden sind diese Anforderungen gegenüber fünf Rechtsfiguren abzugrenzen, denen diese Stellung gerade nicht zukommt.

\section{Subsidiarität}

Das Subsidiaritätsprinzip verlangt, dass eine Aufgabe so nah wie möglich an den betroffenen Bürgerinnen und Bürgern erfüllt wird; dass sie also nur dann überhaupt vom Gemeinwesen übernommen wird, wenn sie im privaten Raum nicht erfüllt werden kann, und dann auf der tiefstmöglichen gliedstaatlichen Ebene. ${ }^{404}$ Zahlreiche Verfassungsbestimmungen werden als Erscheinungen eines solchen Subsidiaritätsgedankens diskutiert: Art. 6405, $12^{406}, 41$ Abs. $1^{407}$, Art. 46 Abs. $3^{408}$, Art. $47^{409}$, Art. 50 Abs. $2^{410}$, Art. 52 Abs. $2^{411}$, Art. 103 BV412. Seit 2008 ist mit Art. 5a BV ein «Grundsatz der Subsidiarität» auch ausdrücklich in der Verfassung verankert. ${ }^{413}$ Der ebenfalls mit der «Neugestaltung des Finanzausgleichs und der Aufgaben zwischen Bund und Kantonen (NFA)» eingeführte Art. 43a Abs. 1 BV ergänzt diesen allgemeinen Grundsatz um eine bundesstaatliche Komponente. ${ }^{414}$

404 Botschaft NFA, 2458; BIAGGINI, Basler Kommentar, Art. 5a Rz. 2; RHINOW/SCHEFER/ UEBERSAX, Schweizerisches Verfassungsrecht, Rz. 596; TSCHANNEN, Staatsrecht, §15 Rz.4, \$19Rz. 8.

405 RHINOW/SCHEFER/UEBERSAX, Schweizerisches Verfassungsrecht, Rz. 222, 2648; SCHWEIZER/MÜLLER, St. Galler Kommentar, Art. 5a Rz.7; TSCHANNEN, Staatsrecht, §6 Rz. 37.

406 HÄFELIN/HALLER/KELLER/THURNHERR, Schweizerisches Bundesstaatsrecht, Rz. 916; RHINOW/SCHEFER/UEBERSAX, Schweizerisches Verfassungsrecht, Rz.3457-3463b; SCHWEIZER/MÜLLER, St. Galler Kommentar, Art. 5a Rz.7.

407 HÄFELIN/HALLER/KELLER/THURNHERR, Schweizerisches Bundesstaatsrecht, Rz. 911; RHINOW/SCHEFER/UEBERSAX, Schweizerisches Verfassungsrecht, Rz.3384; SCHWEIZER/MÜLLER, St. Galler Kommentar, Art. 5a Rz.7.

408 TSCHANNEN, Staatsrecht, \$23 Rz. 12.

409 TSCHANNEN, Staatsrecht, \$15 Rz.4, §23 Rz.12.

410 RHINOW/SCHEFER/UEBERSAX, Schweizerisches Verfassungsrecht, Rz. 647.

411 RHINOW/SCHEFER/UEBERSAX, Schweizerisches Verfassungsrecht, Rz. 930; Bezug nehmend auf alle Formen der Bundesintervention auch HÄFELIN/HALLER/KELLER/ THURNHERR, Schweizerisches Bundesstaatsrecht, Rz.1040.

412 HÄFELIN/HALLER/KELLER/THURNHERR, Schweizerisches Bundesstaatsrecht, Rz. 664.

413 Im Rahmen der sogenannten «Neugestaltung des Finanzausgleichs und der Aufgaben zwischen Bund und Kantonen (NFA)» eingeführt, vgl. dazu in der Botschaft NFA, 2339.

414 RHINOW/SCHEFER/UEBERSAX, Schweizerisches Verfassungsrecht, Rz. 595; TSCHANNEN, Staatsrecht, §19 Rz. 8. 
Seinem offenen Wortlaut zufolge könnte Art. 5a BV eigentlich auch die Aufgabenteilung zwischen Staat und (privater) Gesellschaft betreffen. Die einhellige Lehre beschränkt die Geltung des Artikels aber auf das Verhältnis der verschiedenen Staatsebenen untereinander. ${ }^{415}$ Auch in diesem Verhältnis ist der Subsidiaritätsgrundsatz nur eine nicht justiziable Leitlinie.416

Unabhängig davon, dass Art. $5 \mathrm{a}$ BV prozessrechtlich nicht durchsetzbar ist: Kann eine staatliche Handlung mangels Subsidiarität als verfassungswidrig angesehen werden? Kann also ein Erlass oder ein Rechtsanwendungsakt deswegen bemängelt werden, weil er von der falschen Staatsebene ergriffen wurde? Seinem Gehalt nach beschlägt der Grundsatz zwar sowohl die Zuweisung wie die Ausübung von Kompetenzen. ${ }^{417}$ Keine dieser Vorgaben ist aber rechtlich bindend, Subsidiarität bleibt trotz ausdrücklicher Verfassungsbestimmung rechtlich «bedeutungslos, wie übrigens jede Selbstbindung des Verfassungsgebers.»418 Dem Bund steht es frei, diejenigen Aufgaben an sich zu ziehen, die er will, und diejenigen Erfüllungsmassnahmen vorzusehen und zu ergreifen, die ihm angemessen scheinen.

\section{Bedarfsgerechtigkeit und Wirtschaftlichkeit}

Die Verfassung enthält in Art. 43a BV verschiedene «Grundsätze für die Zuweisung und Erfüllung staatlicher Aufgaben». Diese Grundsätze sind allerdings keine bindenden Anforderungen an das staatliche Handeln. Die Absätze 1-3 sind offensichtlich als Vorgaben an die Aufgabenzuweisung formuliert. ${ }^{419}$ Absatz 4 wird sogleich im Zusammenhang mit dem Service public diskutiert (3). Hingegen scheint Absatz 5 seinem Wortlaut nach eigentliche Anforderungen an das Staatshandeln aufzustellen: «Staatliche Aufgaben müssen bedarfsgerecht und wirtschaftlich erfüllt werden.» Den bundesrätlichen Erläuterungen zufolge muss der Satz allerdings ebenfalls auf die Zuweisung, nicht die Erfüllung, staatlicher Aufgaben gemünzt werden: «Das Gemeinwesen, dem eine

415 Botschaft NFA, 2458. BGE138 I 378 E. 8.4 S. 395. RHINOW/SCHEFER/UEBERSAX, Schweizerisches Verfassungsrecht, Rz. 597; TSCHANNEN, Staatsrecht, \$19 Rz.13; BIAGGINI, Basler Kommentar, Art. 5a Rz.16.

416 Botschaft NFA, 2458 («staatspolitische Maxime»); BGE 138 I 378 E. 8.4 S. 395; RHINOW/ SCHEFER/UEBERSAX, Schweizerisches Verfassungsrecht, Rz. 687; SCHWEIZER/MÜLLER, St. Galler Kommentar, Art. 5a Rz. 16; TSCHANNEN, Staatsrecht, \$19 Rz.15. Zu Art. 43a BV gleichlautend auch BIAGGINI, Basler Kommentar, Art. 43a Rz.12.

417 RHINOW/SCHEFER/UEBERSAX, Schweizerisches Verfassungsrecht, Rz. 598-610.

418 TSCHANNEN, Staatsrecht, \$19 Rz. 8.

419 Zum «föderativen Subsidiaritätsprinzip» von Abs. 1 siehe TSCHANNEN, Staatsrecht, \$15 Rz. 4, §19 Rz. 8; zur «fiskalischen Äquivalenz» von Abs. 2 und 3 siehe TSCHANNEN, Staatsrecht, §19 Rz.8a; und allgemein zum Gehalt von Art. 43a BV SCHWEIZER/MÜLLER, St. Galler Kommentar, Art. 43a Rz. 5. 
staatliche Aufgabe zugeteilt werden soll, muss in der Lage sein, diese bedarfsgerecht und wirtschaftlich zu erfüllen.» ${ }^{420}$ Mangelnde Bedarfsgerechtigkeit oder Wirtschaftlichkeit kann allerdings nicht als Rechtsverletzung gerügt werden.

Das Bundesgericht hat es in einem Fall offengelassen, ob der Wirtschaftlichkeitsbegriff von Art. 43a Abs. 5 BV direkten Einfluss auf die rechtsanwendende Verwaltungstätigkeit hat. ${ }^{421}$ Es bleibt also denkbar, dass diese Bestimmung doch als Anforderung für die Ausübung einer bereits zugewiesenen Aufgabe beigezogen wird. Dem steht allerdings entgegen, dass Art. 43a BV in seinem gesamten Umfang als nicht justiziabel konzipiert ist. ${ }^{422}$

Über diese verfassungsrechtliche Verankerung hinaus wird diskutiert, ob andere wirtschaftliche Kriterien den Staat binden. LIENHARD etwa sieht das staatliche Handeln an ein verfassungsrechtliches Effizienzprinzip gebunden. ${ }^{423}$ Jede staatliche Tätigkeit stehe unter dem Vorbehalt genügender Ressourcen, weshalb der Staat verpflichtet sei, seine knappen Mittel effizient einzusetzen. ${ }^{424}$

Die Aufgabenzuweisung und-konkretisierung in Verfassung und Gesetzen stehen allerdings nicht unter dem Vorbehalt ausreichender Mittel. Der Staat hat die ihm zugewiesenen Aufgaben in vollem Umfang und der vorgeschriebenen Qualität zu erfüllen. Wirtschaftlichkeit kann als Leitlinie für die Aufgabenzuweisung dienen, aber die Aufgabenerfüllung selbst mag sie nicht binden.

\section{Grundversorgung}

Gemäss vereinzelten Lehrmeinungen stehe die Pflicht des Staats, eine ausreichende Grundversorgung sicherzustellen, gewissen Privatisierungen entgegen. ${ }^{425}$ Mögliche Rückzüge des Staats aus der «Versorgung mit öffentlichen Gütern (Telekommunikation, Energie, Bahnen usw.)» stünden zwingend in

\footnotetext{
420 Botschaft NFA, 2459.

421 BGer-E 9C_940/2011 vom 21. September 2012, E.3.4, bezüglich Einfluss von Art. 43a Abs. 5 BV auf den krankenversicherungsrechtlichen Begriff der Wirtschaftlichkeit.

422 Botschaft NFA, 2339, 2458; BIAGGINI, Basler Kommentar, Art. 43a bezeichnet dies als die einhellige Auffassung, Rz. 5, 42; sCHWEIZER/MÜLLER, St. Galler Kommentar, Art. 43a Rz.22.

423 LIENHARD, Grundlagen NPM, S. 140 f., leitet dies aus Art. 43a Abs. 5, 126, 170, 178 Abs. 1 BV ab; mit zahlreichen Literaturhinweisen. HOFSTETTER stimmt inhaltlich überein, sieht aber die Notwendigkeit einer ausdrücklichen verfassungsrechtlichen Verankerung, HOFSTETTER, Verhältnismässigkeitsprinzip, Rz. 520.

424 HOFSTETTER, Verhältnismässigkeitsprinzip, Rz. 514; LIENHARD, Grundlagen NPM, S.140.

425 SCHWEIZER/MÜLLER, St. Galler Kommentar, Art. 43a Rz. 17; UEBERSAX, Privatisierung, S. $397 \mathrm{f}$.
} 
einem «Spannungsverhältnis zur Sicherung der Grundversorgung im Sinne des chancengleichen, flächendeckenden und preiswerten Zugangs zu Infrastrukturleistungen» ${ }^{426}$. Nach dieser Konzeption könnte also die staatliche Aufgabenerfüllung als ungenügend gerügt werden, wenn sie die Standards einer ausreichenden Grundversorgung missachtet.

Verfassungsrechtlicher Anknüpfungspunkt dafür ist Art. 43a Abs. 4 BV: «Leistungen der Grundversorgung müssen allen Personen in vergleichbarer Weise offen stehen.» Diese Bestimmung vereint eine soziale mit einer geografischen Dimension: Im ganzen Landesgebiet müssen die Grundversorgungsleistungen allen Personen, unabhängig von ihrer wirtschaftlichen Kaufkraft, zugänglich sein. 427

Diese Bestimmung ist allerdings - wie der gesamte Art.43a BV - nicht justiziabel; sie schafft keine einklagbaren Rechte. 428 Die Pflicht, Leistungen der Grundversorgung zu erbringen, ergibt sich nur aus einzelnen Sachkompetenzen:429 Im Bund sind dies Art. 89 Abs. 1, Art. 92 Abs. 2, Art. 102, 103, 104 Abs. 1 Bst. a, Art. 111 Abs. 1 und Art.117a Abs. 1 BV. 430 Rechtlich verbindliche Ansprüche auf bestimmte Leistungen, die in bestimmter Qualität an bestimmten Orten und zu bestimmten Preisen verfügbar sein müssen, ergeben sich aus den Vorgaben in den ausführenden Sachgesetzen. Die Aufgabenzuweisung und die ausführende Gesetzgebung bestimmen Umfang und Qualität der staatlichen Leistungen. Es gibt keinen Anspruch auf Grundversorgungsleistungen, die über die einzelnen Kompetenzen und gesetzlichen Verpflichtungen des Staats hinausgehen. Der Staat ist zur Erbringung derjenigen Leistungen verpflichtet, die ihm die Verfassung und das Gesetz übertragen.

Der verfassungsrechtliche Begriff der Grundversorgung taugt nicht als Anforderung, aus der Leistungsansprüche abgeleitet werden könnten. ${ }^{431}$ Verschiedene Bestrebungen, weitergehende Anforderungen an die Grundversorgungsleistungen in der Verfassung zu verankern, sind gescheitert. $\mathbf{4 3 2}$

\footnotetext{
426 UEBERSAX, Privatisierung, S. $397 \mathrm{f}$.

427 SCHWEIZER/MÜLLER, St. Galler Kommentar, Art. 43a Rz.17. Zum geografischen Aspekt auch Botschaft NFA, 2459.

428 Botschaft NFA, 2459; BIAGGINI, Basler Kommentar, Art. 43a Rz. 5, 37.

429 BIAGGINI, Basler Kommentar, Art. 43a Rz. 36; SCHWEIZER/MÜLLER, St. Galler Kommentar, Art. 43a Rz.17.

430 SCHWEIZER/MÜLLER, St. Galler Kommentar, Art. 43a Rz.18.

431 Ähnlich auch BIAGGINI, Grundversorgung, S.114.

432 Siehe die Geschäfte 05.3232 und 13.036 in der Bundesversammlung, sowie die im Juni 2016 abgelehnte Volksinitiative, BBl 2016 6779; vgl. die provisorische Nachzeichnung vor endgültiger Abschreibung oder Ablehnung in BIAGGINI, Grundversorgung, S.114; BIAGGINI, Basler Kommentar, Art. 43a Rz.38f.; SCHWEIZER/MÜLLER, St. Galler Kommentar, Art. 43a Rz.19.
} 


\section{Staatsfreie Wirtschaft}

Die Wirtschaftsfreiheit umfasst neben individualrechtlichen Ansprüchen wie der Berufswahl- und Berufsausübungsfreiheit auch einen objektiv-rechtlichen Gehalt: Die Wirtschaftsfreiheit schützt als institutionelle Garantie die staatsfreie, von Marktmechanismen gesteuerte Wirtschaftsordnung (siehe vorne II.6.a.dd). ${ }^{433}$ Die privatwirtschaftliche Sphäre soll von staatlicher Intervention abgeschirmt werden, woraus sich ein leitendes Prinzip der «staatsfreien Wirtschaft» ableitet. ${ }^{434}$ Art. 94 Abs. 1 BV bietet einen konkreten Anknüpfungspunkt für diese Forderung.

Die Beschreibungen dieses Prinzips legen nahe, dass es das staatliche Handeln in ähnlicher Weise bindet wie etwa die Erfordernisse einer gesetzlichen Grundlage und der Verhältnismässigkeit:Jede staatliche Intervention, die sich auf die wirtschaftliche Tätigkeit oder Situation Privater auswirke, muss grundsatzkonform sein. Für grundsatzwidrige Eingriffe braucht es gemäss Art. 94 Abs. 4 BV eine eigene Verfassungsgrundlage oder ein kantonales Regalrecht. Nicht jede Beeinflussung des Wettbewerbs ist aber grundsatzwidrig; untersagt sind nur wettbewerbsverzerrende Eingriffe. 435 Positiv formuliert muss das staatliche Handeln wettbewerbsneutral sein. Dabei kommt es nicht auf die Auswirkungen, sondern die Intention an. Eingriffe in den Wettbewerb dürfen keine wirtschaftspolitischen Motive verfolgen. Solange andere Interessen vorliegen, sind auch wettbewerbsverzerrende Auswirkungen verfassungsmässig.

Diese Ermahnung zur grundsätzlichen Staatsfreiheit des Wirtschaftssystems darf allerdings nicht überschätzt werden:436 Nach dem Verständnis dieser Arbeit ist staatliche Wirtschaftstätigkeit keine zu vermeidende Anomalie, son-

433 Grundlegend dazu VALLENDER, Ordnungspolitischer Grundentscheid, passim; näher HÄFELIN/HALLER/KELLER/THURNHERR, Schweizerisches Bundesstaatsrecht, Rz. 627; KIENER/KÄLIN/WYTTENBACH, Grundrechte, \$31 Rz.9-14; KRÄHENMANN, Privatwirtschaftliche Tätigkeit, S.160; RHINOW/BIAGGINI/SCHMID/UHLMANN, Öffentliches Wirtschaftsrecht, §4 Rz.67-72; voGEL, Staat als Marktteilnehmer, S. 106. Und BGE 138 I 378 E. 6.3.2 S.388f. mit einem umfassenden Literaturüberblick. Dieser Abschnitt ist teilweise angelehnt an eine frühere Publikation: ELSER, Funktioneller Staatsbegriff, S. 71-77.

434 Dazu ausführlich VoGEL, Staat als Marktteilnehmer, S. 107-120; noch auf Basis der aBV zudem KRÄHENMANN, Privatwirtschaftliche Tätigkeit, S.159-164; UHLMANN, Gewinnorientiertes Staatshandeln, S. 175-182.

435 Anstelle vieler, auch zum Folgenden: BIAGGINI, Kommentar BV, Art. 94 Rz. 4 f.; KIENER/ KÄLIN/WYTTENBACH, Grundrechte, \$31 Rz. 55-64; RHINOW/BIAGGINI/SCHMID/UHLMANN, Öffentliches Wirtschaftsrecht, \$5 Rz. 92-99. Grundlegend zur Dogmatik dieses Grundsatzes REICH, Grundsatz der Wirtschaftsfreiheit, passim und insb. S. 429-490.

436 BAUMANN, Wettbewerbsverzerrungen, Rz.360. Indirekt dazu ermahnend auch UHLMANN, Gewinnorientiertes Staatshandeln, S.182. 
dern eine zu begründende Regelmässigkeit.437 In seiner ausführlichsten Bewertung dieser Thematik - dem Glarnersach-Urteil von 2012 - arbeitet das Bundesgericht die umfangreiche Literatur zu dieser Frage auf und resümiert den überwiegenden Konsens, dass es dem Staat nicht grundsätzlich verboten ist, sich unternehmerisch zu betätigen. ${ }^{438}$ Dies entspreche "der gelebten Verfassungspraxis» 439 , und weiter sei mit dem Grundsatz der staatsfreien Wirtschaftsordnung nach Art. 94 Abs. $1 \mathrm{BV}$ «in erster Linie gemeint, dass die private Wirtschaft nicht ohne Rechtfertigung durch den Staat beschränkt wird» $\mathbf{4 4 0}^{4}$

Die reisserische Phrase des Bundesgerichts, es könne «von einem gewissen 'Wettbewerb der Systeme) (zwischen Staats- und Privatunternehmen) gesprochen werden ${ }^{441}$, bedeutet aber nicht, dass die staatliche Aufgabenerfüllung die private Wirtschaftstätigkeit konkurrenzieren soll. Die Phrase ist gemünzt auf den Fall, dass ein staatlicher Akteur wettbewerbliche Tätigkeiten wie ein Privater ausübt und somit funktionell nicht mehr Staat ist. Diese wettbewerbliche Tätigkeit erfolgt dann in Konkurrenz zu allen anderen Wettbewerbern. Das Bundesgericht drückt damit also keine Privilegierung der unternehmerischen Tätigkeit des Staats aus, sondern geht im Gegenteil davon aus, dass sich die nichtstaatlichen, privatwirtschaftlichen Tätigkeiten des Staats im Wettbewerb behaupten müssen, um Bestand haben zu können. ${ }^{442}$ Der «Wettbewerb der Systeme» geht also - in der Konzeption des Bundesgerichts - nicht zulasten der Privaten, sondern fordert vom Staat, sich mit wirtschaftlich lohnendem Handeln unter Konkurrenzbedingungen zu bewähren. ${ }^{443}$

Dass staatliche Akteure ausserhalb ihres Aufgabenbereichs auftreten und private Anbieter konkurrenzieren, ist also keineswegs die Ausnahme. Die Rechtsordnung sieht dies geradezu vor und stellt es lediglich unter eine Rechtfertigungspflicht. In der Literatur wird kritisiert, dies «verkehr[e] den Grundsatz einer staatsfreien Wirtschaft ins Gegenteil»444 und das Bundesgericht schütze damit «primär die unternehmerische Freiheit staatlicher Unter-

437 Zur grundsätzlichen Zulässigkeit unter Rechtfertigungsvorbehalt auch BAUMANN, Wettbewerbsverzerrungen, Rz.32, mit zahlreichen Hinweisen.

438 Siehe die zahlreichen Hinweise in BGE 138 I 378 E. 6.3.2 S.388f.

439 BGE 138 I 378 E. 6.3.3 S. 389. Als Argumente angeführt werden «gewerbliche Betriebe» wie öffentliche Spitäler, Gastbetriebe, Weingüter etc. (S. 389 f.) und neuere Bundesgesetze, die vorsehen, «dass staatliche Unternehmen neben einem allfälligen Monopoloder Service-public-Bereich in Konkurrenz zur Privatwirtschaft weitere Tätigkeiten ausüben können» (S.390).

440 BGE 138 I 378 E.6.3.1 S.387.

441 BGE 138 I 378 E. 8.5 S.396.

442 Ähnlich schon UHLMANN, Gewinnorientiertes Staatshandeln, S. 268.

443 Vgl. empirische Hinweise zur Effizienz privater und staatlicher Akteure unter Wettbewerbsbedingungen bei BAUMANN, Wettbewerbsverzerrungen, Rz.165.

444 HETTICH, Bemerkungen zu BGE 138 I 378, S. 1470. 
nehmen [...] (statt jene der privaten Unternehmen)»445. Diese Kritik bedauert, was verfassungsrechtliche Tatsache ist: Der Grundsatz der staatsfreien Wirtschaft bindet den Staat nicht zurück, er ist eben keine verfassungsrechtliche Anforderung an staatliches Handeln. ${ }^{446}$ Der Staat ist hingegen durch seine übrigen Anforderungen begrenzt. Das Augenmerk der Kritik sollte also nicht auf einer «Durchbrechung» dieses Grundsatzes liegen, sondern auf der Einhaltung der übrigen Kriterien.

Und dort zeigt sich, dass der Fokus auf wettbewerbsneutrales Verhalten die Anforderungen geradezu herabsetzt; wiederum vorexerziert im Glarnersach-Urteil: «Insbesondere im Bereich der wirtschaftlichen Tätigkeit des Staates kann die gesetzliche Grundlage nicht zu detailliert sein, um die unternehmerische Tätigkeit nicht zu behindern ${ }^{447}$; vielmehr reiche es aus, wenn das Gesetz in Befolgung des sogenannten Spezialitätsprinzips «den Sachbereich umschreib[t], in welchem die Tätigkeit erfolgen soll»448 (siehe zum Spezialitätsprinzip hinten \$6/II.1.c). Das öffentliche Interesse wird sodann nicht einmal mehr gesondert geprüft. Schliesslich belege die gesetzliche Grundlage als Abschluss eines demokratischen Prozesses, dass eine bestimmte Tätigkeit als im öffentlichen Interesse eingestuft worden sei. ${ }^{449}$ Auch eine eigentliche Verhältnismässigkeitsprüfung entfällt. Der Staat darf in den Wettbewerb eingreifen, er darf diesen auch verzerren; er darf ihn nur nicht gerade ausschalten. ${ }^{450}$ Das ist eine schwache Grenze, die nur eigentliche Planwirtschaft verbietet. Diese gelockerten Standards erklären sich teilweise aus prozessualen Gründen: Die Prüfung erfolgte nach Art. 5 BV, nicht nach Art. 36 $\mathrm{BV}$, weshalb sich das Bundesgericht gegenüber kantonalem Recht auf eine Willkürprüfung beschränkte. ${ }^{451}$

Das Bundesgericht ist nicht dafür zu kritisieren, dass es staatliche Wirtschaftstätigkeit grundsätzlich für zulässig hält, sondern dass es die daran gestellten Anforderungen derart zurückhaltend anwendet. ${ }^{452}$

Eines darf aber nicht vergessen werden: Sofern der Staat im Wettbewerbsbereich Aufgaben wahrnimmt, zu denen das Gesetz ihn nicht verpflichtet,

445 BIAGGINI, Bemerkungen zum Glarnersach-Urteil, S. 672.

446 Ähnlich auch BAUMANN, Wettbewerbsverzerrungen, Rz.48.

447 BGE 138 I 378 E.7.2 S.392.

448 BGE 138 I 378 E. 7.2 S. 392. Zu den herabgesetzten Anforderungen an die gesetzliche Grundlage in diesem Fall auch BIAGGINI, Bemerkungen zum Glarnersach-Urteil, S. 671; KRAEMER/STÖCKLI, Grenzenlose Staatswirtschaft?, S. 30 f., 35.

449 BGE 138 I 378 E. 8.3 S. 394.

450 BGE 138 I 378 E. 8.7 S. $397 \mathrm{f}$.

451 KRAEMER/STÖCKLI, Grenzenlose Staatswirtschaft?, S. 36.

452 BIAGGINI, Kommentar BV, Art. 94 Rz.6a. In diese Richtung auch KRAEMER/sTöCKLI, Grenzenlose Staatswirtschaft?, S. 35. 
ist er funktionell nicht mehr Staat. ${ }^{453} \mathrm{Er}$ ist lediglich noch ein staatlicher Akteur im organisatorischen Sinn. Den drei Anforderungen Gesetzmässigkeit, öffentliches Interesse und Verhältnismässigkeit ist der Markteintritt des Staats unterworfen. Bei der marktwirtschaftlichen Tätigkeit selbst untersteht der funktionelle Nicht-Staat denselben Regeln wie ein Privater.

\section{Amtssprachen}

Art. 70 Abs. 1 BV verpflichtet den Staat, sich im Verkehr mit dem Bürger einer der offiziellen Amtssprachen zu bedienen. ${ }^{454}$ Der Verfassungsartikel knüpft dies allerdings nicht an die Erfüllung einer staatlichen Aufgabe und stellt daher keine Anforderung an sämtliches Staatshandeln auf. Der Gesetzgeber hat diesen Spielraum genutzt, indem er Organisationen und Personen ausserhalb der (zentralen und dezentralen) Bundesverwaltung generell von den Bindungen des Sprachengesetzes (SpG) ausgenommen hat. 455 Der Bundesrat fordert immerhin, dass bei einer Aufgabenübertragung auf einen solchen Akteur geprüft wird, ob dem Sprachengesetz gleichwertige Anforderungen aufgestellt werden sollen. 456

453 Ähnlich auch BAUMANN, Wettbewerbsverzerrungen, Rz. 54.

454 BIAGGINI, Kommentar BV, Art.70 Rz.3; KÄGI-DIENER, St. Galler Kommentar, Art.70 Rz.17; TSCHANNEN, Staatsrecht, §14 Rz. 8.

455 Art. 4 Abs.1 e contrario SpG.

456 Art. $1 \mathrm{SpV}$. 


\section{Zwischenfazit: \\ Jede Erfüllung staatlicher Aufgaben ist gleich rechtfertigungspflichtig}

Die Verfassung enthält einen abschliessenden Katalog von Anforderungen, die immer und nur gelten, wenn staatliche Aufgaben erfüllt werden. Es sind dies die rechtsstaatlichen Grundsätze von Art. 5 BV (Gesetzmässigkeit, öffentliches Interesse, Verhältnismässigkeit und gewisse Teilgehalte des Grundsatzes von Treu und Glauben) sowie die Grundrechte. Dass staatliches Handeln zudem der Staatshaftung unterliegt sowie geplant und koordiniert werden muss, ist zwar technisch gesehen auch als solche Anforderung einzustufen, ist aber für die Fragestellung dieser Arbeit nicht weiter relevant.

Diese Anforderungen bilden die Gesamtheit der staatlichen Sonderpflichten und sind somit das verfassungsrechtliche Gegenstück zum staatlichen Vorrecht, Zwang gegenüber Privatpersonen ausüben zu dürfen.

Die verfassungsrechtlichen Anforderungen dienen der Legitimierung des staatlichen Handelns. Immer wenn staatliche Macht ausgeübt wird, schützen sie Private. Deshalb prägen sie den funktionellen Staatsbegriff der Verfassung: Sie sind, was den Staat von den Privaten unterscheidet, und ihre Geltung hängt gerade an der Staatlichkeit der Tätigkeit, nicht an der Staatlichkeit der Akteure oder Instrumente. Sie gelten denn auch grundsätzlich unterschiedslos: in demselben Umfang und derselben Intensität, unabhängig von der Art und Weise der Aufgabenerfüllung.

Die Erfüllungsprivatisierung ist gerade von der Erwartung geprägt, dennoch relevante Freiheiten gegenüber der klassisch staatlichen Aufgabenerfüllung zu erreichen. Bevor untersucht werden kann, inwiefern diese Erwartung berechtigt ist (hinten \$\$5 und 6), muss zunächst herausgearbeitet werden, in welcher Hinsicht die Erfüllung staatlicher Aufgaben privatisiert werden kann (sogleich \$4). 


\section{§4 In welcher Hinsicht kann die Erfüllung staatlicher Aufgaben privatisiert werden?}

Diese Arbeit behandelt die Probleme und Spielräume, die entstehen, wenn die Erfüllung staatlicher Aufgaben privatisiert wird. Der Prüfmassstab wurde soeben in $\$ 3$ herausgearbeitet: Es sind die verfassungsrechtlichen Anforderungen, die für jede Erfüllung staatlicher Aufgaben gelten. Diese Anforderungen gelten - aus Sicht der Verfassung - immer gleich, unabhängig von der Art und Weise der Aufgabenerfüllung.

Privatisierungsbestrebungen fallen in eine von zwei Arten: die Aufgabenprivatisierung und die Erfüllungsprivatisierung (siehe vorne \$2/II.4). Eine Aufgabenprivatisierung liegt vor, wenn der Staat seine Verantwortung für einen Aufgabenbereich aufgibt, wenn er also eine bestimmte Zielerreichung weder selber befördern noch gewährleisten muss. Die Frage nach den Problemen und Spielräumen stellt sich bei dieser Aufgabenprivatisierung nicht: Entfällt die staatliche Aufgabe, entfallen auch die verfassungsrechtlichen Anforderungen an staatliches Handeln.

Prüfgegenstand dieser Arbeit bildet nur die Erfüllungsprivatisierung, also die privatisierte Erfüllung staatlicher Aufgaben. Dieser Gegenstand ist erklärungsbedürftig, besonders wegen des funktionellen Staatsverständnisses, das der Bundesverfassung (und dieser Arbeit) zugrunde liegt.

Eine Tätigkeit ist dann staatlich, wenn sie der Erfüllung staatlicher Aufgaben dient. Eine Tätigkeit zu privatisieren (also zu «entstaatlichen»), würde also bedeuten, den Staat aus der Verantwortung für die Aufgabe zu entlassen: eine vormals staatliche Aufgabe zu privatisieren. Dies stellt eine Aufgabenprivatisierung dar.

Bei der Erfüllungsprivatisierung bewegt sich die Verschiebung hingegen entlang eines Spektrums: von typisch staatlichen zu privatähnlichen Erfüllungsmodi. Die (weiterhin staatliche) Aufgabe wird auf eine Art und Weise erfüllt, die von aussen betrachtet privat wirkt - als würde keine staatliche Aufgabe erfüllt. 
Dieser Paragraf arbeitet heraus, was es bedeutet, die Aufgabenerfüllung so zu gestalten, dass sie wie privates Handeln erscheint. Es gibt jedenfalls nicht die Erfüllungsprivatisierung, sie kann vielmehr bei jedem Aspekt der Aufgabenerfüllung ansetzen. Zuerst ist der Ansatzpunkt solcher Vorgänge herauszuschälen: die Aufgabenerfüllung (I). Danach werden die Kennzeichen privatisierter Organisations-, Handlungs- und Finanzierungsformen aufgezeigt(II, III und IV).

\section{Ansatzpunkt: Aspekte der Aufgabenerfüllung}

Um die Erfüllungsprivatisierung beschreiben zu können, muss zunächst deren Ansatzpunktgeklärt sein: die verschiedenen Aspekte der Aufgabenerfüllung. Die Tätigkeiten des Staats sind vielfältig, und sie alle dienen in gewisser Weise der Erfüllung staatlicher Aufgaben - nicht immer gleich unmittelbar und effizient, aber der Staat arbeitet nicht im Vakuum und nicht zum Selbstzweck. Es gilt, die Erfüllung staatlicher Aufgaben zu strukturieren und gegenüber unter- und beigeordneten Handlungen abzugrenzen .

In einem ersten Schritt sind diejenigen Verwaltungstätigkeiten zu identifizieren, die einen unmittelbaren Beitrag zur Erfüllung staatlicher Aufgaben leisten.

\section{Unmittelbare Aufgabenerfüllung}

Die Lehre unterscheidet vier Arten der Erfüllung von Verwaltungsaufgaben. ${ }^{457}$

- Die Eingriffsverwaltung meint Tätigkeiten, mit denen der Staat Private verpflichtet oder belastet und damit ihre Rechte und Freiheiten regelt. Die Verwaltung handelt etwa dann eingreifend, wenn sie Auflagen verhängt für den Betrieb eines Restaurants oder Sprachkenntnisse abprüft im Rahmen eines ausländerrechtlichen Verfahrens.

- Mit Handlungen der Leistungsverwaltung unterstützt und fördert der Staat Private, indem er ihnen Sach-, Geld- oder Dienstleistungen 458 zukommen lässt.

457 Mit unterschiedlicher Terminologie, aber inhaltlich deckungsgleich, auch zum Folgenden: «Formen der Verwaltungstätigkeit» bei HÄFELIN/MÜLLER/UHLMANN, Allgemeines Verwaltungsrecht, Rz. 27, 33-40; «Kategorien von Verwaltungstätigkeiten» bei JAAG, Dezentralisierung und Privatisierung, S.35-37; «Arten der Aufgabenerfüllung» bei TSCHANNEN/ZIMMERLI/MÜLLER, Allgemeines Verwaltungsrecht, §4; ferner TSCHANNEN, Systeme, Rz. 22-37; «Formen der Verwaltungstätigkeit» bei HÄFELIN/MÜLLER/ UHLMANN, Allgemeines Verwaltungsrecht, Rz. 27, 33-40.

458 Wendung übernommen von TSCHANNEN/ZIMMERLI/MÜLLER, Allgemeines Verwaltungsrecht, §4 Rz. 4 . 
- Die Bedarfsverwaltung (oder: administrative Hilfstätigkeit) stellt das Personal und die Sachmittel bereit, die zur Erfüllung von Verwaltungsaufgaben notwendig sind. Solche Hilfstätigkeiten sind beispielsweise die Miete von Büroräumlichkeiten oder die Anstellung von Dossierverantwortlichen und leitenden Angestellten.

- Die wirtschaftende Verwaltung schliesslich ist ein Sammelbegriff, der zwei verschiedene Vorgänge verbindet: die Verwaltung des Finanzvermögens, also der werterhaltende oder gewinnmehrende Umgang mit staatlichen Vermögenswerten, und das Angebot von marktwirtschaftlichen Diensten und Gütern.

Mit der marktwirtschaftlichen Tätigkeit des Staates rückt die wirtschaftende Verwaltung in die Nähe der Leistungsverwaltung. ${ }^{459}$ Der Begriff ist deshalb zu präzisieren: Nur eigentliche Wettbewerbsleistungen, zu deren Erbringung der Staat nicht verpflichtet ist, sollten zur wirtschaftenden Verwaltung gezählt werden. ${ }^{460}$ Die Service-public-Leistungen im staatlichen Pflichtbereich etwa sind als Leistungsverwaltung anzusehen. ${ }^{461}$ Leistungserbringung «am Markt» ist kein geeignetes Abgrenzungskriterium: Sind landwirtschaftliche Subventionen - ein typisches Beispiel der Leistungsverwaltung - etwa kein «Darreichen von Leistungen am (Landwirtschafts-)Markt»? Bewegt sich der Bund mit dem Betrieb eigener Hochschulen nicht auf dem (Bildungs-) Markt?

Diese vier Tätigkeitsarten lassen sich in zwei Gruppen einordnen: Tätigkeiten der Eingriffs- und Leistungsverwaltung leisten einen unmittelbaren Beitrag zur Aufgabenerfüllung, ${ }^{462}$ während diese Beziehung bei der Bedarfsund der wirtschaftenden Verwaltung nur mittelbar ist. ${ }^{463}$ Die Tätigkeiten der letzterenbeiden ArtengehörennichtzurVerwaltungimfunktionellenSinn:464

459 JAAG, Dezentralisierung und Privatisierung, S. 36 (dort als «fiskalische Wettbewerbswirtschaft» bezeichnet); TSCHANNEN/ZIMMERLI/MÜLLER, Allgemeines Verwaltungsrecht, \$4 Rz.14.

460 Vgl. Präzisierung bei TSCHANNEN, Systeme, Rz.33.

461 Monopol- und Wettbewerbsbereich vermischend hingegen TSCHANNEN/ZIMMERLI/ MÜLLER, Allgemeines Verwaltungsrecht, §4 Rz.13 f.

462 TSCHANNEN/ZIMMERLI/MÜLLER, Allgemeines Verwaltungsrecht, §4 Rz. 5;

463 Zur Bedarfsverwaltung HÄFELIN/MÜLLER/UHLMANN, Allgemeines Verwaltungsrecht, Rz. 40; TSCHANNEN/ZIMMERLI/MÜLLER, Allgemeines Verwaltungsrecht, §4 Rz. 9; VPB 60.1, E. 4. Zur wirtschaftenden Verwaltung RHINOw, Verfügung, Verwaltungsvertrag und privatrechtlicher Vertrag, S. 297f.; TSCHANNEN, Systeme, Rz.32; mit der Einschränkung im vorangehenden, kleingeschriebenen Absatz auch TSCHANNEN/ZIMMERLI/MÜLLER, Allgemeines Verwaltungsrecht, §4 Rz.14.

464 TSCHANNEN, Systeme, zur wirtschaftenden Verwaltung ausdrücklich Rz. 32, zur Bedarfsverwaltung aus dem Kontext Rz. 30. Sinngemäss auch DUBEY/ZUFFEREY, Droit administratif général, Rz.157, unter dem Titel der nicht hoheitlichen Verwaltungstätigkeit. 
Sie haben zum Ziel, die finanziellen, gegenständlichen und personellen Voraussetzungen für die Aufgabenerfüllung zu beschaffen.

Diese Arbeit nimmt vorwiegend die Eingriffs- und die Leistungsverwaltung ins Visier. Dies aus zwei Gründen: Erstens greifen die verfassungsrechtlichen Anforderungen bei der bloss mittelbaren Aufgabenerfüllung nicht. Das Gesetzmässigkeitsprinzip (siehe vorne \$3/II.2) bindet zwar auch die Bedarfsund die wirtschaftende Verwaltung. Es beschlägt allerdings vor allem die prinzipielle Ermächtigung zu solcher Tätigkeit: Die Befugnis, «für die betriebsnotwendigen Personal- und Sachmittel zu sorgen, folgt aus den Sachgesetzen, welche die einzelnen Verwaltungsaufgaben regeln»465, also aus der Aufgabe selbst. Welcher Umgang mit den Staatsfinanzen gesetzmässig ist, ergibt sich aus den einschlägigen Bestimmungen zum Finanzhaushalt. ${ }^{466}$ Wollen sich staatliche Organe marktwirtschaftlich - ausserhalb ihres Aufgabenbereichs - betätigen, brauchen sie dafür zwar eine gesetzliche Grundlage, die mit dem Grundsatz der Wirtschaftsfreiheit konform ist, unterliegen abgesehen davon aber gerade nicht den verfassungsrechtlichen Anforderungen, die bei der Aufgabenerfüllung greifen (siehe vorne \$3/III.4). 467

Zweitens ist die Eingriffs- und Leistungsverwaltung von grösserem Interesse, weil hier die Verbindung zu den Adressaten der Verwaltungstätigkeit direkter ist. Private sind hier als Eingriffserdulder ${ }^{468}$ und Leistungsempfänger betroffen, 469 während sie bei den mittelbaren Erfüllungsarten dem Staat als gleichberechtigte Partner im zivilen Rechtsverkehr gegenübertreten: als Bleistiftverkäufer, Jobinteressenten, Bürovermieter oder Mitkonkurrenten. «Eingriffs- und Leistungsverwaltung unterscheiden sich [zwar] danach, wie die rechtsverhältnisbestimmende Verwaltungshandlung beim Bürger ‘ankommt» ${ }^{470}$, doch in beiden Fällen kommen Handlungen beim Privaten an, was gerade nicht der Fall ist bei der Bedarfsverwaltung, die sich mit Betriebsinterna der Verwaltung beschäftigt. ${ }^{471}$ Ein Bleistiftverkäufer etwa geht zwar

\footnotetext{
465 TSCHANNEN/ZIMMERLI/MÜLLER, Allgemeines Verwaltungsrecht, §19 Rz. 29.

466 TSCHANNEN/ZIMMERLI/MÜLLER, Allgemeines Verwaltungsrecht, §19 Rz.31 erstes Lemma

467 TSCHANNEN/ZIMMERLI/MÜLLER, Allgemeines Verwaltungsrecht, §19 Rz.31 zweites Lemma.

468 Danke an meinen früheren Kollegen am Institut für öffentliches Recht der Universität Bern, Karl-Marc Wyss, für diese Wortschöpfung. In der Folge steht das vereinfachende Wort «Verwaltungsadressaten» als Sammelbegriff für Eingriffserdulder und Leistungsempfänger.

469 TANQUEREL spricht allgemein von den Verwalteten («administrés»), TANQUEREL, Droit administratif, Rz.11, 15, 20.

470 TSCHANNEN, Systeme, Rz. 22.

471 Anders JAAG, Dezentralisierung und Privatisierung, S.37, der bei faktischen Auswirkungen auf die Rechtsstellung Privater von «Bedarfsverwaltung mit Aussenwirkung» spricht.
} 
eine zivilrechtliche Beziehung zum Staat ein, doch seine rechtliche Stellung als Machtunterworfener dieses Gemeinwesens ist damit nicht berührt.

Diese Arbeit betrachtet die Aufgabenerfüllung - und damit auch die Erfüllungsprivatisierung - aus der Sicht der betroffenen Privaten (dazu näher hinten 3). Die Probleme und Spielräume, die bei der Einhaltung der verfassungsrechtlichen Anforderungen entstehen, zeigen sich zuvorderst in diesem Verhältnis: der staatlichen Aufgabenerfüllung gegenüber Privaten.

\section{2. «Nebenaktivitäten» - eine neue Kategorie des Bundesgerichts?}

Das Bundesgericht hat die Unterscheidung zwischen mittelbarer und unmittelbarer Aufgabenerfüllung in einem Urteil von 2013 durch eine neuartige Kategorie aufgeweicht: Tätigkeiten im «privatrechtlich bewirtschafteten Werbebereich» der Schweizerischen Radio- und Fernsehgesellschaft (SRG) wurden als grundrechtsgebundene Erfüllung staatlicher Aufgaben eingestuft (siehe auch vorne \$3/II.6.b.cc). ${ }^{472}$ Das Bundesgericht erachtete dies nicht etwa als reine Mittelbeschaffung oder Einnahmenmehrung, sondern stellte die Werbetätigkeit als «Nebenaktivität zur Finanzierung ihrer Programme» in direkten Bezug zur unmittelbaren Aufgabenerfüllung.

Die weitere Rechtsprechung wird weisen müssen, wie dieser Entscheid einzustufen ist. Sollte jede Geldbeschaffung als der direkten Aufgabenerfüllung unmittelbar angegliedert bestätigt werden, müssten die Kategorien der Bedarfs- und der wirtschaftenden Verwaltung aufgelöst werden. Weniger dogmatischen Aufräumbedarfbereitet die Lesart, dass die Kategorie der «Nebenaktivität» den Besonderheiten des audiovisuellen Medienangebots geschuldet ist. Der staatliche Informations- und Unterhaltungsauftrag, den die SRG erfüllt, ist ohne Werbung undenkbar. In dieser Staatsaufgabe ist diese konkrete Finanzierung also bereits eingeschrieben.

Welchen Weg das Bundesgericht in dieser Frage künftig einschlagen wird, ist noch nicht absehbar. Jedenfalls gibt es einen Anhaltspunkt, dass es die Bedeutung der Frage nicht vollständig erfasst. Das Bundesgericht belegt seine Einstufung der «Nebenaktivität» an der zitierten Stelle mit einem Verweis auf BGE 138 I 274 (SBB-Plakatfall, siehe vorne \$3/II.6.b.bb). Die Werbetätigkeit in jenem Fall hat aber eine andere verwaltungsrechtliche Qualität: Gestützt auf Art. 63 i.V.m 62 Abs. 1 Bst. d EBG ist die Bewirtschaftung der Plakatflächen an Bahnhofswänden als eigentliche Verwaltungsaufgabe anzusehen. So hielt das Bundesgericht denn auch unmissverständlich fest: «Insofern handelt es sich dabei um eine der unmittelbaren Erfüllung der Verwaltungsaufgabe des 
öffentlichen Verkehrs gewidmete öffentliche Sache i.e.S.»473 Der Prüfzusammenhang des öffentlichen Sachenrechts ist für diese Frage nicht weiter entscheidend - relevant ist, dass im SBB-Plakatfall die Werbetätigkeit als Leistungsverwaltung erscheint (Bereitstellung und Bewirtschaftung von Bahninfrastruktur). Im SRG-Urteil war die Werbetätigkeit gerade nicht unmittelbare Aufgabenerfüllung, sondern nur «Nebenaktivität zur Finanzierung der Programme». Das Bundesgericht scheint diesen Unterschied zu verkennen - oder ihn (teilweise) bewusst zu überdecken. Es bleibt somit offen, ob das Bundesgericht jeden Mittelerwerb durch Werbung künftig als unmittelbare Aufgabenerfüllung ansehen wird oder die dogmatische Zweideutigkeit des SRG-Urteils alleine stehen bleiben wird.

\section{Aufgabenerfüllung aus Sicht der betroffenen Privaten}

Die Verfassung unterstellt staatliche Tätigkeit besonderen Anforderungen, weil die staatliche Machtausübung auf Private rechtfertigungsbedürftig ist (siehevorne \$3/I.2).

Nicht jedes Leeren der Mailbox, jedes Teammeeting und jedes Hinkritzeln einer Post-it-Notiz sind von diesem Standpunkt aus als rechtlich relevante Erfüllungshandlung anzusehen (siehe zu dieser Grenzziehung bei den Realakten hinten III.2.a.dd). Die verfassungsrechtlichen Anforderungen an das staatliche Handeln greifen immer dann, wenn die Erfüllungshandlungen auf Privatpersonen einwirken, wenn ein Einzelner von staatlichem Handeln betroffen ist. Nicht nur der (zum Standardbeispiel gewordene) Kauf von Bleistiften als Sinnbild für die Bedarfsverwaltung fällt damit weg. Auch untergeordnete Handlungen in Vorbereitung, Ausführung und Nachbearbeitung der Eingriffs- und Leistungsverwaltung müssen ausgespart werden.

Das BUNDESAMT FÜR JUSTIZ hat die Tätigkeitsbereiche des Bundes in einem Gutachten von 1995 zu drei Gruppen systematisiert: Tätigkeiten mit Rechtswirkungen gegenüber Dritten; Tätigkeiten ohne Rechtswirkungen, aber dennoch mit Aussenwirkung sowie Tätigkeiten «aufbetrieblicher Ebene, das heisst ohne «direkte` Aussenwirkung, zur Beschaffung oder Instandhaltung der notwendigen Mittel für die Erfüllung» der anderen beiden Tätigkeitstypen. ${ }^{474}$ Das Abgrenzungskriterium der Aussenwirkung leitet die folgenden Ausführungen. Die Zweiteilung nach Rechtswirkungen oder bloss faktischen Wirkungen gehört zu den Handlungsformen (hinten III.1).

473 So ausdrücklich BGE 138 I 274 E.1.4 S. 278, auf eine frühere Fassung des EBG Bezug nehmend.

474 BJ, Leistungsverwaltung, Ziff. 4. 
Im Folgenden sollen Privatisierungen derjenigen Erfüllungsaspekte betrachtet werden, mit denen Private in direkten Kontakt kommen, namentlich:

- die Organisationsform von Verwaltungsträgern, die Privaten gegenüber als Träger staatlicher Aufgaben auftreten und staatliche Macht ausüben (II);

- die Handlungsformen, denen sich Verwaltungsträger bedienen, um Privaten Leistungen zu erbringen oder in ihre Rechte und Freiheiten einzugreifen (III);

- und schliesslich die Finanzierungsformen, mit denen Private für empfangene Leistungen und erduldete Eingriffe bezahlen (IV).

Diese Arbeit erörtert die personellen und prozessualen Erfüllungsaspekte nicht gesondert. Die personalrechtliche Stellung von Verwaltungsangestellten ist eine innerbetriebliche Angelegenheit, die unter die Bedarfsverwaltung fällt. Die prozessrechtlichen Folgen des Verwaltungshandelns beeinflussen die Durchsetzung der verfassungsrechtlichen Anforderungen. Daher hätten sie es verdient, gesondert untersucht zu werden. Diese Arbeit fokussiert aber auf den materiellen Gehalt dieser Anforderungen, weniger auf ihre Durchsetzung. Prozessuale Aspekte der Aufgabenerfüllung werden allerdings im Zusammenhang mit den Handlungsformen aufgenommen (siehe hinten III.3.i), soweit sie sich daraus ergeben.

Vor der Privatisierung der Organisationsformen (II) folgt ein Einschub zu aktuellen Verwaltungsreformen, um diese in die hier verfolgte, vom Privaten ausgehende Betrachtung der Aufgabenerfüllung einzuordnen.

\section{Einschub: Abgrenzung von Reformen der wirkungsorientierten Verwaltungsführung}

Die Begriffsklärung in § 2 hat bereits auf die Nähe der Privatisierung zum New Public Management (NPM) hingewiesen: NPM kann als Sammelbegriff für vielgestaltige Vorschläge zur Verbesserung der staatlichen Aufgabenerfüllung verstanden werden (siehe vorne \$2/II.6.e). Die Motivation hinter NPM-Bestrebungen mag sich grundlegend von Privatisierungen unterscheiden (bessere Verwaltung statt weniger Staat), 475 doch in den Erscheinungsformen ähneln sich die beiden Phänomene.

Die Weiterentwicklung des NPM im Bund heisst «Neues Führungsmodell für die Bundesverwaltung (NFB)» und wurde auf Jahresbeginn 2016 einge- 
führt.476 Diese Reform ersetzte die Zweiteilung von herkömmlichen und FLAG-Einheiten der Zentralverwaltung durch ein «einheitliches, ziel- und ergebnisorientiertes Führungsmodell ${ }^{477}$. Den Kern des NFB bilden fünf Instrumente, welche die bisherigen Führungsmittel innerhalb der Bundesverwaltung sowie die Aufsichts- und Steuerungsmöglichkeiten der Bundesverwaltung anpassen und erweitern: (1) erweiterter Legislaturfinanzplan; (2) mit integriertem Aufgaben- und Finanzplan angereicherter Voranschlag; (3) Leistungsvereinbarungen zwischen Departementsvorsteherin und Amtsdirektor; (4) Anreizinstrumente für Verwaltungseinheiten im Hinblick auf wirtschaftliches Verhalten, insbesondere umfassender Einsatz von Globalbudgets; sowie (5) weiter ausgebaute Kosten- und Leistungsrechnungen auf Stufe der Verwaltungseinheiten. ${ }^{478}$

Diesen Instrumenten liegt zugrunde, dass die Aufgaben jeder einzelnen Verwaltungseinheit (der Zentralverwaltung) in Leistungsgruppen zusammengefasst und mit messbaren Leistungszielen in den Voranschlag und in die weiteren Planungsinstrumente aufgenommen werden. ${ }^{479} \mathrm{Gemäss} \mathrm{der} \mathrm{neu}$ eingefügten Legaldefinition werden «Leistungen einer Verwaltungseinheit, mit denen gleichartige Ziele erreicht werden sollen» (Art. 3 Abs. 7 FHG), in Leistungsgruppen zusammengefasst, und die Berichterstattung über diese Gruppen hat insbesondere «Ziele, Messgrössen und Kontextinformationen» (Art. 11 Abs. 5 Bst. a FHG) zu umfassen.

Auch die in Art. 38a RVOG neu vorgesehenen Leistungsvereinbarungen, welche die Departemente mit den einzelnen Verwaltungseinheiten ${ }^{480}$ abschliessen, fassen die zu erfüllenden Aufgaben zu Leistungsgruppen zusammen und bauen auf messbaren Zielvorgaben auf: Meilensteine, Termine, Leistungs- und Wirkungsziele mit Messgrössen und Sollwerten (Art. 22a Abs.1 RVOV). Dieser vorgeschriebene Inhalt von Leistungsvereinbarungen soll sicherstellen, dass die «politische Zielsetzung und die interne Leistungspla-

476 AS 20151583; siehe auch Botschaft NFB.

477 Botschaft NFB, 768 und 774 f. eingehend zur Abkehr vom «dualen Führungsmodell». Dazu auch SÄGESSER, Verwaltungsorganisationsrecht, S. $468 \mathrm{f}$., der insbesondere auf die Ausnahmen hinweist: Parlamentsdienste, Gerichtsverwaltungen und die Eidgenössische Verwaltungskontrolle sind ausserhalb des einheitlichen Führungsmodells.

478 Botschaft NFB, 769 f. (Übersicht), 786-797 (Detailbeschrieb der Instrumente), 797-800 (Verhältnis zu bisherigen Instrumenten), 800-804 (Rolle der Bundesverwaltung).

479 Zur Funktionsweise der Leistungsgruppen als Grundlage des NFB siehe Botschaft NFB, 786 f.; zu ihrer Stellung in der Finanzberichterstattung: 792.

480 Die Einheiten der Zentralverwaltung sowie diejenigen Einheiten der dezentralen Verwaltung, die keine eigene Rechnung führen (Art. 38a Abs.1 RVOG), mit zwölf Ausnahmen (Art. 22a Abs. 2 RVOV). 
nung miteinander verbunden» werden. ${ }^{481}$ Der Bundesrat spricht vom zentralen «Steuerungsinstrument an der Schnittstelle zwischen Politik und Verwaltung», was für sich genommen wenig heisst, ${ }^{482}$ aber immerhin die Erkenntnis ausdrückt, dass der Gesetzgeber auf die Tätigkeit der Verwaltung einwirken kann und muss, um zu bestimmen und zu kontrollieren, wie die Aufgaben erfüllt werden, die er ihr übertragen hat.

Das NFB reagiert auf die grundlegende Kritik, die früheren Ausprägungen des New Public Management entgegengehalten wurde: die übertriebene Erwartung einer Trennung von parlamentarischer Steuerung und exekutiver Umsetzung. Oder wie es TSCHANNEN ausdrückt: «Der Gesetzgeber wird es sich nicht nehmen lassen, über das «Grundsätzliche` und (Wesentliche` hinaus fallweise in den «operativen` Bereich einzugreifen und Einzelheiten zu regeln, die er für politisch wichtig hält.» ${ }^{483}$ LIENHARD spricht von verworfenen NPMReformen, «die sich in der Umsetzung an der Nahtstelle zwischen Exekutive und Legislative nicht bewährt haben»484. Das NFB kehrt «von einer strikten Rollentrennung zwischen Parlament, Regierung und Verwaltung [ab und] geht demgegenüber davon aus, dass die strategische und die operative Führung eng miteinander verbunden sind.»485 Das neue Führungsmodell gibt demnach der Bundesversammlung das Recht, neben finanziellen Vorgaben im jährlichen Voranschlag «Ziele, Messgrössen und Sollwerte» für einzelne Leistungsgruppen vorzugeben (Art. 29 FHG, insb. Abs. 2 Bst. a) und damit «dort Näheres zu bestimmen, wo [sie] dies für notwendig erachtet.»486

Zwei Beispiele zur Handhabung der quantifizierbaren Zielvorgaben:

- Das Umweltschutzrecht sieht vor, «Menschen, Tiere und Pflanzen, ihre Lebensgemeinschaften und Lebensräume gegen schädliche oder lästige Einwirkungen» u.a. mittels Immissionsgrenzwerten zur Begrenzung der Lärmbelastung zu schützen. ${ }^{487}$ Der Bundesrat hat zu diesem Zweck für verschiedene Lärmquellen Immissionsgrenzwerte vorgesehen (Anhänge 3-9 LSV) und das Bundesamt für Umwelt (BAFU) führt eine nationale Übersicht über die Lärmbelastung (Art. 45a LSV). Im «Voranschlag mit integriertem Aufgaben- und Finanzplan 2021-2023 der Verwaltungsein-

\footnotetext{
481 Botschaft NFB, 833; SÄGESSER, Verwaltungsorganisationsrecht, S. 469.

482 Wie muss man sich die Steuerung einer Schnittstelle vorstellen? Meinen «Politik und Verwaltung» nicht eher Rechtsetzung und Rechtsvollzug bzw. -anwendung?

483 TSCHANNEN/ZIMMERLI/MÜLLER, Allgemeines Verwaltungsrecht, §5 Rz. 43 erstes Lemma.

484 LIENHARD, Neues Führungsmodell für die Bundesverwaltung, S. 248.

485 Botschaft NFB, $785 \mathrm{f}$.

486 LIENHARD, Neues Führungsmodell für die Bundesverwaltung, S. 259.

487 Art.1 Abs.1, Art.11, Art.13 Abs. 1 und Art.15 USG.
} 
heiten» 488 erscheint für das BAFU in der «Leistungsgruppe 2: Immissionsschutz» das Ziel «Umweltbelastung Lärm: Die messbare Belastung der Umwelt durch Lärm wird reduziert», welches mit der folgenden messbaren Zielgrösse quantifiziert wird: «Anzahl der geschützten Personen vor Strassenlärm (übrige Strassen; Ziel bis 2022: 190'900 Personen)»489.

- Die Vorgabe des Zweitwohnungsgesetzes, den Anteil von Zweitwohnungen am gesamten Wohnungsbestand für jede Gemeinde in einem Wohnungsinventar festzustellen, ${ }^{490}$ wird präzisiert im Voranschlag unter «Leistungsgruppe 1: Raum- und Verkehrsentwicklung» des Bundesamts für Raumentwicklung (ARE) unter dem Ziel «Raumplanungsrecht: Das Raumplanungsrecht wird problemadäquat weiterentwickelt und der korrekte Vollzug sichergestellt»: «Gemeinden mit einem Zweitwohnungsanteil von mehr als $20 \%$ : Termingerechte Publikation (31.3.) auf Webseite ARE (ja/nein).»491 Diese quantifizierte Zielvorgabe erlaubt es, die Aufgabenerfüllung zu messen; Stichtag und Zuständigkeit ergeben sich freilich bereits aus der Zweitwohnungsverordnung (Art. 2 Abs. 2 ZWV).

Ein weiteres Merkmal des NFB ist die Überzeugung, dass mit grösseren Handlungsspielräumen die Verwaltungseinheiten zu einem wirtschaftlicheren Umgang mit ihren Finanzen bewegt werden können. ${ }^{492}$ Globalbudgets, die Ermächtigung zur Reservenbildung und -auflösung sowie die Möglichkeit, Kredite zu überschreiten (bei entsprechender Kompensation) oder zu anderen Aufwandsposten zu verschieben, sollen zu einer «erhöhten betrieblichen Flexibilität» führen. Diese Spielräume und Flexibilitäten sind typisch für Privatisierungen der Organisationsformen (siehe hinten II.4).

Die verschiedenen Steuerungs- und Führungsmittel betreffen allesamt die Innensicht der Bundesverwaltung und verknüpfen dabei verschiedene Themen: ${ }^{493}$ zwischen Legislaturplanung und Leistungsvereinbarung; zwischen Aufgaben und Wirkungen sowie zwischen jährlicher und mehrjähriger Steuerung. Die gesetzlichen Aufgabenbestimmungen werden so weit formalisiert und katalogisiert, dass sie im interbehördlichen Verkehr in mehrjährigen Planungshorizonten verortet und in Berichten referenziert werden können. Die Aufgabenerfüllung gegenüber Privaten - etwa als Lärmbelästigte

\footnotetext{
488 Nicht amtlich publiziert, abrufbar unter www.efv.admin.ch/efv/de/home/ finanzberichterstattung/finanzberichte/budget.html.

489 Band 2B des Voranschlags, Seite 319.

490 Art. 5 Abs.1ZWG i.V.m. Art. 2 Abs.2ZWV.

491 Band 2B des Voranschlags, S. 338.

492 Vgl. Botschaft NFB, 794-796.

493 Dazu LIENHARD, Neues Führungsmodell für die Bundesverwaltung, S. 252.
} 
oder Emittenten oder als Besitzer oder Bauherren von Zweitwohnungen - ist dadurch nicht betroffen. Diese NFB-Instrumente sind somit keine Erfüllungsaspekte, die für die Zwecke dieser Arbeit von weiterer Relevanz wären.

Diese neueste Verwaltungsreform bringt also keine bahnbrechenden Neuerungen in die Erscheinungen der Erfüllungsprivatisierungen. Sie verdeutlicht aber die erneuerte Absicht von Bundesrat und Bundesversammlung, die Wirkung der Verwaltungstätigkeit zu messen und nicht auf die Übertragung von Aufgaben allein zu vertrauen. Der Bundesrat anerkennt zwar, dass "zwischen den Leistungen einer Verwaltungseinheit und den angestrebten wirtschaftlichen, gesellschaftlichen oder ökologischen Entwicklungen kaum je eine monokausale Beziehung bestehen dürfte» und deshalb die «Messung der Wirkung (Outcome) von Leistungsgruppen nur in den seltensten Fällen sinnvoll sein» wird. ${ }^{494}$ Dennoch verwarf er eine «Rückkehr zur reinen Input-Steuerung», insbesondere weil ein solcher «Rückschritt, [...] nur schwer zu begründen wäre» im Kontext von Verwaltungsreformen in vergleichbaren Ländern, in den Kantonen und im Bund selbst. ${ }^{495}$ Mit anderen Worten wird das Weitertreiben von Reformbestrebungen, die auf der Messung von Unmessbarem beruhen, damit begründet, dass solche Reformen bereits im Gang sind. 


\section{Privatisierung der Organisationsformen}

Die Organisationsformen der Verwaltung sind die rechtliche Gestalt, die der Staat sich gibt, um Privaten gegenüber aufzutreten. Dieser Abschnitt stützt sich auf die etablierte Dogmatik der Verwaltungsorganisation, ${ }^{496}$ und verzichtet wenn immer möglich auf die Schaffung neuer Kategorien.

Die organisatorischen Erfüllungsaspekte zu privatisieren, bedeutet, die staatliche Erfüllungsarbeit in ein Gewand zu hüllen, das privaten Organisationen ähnelt. Quasiprivate Organisationsformen können beim Empfänger einer staatlichen Leistung bzw. der Erdulderin eines staatlichen Eingriffs das Bild erwecken, es mit einem privaten Unternehmen zu tun zu haben.

Zunächst ist der Begriff der Organisationsformen zu umreissen (1), bevor die bekannten Formen aufgelistet werden (3) - ohne zu viel auf Ordnungsmodelle zu setzen (2). Danach lassen sich die Kennzeichen derjenigen Organisationsformen diskutieren, die privaten Formen ähneln (4) - dies ergibt ein Bild der Erfüllungsprivatisierung in organisatorischer Hinsicht.

\section{Begriff und Bedeutung der Organisationsformen}

Zunächst muss die Flughöhe dieser organisatorischen Betrachtungen bestimmt werden. Vom funktionellen Staatsverständnis ausgehend interessieren zuvorderst die Verwaltungsträger und nur nachgeordnet die Verwaltungseinheiten und Verwaltungsfunktionäre. 497

«Ein Akteur heisst Verwaltungsträger, sofern und soweit er in eigenem Namen Verwaltungsaufgaben besorgt und in dieser Eigenschaft Träger von Rechten und Pflichten ist [...]. Damit ist der Verwaltungsträger begriffsnotwendig Rechtssubjekt, in der Regel juristische Person.»498 Verwaltungsträger sind diejenigen Subjekte, denen die «Befugnisse und Obliegenheiten» $499 \mathrm{im}$ Zusammenhang mit der Erfüllung staatlicher Aufgaben zugerechnet werden können. Verwaltungsträger sind mit anderen Worten diejenigen organisato-

496 Vgl. HÄFELIN/MÜLLER/UHLMANN, Allgemeines Verwaltungsrecht, §22-24; TANQUEREL, Droit administratif, § 2; TSCHANNEN/ZIMMERLI/MÜLLER, Allgemeines Verwaltungsrecht, §5-10.

497 Zum Zusammenspiel dieser drei Begriffe TSCHANNEN, Systeme, Rz. 46-52; TSCHANNEN/ ZIMMERLI/MÜLLER, Allgemeines Verwaltungsrecht, §5 Rz. 4-10. In der französischsprachigen Schweizer Lehre sind diese Begriffsstufen weniger gebräuchlich; TANQUEREL, Droit administratif, Rz. 78 f., verwendet «entités administratives» als Überbegriff, übersetzt «Verwaltungsträger» mit «titulaires du pouvoir administratif», verwendet aber «autorité administrative» an dessen Stelle.

498 TSCHANNEN, Systeme, Rz. 46, ohne die Hervorhebungen im Original.

499 TSCHANNEN/ZIMMERLI/MÜLLER, Allgemeines Verwaltungsrecht, §5 Rz. 4. 
rischen Einheiten, die Privaten gegenüber als Träger eigener staatlicher Aufgaben auftreten. Sie tragen die Verantwortung für die Erfüllung der ihnen übertragenen Aufgaben.

Verwaltungseinheiten hingegen sind interne Gliederungen, Teile eines Verwaltungsträgers. ${ }^{500}$ Die Einheiten erfüllen die Aufgaben des Verwaltungsträgers, in den sie eingegliedert sind, nach Massgabe ihrer sachlichen und örtlichen Zuständigkeit. Sie sind nicht rechtsfähig; ihr Handeln verpflichtet und berechtigt nicht sie selbst, sondern immer nur den übergeordneten Verwaltungsträger.

Verwaltungseinheiten sind konstruierte organisatorische Grössen; Verwaltungsaufgaben effektiv verrichten können nur natürliche Personen. Diese Menschen werden als Verwaltungsfunktionäre bezeichnet. ${ }^{01}$ Verwaltungsfunktionär ist jede Person, die «als Verwaltung» Aufgaben wahrnimmt - das heisstauch beliehene Private, denen eine staatliche Aufgabe übertragen ist. 502

Private nehmen das Staatshandeln zwar in Gestalt dieser Einzelpersonen wahr, jegliche Rechtsverhältnisse zwischen Staat und Bürger entstehen jedoch gegenüber dem Verwaltungsträger, weshalb diese Arbeit auf diese Ebene fokussiert. Die rechtliche Relevanz der Verwaltungseinheiten liegt vor allem im Verwaltungsorganisationsrecht, diejenige der Verwaltungsfunktionäre bei der strafrechtlichen und disziplinarischen Verantwortlichkeit.503

\section{Beschränkte Nützlichkeit von Organisationsmodellen}

Verwaltungsträger können in verschiedene rechtliche Formen gekleidet werden (dazu sogleich 3). Für die Zwecke dieses Paragrafen ist es nicht notwendig, ein Organigramm der Verwaltung zu zeichnen. Es reicht aus, die verschiedenen Organisationsformen und ihre jeweilige rechtliche Bedeutung zu kennen.

An Versuchen, dieSumme von Verwaltungsträgern zu ordnen, mangelt es nicht. 504 Im neuen Jahrtausend führte der Bundesrat bereits zwei Ordnungsmodelle ein: das Vier-Kreise-Modell anlässlich der NPM-inspirierten Einführung der FLAG-Verwaltungseinheiten ${ }^{505}$ und später die Idealtypologie nach

500 TSCHANNEN, Systeme, Rz.48; TSCHANNEN/ZIMMERLI/MÜLLER, Allgemeines Verwaltungsrecht, §5 Rz. 6 f.; auch zum Folgenden.

501 TSCHANNEN/ZIMMERLI/MÜLLER, Allgemeines Verwaltungsrecht, §5 Rz. 9 f., mit den alternativen Begriffen Organwalter oder Amtsinhaber.

502 TSCHANNEN/ZIMMERLI/MÜLLER, Allgemeines Verwaltungsrecht, §5 Rz.10a.

503 TSCHANNEN, Systeme, Rz. 48 a.E. und 52.

504 Ausführlich dazu TSCHANNEN, Organigramm, passim; VOGEL, Einheit der Verwaltung, \$4.

505 Bericht FLAG, 3538 f.; dazu auch LIENHARD, Grundlagen NPM, S. 71-73. 
Aufgabenart im Corporate-Governance-Bericht ${ }^{506}$. Beide Modelle entfalteten nicht die erwünschte normative Wirkung: Der Gesetzgeber wählt weiterhin die ihm im Einzelfall passendste Form für ausgelagerte Aufgabenträger. ${ }^{507}$ Das geltende Verwaltungsorganisationsrecht teilt die Bundesverwaltung in drei Teile (mit uneinheitlicher Terminologie): zentrale, dezentrale und ausserhalb/nicht angehörig/extern. ${ }^{508}$ Anhand welcher Kriterien diese Trennlinien zu ziehen sind, birgt einige Schwierigkeiten. Die Grenze zwischen Zentralverwaltung und dezentraler Verwaltung lässt sich am verlässlichsten anhand der Rechtspersönlichkeit ziehen. ${ }^{509}$ Nur rechtsfähige Gebilde können Aufgabenträger sein. Die RVOV grenzt dezentrale von externen Aufgabenträgern entlang weniger deutlichen Kriterien ab: einerseits der Aufgabentypologie des Corporate-Governance-Berichts und anderseits des Empfangs von Finanzhilfen oder Abgeltungen nach Subventionsgesetz. 510

Die Abwesenheit eines verbindlichen Organigramms, das alle Träger staatlicher Aufgaben abbildet, und die Unschärfe der Zuordnungen zu den verschiedenen Verwaltungsbereichen sind nicht weiter besorgniserregend. Organisatorische Einteilungen müssen über die eintretenden Rechtsfolgen Auskunft geben. Zu diesen Rechtsfolgen zählen etwa: Leitungs- und Aufsichtsbefugnisse, Rechtsverhältnisse zu Dritten, Zuständigkeitsbereich, Unterstellung unter Finanzhaushaltsrecht, Partei- und Prozessfähigkeit, haftungsrechtliche Stellung. 511

Mit Blick auf die verfassungsrechtlichen Anforderungen ist es nicht zwingend, die handelnde Staatsstelle auf einem Organigramm verordnen zu können; es reicht zu wissen, wofür sie zuständig ist, welche Rechtsverhältnisse sie zu begründen vermag und auf welchem Verfahrensweg gegen ihr Handeln zu rekurrieren ist. Diese rechtlichen Konsequenzen der gewählten Organisationsform ergeben sich durch die jeweilige spezialgesetzliche Grundlage: Mit Ausnahme der Einheiten der Zentralverwaltung wird jeder Verwaltungsträger mit eigenem Errichtungsakt gegründet. Durch die Wahl der Organisations-

506 Corporate-Governance-Bericht, 8234 und 8258-8265; ausdrücklich als Weiterentwicklung des Vier-Kreise-Modells konzipiert (S. $8249 \mathrm{f}$.).

507 Die Postreform von 2010 als Beispiel anführend TSCHANNEN, Organigramm, S. 520 f. Kritisch auch VOGEL, Einheit der Verwaltung, S. 75f. (ausdrücklich auf Vier-Kreise-Modell bezogen, aber generell skeptisch gegenüber «einer strikten Kategorisierung und [...] der einseitigen betriebswirtschaftlichen Betrachtung»).

508 Art. 2 Abs. 3 und 4 RVOG, Art. 6 RVOV.

509 So TSCHANNEN, Organigramm, S. 528-530.

510 Art. 6 Abs. 2 und 3 RVOV. Kritisch zur Vermischung von Kriterien TSCHANNEN, Organigramm, S. 522.

511 Zusammengestellt aus TSCHANNEN, Organigramm, S. 525 f.; und vOGEL, Einheit der Verwaltung, S.71. 
formen sind zwar «bestimmte Rechtserwartungen» begründet, die der Gesetzgeber aber «jederzeit relativieren und durchkreuzen» kann. ${ }^{512}$ So wird etwa der Autonomiegrad dezentraler Verwaltungsträger im Einzelfall gesetzlich bestimmt. ${ }^{513}$ Organigramme können dem Gesetzgeber als Werkzeugkasten dienen, das metaphorische Haus zimmert dieser aber immer nach eigenem Bauplan.514

\section{Einzelne Organisationsformen}

Organisationsformen sind laut VOGEL «rechtliche Typisierungen des Aussenund Innenlebens von Organisationen».515 Jede gefestigte Rechtsform verfügt über für sie typische Eigenschaften, womit aber nicht gesagt ist, dass sich die verschiedenen Formen in jedem Punkt unterscheiden. Die verschiedenen Verwaltungsträger ausserhalb der Zentralverwaltung werden also nicht zu einem Modell geordnet, sie stehen begrifflich nebeneinander. ${ }^{516}$

Im Folgenden werden diejenigen Organisationsformen von Verwaltungsträgern aufgelistet, die über eine gefestigte Bezeichnung verfügen. Die Aufzählung entspricht der aktuellen Praxis der Verwaltungsorganisation auf Bundesebene - zu beachten ist allerdings, dass das öffentliche Recht keinen Numerus clausus der Organisationsformen kennt, im Unterschied zum Privatrecht. ${ }^{517}$ Der Gesetzgeber kann jederzeit neue Formen schaffen.

\section{a. Zentralverwaltung}

Zentralverwaltung bedeutet «die Zusammenfassung von Verwaltungseinheiten eines Verwaltungsträgers zu einem geschlossenen, hierarchisch durchstrukturierten Gefüge» ${ }^{518}$. Die Einheiten der zentralen Bundesverwaltung heissen Ämter und können zu Gruppen zusammengefasst werden. ${ }^{519}$ Prägendes und kennzeichnendes Merkmal der Zentralverwaltung ist die Hierarchie. Sie bedeutet viererlei:520 (1) Jede Verwaltungseinheit ist einer anderen

512 TSCHANNEN, Organigramm, S. 525. Ähnlich auch TSCHANNEN, Systeme, Rz. 56.

513 TANQUEREL, Droit administratif, Rz.127.

514 Ähnlich auch TSCHANNEN, Organigramm, S. 525.

515 VOGEL, Einheit der Verwaltung, S. 71.

516 Die beschränkten «Möglichkeiten für eine dogmatische Typisierung» betont auch VOGEL, Einheit der Verwaltung, S.72.

517 VOGEL, Einheit der Verwaltung, S. $76 \mathrm{f}$.

518 TSCHANNEN, Systeme, Rz. 57.

519 Art. 2 Abs. 2 RVOG. Dazu auch TSCHANNEN/ZIMMERLI/MÜLLER, Allgemeines Verwaltungsrecht, \$6 Rz. 2; ferner VOGEL, Einheit der Verwaltung, S. 225 f.

520 Eigene Zusammenstellung. Zum Ganzen ausführlich HÄFELIN/MÜLLER/UHLMANN, Allgemeines Verwaltungsrecht, Rz.1569-1577, 1594; TSCHANNEN/ZIMMERLI/MÜLLER, Allgemeines Verwaltungsrecht, §6 Rz.4-7; VoGEL, Einheit der Verwaltung, S. 137 f., 226-229. 
untergeordnet; (2) Aufgabenverantwortung und Entscheidungsmacht münden an der Spitze der Hierarchie, beim Bundesrat; (3) übergeordnete Einheiten haben gegenüber den untergeordneten besondere Aufsichts- und Eintrittsrechte; (4) Einheiten sind in ihrer gegenseitigen Kommunikation auf vorgegebene Dienstwege verpflichtet.

Einheiten der Zentralverwaltung sind etwa das Schweizerische Bundesarchiv und das Bundesamt für Umwelt. ${ }^{221}$

\section{b. Weisungsfreie Einheiten der Bundesverwaltung}

Eine Gruppe von Verwaltungseinheiten nehmen eine ungewöhnliche Stellung ein: Sie sind nicht rechtsfähig und deshalb keine eigenen Verwaltungsträger; sie erfüllen Aufgaben der Zentralverwaltung (oder eines anderen Verwaltungsträgers), unterstehen aber nicht derselben Hierarchie. ${ }^{522}$ Insbesondere entfällt die Dienstaufsicht, was sie von Weisungen übergeordneter Behörden befreit. ${ }^{23}$ Erwähnenswerte Organisationsformen, die als weisungsfreie Einheiten der Bundesverwaltung angesehen werden, sind öffentliche Beauftragte (etwa der Eidgenössische Datenschutz- und Öffentlichkeitsbeauftragte ${ }^{524}$ ), Kontrollstellen (im Bund nur die Eidgenössische Finanzkontrolle525) und Behördenkommissionen ${ }^{526}$ (z.B. die Elektrizitätskommission $\left.{ }^{527}\right) .528$

\section{c. Öffentlichrechtliche Anstalten}

Öffentlichrechtliche Anstalten sind die klassische Organisationsform für die Leistungsverwaltung. So sind etwa die Eidgenössischen Technischen Hochschulen (Bildungsleistungen) und die Schweizerische Unfallversicherungsanstalt (Versicherungsleistungen) als Anstalten ausgestaltet. ${ }^{529}$ In diesen Fällen werden Anstalten per Spezialgesetz errichtet, um Benutzern den Zugang zu einer Einrichtung bereitzustellen. Das Bundesrecht setzt Anstalten jedoch

\footnotetext{
521 Siehe Ziff. B.II.1.4 und B.VII.1.7 im Anhang 1 zur RVOV.

522 Siehe zum Ganzen HÄFELIN/MÜLLER/UHLMANN, Allgemeines Verwaltungsrecht, Rz.1626-1632; TSCHANNEN/ZIMMERLI/MÜLLER, Allgemeines Verwaltungsrecht, \$6 Rz. 8-12; VOGEL, Einheit der Verwaltung, $\$ 8$.

523 So ausdrücklich Art. 7a Abs. 2 RVOV.

524 Art.26 Abs.3 DSG.

525 Art.1FKG.

526 Die andere Form der ausserparlamentarischen Kommission, die Verwaltungskommission, ist hier nicht relevant, sie übt lediglich beratende und vorbereitende Funktionen aus (Art. 8a RVOV).

527 Art.21 StromVG.

528 TSCHANNEN, Organigramm, S. 530 f. Zu diesen drei Formen ausführlich voGEL, Einheit der Verwaltung, §9-11; und ferner TSCHANNEN/ZIMMERLI/MÜLLER, Allgemeines Verwaltungsrecht, \$6 Rz. 8-12.

529 Art.1ETH-Gesetz; Art.61 UVG.
} 
für allerlei Zwecke ein, so auch für Zulassungs- und Aufsichtsaufgaben;530 wie etwa das Schweizerische Heilmittelinstitut Swissmedic und die Eidgenössische Finanzmarktaufsicht FINMA ${ }^{531}$. Die öffentlichrechtliche Anstalt lässt sich daher «nicht nach dem Gegenstand ihrer Tätigkeit definieren.»532 Begriffsbildend ist vielmehr ihre Rechtsfähigkeit. Nicht rechtsfähige «Anstalten» sind bloss betriebliche Einheiten eines anderen Verwaltungsträgers. 533

\section{d. Öffentlichrechtliche Körperschaften}

Öffentlichrechtliche Körperschaften sind rechtsfähige Verbindungen von Mitgliedern, die auf dem Wege der Selbstverwaltung Verwaltungsaufgaben erfüllen.534 Selbstverwaltung bedeutet, dass die Körperschaftsmitglieder selbst über die Erfüllung der ihnen übertragenen Aufgaben bestimmen. Nach dem Grund zur Mitgliedschaft werden drei Arten unterschieden: Gebietskörperschaft (Mitgliedschaft hängt an Wohnsitz), Personalkörperschaft (persönliche Eigenschaft, wie etwa bestimmte wirtschaftliche Tätigkeit) und Realkörperschaften (Eigentum einer Sache).

Im Bund ist beispielsweise Schweiz Tourismus als öffentlichrechtliche Körperschaft verfasst. ${ }^{535}$

\section{e. Öffentlichrechtliche Genossenschaften}

Eine sonderliche und seltene Organisationsform sind die öffentlichrechtlichen Genossenschaften. Sie sind privatrechtlich als Einrichtungen des öffentlichen Rechts vorgesehen (Art. 829 OR). ${ }^{336}$ Das einzige Beispiel ist gegenwärtig die Schweizerische Gesellschaft für Hotelkredit, 537 die ihren Aufgaben nach wohl auch als Personalkörperschaft ausgestaltet werden könnte. ${ }^{538}$

530 Vgl. TSCHANNEN/ZIMMERLI/MÜLLER, Allgemeines Verwaltungsrecht, §7 Rz. 6.

531 Art.68 HMG; Art. 4 FINMAG.

532 VOGEL, Einheit der Verwaltung, S.282.

533 TSCHANNEN/ZIMMERLI/MÜLLER, Allgemeines Verwaltungsrecht, §7 Rz. 10; VOGEL, Einheit der Verwaltung, S. 281 f. Etwas undeutlich zur Abgrenzung HëFELIN/MÜLLER/ UHLMANN, Allgemeines Verwaltungsrecht, Rz.1667.

534 Zum Folgenden HÄFELIN/MÜLLER/UHLMANN, Allgemeines Verwaltungsrecht, Rz. 16331658; TSCHANNEN/ZIMMERLI/MÜLLER, Allgemeines Verwaltungsrecht, §8 Rz.10; vOGEL, Einheit der Verwaltung, $\$ 13$.

535 Art.1 Abs. 1 des Bundesgesetzes über Schweiz Tourismus vom 21. Dezember 1955 (SR 935.21).

536 HÄFELIN/MÜLLER/UHLMANN, Allgemeines Verwaltungsrecht, Rz.1705 f.

537 Art. 2 des Bundesgesetzes über die Förderung der Beherbergungswirtschaft vom 20. Juni 2003 (SR 935.12).

538 Vgl. voGEL, Einheit der Verwaltung, S. 323, der Genossenschaften generell den Körperschaften beiordnet. 


\section{f. Öffentlichrechtliche Stiftungen}

Öffentlichrechtliche Stiftungen erfüllen Verwaltungsaufgaben, indem sie finanzielle Beiträge ausrichten. ${ }^{539}$ Sie sind Vermögensansammlungen, die für einen bestimmten Zweck errichtet werden («abgesondertes Zweckvermögen» ${ }^{400}$ ). Stiftungen haben keine Benutzer oder Mitglieder, sondern «Destinatäre» ${ }^{541}$. Die Trägerschaft des Schweizerischen Nationalparks ist beispielsweise als öffentlichrechtliche Stiftung ausgestaltet. ${ }^{542}$

\section{g. Öffentlichrechtliche Aktiengesellschaften}

Sollen staatliche Aufgabenträger am privatwirtschaftlichen Markt teilnehmen, kann sich der Staat des Gesellschaftsrechts bedienen (sogleich $h$ ), oder er kann sich lediglich inspirieren lassen von den privatrechtlichen Organisationsformen und per Gesetz eigene Formen schaffen. «Die Form der spezialgesetzlichen Aktiengesellschaft ermöglicht es dem Bund, die Grundordnung des zivilistischen Aktienrechts den besonderen Bedürfnissen eines öffentlichen Unternehmens anzupassen.»543

In der Verwaltungspraxis des Bundes werden Träger von Grundversorgungsaufgaben (Service public) als spezialgesetzliche Aktiengesellschaften ausgestaltet. ${ }^{544}$ Damit drückt der Bund aus, dass diese Organisationsform zugleich staatliche Aufgaben wahrnimmt wie auch im privatwirtschaftlichen Wettbewerb tätig sein soll. Diese «Sowohl-als-auch»-Motivation führte der Bundesrat etwa bei der jüngsten Postreform ${ }^{545}$ an: Die Form der Aktiengesellschaft soll der Post Kapitalmarkt- und Kooperationsfähigkeit vermitteln, das heisst die «Fähigkeit[,] eigene Finanzmittel zu beschaffen oder Fremdmittel aufzunehmen [und] Allianzen einzugehen.»546 Gleichzeitig können Zweckbestimmung und Eignerverhältnisse «auf die spezifischen Bedürfnisse des Bundes und dessen öffentliche Interessen an den

539 Zum Folgenden HÄFELIN/MÜLLER/UHLMANN, Allgemeines Verwaltungsrecht, Rz. 16851694; TSCHANNEN/ZIMMERLI/MÜLLER, Allgemeines Verwaltungsrecht, \$9; VOGEL, Einheit der Verwaltung, S. 297-306.

540 TSCHANNEN/ZIMMERLI/MÜLLER, Allgemeines Verwaltungsrecht, §9 Rz. 2.

541 TSCHANNEN, Systeme, Rz.64 a.E.

542 Art. 2 des Bundesgesetzes über den Schweizerischen Nationalpark im Kanton Graubünden vom 19. Dezember 1980 (Nationalparkgesetz; SR 454).

543 TSCHANNEN/ZIMMERLI/MÜLLER, Allgemeines Verwaltungsrecht, §10 Rz.11, zum Ganzen Rz. 9-11; HÄFELIN/MÜLLER/UHLMANN, Allgemeines Verwaltungsrecht, Rz.1695-1701; VOGEL, Einheit der Verwaltung, S. 324.

544 So die Post, SBB und Swisscom; siehe jeweils Art. 2 Abs.1 POG, SBBG und TUG. Dazu auch TSCHANNEN/ZIMMERLI/MÜLLER, Allgemeines Verwaltungsrecht, §10 Rz.10.

545 Art. 2 Abs. 1 POG zur rechtlichen Stellung der Post.

546 Botschaft POG, 5278. 
Aufgaben der Schweizerischen Post ausgerichtet werden.»547 Für den Bundesrat waren somit besonders die «Informations- und Einflussrechte» ausschlaggebend für sein Abweichen von einer «rein privatrechtlichen Konzeption».548

\section{h. Privatrechtliche Gesellschaften}

Schliesslich können privatrechtliche Gesellschaften Verwaltungsaufgaben wahrnehmen. ${ }^{549}$ Der Staat kann dabei auf vorbestehende juristische Personen zurückgreifen, solche gründen oder sich an solchen beteiligen. ${ }^{550}$ Alle Formen des Privatrechts stehen dem Gemeinwesen zur Verfügung, wobei meist die Aktiengesellschaft, seltener auch die Genossenschaft zum Zug kommt. 551 Rechtsgestalt und innere Organisation der Verwaltungsträger richten sich ausschliesslich nach dem zivilen Gesellschaftsrecht, mit Ausnahme der ausdrücklichen Möglichkeit des Gemeinwesens, Vertreter in die Gesellschaftsorgane zu entsenden (Art. 762 Abs. 1 OR für die Aktiengesellschaft und Art. 926 Abs. 1 für die Genossenschaft).

In der Lehre werden die privatrechtlichen Verwaltungsträger gelegentlich weiter unterschieden nach dem Grad der finanziellen Beteiligung des Gemeinwesen, sprich, ob das Gesellschaftskapital aus Steuermitteln oder aus privater Hand bestritten wird: «Echte Private» kommen ohne staatliche Beteiligung aus, in gemischtwirtschaftlichen Unternehmen stehen privates und öffentliches Kapital Seite an Seite, während öffentliche Unternehmen gänzlich im Besitz der öffentlichen Hand sind. ${ }^{552}$ Rein private Aufgabenträger ohne staatliche Beteiligung sind etwa die ORS Service AG oder die Securitas AG, die im Auftrag des Staatssekretariats für Migration Asylsuchende betreuen bzw. die Sicherheit in Asylzentren gewährleisten. ${ }^{553}$ Ein öffentliches Unternehmen im vollumfänglichen Bundesbesitz ist dagegen etwa die RUAG Holding AG, eine Beteiligungsgesellschaft, welche die Rüstungsunter-

\footnotetext{
547 Botschaft POG, $\mathbf{5 2 7 9 .}$

548 Botschaft POG, 5278f. Ähnlich, aber etwas weniger deutlich schon bezüglich Swisscom, Botschaft Umwandlung PTT, 1316, 1333 (damals noch «Telekommunikationsunternehmung»); bei der Bahnreform von 1998 siehe Botschaft Bahnreform, 944.

549 Allgemein dazu HÄFELIN/MÜLLER/UHLMANN, Allgemeines Verwaltungsrecht, Rz.1707-1712; TSCHANNEN/ZIMMERLI/MÜLLER, Allgemeines Verwaltungsrecht, §10 Rz.3-8.

550 TSCHANNEN, Systeme, Rz. $69 \mathrm{f}$.

551 HÄFELIN/MÜLLER/UHLMANN, Allgemeines Verwaltungsrecht, Rz.1707; TSCHANNEN/ ZIMMERLI/MÜLLER, Allgemeines Verwaltungsrecht, §10 Rz.3.

552 BAUMANN, Wettbewerbsverzerrungen, Rz.79, 82; TSCHANNEN, Systeme, Rz. $69 \mathrm{f}$.

553 Beispielsweise SEM, Temporäre Unterkunft für Asylsuchende in Boltigen, Medienmitteilung vom 28. Oktober 2016.
} 
nehmen des Bundes umfasst. ${ }^{554}$ Ein gemischtwirtschaftliches Unternehmen, an dem der Bund nur eine Mehrheitsbeteiligung hält, ist dagegen etwa die Swisscom AG. 555

\section{Kennzeichen der Erfüllungsprivatisierung in organisatorischer Hinsicht}

Erfüllungsprivatisierung bedeutet, staatliches Handeln wie privates erscheinen zu lassen. Erfüllungsprivatisierung in organisatorischer Hinsicht heisst also, dass Verwaltungsträger so ausgestaltet werden, dass sie wie private Akteure erscheinen. Was zeichnet die privatisierten Organisationsformen aus? Während die Zentralverwaltung «für Bürger und Politik als Staat erkennbar [ist, ...] durchtrennt jede «Ausgliederung〉 ein Stück weit das rechtliche Band zwischen dem Gemeinwesen und Teilen seiner Verwaltung, schwächt unweigerlich die Führungskraft der Regierung und mindert im gleichen Zug ihre Verantwortlichkeit gegenüber dem Parlament.» 556 Woran dies im Einzelnen festzumachen ist, wird folgend herausgearbeitet.

\section{a. Vorab:Die privatisierten Organisationsformen}

Um präzise Aussagen über die Erfüllungsprivatisierung zu treffen, muss der Prüfgegenstand möglichst genau feststehen. Es muss also bezeichnet werden, welche Organisations-, Handlungs- und Finanzierungsformen als privatisiert im Sinne dieser Arbeit gelten (siehe auch hinten III.3.a und hinten IV.4.a).

Es ist unmöglich, eine eindeutige Grenze zu ziehen zwischen staatlichen und privatisierten Formen. Nur bezüglich der Aufgaben gilt die binäre Einteilung zweier abgetrennter Sphären: Was der Erfüllung staatlicher Aufgaben dient, ist staatliche Tätigkeit, alles andere ist privat. Die Modalitäten der Aufgabenerfüllung liegen auf einem Spektrum (siehevorne \$2/I.3). Die Staatlichkeit hängt gerade nicht an der Art und Weise der Aufgabenerfüllung, sondern nur an der zu erfüllenden Aufgabe.

Dennoch muss diese Arbeit eine Grenze ziehen, damit Gewissheit darüber besteht, was mit Erfüllungsprivatisierung gemeint ist. Nicht nur neuartige Organisationsformen und privatrechtliche Gesellschaften werden im Folgenden als privatisiert behandelt: Alle Aufgabenträger ausserhalb der Zentralverwaltung sind im Sinne dieser Arbeit privatisierte Organisationsformen.

\footnotetext{
554 Art. 3 Abs.1BGRB. Vgl. zu den Beteiligungsverhältnissen Bericht Bundesfinanzen 2016, 5271; Corporate-Governance-Bericht, 8300.

555 Art. 3 Abs. 1 TUG. Vgl. zu den Beteiligungsverhältnissen Strategische Ziele Swisscom; Corporate-Governance-Bericht, 8300.

556 TSCHANNEN, Organigramm, S. 528.
} 
Die folgenden Kennzeichen gelten nicht für alle diese Formen gleichermassen, bei einigen sind sie ausgeprägter als bei anderen, doch wenn pauschal von privatisierten Aufgabenträgern oder Organisationsformen gesprochen wird, erfasst dies alle Organisationsformen von soeben 3.c-h. Denn diese Formen gleichen privaten Organisationen.

\section{b. Grundlegend: Einzelfallregelung der Rechtsfolgen}

Das Verwaltungsorganisationsrecht (namentlich RVOG und RVOV) bestimmt die Rechtsfolgen der jeweiligen Organisationsform nur für die Zentralverwaltung einheitlich und abschliessend. Am deutlichsten wird dies in den Bestimmungen über die Aufsicht:

- Art. 8 Abs. 3 und 4 RVOG: «[Der Bundesrat] übt die ständige und systematische Aufsicht über die Bundesverwaltung aus. Er beaufsichtigt nach Massgabe der besonderen Bestimmungen die dezentralisierten Verwaltungseinheiten und die Träger von Verwaltungsaufgaben des Bundes, die nicht der Bundesverwaltung angehören.»

- Art. 24 Abs. 2 und 3 RVOV: «Die Aufsicht über die zentrale Bundesverwaltung ist umfassend.» Dagegen wird die Aufsicht über alle anderen Verwaltungsträger «in Gegenstand, Umfang und Grundsätzen durch die Spezialgesetzgebung geregelt und richtet sich nach dem jeweiligen Grad der Autonomie» 557 .

Gemäss Art.38 RVOG bestehen nur innerhalb der Departemente «uneingeschränkte Weisungs-, Kontroll- und Selbsteintrittsrechte». Ausserdem sieht Art. 28 RVOV vor, dass nur für die Departemente (und die Bundeskanzlei) Organisationsverordnungen erlassen werden, womit ein geordneter Rahmen für die abschliessende Regelung verschiedener Rechtsfragen ausserhalb der Zentralverwaltung entfällt. Diese Fragen müssen bei allen anderen Verwaltungsträgern einzeln geregelt werden. Die Grundlage dazu ist in den jeweiligen Errichtungsakten zu finden: Im Spezialgesetz bzw. in den Statuten. Soweit Verwaltungsträger gesetzlich errichtet werden, sind die Details der organisatorischen Bindung zum Trägergemeinwesen einseh- und nachvollziehbar, und sie unterliegen demokratischer Kontrolle. Überträgt hingegen das Gemeinwesen staatliche Aufgaben an vorbestehende Privatrechtssubjekte, entfällt diese Transparenz. Die Leistungsaufträge sind nicht immer einsehbar, und es gibt keine Gewissheit darüber, dass alle organisatorischen Fragen geregelt sind.

Für privatisierte Organisationsformen ist typisch, dass sie einander ungleich ausgestaltet sind und keine Übersicht über ihre Merkmale besteht. Die

557 Dazu auch TANQUEREL, Droit administratif, Rz.132. 
folgenden zwei Kennzeichen sind jedenfalls typische Abweichungen von den klassisch staatlichen Organisationsformen.

\section{c. Eingeschränkte Aufsichtsmittel}

Die Verwaltungseinheiten der Zentralverwaltung unterstehen einer Dienstaufsicht, was der übergeordneten Behörden insbesondere zwei Machtmittel in die Hand gibt: das Recht, verbindliche Weisungen (Dienstbefehle) zu erteilen, sowie das Recht, in die Aufgabenerfüllung einzugreifen und sogar Entscheide im Zuständigkeitsbereich der untergeordneten Behörde aufzuheben. ${ }^{558}$ Mit der Dienstaufsicht kann zusätzlich die Angemessenheit einer Verwaltungshandlung hinterfragt werden. Es reicht, dass die übergeordnete Behörde eine andere Entscheidung für opportuner oder zweckmässiger hält; 559 die Verletzung einer objektiven Pflicht ist nicht verlangt. Eine weitgehende Form des Eingriffs in die Aufgabenerfüllung ist der sogenannte Selbsteintritt: Das Recht der Aufsichtsbehörde, eine Angelegenheit zur eigenen Erfüllung an sich zu ziehen. ${ }^{560}$

Demgegenüber unterstehen Verwaltungsträger ausserhalb der Zentralverwaltung nur einer Verbandsaufsicht. Diese ist gegenüber der Dienstaufsicht dreifach reduziert:561

- Sie richtet sich nur an den Verwaltungsträger insgesamt und greift nicht auf einzelne Einheiten oder Funktionäre durch;

— inhaltliche Weisungen für Materien im Zuständigkeitsbereich des Verwaltungsträgers sind nicht vorgesehen;

- und ihr Massstab sind rechtliche Vorgaben, nicht Fragen der Angemessenheit.

Was die Aufsicht betrifft, verläuft die grundlegende Trennlinie zwischen zentraler und dezentraler Verwaltung: Dort endet die Dienstaufsicht. Privatrechtliche Aufgabenträger heben sich zusätzlich gegen öffentlichrechtliche ab, indem ihr Aufsichtsregime nicht in Gesetzen publiziert, sondern in Aufträgen, Vereinbarungen und Strategien festgeschrieben ist.

558 HÄFELIN/MÜLLER/UHLMANN, Allgemeines Verwaltungsrecht, Rz.1575f. (dort Weisungsrecht und Dienstaufsicht getrennt); TSCHANNEN/ZIMMERLI/MÜLLER, Allgemeines Verwaltungsrecht, §5 Rz.32.

559 MÜLLER, Basler Kommentar, Art. 187, Rz.9.

560 Art. 47 Abs. 4 RVOG. SÄGESSER, Kommentar RVOG, Art. 47 Rz. 36; TSCHANNEN/ZIMMERLI/ MÜLLER, Allgemeines Verwaltungsrecht, §6 Rz.7. Ferner auch HÄFELIN/MÜLLER/ UHLMANN, Allgemeines Verwaltungsrecht, Rz.1576; TSCHANNEN, Systeme, Rz.60.

561 TSCHANNEN/ZIMMERLI/MÜLLER, Allgemeines Verwaltungsrecht, §5 Rz. 33 (dort auch Organisationsaufsicht genannt); TSCHANNEN, Systeme, Rz.66. 
Dass die Aufsicht je nach Organisationsform unterschiedlich intensiv ist, steht dem Verfassungswortlaut von Art. 187 Abs. 1 Bst. a BV entgegen. Diese Bestimmung trägt dem Bundesrat auf, «die Bundesverwaltung und die anderen Träger von Aufgaben des Bundes» zu beaufsichtigen - unterschiedslos. ${ }^{562}$ Die eingeschränkte Verbandsaufsicht gegenüber privatisierten Aufgabenträgern muss daher kompensiert werden durch spezifische Aufsichtsmittel, die dem Gemeinwesen angemessene Steuerungsmöglichkeiten gewähren. ${ }^{563}$ Die Lehre hält eine Reihe von Instrumenten für geeignet, den verfassungsrechtlichen Auftrag einer genügenden Aufsicht zu erfüllen: ${ }^{564}$ Die Genehmigung von Statuten und Reglementen; die Genehmigung von Einzelakten und Sachentscheiden; besondere Einsichts- und Auskunftsrechte; die Wahl von Behördenvertretern, besondere Einspruchsrechte dieser Vertreter und deren Abberufung; die Genehmigung von Budget, Jahresrechnung und Geschäftsbericht; Ersatzvornahmen; die Überprüfung der Zielerreichung; und vermehrt aktienrechtliche Einwirkungsinstrumente.

Es darf dabei nicht vergessen werden, dass diese Aufsichtsmittel nur eine behelfsmässige Korrektur auf einem tieferen Einflussniveau darstellen. Nur weil die gewählten privatrechtlichen Organisationsformen solcher Einflussnahme grundsätzlich entgegenstehen, wird es überhaupt nötig, solche Instrumente vorzusehen. Diese Aufsichtsmittel bedeuten insbesondere eine Verschiebung von ständiger Leitung zu periodischer Steuerung hin (dazu sogleich d).

Die eingeschränkte Aufsicht gegenüber privatisierten Verwaltungsträgern lässt das entstehen, was gemeinhin als Autonomie bezeichnet wird: grössere Spielräume bei der Aufgabenerfüllung und der inneren Organisation, ein loseres Band zum Trägergemeinwesen. ${ }^{565}$ Autonomie heisst «Entscheidungs-

562 MÜLLER, Basler Kommentar, Art.187 Rz. 8. Ohne dies weiter zu begründen, liest der Bundesrat unterschiedliche Aufsichtsintensitäten in diese Bestimmung hinein, gegenüber externen Aufgabenträgern sei bloss eine «angemessene Aufsicht» auszuüben, Botschaft BV, 422. Die Unterschiedslosigkeit in der Verfassung kritisierend BIAGGINI, Kommentar BV, Art. 187 Rz. 5.

563 Die Bedeutung dieser Mittel betonend etwa LIENHARD, Ausgelagerte Aufgabenerfüllung, S.1170 f. Ferner BIAGGINI, Kommentar BV, Art.187 Rz. 5.

564 Folgende Auflistung zusammengestellt aus BIAGGINI, St. Galler Kommentar, Art. 187 Rz.15; LIENHARD, Ausgelagerte Aufgabenerfüllung, S.1170 f.; MÜLLER, Basler Kommentar, Art. 187 Rz.13. Ferner FREUDIGER, Anstalt oder Aktiengesellschaft?, S. 104-106 (betreffend Anstalten) und S.113-119 (betreffend Aktiengesellschaften). Vgl. SPANNOWSKY, Verantwortung der öffentlichen Hand, S. 1078, für eine frühe Forderung solcher Kompensationsmassnahmen aus der deutschen Lehre.

565 Vgl. dazu LIENHARD, Corporate Governance, S. 56 f.; TSCHANNEN/ZIMMERLI/MÜLLER, Allgemeines Verwaltungsrecht, §5 Rz. 34. Ansatzweise auch BIAGGINI, Kommentar BV, Art. 187 Rz. 5. 
und Auswahlfreiheit [...] in der Art und Weise der Aufgabenbesorgung» 566 welche nicht positiv geschaffen wird, sondern im Schatten schwächerer Aufsicht entsteht.

Solche Freiheiten sind kein Zufall. Der Gesetzgeber gewährt sie in der Hoffnung auf bürgernahe Aufgabenerfüllung, insbesondere bei Aufgaben «von ausgeprägtem Dienstleistungscharakter oder besonderer Orts- und Sachnähe»567. Die weniger intensive Aufsicht und die grössere Autonomie dieser Aufgabenträger sind nicht bloss hinzunehmende Konsequenzen der privatisierten Organisationsform - sie sind gerade der Zweck der organisatorischen Erfüllungsprivatisierung. ${ }^{568}$ Mehr Selbständigkeit soll der Aufgabenerfüllung dienen, indem flexible Lösungen gefunden werden können, die den Bedürfnissen der Verwaltungsadressaten entsprechen und nicht bloss gesetzliche Vorgaben umsetzen, die von der konkreten Erfüllungshandlung weit entfernt sind (siehe zur Flexibilität der privatisierten Handlungsformen hinten III.3.b).

\section{d. Periodische Steuerung statt ständiger Leitung}

Wenn privatisierte Organisationsformen staatliche Aufgaben wahrnehmen, sind nicht nur die Aufsichtsmittel eingeschränkt, die Rolle des Bundesrats als oberste Leitungsbehörde der Verwaltung ändert sich sogar grundlegend. Die Zentralverwaltung wird vom Bundesrat beaufsichtigt und geleitet.569 Die uneingeschränkte Dienstaufsicht bedeutet, dass der Bundesrat jederzeit auf die laufende Aufgabenerfüllung durchgreifen kann. ${ }^{570}$ Bevor eine Verfügung eröffnet wird, während die Informationen für einen Bericht zusammengestellt werden, während die Parameter für einen Beschaffungsauftrag ausgearbeitet werden - jederzeit und laufend kann die Verwaltungsspitze auf untergeordnete Einheiten durchgreifen und die Aufgabenerfüllung der einzelnen Verwaltungsfunktionäre beeinflussen.

Die besonderen Aufsichtsmittel hingegen, die gegenüber privatisierten Aufgabenträgern zum Einsatz kommen, formalisieren letztlich nur Zeitpunkt und Gefäss der vorgängigen Steuerung oder nachträglichen Kontrolle: In welchen Abständen werden welche Berichte verfasst, welche Ziele vorgegeben

566 TSCHANNEN, Systeme, Rz. 65, ohne die Hervorhebung im Original.

567 TSCHANNEN, Systeme, Rz. 65. In diesem Sinn auch TSCHANNEN/ZIMMERLI/MÜLLER, Allgemeines Verwaltungsrecht, \$5 Rz. 35.

568 Ähnlich auch ABEGG/FREI, Wirtschaftsfreiheit, S. 291.

569 Art.178 Abs.1 BV. BIAGGINI, Kommentar BV, Art.187 Rz. 5; TSCHANNEN, Organigramm, S. $524 \mathrm{f}$.

570 Art. 47 Abs. 4 RVOG sieht ausdrücklich die «jederzeitige» Eingriffsmöglichkeit vor, vgl. säGESSER, Kommentar RVOG, Art. 47 Rz. 38. 
oder welche Handlungen genehmigt? Die Funktionsweise dieser Art von Aufsicht kann mit folgenden Beispielen illustriert werden:

- Post. Die Schweizerische Post AG ist als öffentlichrechtliche Aktiengesellschaft organisiert (Art. 2 Abs. 1 POG). Im Sinne der Eignerstrategie legt der Bundesrat «jeweils für vier Jahre fest, welche strategischen Ziele der Bund als Eigner der Post erreichen will» (Art. 7 Abs. 1 POG). Die strategischen Ziele sehen selber folgende Berichterstattung vor:571 Die Post pflegt einen vierteljährlichen Informationsaustausch mit Vertreterinnen und Vertretern des Bundes und erstattet dem Bundesrat im Anschluss an ihren jährlichen Geschäftsbericht zusätzlich Bericht über die Erreichung der strategischen Ziele. Dieser Bericht der Post ist nicht öffentlich einsehbar. Der Bundesrat veröffentlicht jedoch seinerseits jährliche Kurzberichte über diese Zielerreichung für sämtliche bundesnahen Betriebe. 572 Eine weitere Einflussnahme des Bundes erfolgt über die Wahl des Verwaltungsrats gemäss Art. 13 Abs. 2 Bst. b POG. Bei der Post ist ausserdem interessant, dass der Bundesrat in Art. 3 VPOG für die Steuerung und Kontrolle der Grundversorgungspflicht grösstenteils auf angemessene Regeln in den Statuten verweist, welche wiederum nicht öffentlich einsehbar sind.

- RUAG. Die bundeseigenen Rüstungsunternehmen sind in einer privatrechtlichen Aktiengesellschaft zusammengefasst, der RUAG Holding AG. ${ }^{573}$ Sie unterliegt einem vergleichbaren Aufsichtsregime wie die Post. Der Bundesrat legt wiederum vierjährige strategische Ziele fest und erwartet im Gegenzugjährliche Berichterstattung sowie einen vierteljährlichen Informationsaustausch. ${ }^{574}$ Das Gesetz verpflichtet die Rüstungsunternehmen, ihre Aufgaben «unter Beachtung marktwirtschaftlicher Grundsätze» zu erfüllen (Art. 2 BGRB). Im Unterschied zu den öffentlichrechtlichen Aktiengesellschaften wählt der Bundesrat die Mitglieder des Verwaltungsrats nicht vollends, sondern sichert sich nur eine seinen Interessen entsprechende Vertretung (Art. 4 BGRB). Eine Änderung der Holdingstruktur auf das Jahr 2020 wurde angekündigt, aber noch nicht in den gesetzlichen Grundlagen oder den strategischen Zielen vollzogen. ${ }^{575}$

571 Strategische Ziele Post, Ziff. 7.

572 Nicht amtlich publiziert, abrufbar unter www.uvek.admin.ch/uvek/de/home/uvek/ bundesnahe-betriebe/zielvorgaben-und-zielerreichung.html.

573 Als Privatrechtssubjekt nicht gesetzlich begründet; vgl. allerdings Art. 1 und 3 BGRB.

574 Strategische Ziele RUAG, Ziff. 5.

575 Zum Stand der Dinge per 2019 siehe die Stellungnahme des Bundesrates auf die Interpellation 19.3786 von Nationalrätin Priska Seiler Graf vom 20. Juni 2019. 
- Swissmedic. Das Schweizerische Heilmittelinstitut ist als öffentlichrechtliche Anstalt verfasst (Art. 68 Abs. 2 HMG). Im Unterschied zu den beiden vorangehenden Beispielen nimmt der Bundesrat seine Eignerstrategie gegenüber der Anstalt zurückhaltend wahr. Zwar wurde auf 2019 das Aufsichts- und Leitungsregime des Bundes gegenüber Swissmedic von Leistungsaufträgen auf strategische Ziele umgestellt. Im Unterschied zu Post und RUAG erlässt Swissmedic aber ihre Ziele selbst. Der Institutsrat erarbeitet sie, unterbreitet sie dem Bundesrat zur Genehmigung und erstattet jährlich Bericht. ${ }^{576}$ Der Institutsrat berichtet jährlich über die Zielerreichung. Dieser Bericht sowie eine allfällige bundesrätliche Antwort darauf dürften nicht öffentlich einsehbar sein. Der Zielkatalog enthält eher abstrakte «programmatische» Vorgaben, wie etwa: «Die Swissmedic erfülltim Rahmen der politischen Vorgaben ihre Aufgaben effizient, transparent und unabhängig»577. Darauf folgen "Aufgaben- und unternehmensbezogene Ziele», wie etwa «zeitkritische Prozesse sind beschleunigt».578 Seit der Umstellung auf strategischeZiele ist unklarer, ob und wie häufig der Bundesrat mitGremien der Anstaltzusammentrifftund dieZielerreichungbespricht. ${ }^{579}$

Diese Beispiele verdeutlichen die periodische, punktuelle Qualität der bundesrätlichen Steuerung solcher Aufgabenträger. Die Entscheidungsspielräume, die mit der sogenannten Autonomie einhergehen, bringen vor allem eine zeitliche Freiheit. Der Zeitpunkt der inhaltlichen Kontrolle verschiebt sich von laufender Einflussnahme zu periodischer Bewertung. Diese zeitliche Verschiebung lässt die inhaltliche Freiheit erst entstehen. Eine bundesrätliche Stellungnahme zu einem Jahresbericht oder ein Diskussionsbeitrag eines Bundesvertreters an einer Verwaltungsratssitzung werden nicht regelmässig inhaltlich detaillierte Vorgaben enthalten über Vorgänge, die eine gewisse Zeit zurückliegen.

Nebenbei: Auch innerhalb der Zentralverwaltung macht der Bundesrat Schritte von laufender Leitung zu periodischer Steuerung: Das Neue Führungsmodell der Bundesverwaltung (NFB, siehe vorne I.4) hat Leistungsvereinbarungen als zentrales Führungsinstrument gegenüber allen Einheiten der Zentralverwaltung eingeführt. ${ }^{580}$ Es wird sich weisen müssen, ob dies zu einem zurückhaltenderen Umgang mit dem Selbsteintrittsrecht führen wird - die organisatorischen Vorarbeiten dazu sind jedenfalls geleistet.

\footnotetext{
576 Art.72a Abs.1 HMG, wiederholt in Strategische Ziele Swissmedic, S.1.

577 Strategische Ziele Swissmedic, Ziff.1, S. 3.

578 Strategische Ziele Swissmedic, Ziff. 2, S. 4-10, 6.

579 Vgl. zur vorherigen Regelung Leistungsauftrag Swissmedic, Ziff. 10.1.1.

580 Sowie gegenüber denjenigen dezentralen Verwaltungseinheiten, die keine eigene Rechnung führen; Art. 38a Abs. 1 RVOG.
} 


\section{Zwischenfazit: Grössere Spielräume und nur punktuelle Kontrolle}

Gesetz ${ }^{581}$ und Leistungsaufträge geben das «Was» vor: Welche Leistungen müssen in welcher Qualität erbracht werden. Privatisierte Verwaltungsträger sind mit grösserer Freiheit für das «Wie» der Aufgabenerfüllung ausgestattet. Die veränderte Rechtsform bringt veränderte Aufsichtsmittel mit sich. Die Autonomie bzw. Selbständigkeit dieser Organisationsformen zeigt sich im Kern darin, dass der Bundesrat seine Dienstaufsicht und damit sein Recht zum Selbsteintritt verliert. Er kann nicht mehr in gleicher Weise auf die laufende Arbeit eingreifen. Seine Einflussnahme verlagert sich auf Berichte sowie Diskussionen und Stellungnahmen über diese Berichte. 


\section{Privatisierung der Handlungsformen}

Die staatliche Aufgabenerfüllung zu betrachten, heisst, den Staat aus der Sicht der Adressaten seines Handelns zu betrachten. Mehr noch als die Organisationsform der Verwaltungsträger (soeben II) ist dabei die rechtliche Gestalt der einzelnen Erfüllungshandlungen ausschlaggebend. Erfüllungsprivatisierung kann neben den organisatorischen auch bei diesen instrumentellen Aspekten der Aufgabenerfüllung ansetzen - mit anderen Worten kann dem Staat erlaubt werden, seine Aufgaben mittels privatisierter Handlungsformen zu erfüllen. Privatisierung in instrumenteller Hinsicht meint dabei Handlungsformen, die eher für privates denn für staatliches Handeln typisch sind.

Dieser zweite Erfüllungsaspekt ist gesondert vom ersten, organisatorischen zu betrachten, denn die Rechtsgrundlage eines Verwaltungsträgers ist noch nicht bestimmend für die verfügbaren Handlungsformen. 582

Wie schon bei den Organisationsformen soll an dieser Stelle keine neue Dogmatik entworfen werden; es geht um Einordnung und Übersicht. Bevor die einzelnen Instrumente aufgelistet (2) und die Kennzeichen der privatisierten Handlungsformen herausgearbeitet werden (3), muss allerdings eine begriffliche Eingrenzung erfolgen (1).

\section{Begriff und Bedeutung der Handlungsformen}

Sollen die Instrumente des staatlichen Handelns untersucht werden, muss ein Schwellenwert definiert werden: Nicht jeder Handgriff, jede E-Mail, jede Datenbankabfrage einer Verwaltungsfunktionärin kann als Erfüllungshandlung angesehen werden, deren Form definiert und rechtsfolgenbegründend wäre. Nein, die Lehre von den Handlungsformen dient vielmehr dazu, im «Kontinuum der administrativen Handlungsketten» 583 formalisierte Momente festzumachen. Die Aufgabenerfüllung ist ein fortschreitender Prozess, $584 \mathrm{der}$ irgendwo einen Anfang nimmt und mit Zwischenschritten an einem anderen Ort ein Ende findet.

Diese formalisierten Momente sind aber nicht auf Rechtsakte zu verkürzen; auch Handlungen, die bloss auf eine Veränderung der faktischen Lage zielen, sogenannte Realakte, ${ }^{585}$ sind eine Kategorie von Handlungsformen. Entscheidend ist nicht, ob eine Handlung einen rechtlichen oder tatsächlichen

582 TSCHANNEN, Systeme, Rz.56.

583 TSCHANNEN, Systeme, Rz.122.

584 TSCHANNEN, Systeme, Rz.124.

585 HÄFELIN/MÜLLER/UHLMANN, Allgemeines Verwaltungsrecht, Rz.1408; TSCHANNEN/ ZIMMERLI/MÜLLER, Allgemeines Verwaltungsrecht, §38 Rz.1. 
Erfolg anstrebt, sondern welche prozessrechtlichen Folgen sie zeitigt. Aus Sicht des Verwaltungsadressaten sind vor allem Handlungen interessant, die ihn direkt betreffen und die er gerichtlich einfordern oder anfechten kann.

Gerade wegen dieser Adressaten-Optik können innerbehördliche Handlungsformen ausgespart werden. Auch dort treten zwar vermehrt Formen auf, die zivilrechtlichen Formen ähneln, wie etwa die Leistungsvereinbarung, die hierarchischere Anweisungen ersetzt. 586 Wie aber eingehend unter I ausgeführt, beschränkt sich diese Arbeit auf Privatisierungsphänomene, welche bei der unmittelbaren, nach aussen gerichteten Erfüllung von Verwaltungsaufgaben ansetzt.

Ebenfalls abzugrenzen sind die Handlungsformen von den Verwaltungsrechtsverhältnissen. Diese bezeichnen die «von verwaltungsrechtlichen Befugnissen und Verpflichtungen geprägte konkrete Beziehung zwischen einem Verwaltungsträger auf der einen und Privaten (oder einem weiteren Verwaltungsträger) auf der anderen Seite aus Anlass und zum Zweck der unmittelbaren Erfüllung von Verwaltungsaufgaben.» ${ }^{587}$ Sie umfassen die gesamte Dauer der Beziehung zwischen Handlungsadressat und Verwaltungsträger und beschlagen auch den Inhalt der geregelten Rechte und Pflichten. ${ }^{588} \mathrm{Hier}$ stehen aber einzelne Instrumente und deren Kennzeichen im Fokus; dies gerade auch, um den verwaltungsrechtlichen Inhalt dieser Rechtsbeziehungen auszublenden. ${ }^{589}$ Nicht die wandelnde Aufgabenerfüllung in einzelnen Gebieten des besonderen Bundesverwaltungsrechts sind Gegenstand dieser Arbeit, sondern veränderte Handlungsformen insgesamt.

Die instrumentellen Aspekte der Aufgabenerfüllungen nehmen Gestalt an als Handlungsformen, deren sich Verwaltungsträger bedienen. Das gewählte Handlungsinstrument zeitigt rechtliche Folgen bezüglich Zuständigkeit, Verfahren, Steuerungswirkung, Rechtsbeständigkeit und Rechtsschutz. ${ }^{590}$ Die Wahl des Instruments beantwortet verschiedene Rechtsfragen, wie: «[A]nhören oder nicht?, das Einverständnis in der Sache suchen?, eröffnen, publizieren?, usf.»591

586 Siehe dazu TANQUEREL, Droit administratif, Rz.987; TSCHANNEN/ZIMMERLI/MÜLLER, Allgemeines Verwaltungsrecht, §33 Rz.16; in Abgrenzung von verwaltungsrechtlichen Verträgen.

587 TSCHANNEN, Systeme, Rz.175, ohne die Hervorhebungen im Original.

588 HÄFELIN/MÜLLER/UHLMANN, Allgemeines Verwaltungsrecht, Rz. 736; TSCHANNEN/ ZIMMERLI/MÜLLER, Allgemeines Verwaltungsrecht, §43 Rz. 7 .

589 Vgl.ähnlichen Zugang zur Handlungsformenlehre bei TSCHANNEN/ZIMMERLI/MÜLLER, Allgemeines Verwaltungsrecht, \$27 Rz.1.

590 HÄFELIN/MÜLLER/UHLMANN, Allgemeines Verwaltungsrecht, Rz. 841; TSCHANNEN, Systeme, Rz.126.

591 TSCHANNEN, Systeme, Rz.123. 
Die Zahl der Instrumente und deren rechtliche Konsequenzen sind durch den Gesetzgeber begrenzt. Aus dem Gesetzmässigkeitsprinzip folgt ein Numerus clausus von Instrumenten, deren sich Verwaltungsträger zu bedienen haben. ${ }^{592}$ Dem Gesetzgeber steht es aber frei, der Verwaltung neue Instrumente an die Hand zu geben.

Im Folgenden werden die gängigen Handlungsformen in drei Gruppen erörtert: unterteilt nach ihrer Nähe zum Handlungsadressaten zunächst die Einzelakte (2.a) und die generell-abstrakten Instrumente (2.b). Es mag untypisch sein, auch generell-abstrakte Formen als Instrumente der unmittelbaren Aufgabenerfüllung abzuhandeln - doch können sie nicht ausgespart werden, da Verwaltungsaufgaben auch ohne individuell-konkrete Handlungen unmittelbar und vollständig erfüllt werden können. Sachgesetzliche Instrumente sui generis, wie etwa Pläne, werden sodann als eigenständige dritte Gruppe behandelt (2.c).

Die Zuordnung von Verwaltungshandlungen zu bestimmten Handlungsformen erfolgt entlang von Strukturmerkmalen. Die Abgrenzung ist materiell: Nicht die äussere Bezeichnung ist begriffsbildend, sondern die inhaltliche Struktur. Die einzelnen Instrumente können zudem Formvorschriften unterliegen - deren Nichtvorhandensein die Zuordnung einer Handlung zum jeweiligen Instrument aber gerade nicht hindert.593

Diesen strukturellen Merkmalen folgend lässt sich eine modellartige Ordnung aufzeigen:594 Zunächst sind nach der Art des angezielten Erfolgs Real- und Rechtsakte abzutrennen; bei den Rechtsakten dann nach dem Adressat die innen- und aussengerichteten Instrumente und bei den aussengerichteten dann nach der Entscheidungshoheit die einseitigen und die einvernehmlichen Instrumente. Eine diese Gabelung überlagernde Einteilung liesse sich noch nach der Rechtsgrundlage der Rechtsakte vollziehen: Die privatrechtlichen bildeten dann eine eigene Kategorie - sie sind aber immer aussengerichtet und einvernehmlich und lassen sich daher getrost in die soeben aufgefächerte Ordnung eingliedern.

592 TSCHANNEN, Systeme, Rz.128.

593 Deutlich bei der Verfügung, mit Ausnahme seltener Nichtigkeitsfälle, siehe TSCHANNEN/ ZIMMERLI/MÜLLER, Allgemeines Verwaltungsrecht, \$28 Rz.18, \$29 Rz. 3.

594 TSCHANNEN/ZIMMERLI/MÜLLER, Allgemeines Verwaltungsrecht, §27 Rz. 7-11. Etwas rudimentärer auch HÄFELIN/MÜLLER/UHLMANN, Allgemeines Verwaltungsrecht, \$12. 


\section{Einzelne Handlungsformen}

\section{a. Individuell-konkrete Instrumente}

aa. Verfügung

Eine Handlung ist als Verfügung anzusehen, wenn sie sechs strukturelle Merkmale aufweist: ${ }^{595}$ Mit der (1) Anordnung einer Behörde wird in einem (2) Einzelfall ein (3) Rechtsverhältnis geregelt; dies auf (4) einseitige und (5) verbindliche Weise und (6) abgestützt auf öffentliches Recht.596

Die Verfügung ist das stereotype Handlungsinstrument der Verwaltung;597 und zwar weil sie die Bedürfnisse des Verwaltungshandelns am besten deckt:598 Sie münzt generell-abstraktes Recht, auf das sich alles Verwaltungshandeln stützen muss (Gesetzmässigkeitsprinzip), in eine verbindliche Anordnung um, die Stellung und Verhalten eines Verwaltungsadressaten prägt. Der rechtsstaatliche Rahmen des Verfassungsstaats wird dadurch für den Alltag passend gemacht.

Kennzeichnend für die Verfügung ist weiter deren Hoheitlichkeit, oder wie es HANGARTNER ausdrückt:

«Damit die Verwaltungsbehörden die Verwaltungsaufgaben erfüllen können, sind sie mit Hoheitsgewalt ausgestattet; sie können einseitig Anordnungen treffen, also verfügen und entscheiden, und ihre Anordnungen nach Massgabe der Gesetzgebung nötigenfalls zwangsweise durchsetzen. In der Ausübung von Hoheitsgewalt unterscheidet sich behördliches Handeln grundlegend von der Tätigkeit der Privaten, die miteinander aufgrund zivilrechtlicher Verträge verkehren.»599

Diese Hoheitlichkeit manifestiert sich etwa im «Privileg [des Verwaltungsträgers], Rechtsverhältnisse einseitig festzulegen» ${ }^{600}$. Das Verfahren auf Erlass einer Verfügung kann auch ohne Zustimmung des Adressaten abgeschlossen werden; dem Privaten stehen danach Rechtsschutzmittel zur Durchsetzung seiner Interessen zur Verfügung.

595 Für bundesrechtliche Verfügungen ergeben sich diese Merkmale aus Art. 5 VwVG; siehe dazu ausführlich HÄFELIN/MÜLLER/UHLMANN, Allgemeines Verwaltungsrecht, Rz. 849852; TANQUEREL, Droit administratif, Rz. 784-808; TSCHANNEN/ZIMMERLI/MÜLLER, Allgemeines Verwaltungsrecht, §28 Rz.16-46.

596 Nebenbei: Die Strukturmerkmale der Verfügung lassen sich mit dem Akronym AbER EVA merken.

597 TSCHANNEN/ZIMMERLI/MÜLLER, Allgemeines Verwaltungsrecht, §27 Rz.12, §33 Rz.1.

598 Vgl. die Funktionen der Verfügung als Grund für deren Vorrang vor den anderen Instrumenten bei TSCHANNEN/ZIMMERLI/MÜLLER, Allgemeines Verwaltungsrecht, \$27 Rz.12.

599 HANGARTNER, Verwaltung durch Private, S. 4.

600 TSCHANNEN/ZIMMERLI/MÜLLER, Allgemeines Verwaltungsrecht, \$28 Rz. 6; vgl. auch Rz.15. 
Die Verfügung dient ausserdem als Ansatzpunkt für das Verwaltungsverfahren und den Rechtsschutz. ${ }^{601}$ Sie gewährt Rechtssicherheit unter anderem dadurch, dass sie einen urteilsähnlichen Vollstreckungstitel darstellt. ${ }^{602}$ Beim Vertrag hingegen muss im Falle der Nichterfüllung erst auf dessen Erfüllung geklagt werden.

\section{bb. Öffentlichrechtlicher Vertrag}

Der öffentlichrechtliche Vertrag (oder: Verwaltungsvertrag) unterscheidet sich in einem grundlegenden Punkt von der Verfügung: Er kommt durch gegenseitige Übereinstimmung zustande und ist daher kein hoheitliches Instrument. ${ }^{603}$ Er ist ein zwei- oder mehrseitiges Instrument zwischen vermeintlich gleichgestellten Vertragsparteien.604

Bei der innerbehördlichen Variante, dem koordinationsrechtlichen Verwaltungsvertrag, ist diese Zweiseitigkeit unproblematisch. Zwei (oder mehrere) Verwaltungsträger einigen sich untereinander über ihre Zusammenarbeit bzw. Aufgabenteilung. ${ }^{605}$ Jedoch können auch mit solchen Verträgen unmittelbar Verwaltungsaufgaben erfüllt werden, so etwa wenn öffentliche Spitäler mit Krankenversicherern Verträge schliessen (nach Art. 43 Abs. 4 KVG) oder wenn der Bund mit dem Nationalmuseum den Umgang mit Sammlungsgegenständen regelt (nach Art. 15 Abs. 1 und 5 MSG).

Problematischer sind hingegen öffentlichrechtliche Verträge, die zwischen Verwaltungsträgern und Verwaltungsadressaten geschlossen werden. Deren widersprüchliche Bezeichnung als subordinationsrechtliche Verwaltungsverträge deutet bereits auf die inhärente Spannungslage hin. ${ }^{606}$ Verwaltungshandeln ist begriffsnotwendigerweise autoritativ: Der Staat übt seine Macht gegenüber Privaten aus, ${ }^{607}$ während Verträge unter gleichgestellten, gleichberechtigten Partnern geschlossen werden.

Abgesehen davon, dass weder die Wahl der Vertragsform noch der Vertragsinhalt widerrechtlich sein dürfen, braucht es eine besondere Rechtfer-

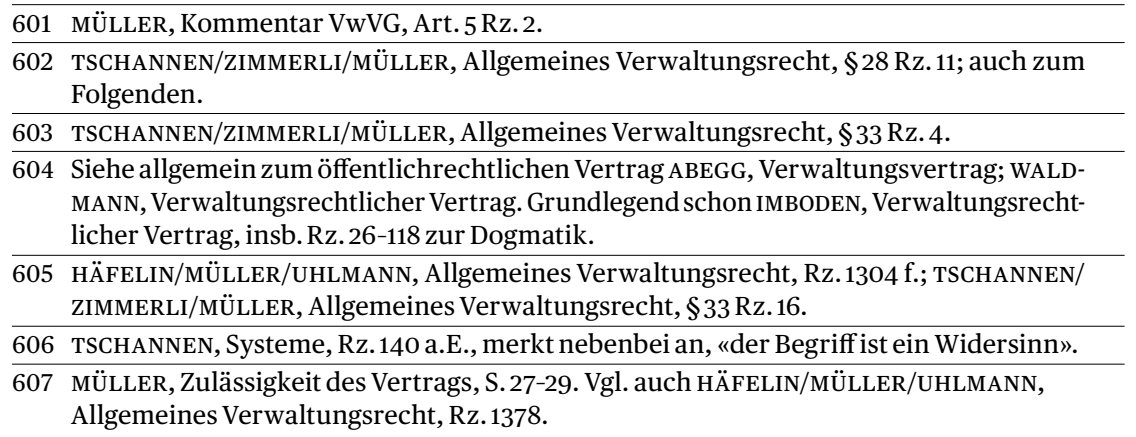


tigung, um dieses Instrument benützen zu dürfen:608 Die Vertragsform muss besser geeignet sein, die anstehende Verwaltungsaufgabe zu erfüllen als eine Verfügung. ${ }^{609}$ Als mögliche solche Rechtfertigung werden etwa Fälle angeführt, in denen der Verwaltungsträger eine dauerhafte gegenseitige Bindung etablieren will.610 Ein einvernehmliches Ende nach gemeinsamer Aushandlung, wie das die Vertragsform erlaubt, soll wohl die Chance auf fortdauernde Zusammenarbeit erhöhen. Eine zweite Konstellation, die diese bessere Eignung ausdrücken soll, ist das konsensuale Konkretisieren eines Ermessensspielraums. ${ }^{611}$ Auch im Verfügungshandeln werden mitunter Spielräume konkretisiert; die einvernehmliche Auswahl einer von verschiedenen denkbaren Optionen soll wohl die Chancen erhöhen, dass der Private sich mit dem gefundenen Ergebnis zufriedengibt und es respektiert. Die «Kooperation zwischen Staat und Privaten soll die Akzeptanz staatlicher Regelungen und damit deren Durchsetzungschancen verbessern.»612

Die Motivation für die Wahl der Vertragsform liegt also gerade im strukturellen Unterschied zur Verfügung: Ein Verwaltungsträger verspricht sich einen Vorteil davon, nichthoheitlich aufzutreten. Der Anstrich eines ebenbürtigen Auftritts Privaten gegenüber soll ihm seine Aufgabenerfüllung erleichtern (siehe dazu hinten 3.e).

So viel zu den erhofften Vorteilen der Vertragsform. Ihr Preis ist, dass ein ausgebildetes Vertragsrecht des öffentlichen Rechts fehlt. Bei der bundesrechtlichen Verfügung findet das Verwaltungsverfahren nach VwVG Anwendung, beim privatrechtlichen Vertrag das OR - ein Gegenstück beim öffentlichrechtlichen Vertrag gibt es nicht. ${ }^{613}$ Auch wenn das Ergebnis nicht ganz so dramatisch ausfallen mag, wie das TSCHANNEN befürchtet («So drohen sich schon kleine Störungen im Vertragsverhältnis zu Wüsten der Ratlosigkeit auszuwachsen»614), so bleibt ein gewisses Mass an Rechtsunsicherheit.615

\footnotetext{
608 HÄFELIN/MÜLLER/UHLMANN, Allgemeines Verwaltungsrecht, Rz.1310-1319; TSCHANNEN/ ZIMMERLI/MÜLLER, Allgemeines Verwaltungsrecht, §33 Rz. 20-30.

609 TSCHANNEN, Systeme, Rz.142 f.

610 HÄFELIN/MÜLLER/UHLMANN, Allgemeines Verwaltungsrecht, Rz.1315; TSCHANNEN/ ZIMMERLI/MÜLLER, Allgemeines Verwaltungsrecht, \$33 Rz. 25.

611 TSCHANNEN/ZIMMERLI/MÜLLER, Allgemeines Verwaltungsrecht, §33 Rz. 26; TSCHANNEN, Systeme, Rz.142.

612 HÄFELIN/MÜLLER/UHLMANN, Allgemeines Verwaltungsrecht, Rz.1307.

613 TSCHANNEN/ZIMMERLI/MÜLLER, Allgemeines Verwaltungsrecht, §34 Rz. 4; WALDMANN, Verwaltungsrechtlicher Vertrag, S. 9-13.

614 TSCHANNEN, Systeme, Rz.142.

615 Siehe HÄFELIN/MÜLLER/UHLMANN, Allgemeines Verwaltungsrecht, Rz.1348-1375 zu fehlerhaften Verträgen; sowie TSCHANNEN/ZIMMERLI/MüLLER, Allgemeines Verwaltungsrecht, §34 zu einzelnen Verfahrensfragen und §35 zur Auslegung, Rechtsbeständigkeit und Durchsetzung.
} 
Dazu tritt eine weitere Schwierigkeit: Schliesst ein Verwaltungsträger einen Vertrag mit einem Privaten ab, ist nicht immer auf Anhieb erkennbar, ob es sich um einen öffentlich- oder privatrechtlichen Vertrag handelt. ${ }^{616}$ Die Rechtsnatur des Verwaltungsträgers ist jedenfalls nicht entscheidend.

Die Schwierigkeit, Vereinbarungen zwischen Behörden und Privaten zu klassifizieren, zeigt sich etwa bei den sogenannten Gentlemen's Agreements, wie sie vereinzelt im Umweltrecht geschlossen wurden. Das sind Vereinbarungen zwischen einer Behörde und Wirtschaftsverbänden mit dem Zweck, den Erlass von generell-abstrakten Regeln zu vermeiden. ${ }^{617}$ Die Privaten verpflichten sich, bestimmte Standards einzuhalten, ${ }^{618}$ und dürfen im Gegenzug darauf vertrauen, dass ihre Tätigkeit keinen neuen Rechtsvorschriften unterworfen wird. Das Bundesgericht bezeichnet dieses Instrument als «acte de planification indicative consensuelle»619. Die künftige Gestaltung eines Rechtsgebiets soll also einvernehmlich mit Privaten geregelt werden. Es ist unklar, ob diese Agreements als öffentlichrechtliche Verträge, privatrechtliche Verträge oder gar als eigenständiges Handlungsinstrument anzusehen sind. ${ }^{620}$

\section{cc. Privatrechtlicher Vertrag}

Staatliche Tätigkeit kann jedwede Form annehmen - entscheidend für ihre staatliche Qualität ist einzig die Erfüllung staatlicher Aufgaben. Daher ist es nicht weiter überraschend, dass sich Verwaltungsträger mitunter auch zivilrechtlicher Handlungsformen bedienen, namentlich des Vertrags nach Art. 1-4O OR. ${ }^{621}$ Hier interessiert nur dasjenige Verwaltungshandeln, das unmittelbar Verwaltungsaufgaben erfüllt. Nicht weiter bemerkenswert ist der Einsatz privatrechtlicher Verträge in Konstellationen, in denen der Staat als Privater auftritt. ${ }^{622}$ Wie anders als privatrechtlich soll zum Beispiel der viel zitierte Bleistiftkauf für das Verwaltungsbüro abgewickelt werden?

616 TSCHANNEN/ZIMMERLI/MÜLLER, Allgemeines Verwaltungsrecht, §33 Rz.13; zum Folgenden Rz.14. Es kann auch vorkommen, dass die Parteien «unbeabsichtigterweise» einen öffentlichrechtlichen Vertrag abschliessen, HÄFELIN/MÜLLER/UHLMANN, Allgemeines Verwaltungsrecht, Rz.1383.

617 Siehe allgemein dazu POLTIER, Gentlemen's agreements; RUCH, Informalisierung, S. 521.

618 In BGE 118 Ib 367 etwa bestimmte PVC-Grenzwerte bei der Produktion von Plastik-Trinkflaschen, E. 5d/bb S. 376 .

619 BGE118 Ib 367 E.9b S.380, unter Bezugnahme auf POLTIER, Gentlemen's agreements, S.388.

620 HÄFELIN/MÜLLER/UHLMANN, Allgemeines Verwaltungsrecht, Rz.1422, zählen sie mit anderen Beispielen des «informellen Verwaltungshandelns» zu den Realakten.

621 Dies ist denn auch nicht nur ein neues Phänomen, vgl. etwa «Privatrechtliche Tätigkeit als Handlungsform der Verwaltung» bei HANGARTNER, Öffentlich-rechtliche Bindungen, S. 133-135.

622 TSCHANNEN/ZIMMERLI/MÜLLER, Allgemeines Verwaltungsrecht, §42 Rz. 5. Etwas weniger deutlich auch bei HÄFELIN/MÜLLER/UHLMANN, Allgemeines Verwaltungsrecht, Rz.1384-1389. 
Im Bereich der unmittelbaren Aufgabenerfüllung kommen privatrechtliche Verträge ausschliesslich in der Leistungsverwaltung zum Einsatz, nie in der Eingriffsverwaltung, die ihrem Zweck nach hoheitlichen Handlungscharakter voraussetzt. Privatrechtliche Verträge tauchen daher bei öffentlichen Dienstleistungen auf, in denen die Verwaltungsadressaten als Kunden erscheinen: etwaim Eisenbahnverkehr, der Energielieferung und dem Postwesen.623 Diese Fälle sind zu unterscheiden von wettbewerblichen Dienstleistungen «staatlicher» Unternehmen, die mangels funktioneller Staatlichkeit selbstredend privatrechtlich zu gestalten sind.

Der privatrechtliche Vertrag als Instrument zur Erfüllung staatlicher Aufgaben ist doppelt rechtfertigungsbedürftig: Erstens muss die einvernehmliche Form begründet werden, wenn doch die hoheitliche Verfügung typisch wäre;624 und zweitens läge mit dem öffentlichrechtlichen Vertrag eine verwaltungsnähere Alternative bereit. Wann also sind privatrechtliche Verträge zulässig? Wenn einer der folgenden drei Fälle vorliegt: Das einschlägige Sachgesetz schreibt zivilrechtliche Rechtsverhältnisse vor; es verweist auf dieZiviljustiz; oder - in Abwesenheit einer spezialgesetzlichen Erklärung - wenn «vernünftigerweise nur die Handlungsformen des Zivilrechts in Frage kommen» ${ }^{625}$.

Dem Gesetzgeber steht es selbstverständlich frei, das Zivilrecht für geeigneter zu halten und die Verwaltung auf dessen Instrumente verweisen. So soll etwa die Schweizerische Post AG ihre Kundenbeziehungen privatrechtlich gestalten (Art.11 Abs.1 POG), wie auch die Rechtsbeziehungen der Betreiberin des Domain-Namen-Registers dem Privatrecht unterstehen (Art. 22 Abs. 1 VID) ${ }^{\mathbf{6 2 6}}$.

Fehlt ein ausdrücklicher gesetzlicher Verweis auf das Privatrecht, ist eine gewisse Alternativlosigkeit vorausgesetzt. Verwaltungsträger dürfen nur dann privatrechtliche Verträge schliessen, wenn etwas anderes unvernünftig wäre. Die Lehre führt zwei Beispiele für diese Situation an: Wenn beliehene Private Ersatzvornahmen ausführen 627 und die nach aussen gerichtete Vergabe im Beschaffungswesen. ${ }^{628}$

623 HÄFELIN/MÜLLER/UHLMANN, Allgemeines Verwaltungsrecht, Rz.1390 f.; TSCHANNEN/ ZIMMERLI/MÜLLER, Allgemeines Verwaltungsrecht, \$42 Rz. 4.

624 Vgl. auch HÄFELIN/MÜLLER/UHLMANN, Allgemeines Verwaltungsrecht, Rz.1380; wonach Verwaltungshandeln grundsätzlich und vorrangweise auf öffentlichrechtlichen Wegen erfolgen soll.

625 TSCHANNEN/ZIMMERLI/MÜLLER, Allgemeines Verwaltungsrecht, §42 Rz. 3, ohne die Hervorhebung im Original; auch insgesamt zur Zulässigkeit.

626 Vgl. auch BGE 131 II 162 E. 2.2 S.165f.

627 TSCHANNEN/ZIMMERLI/MÜLLER, Allgemeines Verwaltungsrecht, \$42 Rz. 3 a.E.

628 TSCHANNEN, Systeme, Rz.149 a.E. Der behördeninterne Teil des Beschaffungsverfahrens, der Entscheid über den Zuschlag an einen Bewerber, erfolgt allerdings als Verfügung; ebd. Rz. 30 . 
Wie schon beim öffentlichen Vertrag hängt auch hier die Zulässigkeit des Instruments zu einem grossen Teil an der Motivation des Verwaltungsträgers, dieses zu benutzen. Reicht im ersten Fall die Einschätzung, der Vertrag sei besser geeignet, muss er in der privatrechtlichen Variante einzig geeignet sein, die anstehende Aufgabe zu lösen. Der Staat mag privatrechtliches Instrumentarium vorziehen, «um für die Bewältigung einer bestimmten Aufgabe eine flexible und speditive Betriebsführung zu erlangen»629, doch reicht dies streng genommen als Rechtfertigung nicht aus. Dadurch ist bloss belegt, dass das Privatrecht Vorteile bringt, nicht dass es unausweichlich ist.

Ohnehin vermag die Wahl privatrechtlicher Instrumente das öffentliche Recht nicht vollends zu verdrängen. So bleibt etwa die Beziehung zwischen der Billag AG und den Gebührenzahlern öffentlichrechtlich (kraft der zugrundeliegenden Staatsaufgabe), unabhängig von der privatrechtlichen Gebührenrechnung. ${ }^{630}$ Und im Beschaffungsrecht, dem typischen Beispiel für den Einsatz dieses Handlungsinstruments, verlangt die sogenannte Zwei-Stufen-Theorie, dass die innerbehördliche Entscheidung über die Vergabe eines staatlichen Auftrags mittels Verfügung gefällt wird, bevor nach aussen hin ein privatrechtlicher Vertrag geschlossen wird. ${ }^{631}$ «Das öffentliche Recht bestimmt also, mit wem der Staat welchen Vertrag zu welchen Bedingungen abschliesst.»632

\section{dd. Realakte}

Realakte sind Handlungen zur Erfüllung von Verwaltungsaufgaben, die in erster Linie eine Veränderung der tatsächlichen, nicht der rechtlichen Lage anstreben. ${ }^{633}$ Entscheidend für diese Klassifizierung ist der angestrebte Erfolg, nicht die erzielte Wirkung. ${ }^{634}$ Es ist selbstverständlich, dass auch Verwaltungshandelnmittels Realaktenunmittelbar Verwaltungsaufgaben erfüllen kann ${ }^{635}$ (vgl. vorne I.1) und den verfassungsrechtlichen Anforderungen (vorne \$3) unterliegt. Nicht nur Rechtsakte stellen staatliche Machtausübung dar - im Gegenteil: Tatsächliches Durchgreifen auf Private beschränkt Freiheiten mitunter stärker und ist daher ebenso rechtfertigungsbedürftig.

629 HÄFELIN/MÜLLER/UHLMANN, Allgemeines Verwaltungsrecht, Rz.1379.

630 BGE140 II 80 E. 2.5 S. 86-88. Seit 2019 erhebt die Serafe AG die RTVG-Abgabe; vgl. BAKOM, Radio- und TV-Abgaben werden künftig von der Serafe AG erhoben, Medienmitteilung vom 10. März 2017.

631 Vgl. Art.22 BöB.

632 HÄFELIN/MÜLLER/UHLMANN, Allgemeines Verwaltungsrecht, Rz.1392.

633 HÄFELIN/MÜLLER/UHLMANN, Allgemeines Verwaltungsrecht, Rz.1408; HÄNER, Praxiskommentar VwVG, Art. 25a Rz. 6; TSCHANNEN/ZIMMERLI/MÜLLER, Allgemeines Verwaltungsrecht, §38 Rz.1.

634 TSCHANNEN/ZIMMERLI/MÜLLER, Allgemeines Verwaltungsrecht, \$38 Rz. 4.

635 HÄFELIN/MÜLLER/UHLMANN, Allgemeines Verwaltungsrecht, Rz.1409. 
Einen Schritt zurück machend liesse sich also fragen, warum Verwaltungshandeln nach der Art des angestrebten Erfolgs zweigeteilt wird. In der Substanz will nämlich auch ein Rechtsakt die tatsächliche Welt beeinflussen. ${ }^{636}$ Die Betriebsbewilligung will bezwecken, dass der Gesuchsteller sein Geschäft tatsächlich betreiben kann. Mit der Verweigerung einer Rodungsbewilligung soll bezweckt werden, dass bestimmte Bäume nicht gefällt werden.

Von der Wirkung auf den Verwaltungsadressaten her betrachtet ist die dogmatische Zweiteilung vielleicht überzeichnet - doch sie bleibt bedeutsam vor allem wegen der verfahrensrechtlichen Komponente. Rechtsakte geben direkten Anlass für Verfahren zu vorgängigem und nachträglichem Rechtsschutz: Sei es das rechtliche Gehör im Verwaltungsverfahren oder die Verfügung als Anfechtungsobjekt für Beschwerden. ${ }^{637}$ Auch Realakte können die Rechtsstellung Privater berühren, was ein potentielles Rechtsschutzdefizit entstehen lässt: Ein Realakt verkürzt Rechte, bildet aber kein Anfechtungsobjekt.638 Dieses Problem des nachträglichen Rechtsschutzes besteht im Bund nicht mehr: Art. 25a VwVG erlaubt es den Betroffenen eines Realakts, eine anfechtbare Verfügung zu verlangen, sofern sie über ein schutzwürdiges Interesse verfügen.

Der Realakt ist dabei kein Handlungsinstrument an sich, sondern lediglich eine Sammelbezeichnung für eine ganze Kategorie von Instrumenten. ${ }^{639}$ Es besteht keine gefestigte Dogmatik zu den einzelnen Instrumenten, die einen Taterfolg bezwecken. Daher ist es angebracht - und in der Lehre auch durchaus üblich -, den Realakt gesamthaft als Form des Verwaltungshandelns zu behandeln. Allerdings lassen sich Untergruppen von Realakten herausschälen, die sich insbesondere dadurch auszeichnen, ob und wie sie mit Rechtsakten zusammenhängen.

- Vollstreckungshandlungen sind Realakte, die auf eine Verfügung folgen und der zwangsbewehrten Durchsetzung der darin enthaltenen Rechte und Pflichten dienen. ${ }^{640}$ Vollstreckungshandlungen sind also rechtsaktbezogene ${ }^{641}$ Realakte.

- Mit vollstreckenden Realakten verwandt sind unmittelbare Vollzugshandlungen - mit dem gewichtigen Unterschied der fehlenden vorgängigen

636 TSCHANNEN, Systeme, Rz.130.

637 Vgl. TSCHANNEN/ZIMMERLI/MÜLLER, Allgemeines Verwaltungsrecht, §38 Rz.3.

638 HÄFELIN/MÜLLER/UHLMANN, Allgemeines Verwaltungsrecht, Rz.1425-1427.

639 Realakt als Oberbegriff auch bei TSCHANNEN/ZIMMERLI/MÜLLER, Allgemeines Verwaltungsrecht, $\$ 38 \mathrm{Rz} .1$.

640 HÄFELIN/MÜLLER/UHLMANN, Allgemeines Verwaltungsrecht, Rz.1411 f.; TSCHANNEN/ ZIMMERLI/MÜLLER, Allgemeines Verwaltungsrecht, §38 Rz. 9.

641 TSCHANNEN, Systeme, Rz.165; TSCHANNEN/ZIMMERLI/MÜLLER, Allgemeines Verwaltungsrecht, §38 Rz.15. 
Verfügung. ${ }^{642}$ Dies sind somit rechtsaktvertretende ${ }^{643}$ Realakte, die aus Gründen zeitlicher und sachlicher Dringlichkeit nicht auf eine vorgängige Verfügung warten können. Dieses Instrument wird auch als antizipierte Ersatzvornahme bezeichnet und setzt unmittelbar drohende Gefahren oder bereits eingetretene Störungen voraus. ${ }^{644}$ Gerade diese Konstellationen würden ohne die Hilfskonstruktion von Art. 25a VwVG ein untragbares Rechtsschutzdefizit verursachen - greifen sie doch rechtsaktgleich in Rechte und Pflichten ein. ${ }^{645}$

- Informelle Absprachen sind formlose Vereinbarungen zwischen Verwaltungsträgern und Adressaten. ${ }^{646}$ Sie sind rechtsaktvermeidend ${ }^{647}$, denn an ihrer Stelle könnten auch Verträge geschlossen werden. Dieses Instrument - wie Realakte allgemein - dürfen nicht der Umgehung gebotener Rechtsakte dienen. ${ }^{648}$ Solche Absprachen sind nur insofern rechtsaktvermeidend, als an ihrer Stelle Rechtsakte hätten geschlossen werden können, aber nicht hätten müssen. Wo etwa eine Verfügung unerlässlich ist, kann kein Tathandeln an ihre Stelle treten.

- Ohne besonderen Bezug zu Rechtsakten sind Auskünfte und Zusicherungen. Mit diesem Instrument informiert ein Verwaltungsträger Private über einen Sachverhalt oder über sein künftiges Verhalten. ${ }^{649}$ Auskünfte und Zusicherungen wollen zwar keine Rechtsfolgen zeitigen, begründen aber regelmässig eine schützenswerte Vertrauensgrundlage. ${ }^{650}$

- Mit Empfehlungen und Warnungen versuchen Verwaltungsträger, privates Verhalten zu lenken, indem sie sich über die «faktische Ratsamkeit bestimmter Verhaltensweisen» ${ }^{651}$ äussern. ${ }^{652}$ Solche Äusserungen sind

642 HÄNER, Praxiskommentar VwVG, Art. 25a Rz. 7; TSCHANNEN/ZIMMERLI/MÜLLER, Allgemeines Verwaltungsrecht, \$38 Rz.10.

643 TSCHANNEN, Systeme, Rz.169; TSCHANNEN/ZIMMERLI/MÜLLER, Allgemeines Verwaltungsrecht, §38 Rz.17.

644 Siehe dazu TSCHANNEN/ZIMMERLI/MÜLLER, Allgemeines Verwaltungsrecht, §32 Rz. 27.

645 TSCHANNEN, Systeme, Rz.169.

646 HÄFELIN/MÜLLER/UHLMANN, Allgemeines Verwaltungsrecht, Rz.1422; HÄNER, Praxiskommentar VwVG, Art. 25a Rz. 7; TSCHANNEN/ZIMMERLI/MÜLLER, Allgemeines Verwaltungsrecht, §38 Rz.13.

647 TSCHANNEN, Systeme, Rz. 168; TSCHANNEN/ZIMMERLI/MÜLLER, Allgemeines Verwaltungsrecht, §38 Rz.16.

648 TSCHANNEN/ZIMMERLI/MÜLLER, Allgemeines Verwaltungsrecht, §38 Rz.19.

649 HÄFELIN/MÜLLER/UHLMANN, Allgemeines Verwaltungsrecht, Rz. 1413; HÄNER, Praxiskommentar VwVG, Art. 25a Rz. 7; TSCHANNEN/ZIMMERLI/MÜLLER, Allgemeines Verwaltungsrecht, §38 Rz.11.

650 Vgl. TSCHANNEN/ZIMMERLI/MÜLLER, Allgemeines Verwaltungsrecht, §22 Rz.11, 15.

651 TSCHANNEN/ZIMMERLI/MÜLLER, Allgemeines Verwaltungsrecht, §39 Rz. 3; allgemein auch Rz.1.

652 Siehe auch FLÜCKIGER, Actes non obligatoires, Rz. 43 f., 47. 
nicht rechtlich verbindlich, können aber dennoch erhebliche rechtliche Tragweite haben, indem sie faktische Zwänge erzeugen, die im Ergebnis Rechtsverboten gleichkommen. ${ }^{653}$ Ein Beispiel für Warnungen findet sich etwa im Lebensmittelrecht, wo Art. 54 LMG dem Bundesamt für Lebensmittelsicherheit und Veterinärwesen (BLV) die Kompetenz gibt, die Öffentlichkeit über unsichere Lebensmittel und Gebrauchsgegenstände zu informieren und Verhaltensempfehlungen abzugeben. ${ }^{654}$ So warnte das BLV etwa vor falsch verpackten Muffins, die Allergiker gefährden könnten, und vor Listerien in Entenleberpasteten. ${ }^{655}$ Das Handlungsinstrument der Empfehlung ist zentral für die Aufgabenerfüllung des Eidgenössischen Datenschutz- und Öffentlichkeitsbeauftragten (EDÖB).656 Er kann privaten Unternehmen per Empfehlung bestimmte Verhaltensweisen nahelegen - und bei Verpassen einer Frist den Rechtsweg über das Bundesverwaltungsgericht androhen (Art. 29 Abs. 4 DSG). So empfahl der EDÖB im Jahr 2009 etwa, dass Google Aufzeichnung und Aufschaltung von Bildern für den Google-Street-View-Dienst einschränke; woraufhin Google Beschwerde führte und das Bundesgericht letztinstanzlich bestätigte, dass der EDÖB für den Erlass solcher Empfehlungen zuständig sei. 657

Sodann wird oft auch die einfache Tathandlung, sogenannt schlichtes Verwaltungshandeln, als Unterart des Realakts genannt. ${ }^{658}$ Darin ist aber eher das Sammelbecken jeder auf Aufgabenerfüllung gerichteter Verwaltungstätigkeit zu sehen. Bei jedem Dokumentdrucken und Telefonabheben von Handlungsformen zu sprechen, wäre übertrieben. Denn Verwaltungshandeln ist nur insofern für diese Arbeit bedeutsam, als es von Verwaltungsadressaten wahrnehmbar ist und deren (rechtliche oder tatsächliche) Stellung berührt

653 MÜLLER, Informales Staatshandeln, S. 536. Vgl. ferner MÜLLER-GRAF, Informalisierung, S. $173 \mathrm{f}$.

654 Das Bundesamt ist insbesondere zuständig, wenn die Bevölkerung mehrerer Kantone gefährdet ist; ansonsten sind die kantonalen Vollzugsbehörden zuständig. Vgl. dazu FLÜCKIGER, Actes non obligatoires, Rz. 47.

655 Siehe öffentliche Warnungen vom 19. Juni 2017 (Nussmuffins des Allergiker-Labels aha! falsch verpackt) und vom 3. Januar 2020 (Listerien in «Entenleberpastete mit Porto» und «Schweineleberpastete mit Steinpilzen» von Madrange); einsehbar unter www.blv.admin. ch/blv/de/home/lebensmittel-und-ernaehrung/rueckrufe-und-oeffentliche-warnungen. html.

656 Art.14 BGÖ und Art. 29 DSG geben ihm entsprechende Kompetenzen.

657 Siehe Empfehlung des EDÖB vom 11. September 2009 sowie BGE 138 II 346 E. 3.3 S. 352 f. und E. 10.7 S. 374 .

658 HÄNER, Praxiskommentar VwVG, Art. 25a Rz. 7; TSCHANNEN/ZIMMERLI/MÜLLER, Allgemeines Verwaltungsrecht, $\$ 38$ Rz. 8. 
(siehe die Einleitung vorne in I.3). Vorbereitende Tathandlungen, die in einem der eigentlichen Instrumente münden, überschreiten diese Schwelle nicht.

Schlichtes Verwaltungshandeln, wie alle Realakte, zeichnet sich durchwegs durch seine öffentlichrechtliche Verwurzelung aus. Formloses Handeln auf privatrechtlichem Boden müsste als zivilrechtlicher Realakt abgesondert werden: Dies ist allerdings eine Handlungskategorie, die nicht dogmatisch aufgearbeitet ist.

\section{b. Generell-abstrakte Instrumente}

Generell-abstraktes Staatshandeln ist einzelfallübergreifend. Es betrifft eine Vielzahl von Personen in einer Vielzahl von Situationen. ${ }^{659}$

Es mag zunächst überraschen, aber die unmittelbare Aufgabenerfüllung muss nicht zwingend die Gestalt von Einzelakten annehmen. Verwaltungsaufgaben können bereits mit allgemeinverbindlichen Akten vollständig erfüllt sein, ohne dass in Einzelfällen Rechte gewährt oder Pflicht auferlegt werden müssten.

Dies gilt grundsätzlich für sämtliche Erlasse. Eine gesetzliche Bewilligungspflicht beispielsweise erfüllt ihren Zweck nicht erst durch die Gewährung oder Verweigerung einzelner Bewilligungen. Bereits die Definition der Bewilligungsvoraussetzungen steuert das private Handeln. Und auch ein strafbewehrtes Verbot ist bereits in seiner gesetzlichen Formulierung eine unmittelbare Aufgabenerfüllung, wirkt es doch schon vor und unabhängig von der Verhängung einer Busse.

Im vorliegenden Zusammenhang steht die unmittelbare Aufgabenerfüllung im Vordergrund, weshalb einzig Erlasse der Regierung und der Verwaltung als Handlungsformen betrachten werden. Aus Sicht des Verwaltungsträgers geben die Erlasse des Parlaments seinen Handlungsspielraum vor, während Verwaltungserlasse diesen Spielraum ausschöpfen. ${ }^{660}$

\section{aa. Rechtsverordnung}

Die klassische und nicht weiter erklärungsbedürftige Variante eines generellabstrakten Instruments ist die Rechtsverordnung. Für die Aufgabenerfüllung aus der Sicht der Handlungsadressaten ist die Verwaltungsverordnung, welche

659 TSCHANNEN/ZIMMERLI/MÜLLER, Allgemeines Verwaltungsrecht, §13 Rz. 8. Vgl. auch «generell-abstrakt» als Rechtsbegriff in Art. 22 Abs. 4 ParlG und die dazugehörigen Ausführungen der SPK-N, Bericht ParlG, $3536 \mathrm{f}$.

660 In diesem Sinn TSCHANNEN/ZIMMERLI/MÜLLER, Allgemeines Verwaltungsrecht, \$40 Rz.1, die von einer «Trennlinie» im «Rechtsverwirklichungsprozess» sprechen. 
innengerichtet ausschliesslich Behörden adressiert, nicht weiter relevant. 661 Innerhalb der Rechtsverordnungen sind weiter die gesetzesvollziehenden von den gesetzesvertretenden Verordnungen zu scheiden: Rechtsverordnungen anstelle von Parlamentserlassen (vertretende) gehören eher zu den Rechtsgrundlagen, während vollziehende Verordnungen bereits den Schritt hin zur Ermessensausübung und Aufgabenerfüllung machen.662 Gesetzesvollziehende Verordnungen führen die gesetzlichen Vorgaben aus, ohne neue Rechte und Pflichten zu schaffen; das macht sie zu einem «Instrument der Verwaltungspraxis» statt zu einem Rechtsetzungsinstrument, wie dies die meisten generell-abstrakten Erlasse sind. ${ }^{663}$

Die Rechtsverordnung ist ein klassisches Instrument des Staatshandelns und weist zahlreiche Parallelen zur Verfügung auf: Sie regelt einseitig, verbindlich und zwangsweise durchsetzbar die Rechtsstellung Privater. Mit dem soeben diskutierten, aber zentralen Unterschied, dass diese Regelung nicht nur einzelne Personen in bestimmten Sachverhalten betrifft, sondern allgemeingültig ist.

\section{bb. Insbesondere: Tendenz zu finalen Normen}

Rechtsnormen sind typischerweise im Wenn-dann-Schema verfasst: Wenn die Voraussetzungen A, B und C vorliegen, dann folgen die Rechtsfolgen $\mathrm{X}$ und Y.664 Die Lehre beobachtet eine Tendenz zu einer anderen Art von Norm: «Die klassische Konditionalnorm, die Tatbestand und Rechtsfolge enthält, wird mehr und mehr von der Finalnorm und der offenen Rechtsnorm abgelöst, die lediglich die Ziele angeben, die die Behörden zu verfolgen haben» 665 . Diese Tendenz lässt befürchten, dass entfesselte Aufgabenträger freier und unvorhersehbar Pflichten auferlegen und freihändige Verhaltenslenkung betreiben, indem sie Konsequenzen für bestimmtes Verhalten definieren, die nicht gesetzgeberisch vorgesehen sind.

Diese Entwicklung, soweit sie denn stattfindet, ist allerdings nicht weiter problematisch. Finale Normen können konditionale Normen nur ergänzen, vor allem weil sie als «reine Wertungs- und Entscheidungshilfe» 666 nicht als Grundlage für Verfügungen dienen können. Normen, gestützt auf die verbindliche

\footnotetext{
661 Siehe dazu an Stelle vieler TSCHANNEN/ZIMMERLI/MÜLLER, Allgemeines Verwaltungsrecht, \$14 Rz.10 f.

662 TSCHANNEN, Systeme, Rz.139. Zur Unterscheidung der beiden Arten ausführlicher TSCHANNEN/ZIMMERLI/MÜLLER, Allgemeines Verwaltungsrecht, \$14 Rz.19-27.

663 TSCHANNEN/ZIMMERLI/MÜLLER, Allgemeines Verwaltungsrecht, §14 Rz. 23.

664 TSCHANNEN, Systeme, Rz.104.

665 RUCH, Informalisierung, S. 518.

666 TSCHANNEN, Systeme, Rz.105.
} 
Verhaltenslenkung betrieben werden soll, müssen die angeordneten Rechte und Pflichten selber vorsehen. ${ }^{667}$ Normen ohne Rechtsfolgen, eben finale Normen mit blossen Ziel- und Grundsatzbestimmungen, tun dies nicht.

Die finale Rechtsetzung kommt denn auch «vorab verwaltungsintern in Frage [...], in der Regel jedoch nicht im Aussenverhältnis zu Bürgerinnen und Bürgern» 668 - jedenfalls ist sie untauglich, um in private Rechtspositionen einzugreifen oder Leistungen in Form von Rechtsansprüchen zu erbringen. Allerdings zeichnen sich auch andere privatisierte Handlungsformen dadurch aus, dass sie auf freiwillige Befolgung und erhöhte Akzeptanz seitens der Privaten setzen, statt verbindliche Anordnungen zu treffen (siehe hinten 3.e). Insofern ist nicht auszuschliessen, dass finale Normen als privatisiertes Instrument eingesetzt werden, um das Verhalten Privater immerhin indirekt zu beeinflussen.

\section{cc. Ausgelagerte Rechtsetzung und Rechtsanwendung unter beibehaltenen Einflussmöglichkeiten}

Eine weitere Gruppe von Handlungsformen zeichnet sich durch eine mehrfach verstrebte Konstruktion aus, die die Aufgabenverantwortung aufteilt: Das Gesetz überträgt Verwaltungsaufgaben auf private Organe, behält originär staatlichen Akteuren aber begleitende Aufsichtskompetenzen über die ausgelagerte Tätigkeit vor. Solche Konstruktionen werden vorab bei der Rechtsetzung und beim Vollzug eingesetzt und erscheinen in zweitypischen Ausprägungen: als sogenannte regulierte Selbstregulierung669, wenn vorab Normsetzungsaufgaben übertragen werden, und als sogenannte akkreditierte Zertifizierung670, wenn Prüf- und Kontrollaufgaben im Fokus stehen.

Der Staat überträgt eine bestimmte Aufgabe an Private - sei dies, Regeln zu erlassen oder die Befolgung bestimmter Kriterien zu überprüfen -, um daraufhin diese privatisierte Aufgabenerfüllung selbst anzuleiten oder zu beaufsichtigen. Der Staat gibt also einen Teil seiner Aufgabenverantwortung an nichtstaatliche Organisationen und Personen ab, behält aber die Kontrolle bei sich. ${ }^{671}$

667 TSCHANNEN, Systeme, Rz.104. Ähnlich auch RUCH, Informalisierung, S. 518.

668 MOSIMANN, New Public Management, S. 350.

669 Begriffsübersicht m.w.H. bei GILI, Selbstregulierung, S.11 f.; HETTICH, Kooperative Risikovorsorge; ZYSSET, Selbstregulierung, Rz.14-186. Ferner HÄFELIN/MÜLLER/UHLMANN, Allgemeines Verwaltungsrecht, Rz.120; MARTI, Aufgabenteilung, S.1157-1159. Grundlegend dazu aus der deutschen Lehre THOMA, Selbstregulierung, passim.

670 Siehe die grundlegende Begriffsbildung bei BÜREN, Akkreditierte Zertifizierung, S. 7-19.

671 WALTHER, Kooperative Steuerungsinstrumente, passim, spricht von kooperativen Steuerungsinstrumenten. 
Der Handlungsformenlehre ist es neu, Erscheinungen wie die regulierte Selbstregulierung oder die akkreditierte Zertifizierung zu einer Gruppe zusammenzufassen. Für die Zwecke dieser Arbeit werden die soeben beschriebenen und teils diffusen Erscheinungen verdichtet zu einem Typus Handlungsinstrument, das zur Erfüllung staatlicher Aufgaben eingesetzt wird.

Als begriffsbildend für diesen Typus wird hier die bereits angetönte Doppelkonstruktion von Auslagerung und beibehaltenen Einflussmöglichkeiten vorgeschlagen. Daneben tritt eine typische Folge dieser Handlungsformen: geteilte Aufgabenverantwortung. Der Staat setzt diese Art von Instrument ein, um einen Teil seiner Verantwortung für die Regulierung von gesellschaftlichen oder wirtschaftlichen Bereichen mit privaten Akteuren zu teilen ${ }^{672}$ oder um wenigstens Private mit der Einhaltung der staatlichen Regulierung zu betrauen. Entweder sollen Private im staatlichen Auftrag und unter staatlicher Anleitung Regeln setzen; oder Private sollen die Einhaltung der Anforderungen einer staatlichen Regulierung überprüfen und durchsetzen.

Zum besseren Verständnis einige Beispiele:

- Die Übertragungvon Normsetzungsaufgaben ist im Finanzwesen verbreitet und im Geldwäschereigesetz als zentrales Instrument der Aufgabenerfüllung verankert (Art. 7 Abs. 3 FINMAG; Art. 18, 24-28 GwG). Die Konstruktion ist dabei wie folgt: Die FINMA (als staatliche Behörde ausgestaltete Finanzmarktaufsicht) erteilt privaten Stellen auf deren Gesuch hin die Anerkennung als Selbstregulierungsorganisation. ${ }^{673}$ Diese Organisationen erlassen daraufhin Reglemente ${ }^{674}$ zur Durchsetzung des Gesetzeszwecks und der öffentlichen Interessen und mithin funktionell in Erfüllung staatlicher Aufgaben. ${ }^{675}$ Die FINMA genehmigt diese Reglemente ${ }^{676}$ und bleibt ausserdem dafür verantwortlich, dass die in diesen Reglementen festgesetzte Ordnung auch tatsächlich durchgesetzt wird; und sie beaufsichtigt die Selbstregulierer entsprechend. ${ }^{677}$ Das Instrument der Selbstregulierung ermöglicht es laut Bundesgericht, dass feingliedrige, branchenspezifische Besonderheiten abweichend von den einheitlichen Vorgaben von rechtssatzförmigen Verordnungen geregelt werden. ${ }^{678}$ Wie das Gericht

\footnotetext{
672 Zur Aufgabenteilung bei der Rechtsetzung MARTI, Aufgabenteilung, passim.

673 Art. 18 Abs. 1 Bst. a, Art. 24 Abs. 1 GwG. Siehe dazu BOVET/HÉRITIER LACHAT, Intermédiares financiers, Rz.141, 144-148; HETTICH, Kooperative Risikovorsorge, Rz. 541 f.

674 Art.25 GwG.

675 BGer-E 2C_887/2010 vom 28. April 2011, E. 6.1; BGE 143 II 162 E. 2.3 S. 167 f.

676 Art.18 Abs.1Bst.c GwG.

677 Art.18 Abs.1Bst.d und b GwG.

678 BGE 143 II 162 E. 3.2.6 S. 174.
} 
in demselben grundlegenden Urteil zur Stellung der Selbstregulierung im Geldwäschereirecht festhielt, sei die FINMA aber «letztlich dafür verantwortlich, dass die Selbstregulierungsorganisationen sich bei den von ihnen gewählten Erleichterungen nicht vom Gesetzeszweck entfernten»679.

- Die zweite Ausprägung dieses Instruments, die Übertragung von Prüfaufgaben mit mehrfach verstrebter staatlicher und privater Aufsicht, ist gerade im Bildungswesen weit verbreitet. Eigens geschaffene Akkreditierungsorgane prüfen, ob neue Organisationen als Bildungsinstitutionen anerkannt werden sollen. In diesem Sinn funktioniert etwa die Schweizerische Agentur für Akkreditierung und Qualitätssicherung AAQ, welche gemäss Art. 22 HFKG als unselbständige Anstalt die ihr übertragenen Aufgaben wahrnimmt. 680 Die inhaltlichen Kriterien für den Akkreditierungsentscheid sind von einer ihr übergeordneten Stelle rechtssatzförmig verankert. 681

Diese privatisierten Handlungsformen bringen auf den ersten Blick nachteilige Unsicherheiten mit sich: Wer ist verantwortlich für die Aufgabenerfüllung?682 Sind Selbstregulierungsorgane staatliche Aufgabenträger? Verliert die staatliche Behörde an Einwirkungsmöglichkeiten durch diese Konstruktion?683 Diese Unsicherheiten sollen aufgewogen werden durch die Motivation hinter dieser Konstruktion: Erstens soll Selbstregulierung das Subsidiaritätsprinzip verwirklichen, indem der Staat die Regulierung Privaten überlässt und nur soweit nötig eingreift. ${ }^{684}$ Zweitens soll die Regelung im Ergebnis bedarfsgerechter sein, indem die Regulierungsverantwortung auf private Organe übertragen wird, die «mit den sich in ihrem Tätigkeitsbereich ergebenden speziellen Problemen besonders eng vertraut» 685 sind. ${ }^{686}$ Die Doppelkonstruktion soll es insbesondere ermöglichen, «die Innovationsoffenheit,

679 BGE 143 II 162 E.3.2.5 S. 173.

680 Eine ähnliche Regelung besteht im Bereich der universitären Medizinalberufe, siehe Art. 22-31a MedBG.

681 Verordnung des Hochschulrates über die Akkreditierung im Hochschulbereich vom 28. Mai 2015 (Akkreditierungsverordnung HFKG, SR 414.205.3). Andere Beispiele sind etwa die akkreditierte Prüfung von Konformität im Bereich der technischen Handelshemmnisse, siehe Art. 18 THG.

682 HÄFELIN/MÜLLER/UHLMANN, Allgemeines Verwaltungsrecht, Rz.1844.

683 HETTICH, Kooperative Risikovorsorge, Rz. 545, erwartet etwa den Verlust an Effektivität.

684 GILI, Selbstregulierung, S. 139; HÄFELIN/MÜLLER/UHLMANN, Allgemeines Verwaltungsrecht, Rz.345; KUSTER, Selbstregulierungsorganisationen, S.1503 f.

685 Botschaft GwG, 1145.

686 GILI, Selbstregulierung, S. 140-142, 147. Zur Bedeutung von Fachwissen bei Akkreditierung und Zertifizierung auch HÄFELIN/MÜLLER/UHLMANN, Allgemeines Verwaltungsrecht, Rz.1843. 
Lernfähigkeit und Anpassungsfähigkeit der Selbstregulierung zu bewahren, ohne diese den Eigeninteressen der Akteure auszuliefern»687. Das Beste beider Welten also.

\section{dd. Einschub: Die missverständlichen Begriffe private Normsetzung und Selbstregulierung}

Die soeben beschriebene Gruppe von Handlungsformen verleitet zu missverständlicher Begriffsnutzung. Sie zeichnen sich durch ein (gestaffeltes und teils mehrschichtiges) Zusammenwirken von klassisch staatlichen und originär privaten Akteuren aus. Sie bleiben aber Erscheinungsformen staatlicher Aufgabenerfüllung. Die privaten Akteure sind nur bezüglich ihrer Organisationsform privat. Soweit sie gesetzlich zur Vornahme bestimmter Tätigkeiten nach vorgegebenen Kriterien verpflichtet werden, sind sie in funktioneller Hinsicht Staat. Es wäre also falsch, in diesem Zusammenhang von privater Tätigkeit zu sprechen. Die Begriffe private Normen ${ }^{688}$ oder Selbstregulierung ${ }^{689}$ sind daher zumindest missverständlich, wenn nicht gar irreführend.

Mit den soeben unter cc beschriebenen Instrumenten werden privatrechtliche Akteure, seien sie vorbestehend oder eigens gegründet, in die staatliche Aufgabenerfüllung einbezogen. Rechtsetzungs- und Rechtsanwendungsaufgaben werden an Private delegiert, die dadurch zu funktionell staatlichen Akteuren werden. Für diesen Vorgang gibt es einen etablierten Begriff: die Beleihung (siehe dazu vorne \$2/I.2.a). 690

Selbstregulierung ist dann eine treffende Bezeichnung, wenn private Akteure «kollektive Selbstbindungspflichten» ${ }^{691}$ beschliessen. Ein Beispiel ${ }^{692}$ dafür sind etwa sogenannte Regionallabels für Lebensmittel, soweit es sich um Richtlinien ohne gesetzliche Verbindlichkeit handelt, die von grossen Detailhändlern festgesetzt und von Produzenten und Lieferanten befolgt werden. Sobald aber die beschlossenen Regeln Bindungswirkung für Personen

687 HETTICH, Kooperative Risikovorsorge, Rz. 516. In der deutschen Lehre wird mit «Selbstregulierung» auch die Hoffnung auf beschleunigte Entscheidungsprozesse verbunden, siehe DOLDE, Verwaltungsverfahren und Deregulierung, S. 863.

688 Z.B. bei BÜREN, Akkreditierte Zertifizierung, S. 259; MARTI, Aufgabenteilung, S.1160-1162.

689 Z.B. bei BÜREN, Akkreditierte Zertifizierung, S.127 f.; ERRASs, Technikregulierungen, S. 63, 67 f.; HETTICH, Kooperative Risikovorsorge, Rz. 472, 512, 514; WYSS, Öffentliche Interessen, S.100-106.

690 So auch HÄFELIN/MÜLLER/UHLMANN, Allgemeines Verwaltungsrecht, Rz.1840 (speziell zu Akkreditierung und Zertifizierung). Siehe allgemein zur Beleihung ebd., Rz.1821-1839 und TSCHANNEN/ZIMMERLI/MÜLLER, Allgemeines Verwaltungsrecht, §10 Rz.12-15.

691 HETTICH, Kooperative Risikovorsorge, Rz. 513.

692 FLÜCKIGER, Actes non obligatoires, Rz. 71; HETTICH, Kooperative Risikovorsorge, Rz. 513; RUCH, Regulierungsfragen, S. 419. 
und Organisationen haben, die nicht aktiv am Regulierungsprozess teilhatten, verliert das «Selbst» des Begriffs seine Richtigkeit. ${ }^{693}$ Wo der Staat private Akteure damit beauftragt, allgemein verbindliche Regeln zu setzen, ist dies staatliche Rechtsetzung.

Wiederum scheiden sich private und staatliche Tätigkeiten entlang eines funktionellen Grats: Dient die Regelsetzung der Erfüllung staatlicher Aufgaben, handelt es sich um die (soeben in cc beschriebene) Doppelkonstruktion von Beaufsichtigung ausgelagerter Rechtsetzungstätigkeit.694 Hingegen ist es eine Erscheinung der Privatautonomie, wenn private Akteure unter sich Regeln des privaten Rechts- und Geschäftsverkehrs erlassen, zu denen sie der Staat nicht verpflichtet. ${ }^{695}$ In diesen Fällen der «echten», privaten Selbstregulierung überträgt der Staat keine Aufgaben an Private, sondern beschränkt sich darauf, die rechtliche Rahmenordnung für privates Handeln bereitzustellen. ${ }^{696}$

Kurzum: Es empfiehlt sich, die Bezeichnung von Selbstregulierung zu vermeiden. Wo sie treffend ist (ausserhalb des Bereichs staatlicher Aufgaben), drückt sie Selbstverständliches aus: Private binden sich untereinander selbst. Wo die Bezeichnung eine spezifische Ausprägung staatlicher Regelbildung ist, ist sie irreführend. Staatliche Akteure beschränken sich zwar auf weniger detaillierte Regelsetzung, übertragen aber privaten Akteuren einen Teil der staatlichen Aufgabenverantwortung. Die Selbstregulierung erscheint dann funktionell als staatliches Handeln, genauer als privatisierte Erfüllung staatlicher Aufgaben.

\section{c. Andere sachgesetzliche Instrumente, insbesondere Pläne}

Wie eingangs festgehalten (vorne 1), dürfen sich Verwaltungsträger nur derjenigen Instrumente bedienen, die gesetzlich vorgesehen sind.697 Der formell zwar bestehende Numerus clausus von Handlungsformen wird dadurch in der Praxis aufgelöst in eine unüberblickbare Summe von eigens geschaffenen Sonderinstrumenten. Diese Instrumente sind weder eindeutig individuell-konkret (vorne 2.a) noch generell-abstrakt (soeben 2.b), weshalb sie hier als eigenständige Kategorie aufgeführt werden.

Die Lehre des Allgemeinen Verwaltungsrechts versucht Ordnung in die Vielfalt zu bringen, indem sie eine Gruppe von Instrumenten ansatzweise

693 Vgl. GILI, Selbstregulierung, S.13, m.w.H. auf die Kritik am Begriff.

694 Etwas weniger deutlich, aber in diesem Sinn auch HETTICH, Kooperative Risikovorsorge, Rz. 515.

695 GILI, Selbstregulierung, S. 10.

696 HETTICH, Kooperative Risikovorsorge, Rz. 514.

697 TSCHANNEN, Systeme, Rz.128. 
dogmatisch verdichtet zum Plan. ${ }^{698}$ Dieser sei definiert als «die zweckgerichtete, auf Steuerung künftigen Handelns angelegte Zusammenfassung zukunftsbezogener Aussagen» ${ }^{699}$.

Es gibt allerdings nicht den einen Plan als gefestigtes Handlungsinstrument. 700 Vielmehr werden seine rechtliche Bedeutung und Kennzeichen jeweils einzeln sachgesetzlich erst gebildet. So ergibt sich etwa auch die verfahrensrechtliche Behandlung - wie etwa Fragen des rechtlichen Gehörs und der Eröffnung - erst aus dem Sachgesetz. ${ }^{701}$ Was dogmatisch als Plan bezeichnet werden kann, muss im Sachgesetz nicht immer so heissen; auch Instrumente mit den Bezeichnungen Konzept, Leitbild, Programm oder Ähnliches fallen in diese Instrumentengruppe. ${ }^{702}$ Zudem muss bei jeder dieser Erscheinungen im Einzelnen beurteilt werden, ob sie nur behördenverbindlich sind und daher als ausschliesslich verwaltungsinterne Akte gar nicht Teil der Aufgabenerfüllung sind, wie sie in diesem Paragrafen behandelt wird (siehe einleitend I).

Am besten dogmatisch ausgebildet ist der Plan im Raumplanungsrecht: als Richtplan und als Nutzungsplan. ${ }^{703}$ Andere sachgesetzliche Instrumente tauchen etwa in folgenden Gebieten des Besonderen Bundesverwaltungsrechts auf:

- Der Bund erfüllt seine Aufgaben im Sicherheitsrecht unter anderem dadurch, dass er «ein Leitbild der Massnahmen zum Schutz von Bundesbehörden» erlässt (Art. 5 Bst. a BWIS).

- Gleichstellungsrecht. Das Gleichstellungsgesetz (GlG) sieht etwa vor, dass der Bund «öffentlichen oder privaten Institutionen, die Programme zur Förderung der Gleichstellung von Frau und Mann im Erwerbsleben durchführen, Finanzhilfen gewähren [und] selbst Programme durchführen» kann (Art. 14 Abs. 1 GlG). Daneben kann sich der Bund auch an «Projekten von gesamtschweizerischer Bedeutung beteiligen» (Art. 16 Abs. 2 Bst. d GlG). Inwiefern sich Programme von Projekten unterscheiden, bleibt unklar - in der Verordnung über Finanzhilfen nach dem

698 Die Literatur behandelt den Plan nicht einheitlich. HÄFELIN/MÜLLER/UHLMANN, Allgemeines Verwaltungsrecht, Rz.949-962, behandeln ihn nur im Zusammenhang der Raumplanung. Eigenständige Behandlung bei TANQUEREL, Droit administratif, §15.

699 TSCHANNEN/ZIMMERLI/MÜLLER, Allgemeines Verwaltungsrecht, §36 Rz. 6.

700 TSCHANNEN, Systeme, Rz. 155. Ähnlich auch TANQUEREL, Droit administratif, Rz.1087f.

701 TSCHANNEN/ZIMMERLI/MÜLLER, Allgemeines Verwaltungsrecht, §36 Rz.11.

702 TSCHANNEN/ZIMMERLI/MÜLLER, Allgemeines Verwaltungsrecht, §36 Rz. 7.

703 HÄFELIN/MÜLLER/UHLMANN, Allgemeines Verwaltungsrecht, Rz.949-962; TANQUEREL, Droit administratif, Rz.1101-1132; TSCHANNEN/ZIMMERLI/MÜLLER, Allgemeines Verwaltungsrecht, §37; TSCHANNEN, Richtplan, passim. 
Gleichstellungsgesetz ${ }^{704}$ werden die Begriffe zum Teil austauschbar verwendet.

— Im Natur- und Heimatschutzrecht gehört insbesondere «die Planung [...] von Werken und Anlagen» zu Erfüllung von Bundesaufgaben (Art. 2 Abs. 1 Bst. a NHG).

Viel Planungs- und Projektierungsbedarfbesteht vor allem im Verkehrsrecht. Neue Nationalstrassen etwa werden zuerst geplant (Art. 9-11 NSG), dann in einer generellen Projektierung ausgearbeitet (Art.12-20), bevor ein Ausführungsprojekt (Art. 21) dem Plangenehmigungsverfahren zugeführt werden kann (Art. 26a-28a). Auch Eisenbahnanlagen dürfen «nur mit einer Plangenehmigung erstellt oder geändert werden» (Art. 18 Abs. 1 EBG). Im Eisenbahnwesen werden zudem grössere Entwicklungen mittels Konzepten entwickelt: Zum Beispiel das NEAT-Konzept (Art. $3^{\text {bis }}$ AtraG), das Hochgeschwindigkeitverkehrs-Anschluss-Konzept (Art. 3 HGVAnG) oder gesamthaft zur Förderung des öffentlichen Verkehrs das Konzept BAHN $2000^{705}$.

Wie schon bei der ausgelagerten Rechtsetzung und Rechtsanwendung unter beibehaltenen Einflussmöglichkeiten (siehevorne 2.b.cc) gilt auch beim Plan, dass die Unterschiede gewichtiger als die Gemeinsamkeiten sein können und dass die rechtlichen Eigenschaften des jeweiligen Instruments von der einzelnen gesetzlichen Ausgestaltung abhängen. Hier zeigt sich die Gestaltungsmacht des Gesetzgebers: Er kann neue Formen schaffen und muss das Recht nicht innerhalb vorgezeichneter Linien der Dogmatik setzen.

\section{Kennzeichen der Erfüllungsprivatisierung in instrumenteller Hinsicht}

Handlungsformen sind das «In-Form-Bringen einer konkreten Verwaltungshandlung» $\mathbf{7 0 6}$ - sie übersetzen Verwaltungsaufgaben in Rechte, Pflichten und tatsächliche Veränderungen. Je nach gewählter Form unterscheiden sich die Rechtsfolgen. Diese Folgen lassen sich grob in zwei Aspekte einteilen: Verfahren (wie rechtliches Gehör, Mitwirkung, Einseitigkeit, Eröffnung, Publikation und Rechtsschutz) und Steuerungswirkung (wie Verbindlichkeit, Durchsetzbarkeit, Beständigkeit und Abänderlichkeit). ${ }^{707}$ Im Folgenden

704 Vom 22. Mai 1996 (SR151.15).

705 Siehe das immer noch in Kraft stehende Bundesgesetz betreffend das Konzept BAHN 2000 vom 19. Dezember 1986 (SR 742.100).

706 TSCHANNEN, Systeme, Rz.123.

707 Vgl. HÄFELIN/MÜLLER/UHLMANN, Allgemeines Verwaltungsrecht, Rz. 841; TSCHANNEN, Systeme, Rz.126. 
wird herausgearbeitet, inwiefern die Privatisierung der instrumentellen Aspekte der Aufgabenerfüllung diese Rechtsfolgen beschlägt.

\section{a. Vorab: Die privatisierten Handlungsformen}

Damit Gewissheit über den Gegenstand der Erfüllungsprivatisierung besteht, müssen die privatisierten von den staatlichen Handlungsformen abgegrenzt werden. Wie schon vor den Kennzeichen der privatisierten Organisationsformen ausgeführt (siehe vorne II.4.a), ist auch hier eine klare Grenzziehung unmöglich, da die Aufgabenerfüllung auf einem Spektrum liegt und nicht binär der staatlichen oder der privaten Sphäre zufällt - im Gegensatz zur Aufgabenzuweisung. Für die Zwecke dieser Arbeit gelten die Verfügung und die Rechtsverordnung als staatliche Handlungsformen und von den Realakten die Vollstreckungs- und Vollzugshandlungen (vorne 2.a.aa und 2.b.aa sowie teilweise 2.a.dd). Alle anderen Instrumente sind privatisierte Handlungsformen; sie ähneln dem privaten Handeln. Auch hier gilt also, dass nicht nur neuartige Phänomene Privatisierungen darstellen ${ }^{708}$ und dass die folgenden Kennzeichen je nach betroffenem Instrument unterschiedlich ausgeprägt sind.

\section{b. Gestaltungsfreiheit im Einzelfall}

Privatisierte Handlungsformen zeugen in doppelter Weise von mehr Gestaltungsfreiheit für die Verwaltung: Erstens sind sie ein Zeichen dafür, dass der Gesetzgeber der Verwaltung neue Mittel an die Hand gibt, statt sie darauf zu verpflichten, ihre Tätigkeit innerhalb der klassisch staatlichen Instrumente zu entfalten. Nicht die Instrumente steuern das Handeln, sondern das Handeln die Instrumente. Und zweitens lassen die privatisierten Handlungsformen eine freiere, vielfältigere Ausgestaltung zu. Markant unterschiedliche Vereinbarungen passen unter das Begriffsdach «Vertrag», während Verfügungen einander ähnlicher sind.

Diese beiden Facetten der grösseren Gestaltungsfreiheit sollen nun näher ausgeführt werden.

Zum ersten Punkt: Die Verwaltung darf sich nur derjenigen Instrumente bedienen, die ihr der Gesetzgeber an die Hand gibt - so fordert es das Gesetzmässigkeitsprinzip. ${ }^{709}$ Es steht dem Gesetzgeber aber frei, neue Instrumente zu schaffen. Kennzeichnend dafür sind die soeben unter 2.c aufgeführten sachgesetzlichen Instrumente. Per Gesetz wird die Verwaltung angewiesen,

708 Vgl. IMBODEN, Verwaltungsrechtlicher Vertrag, Rz. 22, mit Verweis auf «ein enges Ineinandergreifen privatrechtlicher und öffentlichrechtlicher Gestaltung» im Gemeinderecht und den «bürgerlichen Genossenschaftsgeist».

709 TSCHANNEN, Systeme, Rz. 128. 
Leitbilder, Projekte und Konzepte zu schaffen. Je eigene Formen, deren Rechtsfolgen neu definiert werden können. Wäre die Verwaltung angehalten, ihre Planungen entweder in eine generell-abstrakte Rechtsverordnung zu giessen oder auf dem Verfügungsweg verbindliche Rechtswirkungen auszulösen, wäre ihre Gestaltungsfreiheit kleiner.

Ähnlich verhält es sich bei den ausgelagerten Rechtsetzungs- und Rechtsanwendungsinstrumenten (soeben 2.b.cc) - sie zwingen die Verwaltung weder zur vollständigen Übernahme der Regelungsverantwortung, noch beschränken sie den Staat auf eine reine Aufsichtsfunktion. Stattdessen schaffen sie einen dritten, mittleren Weg mit neuen Möglichkeiten.

Diese neuen Möglichkeiten sind kennzeichnend für den zweiten Aspekt der grösseren Gestaltungsfreiheit: Privatisierte Handlungsformen lassen sich flexibler ausgestalten. Rechtsaktvertretende und rechtsaktersetzende Realakte erlauben der Verwaltung eine flexiblere Problemlösung als die Verfügungen, an deren Stelle sie treten. Mit Warnungen und Empfehlungen kann die Verwaltung das private Verhalten auf genau die Art lenken, die ihr am geeignetsten erscheint, statt sich für verbindliche Anordnungen entscheiden zu müssen. Würden Rechtspflichten auferlegt, etwa mit einer Bewilligung in Verfügungsform, müssten Voraussetzungen und Durchsetzungsmechanismen definiert werden. Empfehlungen und Warnungen lassen sich offener und weniger starr ausgestalten. Ebenso verhält es sich bei informellen Absprachen, die an die Stelle von Verträgen und Verfügungen treten.

Was ist die Konsequenz dieser grösseren Gestaltungsfreiheit? «Formfreiheit mindert den Verfahrensaufwand und verheisst Zeitgewinn.»710 Dadurch können Verfahrensgarantien nicht oder nur weniger greifen, und der mangelnde Vollstreckungstitel schafft Probleme für den Vollzug. Vollzugsprobleme können sich etwa in geringerer Vorhersehbarkeit und Rechtssicherheit zeigen, indem spontane Lösungen ausserhalb gefestigter Abläufe kreiert werden müssen.

Dass sich die Verwaltung nur derjenigen Formen bedienen darf, die ihr der Gesetzgeber anbietet, soll das Verwaltungshandeln rationalisieren.711 Verwaltungshandeln bedeutet den Umgang mit komplexen Sachverhalten und Problemstellungen. Die Handlungsformen geben Verfahrensschritte vor, die dieses Handeln strukturieren. In welcher Form wird der Sachverhalt ermittelt? Wann und wozu wird den Verwaltungsadressaten rechtliches Gehör gewährt? Wann und in welcher Form begründet die Verwaltung ihr Handeln?

710 TSCHANNEN, Systeme, Rz. 168; auch zum Folgenden.

711 Dazu ausführlich TSCHANNEN/ZIMMERLI/MÜLLER, Allgemeines Verwaltungsrecht, §28 Rz.13. 
Wie wird ihr Handeln publiziert? Klassisch staatliche Instrumente wie Verfügungen und Rechtsverordnungen beantworten diese Fragen auf die immer gleiche, vorhersehbare Weise. Bei sachgesetzlichen Einzelformen und anderen neuen Instrumenten besteht grössere Unsicherheit. Die Rechtsfolgen und verfahrensrechtlichen Konsequenzen müssen in einer neuen Praxis erst geprägt werden.

Diese Konsequenzen sind gewollt, sie zeugen von einer bewussten Entfernung vom öffentlichen Recht. Oder, wie es TSCHANNEN anhand des privatrechtlichen Vertrags pointierter ausdrückt: «Mit dem privatrechtlichen Vertrag soll der Verwaltungsträger ja Gestaltungsautonomie gewinnen, die er auf dem Boden des öffentlichen Rechts nicht hätte. So trägt der Wechsel ins Privatrecht eben doch den Stempel einer kleinen Flucht - dogmatisch zwar verpönt, politisch aber legalisiert.» ${ }^{712}$

\section{c. Vermeintliche Aufgabe von Machtvorsprung}

Das öffentliche Recht zeichnet sich durch seine zwingende Natur aus. ${ }^{713}$ Mit dem Besonderen Verwaltungsrecht regelt der Staat, wie er seine Ziele und Interessen befördert - nämlich indem es staatliche Aufgaben definiert. Das Staatsrechtregelt dabeidie Organisation und verbindlicheZuständigkeiten. ${ }^{714}$

Wo staatliche Aufgaben erfüllt werden, besteht ein Machtgefälle. Daran gibt es keinen Weg vorbei, und das gilt auch bei Handlungsformen, die als Vereinbarungslösung daherkommen. ${ }^{715}$ Auf zwingende Vorgaben zu verzichten, würde bedeuten, die staatliche Verantwortung für die Beförderung seiner Ziele und Interesse zu negieren. Ob staatliche Aufgaben wünschbar sind und im demokratischen Interesse aller Machtunterworfenen liegen, wurde mit deren Festlegung im politischen Prozess abschliessend beantwortet. Die Zustimmung der Verwaltungsadressaten darf die Erfüllung staatlicher Aufgaben nicht aufhalten.

Die vorne beschriebenen Handlungsformen (2) berücksichtigen diese Überlegungen uneinheitlich. Ein Kennzeichen für privatisierte Instrumente ist gerade, dass sie das Machtgefälle vermeintlich einebnen. Der Staat scheint seinen Machtvorsprung aufzugeben, wenn er mit den Verwaltungsadressaten Verträge schliesst.716 Und er scheint seinen Machtvorsprung aufzugeben,

712 TSCHANNEN, Systeme, Rz.150.

713 HÄFELIN/MÜLLER/UHLMANN, Allgemeines Verwaltungsrecht, Rz. 49.

714 TSCHANNEN/ZIMMERLI/MÜLLER, Allgemeines Verwaltungsrecht, \$1 Rz. 20, 23.

715 Vgl. diese Diskussion unter dem Begriff Kräftegleichgewicht bei TSCHANNEN, Privatisierung, S. 238.

716 So HÄFELIN/MüLLER/UHLMANN, Allgemeines Verwaltungsrecht, Rz.1307; Bezug nehmend auf TSCHANNEN/ZIMMERLI/MÜLLER, Allgemeines Verwaltungsrecht, §28 Rz.15. 
wenn er zwingende Rechtsetzung und -anwendung in vermeintlich private Selbstregulierung und Zertifizierung hüllt. Der Staat gibt vor, Privaten auf Augenhöhe zu begegnen, wenn er konsensual mit ihnen verhandelt oder ihnen Rechtsetzungs- und Rechtsanwendungsaufgaben zur eigenen Erfüllung übergibt. Doch kann diese Augenhöhe bei der Erfüllung staatlicher Aufgaben nie bestehen - unabhängig vom Handlungsinstrument.

Und da das Machtgefälle zwischen dem Staat und den Rechtsunterworfenen unumgänglich ist, sind diese daran interessiert zu wissen, wann und wie sie zwingendem Recht unterworfen sind - also wann sie es mit dem Staat zu tun haben. Denn ausserhalb dieses Bereichs, in dem der Staat ihnen gegenüber einen Machtvorsprung besitzt, können sie frei und autonom ihre Interessen mit und gegenüber anderen Privaten verfolgen. ${ }^{717}$ Verwaltung und Verwaltungsadressat haben also spiegelbildliche Bedürfnisse: Der Staat möchte die generell-abstrakten und zwingenden Vorgaben des öffentlichen Rechts im Einzelfall verbindlich umsetzen; die Bürger wünschen Gewissheit darüber, was diese Vorgaben für ihre Rechte, Pflichten und tatsächlichen Umstände bedeuten.

Weshalb wählt ein Verwaltungsträger trotzdem privatisierte Handlungsformen? Am Beispiel des Verwaltungsvertrags: Nicht weil sie mehr bewirken könnten - der öffentliche Vertrag entfaltet dieselbe Wirkung wie eine Verfügung, und wenn immer ein Vertrag abgeschlossen wird, könnte auch verfügt werden. ${ }^{718}$ Nüchtern betrachtet bleibt wenig Positives am «konsensualen Konkretisieren»: Der Verwaltungsträger gibt Entscheidungsmacht auf und gewinnt dadurch höhere Chancen auf Nicht-durchsetzen-Müssen.

Nicht etwa steigt die Durchsetzbarkeit, nein; vielmehr hofft man, sich mit vertraglichem Handeln von der Pflicht zu befreien, eine rechtliche Regelung auf rechtlichem Weg durchsetzen zu müssen. Wenn die Verwaltungsadressaten in dieser Form am Verwaltungshandeln teilhatten, werden sie seltener gegen die vereinbarte Lösung rekurrieren, und der Verwaltungsträger muss nicht den beschwerlichen rechtsstaatlichen Weg gehen, seine Interessen gerichtlich durchzusetzen oder zu verteidigen (siehe hinten e).

Wie die privatisierten Handlungsformen das Verhalten der Verwaltungsadressaten beeinflussen, müsste empirisch untersucht werden, was diese rechtswissenschaftlich-theoretische Arbeit nicht zu leisten vermag. Die Analyse in den $\$ \S 5$ und 6 baut dennoch auf den soeben aufgestellten Vermutungen auf. 


\section{d. Geringere Steuerungskraft}

Während der Staat mit der instrumentellen Erfüllungsprivatisierung nur vermeintlich seinen Machtvorsprung aufgibt (soeben c), verzichtet er tatsächlich auf einen Teil seiner Steuerungskraft. Privatisierte Handlungsformen sind weniger verbindlich, weniger leicht durchsetzbar und einfacher abänderlich als hoheitliche Instrumente. Allgemein ausgedrückt übt der Staat weniger Zwang aus bei der Ausgestaltung der Rechte und Pflichten der Verwaltungsadressaten. Dieser Zwangsverzicht beschlägt die soeben angesprochenen drei Elemente der Steuerungskraft staatlichen Handelns: Trifft die Verwaltung Anordnungen, an die sich Private halten müssen (Verbindlichkeit)? Kann die Verwaltung die Einhaltung dieser Anordnungen erzwingen (Durchsetzbarkeit)? Und unter welchen Voraussetzungen sind die Anordnungen abänderlich (Rechtsbeständigkeit)?

Die geringere Verbindlichkeit zeigt sich etwa bei informellen Absprachen und Empfehlungen (soeben 2.a.dd). Mit einer Empfehlung versuchen staatliche Stellen, privates Verhalten zu lenken, indem sie sich über die «faktische Ratsamkeit bestimmter Verhaltensweisen» 719 äussern. Solche Äusserungen sind nicht rechtlich verbindlich, können aber dennoch erhebliche rechtliche Tragweite haben, insbesondere indem sie faktische Zwänge erzeugen, die im Ergebnis Rechtsverboten gleichkommen. ${ }^{720}$ Der Staat nimmt so eine neue Rolle ein: Er bedient sich nicht des faktischen Handelns, weil ihm rechtsförmiges Handeln nicht offenstünde (so typisch bei Realakten), sondern weil er seinen traditionellen Instrumenten freigewählt flexible, unverbindliche Formen beiseitestellen will. ${ }^{721}$ Es liesse sich kritisch fragen, warum der Staat sich nicht einfach entscheide, privates Verhalten entweder verbindlich zu regeln oder dann der Privatautonomie zu überlassen. Indem er mit unverbindlichen Mitteln zu steuern versucht, wird er somit weniger strikt und einschneidender zugleich.

Die geringere Durchsetzbarkeit wird insbesondere beim Vertrag deutlich. Die Verfügung ist bereits ein urteilsähnlicher Vollstreckungstitel, «während beim privatrechtlichen Vertrag im Falle des Verzugs erst noch auf Erfüllung geklagt werden muss» ${ }^{722}$. Will der Staat also die Rechte und Pflichten, die er mit einem Verwaltungsadressaten vertraglich geregelt hat, durchsetzen, muss er ein erneutes Rechtsverfahren einleiten.

719 TSCHANNEN/ZIMMERLI/MÜLLER, Allgemeines Verwaltungsrecht, §39 Rz. 3; allgemein auch Rz.1.

720 MÜLLER, Informales Staatshandeln, S. 536.

721 Vgl. MÜLLER-GRAF, Informalisierung, S. 105-107 zum «Alternativverhältnis» von unverbindlichen zu rechtlichen Handlungsformen.

722 TSCHANNEN/ZIMMERLI/MÜLLER, Allgemeines Verwaltungsrecht, §28 Rz.11. 
Wählt der Staat statt der Verfügung ein anderes Instrument, muss er verschiedene Fragen der Rechtsbeständigkeit im Einzelfall klären, die ansonsten bereits mit der Wahl der Verfügungsform geregelt wären. So sind etwa Wiedererwägung und Widerruf bei Verfügungen klar geregelt; bei einem Vertrag etwa muss vor Gericht auf Ungültigerklärung geklagt werden.723 Beim öffentlichrechtlichen Vertrag entsteht ein Spannungsverhältnis zwischen «Interesse an der Verwirklichung des objektiven Rechts» und «Vertrauen in die Beständigkeit des Vertrags» ${ }^{24}$. Verfügungen müssen abgeändert werden, wenn sie nachträglich rechtswidrig oder nicht mehr sachverhaltsgerecht werden. Bei Verträgen besteht grundsätzlich ein Vertrauen in deren Beständigkeit. Der Staat steht also vor einem Dilemma: Duldet er rechtswidrige Zustände, oder enttäuscht er Erwartungen, die er durch die Wahl eines privatisierten Instruments geweckt hat?

Mit diesem Kennzeichen ist nur belegt, dass die Steuerungskraft der Instrumente abnimmt, nicht zwingend deren Steuerungswirkung. Mit diesen Instrumenten strebt die Verwaltung weiterhin an, die rechtliche und faktische Lage von Privaten zu steuern. Die geringere Steuerungskraft kann durch gesteigerte Wirkung dank freiwilliger Befolgung kompensiert werden (dazu sogleiche).

\section{e. Gesteigerte Wirkungserwartung durch höhere Akzeptanz und freiwillige Befolgung}

Die geringere Steuerungskraft ist kein Versehen - der Staat setzt bewusst auf weniger zwingende Mittel und erhofft sich im Gegenzug eine höhere Akzeptanz und freiwillige Befolgung der angeordneten Pflichten. Was kümmert den Staat also die umständlicher werdende Durchsetzbarkeit, wenn die Verwaltungsadressaten ihre Pflichten zwanglos befolgen, weil sie sich ernst genommen und auf Augenhöhe begegnet fühlen.

Diese Überlegung ist eine der wichtigsten Begründungen für die Wahl der Vertragsform vor der Verfügung. Damit diese Form gewählt werden darf, muss sie (im Fall des öffentlichrechtlichen Vertrags) besser geeignet oder (bei der zivilrechtlichen Variante) vernünftigerweise unumgänglich sein. ${ }^{725}$ Zumindest die bessere Eignung lässt sich damit begründen, dass Kooperation zwischen Staat und Privaten «die Akzeptanz staatlicher Regelungen und damit

723 TSCHANNEN/ZIMMERLI/MÜLLER, Allgemeines Verwaltungsrecht, §35 Rz.14, siehe auch \$31 Rz. 8f., §35 Rz.3-13.

724 TSCHANNEN/ZIMMERLI/MÜLLER, Allgemeines Verwaltungsrecht, §35 Rz.9. Ähnlich auch HÄNER, Einwilligung, S. 61.

725 Siehe TSCHANNEN, Systeme, Rz.142 f. und TSCHANNEN/ZIMMERLI/MÜLLER, Allgemeines Verwaltungsrecht, $\$ 42 \mathrm{Rz} .3$. 
deren Durchsetzungschancen verbesser[t]»726. Kooperative Handlungsformen zeichnen sich unter anderem dadurch aus, dass ihr Entstehen und Abschliessen zweiseitig ist; Private wirken mit und haben an der Festlegung der Rechte und Pflichten teil.

Bei anderen privatisierten Handlungsformen erwartet der Staat eine gesteigerte Wirkung, indem Private freiwillige, unverbindliche und informelle Regelungen mehr respektieren. An informelle Absprachen würden sich Private etwa halten aus Dankbarkeit, kein förmliches Verfahren durchlaufen zu müssen. Ähnliche Überlegungen dürften bei Auskünften und Zusicherungen zutreffen. Die in dieser Arbeit eingesetzte Methodik kann diese Vermutungen über die verhaltenspsychologischen Auswirkungen des Staatshandelns allerdings nicht empirisch prüfen.

Eine ähnliche Überlegung kann hinter der Wahl finaler Normen (anstelle konditionaler Normen) stehen: Private schätzen die unverbindliche Lenkung über Zielvorgaben und beachten diese, ohne dass der Staat klare Pflichten formulieren und durchsetzen müsste.

Zudem verspricht sich der Staat wohl eine gesteigerte Wirkung bei denjenigen Formen, bei denen er betroffene Private in die Setzung und Anwendung der sie betreffenden Regelungen miteinbezieht (siehe vorne 2.b.cc). Bei den ausgelagerten und überwachten Formen der Normsetzung und Prüfung werden die Verwaltungsadressaten selbst oder ihre Vertreter aktiv - was ihre Bereitschaft zur Befolgung steigern sollte. ${ }^{727}$

Diese Beispiele und Mutmassungen zeigen es: Dieses Kennzeichen ist nicht handfest rechtswissenschaftlich. Es handelt sich vielmehr um Erwartungen und Hoffnungen der Verwaltung. Die Lehre diskutiert diese Motive vor allem beim Wechsel von der Verfügung zum Vertrag. Oder wie es TSCHANNEN lapidar mutmasst: «[D]er Hang zur Vertragsform im Verwaltungsrecht habe vor allem psychologische Gründe» 728 .

\section{f. Inkaufnahmevon Interessenkonflikten}

Je weniger verbindlich, hoheitlich und einseitig die Verwaltung handelt, umso mehr ist sie auf die Kooperation der Verwaltungsadressaten angewiesen, um die verwaltungsrechtlichen Rechte und Pflichten durchzusetzen. Bei gewissen privatisierten Handlungsformen nimmt die Verwaltung Interessenkonflikte in Kauf, die dieser Durchsetzung entgegenlaufen könnten. ${ }^{729}$

726 HÄFELIN/MÜLLER/UHLMANN, Allgemeines Verwaltungsrecht, Rz.1307.

727 GILI, Selbstregulierung, S.146f.

728 TSCHANNEN/ZIMMERLI/MÜLLER, Allgemeines Verwaltungsrecht, \$33 Rz. 28 a.E.

729 Vgl. sinngemäss HETTICH, Kooperative Risikovorsorge, Rz. 637. 
Besonders deutlich zeigt sich dies bei Formen der ausgelagerten Rechtsetzung und Rechtsanwendung (siehe soeben 2.b.cc). Dort bezieht der Staat die Verwaltungsadressaten selbst oder deren Vertreter in die Normsetzung oder die Prüf- und Kontrollaufgaben ein. Die Privaten haben dabei ein Interesse an einer möglichst lockeren Handhabung der rechtlichen Grundlagen oder an einer möglichst wohlwollenden Kontrolle der Einhaltung derselben. Die Privaten kommen dabei in eine Doppelrolle: als Adressaten einer Regelung, die sie selber mitprägen oder kontrollieren.

Der Staat nimmt dieses gegenläufige Interesse in Kauf und baut es direkt in die Aufgabenerfüllung ein, weil er sich davon Kooperation und Akzeptanz verspricht. ${ }^{730}$ Diese Erwartung mag zutreffen - was bleibt, ist, dass der Staat einen Interessenkonflikt gewissermassen institutionalisiert.

\section{g. Einbindung von externem Fachwissen}

Einige der privatisierten Handlungsformen zeichnen sich durch die Auslagerung von verwaltungseigenem Fachwissen aus. Wenn private Organisationen mit staatlichen Aufgaben betraut werden, weil sie näher an der Praxis und auf dem aktuellsten Wissensstand sind, muss deren Fachwissen mit massgeschneiderten Handlungsformen wieder in die Verwaltungsarbeit einbezogen werden. ${ }^{731}$

Dafür sind insbesondere die ausgelagerte Normsetzung und Prüftätigkeit mit beibehaltenen Einflussmöglichkeiten der Verwaltung geeignet. Diese Instrumente werden gerade besonders in Bereichen eingesetzt, die «eine hohe Dynamik, hohe Komplexität oder grosse Wissenslücken» ${ }^{732}$ aufweisen.

Diese Motivation steht auch hinter anderen privatisierten Handlungsformen. Wenn die Verwaltung selbst einen Sachbereich für zu komplex hält, kann dies ihre Bereitschaft steigern, lieber mittels Verträgen zu kooperieren, statt mit Verfügungen zu ordnen, oder lieber unverbindlich zu planen oder abzusprechen, denn rechtssatzförmig zu gestalten.

Die Zurückdrängung der Verfügung als die prägende, typische Handlungsform der Verwaltung ist symptomatisch für die Annahme, die Verwaltung sei selber nicht kompetent genug, eine Frage abschliessend und hoheitlich zu regeln.

730 Vgl. GILI, Selbstregulierung, S.146 f.

731 Diese Überlegungen besonders im Bereich der Medizin betonend GILI, Selbstregulierung, S.140-142.

732 HETTICH, Kooperative Risikovorsorge, Rz. 636. 


\section{h. Erschwerte Abgrenzung zwischen staatlicher Aufgabenerfüllung und privater Tätigkeit}

Staatstätigkeit definiert sich über die Staatlichkeit der erfüllten Aufgabe, nicht über die rechtliche Einordnung der handelnden Akteure oder der Instrumente, die diese einsetzen. Auch mit privatrechtlichen Verträgen und durch private Akkreditierungsstellen können staatliche Aufgaben erfüllt werden. Doch der Einsatz privatisierter Handlungsformen erschwert die Abgrenzung zwischen staatlicher und privater Tätigkeit. ${ }^{733}$

Die Grenze ist dünn zwischen freiwilliger Befolgung öffentlichen Rechts und dem Erlass von Selbstregulierungsnormen - nach aussen können beide Tätigkeiten identisch erscheinen. Ob im zweiten Fall ein staatliches Handeln vorliegt, hängt daran, ob der Erlass dieser Reglemente gesetzlich befohlen ist (siehe vorne 2.b.cc). ${ }^{734}$ Denn daran entscheidet sich die Aufgabenqualität: an der Übertragung einer Tätigkeit mit der Pflicht, sie auch zu erfüllen (siehe vorne $2 /$ I.2.b).

In Kenntnis der jeweiligen Rechtsgrundlage bleibt die Grenze also weiterhin einwandfrei zu ziehen. Doch im Rechtsverkehr wird es für Private schwieriger zu erkennen, mit wem sie es zu tun haben. Mitunter kann es sogar für die Aufgabenträger selber schwerer erkennbar werden, ob und wie weit sie staatliche Aufgaben wahrnehmen.

\section{i. Ziviles Verfahrensrecht bei zivilrechtlichen Verträgen}

Während einige der soeben aufgeführten Kennzeichen eher im Bereich der Beweggründe und Erwartungen liegen, gibt es auch handfest juristische Konsequenzen aus der Wahl privatisierter Handlungsformen. Dies trifft insbesondere auf das Verfahrensrecht zu. Die Wahl des Instruments kann den zivilen Verfahrensweg mit sich bringen oder zumindest Unsicherheit über das einschlägige Verfahrensrecht.

Ziviles Verfahrensrecht kommt etwa zum Zug bei Streitigkeiten über den Inhalt privatrechtlicher Verträge. ${ }^{735}$ Unsicherheit besteht vor allem im Bereich des Beschaffungswesens, wo die Theorie im Raum steht, es müsse ein verwaltungsinterner Willensbildungsprozess vom Vertragsabschluss abgeschieden werden, der dem öffentlichen Verfahrensrecht zugänglich ist. ${ }^{736}$ «Es liegt

733 Siehe bezüglich Zertifizierung im Produktesicherheitsbereich BÜREN, Akkreditierte Zertifizierung, S. 217-221.

734 Wie z.B. im Bereich der Finanzmarktaufsicht, vgl. Art. 25 GwG.

735 HÄFELIN/MÜLLER/UHLMANN, Allgemeines Verwaltungsrecht, Rz.1393.

736 Siehe zu dieser Zweistufentheorie ausführlich HÄFELIN/MÜLLER/UHLMANN, Allgemeines Verwaltungsrecht, Rz.1394-1403. 
nahe, mindestens in Fällen mit ausgewiesenem Rechtsschutzbedarf auf die Zweistufentheorie (und weitere Prinzipien des öffentlichen Beschaffungsrechts) zurückzugreifen.» ${ }^{737}$ Das Bundesgericht übernimmt diese Linie aber nicht vollends. Es bleibt also denkbar, dass gewisse Lücken im Rechtsschutz bestehen bleiben. Im Unterschied zur Verwaltungsrechtspflege geht es im Zivilprozess nämlich nicht um die «objektive Rechtmässigkeit des Verwaltungshandelns», sondern «ausschliesslich um den Schutz subjektiver Rechte der Parteien».738

Ausserhalb der privatrechtlichen Verträge herrscht weiterhin das öffentliche Verfahrensrecht vor. Für die - dem Erscheinen nach - privatrechtlichen Vorgänge der ausgelagerten Rechtsetzung und der Akkreditierung wählt der Gesetzgeber mitunter den Weg über Verfügungen der letztendlich verantwortlichen Verwaltungseinheit, womit dann der Rechtsschutz über die Bundesrechtspflege läuft.739

\section{Zwischenfazit: Die Verwaltung verzichtet auf hoheitliche Vorrechte und hofft auf dankbare Verwaltungsadressaten}

Die instrumentelle Erfüllungsprivatisierung ist geprägt vom freiwilligen und kreativen Verzicht auf die klassischen Werkzeuge des Staats. Freiwillig, weil privatrechtliche Instrumente nie alternativlos sind. Und kreativ, weil immer neue (Einzelfall-)Instrumente geschaffen werden, um Verfügung und Rechtssatz zu vermeiden. Die Wahl von privatisierten Handlungsformen führt dazu, dass der Staat Privaten vermeintlich auf Augenhöhe begegnet. Er verhandelt mit ihnen, einigt sich mit ihnen auf ihre Rechte und Pflichten, übergibt ihnen die sie betreffende Regelung zum eigenen Erlass und Vollzug. Dies nicht, weil die hoheitlichen Mittel ungeeignet wären, sondern weil er sich davon höhere Akzeptanz seiner Tätigkeit verspricht.

Die Verwaltung scheint dabei anzunehmen, sie müsse ihre Staatlichkeit möglichst verdecken, um überhaupt noch etwas zu erreichen. Sie könne ihre staatlichen Aufgaben also am besten erfüllen, wenn sie nicht als Staat wahrgenommen wird. Dass diese Herangehensweise zu Instrumenten führt, die Verwirrung und Interessenkonflikte stiften, liegt auf der Hand - ist aber ausdrücklich gewollt, im Dienste unkomplizierter und effektiver Verwaltungsarbeit.

737 HÄFELIN/MÜLLER/UHLMANN, Allgemeines Verwaltungsrecht, Rz.1403.

738 E contrario TSCHANNEN/ZIMMERLI/MÜLLER, Allgemeines Verwaltungsrecht, §28 Rz.14. 739 Siehe etwa Art. 54 FINMAG und Art. 65 HFKG. 


\section{Privatisierung der Finanzierungsformen}

Private nehmen den Staat über seine Organisationsformen wahr und über die Instrumente, die er einsetzt. Ebenso direkt betrifft sie aber die Art und Weise, wie staatliche Tätigkeit finanziert wird. Für den Staat ist typisch, dass er über Steuermittel verfügt, mit denen er seine Aufgaben erfüllt. In diesem Abschnitt ist zu untersuchen, wie es aussieht, wenn die Finanzierungsformen privatisiert werden. Wenn also der Staat seine Aufgabenerfüllung auf eine Weise finanziert, die privater Tätigkeit ähnelt.

Wiederum geht es nicht um die Schaffung neuer dogmatischer Strukturen. Zunächst wird der Gegenstand dieses Abschnitts definiert (1). Bevor die einzelnen Arten der privatisierten Finanzierung staatlicher Aufgaben dargestellt werden (3), erfolgt eine Übersicht der Systematik der öffentlichen Abgaben (2). Die gefestigte Dogmatik zu den öffentlichen Abgaben dient hier also als Ausgangspunkt. Wie bei den organisatorischen und instrumentellen Aspekten auch werden am Ende die Kennzeichen der Erfüllungsprivatisierung in finanzieller Hinsicht herausgearbeitet (4).

\section{Begriff und Bedeutung der Finanzierungsformen}

Die finanziellen Erfüllungsaspekte beschlagen die Finanzierung der Aufgabenerfüllung aus Sicht der Verwaltungsadressaten. Wer deckt die Kosten, die bei der Erfüllung staatlicher Aufgaben anfallen? Wer zahlt Löhne, Mieten, Materialkosten und andere Aufwendungen? Und nach welchen Massstäben richtet sich die Kostendeckung?

Aus Sicht der Verwaltungsadressaten sind zwei Teilaspekte besonders bedeutsam: Inwiefern hängt ihr Beitrag an die staatliche Aufgabenerfüllung von ihrem eigenen Verhalten ab? Wie steht ihr Beitrag im Verhältnis zu anderen Verwaltungsadressaten? Sprich: Zahlen sie nur für staatliche Tätigkeiten, die sie verursachen und benutzen, und zahlen sie gleich viel wie andere Private?

Privatisierte Finanzierungsformen bedeuten eine Verschiebung der hauptsächlichen Kostentragepflicht: Mit der klassisch staatlichen Finanzierung, der Steuererhebung nach dem Gemeinlastprinzip finanzieren Private das Gemeinwesen insgesamt, und dieses deckt dann die Kosten für die einzelnen Erfüllungstätigkeiten. Wird diese Finanzierung privatisiert, tragen Private direkt die Kosten für das Verwaltungshandeln: Mit Gebühren und Abgaben, die aus dem Empfang staatlicher Leistung oder der Erduldung staatlicher Eingriffe anfallen. Bei der privatisierten Finanzierung kommt nicht mehr der Staat selbst für die Kosten der Erfüllung seiner Aufgaben auf. 
Dieser Vorgang ist als Finanzierungsprivatisierung740 bekannt (siehe vorne \$2/II.5.e). Finanzielle Erfüllungsprivatisierung heisst also, dass das Budget eines Aufgabenträgers ausserhalb des üblichen Staatshaushalts liegt, die Aufgabenerfüllung mithin nicht über Steuermittel finanziert wird. Dann wird die Aufgabenerfüllung zu einem Teil dem Verursacher angelastet. Leistungsbezüger zahlen die vollen Kosten der beanspruchten Dienste individuell. Die staatliche Finanzierung des Verwaltungsbetriebs kann ganz eingestellt werden, wie unter Umständen auch die staatliche Vorfinanzierung der nötigen Infrastruktur. ${ }^{741}$

Diese Verlagerung der Kostentragepflicht verfolgt das Ziel, die Aufgabenerfüllung "wirtschaftlicher» ${ }^{742}$, sprich günstiger, zu gestalten. Steuerfinanzierte Leistungen würden als gratis empfunden und deshalb überbenutzt. Die Bürger sollen zu Verbrauchern werden, die kostenbewusst und gezielt konsumieren und so insgesamt die Nachfrage nach staatlichen Leistungen senken. ${ }^{743}$ Diese Spielart der Privatisierung ist am direktesten mit der Erwartung verbunden, die Staatstätigkeit würde dadurch günstiger.

Die finanziellen Aspekte der Aufgabenerfüllung liegen quer zu den organisatorischen und den instrumentellen. Während sich diese zwei Aspekte auf die unmittelbare Aufgabenerfüllung beziehen, verläuft bei der Finanzierung die Trennlinie zwischen mittelbarer und unmittelbarer Aufgabenerfüllung. Es geht um die Bereitstellung der Mittel für die Aufgabenerfüllung also um Bedarfs- und wirtschaftende Verwaltung. Dazu kommt, dass die Erhebung von Steuern und Abgaben selbst eine Form der Eingriffsverwaltung darstellt. 744

Ebenso muss die privatwirtschaftliche Tätigkeit des Staats miteinbezogen werden; auch sie kann direkt die Aufgabenerfüllung (mit-)finanzieren. Wenn der Staat seine Aufgabenerfüllung quersubventioniert mit Erträgen aus freigewählter Erwerbstätigkeit im Wettbewerb (und damit ausserhalb seines Aufgabenbereichs), ist dies eine Form der privatisierten Finanzierung: Konsumenten finanzieren dann Strukturen, die der Erfüllung staatlicher Aufgaben dienen.

Allerdings ist die Finanzierungsprivatisierung von der Vermögensprivatisierung abzugrenzen. Bei diesem Vorgang veräussert der Staat eigene Vermö-

740 HÄFELIN/MÜLLER/UHLMANN, Allgemeines Verwaltungsrecht, Rz. 1871; TSCHANNEN/ ZIMMERLI/MÜLLER, Allgemeines Verwaltungsrecht, \$11 Rz. 4 a.E.

741 TSCHANNEN, Privatisierung, S. 213.

742 Etwa FREY, Drei Stossrichtungen, S. 343; MÜLLER/JENNI, Privatisierung, S.1072.

743 HANDSCHIN/SIEGENTHALER, Privatisierung, S. 512, identifizieren «nachfrageoptimierten Ressourceneinsatz» als Hauptmotiv hinter Privatisierungen.

744 TSCHANNEN/ZIMMERLI/MÜLLER, Allgemeines Verwaltungsrecht, §4 Rz.10. 
genswerte, um Einnahmen zu erzeugen. ${ }^{745}$ Solche Veräusserungen können die Aufgabenerfüllung finanzieren, weil sie die Staatskasse äufnen, doch sind sie ein gesondertes Phänomen, das mit der Privatisierung der Aufgabenerfüllung an sich nicht direkt verbunden ist.

\section{Das System der öffentlichen Abgaben}

«Öffentliche Abgaben sind alle öffentlichrechtlichen Geldleistungen, die das GemeinwesengestütztaufseineFinanzkompetenz den Privaten auferlegt.»>746 Das System der öffentlichen Abgaben ist also konzipiert, um zu erörtern, welche Gelder der Staat einziehen darf. Die finanziellen Aspekte der Aufgabenerfüllung gehen aber darüber hinaus. Sie beschlagen jegliche Quelle, die die Erfüllung staatlicher Aufgabe finanziert. Als Ausgangspunkt soll hier dennoch das System der öffentlichen Abgaben rekapituliert werden.

Der Bund braucht eine Grundlage in Verfassung oder Gesetz, um öffentliche Abgaben zu erheben, ein Numerus clausus von Abgabearten besteht aber nicht. ${ }^{747}$ Daher kann es vorkommen, dass Abgaben im Einzelfall schwierig einzuordnen sind. Grundsätzlich lassen sich aber drei Kategorien von öffentlichen Abgaben ausscheiden:

- Abgaben, die ohne Verbindung zu einer staatlichen Leistung stehen, heissen Steuern. Dies sind voraussetzungslos geschuldete Abgaben. ${ }^{748}$ Abhängig davon, ob die Steuern der allgemeinen Staatskasse zufliessen oder für bestimmte Verwendungszwecke vorgemerkt sind, lassen sie sich in allgemeine und Sondersteuern unterscheiden. ${ }^{749}$

- Als Gegenstück dazu werden alle Abgaben, die als Gegenleistung für eine bestimmte staatliche Leistung erhoben werden, als Kausalabgaben kategorisiert. ${ }^{750}$ In diese Kategorie fallen Gebühren, Vorzugslasten, Ersatzabgaben und Mehrwertabgaben. ${ }^{751}$ In direktestem Bezug zur Erfüllung

745 HÄFELIN/MÜLLER/UHLMANN, Allgemeines Verwaltungsrecht, Rz. 1871.

746 TSCHANNEN/ZIMMERLI/MÜLLER, Allgemeines Verwaltungsrecht, §57 Rz.1. Ähnlich auch HÄFELIN/MÜLLER/UHLMANN, Allgemeines Verwaltungsrecht, Rz. 2753.

747 TSCHANNEN/ZIMMERLI/MÜLLER, Allgemeines Verwaltungsrecht, §57 Rz. $2 \mathrm{f}$.

748 BIAGGINI, Kommentar BV, Art. 127 Rz. 5; HÄFELIN/MÜLLER/UHLMANN, Allgemeines Verwaltungsrecht, Rz.2758, 2830; TSCHANNEN/ZIMMERLI/MÜLLER, Allgemeines Verwaltungsrecht, $\$ 57$ Rz.7.

749 TSCHANNEN/ZIMMERLI/MÜLLER, Allgemeines Verwaltungsrecht, §57 Rz. 8-10.

750 HÄFELIN/MÜLLER/UHLMANN, Allgemeines Verwaltungsrecht, Rz. 2758; TSCHANNEN/ ZIMMERLI/MÜLLER, Allgemeines Verwaltungsrecht, \$57 Rz.18; WIEDERKEHR, Kausalabgaben, S.9.

751 TSCHANNEN/ZIMMERLI/MÜLLER, Allgemeines Verwaltungsrecht, §57 Rz. 20-38. Abweichend bzgl. Mehrwertabgaben WIEDERKEHR, Kausalabgaben, S. 5. 
staatlicher Aufgaben stehen dabei die Gebühren, welche leistungs- und meist auch kostenabhängig erhoben werden. Sie fallen nur an, wenn tatsächlich eine staatliche Leistung beansprucht wird, und sie sollen die genau dadurch entstehenden Kosten decken. ${ }^{752}$

- Als dritte Kategorie werden die Gemengsteuern ausgeschieden. Sie sind Abgaben, die zwar als Gegenleistung für eine staatliche Leistung anfallen, aber nicht kostenabhängig sind. ${ }^{753}$ Sie sind also dem Entstehungsgrund nach den Kausalabgaben nahe, liegen aber höher, als es bei diesen zulässig wäre. Zum Beispiel kann das kantonale Recht den Tarif für Notariatsleistungen höher ansetzen, als es der vom Notar geleistete Aufwand rechtfertigen würde. ${ }^{754}$

Diese Kategorisierung ist rechtlich bedeutsam, weil je nach Abgabeart andere Prinzipien zur Anwendung kommen. Für die Steuern setzt die Verfassung selbst die einschlägigen Prinzipien fest. Art.127 Abs. 2 BV verpflichtet die Steuererhebung auf die Grundsätze der Allgemeinheit, der Gleichmässigkeit und der wirtschaftlichen Leistungsfähigkeit. Die ersten beiden Prinzipien gebieten, dass alle Personen in gleicher Weise der Steuerordnung unterliegen, ohne Steuerprivilegien, und dass die Rechtsgleichheit auch in der Besteuerung beachtet wird. 755 «Besteuerung nach wirtschaftlicher Leistungsfähigkeit» ist ein Ausdruck der Verhältnismässigkeit und bedeutet, Geringverdienende leichter und Gutverdienende stärker zu belasten. Pauschalisierungen sind dabei zulässig, eine individuelle Steuerberechnung ist nicht gefordert. 756

Bei den gegenleistungsbasierten Kausalabgaben kommen zwei andere Prinzipien zur Anwendung: das Kostendeckungs- und das Äquivalenzprinzip. Ihnen kommt aber ein anderes Gewicht zu als den steuerlichen Prinzipien: Sie gelten nämlich nicht generell, sondern sind lediglich mögliche Kompensationsmechanismen für eine gelockerte gesetzliche Grundlage; sie können

752 HÄFELIN/MÜLLER/UHLMANN, Allgemeines Verwaltungsrecht, Rz. 2764; TSCHANNEN/ ZIMMERLI/MÜLLER, Allgemeines Verwaltungsrecht, \$57 Rz. $20 \mathrm{f}$.

753 BGE 140 I 176 E. 5.3 S. 181; HÄFELIN/MÜLLER/UHLMANN, Allgemeines Verwaltungsrecht, Rz. 2866; TSCHANNEN/ZIMMERLI/MÜLLER, Allgemeines Verwaltungsrecht, §57 Rz. 39.

754 BGE130 III 225 E. 2.5 S. 230; nebenbei: «Die Zulässigkeit einer Gemengsteuer beruht hier darauf, dass das Bundeszivilrecht die Steuerhoheit der Kantone nicht beschränkt».

755 BIAGGINI, Kommentar BV, Art.127 Rz. 8, 10 f.; TSCHANNEN/ZIMMERLI/MÜLLER, Allgemeines Verwaltungsrecht, \$58 Rz. $5 \mathrm{f}$.

756 BIAGGINI, Kommentar BV, Art. 127 Rz.12; TSCHANNEN/ZIMMERLI/MÜLLER, Allgemeines Verwaltungsrecht, $\$ 58$ Rz. 7; insbesondere sind degressive Einkommenssteuern verboten. Kursorisch auch HÄFELIN/MÜLLER/UHLMANN, Allgemeines Verwaltungsrecht, Rz. 2845 . 
also einen Mangel im Gesetzmässigkeitsprinzip beheben. ${ }^{757}$ «Das Kostendeckungsprinzip besagt, dass die Gesamteingänge an Abgaben den Gesamtaufwand für den betreffenden Verwaltungszweig nicht oder höchstens geringfügig überschreiten sollen» ${ }^{758}$. Das Kostendeckungsprinzip bindet also den Verwaltungsaufwand insgesamt an die erhobenen Abgaben - eine Abgabe soll nicht mehr einbringen, als im betreffenden Verwaltungszweig aufgewendet wird. Das Äquivalenzprinzip beschlägt dagegen die einzelne Kausalabgabe: «Es bestimmt, dass eine Gebühr nicht in einem offensichtlichen Missverhältnis zum objektiven Wert der Leistung stehen darf und sich in vernünftigen Grenzen halten muss.» ${ }^{759}$ Es konkretisiert damit das Verhältnismässigkeitsprinzip und das Willkürverbot.

Das Kostendeckungs- und das Äquivalenzprinzip scheinen eher den Gehalt von optionalen Argumenten aufzuweisen denn von standfesten Prinzipien, die diesen Namen verdienen. ${ }^{760}$ Der Gesetzgeber ist bei der Ausgestaltung von Kausalabgaben weitgehend frei, welche Kosten in welchem Ausmass damit gedeckt werden sollen.

«Bei der Prüfung der Frage, ob eine Kausalabgabe nicht in einem offensichtlichen Missverhältnis zur erbrachten Leistung steht, ist auch dem Umstand Rechnung zu tragen, ob diese von Gesetzes wegen die vollen Kosten der Behörde zu decken bzw. eine rein verursacherorientierte Finanzierung bezweckt [...]. Eine derart weitgehende finanzielle Belastung - im Sinne einer uneingeschränkten Verursacherfinanzierung - kann unter Umständen das Äquivalenzprinzip verletzen, wenn der Wert der staatlichen Leistung bzw. Handlung nicht allein dem Abgabepflichtigen zugerechnet werden kann bzw. wenn die vom Staat erbrachte Leistung auch der Verwirklichung öffentlicher Interessen dient» ${ }^{761}$.

Kausalabgaben sind generell eine Möglichkeit, die Kosten der Verwaltungstätigkeit auf Private zu überwälzen.

757 Anstelle vieler BGE 143 II 283 E. 3.5 S 292. Ferner BIAGGINI, Kommentar BV, Art. 127 Rz. 6; HÄFELIN/MÜLLER/UHLMANN, Allgemeines Verwaltungsrecht, Rz. 2762, 2806.

758 Anstelle vieler BGE 143 II 283 E. 3.7.1 S. 293. Ferner BIAGGINI, Kommentar BV, Art. 127 Rz. 6; HÄFELIN/MÜLLER/UHLMANN, Allgemeines Verwaltungsrecht, Rz. 2760; TSCHANNEN/ZIMMERLI/MÜLLER, Allgemeines Verwaltungsrecht, §58 Rz.13.

759 Anstelle vieler BGE 143 I 147 E. 6.3.1. S.158; auch zum Folgenden. Ferner HÄFELIN/ MÜLLER/UHLMANN, Allgemeines Verwaltungsrecht, Rz. 2761.

760 WIEDERKEHR, Legalitätsprinzip im Kausalabgaberecht, S. 50. Ansatzweise in diese Richtung auch TSCHANNEN/ZIMMERLI/MÜLLER, Allgemeines Verwaltungsrecht, §59 Rz.13.

761 WIEDERKEHR/RICHLI, Praxis des allgemeinen Verwaltungsrechts, Rz. $566 \mathrm{f}$. 


\section{Einzelne Finanzierungsformen}

Im Folgenden werden einzelne Finanzierungsformen untersucht, um herauszuarbeiten, welche als privatisierte Aufgabenerfüllung eingestuft werden können. Das soeben rekapitulierte, bewährte System der öffentlichen Abgaben dient dabei als Ausgangspunkt. Steuern werden im Folgenden nicht weiter vertieft.

\section{a. Verwaltungsgebühren und Benutzungsgebühren}

Mit Verwaltungsgebühren zahlen Verwaltungsadressaten für Amtshandlungen, die sie veranlasst haben, etwa für die Erstellung einer Ausweiskopie oder die Ausstellung einer Bewilligung, um die sie ersucht haben.762 Benutzungsgebühren fallen an für die Inanspruchnahme öffentlicher Einrichtungen, wie etwa Studiengebühren oder Parkgebühren. ${ }^{763}$ Beide Gebührenarten sind also direkte Entgelte für staatliche Tätigkeiten oder Angebote. ${ }^{764}$

Solche Gebühren können einen Bestandteil eines selbsttragenden Finanzierungsmodells darstellen, der von anderen Abgabearten ergänzt wird. So erheben etwa die FINMA und die Swissmedic Verwaltungsgebühren für ihre Aufsichtsleistungen und Kontrollen. ${ }^{765}$ Jährliche Pauschalabgaben decken sodann die Kosten, die von diesen Verwaltungsgebühren nicht gedeckt sind. 766

Es mag seltsam anmuten, diese geradezu stereotyp staatlichen Finanzierungsformen unter dem Stichwort Finanzierungsprivatisierung anzuführen. Sie gehören aber hierhin, weil sie Ausdruck eines bewussten Entscheids sind: Die Kosten, die für Ausweise, Bewilligungen, Bildungseinrichtungen und Parkplätze anfallen, könnten auch über die allgemeinen Steuermittel gedeckt werden. Stattdessen setzt der Staat auf das Verursacherprinzip und bindet das Empfangen einer staatlichen Leistung an eine Kostenfolge. Bei Benutzungsgebühren ist es denn auch gestattet, mit ihrer Höhe einen Lenkungseffekt zu verfolgen, ${ }^{767}$ also Anreize zu setzen für das Ob und Wie der Inanspruchnahme staatlicher Angebote.

762 TSCHANNEN/ZIMMERLI/MÜLLER, Allgemeines Verwaltungsrecht, §57 Rz. 23; WIEDERKEHR, Kausalabgaben, S. 38.

763 TSCHANNEN/ZIMMERLI/MÜLLER, Allgemeines Verwaltungsrecht, § 57 Rz. 25; WIEDERKEHR, Kausalabgaben, S. $40 \mathrm{f}$.

764 Weitere Beispiele bei FREY, Drei Stossrichtungen, S.344f., 348 f., 351 f.: Kehrichtabfuhr, Kehrichtverbrennung, Kläranlagen, öffentlicher Verkehr (benutzergerechte Tarifpolitik).

765 Art.15 Abs.1Satz1 FINMAG und Art.65 Abs.1 HMG.

766 So ausdrücklich Art. 15 Abs. 1 Satz 2 FINMAG und Art. 65 Abs. 4 und 5 HMG.

767 TSCHANNEN/ZIMMERLI/MÜLLER, Allgemeines Verwaltungsrecht, §57 Rz. 27. 


\section{b. Abgaben zur Kostenanlastung zwischen Steuern und Kausalabgaben}

Die dogmatisch bewährte Ordnung der öffentlichen Abgaben geht von einer klaren Zweiteilung auf: auf der einen Seite die voraussetzungslosen Steuern, auf der anderen die an Gegenleistungen geknüpften Kausalabgaben. Mit zwei Abgabearten wird diese Trennlinie von beiden Seiten her aufgeweicht. ${ }^{768}$ Es handelt sich um die (begrifflichen wie konzeptuellen Ungetüme) Kostenanlastungssteuer und Kostenanlastungskausalabgabe.

Die Kostenanlastungssteuer ist eine Sondersteuer, die einer bestimmten Gruppe von Personen auferlegt wird, weil sie «zu bestimmten Aufwendungen des Gemeinwesens eine nähere Beziehung aufweisen als die Gesamtheit der Steuerpflichtigen $\gg 769$. Es ist allerdings nicht vorausgesetzt, dass diese Personen «einen konkreten besonderen Nutzen» haben, die «abstrakte Interessenlage des belasteten Personenkreises» reicht aus. ${ }^{770}$ Deshalb handelt es sich um voraussetzungslose Abgaben, also Steuern, die aber zweckgebunden erhoben werden.771 Gängige Beispiele sind etwa Kurtaxen, Motorfahrzeugsteuern und Strassenreinigungsabgaben. ${ }^{772}$

Kostenanlastungskausalabgaben sind hingegen an Voraussetzungen geknüpfte Abgaben - nur dass die Voraussetzung lockerer ist als bei gewöhnlichen Kausalabgaben. Die vermeintlich harte Unterscheidung ist aber in Wahrheit eine weiche: Der Konnex zum besonderen Nutzen bestimmter Personen ist bei dieser Abgabe nur etwas enger als bei der steuerlichen Variante. ${ }^{773}$ Nämlich ist auch hier kein einzeln nachweisbarer Nutzen verlangt, er liegt vielmehr zwischen individuumsbezogenem und gruppenbezogenem Sondervorteil. 774 Die Relevanz dieser Abgabeart liegt vor allem darin, dass sie eine Aufweichung des Äquivalenzprinzips bedingt: Das Verhältnis zum erhaltenen Nutzen wird an einer «qualifizierten Gruppenäquivalenz» gemessen. ${ }^{775}$ So hält es auch das Bundesgericht: Es «qualifiziert nicht jede Abgabe, der kein individueller Sondernutzen der Abgabepflichtigen gegenübersteht, als Steuer. [...] In der Lehre wird denn auch postuliert, dass Sonderabgaben mit besonderem, gruppen-

\footnotetext{
768 WIEDERKEHR/RICHLI, Praxis des allgemeinen Verwaltungsrechts, Rz.966.

769 TSCHANNEN/ZIMMERLI/MÜLLER, Allgemeines Verwaltungsrecht, §57 Rz.13. Ähnlich auch HÄFELIN/MÜLLER/UHLMANN, Allgemeines Verwaltungsrecht, Rz. 2839.

770 WIEDERKEHR, Kausalabgaben, S. 22.

771 TSCHANNEN/ZIMMERLI/MÜLLER, Allgemeines Verwaltungsrecht, §57 Rz.13; WIEDERKEHR, Kausalabgaben, S. 24.

772 TSCHANNEN/ZIMMERLI/MÜLLER, Allgemeines Verwaltungsrecht, \$ 57 Rz.13; WIEDERKEHR, Kausalabgaben, S. 22.

773 WIEDERKEHR/RICHLI, Praxis des allgemeinen Verwaltungsrechts, Rz. 963.

774 TSCHANNEN/ZIMMERLI/MÜLLER, Allgemeines Verwaltungsrecht, §57 Rz.14.

775 WIEDERKEHR/RICHLI, Praxis des allgemeinen Verwaltungsrechts, Rz. 962.
} 
bezogenem Zurechnungszusammenhang, wiegewisse Aufsichtsabgaben, nicht den Steuern, sondern den Kausalabgaben zuzurechnen seien» ${ }^{776}$.

Ein aktuelles Beispiel für eine solche Kostenanlastungskausalabgabe ist die Abgabe für Radio und Fernsehen nach Art. 68 RTVG. Sie knüpft zwar kausal an den Empfang von Radio- und Fernsehangeboten an, wird aber pauschal bei allen Haushalten und Unternehmen erhoben. ${ }^{777}$

Mit diesen Abgabearten «wird das bipolare System zwischen Steuern und Kausalabgaben erheblich aufgeweicht»778. Hier verwischt auch die Trennlinie des Objekts der Aufgabenfinanzierung: Mit Steuern werden ganze Verwaltungsträger finanziert, mit Kausalabgaben einzelne Tätigkeiten.

Diese Aufweichung ist dogmatisch zu bemängeln, ist aber nicht zufällig. Sie dient der Umgehung einer harten rechtsstaatlichen Grenze: Steuern bedürfen (auf Bundesebene) einer Verfassungsgrundlage. ${ }^{779}$ Diese Zwischenformen decken also rechtspolitisch-pragmatische Bedürfnisse: Die Kosten eines Verwaltungsbereichs sollen mit einer pauschal einziehbaren Abgabe gedeckt werden, ohne dass die Erfordernisse an eine neue Steuer eingehalten werden müssen. Dies führt dann zu verfassungswidrigen Bundessteuern, wie etwa Art. 32e USG, Art. 60 Abs. 3 BBG und Art. 9 Abs. 2 LwG. 780

Hier zeigt sich deutlich, dass Dogmatik und Erhebungsprinzipien die Abgabearten nicht prägen und begrenzen können. Der Gesetzgeber schafft jene Formen, die ihm dienen.

\section{c. Insbesondere:Aufsichtsabgaben}

Eine neuere Finanzierungsform setzt sich für dieFinanzierung einer bestimmten Art von Staatsaufgabe durch: die Aufsichtsabgabe. Sie kann als Kostenanlastungskausalabgabe kategorisiert werden. ${ }^{781}$

Die Aufsichtsabgabe soll den Bestand von Kontroll-und Aufsichtsinstanzen sichern, über Verwaltungsgebühren für einzelne Aufsichtsleistungen hinaus. Die Aufgabenträger finanzieren sich mit dieser Abgabe ausserhalb des Staatshaushalts selber. Kennzeichnend für diese Finanzierungsform ist, dass die Verwaltungsadressaten somit die Strukturfinanzierung gewährleis-

776 BVGE 2007/43 E.4.2. S. $557 \mathrm{f}$.

777 HÄFELIN/MÜLLER/UHLMANN, Allgemeines Verwaltungsrecht, Rz. 2874.

778 WIEDERKEHR/RICHLI, Praxis des allgemeinen Verwaltungsrechts, Rz. 966.

779 TSCHANNEN/ZIMMERLI/MÜLLER, Allgemeines Verwaltungsrecht, §59 Rz. 4.

780 Zu USG und BBG ausdrücklich WIEDERKEHR, Sonderabgaben, S. 58; zum LwG analog.

781 HÄFELIN/MÜLLER/UHLMANN, Allgemeines Verwaltungsrecht, Rz. 2776; WIEDERKEHR, Kausalabgaben, S.19; WIEDERKEHR/RICHLI, Praxis des allgemeinen Verwaltungsrechts, Rz.961. Ansatzweise auch TSCHANNEN/ZIMMERLI/MÜLLER, Allgemeines Verwaltungsrecht, \$57 Rz. 14 . 
ten, zusätzlich zu den Gebühren, die bei der tatsächlichen Inanspruchnahme von Leistungen anfallen.

So ist es konzipiert für die Finanzmarktaufsicht ${ }^{782}$, das Heilmittelinstitut Swissmedic ${ }^{783}$ und die Oberaufsichtskommission des Bundes über die kantonale Aufsicht der Vorsorgeeinrichtungen ${ }^{784}$. In diesen Anwendungsfällen erheben die jeweiligen Aufgabenträger Verwaltungsgebühren für tatsächlich erbrachte Dienstleistungen. ${ }^{785}$ Um das Ziel einer selbsttragenden, kostendeckenden Finanzierung zu erreichen, bestreiten jährliche, pauschale Aufsichtsabgaben die von den Gebühren nicht gedeckten Aufwände.

Diese Abgabeart deckt also eine Finanzierungslücke: Die direkte Verursacherfinanzierung reicht nicht aus, und eine Restfinanzierung über den Staatshaushalt ist politisch nicht gewollt. Also werden die Beaufsichtigten erneut zur Kasse gebeten. Das Bundesgericht spricht bei solchen Aufsichtsabgaben von einer Abgabe «für dem Einzelnen nicht zuordenbare Allgemeindienstleistungen» 786 .

Besonders an dieser Finanzierungsform ist auch, dass die Erdulder von staatlichen Eingriffen so für die Aufrechterhaltung der Infrastruktur aufkommen, die für diese Eingriffe benötigt wird. Im Gegenzug sind die Verwaltungsträger finanziell auf die von ihnen Kontrollierten und Beaufsichtigten angewiesen (siehe hinten 4.g).

\section{d. Privatrechtliche Geldleistungen}

Wie eingangs erwähnt, sind die finanziellen Aspekte der Aufgabenerfüllung nicht gleichbedeutend mit dem öffentlichen Abgaberecht (soeben 2). Von öffentlichen Abgaben kann nur gesprochen werden, wo das zugrundeliegende Rechtsverhältnis öffentlichrechtlich ist. ${ }^{787}$ Staatsaufgaben können aber auch mittels privatrechtlicher Instrumente erfüllt werden.

Privatrechtliche Geldleistungen zur Finanzierung staatlicher Aufgabenerfüllung kommen immer dann vor, wenn die Inanspruchnahme einer staatlichen Leistung mit privatrechtlichen Verträgen geregelt wird. ${ }^{788}$ So unterliegen etwa die staatlichen Leistungen im Postwesen ${ }^{789}$ und in der Telekommunikation ${ }^{790}$ dem Zivilrecht.

\begin{tabular}{l}
\hline 782 Art.15 Abs.1 Satz 2 FINMAG. \\
\hline 783 Art.65 Abs. 2 HMG. \\
\hline 784 Art.64c Abs.1 Bst. a BVG. \\
\hline 785 Art.15 Abs.1 Satz1 FINMAG; Art. 65 Abs.1 HMG; Art. 64c Abs.1 Bst. b BVG. \\
\hline 786 Urteil des BGer 2A.345/2006 vom 24. Oktober 2006, E. 3.2.2. \\
\hline 787 TSCHANNEN/ZIMMERLI/MÜLLER, Allgemeines Verwaltungsrecht, §57 Rz.1. \\
\hline 788 TSCHANNEN/ZIMMERLI/MÜLLER, Allgemeines Verwaltungsrecht, §57 Rz. 26. \\
\hline 789 Art.11 Abs.1 POG. \\
\hline 790 Art.18 Abs.1 TUG.
\end{tabular}


Sofern die privatrechtliche Geldleistung eine staatliche Gegenleistung abgilt, gleicht sie ihrer Funktion nach einer Verwaltungs- oder Benutzungsgebühr.

\section{e. Quersubventionierung aus privatwirtschaftlicher Tätigkeit}

Die finanziellen Aspekte der Aufgabenerfüllung beschreiben, wie Private für staatliche Tätigkeit bezahlen. Eine letzte Finanzierungsform ist indirekt: Träger staatlicher Aufgaben bieten ausserhalb ihres Aufgabenbereichs privatwirtschaftliche Wettbewerbsleistungen an und verwenden die Erträge daraus für die Finanzierung ihrer staatlichen Leistungen. Man spricht dabei von Quersubventionierung. ${ }^{791}$

Ein Beispiel dafür ist der Verkauf von Kioskartikeln an Poststellen, dessen Erträge laut Bundesrat einen «Deckungsbeitrag für die Kosten der Grundversorgung» ${ }^{792}$ erzielen sollen. Ein anderes Beispiel sind die Werbeeinnahmen, mit denen die SRG ihr Programm finanziert, das sie im Rahmen ihres staatlichen Leistungsauftrags ausstrahlen muss. ${ }^{793}$ Dies ist eine freigewählte Finanzierungsform, wie es das Bundesgericht ausdrückt: «[Die SRG] kann ihre Programme unter Einhaltung der öffentlichrechtlichen Vorgaben mittels Werbung finanzieren, ist hierzu jedoch nicht verpflichtet.» ${ }^{794}$

Bei dieser Finanzierungsquelle ist die Verbindung der Bezahlenden zur Staatstätigkeit sehr locker. Der Bezahlende ist weder Verursacher, Besteller noch Nutzniesser einer staatlichen Leistung. Er kauft lediglich privatwirtschaftliche Leistungen auf dem freien Markt ein. Diese Finanzierungsform ist auch nicht an einzelne Leistungen gebunden, es handelt sich eher um Strukturfinanzierung, wie dies auch bei Steuern oder Aufsichtsabgaben vorliegt.

\section{Kennzeichen der Erfüllungsprivatisierung in finanzieller Hinsicht}

Die privatisierte Finanzierung der staatlichen Aufgabenerfüllung ist kein neues Phänomen - es zählen Finanzierungsformen dazu, bei denen nicht der Staat über den ordentlichen Staatshaushalt für die Kosten der Aufgabenerfüllung aufkommt. Es gibt dabei aber dogmatisch gefestigte Formen, wie die klassischen Verwaltungsgebühren, und dogmatisch schwierig einzuordnende Mischerscheinungen, wie die Aufsichtsabgaben.

791 Siehe etwa Art.19 Abs.1PG.

792 Bericht Staat und Wettbewerb, S.18. Gemäss Berner Zeitung vom 10. April 2015, Die Post will kein Kiosk mehr sein, S. 13, handle es sich dabei um eine «wichtige Finanzierungsquelle». Angaben zur Höhe des damit erzielten Umsatzes bei BAUMANN, Wettbewerbsverzerrungen, Rz.105.

793 Vgl. die Kompetenz zur Werbeschaltung in Art. 9-11 RTVG.

794 BGE 139 I306 E.3.2.1 S.310. 
Die folgenden Kennzeichen arbeiten heraus, was die Abkehr von der Steuerfinanzierung bedeutet - sowohl aufseiten der Aufgabenträger wie auch der Verwaltungsadressaten.

\section{a. Vorab:Die privatisierten Finanzierungsformen}

Die folgenden Kennzeichen beschreiben, inwiefern sich privatisierte Finanzierungsformen von den staatlichen Formen unterscheiden. Wie schon vor den Kennzeichen der privatisierten Organisations- und Handlungsformen ausgeführt, ist der Gegenstand der Erfüllungsprivatisierung nur schwer zu umreissen (siehe vorne II.4.a und III.3.a). Nur bezüglich der Aufgaben gibt es eine eindeutig staatliche und eine eindeutig private Sphäre, die Erfüllungsformen liegen vielmehr auf einem beliebig variierbaren Spektrum.

Bei den Finanzierungsformen ist die Grenze entlang des Gemeinlastprinzips zu ziehen. Es kommt auf die strukturelle Finanzierung von Aufgabenträgern an: Wer sichert ihren finanziellen Bestand? Privatisiert sind folglich alle Finanzierungsformen, die den Bestand eines Aufgabenträgers nach dem Verursacherprinzip oder nach der marktwirtschaftlichen Nachfrage finanzieren; in der soeben erfolgten Aufzählung also 3.b-e. Diese Finanzierungsformen ähneln der privatwirtschaftlichen Mittelbeschaffung am Markt. Bei den Verwaltungs- und Benutzungsgebühren (3.a) ist die Grenze fliessend: Soweit diese nur einzelne Handlungen abgelten und der Bestand eines Aufgabenträgers aus Steuermitteln gesichert ist, sind es staatliche Finanzierungsformen.

\section{b. Abkehrvom Gemeinlastprinzip}

In grundsätzlicher Weise zeichnet sich die finanzielle Erfüllungsprivatisierung dadurch aus, dass nicht die Gesamtbevölkerung für die Aufgabenerfüllung aufkommt, sondern Personen und Organisationen mit einem besonderen Bezug zur staatlichen Tätigkeit. Der Tendenz nach sollen die Kosten der Staatstätigkeit nicht mehr der Allgemeinheit zur Last fallen, sondern den Verursachern überbürdet werden. ${ }^{795}$

Allerdings kann nicht gesagt werden, dass generell ein Wechsel zum Verursacherprinzip ${ }^{796}$ stattfinde. Denn dieses wird wiederum aufgeweicht durch neuartige Abgabeformen (siehe dazu sogleich c). Kennzeichnend ist aber für alle Formen, dass vermehrt Gruppen und Einzelpersonen aus der Gemeinschaft herausgeschält werden, die für die Kosten der Staatstätigkeit aufkommen sollen.

795 Vgl. zur Gegensätzlichkeit von Gemeinlast- und Verursacherprinzip FRICK, Verursacherprinzip, S. 32 f.; MORELL/VALLENDER, St. Galler Kommentar, Art. 74 Rz. 28.

796 Dazu näher HÄFELIN/MÜLLER/UHLMANN, Allgemeines Verwaltungsrecht, Rz.1871. 
Damit geht einher, dass das Prinzip der wirtschaftlichen Leistungsfähigkeit an Bedeutung verliert. Private zahlen nicht nach ihrer Finanzkraft, sondern nach ihrem Bezug zu staatlichen Eingriffen und Leistungen. Krass ausgedrückt zahlt für den Staat, wer ihn braucht, nicht wer ihn tragen kann.

Privatisierte Finanzierungsformen dienen also zunächst der Vermeidung von Steuern - mit der Befreiung von der Pflicht, eine Verfassungsgrundlage für neue Abgaben vorweisen zu können. Dazu kommt, dass diese Finanzierungsformen einen direkten Bezug herstellen zwischen dem Empfang von Leistungen bzw. dem Erdulden von Eingriffen und der Bezahlung. Die Optik verschiebt sich also: Staatsaufgaben sind nicht mehr in erster Linie Tätigkeiten, die der Staat bereitstellen muss, sondern sie werden zu Konsumgütern.

\section{c. Aufweichung des Verursacherbegriffs}

Kostenanlastungskausalabgaben - und besonders die Aufsichtsabgabe streben formell eine Verursacherfinanzierung an. Doch nur ein ausgeweiteter Verursacherbegriff kann diese Vorgaben einhalten: Nicht mehr einzelne Verursacher mit konkretem Nutzen aus einer Verwaltungshandlung werden ausgeschieden, sondern ganze Personengruppen. ${ }^{797}$

Die Aufsichtsabgaben haben dabei einen Spagat zu vollziehen. Der Bundesrat drückte dies in seiner Botschaft zum neuen Finanzmarktaufsichtsgesetz - wohl unbeabsichtigt deutlich - aus: Es sei «darauf zu achten, dass eine möglichst verursachergerechte Belastung der Aufsichtskosten erfolgt», wobei mit der jährlichen Pauschalabgabe jene Kosten gedeckt würden, «die den beaufsichtigten Personen nicht individuell zugerechnet werden können» 798 . Aufsichtsabgaben sind also ihrer Natur nach nur annähernd verursachergerecht. Oder wie es der Bundesrat bei der Aufsichtsabgabe nach $\mathrm{HMG}$ ausdrückt: «Diese Gebühr deckt vor allem Aufwendungen, die nicht direkt verrechnet werden können» 799 . Auf diese Formulierung des Bundesrats aufbauend stellt das Bundesverwaltungsgericht fest: «Die Verkaufsgebühr [nach Art. 65 Abs. 2 HMG] soll ihrer Bestimmung nach somit zur Deckung von anfallenden Kosten, die keinem «Verursacher` zugeordnet werden können, verwendet werden» 800 .

797 Vgl. BVGE 2007/43 E. 4.2. S. 557 f. HÄFELIN/MÜLLER/UHLMANN, Allgemeines Verwaltungsrecht, Rz.2775; TSCHANNEN/ZIMMERLI/MÜLLER, Allgemeines Verwaltungsrecht, \$57 Rz.14; WIEDERKEHR/RICHLI, Praxis des allgemeinen Verwaltungsrechts, Rz. 962.

798 Botschaft FINMAG, 2843.

799 Botschaft HMG, 3546.

800 BVGer-E C-1858/2011 vom 23. September 2013, E. 6.4.1. 
Es ist keine Neuerscheinung, dass das Verursacherprinzip mehr der Zurechnung von Kosten an einen Abgabepflichtigen dient als der Bemessung. ${ }^{801}$ Kostenanlastungsabgaben lockern aber zusätzlich das Band zwischen Kosten und Verursacher. ${ }^{802}$ Das Verursacherprinzip bekommt dadurch eine neue Qualität: Es dient nicht mehr dazu, staatliche Eingriffe und Kostenübertragungen auf Private zu begrenzen, sondern es wird neu zur Grundlage für zusätzliche Kostentragungen. ${ }^{803}$ Wiederum zeigt sich dabei, dass die gesetzlich geschaffenen Finanzierungsmittel die abgaberechtlichen Prinzipien prägen und nicht umgekehrt. Es wäre beinahe angebracht, die Rede von Prinzipien aufzugeben und stattdessen von Merkmalen zu sprechen.

Allerdings darf das Verursacherprinzip nicht grenzenlos aufgeweicht werden: Der Staat darf etwa Polizeikosten nicht in einer derartigen Höhe überwälzen, dass dadurch die Geltendmachung von grundrechtlichen Ansprüchen vereitelt würde (siehe hinten $\$ 5 / V .3 . b$ ). Die Verursacherfinanzierung lässt sich zwar über direkte Zurechnungszusammenhänge hinaus ausdehnen, sie stösst aber dort an eine Grenze, wo sie eine Abschreckungswirkung entfacht, mittels deren der Staat seine gesetzlichen Pflichten unterschreitet.

\section{d. Gelockerte Steuerung durch das Parlament}

Mit dem Budgetrecht, also der Verabschiedung des Staatshaushalts, ${ }^{804}$ kann die Bundesversammlung auf die Erfüllung der Staatsaufgaben Einfluss ausüben. Sie kann zusätzliche Mittel bereitstellen, um gewisse Tätigkeiten zu fördern, oder im Gegenzug Mittel (und damit politische Unterstützung) entziehen.

Wenn die Budgets von Aufgabenträgern aus dem ordentlichen Staatshaushalt herausgelöst werden, um sie finanziell selbsttragend auszugestalten, verliert das Parlament diese Einflussmöglichkeit. Wie schon bei der organisatorischen Erfüllungsprivatisierung verlagert sich somit die vorgängige Steuerung auf eine nachträgliche Kontrolle im Rahmen der parlamentarischen Oberaufsicht (vgl. vorne II.4.d).

\section{e. Anreize gegen die Verursachung staatlicher Tätigkeit}

Wer einen staatlichen Eingriff erduldet oder eine Leistung empfängt, wird durch die finanzielle Erfüllungsprivatisierung mit einem zusätzlichen Umstand konfrontiert: Geld spielt eine Rolle bezüglich dieser Eingriffe und Leis-

801 wYss, Kausalabgaben, S. 62 f.

802 Vgl. im Unterschied dazu für verursachergerechte Kausalabgaben wYss, Kausalabgaben, S. 83 .

803 Vgl. bundesrechtswidrige Konstellationen bei TSCHANNEN/ZIMMERLI/MÜLLER, Allgemeines Verwaltungsrecht, \$58 Rz. 12.

804 Art.167 BV; Art. 4 und 29 FHG. TSCHANNEN, Staatsrecht, §33 Rz.14. 
tungen. Die Kostentragung wird zu einem Faktor für Verwaltungsadressaten, der als wirtschaftlicher Anreiz verhaltenslenkende Wirkung entfalten kann. Die Optik verschiebt sich wiederum von der Erbringung von Staatsaufgaben zu deren Inanspruchnahme.

Die Inanspruchnahme staatlicher Leistungen mit wirtschaftlichen Anreizen steuern zu wollen, verkennt aber die besondere Konstellation bei staatlichen Aufgaben: In aller Regel besteht gerade kein Markt. So verhält es sich beispielsweise bei den Aufsichtsabgaben, wie sie die FINMA und Swissmedic erheben: Leistungsempfänger sollen mit ihren Beiträgen dem Leistungserbringer Anreize setzen für Qualität, doch haben die Leistungsempfänger keine Marktmacht; sie haben keine Ausweichmöglichkeit. Wollen sie ihre Produkte auf den Markt bringen, brauchen sie die Genehmigung der Aufsichtsbehörden. Man erwartet, die Finanzierungsprivatisierung bringe Marktlogik in die Leistungserbringung, vergisst aber, dass eben gerade kein Markt spielt. 805

\section{f. Anreizzur Monetarisierungjedes Bestandteils des Verwaltungshandelns}

Aufgabenträger, deren Bestand nicht über staatliche Strukturfinanzierung gesichert ist, bekommen einen Anreiz, möglichst jeden Bestandteil ihrer Aufgabenerfüllung zu verrechnen, um ihn über Gebühren finanzieren zu können.

So verhält es sich etwa beim Heilmittelinstitut Swissmedic, das sich gemäss Art. 77 Abs. 3 Bst. c HMG unter anderem aus «Abgeltungen für gemeinwirtschaftliche Leistungen» finanziert. Statt dass der Bund einer Verwaltungseinheit das erforderliche Budget zur Erfüllung ihrer Aufgaben bereitstellt, muss diese Einheit sämtliche Tätigkeiten als abgeltungsfähige Leistungen darstellen. Dieser Anreiz führt dann etwa dazu, dass Swissmedic in ihrer Jahresrechnung die Einträge «Rechtsgrundlagen» und «Strafrecht» als «gemeinwirtschaftliche» «Produkte» ausweist, für die der Bund aufzukommen hat.806

\section{g. Interessenkonflikte aus gegenseitiger Abhängigkeit}

Namentlich die Aufsichtsabgabe lässt einen unausweichlichen Interessenkonflikt entstehen: Der Aufgabenträger ist auf die Zahlungsbereitschaft derjenigen Personen und Institutionen angewiesen, die er überwacht. Und die Privaten sind auf wohlwollende Prüfungen und Entscheide derjenigen Verwaltungsstelle angewiesen, der sie Geld zu überweisen haben.

Auch bei Verwaltungsgebühren besteht dieses Quidproquo, aber mit anderer Bedeutung: Dort werden diese Gebühren nur als Entgelt für einzelne Leistungen erhoben, während der Bestand des Aufgabenträgers an sich über den 
Staatshaushalt gesichert ist. Bei Aufsichtsabgaben soll auch der Strukturerhalt über Gebühren finanziert werden. So zeigt sich etwa die Finanzlage von Swissmedic: In seiner Jahresrechnung weist das Institut die Erträge aus der Verkaufsabgabe als das Dreifache des Bundesbeitrags aus. ${ }^{807}$

Der Interessenkonflikt wird durch das soeben thematisierte Fehlen eigentlicher Marktmechanismen (vorne e) wieder gemindert. Die Beaufsichtigten können nur begrenzt Druck auf die Aufsichtsbehörden ausüben, da sie kaum Ausweichmöglichkeiten haben. Dennoch schreiben diese Finanzierungsformen ein strukturelles Spannungsverhältnis fest. Auf diese Gefahr wurde bezüglich Swissmedic in der Vernehmlassung zum HMG hingewiesen: «Das Kostendeckungsprinzip wurde zwar grundsätzlich begrüsst, gleichzeitig aber auch die Befürchtung geäussert, dass bei einer ausschliesslichen Finanzierung durch die Industrie die Unabhängigkeit des Instituts gefährdet sein könnte.» 808

\section{Zwischenfazit: Staatsaufgaben werden zur wirtschaftlichen Verhandlungsmasse}

Den privatisierten Finanzierungsformen ist gemein, dass sie Staatsaufgaben mit einem Preis versehen, der von der Allgemeinheit überwälzt wird auf Individuen oder Personengruppen. Die finanzielle Erfüllungsprivatisierung verschiebt somit die Optik: weg von der Erfüllungspflicht des Staats hin zur Zahlungspflicht der Privaten. Damit geht einher, dass ökonomische Anreizsysteme in die staatliche Aufgabenerfüllung eingebaut werden, die dort systemfremd sind. Regelmässig sind Staatsaufgaben nicht ein Angebot unter vielen, aus denen ausgewählt werden könnte. Wer um eine Bewilligung ersucht oder eine Monopoldienstleistung einkauft, hat keine Ausweichmöglichkeiten.809 Der jeweilige Aufgabenträger ist die einzige Anlaufstelle. Umgekehrt haben die Aufgabenträger keine Möglichkeit, Qualität oder Umfang ihrer Leistungen an der preislichen Nachfrage auszurichten. Sie sind zur Erbringung ihrer Aufgaben gesetzlich verpflichtet und müssen sich an die verfassungsmässigen Anforderungen an Staatstätigkeit halten.

Ein Beispiel dazu: Im Fernmelderecht können Konzessionen zum Beispiel versteigert werden, wobei die Konzessionsgebühr dann dem angebotenen Betrag entspricht (Art. 39 Abs. 4 FMG). ${ }^{810}$ Somit lässt sich die Nachfrage nach

807 Jahresrechnung Swissmedic, Erfolgsrechnung, S. 4

808 Botschaft HMG, 3468.

809 Vgl. wYss, Kausalabgaben, S. 46.

810 Siehe auch Art. 23 FKV. 
fernmelderechtlichen Konzessionen direkt quantifizieren, doch auch in diesem Fall bleibt die Verwaltungseinheit - konkret das Bundesamt für Kommunikation (BAKOM) - zur Erbringung ihrer Aufgaben gesetzlich verpflichtet. Das Amt kann die Konzessionsvoraussetzungen nicht der Nachfrage anpassen - etwa die Voraussetzungen erhöhen, wenn hohe Konzessionsbeträge angeboten werden.

\section{Zwischenfazit: Erfüllungsprivatisierung gibt staatlichem Handeln den Anstrich privater Flexibilität}

Dieser Paragraf hat dargelegt, welche Aspekte die Erfüllung staatlicher Aufgaben - aus der Sichtweise der Verwaltungsadressaten - aufweist und inwiefern diese Aspekte privatisiert werden können. Es kann resümiert werden, dass sowohl organisatorische, instrumentelle wie finanzielle Aspekte in vielfältiger und nicht von vornherein begrenzter Weise privatisiert werden können. Der Gesetzgeber ist weitgehend frei, die Aufgabenerfüllung so nichtstaatlich auszugestalten, wie er beliebt.

Den verschiedenen Spielarten der Erfüllungsprivatisierung ist der Anstrich privatwirtschaftlicher Funktionsweise gemein. Das staatliche Handeln soll wie privates erscheinen und kann dies auch.

Was sind die Folgen davon? Drei rote Fäden ziehen sich durch die Kennzeichen der verschiedenen Erscheinungen privatisierter Aufgabenerfüllung. Erstens wird die vorgängige Steuerung und Leitung (durch das Parlament oder die Zentralverwaltung) zurückgedrängt zugunsten punktueller, nachträglicher Kontrolle und Aufsicht. Zweitens wird staatliche Aufgabenerfüllung nach aussen (und teilweise auch nach innen) hin unkenntlich gemacht: Der betroffene Private, wie auch die Aufgabenträger selbst, kann nicht immer einwandfrei erkennen, wann und wieweit er es mit Staatsaufgaben zu tun hat. Und zuletzt zeigt sich, dass der Gesetzgeber im Bestreben um einen privater wirkenden Staat nicht davor zurückschreckt, strukturelle Interessenkonflikte festzuschreiben.

Soweit die Ausgangslage. Im Folgenden sind nun die verfassungsrechtlichen Anforderungen auf die verschiedenen Spielarten der Erfüllungsprivatisierung anzuwenden. Die $\$ \S 5$ und 6 arbeiten nun heraus, welche Probleme und Spielräume im Einzelnen aus den jeweiligen Anforderungen entstehen. 


\section{\$ 5 Welche Probleme schaffen die verfassungsrechtlichen Anforderungen für die Erfüllungsprivatisierung?}

Diese Arbeit untersucht, inwiefern die Erwartung von weniger Staat eingelöst werden kann, wenn lediglich die Art und Weise der Erfüllung einer staatlichen Aufgabe privatisiert wird, nicht aber die Aufgaben an sich. Die zwei vorangehenden Paragrafen haben Prüfmassstab und den Prüfgegenstand herausgearbeitet: Den Massstab bilden die verfassungsrechtlichen Anforderungen, die immer dann gelten, wenn staatliche Aufgaben erfüllt werden (\$3). Gegenstand der Prüfung ist die Privatisierung der verschiedenen Aspekte der Aufgabenerfüllung (\$4).

Bei der Prüfung sind drei Befunde denkbar: Die Einhaltung der verfassungsrechtlichen Anforderungen kann bei der privatisierten Aufgabenerfüllung besondere Probleme verursachen - dies ist Inhalt des vorliegenden $\$ 5$. Andererseits ist denkbar, dass die privatisierte Aufgabenerfüllung Spielräume innerhalb und zwischen den verfassungsrechtlichen Anforderungen ausnutzen kann (\$6). Der dritte Befund ist ein Nullergebnis: Bei der Anwendung der verfassungsrechtlichen Anforderungen auf die privatisierte Aufgabenerfüllung treten keine Besonderheiten gegenüber der nichtprivatisierten Aufgabenerfüllung auf - dies wird ebenfalls hier bei § 5 vermerkt, soweit es überhaupt erwähnenswert ist.

Diese Prüfung erfolgt rasterartig: Jede einzelne Anforderung kann bezüglich jedes Aspekts der Erfüllungsprivatisierung zu Problemen oder Spielräumen führen. Die §§ 5 und 6 sind nach den einzelnen Anforderungen gegliedert.

Die folgenden Abschnitte I-V fragen spezifisch danach, ob die Stossrichtungen der jeweiligen Privatisierungserscheinungen den einzelnen Zwecken der verfassungsrechtlichen Anforderungen entgegenstehen. Der Befund «Es entstehen Probleme» kann dabei unterschiedlich ausgeprägt sein. Eine Privatisierung kann mit der verfassungsrechtlichen Anforderung schlechterdings nicht verträglich sein, sodass die Verfassung verletzt wird. Oder die Privatisierungserscheinung kann zwar eine andere Zielrichtung aufweisen 
als die verfassungsrechtliche Anforderung, aber gerade noch mit der Verfassung im Einklang sein. Im ersten Fall spricht diese Arbeit von einem Problem, im zweiten von einer Spannung.

Aus Sicht der Verfassung gelten die Anforderungen grundsätzlich in gleicher Weise unabhängig von den Erfüllungsmodalitäten (siehe vorne \$3/I.3). Egal, in welche organisatorische Hüllen sich der Staat kleidet, welche Instrumente er einsetzt oder wie er sich finanziert - staatliches Handeln unterliegt denselben verfassungsrechtlichen Anforderungen. Und die Anforderungen sehen selber keine Abstufungen oder verschiedene Bindungsintensitäten vor. Hier liegt genau die Crux: Die binäre Sichtweise der Verfassung, die eine staatliche Sphäre von einer privaten abgrenzt, trifft auf die nuancierte Rechtswirklichkeit der vielfältigen Erfüllungsformen. Die Formen der Aufgabenerfüllung können entlang eines Spektrums staatstypisch sein oder identisch wie privates Handeln erscheinen.

Es ist eine wichtige Abgrenzung bezüglich des Erkenntnisinteresses dieser Arbeit vorzunehmen: Es geht nicht um die Zulässigkeit der privatisierten Aufgabenerfüllung. Die verfassungsrechtlichen Anforderungen auf die Erfüllungsprivatisierung anzuwenden, bedeutet, sich mit deren Konsequenzen zu beschäftigen. Die Zulässigkeit von Staatshandeln wird aber mit der Aufgabenzuweisung abschliessend entschieden. Vielmehr geht es um die Frage, welche Konsequenzen sich aus der Erfüllungsprivatisierung ergeben. Dabei wird aufgespürt, inwiefern die privatisierte Erfüllung staatlicher Aufgaben mit den Anforderungen der Verfassung verträglich ist. Da diese Arbeit die Auswirkungen von Privatisierungen auf einer theoretischen Ebene untersucht, vermeidet sie den Begriff Grenze und spricht stattdessen von Problemen und Spannungen. Diese Arbeit kann bloss aufzeigen, wo Privatisierungen zu Komplikationen führen können; sie kann keine abschliessenden Aussagen über deren Verfassungsmässigkeit treffen. 


\section{Probleme aus der Gesetzmässigkeit}

Das Gesetzmässigkeitsprinzip fordert, dass der Staat sein Handeln im Gesetz begründen kann und es daran gebunden ist - «Grundlage und Schranke staatlichen Handelns ist das Recht», wie es Art. 5 Abs. 1 BV in unübertrefflicher Einfach heit sagt. Soweit der Staat seine Aufgaben durch Verwaltungseinheiten der Zentralverwaltung mit hoheitlichen Instrumenten erbringt und dafür mit Steuermitteln aus dem allgemeinen Bundeshaushalt aufkommt, braucht es keine spezialgesetzliche Grundlage für dieses Handeln. Die gesetzliche oder verfassungsmässige Aufgabenzuweisung reicht aus - und RVOG, VwVG und FHG regeln das Übrige. Wenn aber die organisatorischen, instrumentellen oder finanziellen Aspekte der Aufgaben privatisiert werden, wird häufig eine zusätzliche gesetzliche Grundlage erforderlich: Sei es für die Errichtung einer öffentlichrechtlichen Aktiengesellschaft (vorne \$ 4/II.3.g), sei es für eine ausgelagerte und überwachte Normprüfung (Stichwort akkreditierte Zertifizierung; vorne \$ 4/III.2.b.cc), oder sei es für die Finanzierung mittels Aufsichtsabgabe (vorne $\$ 4 / I V .3 . c)$.

Damit entsteht eine grundsätzliche Spannungslage zwischen dem Gesetzmässigkeitsprinzip und der Motivation hinter jeglicher Form der Erfüllungsprivatisierung: Das Erfordernis einer Einzelfallregelung trifft auf den erhofften Zuwachs an Flexibilität, wenn von den klassisch staatlichen Formen abgewichen wird. Privatisierungen können mehr Freiheit in die staatliche Aufgabenerfüllung bringen, wenn sich der Staat etwa auf Rahmengesetzgebung und eine Gewährleistungsverantwortung beschränkt, während ein Teil der Aufgabe an Private abgegeben wird. Der Staat zieht sich aber gerade nicht zurück, wenn er die staatliche Aufgabenerfüllung lediglich in andere Formen giesst. Dann wird für diese Formen eine eigene gesetzliche Grundlage nötig, die den erhofften Freiheiten neue Grenzen setzt und dem Parlament eine einzelfallgenaue Mitsprache (oder - je nach Sichtweise - Einmischung) erlaubt.

Dazu kommt ein grundsätzliches Potential für Spannungslagen, das bezüglich aller verfassungsrechtlichen Anforderungen gilt: Wenn der Staat die Aufgabenerfüllung wie privates Handeln erscheinen lässt, wissen die von diesem Handeln Betroffenen nicht, dass sie Verwaltungsadressaten sind und es mit dem Staat zu tun haben. Und dass das sie betreffende Handeln also den verfassungsrechtlichen Anforderungen unterliegt (siehe hinten VI.1). Diese Spannung zeigt sich beim Gesetzmässigkeitsprinzip mustergültig: Wer nicht weiss, dass eine AG eigentlich Verwaltungsträger ist, dass ein Vertrag seine öffentlichrechtlichen Pflichten ordnet und er mittels Gebühren wegbleibende Steuermittel ersetzt, wird nicht daran denken, nach einer gesetzlichen Grund- 
lage zu verlangen. Dies ermöglicht der privatisierten Aufgabenerfüllung, ausserhalb des Gesetzes zu operieren, solange sie das Vertrauen ihrer Adressaten geniesst. Der Klarheit halber: Konkrete Beispiele für solche Ausbrüche aus dem Gesetzmässigkeitsprinzip gibt es nicht. Hier sei lediglich auf die potentielle Spannungslage hingewiesen.

\section{Insbesondere bei den privatisierten Organisationsformen}

Die soeben erwähnte, generelle Spannungslage zwischen Einzelfallregelung und Flexibilität verdeutlicht sich bei allen Aufgabenträgern, die nicht zur Zentralverwaltung gehören. Die Hoffnung, mehr Flexibilität in der operativen Umsetzung der gesetzlichen Vorgaben zu erhalten, wird zu einem Stück zunichtegemacht, weil die vermeintlich bloss strategische Ebene auf die konkrete Aufgabenerfüllung durchwirkt, indem das Parlament über das Spezialgesetz bestimmt. ${ }^{811}$

Die Rechtsstellung von Verwaltungseinheiten ist nur innerhalb der Zentralverwaltung geregelt. Das trifft insbesondere für die Aufsichtsrechte der Regierung ihnen gegenüber zu (siehe vorne \$ 4/II.4.b), stimmt aber ebenso bezüglich ihrer prozessrechtlichen Stellung und der Rechtsnatur ihrer Beziehungen zu Privaten. Das Gesetzmässigkeitsprinzip verlangt, dass diese Fragen bei ausgelagerten Aufgabenträgern spezialgesetzlich geregelt werden. Eine Regelungslücke entsteht immer dann, wenn der Staat einen Verwaltungsträger nicht per gezielten Rechtsakt errichtet, sondern lediglich Aufgaben auf ein vorbestehendes Rechtssubjekt überträgt. Da diese Übertragungsakte nicht zwingend in Rechtsakten, sondern oft in Verträgen und Leistungsvereinbarungen geschieht, lässt das Gesetzmässigkeitsprinzip hier eine Lücke entstehen. Dadurch entsteht vorab ein Nachvollziehbarkeitsproblem. Wo ein Errichtungsakt fehlt, sind viele Rechtsfragen nur für die mit der Aufgabenübertragung befassten Organe beantwortbar.

Eingeschränkte Aufsichtsrechte oder andere Abweichungen von der Rechtsstellung der Zentralverwaltung sind noch keine Probleme im hier besprochenen Sinn. Die Gesetzmässigkeit verlangt bloss, dass der Staat auf Grundlage und innerhalb des Gesetzes handelt. Es steht ihm frei, seine Zugriffsrechte innerhalb des gesetzlichen Spielraums einzuschränken. 


\section{Insbesondere bei den privatisierten Handlungsformen}

Die privatisierten Handlungsformen können die Bindung des Aufgabenträgers an diegesetzlichen Grundlagen nicht lockern. Die ausgelagerte Normsetzung (siehe vorne \$ 4/III.2.b.cc) muss auf Gesetzesrecht beruhen und darf dieses nicht abändern. ${ }^{812}$ Der Spielraum für Flexibilisierungen, der solche Auslagerungen mitunter antreibt, ist also von vornherein beschränkt. Dasselbe gilt für die Erwartung, dass informelle Handlungen den Staat aus dem starren Normkorsett befreien und ihm flexiblere Einzelfalllösungen ermöglichen können sollen. 813

\section{a. Dogmatisch ungefestigte Sui-generis-Instrumente}

Verwaltungsträger dürfen ihre Aufgaben nur mittels derjenigen Handlungsformen erfüllen, die ihnen der Gesetzgeber an die Hand gibt. ${ }^{814}$ Privatisierte Handlungsformen müssen eigens erschaffen werden. Sui-generis-Konstruktionen wie akkreditierte Zertifizierungen (siehe vorne \$ 4/III.2.b.cc) können sich dabei auf keine gefestigte Dogmatik stützen, ihre Rechtsnatur, Rechtsund Verfahrensfolgen ergeben sich einzig aus der Einzelfallregelung. 815

Selbst oft eingesetzte Instrumente wie der öffentlichrechtliche Vertrag sind dogmatisch nicht gefestigt. ${ }^{816}$ Der Gesetzgeber hat wichtige Rechtsfragen nicht geklärt. Er hätte «wenigstens die wichtigsten allgemeinen Regeln zum Verwaltungsvertrag positiveren müssen», denn aktuell «drohen sich schon kleine Störungen im Vertragsverhältnis zu Wüsten der Ratlosigkeit auszuwachsen.»817 Dies ist ein grundsätzliches Problem von privatisierten Handlungsformen, das tendenziell zu zusätzlichem Aufwand in der Rechtsdurchsetzung führen dürfte - man denke an Auslegungsfragen vor Gericht.

\section{b. Verminderte Steuerungskraft trifft auf zwingendes Recht}

Staatliche Aufgaben erfüllen heisst, zwingendes Recht anwenden. Jede Verwaltungshandlung, die der Erfüllung staatlicher Aufgaben dient, setzt öffentliches Recht um - auch wenn das Handeln selbst dem Privatrecht unterliegt. Die öffentlichrechtlichen Vorgaben des besonderen Verwaltungsrechts sind

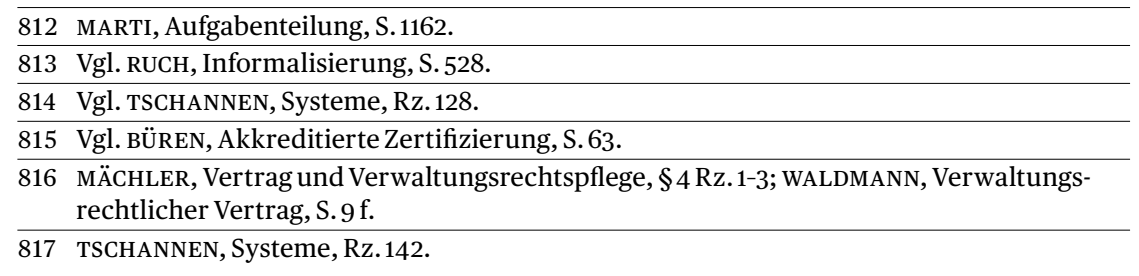


grundsätzlich zwingender Natur - von ihnen kann nicht durch Parteiabsprache abgewichen werden. ${ }^{818}$ Diese Eigenschaft der gesetzlichen Grundlagen, auf der Verwaltungshandeln beruht, führt zu einer grundsätzlichen Spannungslage mit einem Kennzeichen der privatisierten Handlungsformen: ihre geringere Steuerungskraft (siehe vorne \$ 4/III.3.d).

Verträge, Absprachen und andere neue Instrumente sind weniger verbindlich und weniger einfach durchsetzbar. Das bedeutet einerseits, dass die Rechtsbefolgung zu einem grösseren Teil von der Kooperation der Verwaltungsadressaten abhängt als etwa bei Verfügungen und Rechtsverordnungen. Auch dies erhöht tendenziell den Aufwand zur Rechtsdurchsetzung. Der öffentlichrechtliche Vertrag stellt beispielsweise noch keinen vollstreckbaren Rechtstitel dar - im Gegenteil zur Verfügung. Dieser muss erst noch erwirkt werden, oder es muss auf Erfüllung geklagt werden.819

Diese potentiellen Abstriche bei Verbindlichkeit und Durchsetzbarkeit sind aber keine grundsätzlichen Probleme, die aus dem Gesetzmässigkeitsprinzip entstehen. Das Gesetz soll das Staatshandeln bloss legitimieren und begrenzen. Es steht dem Staat frei, von gesetzlichen Vorgaben zugunsten des Privaten abzuweichen, also auf die Durchsetzung zwingenden Rechts zu verzichten. Die Grenze dafür sind bloss gesetzliche Leistungspflichten, die nicht unterschritten werden dürfen. Die selektive Nichtdurchsetzung von Gesetzesbestimmungen gegenüber ausgewählten Verwaltungsadressaten kann allerdings gegenüber dem Gleichbehandlungsgebot Probleme aufwerfen (siehe dazu hinten V.2).

\section{c. Insbesondere: Eingeschränkte Anpassbarkeit an veränderte Rechtslage}

Öffentlichrechtliche Verträge führen zu einem dogmatisch viel bearbeiteten Problem. Entsprechend dem Gesetzmässigkeitsprinzip müssen nachträglich rechtswidrig gewordene Verwaltungsakte an die neue Rechtslage angepasst werden. ${ }^{820}$ Verträge sind aber nur mit der Zustimmung des privaten Vertragspartners abänderlich. ${ }^{821}$ Das widerspricht dem Gesetzmässigkeitsprinzip, weil der Staat aufgrund einer Vereinbarung an eine veraltete Rechtslage gebunden bleibt. Dieses Problem entsteht aus dem Grundsatz von Treu und Glauben, der das Vertrauen in einvernehmlich ausgehandelte Instrumente

818 Vgl. HÄFELIN/MÜLLER/UHLMANN, Allgemeines Verwaltungsrecht, Rz. 49; TSCHANNEN/ ZIMMERLI/MÜLLER, Allgemeines Verwaltungsrecht, §35 Rz. 4.

819 TSCHANNEN/ZIMMERLI/MÜLLER, Allgemeines Verwaltungsrecht, \$28 Rz.11.

820 Siehe die Voraussetzungen dazu bei der Verfügung in HÄFELIN/MÜLLER/UHLMANN, Allgemeines Verwaltungsrecht, \$17; TSCHANNEN/ZIMMERLI/MÜLLER, Allgemeines Verwaltungsrecht, \$31 Rz.19-62.

821 RUCH, Informalisierung, S. 523. 
tendenziell höher gewichtet als die Rechtmässigkeit (siehe hinten IV.2.b). Ausserdem limitiert das den Staat darin, flexiblere und passgenauere Lösungen anzuwenden. Die vermeintliche Bürgernähe hat also die Kehrseite, dass auf Vereinbarung basierende Aufgabenerfüllung starr ist und dadurch auch Ineffizienzen schaffen kann.

\section{d. Problem aus Normdichte bei ausgelagerter Normsetzung}

Einige privatisierte Handlungsformen dienen dazu, externes Fachwissen in die Aufgabenerfüllung einzubinden (siehe vorne \$4/III.3.g). Dieses Ziel kann mit einem Teilgehalt des Gesetzmässigkeitsprinzips in Konflikt geraten: der Normdichte. Die Normdichte fordert so präzise Gesetze, dass Private ihr Verhalten danach richten können. ${ }^{822}$ Das staatliche Handeln soll für Normadressaten der gesetzlichen Grundlagen erwartbar und erkennbar sein.

Geht nun aber der Einbezug externer Fachkenntnisse so weit, dass Branchenspezialisten selber staatliche Regulierung schreiben, kann dies deren Verständlichkeit und Nachvollziehbarkeit beeinträchtigen. ${ }^{823}$

Das Stromversorgungsrecht beispielsweise verweist auf private Normen. 824 Das Elektrizitätsgesetz (EleG) hält den Bundesrat in Art. 3 Abs. 1 an, «Vorschriften zur Vermeidung von Gefahren und Schäden, welche durch Stark- und Schwachstromanlagen entstehen», zu erlassen. Der Bundesrat nimmt diesen Auftrag mit Art. 4 Abs. 1 der Starkstromverordnung 825 wahr, wonach Starkstromanlagen nach den «den anerkannten Regeln der Technik erstellt, geändert, instandgehalten und kontrolliert werden». Die Verordnung führt in Abs. 2 derselben Bestimmung weiter aus: «Als anerkannte Regeln der Technik gelten insbesondere die Normen von IEC und CENELEC. Wo international harmonisierte Normen fehlen, gelten die schweizerischen Normen.» Fussnoten zu diesem Absatz führen die Akronyme der internationalen Organisationen aus; direkte Verweise auf die Normen fehlen aber. Auf den jeweiligen Websites ${ }^{826}$ sind verschiedene Versionen von Standards zu verschiedenen Regelungsbereichen aufgeführt - wobei sich anhand der Titel nicht sofort erschliesst, wovon die verschiedenen Dokumente handeln. Was die schweizerischen Normen betrifft, verweist eine Fussnote auf die Postanschrift des Schweizerischen Informationszentrums für technische Regeln (switec). Dieses

822 BGE 139 I 280 E. 5.1 S. 284; ähnlich schon BGE 109 Ia 273 E. 4d S. 283. Siehe auch HÄFELIN/ MÜLLER/UHLMANN, Allgemeines Verwaltungsrecht, Rz. 342 f.; TSCHANNEN/ZIMMERLI/ MÜLLER, Allgemeines Verwaltungsrecht, §19 Rz.19-21.

823 Vgl. GILI, Selbstregulierung, S.150-152.

824 Vgl. zum Ganzen auch WALTHER, Kooperative Steuerungsinstrumente, S. 206-208.

825 Verordnung über elektrische Starkstromanlagen vom 30. März 1994 (SR 734.2).

826 www.iec.ch; www.cenelec.eu. 
Zentrum verfügt zwar über eine Website mit einer Liste von «harmonisierten Normen», wo dann aber keine Einträge zum StichwortStrom zu finden sind. ${ }^{827}$

Wenn Normadressaten ihr Verhalten nach privaten Normen richten sollen, müssen diese zunächst einmal auffindbar sein. ${ }^{828}$ Und es muss ersichtlich sein, welche Regelung welches Absenders worauf anwendbar ist. Im Beispiel des Stromversorgungsrechts ist dies nicht gewährleistet. Die Starkstromverordnung macht die Regelung sogar noch unübersichtlicher, indem sie in Art. 4 Abs. 3 anfügt: «Bestehen keine spezifischen technischen Normen, so sind sinngemäss anwendbare Normen oder allfällige technische Weisungen zu berücksichtigen.»

Zudem muss auch ausgelagerte Rechtsetzung verständlich sein. Dafür ist neben fachlicher Kenntnis auch Rechtsetzungswissen erforderlich. Von Fachexperten verfasste Normen drohen aufgrund sprachlicher und struktureller Mängel weniger leserlich und verständlich zu werden. ${ }^{829}$

\section{Insbesondere bei den privatisierten Finanzierungsformen}

Bei der Erfüllungsprivatisierung in finanzieller Hinsicht spitzt sich die Spannungslage zwischen Einzelfallregelung und erhoffter Flexibilisierung noch zu, die sich in grundsätzlicher Weise aus der Einhaltung des Gesetzmässigkeitsprinzips ergibt. Privatisierte Finanzierungsformen drängen das Gemeinlastprinzip zugunsten einer vermehrten Verursacherfinanzierung zurück (siehevorne \$ 4/IV.1). Die Aufwände des Verwaltungsträgers werden nicht aus dem regulären Staatshaushalt beglichen; er muss selber Einnahmen generieren. Dies wiederum bedeutet einen Verlust an parlamentarischer Steuerung: Die Einflussnahme mittels Budgetrecht entfällt (siehe vorne \$ 4/IV.4.d).

Das Erfordernis einer gesetzlichen Grundlage führt nun aber dazu, dass das Parlament jede privatisierte Finanzierungsform als gesetzliche Einzelfallregelung einrichten muss. Der Verwaltungsträger, der unternehmerisch und wendig um seine eigene Finanzierung besorgt sein soll, entkommt also der parlamentarischen Einflussnahme nicht. Diese verschiebt sich lediglich von einer begleitenden Kontrolle zu einer vorgängigen Steuerung: Der Gesetzgeber wägt Vor- und Nachteile einer konkreten Aufsichtsabgabe ab, richtet sie ein und überlässt den Verwaltungsträger dann sich selbst.

Privatisierte Finanzierungsformen stellen den Verwaltungsadressaten gegenüber Anreize auf, die tendenziell von der Inanspruchnahme staatlicher

827 www.switec.info/de/neue-harmonisierte-normen.

828 GILI, Selbstregulierung, S.151f.

829 GILI, Selbstregulierung, S. 152. 
Leistungen abraten sollen: Verwaltungsgebühren, Kostenanlastungssteuern, Aufsichtsabgaben - sie alle versehen die Verursachung staatlicher Tätigkeit mit einem Preis (siehe vorne \$ 4/IV.4.e). Gesetzliche Leistungspflichten drohen somit zu einem blossen Leistungsangebot heruntergestuft werden. Ohne ökonomische Kategorien zu bemühen, kann dies als Verschiebung von gemeinnützigen Gütern zu Konsumgütern bezeichnet werden.

Ein eigentliches Problem im Sinn dieses Paragrafen entsteht aber nur, wenn der Verwaltungsträger mit solchen Anreizen eine gesetzliche Leistungspflicht unterschreitet. Dies kann etwa vorliegen, wenn Kostentragepflichten eine Abschreckungswirkung entfalten, die sich wie eine Grundrechtsbeschränkung auswirkt (siehe hinten V.3.b). Sofern das Leistungsangebot bezüglich Zugänglichkeit und Qualität den gesetzlichen Vorgaben entspricht und die Anreize einer spezialgesetzlich vorgesehenen Finanzierungsform entspringen, ist daran aber nichts auszusetzen. 


\section{Probleme aus dem öffentlichen Interesse}

Ein staatlicher Eingriff ist selten alleine aufgrund eines unzureichenden öffentlichen Interesses ungerechtfertigt- dennoch ist der Grundsatz des öffentlichen Interesses (aus Art. 5 Abs. 2 BV) neben dem Gesetzmässigkeitsund dem Verhältnismässigkeitsprinzip die wichtigste Gewährleistung für legitimes Staatshandeln. Dieser Grundsatz fordert, dass jedes Staatshandeln auf einem zulässigen öffentlichen Interesse gründet (siehevorne \$3/II.3). Damit ist immerhin gesagt, dass ein privates Interesse oder Sondergruppeninteressen allein Staatstätigkeit nicht legitimieren. Vielmehr muss der Staat immer ein Interesse verfolgen, das «die Öffentlichkeit (verstanden als Allgemeinheit, Bevölkerung, Publikum) für erstrebens- oder erhaltenswert hält»830. Die Festlegung, welche Interessen diesen Massstab erfüllen, erfolgt im politischen Prozess.

Nach diesem Resümee können diegrundsätzlichen Spannungslagen untersucht werden, zu denen die Einhaltung dieser verfassungsrechtlichen Anforderung führt. Das Ergebnis sei vorab festgehalten: Das öffentliche Interesse steht der Erfüllungsprivatisierung nicht grundsätzlich entgegen.

Zunächst ist zwar anzunehmen, dass die grössere Flexibilität und der zunehmend private Auftritt des Staats zu einer generellen Spannungslage führen müssen mit der Bindung auf öffentliche Zwecke. Staatliche Aufgaben liegen in gesamtgesellschaftlicher Verantwortung; Organisations-, Handlungs- und Finanzierungsformen, die der Sphäre der Privatautonomie entlehnt sind, lassen da an einen Zielkonflikt denken. Etwa wenn Verwaltungsträger mit ökonomischen Anreizen dazu bewegt werden, die aufwändigen und nicht lohnenden Teile der Aufgabenerfüllung zurückzudrängen. Der Grundsatz des öffentlichen Interesses ist diesbezüglich aber ein schwaches Hindernis. Er fordert nur, dass überhaupt ein öffentliches Interesse vorliegt, nicht dass Staatstätigkeit einzig auf dieses ausgerichtet ist, oder auch nur, dass es gegenüber anderen Interessen überwiegen muss.

Nicht jeder Effekt einer Privatisierungsbestrebung, der Staatszielen vermeintlich entgegenläuft, ist deshalb verfassungswidrig. Und insbesondere darf diese Anforderung nicht zum alles übergreifenden Gemeinwohl überhöht werden. Es ist gerade kein verfassungsmässiges Gebot, dass jedes Staatshandeln in der Summe das Gemeinwohl befördern muss. Nochmals: Ein öffentliches Interesse genügt, es muss nicht das einzige handlungsrelevante Motiv sein. Das öffentliche Interesse fordert immerhin, dass auch die privatisierte Aufgabenerfüllung auf die dahinterliegenden gesamtgesellschaftlichen Ziele

830 TSCHANNEN/ZIMMERLI/MÜLLER, Allgemeines Verwaltungsrecht, §20 Rz. 2. 
ausgerichtet bleibt. Denn aufgrund solcher Ziele wird ein Tätigkeitsbereich überhaupt erst dem Staat überantwortet und somit zur staatlichen Aufgabe.

\section{Insbesondere bei den privatisierten Organisationsformen}

\section{a. Spannung aus Zweckbindung wegen eingeschränkter Aufsicht und weniger enger Kontrolle}

Wenn Staatsaufgaben an Rechtssubjekte ausserhalb der Zentralverwaltung übertragen werden, führt dies tendenziell zu einer weniger intensiven Aufsicht durch die Regierung und zu einer bloss periodischen Steuerung statt ständiger Begleitung (siehe zu diesen Kennzeichen vorne \$ 4/II.4.cundd). Wie auch immer die Einflussnahme des Parlaments und der Regierung auf ausgelagerte Aufgabenträger im Einzelnen ausgestaltet ist, ihre laufende Verwaltungsarbeit wird weniger eng begleitet als bei Einheiten der Zentralverwaltung.

Dies führt zu Spannungen mit dem Grundsatz des öffentlichen Interesses: Ob der privatisierte Aufgabenträger die öffentlichen Interessen befördert, die für die Designation seines Tätigkeitsbereichs als staatliche Aufgabe entscheidend waren, wird nur noch vorab kontrolliert (mit dem Errichtungsakt) und dann periodisch überprüft (etwa bei der jährlichen Evaluation der Zielerreichung nach Leistungsvereinbarung). Dadurch nimmt die Gewähr ab, dass der Aufgabenträger in seiner laufenden Tätigkeit zuvorderst die öffentlichen Interessen befördert.

Ausserdem nehmen die Möglichkeiten ab, bei Bedarf korrigierend einzugreifen. Sollte sich ein privatisierter Aufgabenträger von den Zielen entfernen, die seine Arbeit überhaupt legitimieren, wird dies erst bei der periodischen Kontrolle aufscheinen, wenn eine grundlegende Korrektur vielleicht schon nicht mehr möglich ist. Innerhalb der Zentralverwaltung hingegen erlaubt es die Dienstaufsicht einer übergeordneten Behörde, direkt in die laufende Aufgabenerfüllung einzugreifen. ${ }^{831}$

\section{b. Spannung aus Renditezielen}

Eine Spannung entsteht insbesondere bei zwei Organisationsformen: der öffentlichrechtlichen Aktiengesellschaft und den privatrechtlichen Gesellschaften (siehevorne \$ 4/II.3.g und h). Immer dann, wenn staatliche Aufgaben an Organisationen mit Renditeziel übertragen werden, könnte dieses privatökonomische Ziel die öffentlichen Interessen verdrängen, die der Aufgaben- 
übertragung zugrunde liegen. Diese Spannung dürfte in den allermeisten Fällen aber ein bloss potentielles Problem bleiben.

Zur Rekapitulation: Das Bundesgericht hat 2013 das Eintreiben von Werbegeldern als mittelbare Staatsaufgabe der schweizerischen Radio- und Fernsehgesellschaft (SRG) eingestuft:

«Nach Art. 35 Abs. 2 BV ist an die Grundrechte gebunden und hat zu deren Verwirklichung beizutragen, wer staatliche Aufgaben wahrnimmt. Dies ist bei der SRG im Rahmen ihres programmrechtlichen Auftrags im an sich von ihr privatrechtlich bewirtschafteten Werbebereich der Fall, da dieser als Nebenaktivität zur Finanzierung ihrer Programme dient [...].»832

Konsequent weitergedacht bedeutet diese Wertung, dass die privatwirtschaftliche Mittelbeschaffung ein legitimes öffentliches Interesse darstellt. Aus dieser Rechtsprechungslinie ist also zu folgern, dass tendenziell auch fiskalische Motive allein staatliches Handeln legitimieren können. So deutlich wurde das Bundesgericht bisher nicht. Aber schon heute ist unbestritten, dass fiskalische Interessen (neben anderen öffentlichen) zulässige Interessen sind (siehe vorne \$3/II.3.a).

Auch wenn Art. 5 Abs. 2 BV Renditezielen nicht grundsätzlich entgegensteht, führt diese verfassungsrechtliche Anforderung zu Interessenkonflikten. Das Renditeziel kann nämlich andere öffentliche Interessen zurückdrängen. Die Erfüllung der gesetzlich vorgegebenen Staatsaufgaben sollte im Interesse der AG liegen - diese Aufgabe ist ihr übertragen. Wenn die Besorgung der Aufgabe das gleichzeitig bestehende Renditeziel gefährdet, dürfte dies die Erfüllung staatlicher Aufgaben nicht effizienter machen. Der Staat muss diesen Interessenkonflikten nämlich vorbeugen oder sie entschärfen, etwa indem er Verwaltungsräte ein- oder absetzt. ${ }^{833}$

Renditeziele sind zwar nicht grundsätzlich problematisch, sie drängen aber den eigentlichen Zweck eines Aufgabenträgers - die Erfüllung staatlich übertragener Aufgaben - hinter privatwirtschaftlich geprägte Überlegungen zurück. 834

c. Kein grundsätzliches Problem aus der «Kohärenz des staatlichen Handelns» BIAGGINI sieht in Auslagerungen eine grundsätzliche Gefahr für die «Kohärenz staatlichen Handelns» 835: «Verselbständigte Fachbehörden» weisen eine für

832 BGE 139 I 306 E.3.2.2 S.311.

833 Vgl. BÜHLER, Corporate Governance, S. $516 \mathrm{ff}$.

834 So etwa im Glarnersach-Fall, BGE 138 I 378 E. 8.6.2 S.397.

835 BIAGGINI, Entwicklungen im Verfassungsrecht, S. 35, auch zum Folgenden. 
sie «typische «Eindimensionalität» der Aufgabenstellung» auf, welche in einem gewissen Konflikt zur mehrdimensionalen Gemeinwohlbindung des Staats stehe.

Ein Problem könnte darin bestehen, dass bei der organisatorischen Erfüllungsprivatisierung der Blick auf die Gesamtheit staatlicher Aufgaben verloren zu gehen droht. Staatstätigkeit erfolgt nie in einem Vakuum, sondern sie dient der Förderung der staatlichen Ziele insgesamt. Vor dem Hintergrund des öffentlichen Interesses ist diese Entwicklung nur vermeintlich ein Problem. Die Verfassung lässt es zu, dass verschiedene öffentliche und private Interessen einander konkurrenzieren; mit der Interessenabwägung innerhalb der Verhältnismässigkeitsprüfung hält die Verfassung gar ein Instrument für die Abwicklung solcher Konkurrenzen bereit. Dass bei der einen Verwaltungstätigkeit nicht nur das eine, eindimensionale Ziel befördert wird, mag für die Effizienz des Staats als ausgeklügelte Maschine nachteilig sein, hält aber vor der Verfassung ohne Weiteres stand.

\section{Insbesondere bei den privatisierten Handlungsformen}

\section{a. Grundsätzliche Spannung zwischen Gestaltungsfreiheit und Zweckbindung}

Auch privatisierte Handlungsformen führen zu einer grundsätzlichen Spannungslage zwischen erhoffter Flexibilität und der Zweckbindung an öffentliche Interessen.

Die privatisierten Handlungsformen zeichnen sich durch einen Zuwachs an Gestaltungsfreiheit im Einzelfall aus (siehe vorne \$ 4/III.3.b). Die Abkehr von einseitigen, hoheitlichen und verbindlichen Instrumenten soll dem Aufgabenträger eine flexiblere, freiere Aufgabenerfüllung ermöglichen. Der Verzicht auf hoheitliche Instrumente soll die Akzeptanz und freiwillige Befolgung seitens der Verwaltungsadressaten befördern (siehe zu dieser gesteigerten Wirkungserwartung vorne \$4/III.3.e).

Tendenziell mögen Verwaltungsträger diegrössere Freiheit dazu nutzen, ihre Aufgaben möglichst ressourcenschonend zu erfüllen - und nicht möglichst zwecktauglich bezogen auf das öffentliche Interesse. Inwiefern dadurch die Aufgabenerfüllung die öffentlichen Interessen vernachlässigt, kann nur im Einzelfall untersucht werden. Den Massstab dafür gibt die Interessenabwägung unter dem Titel der Verhältnismässigkeit vor (siehe hinten III). 


\section{b. Einbezug von Fachexperten kann Sondergruppeninteressen über öffentliche Interessen stellen}

Von den in dieser Arbeit erläuterten privatisierten Instrumenten für die Erfüllung staatlicher Aufgaben bereitet die ausgelagerte Rechtsetzung und Rechtsanwendung unter beibehaltenen Einflussmöglichkeiten die vielfältigsten Herausforderungen (siehe vorne \$ 4/III.2.b.cc).

Dieser Sammelbegriff vermeidet die sonst gebräuchlichen Bezeichnungen «regulierte Selbstregulierung» und «akkreditierte Zertifizierung», um etwas zu verdeutlichen: Wie auch immer das Konstrukt heisst, Private werden dadurch Träger staatlicher Aufgaben. Sie werden nämlich beauftragt, Teilaufgaben in der Setzung oder Anwendung staatlicher Normen wahrzunehmen. Nicht nur die Normen sind dabei staatlich, auch sind deren Setzen und Anwenden staatliche Aufgaben. So ist etwa der Erlass von Reglementen durch Selbstregulierungsorganisationen im Geldwäschereirecht ${ }^{836}$ ebenso als staatliche Aufgabe einzustufen wie die Akkreditierung von Studienprogrammen durch die dafür zuständige Agentur ${ }^{837}$.

Doch diese und andere Vorgänge der ausgelagerten und überwachten Normsetzung und -anwendung bergen grundsätzliches Spannungspotential. Die auf öffentlichen Interessen fussende Regulierungs- und Prüftätigkeit wird auf Akteure übertragen, die den betroffenen Verwaltungsadressaten näher stehen als dem Staat. Zu den vorherigen Beispielen: Den Selbstregulierungsorganisationen nach GwG sind insbesondere Finanzintermediäre mit den erforderlichen Fachkenntnissen angeschlossen;838 und die Mitglieder des Akkreditierungsrats nach HFKG «vertreten insbesondere die Hochschulen, die Arbeitswelt, die Studierenden, den Mittelbau und den Lehrkörper.»839

Private Interessen und Sondergruppeninteressen sind gerade keine zulässigen öffentlichen Interessen nach Art. 5 Abs. 2 BV (siehe vorne \$3/II.3). ${ }^{840}$ Werden Fachexperten und Betroffene selbst zu Gesetzgebern und Rechtsanwendern, liegt die Vermutung nahe, dass tendenziell Sondergruppeninteressen über gesamtgesellschaftliche, öffentliche Interessen gestellt werden. ${ }^{841}$ Auf jeden Fall verschiebt sich die Gewährleistung der öffentlichen Zweckbindung auf die periodische Aufsicht, Kontrolle und potentielle Anpassungen der Rahmengesetzgebung (ähnlich wie vorne in 1.a betreffend die Organisationsformen).

\footnotetext{
836 Siehe Art. 24 f. GwG.

837 Art. 22 HFKG, Art. 5 Akkreditierungsverordnung HFKG (Fn. 681).

838 Art. 26 Abs.1 i.V.m. Art. 24 Abs.1 Bst.c Ziff.1 GwG.

839 Art. 21 Abs.1Satz1 HFKG.

840 BIAGGINI, Kommentar BV, Art. 5 Rz. 15.

841 In der deutschen Lehre ähnlich auch THOMA, Selbstregulierung, S. 473.
} 
c. Nurvermeintliche Spannung zwischen privatem Auftreten und Gemeinwohl Mehrere Kennzeichen der Erfüllungsprivatisierung in instrumenteller Hinsicht gründen darin, dass der Staat vermehrt wie ein Privater auftritt: Er verzichtet auf seinen (in der Sache unveräusserlichen) Machtvorsprung und auf die verbindliche, einseitige, hoheitliche Anordnung von Rechten und Pflichten (vgl. vorne die Kennzeichen bei \$4/III.3.c, dund h).

Dieses Auftreten steht in Spannung zum öffentlichen Interesse. Es könnte befürchtet werden, die privatisierten Handlungsformen drängten die originär öffentlichen Interessen in den Hintergrund, wenn Staatstätigkeit zum verhandlungsbasierten Austarieren zwischen gleichgestellten Akteuren wird. Doch die Anforderung von Art. 5 Abs. 2 BV heisst nur, dass ein öffentliches Interesse die Staatstätigkeit legitimieren können muss. Dass private Interessen ebenso hoch gewichtet werden, widerspricht dieser Anforderung nicht, solange ein zulässiges öffentliches Interesse vorliegt. Im Gegenteil, dies ist ganz im Sinne der Anforderung.

Privatautonome Handlungen sind zwar grundlegend nicht dem Gemeinwohl verpflichtet. ${ }^{842}$ Doch ist daraus noch kein Problem im hier diskutierten Sinn abzuleiten. Es mag seltsam sein, dass sich der Staat Vorteile daraus verspricht, die Eigenheiten seiner Wirkungsweise dem Anschein nach aufzugeben, doch verfassungsrechtlich stossend ist das nicht.

Gewiss, Verträge und wegdelegierte Normsetzungen lockern tendenziell die Gemeinwohlbindung. Doch ist dies, zumindest an den verfassungsrechtlichen Anforderungen gemessen, nicht grundsätzlich zu bemängeln. Die Verhältnismässigkeitsprüfung kann sicherstellen, dass weiterhin genügend gewichtige öffentliche Interessen das Staatshandeln legitimieren.

\section{Insbesondere bei den privatisierten Finanzierungsformen}

Die Anforderung eines genügenden öffentlichen Interesses steht der Erfüllungsprivatisierung in finanzieller Hinsicht nicht grundsätzlich entgegen. Fiskalische Interessen sind zulässige öffentliche Interessen (siehe vorne \$3/ II.3.a). Der jüngeren Rechtsprechungslinie folgen könnten gar fiskalische Interessen alleine ausreichen.

Die Verschiebung der Aufgabenfinanzierung vom Gemeinlastprinzip weg zu einer als Gegenleistung ausgestalteten Verursacherfinanzierung steht somit der Zweckrichtung von Art. 5 Abs. 2 BV nicht entgegen. Die verfassungsrechtlich relevante Verschiebung findet an anderer Stelle statt: Indem die 
mittelbare Aufgabenerfüllung (also die Mittelbeschaffung) im Rahmen der Grundrechtsbindung tendenziell der unmittelbaren Aufgabenerfüllung gleichgestellt wird, verwischt die Grenze zwischen Leistungs- und Bedarfsverwaltung (siehe vorne \$3/II.6.b.cc und \$4/I.2).

\section{a. Kein grundsätzliches Problem aus Abkehr von Gemeinlastprinzip}

Die Abkehr vom Gemeinlastprinzip (siehe zu diesem Kennzeichen vorne \$4/ IV.4.b) ist mit dem öffentlichen Interesse grundsätzlich vereinbar. Zwar böte die Finanzierung über den alltäglichen Staatshaushalt Gewähr dafür, dass die Staatstätigkeit auf die gesamtgesellschaftlichen Staatsziele ausgerichtet bleibt. Aber diese verfassungsrechtliche Anforderung bedeutet gerade keine einschränkende Bindung an ein übergreifendes Gemeinwohl. Im Gegenteil muss für jedes Staatshandeln und jede einzelne Einschränkung privater Rechtsstellungen ein konkretes legitimierendes Ziel benannt werden.

Muss ein Verwaltungsträger seinen Etat selber bestellen, etwa mit Gebühren, Kostenanlastungssteuern oder Aufsichtsabgaben, ändert dies nichts an seiner Pflicht, die ihm anvertrauten Aufgaben zu erfüllen. Die Verschiebung parlamentarischer Kontrolle weg vom Bereich des Budgetrechts bedeutet zwar tendenziell eine weniger intensive Kontrolle, ob und wie treffgenau die vorgegebenen Zwecke gefördert werden. Doch dies ist eine blosse Spannung und führt nicht zu Problemen mit Blick auf die Anforderungen der Verfassung (vgl. vorne I.3). Das öffentliche Interesse wird weiterhin im politischen Prozess definiert, indem das Parlament seinen Einfluss über die Einzelfallregelung ausübt - sprich die spezialgesetzliche Aufgabenübertragung und die Einrichtung besonderer, privatisierter Finanzierungsformen.

Allerdings kann ein Problem aus der Grundrechtsbindung entstehen, wenn die Verursacherfinanzierung die Geltendmachung grundrechtlicher Ansprüche vereitelt (siehe dazu hinten V.3.b).

\section{b. Spannung aus verschobener Optik von Verwaltungsadressaten zu Kunden}

Wenn Verwaltungsadressaten für staatliche Eingriffe und Leistungen zahlen, werden sie von Bürgern zu Kunden. Die Optik der Aufgabenerfüllung verschiebt sich von der Regelung öffentlichrechtlicher Pflichten und der Erbringung von Leistungen im Dienste der Allgemeinheit hin zum privatökonomischen Quidproquo von Leistung für Gegenleistung. Der Wert einer Verwaltungshandlung liegt dann im Entgelt, das sie generiert, nicht im öffentlichen Interesse, das sie befördert.

Ähnlich wie beim Einbezug von Fachexperten in die Rechtsetzung und Rechtsanwendung droht dies Sonderinteressen über genuin öffentliche Interessen zu stellen (siehe dazu vorne 2.b). Zur Illustration eine sehr treffende 
Zuspitzung aus der US-amerikanischen Lehre: «Thus, the definition of service to clients becomes one of particular services to particular clients as voters and members of interest groups, rather than service to citizens in their role as citizens." "843 Staatstätigkeit werde durch solche Vorgänge zur bestimmten Leistung für bestimmte Kunden - verstanden als Wähler und Interessenvertreter.

Ein Beispiel: Swissmedic fördert nicht mehr zuerst die öffentliche Gesundheit durch ihre Arzneimittelkontrollen, sondern bedient die Interessen der Antragssteller, die ihren Betrieb sichern. Doch solange Swissmedic, was unbestritten ist, auch weiterhin öffentliche Interessen befördert, ist aus Sicht der Verfassung eine grundsätzliche «Kundenorientierung» nicht zu bemängeln.

\section{c. Kein grundsätzliches Problem aus ökonomischen Anreizen}

Die verursacherbasierte, auf Leistung und Gegenleistung aufbauende Finanzierung soll die Staatstätigkeit effizienter und günstiger machen. Zu diesem Zweck sind die privatisierten Finanzierungsformen von Anreizen geprägt sowohl an Private, Staatstätigkeit zu vermeiden, wie auch an Aufgabenträger, alle Teilaspekte ihrer Tätigkeit zu monetarisieren (vgl. die Kennzeichen vorne in \$4/IV.4.e undf).

Dass damit ökonomische Kräfte das Verwaltungshandeln durchdringen, ist nicht per se problematisch. Auf öffentlichen Interessen zu fussen, heisst nicht zwingend, rein idealistisch und in keinem Fall ökonomisch motiviert zu sein. Die neuere Rechtsprechungslinie, die Mittelbeschaffung als «Nebenaktivität» zur Finanzierung der Haupttätigkeit ansieht (siehe vorne \$ 4/I.2), zeigt es deutlich: Die langfristige finanzielle Absicherung darf bei jeder Aufgabenerfüllung mitgedacht bzw. ihr gar vorangestellt werden.

Dadurch rücken die öffentlichen Interessen, die hinter der zugewiesenen Staatsaufgabe liegen, vorübergehend in den Hintergrund. Die Optik der Staatsaufgaben lautet: «Diese Leistung ist wichtig, sie muss erbracht werden.» Die Verursacherfinanzierungs-Optik lautet: «Der Bürger soll sich zweimal überlegen, ob er die Leistung wirklich braucht.»

Die Verursacherfinanzierung soll die Nachfrage nach der entsprechenden staatlichen Leistung senken. ${ }^{844}$ Wenn die Erfüllung einer Aufgabe steuerfinanziert ist, werden mehr Leute davon profitieren wollen, weil der Zusammenhang zwischen Leistungsbezug und Steuern nur indirekt ist. Diese Optik verkennt aber, dass staatliche Aufgaben erfüllt werden müssen. Verfassungsund Gesetzgeber haben entschieden, dass an ihrer Erfüllung ein öffentliches Interesse besteht. Sollen nun finanzielle Anreize von der Inanspruchnahme

843 PETERS, Failure of Managerial Reform, S. 43.

844 FREY, Drei Stossrichtungen, S. $343 \mathrm{f}$. 
einer staatlichen Leistung abraten, widerläuft das diesem Interesse. Doch solange das öffentliche Interesse weiterhin vorliegt und die Staatstätigkeit im Ganzen legitimiert, entsteht hier keine problematische Spannung aus Art. 5 Abs. 2 BV.

Ein Problem entsteht nur, wenn aufgrund ökonomischer Anreize gesetzliche Leistungspflichten unterschritten werden (siehe vorne I.3 und hinten V.3.b). Wenn hingegen die ökonomischen Anreize aufzeigen, dass an einer bestimmten staatlichen Leistung gar kein Interesse besteht, weil sie falsche Sachverhalte oder falsche Bezüger abdeckt, eröffnet dies eine Diskussion auf anderer Ebene: Dann muss geprüft werden, ob an der fraglichen Leistung weiterhin ein öffentliches Interesse besteht. Falls nicht, kann die Leistungspalette zurückgefahren, sprich ein Teil der Aufgabe privatisiert werden. Die Diskussion verlagert sich somit auf die Änderung der gesetzlichen Pflichten, bestimmte Tätigkeiten als staatliche Aufgaben zu erfüllen. 


\section{Probleme aus der Verhältnismässigkeit}

Art. 5 Abs. 2 BV verlangt für staatliches Handeln zweierlei: ein öffentliches Interesse und Verhältnismässigkeit. Der erste Teilgehalt ist schon dann erfüllt, wenn sich staatliches Handeln überhaupt auf ein zulässiges öffentliches Interesse stützen kann. Die Verhältnismässigkeit baut darauf auf und fordert zusätzlich, dass das staatliche Handeln im Einzelfall dieses Interesse auch tatsächlich befördert und dass das öffentliche Interesse entgegenstehende private Interessen überwiegt (siehe vorne \$3/II.4). Das Verhältnismässigkeitsprinzip operationalisiert das öffentliche Interesse also für den einzelnen Fall einer staatlichen Handlung, die Rechtspositionen Privater betrifft: Die Verfassung misst den Staat nicht nur an den Motiven, auf denen seine Tätigkeiten beruht, sondern auch an den Wirkungen, die sie zeitigen.

Werden diese inhaltlichen Anforderungen nun auf die verschiedenen Erscheinungsarten der Erfüllungsprivatisierung angewendet, ist zunächst grundlegend festzustellen: Wo immer beim öffentlichen Interesse eine Spannungslage besteht, kommt dem Verhältnismässigkeitsprinzip eine grössere Bedeutung zu. Denn nur es kann beantworten, ob im Einzelfall ein Problem entsteht. Immer wenn aufgabenfremde Motive, Interessen und Anreize in die Aufgabenerfüllung hineinwirken, entsteht ein genereller Verdacht, dass die verfassungsrechtlichen Anforderungen zu Problemen führen können. Nur die Verhältnismässigkeitsprüfung im Einzelfall kann diesen Verdacht ausräumen. Dies trifft insbesondere dort zu, wo Interessenkonflikte und eingebundene Sonderinteressen zu den Kennzeichen der Erfüllungsprivatisierung gehören (siehe die Kennzeichen \$ 4/III.3.fund g sowie \$ 4/IV.4.g und die entsprechenden Spannungen aus dem öffentlichen Interesse vorne bei II.2.b und II.3.c).

Staatliches Handeln muss die öffentlichen Interessen, die es legitimieren, tatkräftig und zielgenau befördern. Die Erfüllungsprivatisierung darf nicht dazu führen, dass die staatliche Aufgabenerfüllung zur Schrotflinte mutiert, die das öffentliche Interessen nur noch zufällig und nebenbei trifft.

\section{Keine besonderen Probleme bei der Privatisierung der organisatorischen Erfüllungsaspekte}

Das Verhältnismässigkeitsprinzip entfaltet seine bindende Wirkung auf der Ebene des konkreten Einzelfalls. Daher verursacht die Organisationsprivatisierung alleine noch keine Probleme, die hier erwähnenswert wären. Die potentiell problematischen Auswirkungen entstehen erst aus einzelnen Erfüllungshandlungen - also bei den privatisierten Handlungsformen (sogleich 2). 
Wenn Verwaltungsträger so organisiert werden, dass sie (zum Teil auch) Renditeziele erreichen und ihren strukturellen Erhalt selber sichern müssen, kann dies potentiell zu Interessenkonflikten führen (siehevorne II.1.b). Hier wird dies als Konsequenz der Erfüllungsprivatisierung in finanzieller Hinsicht abgehandelt (sogleich 3). Erst durch die einzelne Finanzierungsform, sprich durch die konkret auferlegte Gebühr, entstehen Spannungen aus dem Verhältnismässigkeitsprinzip, nicht schon aus der entsprechenden Organisationsform.

\section{Insbesondere bei den privatisierten Handlungsformen}

\section{a. Quasiprivates Auftreten kommt tendenziell privaten Interessen zugute}

Werden die instrumentellen Aspekte staatlicher Aufgabenerfüllung privatisiert, führt dies in verschiedener Hinsicht zu einem staatlichen Handeln, das in seinem Erscheinen und seinen Wirkungen privatem Handeln ähnelt. Sei es durch quasiprivatautonome Gestaltungsfreiheit oder durch den freiwilligen Verzicht auf staatliche Steuerung zugunsten unverbindlicher, nur beschränkt durchsetzbarer Instrumente - der Staat gibt dadurch seinen Machtvorsprung dem Anschein nach auf, womit er mitunter schwerlich von privaten Akteuren zu unterscheiden ist (vgl. vorne die Kennzeichen \$ 4/III.3.b-d und h).

Indem der Staat Instrumente einsetzt, die nicht von einseitiger und verbindlicher Rechtsdurchsetzung geprägt sind, schwächt er tendenziell seine Fähigkeit, seine Interessen direkt und präzise zu erreichen. Auch eine informelle Absprache oder eine amtliche Warnung muss die vorgegebenen Interessen wirkungsvoll fördern. Dass sie dies grundsätzlich kann, wird hier nicht bestritten. Dass solche Instrumente private Interessen auf neue und vielleicht überraschend beschränkende Art treffen, ist sogleich unter $b$ zu thematisieren.

Der quasiprivate Auftritt alleine führt nicht zu einem Problem. Im Gegenteil, er dürfte erreichen, dass das staatliche Handeln auch private Interessen befördert und die öffentlichen Interessen schonend (weil weniger steuerungskräftig) umsetzt. Die Effektivität - und damit die Zwecktauglichkeit - mag im Einzelnen leiden, doch ist dies nicht in grundsätzlicher Weise ein Problem mit Blick auf die Verfassung. Die eingesetzten Mittel müssen geeignet sein, zur staatlichen Zielerreichung beizutragen, es müssen aber nicht die einzigen geeigneten Mittel sein. ${ }^{845}$ Laut Bundesgericht ist eine Massnahme schon dann geeignet, wenn sie «im konkreten Fall mit einer minimalen Wahrscheinlichkeit [...] ihren Zweck zu erfüllen» ${ }^{846}$ vermag.

845 Vgl. HÄFELIN/MÜLLER/UHLMANN, Allgemeines Verwaltungsrecht, Rz. 522. 846 BGE 135 II 105 E. 2.3.3 S.109. 


\section{b. Problem aus neuen Formen staatlicher Beeinflussung}

$\mathrm{Zu}$ den Kennzeichen privatisierter Handlungsformen gehört auch, dass sie die reduzierte Steuerungskraft mit einer erhofft stärkeren Steuerungswirkung kompensieren (siehe vorne \$4/III.3.e). Die geringere Verbindlichkeit und der Verzicht auf hoheitliche Anordnungen seitens des Staats sollen Private dazu bewegen, das Staatshandeln besser zu akzeptieren und freiwillig zu befolgen.

Mit dieser Verschiebung von Steuerungskraft zu Steuerungshoffnung reduziert der Staat allerdings nur vermeintlich seine Eingriffe in private Rechtspositionen. Die Eingriffe mögen weniger direkt sein, weniger einschneidend sind sie deswegen noch nicht. Mit welchen Mitteln auch immer - die Erfüllung staatlicher Aufgaben bedingt die Steuerung privaten Verhaltens. Müssten die Rechte Privater nicht eingeschränkt werden, müssten keine neuen Pflichten oder Ansprüche verteilt werden, entfiele die Legitimation für das staatliche Handeln komplett: Dann mangelte nämlich das öffentliche Interesse; wo Private sich selbst überlassen werden können, braucht es den Staat von vornherein nicht.

Die weniger verbindlichen Instrumente bringen das grundsätzliche Problem mit sich, weniger zwecktauglich und weniger zielgenau zu sein. Statt ein bestimmtes Verhalten zu verbieten oder für bewilligungspflichtig zu erklären, sollen Anreize dasselbe Ziel erreichen. Diese Regulierungslogik basiert auf dem verhaltensökonomischen Konzept des «Nudging» (Anstupsen). ${ }^{847} \mathrm{Um}$ das Verhalten von Privaten zu lenken, müsse ihnen der Staat nicht paternalistische Gebote und Verbote aufstellen, er könne stattdessen ihre «Entscheidarchitektur» mit Anreizen beeinflussen, ohne Entscheidmöglichkeiten ganz zu verschliessen. Das typische Beispiel ist für das Schweizer Recht hypothetisch: Statt gesundheitsgefährdende Lebensmittel in Schulkantinen per Gesetz zu verbieten, werden sie bloss weniger gut sichtbar platziert. ${ }^{848}$ Diese Regelungslogik bereits umgesetzt haben hingegen das Bundesgesetz und die dazugehörige Verordnung über das elektronische Patientendossier. ${ }^{849}$ Statt verbindlich zu regeln, welche Gesundheitspersonen auf welche vertraulichen Dokumente Zugriff haben oder diese Zugriffsfrage völlig offenzulassen, hat sich der Gesetzgeber für bestimmte Voreinstellungen entschieden. ${ }^{850} \mathrm{Im}$

847 Grundlegend THALER/SUNSTEIN, Nudge. Das Konzept in der schweizerischen Rechtswissenschaft rezipierend HEINEMANN, Recht, Ökonomie und Realität, S. 33 f.; HETTICH, Kooperative Risikovorsorge, Rz.101; auch zum Folgenden.

848 HEINEMANN, Recht, Ökonomie und Realität, S. 33; SCHNIDER, Schutz des Arbeitnehmers, S. $136 \mathrm{f}$.

849 EPDG bzw. EPDV.

850 SPRECHER, Datenschutz und Big Data, S. $530 \mathrm{f}$. 
entsprechenden Formular sind etwa bestimmte Zugriffsrechte standardmässig gesetzt; wer das Formular ausfüllt, kann die Einstellungen aber ändern. Patientinnen und Patienten haben weiterhin alle Entscheidmöglichkeiten, doch versucht der Staat, ihre Entscheidung zu beeinflussen.

Wählt der Staat unverbindliche Instrumente, scheint dies im Rahmen der Verhältnismässigkeit zunächst den privaten Interessen zugutezukommen. Eine Warnung ist weniger einschneidend als ein Verbot, eine Voreinstellung in einem Formular überlässt dem Betroffenen mehr Freiheit als eine gesetzliche Anordnung. Doch müssen auch diese Handlungsformen an ihrer Tauglichkeit gemessen werden, die angepeilten öffentlichen Interessen zu fördern. ${ }^{851}$ Es mag sich dabei um milde Mittel handeln, doch vielleicht fehlt ihnen bereits die Eignung.

Die vermeintlich flexiblere und bedarfsgerechte Lösung, starre Regeln zugunsten informeller Kommunikation zurückzudrängen, beinhaltet also auch die Gefahr überschiessender staatlicher Eingriffe. ${ }^{852}$

\section{Insbesondere bei den privatisierten Finanzierungsformen}

Bei denjenigen Finanzierungsformen, die als Gegenleistung für eine staatliche Leistung ausgestaltet sind, kann das Äquivalenzprinzip Mängel in der gesetzlichen Grundlage ausbessern. ${ }^{853}$ Dies bedeutet aber keine Verschärfung der Verhältnismässigkeitsprüfung, im Gegenteil: Das Äquivalenzprinzip lässt bei der privatisierten Aufgabenfinanzierung Spielräume entstehen, die desto grösser werden, je mehr sie das Verursacherprinzip aufweichen (siehe vorne das Kennzeichen \$ 4/IV.4.c). Diese Spielräume werden im folgenden Paragrafen unter dem Gesetzmässigkeitsprinzip abgehandelt (siehe hinn ten $\$ 6 / I V .3 . a)$.

Eine Spannung entsteht hingegen, wenn privatisierte Aufgabenträger Renditeziele erreichen müssen oder Anreize sie dazu anhalten, jeden Teilaspekt ihrer Aufgabenerfüllung als verrechnungsfähige Leistung zu monetarisieren (vgl. vorne II.1.b und das Kennzeichen \$ 4/IV.4.f). Dies sind mittelbare Zwecke: Der Aufgabenträger muss Mittel generieren, um seine Aufgaben überhaupt erst erfüllen zu können. Mit Blick auf die öffentlichen Interessen mag dies nicht weiter problematisch sein, da ja weiterhin inhaltliche (sprich aufgabenbezogene) Interessen vorliegen und wohl sogar rein fiskalische Motive zulässig wären. Anders liegt dies bei der Verhältnismässigkeit.

851 Vgl. RUCH, Informalisierung, S. 525f.

852 Vgl. SPRECHER, Datenschutz und Big Data, S. $531 \mathrm{f}$.

853 Anstelle vieler BGE 143 I 147 E. 6.3.1 S.158. 
Die Verfassung fordert hier, dass das Staatshandeln seine Ziele im Einzelfall tatkräftig befördert, und zwar möglichst zielgenau (also nicht daneben- oder überschiessend).

Dient die Verwaltungstätigkeit mehr der Mittelbeschaffung als der eigentlichen Aufgabenerfüllung, führt dies zu weniger zielgerichteten und schonenden Eingriffen in private Rechtspositionen. Es darf angenommen werden, dass zusätzlicher Aufwand als Leistung verrechnet wird, wie etwa wenn die Swissmedic in ihrer Jahresrechnung «Rechtsgrundlagen» und «Strafrecht» als gemeinwirtschaftliche «Produkte» ausweist (siehe vorne \$ 4/IV.4.f). ${ }^{854}$ Ebenso ist davon auszugehen, dass privat- oder öffentlichrechtliche Aktiengesellschaften vermehrt rentable Tätigkeiten verfolgen und dies im betriebseigenen Ressourcenkampf die originär staatliche Aufgabenerfüllung potentiell konkurrenziert. 


\section{Probleme aus den Teilgehalten des Grundsatzes von Treu und Glauben}

Der Grundsatz von Treu und Glauben ist zwar eine allgemeine Verhaltensregel im gesamten Rechtsverkehr, doch er enthält vier Teilgehalte, die die staatliche Aufgabenerfüllung in Gestalt verfassungsrechtlicher Anforderungen binden: den Schutz des berechtigten Vertrauens in (an sich unrichtiges) Staatshandeln und die drei Verbote widersprüchlichen, täuschenden und rechtsmissbräuchlichen Verhaltens (siehe vorne \$3/II.5. a und b). Diese verschiedenen Anforderungen auf einen Satz gebracht, bedeutet Treu und Glauben, dass Verwaltungsadressaten darauf vertrauen dürfen, dass der Staat verlässlich handelt.

Die Erfüllungsprivatisierung steht diesen (Teil-)Anforderungen von Treu und Glauben nicht entgegen. Die Motivation und Stossrichtung der privatisierten Aufgabenerfüllung reibt sich nicht an der Forderung, dass Private in die Verlässlichkeit des Staats vertrauen dürfen.

Die privatisierte Aufgabenerfüllung erschwert zwar treugemässes Staatshandeln nicht grundsätzlich, doch bringt sie die Verwaltungsadressaten in eine problematische Lage: Ihr Vertrauen in eine Handlung kann nur geschützt werden, wenn sie wissen, dass diese eine staatliche Handlung ist und daher eine Vertrauensgrundlage im Sinne dieser verfassungsrechtlichen Anforderung darstellt. 855 Inwiefern die privatisierten Handlungsformen somit den Vertrauensschutz gefährden, wird hinten in 2. $a$ thematisiert.

\section{Keine besonderen Probleme bei der Privatisierung der organisatorischen Erfüllungsaspekte}

Die Teilgehalte des Grundsatzes von Treu und Glauben sind jeweils nur auf einzelne Handlungen anwendbar. Nur im konkreten Bezug zu einzelnen Verwaltungsadressaten kann schutzwürdiges Vertrauen entstehen und kann sich der Staat potentiell treuwidrig verhalten. Aus privatisierten Organisationsformen allein entstehen mit Blick auf diese verfassungsrechtliche Anforderung also keine Probleme.

855 Siehe zur Vertrauensgrundlage HÄFELIN/MÜLLER/UHLMANN, Allgemeines Verwaltungsrecht, Rz.668-675; TSCHANNEN/ZIMMERLI/MÜLLER, Allgemeines Verwaltungsrecht, §22 Rz.15, 19. 


\section{Insbesondere bei den privatisierten Handlungsformen}

Der Staat darf auch dann nicht widersprüchlich, täuschend oder rechtsmissbräuchlich handeln, wenn er privatisierte Handlungsformen einsetzt. Die grössere Gestaltungsfreiheit (siehe vorne \$ 4/III.3.b) erhöht zwar tendenziell das Risiko von widersprüchlichem Handeln, da der Staat in freien Verhandlungen und formlosen Äusserungen verschiedene Taktiken ausprobieren könnte. Doch dass dieses Verhalten der Verfassung entgegensteht, ist keine Besonderheit dieser Instrumente.

Ebenso verhält es sich, wenn der Staat Private mit neuen Methoden zur freiwilligen Befolgung seiner Vorgaben bewegen will (siehe vorne \$4/III.3.e und soeben III.2.b). Dass staatliche Aufgabenerfüllung darauf beruht, Private zu gewissem Verhalten anzuregen bzw. davon abzuhalten, ist nicht auf privatisierte Handlungsformen beschränkt. Die - immer geltende - Grenze dafür ist das Verbot täuschenden Verhaltens.

\section{a. Potentielle Probleme aus erschwerter Erkennbarkeit staatlicher Instrumente}

Was den Teilgehalt des Vertrauensschutzes betrifft, so ergeben sich keine Unterschiede aus dem Einsatz privatisierter Handlungsformen. Sogar formlose Handlungen wie schlichte Informationshandlungen ${ }^{856}$ oder eine mündliche Auskunft ${ }^{857}$ können Vertrauensgrundlagen bilden. Es ist einzig gefordert, dass «l'autorité soit intervenue dans une situation concrète à l'égard de personnes déterminées» ${ }^{858}$, dass also die staatliche Äusserung auf einen konkreten Sachverhalt bezogen ist und gegenüber Einzelpersonen vorbehaltlos kommuniziert wird. ${ }^{859}$ Dies kann etwa mittels Empfehlungen und Verträgen ebenso erfolgen wie mittels Verfügung.

Die privatisierten Handlungsformen führen dann zu Problemen, wenn sie nicht als staatliche Auskunft erkennbar sind und Private also gar nicht auf die Idee kommen, den Schutz ihres berechtigten Vertrauens darin gestützt auf Art. 5 Abs. 3 oder Art. 9 BV einzufordern. Mit einvernehmlichem und informellem Handeln bemüht sich der Staat um einen bewusst privatähnlichen Auftritt (siehe vorne die Kennzeichen \$ 4/III.3.cund h).

Um Missverständnisse zu vermeiden: Die Erkennbarkeit einer staatlichen Handlung betrifft nicht die Frage, ob der Anspruch auf Vertrauensanspruch besteht, sondern nur, ob Verwaltungsadressaten diesen Anspruch geltend machen.

856 TSCHANNEN/ZIMMERLI/MÜLLER, Allgemeines Verwaltungsrecht, \$22 Rz.15.

857 HÄFELIN/MÜLLER/UHLMANN, Allgemeines Verwaltungsrecht, Rz. 668.

858 BGE 131 II 627 E. 6.1 S.637.

859 Kritisch dazu WEBER-DÜRLER, Vertrauensschutz, S. 205-207. 


\section{b. Probleme aus dem stärkeren Vertrauen in Beständigkeit von Verträgen}

Privatisierte Handlungsformen haben eine geringere Steuerungskraft, etwa indem sie weniger einfach an eine veränderte Rechtslage angepasst werden können (siehevorne \$ 4/III.3.d). Die Bedeutung einer Rechtsänderung schwindet, wenn einvernehmlich entstandene Verträge an die alte Rechtslage gebunden bleiben, während Verfügungen eher wiedererwägt würden.

Dies ist ein Problem für die Gesetzmässigkeit des konsensual handelnden Staats: Verträge können so nachträglich rechtswidrig werden. Die Änderung nachträglich rechtswidrig gewordener Rechtsakte bedingt in jedem Fall eine Interessenabwägung zwischen Gesetzmässigkeits- und Vertrauensinteressen. ${ }^{860}$ Bei einvernehmlich ausgehandelten Instrumenten, wie eben Verträgen, dürfte das Vertrauen in deren Fortbestand das entgegenstehende Interesse einer gesetzeskonformen Anpassung regelmässig überwiegen. ${ }^{861}$ Das Bundesgericht spricht davon, der Schutz des berechtigten Vertrauens in einen Vertrag sei «tendenzmässig» 862 stärker zu gewichten als bei einer Verfügung.

Kurz: Das Problem manifestiert sich beim Gesetzmässigkeitsprinzip, es entsteht aber aus dem Grundsatz von Treu und Glauben. Indem der Staat vorgibt, flexibel und quasiprivatautonom mit Verwaltungsadressaten zu verhandeln, verpflichtet er sich, rechtswidrig oder unsachgemäss werdende Ergebnisse im Interesse des Vertrauensschutzes zu bewahren.

\section{Insbesondere bei der Privatisierung der finanziellen Erfüllungsapekte}

Die privatisierten Finanzierungsformen sollen nicht nur den Staat zu effizientem und gewinnbringendem Handeln anregen, sie sollen Private auch davon abhalten, staatliche Eingriffe und Leistungen zu verursachen (siehe zu diesem Anreizvorne \$ 4/IV.4.e).

Dieses Privatisierungsmotiv steht in Spannung zum Rechtsmissbrauchsverbot. Der Staat darf die Rechtsansprüche Privater nicht mit finanziellen Hürden vereiteln. Wenn verursachergerechte Kostentragepflichten dazu führen, dass Private auf ihre Rechte verzichten, verhält sich der Staat ähnlich rechtsmissbräuchlich, wie wenn er Rechtsansprüche durch prozessuale

\footnotetext{
860 Siehe speziell für öffentlichrechtliche Verträge TSCHANNEN/ZIMMERLI/MÜLLER, Allgemeines Verwaltungsrecht, §35 Rz. 9; WEBER-DÜRLER, Vertrauensschutz, S. 226.

861 Vgl. WALDMANN, Verwaltungsrechtlicher Vertrag, S.18; WEBER-DÜRLER, Vertrauensschutz, S. 226.

862 BGE 138 V 147 E. 2.4 S. 150; konkret ging es um einen Vergleich (in der Form eines öffentlichrechtlichen Vertrags), S.149.
} 
Verzögerungen vereitelt.863 Das Rechtsmissbrauchsverbot verbietet es dem Staat, die Privaten zu schikanieren, wenn sie ihre Rechte durchsetzen möchten. Aus der Grundrechtsbindung kann ein gesondertes Problem entstehen, das hinten in V.3.b ausgeführt wird: Finanzielle Hindernisse können faktisch in grundrechtliche Ansprüche eingreifen, weil sie eine Abschreckungswirkung entfalten. 


\section{Probleme aus der Grundrechtsbindung}

Jede Erfüllung staatlicher Aufgaben muss die Grundrechte der betroffenen Privaten beachten. Die Grundrechtsbindung gilt - ohne Unterscheidung nach der Rechtsnatur der Organisations- oder Handlungsformen - immer dann, wenn staatliche Aufgaben wahrgenommen werden (Art. 35 Abs. 2 BV; siehe vorne \$3/II.6.b). Ein Teil der Lehre hält es für zulässig, den Umfang der Grundrechtsbindung bei der privatisierten Aufgabenerfüllung auf die rechtsstaatlichen Garantien zu beschränken. Doch entspringt diese einschränkende Dogmatik zur Grundrechtsgeltung einer fehlgeleiteten Interpretation der Rechtsprechung: Dass vorab die Geltung von Gleichbehandlungsgebot und Willkürverbot bestätigt wurde, hat mit den typischen Gefährdungslagen zu tun, die das Bundesgericht zu bewältigen hatte. Eine dogmatische Beschränkung der Grundrechtsgeltung ist nicht auszumachen (siehe hinten \$6/VI.1).

Hier kann nicht im Einzelnen aufgelistet werden, welche Erscheinungen der Erfüllungsprivatisierung welche grundrechtlichen Ansprüche einschränken. Dies kann nur unter Kenntnis des konkreten Sachverhalts beurteilt werden. Grundrechte vermitteln keinen absoluten Schutz vor staatlichen Eingriffen, sondern geben vielmehr ein qualifiziertes Rechtfertigungsschema vor, an dem diese Eingriffe zu messen sind (siehe vorne \$3/II.6.a).

Die Grundrechte lassen ein Problem im hier behandelten Sinn jedenfalls immer dann entstehen, wenn die Erfüllungsprivatisierung die Grundrechtsbindung verkompliziert - und den Grundrechtsschutz damit insgesamt gefährdet - oder wenn die Zielrichtung der Privatisierung den jeweiligen Schutzzwecken einzelfallübergreifend entgegensteht.

\section{Insbesondere bei den privatisierten Organisationsformen}

\section{a. Strapazierte Dogmatik zur Grundrechtsbindung bei Organisationen mit gemischten Aufgaben}

Wie vorne in \$3/II.6.b ausführlich herausgearbeitet, ist der Verfassung ein funktioneller Ansatz bei der Grundrechtsbindung zu entnehmen: Die Grundrechte gelten immer, wenn - aber nur soweit - eine Tätigkeit der Erfüllung staatlicher Aufgaben dient. Dogmatisch bedeutsam wird diese Festlegung auf den funktionellen Ansatz und damit die Ablehnung des personellen Ansatzes bei Rechtssubjekten, die neben staatlichen Aufgaben auch private Tätigkeiten ausüben.

Gerade öffentlichrechtliche Aktiengesellschaften und privatrechtliche Gesellschaften, denen staatliche Aufgaben übertragen werden, sollen die 
staatlichen Aufgaben mittels marktwirtschaftlicher Funktionsweise effizienter, kundengerechter und günstiger wahrnehmen können. Dass diese Effizienzgewinne im staatlichen Aufgabenbereich nicht zulasten der Grundrechtsbindung gehen dürfen, ist unbestritten. Zu dogmatischen Diskussionen Anlass gibt aber die rein privatwirtschaftliche Tätigkeit von Trägern staatlicher Aufgaben. Dort besteht die Gefahr, dass der gemischte Dienstleister rein private Wettbewerber benachteiligt. Dies waren etwa die Rügen im Glarnersachund im SWITCH-Fall (siehe dazu vorne \$3/III.4 und \$3/II.6.b.cc). 864

Würden Grundrechte dem personellen Ansatz nach auch im nichtstaatlichen Bereich greifen, wäre der effizienzversprechende Spielraum für Aufgabenträger mit gemischtem Tätigkeitsbereich von vornherein beschränkt. Die neuere Kasuistik zeigt aber, dass das Bundesgericht einer Verschlechterung des Grundrechtsschutzes durch eine vorsichtige Ausdehnung der Grundrechtsbindung vorbeugen will. Während das Bundesgericht formell am funktionellen Kriterium der Aufgabe festhält, weitet es die Grundrechtsbindung aus, indem es die Mittelbeschaffung als Teil der eigentlichen Staatsaufgabe ansieht (siehevorne \$3/II.6.a.cc). Tendenziell wird damit die gesamte Bedarfsverwaltung auch der Grundrechtsbindung unterzogen.

Kurz: Es gibt eine grundsätzliche Spannungslage. Auf der einen Seite muss die Erfüllung staatlicher Aufgaben in jeder Erscheinungsform an die Grundrechte gebunden bleiben. Auf der anderen Seite werden die Träger staatlicher Aufgaben vermehrt wie privatwirtschaftliche Unternehmen ausgestaltet und ihnen reine Wettbewerbstätigkeit ermöglicht. Grundrechtsbindung im Kern staatlicher Tätigkeit trifft also auf Organisationsformen, die von Flexibilität und Gestaltungsfreiheit geprägt sind. Dies führt zu dogmatischen Verrenkungen.

Dies zeigt sich etwa auch bei der spiegelbildlichen Frage der Grundrechtsträgerschaft. Können sich (gemischte) Aufgabenträger ihrerseits auf deren Schutz berufen, wenn sie nicht an die Grundrechte gebunden sind? Wie das Bundesgericht vor Kurzem festhielt: «Die Rechtsprechung hat bisweilen auch privatrechtlich organisierten Organisationen, welche öffentliche Aufgaben wahrnehmen, die Berufung auf die Wirtschaftsfreiheit zugestanden, wenn sie ausserhalb ihrer öffentlichrechtlichen Aufgabe handeln, [...] die Frage bezüglich öffentlich beherrschter Unternehmen aber ausdrücklich offengelassen»865. Würde es gemischtwirtschaftlichen Unternehmen, die nicht privatrechtlich konstituiert sind, tatsächlich abgesprochen, sich auf die Grundrechte (besonders die Wirtschaftsfreiheit) zu berufen,

864 BGE 138 I 378 E. 4 S. 383; 138 I 289 E. 2.4 und 2.5 S. 292 f.

865 BGE 142 II 369 E.6.3.2 S.387. 
entstünde eine schwer zu rechtfertigende Lücke: Sie wären im wettbewerblichen Bereich weder grundrechtsgebunden noch -berechtigt. ${ }^{866}$

\section{b. Spannungen aus Renditezielen und Quersubventionierungen}

Auf der Ebene der öffentlichen Interessen oder der Verhältnismässigkeit schaffen Renditevorgaben an Träger staatlicher Aufgaben keine grundsätzlichen Probleme. Auch Zielkonflikte zwischen fiskalischen und inhaltlich öffentlichen Interessen sind nicht weiter problematisch, wenn diese vom Gesetzgeber so vermischt werden. Dort dürften zwar grundsätzliche Spannungen mit der Wirtschaftsfreiheit auftreten, was aber der Gesetzgeber billigend in Kauf nehmen kann. In diesem Sinn äusserte sich das Bundesgericht etwa in den Fällen Glarnersach und SWITCH. Gemünzt auf Wettbewerbsverzerrungen meinte es dabei: «Diese Schwierigkeiten sind aber durch die rechtliche Konstruktion und die damit verbundene Doppelstellung der [SWITCH] bedingt und somit zwangsläufig vorhanden.»867

Marktverzerrungen sind unumgänglich und müssen hingenommen werden, wenn per gesetzliche Einrichtung dieselbe Organisationseinheit staatliche und private Aufgaben wahrnimmt. ${ }^{868}$ Ehemalige (oder gegenwärtige) Staatsmonopolisten etwa haben einen inhärenten Wettbewerbsvorteil gegenüber privaten Konkurrenten: Zugang zum grösseren Kundenstamm. Die kantonale Gebäudeversicherung hat bereits jeden Hausbesitzer in der Kundenkartei und der staatlich beherrschte Mobilfunkanbieter jeden Benutzer eines Festnetzanschlusses. ${ }^{869}$

Der staatliche Bereich wirkt unweigerlich in die privatwirtschaftliche Tätigkeit hinein, wie sich am Beispiel der Schweizerischen Post AG illustrieren lässt. Art. 19 Abs. 1 PG verbietet der Post zwar die Quersubventionierung von Wettbewerbsleistungen mit Erträgen aus dem Monopolbereich. Doch auch wenn die Post Kioskartikel zu marktüblichen Preisen anbietet, profitiert diese privatwirtschaftliche Tätigkeit von der Erfüllung von Staatsaufgaben: Die Ladenfläche steht da, weil die Post einen Grundversorgungsauftrag zu erfüllen hat. Die meisten Kunden, die Kioskartikel bei der Post kaufen, sind vermutungsweise wegen Post-Dienstleistungen (darunter auch Grundversorgungs-Leistungen) dort. Auch wenn die Post also ihre Schokoladenriegel-

866 Dieses Ergebnis befürwortend, ohne diese Lücke ausdrücklich zu thematisieren, ABEGG/ FREI, Wirtschaftsfreiheit, S.296.

867 BGE 138 I 289 E. 2.8.2 S. 295. Sinngemäss auch BGE 138 I 378 E. 9.4 S. 401.

868 Etwas weniger resigniert BAUMANN, Wettbewerbsverzerrungen, Rz.350, 900-902, wonach das Bundesgericht insbesondere die Quersubventionierung als griffigeres Kriterium ausgestalten könne.

869 Vgl. zu dieser Problematik auch KÜHNI, Weder staatlicher Fisch noch Vogel AG. 
Preise nicht mit Briefmarkenerträgen künstlich absenkt, profitiert ihr privates Geschäft vom staatlichen Monopol. Der Bundesrat betont freilich die finanziellen Vorteile für die umgekehrte Richtung: Die Post vertreibe weitere Produkte, um mittels «Skalen-, Netzwerk- oder anderen Effizienzvorteilen [...] einen höheren Gewinn oder Deckungsbeitrag für die Kosten der Grundversorgung» 870 zu erzielen. Allerdings muss auch der Bundesrat anerkennen, dass es sich «in der Praxis als schwierig gestaltet»871, eine Quersubventionierung faktisch zu verhindern, wenn staatliche Unternehmen ihre Infrastruktur für Dienstleistungen ausserhalb der Grundversorgung einsetzen.

\section{c. Probleme bezüglich Durchsetzung der Grundrechtsbindung wegen gelockerter Aufsicht}

Auch wenn die Geltung der Grundrechte unbestritten ist, so erwachsen aus der organisatorischen Privatisierung Probleme in der Durchsetzung dieser Grundrechtsbindung. Übergeordnete Verwaltungseinheiten können in der Zentralverwaltung mittels Führung und Kontrolle beeinflussen, inwiefern die alltägliche Aufgabenerfüllung grundrechtliche Schutzbereiche berührt. Bei privatisierten Aufgabenträgern muss der Staat einen wirksamen Grundrechtsschutz mit anderen Mitteln sicherstellen: «Afin que cette exigence [Grundrechtsbindung] soit effective, l'Etat se doit d'assurer une certaine surveillance voire un certain contrôle sur les activités déléguées»872.

Die ausgelagerte Aufgabenerfüllung führt zwar nicht unweigerlich zu mehr Grundrechtseingriffen, doch wäre die zentralstaatliche Erfüllung immerhin eine Garantie dafür, dass die betrauten Stellen und Organisationen genügend auf grundrechtliche Belange sensibilisiert sind. In diesem Sinn bemängelt etwa GÄCHTER, dass in der ATSG-Revision vom 16. März $2018^{873}$ gerade die grundrechtlich heikle Observation von Versicherten an «externe Spezialisten» übertragen werden kann. Die verwaltungsinterne Besorgung dieser Aufgabe «hätte den Vorteil, dass professionell geschulte Polizistinnen und Polizisten, die in ihrer Ermittlungstätigkeit auch an die Vorgaben der Strafprozessordnung gebunden sind, mit den heiklen Eingriffen in die Privatsphäre betraut wären - statt auf privatrechtlicher Grundlage agierende Externe, deren Vorgehen nur sehr indirekt überwacht werden kann. Offenbar war aber - was wiederum zu Bedenken Anlass gibt - gerade die soeben genannte Bindung und Kontrolle nicht gewollt.»874

870 Bericht Staat und Wettbewerb, S. 18.

871 Bericht Staat und Wettbewerb, S.11.

872 BGE 133 I 49 E.3.2 S. 56.

873 AS 20192829.

874 GÄCHTER, Schnell, schwammig, schlecht, S. $219 \mathrm{f}$. 


\section{Insbesondere bei den privatisierten Handlungsformen}

Die privatisierten Handlungsformen bringen dem Staat in erster Linie grösseren Gestaltungsspielraum und Flexibilität (siehe vorne das Kennzeichen \$4/ III.3.b). Insofern tritt die Erfüllungsprivatisierung in einen grundsätzlichen Zielkonflikt mit der Rechtsgleichheit nach Art. 8 Abs. 1 BV.

Eine rechtsungleiche Behandlung kann etwa darin liegen, dass zwingendes öffentliches Recht nur selektiv durchgesetzt wird. Wenn der Staat konsensuale Mittel dazu einsetzt, das Verwaltungsrecht in einem Fall strenger anzuwenden als im anderen, kann dies die Rechtsgleichheit verletzen. Bei hoheitlichen Instrumenten wie der Verfügung gewährt die einseitige Entscheidungsgewalt der Behörde, dass Ermessensspielräume rechtsgleich wahrgenommen werden. ${ }^{875}$ Der Gestaltungsspielraum, den privatisierte Instrumente bieten, muss rechtsgleich benutzt werden.

Dies ist gewissermassen auch ein Ausfluss der privatisierten Organisationsformen: Innerhalb der hierarchisch geordneten Zentralverwaltung können Verwaltungsverordnungen die rechtsgleiche Ermessensausübung sicherstellen. 876

Der Anspruch auf rechtsgleiche Rechtsanwendung besteht aber nur gegenüber ein und derselben Behörde (siehe vorne \$3/II.6.a.bb). Die Ermessensausübung desselben Aufgabenträgers droht aber uneinheitlich zu werden, wenn er mit Verträgen und informellen Instrumenten den Verwaltungsadressaten unterschiedliche Mitsprache ermöglicht und zu unterschiedlichen Rechtsanwendungen gelangt. Gerade Verhandlungslösungen können die Rechtsgleichheit verletzen, wenn sie in vergleichbaren Situationen je nach Verhandlungsgeschick der Verwaltungsadressaten unterschiedliche Rechte und Pflichten hervorbringen.

\section{Insbesondere bei den privatisierten Finanzierungsformen}

\section{a. Gefährdete Rechtsgleichheit bei der Kostenanlastung nach aufgeweichter Verursacherfinanzierung}

Die Privatisierung der finanziellen Aspekte der Aufgabenerfüllung kann mit der Rechtsgleichheit von Art. 8 Abs. 1 BV in Konflikt geraten. Die verursachergerechten Finanzierungsformen beachten das Gleichbehandlungsgebot zwar grundsätzlich besser als die Finanzierung nach dem unterschiedslosen Gemeinlastprinzip. Denn sie erlauben eine Kostentragung, die den konkreten

875 TSCHANNEN, Systeme, Rz.145.

876 Ähnlich (ohne Bezug auf Ermessen) voGEL, Einheit der Verwaltung, S.151. 
Umständen angemessen ist: Wer mehr von einer staatlichen Leistung profitiert, zahlt auch mehr dafür. ${ }^{877}$

Allerdings entstehen Probleme bei den Mischfinanzierungen: den Kostenanlastungsabgaben und -steuern (siehe dazu vorne \$ 4/IV.3.b). Diese Finanzierungen berücksichtigen nicht durchgehend, wie stark jemand von einer staatlichen Leistung profitiert. Gleichzeitig fehlt ihnen aber die besondere Legitimation durch eine Verfassungsgrundlage, wie sie Steuern aufweisen können. Diese Finanzierungsformen weichen das Verursacherprinzip auf, indem der Kreis der Abgabepflichtigen über diejenigen Nutzer hinausgeht, die nachweisliche einen besonderen Nutzen aus einer staatlichen Leistung ziehen (siehe vorne \$4/IV.4.c).

Dieses Problem entschärft sich dadurch, dass das Abgaberecht ohnehin von Pauschalisierungen geprägt ist und hier das Gesetzmässigkeitsprinzip eine grössere Bindungskraft entfaltet als die Grundrechte. 878

\section{b. Abkehr vom Gemeinlastprinzip kann Grundrechtsansprüche vereiteln}

Die Verursacherfinanzierung setzt der zahlungspflichtigen Privatperson einen Anreiz, staatliche Leistungen nicht in Anspruch zu nehmen (siehe vorne \$ 4/ IV.4.e). Die Abkehr vom Gemeinlastprinzip lässt eine Spannung zur Grundrechtsbindung entstehen, wenn die Verursacherfinanzierung die Berufung auf gesetzlich garantierte Rechte vereitelt. Die drohenden Kostenfolgen können Träger von Grundrechten davon abhalten, ihre Ansprüche auszuüben. Dann führt die Finanzierungsform zu einem faktischen Grundrechtseingriff. 879

Das Bundesgericht beschreibt das Problem, das aus der Grundrechtsbindung bei der Abkehr vom Gemeinlastprinzip entsteht, wie folgt:

«[Es] ist festzuhalten, dass die Meinungs- und Versammlungsfreiheit nicht nur durch direkte Eingriffe wie Verbote und Sanktionen beeinträchtigt werden können. Denkbar sind auch mittelbare Beeinträchtigungen dieser Grundrechte in dem Sinne, dass der Betroffene sich aufgrund einer behördlichen Reaktion nicht mehr getraut, erneut vom Grundrecht Gebrauch zu machen. In Rechtsprechung und Lehre wird in diesem Zusammenhang vom sog. «chilling effect〉 (auch «effet dissuasif)) gesprochen [...]. Die Ausübung der Grundrechte darf durch negative Begleiterscheinungen nicht derart beschränkt werden, dass von einer Abschreckungswirkung oder einem Einschüchterungseffekt zu sprechen ist. Ein solcher besteht im vorliegenden

877 Vgl. HÄNER, Privatisierung, S. 430.

878 Vgl. TSCHANNEN/ZIMMERLI/MÜLLER, Allgemeines Verwaltungsrecht, §58 Rz.11, 21.

879 HUSMANN, Demokratiefeindliche Polizeikostenüberwälzung, S. 152; BGE 143 I 147 E. 3.1 S.151. 
Zusammenhang dann, wenn für die Ausübung eines ideellen Grundrechts Polizeikosten verrechnet werden, welche die Grundrechtsberechtigten von der Grundrechtswahrnehmung abhalten» ${ }^{880}$.

Die Abschreckungswirkung bedeutet aber nur, dass die privatisierte Finanzierungsform in grundrechtliche Ansprüche eingreifen kann, nicht dass damit Grundrechte verletzt wären. Es kommen die üblichen Rechtfertigungsgründe von Art. 36 BV zum Zug. Insbesondere entschärft sich das Problem, wenn die gesetzliche Grundlage die Voraussetzungen für die Kostentragepflicht genügend vorhersehbar nennt. 881

Dass ein Verwaltungsträger seinen Etat selber bestellen muss, etwa mit Gebühren, Kostenanlastungssteuern oder Aufsichtsabgaben, ändert nichts an seiner Pflicht, die ihm anvertrauten Aufgaben zu erfüllen. Der Anreiz, ein Recht nicht zu beanspruchen, kann wegen der Grundrechtsbindung der direkten Einschränkung dieses Rechts gleichkommen. In beiden Fällen müssen allfällige Grundrechtsbeschränkungen gesetzlich abgestützt, im öffentlichen Interesse und verhältnismässig sein. 


\section{Querschnittsprobleme bei der Einhaltung der verfassungsrechtlichen Anforderungen}

Die vorangehenden Abschnitte I-V haben für jede der verfassungsrechtlichen Anforderungen an staatliches Handeln geprüft, welche Probleme entstehen können, wenn verschiedene Aspekte der Aufgabenerfüllung privatisiert werden. Es wurde dabei spezifisch geprüft, ob die Stossrichtungen der jeweiligen Privatisierungserscheinungen den einzelnen Zwecken der verfassungsrechtlichen Anforderungen entgegenstehen. Handfeste Probleme mussten nur vereinzelt festgestellt werden; meist waren bloss Spannungen festzustellen, die potentiell zu Problemen werden können. Die Erfüllungsprivatisierung verunmöglicht es in keiner Konstellation von vornherein, die verfassungsrechtlichen Anforderungen weiter einzuhalten.

Dass die zahlreichen Spannungen und Probleme die Einhaltung der verfassungsrechtlichen Anforderungen nicht tatsächlich gefährden, hängt allerdings von drei grundlegenden Vorbehalten ab:

- Das privatisierte Staatshandeln muss als staatliches Handeln erkannt werden, damit die verfassungsrechtlichen Anforderungen überhaupt angerufen werden.

- Soweit das zivile Verfahrensrecht zur Anwendung kommt, dürfen Rechtsschutzdefizite die Durchsetzung der verfassungsrechtlichen Anforderungen nicht verunmöglichen.

- Und die zusätzlichen Aufsichts- und Kontrollmechanismen, welche die ausgelagerte Aufgabenerfüllung nötig macht, müssen tatsächlich eingerichtet und befolgt werden.

Diese drei Vorbehalte werden hier als Querschnittsprobleme diskutiert. Sie betreffen alle Erscheinungen der Erfüllungsprivatisierung und überlagern die Probleme, die bezüglich der einzelnen verfassungsrechtlichen Anforderungen entstehen.

\section{Erschwerte Erkennbarkeit des Staats gefährdet die Anrufung der verfassungsrechtlichen Anforderungen}

Die verfassungsrechtlichen Anforderungen sind staatliche Sonderpflichten; sie binden ausschliesslich den Staat und bilden das Gegenstück zu seinen besonderen Rechten, insbesondere der Möglichkeit, gegenüber seinen Bürgern Zwang auszuüben (siehe vorne \$3/I.2). Diese rechtliche Sonderordnung gilt immer, wenn der Staat handelt. Wie er sich dabei organisiert, finanziert 
und welcher Instrumente er sich bedient, ist unerheblich. Die Geltendmachung der Anforderungen jedoch hängt davon ab, dass allfällige Verletzungen gerügt und gerichtlich durchgesetzt werden.

Die Erfüllungsprivatisierung lässt den Staat wie einen privaten Akteur erscheinen. Er organisiert sich als unternehmerische Gesellschaft, er lässt Betroffene ihre eigenen Regeln setzen oder schliesst Verträge mit ihnen, und er erwirtschaftet Zahlungen für seine Leistungen. Gerade bei den instrumentellen Aspekten der Erfüllungsprivatisierung ist dieser private Anstrich Konzept: Er soll die Akzeptanz und freiwillige Befolgung staatlicher Anordnungen steigern (vgl.vorne das Kennzeichen \$ 4/III.3.e).

Wenn dieser private Auftritt die Verwaltungsadressaten vergessen lässt, dass sie es mit dem Staat zu tun haben, werden sie auch nicht den Schutz der Verfassung suchen. Viele werden nicht daran denken, eine generell-abstrakte Warnung anzufechten, weil sie Grundrechte berühren könnte. Und wenn sie dies tun, könnte ihnen die Unverbindlichkeit dieser Handlungsform im Weg stehen.

So lag es in einem Bundesgerichtsfall, in dem die gesetzlichen Vertreter von 35 Kindern und Jugendlichen eine Rüge gegen die Aids-Präventionskampagne «Love Life» des BAG erhoben hatten (BGE 144 II 233). Das Bundesgericht stützte den Entscheid des BAG, über die Kampagne (ein Realakt) keine anfechtbare Verfügung nach Art. 25a VwVG zu erlassen. Es hielt fest: «Rechtsschutz gegen aussenwirksame Informationshandlungen von generellabstrakter Struktur liegt indes nicht ohne Weiteres auf der Hand» ${ }^{882}$. Die Beschwerdeführer müssten eine besondere Nähe zum Realakt aufweisen, 883 wofür es «eines Zurechnungszusammenhangs, einer adäquaten Kausalität, zwischen Handlung und Berührung in Rechte und Pflichten ${ }^{884}$ bedürfe. Das Bundesgericht sprach den Kindern und Jugendlichen ab, von der Informationshandlung des BAG besonders betroffen zu sein, weil sie «mit solchen Inhalten ohnehin in Berührung kommen»885. Die Rechte von Privatpersonen sind im Ergebnis also deshalb nicht direkt betroffen, weil der Staat wie ein Privater handelt - seine Äusserungen unterscheiden sich nicht von anderen im öffentlichen Raum.

Dieses Beispiel bekräftigt, dass der Staat mit unverbindlichen Handlungsformen die Rechtsstellung Privater gerade nicht direkt berührt, und dürfte daher die Annahme von Verwaltungsadressaten nähren, dass sie Warnungen, 
informelle Absprachen oder sonstige formlose Kommunikationshandlungen nicht auf die Verletzung ihrer Rechte überprüfen lassen können.

Kurz und plakativ ausgedrückt: Die verfassungsrechtlichen Anforderungen laufen allesamt ins Leere, wenn sie nicht angerufen werden. Der erhoffte Freiraum, den die Privatisierung bringen soll, liegt also nicht darin, dass die Bindungen der Verfassung nicht mehr gälten, sondern dass der privat eingekleidete Staat nicht darauf verpflichtet wird.

\section{Rechtsschutzdefizite des zivilen Verfahrensrechts}

Das Spezialgesetz kann die Rechtsbeziehungen eines Aufgabenträgers dem Zivilrecht unterstellen - er hat dies etwa für die Post ${ }^{886}$, die Swisscom ${ }^{887}$ und die Stiftung SWITCH ${ }^{888}$ getan. Verwaltungsadressaten müssen den zivilen Verfahrensweg beschreiten, wenn sie sich gegen die Aufgabenerfüllung dieser Aufgabenträger beschweren wollen. Darüber hinaus kommt das Zivilprozessrecht immer dann zur Anwendung, wenn Staatsaufgaben mittels privatrechtlicher Verträge erfüllt werden (siehe vorne \$4/III.3.i).

Worin unterscheidet sich der Zivilprozess vom öffentlichen Verfahren? Das zivile Verfahrensrecht ist insbesondere vom Verhandlungsgrundsatz und vom Dispositionsgrundsatz geprägt (Art. 55 und 58 ZPO, jeweils Abs. 1). Tatsachen und Beweise sind von den Parteien vorzubringen und das Gericht ist in seinem Urteil an die Parteianträge gebunden. ${ }^{889}$ Dies könnte theoretisch dazu führen, dass mangelnde öffentliche Interessen nicht geprüft werden, wenn diese Verletzung nicht gerügt wird. Im öffentlichen Verfahrensrecht gilt hingegen der Untersuchungsgrundsatz (vgl. Art.12 VwVG; Art. 37 und 44 Abs. 2 VGG) - die Behörde bzw. das Gericht stellt den Sachverhalt von Amts wegen fest. ${ }^{890}$ Die potentielle Rechtsschutzlücke betrifft alle von den Parteien nicht ausdrücklich gerügten Rechtsverletzungen. Im Verfahren vor Bundesgericht unterliegen allerdings gerügte Grundrechtsverletzungen ebenfalls der Dispositionsmaxime (qua Art. 106 Abs. 2 BGG). 891

Wo der Gesetzgeber auf den privatrechtlichen Verkehr verweist, nimmt er diese Unterschiede im Rechtsschutz in Kauf und legitimiert sie.

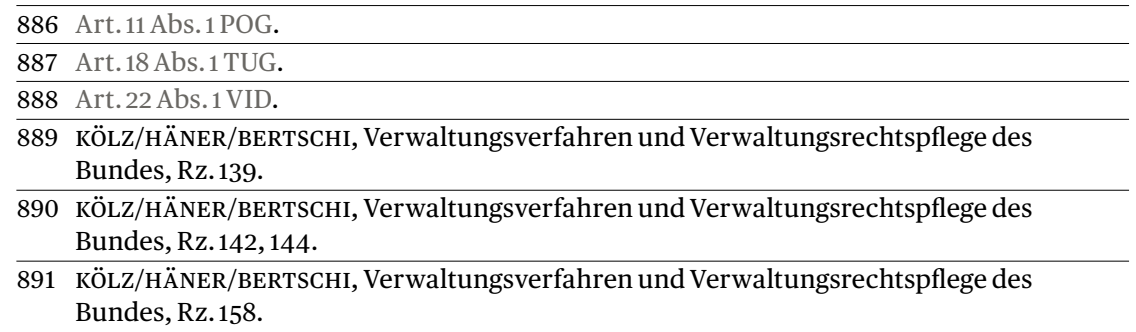


Bei der Verhältnismässigkeit erhalten diese Defizite eine zusätzliche Bedeutung: Diese Anforderung lässt sich erst im Einzelfall durchsetzen - sie bedingt also den Zugang zum Rechtsweg und die Prüfung möglicher Rechtsverletzungen. Doch bedeutet der Wechsel ins zivile Verfahrensrecht insbesondere auch ein grösseres Prozesskostenrisiko. ${ }^{892}$

Das Zivilprozessrecht stellt die Verwaltungsadressaten potentiell schlechter. Dies gilt umso mehr, da sich das Bundesgericht bei der Prüfung der Verhältnismässigkeit grosse Zurückhaltung auferlegt und diese Anforderung in der Praxis auf wenig mehr als eine Willkürprüfung zurückfällt (siehe vorne \$3/II.4.c).

\section{Die Einhaltung der verfassungsrechtlichen Anforderungen bedingt zusätzlichen Aufsichts- und Kontrollaufwand}

Die Erfüllungsprivatisierung zeichnet sich unter anderem dadurch aus, dass die Träger staatlicher Aufgaben mehr Gestaltungsfreiheiten erhalten und ihr Spielraum für eigenständige Entscheidungen grösser wird. Dies betrifft sowohl die organisatorischen Aspekte, wobei Aufgabenträger aus der Verwaltungshierarchie herausgelöst und als eigene Rechtssubjekte organisiert werden, als auch die privatisierten Handlungsformen, mit denen Aufgabenträger formlose Anweisungen geben können oder die öffentlichrechtlichen Pflichten konsensbasiert aushandeln, statt sie einseitig anzuordnen.

Diese Freiheiten bergen das Potential, dass sich die staatliche Aufgabenerfüllung von den gesetzlichen Leistungsvorgaben und der öffentlichen Zweckbindung entfernt (siehe die einzelnen Hinweise vorne in I.3, II.1.a, II.2.a, II.3.b, V.1.b, V.1.c und V.2). Der Machtzuwachs privater Organisationen wird nicht automatisch kompensiert durch stärkere staatliche Kontrolle. ${ }^{893}$ Dass diese Spannung nicht zu einem tatsächlichen Problem wird, bedingt die Einrichtung neuer Aufsichtsbefugnisse der Zentralverwaltung. Dies fordert auch Art. 187 Abs. 1 Bst. a BV.

Und die kompensatorischen Vorkehrungen, die eine genügende Aufsicht sicherstellen sollen, werden in Umfang und Intensität immer hinter der Dienstaufsicht zurückstehen, wie sie innerhalb der Zentralverwaltung gilt. Nur soweit untergeordnete Verwaltungsträger mit Dienstbefehlen und Verwaltungsordnungen angewiesen werden können, besteht eigentliche Leitung;

892 KÖLZ/HÄNER/BERTSCHI, Verwaltungsverfahren und Verwaltungsrechtspflege des Bundes, Rz. 274; MARTI, Kosten im Zivilprozess, Rz.29f.

893 KRUCK, Privatisierung, S. 440; aus deutscher Perspektive am Beispiel von Ratingagenturen im Finanzbereich und privaten Militär- und Sicherheitsfirmen. 
ansonsten müssen sich übergeordnete Stellen mit periodischer und nachträglicher Kontrolle begnügen. ${ }^{894}$ Allfällige Entfernungen von der gesetzlichen Grundlage und vom öffentlichen Interesse können nur wirksam bekämpft werden, wenn der Verwaltungseinheit eine Behörde vorsteht, die über ein Selbsteintrittsrecht verfügt. ${ }^{895}$ Bei autonomen Aufgabenträgern kann nicht von Leitung und Aufsicht in diesem Sinn gesprochen werden, dort bestehen lediglich nachträgliche Einsichts- und Auskunftsrechte der übergeordneten Einheiten sowie eine periodische Berichterstattung. 896 Prägende Einwirkungen auf die Aufgabenerfüllung können dann nur noch mit einer Änderung der spezialgesetzlichen Rechtsgrundlagen bewirkt werden.

Die Selbstkontrolle der ausgelagerten Aufgabenträger birgt das inhärente Problem, dass das Norminteresse dem Interesse der Beaufsichtigten widersprechen kann. ${ }^{897}$ Die ausgelagerten Aufgabenträger streben nach Flexibilität und Autonomie, das Gesetz ist von einem Regelungszweck, einem öffentlichen Interesse geprägt. Somit steht also die Einhaltung der verfassungsrechtlichen Anforderungen unter dem generellen Vorbehalt, dass genügende Kontroll- und Eingriffsinstrumente bestehen, um allfällige Fehlentwicklungen zu korrigieren.

Eine zusätzliche Aufsichtsarchitektur ist in jedem Fall notwendig. Die privatisierte Aufgabenerfüllung verursacht daher zusätzliche Aufwände. ${ }^{898}$ Die Verfassung steht der Privatisierung zwar nicht entgegen, ihre Anforderungen begrenzen aber von vornherein erhoffte Effizienzgewinne. Oder wie es LIENHARD treffend ausdrückt: «Starre Grenzen für Auslagerungen sind kaum auszumachen. Zu berücksichtigen ist aber, dass Auslagerungen allenfalls eine derart intensive Regulierung bzw. Beaufsichtigung erfordern, dass die Aufgabe vorteilhafter weiterhin von der Zentralverwaltung wahrgenommen wird.» 899

Neben dem zusätzlichen Aufsichtsaufwand ist auch zu bedenken, dass ein Weniger an staatlicher Leistung ein Mehr an staatlicher Subvention bedingen kann. ${ }^{900}$

\footnotetext{
894 Vgl. BIAGGINI, Kommentar BV, Art. 187 Rz. 5; VOGEL, Einheit der Verwaltung, S. 154.

895 VOGEL, Einheit der Verwaltung, S. 155 .

896 VOGEL, Einheit der Verwaltung, S. 182.

897 TSCHANNEN, Privatisierung, S. $238 \mathrm{f}$.

898 GILI, Selbstregulierung, S. 145, insb. zur ausgelagerten Rechtsetzung. Vgl. zu Zusatzaufwänden bei der Finanzierungsprivatisierung MÄCHLER, Finanzierung privater Träger, S.1181 f. Ferner aus der deutschen Lehre тномA, Selbstregulierung, S. $474 \mathrm{f}$.

899 LIENHARD, Ausgelagerte Aufgabenerfüllung, S. 1174, ohne die Hervorhebungen im Original. Ähnlich auch DUBEY/ZUFFEREY, Droit administratif général, Rz.1404.

900 TSCHANNEN/ZIMMERLI/MÜLLER, Allgemeines Verwaltungsrecht, §11 Rz.3.
} 


\section{Zwischenfazit: Erfüllungsprivatisierung stellt Freiräume über gesetzliche Leistungspflichten und Zweckbindung}

\begin{tabular}{|c|c|c|c|c|}
\hline \multicolumn{5}{|c|}{ Probleme aus den Anforderungen: Übersichtsdarstellung der rasterartigen Prüfung in $§ 5$} \\
\hline & & \multicolumn{3}{|c|}{ Privatisierung der ... (\$4) } \\
\hline & & Organisationsformen & Handlungsformen & Finanzierungsformen \\
\hline \multirow{6}{*}{ 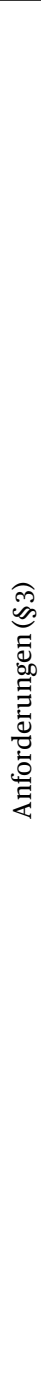 } & $\begin{array}{l}\text { Gesetzmässigkeit } \\
\text { Art. } 5 \text { und } 36 \text {, } \\
\text { jeweils Abs. } 1 \text { BV }\end{array}$ & $\begin{array}{l}\text { Spannung: Einzelfall- } \\
\text { regelung notwendig }\end{array}$ & $\begin{array}{l}\text { Problem: Zusätzlicher } \\
\text { Aufwand wegen Sui- } \\
\text { generis-Instrumenten } \\
\text { Spannung: Verminderte } \\
\text { Steuerungskraft vs. } \\
\text { zwingendes Recht } \\
\text { Problem: Ausgelagerte } \\
\text { Normen schwerer auf- } \\
\text { findbar und verständlich }\end{array}$ & $\begin{array}{l}\text { Spannung: Anreize } \\
\text { zur Kostenvermeidung } \\
\text { vs. Leistungspflichten }\end{array}$ \\
\hline & $\begin{array}{l}\text { Öffentliches } \\
\text { Interesse } \\
\text { Art. } 5 \text { und 36, } \\
\text { jeweils Abs. 2 BV }\end{array}$ & $\begin{array}{l}\text { Spannung: Einge- } \\
\text { schränkte Aufsicht vs. } \\
\text { Zweckbindung } \\
\text { Spannung: Renditeziele } \\
\text { treffen auf Zweckbin- } \\
\text { dung }\end{array}$ & $\begin{array}{l}\text { Spannung: Gestaltungs- } \\
\text { freiheit vs. Zweck- } \\
\text { bindung } \\
\text { Spannung: Fachexper- } \\
\text { ten fördern Sonder- } \\
\text { gruppeninteressen }\end{array}$ & $\begin{array}{l}\text { Spannung: Verwal- } \\
\text { tungsadressaten als } \\
\text { Kunden anzusehen, } \\
\text { fördert Sondergruppen } \\
\text { interessen }\end{array}$ \\
\hline & $\begin{array}{l}\text { Verhältnis- } \\
\text { mässigkeit } \\
\text { Art.5 Abs.2, } \\
\text { Art.36 Abs.3 BV }\end{array}$ & - & $\begin{array}{l}\text { Problem: Nicht direkt } \\
\text { bindende Instrumente } \\
\text { sind weniger geeignet }\end{array}$ & $\begin{array}{l}\text { Spannung: Mittel- } \\
\text { beschaffung als Anreiz } \\
\text { für überschiessendes } \\
\text { Staatshandeln }\end{array}$ \\
\hline & $\begin{array}{l}\text { Treu und Glauben } \\
\text { Art. } 5 \text { Abs. } 3 \text {, } \\
\text { Art. } 9 \text { BV }\end{array}$ & - & $\begin{array}{l}\text { Problem: Vertrauens- } \\
\text { grundlage erschwert } \\
\text { erkennbar } \\
\text { Problem: Beständigkeit } \\
\text { von Verträgen vs. Recht- } \\
\text { mässigkeit }\end{array}$ & $\begin{array}{l}\text { Spannung: Finanzielle } \\
\text { Anreize können } \\
\text { gesetzliche Ansprüche } \\
\text { rechtsmissbräuchlich } \\
\text { vereiteln }\end{array}$ \\
\hline & $\begin{array}{l}\text { Grundrechte } \\
\text { Art. } 35 \text { Abs. 2, } \\
\text { Art. 7-34 BV }\end{array}$ & $\begin{array}{l}\text { Spannung: Überstrapa- } \\
\text { zierte Dogmatik der } \\
\text { Grundrechtsbindung } \\
\text { Spannung: Marktver- } \\
\text { zerrungen bei gemischt- } \\
\text { wirtschaftlichen } \\
\text { Unternehmen } \\
\text { Problem: Gelockerte } \\
\text { Aufsicht gefährdet } \\
\text { Durchsetzung von } \\
\text { Grundrechten }\end{array}$ & $\begin{array}{l}\text { Spannung: Selektive } \\
\text { Nichtdurchsetzung von } \\
\text { zwingendem Recht vs. } \\
\text { Rechtsgleichheit }\end{array}$ & $\begin{array}{l}\text { Spannung: Kosten- } \\
\text { anlastungssteuern und } \\
\text {-abgaben gefährden } \\
\text { Rechtsgleichheit } \\
\text { Problem: Kostentrage- } \\
\text { pflicht schreckt von } \\
\text { Ausübung der Grund- } \\
\text { rechte ab }\end{array}$ \\
\hline & $\begin{array}{l}\text { Querschnitts- } \\
\text { probleme }\end{array}$ & $\begin{array}{l}\text { Erschwerte Erkennbarke } \\
\text { Rechtsschutzdefizite des } \\
\text { Zusätzlicher Aufsichts- ur }\end{array}$ & $\begin{array}{l}\text { t von Staatshandeln } \\
\text { Zivilprozessrechts } \\
\text { id Kontrollaufwand }\end{array}$ & \\
\hline
\end{tabular}


Werden die verfassungsrechtlichen Anforderungen auf die verschiedenen Erscheinungen der Erfüllungsprivatisierung angewendet, ist das Ergebnis zunächst beruhigend. Die Befürchtungen, dass Privatisierungen die rechtsstaatlichen und demokratischen Vorgaben an den Staat zurückdrängen, können grösstenteils entkräftet werden. Die privatisierte Aufgabenerfüllung verunmöglicht es nicht, die verfassungsrechtlichen Anforderungen einzuhalten. Dies steht allerdings unter den soeben angefügten Vorbehalten (siehe VI), dass staatliches Handeln als solches erkannt wird, der Zivilprozess die Rechtsdurchsetzung nicht verunmöglicht und dass die nötigen Aufsichts- und Kontrollinstrumente die Durchsetzung der Anforderungen gewährleisten.

Die Motive der Erfüllungsprivatisierung ziehen systematisch in eine andere Richtung als die Schutzzwecke der Verfassung. Die Verfassung zielt darauf, die Einschränkungen privater Freiheiten möglichst zu begrenzen. Dies bedingt Regelförmigkeit, Nachvollziehbarkeit und Zielgerichtetheit: Die Aufgabenerfüllung sollte direkt darauf ausgerichtet sein, die vorgegebene Aufgabe und die dahinterstehenden Interessen zu befördern. Die Erfüllungsprivatisierung zieht gerade in die gegenteilige Richtung: Sie will dem Aufgabenträger Auswahlmöglichkeiten gewähren. Flexibilität soll passgenaue Lösungen begünstigen, was wiederum die Aufgabenerfüllung insgesamt effizienter machen soll.

Dies führt zu einer Handvoll grundsätzlicher Spannungslagen. Erstens führen Einzelfallregelungen grundsätzlich zu mehr Normsetzungsaufwand, weil jede Privatisierung eine spezialgesetzliche Grundlage benötigt. Zweitens drohen Sondergruppeninteressen das gesamtgesellschaftliche Interesse zu verdrängen, wenn Aufgabenträger adressatenspezifischer handeln, mehr Fachexperten einbeziehen und Bürger als Kunden ansehen. Drittens ist die Effektivität und Zielgenauigkeit des staatlichen Handelns gefährdet, wenn die Aufgabenerfüllung nicht mehr die einzige Zielvorgabe für staatliches Handeln ist. Wenn Gewinne erwirtschaftet werden müssen oder wenn Akzeptanz wichtiger ist als verbindliche Regelungen, wird die Rechtsanwendung ummantelt von Ablenkungen und Zugeständnissen. Viertens steigern Flexibilität und Freiheit in der Rechtsanwendung die Gefahr der rechtsungleichen Behandlung.

Bei all diesen Spannungslagen hängt es aber vom konkreten Einzelfall ab, ob sie zu eigentlichen Problemen werden. Auf die Überprüfung und Korrektur der einzelnen Verwaltungshandlung kommt es also an. Und genau dies ist durch die Erfüllungsprivatisierung gefährdet, da sie die Aufsicht reduziert und die Steuerung auf eine nachträgliche Kontrolle verlagert.

Der Blick auf die Probleme endet also vorsichtig optimistisch mit dem Befund: Die Verfassung kann eingehalten werden, auch wenn es schwieriger 
wird. Nun soll der Blick umgedreht werden auf die Spielräume der verfassungsrechtlichen Anforderungen, welche die privatisierte Aufgabenerfüllung ausnutzen kann. 


\section{§6 Welche Spielräume belassen die verfassungsrechtlichen Anforderungen der Erfüllungsprivatisierung?}

Diese Arbeit untersucht, inwiefern weniger Staat erreicht werden kann, wenn die Art und Weise der Erfüllung von Staatsaufgaben privatisiert wird. Was den Staat auszeichnet, sind die besonderen Anforderungen, die die Verfassung seinem Handeln auferlegt. Es sind diese staatlichen Sonderpflichten, die den Staat grundlegend von Privaten unterscheiden. Diese Anforderungen gelten unabhängig von Organisations-, Handlungs- und Finanzierungsformen immer dann, wenn staatliche Aufgaben erfüllt werden - die Verfassung folgt einem funktionellen Staatsbegriff (siehe vorne \$ $2 / I$ ). Der vorangehende Paragraf $(\$ 5)$ hat die Probleme herausgearbeitet, die aus den verfassungsrechtlichen Anforderungen für die privatisierte Aufgabenerfüllung entstehen. Nun erfolgt die spiegelbildliche Prüfung: Welche Spielräume belassen die verfassungsrechtlichen Anforderungen der Erfüllungsprivatisierung? Nur soweit solche Spielräume bestehen, ist die Erwartung von weniger Staat berechtigt. Nur dann kann die Erfüllungsprivatisierung von Freiräumen profitierten und so zu einer insgesamt effizienteren Aufgabenerfüllung führen.

Die Prüfung erfolgt wiederum rasterartig: Jede Anforderung der Verfassung wird auf die verschiedenen Spielarten der Erfüllungsprivatisierung angewendet (Abschnitte II-VI).

Wie schon bei den Problemen kann auch der Befund «Es bestehen Spielräume» verschiedenartig ausgeprägt sein. Spielräume können ausserhalb oder innerhalb der verfassungsrechtlichen Anforderungen entstehen. Ein Spielraum kann erstens entstehen, wenn die Zweckrichtung einer Privatisierungserscheinung keiner verfassungsrechtlichen Anforderung entgegensteht. Diese allgemeinen Spielräume ausserhalb der Anforderungen werden vorab in I herausgearbeitet.

Zweitens können Spielräume innerhalb der Anforderungen entstehen, und dies wiederum auf zwei Weisen: indem eine verfassungsrechtliche Anforderung Lockerungen vorsieht, die für jede Aufgabenerfüllung gelten oder 
indem die privatisierte Aufgabenerfüllung besondere Lockerungen hervorrufen und ausschöpfen kann.

Für die Fragestellung dieser Arbeit sind vor allem die besonderen Lockerungen interessant, weil sie von der grundsätzlich gleichförmigen und einheitlichen Geltung der verfassungsrechtlichen Anforderungen (siehe vorne \$3/I.3) abweichen.

\section{Allgemein: Spielräume ausserhalb der verfassungsrechtlichen Anforderungen}

Im dritten Paragrafen wurden die verfassungsrechtlichen Anforderungen gegenüber Rechtsfiguren abgegrenzt, die zwar als Prinzipien in der Verfassung verankert sind, aber nicht den Gehalt von Anforderungen haben (siehe vorne \$3/III). Dazu zählen die Subsidiarität; Bedarfsgerechtigkeit und Wirtschaftlichkeit; ein Grundversorgungsauftrag, der über einzelne Leistungspflichten hinausgeht; der Grundsatz einer staatsfreien Wirtschaft; sowie die Bindung des Staats an bestimmte Amtssprachen.

Darüber hinaus entstehen Spielräume an den Grenzen der verfassungsrechtlichen Anforderungen. Denn diese unterziehen das staatliche Handeln bestimmten Voraussetzungen, und sie dürfen nicht ihrerseits zu uferlosen Prinzipien ausgeweitet werden.

Insbesondere verpflichtet die Verfassung den Staat nicht darauf, hoheitlich zu handeln. Sie schreibt ihm keine bestimmten Handlungsformen vor und zwingt ihn auch nicht, immer einseitig und verbindlich zu handeln. Das einfache Gesetz kann zwar einen Vorrang der Verfügungsform vorsehen (so etwa im Kanton Bern in Art. 49 Abs. 1 VRPG-BE). Auch wenn eine entsprechende Bestimmung auf Bundesebene fehlt, «gilt der allgemeine Vorrang der Verfügung auch im Bund» ${ }^{901}$. Dieser Vorrang ist aber nur «allgemein», weil er nicht verpflichtend ist und ihn abweichende Anordnungen im Spezialgesetz wegbedingen können. Die Verfassung hält den Gesetzgeber nicht davon $\mathrm{ab}$, der Verwaltung formlose, konsensuale oder unverbindliche Instrumente an die Hand zu geben. Sie bindet jedes Staatshandeln unterschiedslos an dieselben Anforderungen. Ein grundsätzlicher Spielraum besteht also darin, dass sich der Staat grundsätzlich in private Gewänder hüllen darf. Er kann gegenüber Privaten wie ein Privater wirken, soweit er die staatsspezifischen Pflichten erfüllt. 
Ebenso schuldet der Staat seinen Bürgern keine Übersichtlichkeit über seine Organisation. Die verfassungsrechtlichen Anforderungen beschlagen die je einzelne Verwaltungshandlung, das heisst die konkrete Beschränkung privater Rechtspositionen. Aus der Verfassung ist aber keine Pflicht abzuleiten, das Organigramm der Verwaltungsträger müsse einfach zu zeichnen sein. Immerhin fliesst aus dem Gesetzmässigkeitsprinzip der Anspruch, dass für jede staatliche Tätigkeit eine Grundlage besteht - insofern ist Nachvollziehbarkeit gewährleistet. Ein darüber hinausgehender Anspruch, den Verwaltungsadressaten gegenüber Übersichtlichkeit über die staatlichen Tätigkeiten zu bieten, besteht nicht. Der Bundesrat hielt denn auch fest:

«Entscheide über Auslagerungen sind [...] nicht systematisch, sondern eher aufgrund von Ad-hoc-Überlegungen getroffen worden. In dieser Hinsicht trifft die von der OECD gegenüber ihren Mitgliedstaaten geäusserte Kritik, dass Auslagerungen oft zu einem «administrative zoo geführt haben, auch auf die Schweiz zu.»902

Der Bundesrat nahm dies als Anreiz, die Auslagerung von Staatsaufgaben nach einheitlichen Kriterien zu gestalten (unter dem Stichwort Corporate Governance), als verfassungswidrig ist der verursachte «Zoo» aber nicht anzusehen. Vielmehr gewährt die Verfassung dem Staat gerade den Spielraum, Einzelfallregelungen zu treffen und für jede Aufgabenerfüllung neue Kategorien und neue Instrumente zu schaffen.

Das Öffentlichkeitsgesetz will zwar «die Transparenz über den Auftrag, die Organisation und die Tätigkeit der Verwaltung fördern» (Art.1 Abs. 1 BGÖ). Das BGÖ schafft Transparenz, indem es Privaten das Recht gibt, amtliche Dokumente einzusehen. Und gemäss Art. 164 Abs. 1 Bst. g BV gehören die Grundzüge der staatlichen Organisation in das formelle Gesetz. Doch diese Pflichten bedeuten nicht, dass die Staatsorganisation möglichst einfach oder nachvollziehbar sein muss. ${ }^{903}$ Auch die verfassungsrechtliche Anforderung von Art. 180 Abs. 1 Satz 2 BV fordert einzig, dass der Bundesrat die Staatstätigkeit koordiniert. Diese Pflicht ist aber in das Innere der Verwaltung gerichtet und begründet keine Ansprüche der Verwaltungsadressaten auf eine nachvollziehbare Staatsorganisation. ${ }^{904}$

902 Corporate-Governance-Bericht, 8250.

903 Ähnlich auch TSCHANNEN, Organigramm, S. 518.

904 Vgl. e contrario BIAGGINI, Kommentar BV, Art. 180 Rz. 6 f. 


\section{Spielräume aus der Gesetzmässigkeit}

Das Gesetzmässigkeitsprinzip erfordert, dass sich staatliches Handeln auf eine gesetzliche Grundlage stützen kann, die über genügende demokratische Legitimation verfügt und genügend bestimmt ist, sodass Rechtssicherheitsund Rechtsgleichheitsinteressen gewahrt bleiben (siehe vorne \$3/II.2). 905 Innerhalb dieser Schranken bestehen grosse Spielräume. Insbesondere ist es mit dem Gesetzmässigkeitsprinzip vereinbar, den Verwaltungsträgern grosse Freiräume zu gewähren bezüglich der Art und Weise, wie sie die vorgegebenen Aufgaben erfüllen. Einzig gesetzliche Leistungspflichten können diese Spielräume begrenzen. Wo das Gesetz den Staat verpflichtet, gewisse Leistungen zu erbringen, darf er die Verwaltungsadressaten nicht mittels finanzieller Anreize von der Inanspruchnahme dieser Leistungen abhalten (vorne \$5/II.3.c und \$5/V.3.b).

Im Folgenden stehen diejenigen Lockerungen der Gesetzmässigkeit im Vordergrund, die besonders für die privatisierte Aufgabenerfüllung gelten. Vorab ist aber darauf hinzuweisen, dass das Bestimmtheitsgebot von vornherein sehr offen gestaltet ist. Das Erfordernis einer genügenden Normdichte soll den Verwaltungsadressaten Gewähr für voraussehbares und berechenbares Staatshandeln geben. Das Bundesgericht sagt dazu:

«Das Gebot der Bestimmtheit rechtlicher Normen darf nicht absolut verstanden werden. Der Gesetzgeber kann nicht darauf verzichten, allgemeine und mehr oder minder vage Begriffe zu verwenden, deren Auslegung und Anwendung der Praxis überlassen werden muss. Der Grad der erforderlichen Bestimmtheit lässt sich nicht abstrakt festlegen. Er hängt unter anderem von der Vielfalt der zu ordnenden Sachverhalte, von der Komplexität und der Vorhersehbarkeit der im Einzelfall erforderlichen Entscheidung, von den Normadressaten, von der Schwere des Eingriffs in Verfassungsrechte und von der erst bei der Konkretisierung im Einzelfall möglichen und sachgerechten Entscheidung ab» ${ }^{906}$.

Je vielfältiger, komplexer und unvorhersehbarer ein Aufgabenbereich ist, desto offener darf die entsprechende gesetzliche Grundlage sein. Das Bundesgericht hat dies im Berner Wegweisungsfall für das Polizeirecht ausdrücklich erlaubt: «Die Polizeitätigkeit richtet sich gegen nicht im Einzelnen bestimmbare Gefährdungsarten und Gefährdungsformen in vielgestaltigen und wandelbaren Verhältnissen und ist demnach situativ den konkreten Um-

905 Für den letzten Aspekt ferner HÄFELIN/MÜLLER/UHLMANN, Allgemeines Verwaltungsrecht, Rz.345.

906 BGE 143 I 310 E.3.3.1 S.315. 
ständen anzupassen.»907 Aufgabenbereiche, die ihrer Natur nach unvorhersehbare Herausforderungen mit sich bringen und kreative Lösungen nötig machen, erfordern offenere Normen. Anders gewendet: Das Gesetzmässigkeitsprinzip erlaubt es dem Gesetzgeber, Spielräume für ungebundenes und flexibles Verwaltungshandeln vorzusehen, wo Aufgabenträger diese Spielräume benötigen.

\section{Insbesondere bei den privatisierten Organisationsformen}

\section{a. Zulässigkeit von Einzelfallregelungen als Spielraum}

Das Spezialgesetz muss den organisatorischen Status und die rechtlichen Konsequenzen für privatisierte Organisationsformen je einzeln festlegen (siehe vorne \$ 4/II.4.b). Die Erfüllungsprivatisierung in organisatorischer Hinsicht bedeutet also, bedürfnisgerechte Konstrukte für den jeweiligen Tätigkeitsbereich im Einzelfall zu erschaffen. Damit nützt die Privatisierung der Organisationsformen einen Spielraum aus, den das Gesetzmässigkeitsprinzip bietet. Es erfordert nur, dass die Rechtsfolgen des staatlichen Handelns gesetzlich geregelt sind, nicht dass sie in immer gleicher Art geregelt werden. Das RVOG zeichnet diesen Spielraum der Gesetzmässigkeit nach, indem es nur den Einheiten der Zentralverwaltung dieselben Rechtsfolgen auferlegt (siehe zum Beispiel Art. 38, 45 und 51).

Dieser Spielraum ist einzig durch die Rechtsgleichheit beschränkt, welche einen einzigen Verwaltungsträger in vergleichbaren Situationen zu gleichem Handeln verpflichtet. Art. 8 Abs. 1 BV verbietet es dem Staat aber gerade nicht, dass er verschiedene Aufgabenträger in einander ungleiche Organisationsformen hüllt.

\section{b. Eingeschränkte Aufsicht als Spielraum}

Das Gesetzmässigkeitsprinzip verlangt zudem, dass jede staatliche Aufgabenerfüllung an die rechtlichen Vorgaben gebunden bleibt. Insofern eröffnet Art. 5 Abs. 1 BV hier einen Spielraum: reduzierte Aufsicht gegenüber ausgelagerten Aufgabenträgern. Denn auch die blosse Verbandsaufsicht lässt eine Prüfung des Verwaltungshandelns auf Rechtsverletzungen zu (siehe vorne \$ 4/II.4.b). ${ }^{908}$ Damit stellt auch die eingeschränkte Aufsicht gegenüber privatisierten Aufgabenträgern sicher, dass rechtliche Vorgaben eingehalten werden.

907 BGE 132 I 49 E. 6.2 S. 58.

908 Vgl. aus der Literatur TSCHANNEN/ZIMMERLI/MÜLLER, Allgemeines Verwaltungsrecht, \$5 Rz. 33 . 
Diese Aufsicht kann - soweit gesetzlich vorgesehen - auch in der Form von Leistungsaufträgen und Eignerstrategien erfolgen. Diese Aufsichtsinstrumente gewähren den beaufsichtigten Aufgabenträgern eine grössere Mitsprache, als dies im Rahmen der Dienstaufsicht möglich wäre. Diese Organisationen erreichen durch die eingeschränkte Aufsicht grössere Ermessensfreiheiten und profitieren von einem Spielraum, den ihnen das Gesetzmässigkeitsprinzip gewährt.

\section{c. Unbestimmte Aufgabenübertragungen dank Spezialitätsprinzip}

Verwaltungseinheiten sind immer für denjenigen Aufgabenbereich zuständig, der ihnen gesetzlich übertragen ist. ${ }^{909}$ Die Anforderung an die Bestimmtheit dieser gesetzlichen Übertragung unterscheidet sich je nach Art der Aufgabe. Wie es das Bundesgericht ausdrückt:

«Insbesondere im Bereich der wirtschaftlichen Tätigkeit des Staates kann die gesetzliche Grundlage nicht zu detailliert sein, um die unternehmerische Tätigkeit nicht zu behindern, namentlich dann, wenn damit eine unabhängige staatliche Anstalt betraut wird [m.w.H.]. Die gesetzliche Grundlage muss aber zumindest den Sachbereich umschreiben, in welchem die Tätigkeit erfolgen soll (Spezialitätsprinzip; [m.w.H.]).»910

Sofern also einem staatlichen Aufgabenträger auch marktwirtschaftliche Tätigkeitsbereiche offenstehen und seine Organisationsform autonomes Handeln befördern soll, muss ihm die gesetzliche Grundlage die notwendigen Freiheiten belassen. Indem somit das Gesetzmässigkeitsprinzip auf ein Spezialitätsprinzip reduziert wird, schafft es einen Spielraum für gemischtwirtschaftliche Aufgabenträger. Sie erhalten damit die Möglichkeit, den Umfang ihrer Aufgabe in einem grösseren Mass mitzubestimmen, als dies für Einheiten der Zentralverwaltung möglich ist. Dieser Spielraum bedeutet eine Lockerung der Gesetzmässigkeit, die speziell für privatisierte Organisationsformen gilt und nicht in allgemeiner Weise aus der verfassungsrechtlichen Anforderung fliesst.

Der Spielraum zeigt sich mitunter darin, dass die Aufgabe nicht mehr in einem Rechtssatz genau bestimmt ist, sondern vertraglich geregelt wird. So hält etwa das Fernmeldegesetz fest, dass die «Verwaltung und Zuteilung bestimmter Adressierungselemente Dritten übertragen» werden kann. ${ }^{911}$ Die dazugehörige Verordnung führt sodann aus, dass diese Übertragung auf

909 TSCHANNEN/ZIMMERLI/MÜLLER, Allgemeines Verwaltungsrecht, §5 Rz. 29. Ferner auch BIAGGINI, Polizeiliche Generalklausel, S. 38.

910 BGE 138I378 E.7.2 S.392.

911 Art.28 Abs.2FMG. 
dem Vertragsweg zu erfolgen hat.912 Gemäss Medienmitteilung des zuständigen Departements hat die Stiftung SWITCH die entsprechende Ausschreibung gewonnen und diese Aufgaben übertragen erhalten. ${ }^{913}$ Wie die Aufgaben im Einzelnen aussehen, ist nicht rechtssatzförmig geregelt, der Übertragungsvertrag ist nicht einsehbar. Die Verordnung sagt lediglich: «Die Aufgaben der Registerbetreiberin sind im Rahmen eines Leistungskatalogs zu vereinbaren; zur Überprüfung der Aufgabenerfüllung sind Qualitätsmerkmale festzulegen.» ${ }^{914}$ Auch im Informationssystem über das öffentliche Beschaffungswesen in der Schweiz (www.simap.ch) fehlt eine genaue Aufgabenbeschreibung. Darin ist zwar der Leistungsauftrag öffentlich ausgeschrieben; doch beim Formularpunkt «Detaillierter Aufgabenbereich» steht lediglich «Registerbetreiberin zur Verwaltung der Domain «.ch»»915.

\section{Insbesondere bei den privatisierten Handlungsformen}

Das Bundesgericht setzt bezüglich Normdichte einen weniger strengen Massstab an, soweit der Staat mittels nichthoheitlicher Instrumente handelt: «Unbestimmte Regelungen können insbesondere dann genügen, wenn ein Rechtsverhältnis zur Diskussion steht, welches die Betroffenen freiwillig eingegangen sind oder bei dem die Rechte und Pflichten zwischen Staat und Privaten frei ausgehandelt werden können.»916

Das Gesetzmässigkeitsprinzip bietet also einen besonderen Spielraum für konsensuale Instrumente: Solange die Privaten den Pflichten selber zustimmen, die der Staat ihnen aufbürdet, genügt eine weniger bestimmte gesetzliche Grundlage für die Einschränkung privater Rechtspositionen.917 In solchen Fällen kann eine reine Kompetenznorm genügen. ${ }^{918}$ Die Einwilligung des Privaten kann allerdings das Gesetzmässigkeitsprinzip nicht soweit lockern, dass dadurch die Rechtsgleichheit gefährdet oder staatliche Leistungspflichten unterschritten würden.919

\footnotetext{
912 Art.33 VID.

913 UVEK, Verwaltung der Internet-Domain «.ch` - Switch gewinnt Ausschreibung, Medienmitteilung vom 1. September 2016.

914 Art.37 Abs.1 VID.

915 Siehe die Ausschreibung vom 15. April 2016 zur Projekt-ID 139185 auf www.simap.ch.

916 BGE129 I 161 E. 2.2 S. 163. Vgl. dazu auch RUCH, Informalisierung, S. 522.

917 WIEDERKEHR, Legalitätsprinzip im Kausalabgaberecht, S. 45.

918 HÄNER, Einwilligung, S. 60.

919 HÄNER, Einwilligung, S. 69, 73.
} 


\section{Insbesondere bei den privatisierten Finanzierungsformen}

Sofern die gesetzliche Grundlage einem Aufgabenträger einen aufgabenbezogenen Spielraum einräumt, erstreckt sich dieser auch auf finanzielle Belange der Aufgabenerfüllung, insbesondere auf die Festsetzung von Gebühren. ${ }^{920}$ Bei den privatisierten Finanzierungsformen bietet das Gesetzmässigkeitsprinzip darüber hinaus insgesamt einen beachtlichen Spielraum: Sofern die Höhe einer Abgabe und der Kreis der Abgabepflichtigen sachlich begründet werden können und die Abgabenhöhe in einem vernünftigen Verhältnis zum Wert der staatlichen Leistung steht, sind an die gesetzliche Grundlage keine hohen Anforderungen zu stellen. Dies lässt sich am Verursacherprinzip (sogleich a) und an Marktpreisen (hinten b) illustrieren.

\section{a. Das aufgeweichte Verursacherprinzip weitet die Spielräume des Äquivalenzprinzips aus}

Kostenanlastungssteuern und -abgaben liegen zwischen Steuern und Abgaben. Sie basieren auf der Nähe eines Abgabekreises zu einer staatlichen Leistung und sind damit weder eine Leistung für eine Gegenleistung noch voraussetzungslos geschuldet (siehe vorne \$ 4/IV.3.b). ${ }^{921}$

Soweit solche Abgaben eben nicht als Steuern klassifiziert werden, braucht der Gesetzgeber keine ausdrückliche Verfassungsgrundlage für ihre Erhebung und muss sich generell nicht an die Grundsätze der Besteuerung nach Art. 127 BV halten. Dies bedeutet, dass auch hier das Äquivalenzprinzip die mangelnde Normdichte kompensieren kann. Doch das aufgeweichte Verursacherprinzip wirft dogmatische Wellen:

«Entgegen den reinen Finanzsteuern stehen die Kostenanlastungssteuern freilich nicht völlig ausserhalb eines gewissen, wenn auch nur ansatzweisen Leistungsaustausches. Die Kostenanlastungssteuern finden ihre Legitimation darin, dass die betreffenden Aufwendungen des Gemeinwesens dem abgabepflichtig erklärten Personenkreis eher anzulasten sind als der Allgemeinheit (sog. einfache Gruppenäquivalenz), sei es, weil diese Gruppe von den Leistungen generell stärker profitiert als andere (‘abstrakte Nutzennähe)), sei es, weil sie als hauptsächliche Verursacherin dieser Aufwendungen erscheint (রabstrakte Kostennähe)). [...] Aus der Konzeption einer Kostenanlastungssteuer folgt, dass die Kosten und die anzulastende Steuer in einem engen wechselseitigen Verhältnis stehen.»922

920 MÄCHLER, Finanzierung privater Träger, S.1181 f.

921 Siehe BGer-E 2C_519/2016 vom 4. September 2017, E. 3.5.

922 BGer-E 2C_519/2016 vom 4. September 2017, E. 3.5.4 und 3.5.5, ohne die Hervorhebungen im Original. 
Die abstrakte Nähe zur Leistung reicht also aus, um gewissen Bürgern die Kosten für die staatliche Aufgabenerfüllung aufzubürden - ohne dass die Anforderungen für die Erhebung einer Steuer eingehalten sein müssten.

In einem anderen Urteil schafft das Bundesgericht für solche bloss gruppenäquivalenten Mischabgaben die Kategorie "pauschale Aufsichtsabgabe (für dem Einzelnen nicht zuordenbare Allgemeindienstleistungen)» im Unterschied zu «individuell zurechenbaren Gebühren».923

Diese Mischformen weichen nicht nur den Verursacherbegriff auf, sie schaffen auch neue Spielräume in der Anwendung der Gesetzmässigkeit und der Verhältnismässigkeit. Im Bereich der kostenabhängigen Abgaben fallen diese im Ergebnis auf eine Willkürprüfung zurück. In den Worten des Bundesgerichts: «Es ist nicht notwendig, dass die Gebühren in jedem Fall genau dem Verwaltungsaufwand entsprechen; sie sollen indessen nach sachlich vertretbaren Kriterien bemessen sein und nicht Unterscheidungen treffen, für die keine vernünftigen Gründe ersichtlich sind.»924

In einem abstrakten Normkontrollverfahren befand das Bundesgericht etwa, es könne «jedenfalls nicht von vorneherein [sic!] ausgeschlossen werden, dass eine Kostenauflage von gesamthaft Fr. 30’000.- sich im Einzelfall als verhältnismässig erweisen könnte»925. Auch hier zeigt sich, dass die mangelnde Verhältnismässigkeit am (potentiellen) Einzelfall zu messen ist. Ausserdem muss die Polizei nicht belegen, dass ihr Kostenaufwand im Fall eines solchen Gewaltausbruchs anlässlich einer Grossveranstaltung tatsächlich in dieser Grössenordnung zu liegen käme; es reicht hingegen schon, dass dies nicht von vornherein ausgeschlossen ist - womit hier die Willkürgrenze durchwirkt.

\section{b. Marktpreise ersetzen gesetzliche Bemessungsgrundsätze}

In einer weiteren Konstellation darf die gesetzliche Grundlage bezüglich Abgabenhöhe unbestimmt sein. Wenn es private Konkurrenzangebote für eine staatliche Tätigkeit gibt, braucht das Gesetzmässigkeitsprinzip private Leistungsbezüger nicht vor zu hohen Gebühren zu schützen. Der Marktmechanismus übernimmt dann diese Funktion; ist eine Gebühr zu hoch, weichen Private auf andere Anbieter aus. ${ }^{926}$

Diesen Spielraum aus dem Gesetzmässigkeitsprinzip hat das Bundesgericht mehrfach bestätigt und mit einer Überlegung verbunden, die bereits in

923 BGer-E 2A.345/2006 vom 24. Oktober 2006, E.3.2.2.

924 BGE 141 I 105 E. 3.3.2 S.109.

925 BGE 143 I 147 E. 6.3.1 S.158. Es handelte sich dabei um die gesetzlich festgelegte Höchstgebühr für die Kostenpflicht von Veranstaltern bei Gewaltausbrüchen.

926 UHLMANN, Gewinnorientiertes Staatshandeln, S. 268 f. Vgl. auch BGE 121 I 230 E.3g/dd S. 239 . 
Bezug auf privatisierte Handlungsformen ausgeführt wurde: die Freiwilligkeit der Inanspruchnahme staatlicher Leistungen (siehe vorne 2). Eine offene gesetzliche Grundlage bezüglich Gebührenhöhe sei etwa dann in Ordnung, wenn die Gebühr «ein Entgelt für die freiwillige und kommerzielle Inanspruchnahme einer kostspieligen Infrastruktur» ${ }^{927}$ ist. 928

Diese generelle Lockerung verdeutlicht den grossen Spielraum, der hier speziell den privatisierten Handlungsformen gewährt wird. Der Verweis auf einen Marktpreis stellt lediglich sicher, dass die Privaten zur Bezahlung des verlangten Preises in der Lage sind. Die gesetzliche Grundlage wird also unbestimmt gehalten, damit der Staat den höchsten Preis erzielen kann, der aufgrund seiner Machtposition und der Konkurrenzlage am Markt möglich ist. Dieser Spielraum bedeutet, dass der Staat nicht im demokratischen Prozess der Gesetzgebung festlegt, nach welchen Massstäben sich seine Aufgabenträger zu finanzieren haben, sondern die Verwaltungsadressaten vor die Wahl stellt: «Zahlt, was wir verlangen, oder verzichtet auf unsere Leistung.»

Die Verfassung lässt es mithin zu, dass finanzielle Erwägungen mitprägen können, welche staatlichen Leistungen von den Machtunterworfenen beansprucht werden. DieErfüllungsprivatisierung stösst hier an keine verfassungsrechtlichen Grenzen. 


\section{Spielräume aus dem öffentlichen Interesse}

Art. 5 Abs. 2 BV fordert, dass sich jedes staatliche Handeln auf ein zulässiges öffentliches Interesse abstützen kann. Diese Anforderung ist aber nicht als diffuses Gemeinwohlprinzip zu verstehen, das der Förderung privater Interessen grundsätzlich entgegensteht. Staatliche Tätigkeit darf bloss nicht durch private oder Sondergruppeninteressen allein motiviert sein (siehevorne \$3/II.3 und \$5/II).

Diese Anforderung eröffnet der Erfüllungsprivatisierung einen generellen Spielraum: Private Interessen in der staatlichen Aufgabenerfüllung stärker zu gewichten, liegt im Sinn von Art. 5 Abs. 2 BV. Dieser Spielraum umspannt alle Aspekte der Aufgabenerfüllung.

\section{Insbesondere bei den privatisierten Organisationsformen}

Privatisierte Aufgabenträger dürfen neben dem aufgabenbezogenen Ziel, ein bestimmtes öffentliches Interesse zu befördern, durchaus auch Renditeziele verfolgen. ${ }^{929}$ Dass etwa ein Aufgabenträger in der Form einer privatrechtlichen Aktiengesellschaft unternehmerische Ziele verfolgt, steht der Verfassung nicht entgegen. ${ }^{930}$ Diese verfassungsrechtliche Anforderung verbietet es nicht, öffentliche mit anderen Interessen zu verbinden.

Ein Beispiel dafür: Die SIFEM AG, eine privatrechtliche Aktiengesellschaft in Bundesbesitz, verfolgt öffentliche Interessen im Bereich der Entwicklungszusammenarbeit. ${ }^{931}$ Sie fördert insbesondere Investitionen in Entwicklungsprojekte aus privaten Mitteln. Daneben ist es ausdrückliches Ziel dieses Aufgabenträgers, Gewinn zu erzielen. Gemäss Art. 30d der Verordnung über die internationale Entwicklungszusammenarbeit und humanitäre Hilfe finanziert sich die Gesellschaft «durch eigene Geschäftstätigkeit»932.

Dies steht auch im Zusammenhang mit der Rechtsprechungslinie des Bundesgerichts, wonach fiskalische Interessen nicht nur als öffentliche Interessen zulässig sind, sondern sogar als einziges Interesse staatliche Tätigkeit legitimieren können. Das Bundesgericht hat dies in letzter Konsequenz noch offengelassen, sich aber in der Tendenz so geäussert (siehe vorne \$3/II.3.a).

929 Vgl. BÜHLER, Corporate Governance, S. 518.

930 Siehe dazu KRAEMER/STÖCKLI, Grenzenlose Staatswirtschaft?, S. 37 f., zu BGE 138 I 378 E. 8.5 S. $395 \mathrm{f}$.

931 Art. 6 Abs.1Bst. d des Bundesgesetzes über die internationale Entwicklungszusammenarbeit und humanitäre Hilfe (Fn. 41); Art. 30a f. der Verordnung über die internationale Entwicklungszusammenarbeit und humanitäre Hilfe (Fn. 43) i.V.m Art.15i OV-WBF.

932 So auch ausdrücklich Strategische Ziele SIFEM, Ziff. 3.3. 


\section{Insbesondere bei den privatisierten Handlungsformen}

Wenn der Staat privatisierte Handlungsformen einsetzt, verzichtet er darauf, seinen Machtvorsprung durchzusetzen. Stattdessen zieht er die Sichtweise von Privatpersonen in seine Aufgabenerfüllung ein und setzt auf deren Akzeptanz (siehevorne \$ 4/III.3.c-eundg). Das öffentliche Interesse bietet ganz allgemein den Spielraum, neben öffentlichen auch private Interessen zu berücksichtigen.

Es ist daher verfassungsrechtlich unproblematisch, mittels ausgelagerter Normsetzung die Sichtweise der betroffenen Fachexperten in die Aufgabenerfüllung einzubeziehen (siehe vorne \$ 4/III.2.b.cc). Dieser Spielraum kommt auch unverbindlichen Handlungsformen wie etwa Warnungen und Empfehlungen zugute (siehevorne \$ 4/III.2.a.dd). Die Aufgabenerfüllung muss nicht auf die verbindliche Durchsetzung der öffentlichen Interessen ausgerichtet sein.

\section{Insbesondere bei den privatisierten Finanzierungsformen}

Privatisierte Finanzierungsformen nehmen die Verursacher staatlicher Aufwände in die Pflicht und steuern dadurch ihr Verhalten mit finanziellen Anreizen (vgl. vorne das Kennzeichen \$ 4/IV.4.e). Die Förderung öffentlicher Interessen kann es daher rechtfertigen, Privaten höhere Gebühren aufzuerlegen, um eine bestimmte Regelungsabsicht zu befördern. ${ }^{933}$ So können beispielsweise höhere Kehrichtgebühren den Umweltschutz verbessern. ${ }^{934}$

Das öffentliche Interesse eröffnet der Erfüllungsprivatisierung in finanzieller Hinsicht den Spielraum, wirtschaftliche Anreize als Mittel zur Aufgabenerfüllung einzusetzen. Dieser Spielraum entsteht in allgemeiner Weise aus dem öffentlichen Interesse, er zeigt sich aber besonders bei den privatisierten Finanzierungsformen. Denn gerade diese Formen beziehen das Setzen von Anreizen in die Aufgabenerfüllung ein.

933 FRICK, Verursacherprinzip, S. 44; WYSS, Kausalabgaben, S. 45.

934 FREY, Drei Stossrichtungen, S. 352. 


\section{Spielräume aus der Verhältnismässigkeit}

Die Verhältnismässigkeit misst das staatliche Handeln an den öffentlichen Interessen, die es verfolgt. Ein konkreter staatlicher Eingriff in private Rechtspositionen muss das vorgegebene öffentliche Interesse auch tatsächlich befördern, und dieses Interesse muss entgegenstehende private Interessen im Einzelfall überwiegen (siehe vorne \$3/II.4).

Das Verhältnismässigkeitsprinzip bietet der Erfüllungsprivatisierung einen übergreifenden Spielraum: Die Verwaltungsträger geniessen grosse Freiheit, Interessenabwägungen vorzunehmen. Die gerichtliche Überprüfung der Verhältnismässigkeit erfolgt sehr zurückhaltend (sogleich 1). Ausserdem liegt es gerade im Sinn der Verhältnismässigkeit, die Interessen von Privaten tendenziell stärker zu gewichten, als es die einseitige hoheitliche Aufgabenerfüllung tut. Dies lässt für die privatisierten Handlungs- und Finanzierungsformen weitere Spielräume entstehen (hinten 2 und 3). Die Verhältnismässigkeit entfaltet ihre Wirkung vor allem im konkreten Einzelfall. Deshalb lässt sie keine Spielräume entstehen, die bereits den privatisierten Organisationsformen zugutekämen.

\section{Allgemein: Zurückhaltende Ausübung der gerichtlichen Kognition}

Das Verhältnismässigkeitsprinzip verlangt und vollzieht einen differenzierten Prozess, in dem verschiedene Interessen bezeichnet, gewichtet und gegeneinander abgewogen werden. Gerichte vollziehen diese Abwägung nicht in jedem Fall in grossem Detailgrad. Mitunter werden Verwaltungsentscheidungen stehen gelassen, die aufgrund einer detaillierten Verhältnismässigkeitsprüfung anders hätten ausfallen müssen. Wie Verwaltungsträger ihre Spielräume bei Interessenabwägungen wahrnehmen, überprüft insbesondere das Bundesgericht nur sehr zurückhaltend (siehe vorne \$3/II.4.c). Es betont dabei die «örtlichen Verhältnisse ..., welche die kantonalen Behörden besser überblicken»935. Diese Zurückhaltung hat das Bundesgericht zu der Aussage verallgemeinert, ausserhalb von Grundrechtseingriffen verliere das Prinzip an Aussagekraft. Gestützt auf Art. 5 Abs. 2 BV alleine (ohne die Rückendeckung von Art. 36 Abs. 3), könne das Gericht die Verhältnismässigkeit nur bei offensichtlichen Missachtungen gegenüber kantonalen Behörden durchsetzen.936

935 BGE 142 I 162 E. 3.2.2 S. 165.

936 BGE 134 I 153 E. 4.2.1 S.157; TSCHANNEN/ZIMMERLI/MÜLLER, Allgemeines Verwaltungsrecht, \$21 Rz. 23. 
Dieser Spielraum betrifft insbesondere die Auswahl zwischen mehreren tauglichen Mitteln und die Gewichtung der öffentlichen gegen die privaten Interessen. Die gerichtliche Zurückhaltung lässt die Verhältnismässigkeitsprüfung sinngemäss auf einen Willkürmassstab zurückfallen:937 Das ausgewählte staatliche Mittel zur Erreichung der öffentlichen Interessen darf nicht gerade sachlich unhaltbar sein. Somit kann die Zwecktauglichkeit weiterhin geprüft werden - das staatliche Handeln muss die vorgegebenen Ziele fördern. Bezüglich Erforderlichkeit und Zumutbarkeit kann die Erfüllungsprivatisierung aber von grösseren Spielräumen profitieren.

Dieser Spielraum entsteht zwar nicht aus dem Verhältnismässigkeitsprinzip, vielmehr kreiert ihn die Erfüllungsprivatisierung erst. Allerdings zeigen sich die Konsequenzen dieser eingeschränkten Kognition besonders bei dieser verfassungsrechtlichen Anforderung.

\section{Insbesondere bei den privatisierten Handlungsformen}

\section{a. Einbezug von Fachwissen garantiert die Berücksichtigung privater Interessen}

Mit Handlungsformen, die Normsetzung und Normanwendung auslagern und überwachen, wird das Fachwissen der von den staatlichen Normen betroffenen Branchen in die Aufgabenerfüllung mit einbezogen (vgl.vorne \$4/III.2.b.cc). Dies kann zwar Interessenkonflikte befördern und dazu führen, dass Sondergruppeninteressen über gesamtgesellschaftliche Interessen gestellt werden. Soweit aber weiterhin mindestens ein zulässiges öffentliches Interesse vorliegt, lässt das Verhältnismässigkeitsprinzip solche Erscheinungen frei gewähren. Sie führen nämlich dazu, dass in der Tendenz private Interessen mitberücksichtigt und hoch gewichtet werden. Diesen Spielraum bietet das Verhältnismässigkeitsprinzip nicht generell, er entsteht vielmehr speziell bei der privatisierten Aufgabenerfüllung.

\section{b. Einvernehmliche Handlungsformen garantieren Zumutbarkeit}

Die erhöhte Berücksichtigung privater Interessen zeigt sich auch bei konsensualen Handlungsformen. Mit Verträgen einigen sich zwei Seiten einvernehmlich auf Leistungen und Gegenleistungen, die für beide annehmbar sind. Somit ist zumindest der Teilgehalt der Zumutbarkeit bereits durch das Instrument selbst gewährleistet: Niemand wird sich auf eine Leistung verpflichten lassen, die in keinem vernünftigen Verhältnis zur vereinbarten Gegenleistung

937 Kritik daran von MOOR/FLÜCKIGER/MARTENET, Droit administratif I, S. 813; und MÜLLER, Verhältnismässigkeit, S. $99 \mathrm{ff}$. 
steht. Diese Vermutung, die einen gewissen Spielraum eröffnet, ist dann widerlegt, wenn «die private Partei einem gewissen Drohpotential des Staates nachgeben musste, um einen noch grösseren Nachteil zu vermeiden» ${ }^{938}$.

\section{Insbesondere bei den privatisierten Finanzierungsformen}

\section{a. Äquivalenzprinzip als Kompensat für mangelnde Normdichte schafft Spielraum}

Im Recht der öffentlichen Abgaben erscheint das Verhältnismässigkeitsprinzip in besonderer Ausprägung: als Äquivalenzprinzip. «Es bestimmt, dass eine Gebühr nicht in einem offensichtlichen Missverhältnis zum objektiven Wert der Leistung stehen darf und sich in vernünftigen Grenzen halten muss.» ${ }^{939}$ Immer wenn der Staat kostenabhängige Gebühren einfordert, können diese damit gerechtfertigt werden, dass die Abgabehöhe entweder dem Nutzen für die Verwaltungsadressaten oder dem Kostenaufwand der Verwaltung entspricht (siehe vorne \$ 4/IV.2).940

Dies lässt zunächst erahnen, dass die Anforderung der Verhältnismässigkeit gegenüber privatisierten Finanzierungsformen besondere Durchschlagskraft erhält. Soweit sich der Staat als Erbringer von Leistungen versteht, deren Gegenwert die privaten Leistungsempfänger entgelten, ist die Höhe dieser Gegenleistung durch die Verhältnismässigkeit in Gestalt des Äquivalenzprinzips begrenzt. Die staatliche Leistung muss nämlich im konkreten Einzelfall dem Verwaltungsadressaten individuell zurechenbar sein, dem die Kosten auferlegt werden. ${ }^{941}$ Mit anderen Worten wird Individualäquivalenz gefordert, oder wie es das Bundesgericht ausdrückt: «[L]e montant de la contribution exigée d'une personne déterminée doit être en rapport avec la valeur objective de la prestation fournie à celle-ci» ${ }^{942}$. Eine kostenabhängige Gebühr ist also nur dann verhältnismässig, wenn ihre Höhe dem objektiven Nutzen des konkreten, einzelnen Leistungsempfängers entspricht - soweit die leider bald zu enttäuschende Erwartung an die Verhältnismässigkeit im Abgaberecht.

Das Äquivalenzprinzip (wie auch das Kostendeckungsprinzip) hat im Abgaberecht die Funktion, eine zu wenig bestimmte gesetzliche Grundlage

938 HÄNER, Einwilligung, S.72.

939 Anstelle vieler BGE 143 I 147 E. 6.3.1 S.158.

940 BGE 143 I147 E. 6.3.1 S.158; stellvertretend für die Lehre TSCHANNEN/ZIMMERLI/MÜLLER, Allgemeines Verwaltungsrecht, \$58 Rz. 20.

941 BGE 143 I 147 E. 6.3.1 S.158; BGer-E 2C_798/2017 vom 16. Februar 2018, E. 2.2.2.

942 BGE 143 I 227 E. 4.2.2 S.234. 
zu kompensieren (siehe vorne \$4/IV.2). Mangelnde Normdichte, insbesondere was die Bemessungsgrundsätze und die Abgabehöhe betrifft, kann mit objektiver Individualäquivalenz kompensiert werden. Das Äquivalenzprinzip dient aber eher als optionales Argument für den Verwaltungsträger, der seine Mittel ausserhalb der gesetzlichen Grundlage beschaffen will. Oder wie es WIEDERKEHR ausdrückt: «Der Spielraum, den das Kostendeckungs- und das Äquivalenzprinzip den rechtsetzenden Organen belassen, ist viel zu gross, um ihnen eine Schutz- und Begrenzungsfunktion einzuräumen.»943

Das Verhältnismässigkeitsprinzip bietet also den Spielraum, das Gesetzmässigkeitsprinzip mittels des Äquivalenzprinzips auszuhöhlen. Die verfassungsrechtlichen Anforderungen sind zu wenig starr, als dass sie die Flexibilität und Kreativität in der Mittelbeschaffung einschränken würden. ${ }^{944} \mathrm{Zu}$ diesem Ergebnis kam beispielsweise das Bundesverwaltungsgericht bezüglich der Aufsichtsabgabe, dieSwissmedicgestützt auf Art. 65 Abs. 2 HMG erhebt. ${ }^{945}$

\section{b. Höhergewichtung privater Interessen dank finanzieller Abhängigkeit des Aufgabenträgers}

Die Privatisierung der finanziellen Erfüllungsaspekte kennzeichnet sich unter anderem durch die Inkaufnahme von Interessenkonflikten (siehe vorne das Kennzeichen \$ 4/IV.4.g). Die Verhältnismässigkeit eröffnet hier wiederum den Spielraum, dass private Interessen mitberücksichtigt werden müssen. Mitunter wird ein Verwaltungsträger weniger einschränkend handeln, wenn er auf die strukturelle Finanzierung durch die Verwaltungsadressaten angewiesen ist. Denjenigen, von dessen Zahlungsbereitschaft meine Existenz abhängt, werde ich möglichst zuvorkommend behandeln wollen.

Inwiefern solche Abhängigkeiten die Verwaltungspraxis tatsächlich prägen, müsste empirisch untersucht werden. Dies kann diese Arbeit nicht leisten. Hier muss es bei der Vermutung bleiben, dass die finanzielle Abhängigkeit von den direktbetroffenen Adressaten des eigenen Handelns dazu führen kann, dass die privaten Interessen zulasten der öffentlichen höher gewichtet werden. Damit gewährt die Verhältnismässigkeit den privatisierten Finanzierungsformen einen besonderen Spielraum.

943 WIEDERKEHR, Legalitätsprinzip im Kausalabgaberecht, S. 50, ohne die Hervorhebungen im Original.

944 Vgl. WYSs, Kausalabgaben, S. 87.

945 BVGer-E C-1858/2011 vom 23. September 2013, E. 6.4.1. 


\section{Spielräume aus den Teilgehalten des Grundsatzes von Treu und Glauben}

Der Staat darf nicht widersprüchlich, täuschend oder rechtsmissbräuchlich handeln, und Private dürfen auf Äusserungen des Staats vertrauen. Diese Anforderungen bieten keinen Ansatzpunkt für mögliche Spielräume, die dem Staat grössere Freiheiten in seinem Handeln bieten würden. Aus der Erfüllungsprivatisierung entstehen kaum Besonderheiten bezüglich der Teilgehalte des Grundsatzes von Treu und Glauben, die als verfassungsrechtliche Anforderungen das staatliche Handeln binden (siehe vorne \$3/II.5).

Einzig bezüglich des Vertrauensschutzes ist es vorstellbar, dass die informellen privatisierten Instrumente dem Staat die Möglichkeit bieten, verschiedene Vorgehensweise zu testen, ohne dass eine Vertrauensgrundlage entstehen würde, auf die er verpflichtet werden kann. Da privatisierte Handlungsformen tendenziell über weniger Steuerungskraft verfügen (siehe vorne das Kennzeichen \$ 4/III.3.d), könnte sie der Staat für unverbindliche Äusserungen verwenden. Eine Äusserung der Verwaltung muss sich auf einzelne Personen und konkrete Sachverhalte beziehen, um als Grundlage im Sinn des Vertrauensschutzes zu gelten (siehevorne \$5/IV.2.a). Auch hier ist aber der Spielraum begrenzt; sobald der Staat konkrete Aussagen trifft, seien sie auch in Form einer Warnung oder Empfehlung, können sie eine Vertrauensgrundlage darstellen. Ein Beispiel dazu: Das Bundesamt für Lebensmittelsicherheit und Veterinärwesen (BLV) hat im August 2018 zwei öffentliche Warnungen wegen krankheitserregender Keime in einem schwertförmigen Seifenblasen-Spielzeug ausgesprochen. ${ }^{946}$ Eine solche Warnung gemäss Art. 54 LMG bietet ebenso eine Grundlage für berechtigtes Vertrauen in die Richtigkeit der Angaben, wie wenn das Lebensmittelrecht in solchen Fällen das Verbot des betroffenen Gebrauchsgegenstands vorsähe.

946 Siehe die entsprechenden Medienmitteilungen auf www.blv.admin.ch/blv/de/home/ gebrauchsgegenstaende/rueckrufe-und-oeffentliche-warnungen.html. 


\section{Spielräume aus der Grundrechtsbindung}

Pauschal ausgedrückt vermitteln die Grundrechte keine absolut geschützten Rechte, sondern erfordern bloss qualifizierte Begründungen für die Einschränkung dieser Rechte (siehe vorne \$3/II.6.a). Die Erfüllungsprivatisierung ist von Einzelfallregelungen gekennzeichnet: Statt die üblichen, immer gleichen Formen zu verwenden, wie sie RVOG, VwVG und FHG vorsehen, schaffen Spezialgesetze neue, einzigartige Organisationen, Instrumente und Finanzierungen. Insofern diese Einzelfallregelungen Entscheidungsspielräume vermitteln, kommt den Verwaltungsträgern ein grösseres Ermessen zu. Und diese grösseren Ermessensspielräume müssen dann auch von Gerichten bei der nachträglichen Beurteilung von Grundrechtseingriffen berücksichtigt werden. Die spezialgesetzlich eingeräumten Freiheiten und Autonomiebereiche stellen also einen grundsätzlichen Spielraum bezüglich der Grundrechte dar.

Dennoch ist der Staat auch dann an die Grundrechte gebunden, wenn er seine Aufgaben mit privatisierten Organisations-, Handlungs- oder Finanzierungsformen erfüllt (siehe vorne \$3/II.6.b). Von der Grundrechtsbindung ist aber die Frage zu unterscheiden, ob diese Bindung immer sämtliche Grundrechte umfasst. ${ }^{947}$ Vorab ist daher zu erörtern, ob sich der Grundrechtsschutz durch die Erfüllungsprivatisierung auf gewisse Ansprüche beschränkt (sogleich 1).

Danach folgen spezifische Lockerungen in der Grundrechtsgeltung bezüglich der privatisierten Organisationsformen (2) und Handlungsformen (3); aus den privatisierten Finanzierungsformen entstehen keine erwähnenswerten Spielräume.

\section{Allgemein: Eingeschränkte Grundrechtsgeltung bei privatisierter Aufgabenerfüllung?}

\section{a. Lehre hält Beschränkung aufrechtsstaatliche Garantien für grundsätzlich zulässig}

In der Botschaft zur BV 1999 griff der Bundesrat die Frage auf, ob die Grundrechtsbindung abgestuft werden muss bei der privatisierten Aufgabenerfüllung, und hielt es für «durchaus vorstellbar, dass im einen oder anderen Punkt die Pflichten, die sich aus einem bestimmten Grundrecht ergeben, angepasst werden müssen, wenn gewisse staatliche Aufgaben delegiert werden. Die

947 BIAGGINI, Kommentar BV, Art. 35 Rz.12; HANGARTNER, Öffentlich-rechtliche Bindungen, S.143; KIENER/KÄLIN/WYTTENBACH, Grundrechte, §4 Rz. 69; TSCHANNEN, Staatsrecht, $\$ 7 \mathrm{Rz} .53$. 
Formulierung von [Art. 35 Abs. 2] lässt aber dem Gesetzgeber oder dem Richter genügend Spielraum, Differenzierungen vorzunehmen je nach der zu übertragenden Aufgabe oder deren Träger.» ${ }^{948}$

Der Bundesrat erwähnt dabei Gesetzgeber und Gericht in einem Atemzug, ohne auf diesen wichtigen Unterschied hinzuweisen. Dass das Spezialgesetz den privatisierten Aufgabenträger von gewissen grundrechtlichen Pflichten entbinden kann, ist die eine Sache. Damit ist allerdings nicht beantwortet, ob eine solche Einschränkung in genereller Weise angenommen und auf dem Weg der Rechtsanwendung entschieden werden kann.

Ein überwiegender Teil der Lehre scheint eine generelle Einschränkung auf die rechtsstaatlichen Grundrechtsgarantien, insbesondere das Gleichbehandlungsgebot und das Willkürverbot, für zulässig zu halten. Die entsprechenden Lehrmeinungen scheuen aber davor zurück, die Bindung an andere Grundrechte ausdrücklich abzulehnen: Die Garantien von Art. 8 und 9 BV seien «in erster Linie» 949 anwendbar, stünden «im Vordergrund»950, oder eine Bindung daran könne man «immerhin erwarten» 951 oder sei «vor allem» 952 anerkannt.

\section{b. Doch Beschränkung auf Art. 8 und 9 BV reflektiert bloss typische Gefährdungslage}

Dass der Staat in vorsichtiger Weise nur noch an einen Kern von Grundrechten gebunden wird, wenn er privatisierte Organisations- und Handlungsformen einsetzt, erklärt sich durch die typische Gefährdungslage, die in diesen Fällen vorliegt. Ob der Staat ein gewisses Grundrecht einhalten muss, wird in der Praxis nur dann geklärt, wenn ein Betroffener die Verletzung dieses Rechts rügt. Grundrechte, die in konkreten Fällen nicht zur Diskussion stehen, rücken damit in den Hintergrund - ohne dass dadurch geklärt wäre, dass der Staat nicht auch an sie gebunden ist. 953

948 Botschaft BV, 193, Bezug nehmend auf den gleichlautenden Art.31 Abs. 2 des Verfassungsentwurfs vom 20. November 1996. Ohne weiteren Kommentar auf diese Stelle verweisend auch HÄFELIN/HALLER/KELLER/THURNHERR, Schweizerisches Bundesstaatsrecht, Rz. 277.

949 JAAG, Dezentralisierung und Privatisierung, S. 43.

950 HANDSCHIN/SIEGENTHALER, Privatisierung, S. 414.

951 TSCHANNEN, Staatsrecht, \$7 Rz. 50. Ähnliche Formulierung in TSCHANNEN, Systeme, Rz.150: «Am Ende wird man sich wohl mit der Forderung begnügen müssen [...].»

952 HANGARTNER, Öffentlich-rechtliche Bindungen, S. 143, sogar nur auf das Willkürverbot beschränkt.

953 In diese Richtung auch HÄSLER, Geltung der Grundrechte, S. 126; JAAG, Dezentralisierung und Privatisierung, S. 44. 
Wenn vor Bundesgericht gerügt wird, ein privatisierter Aufgabenträger verletze Grundrechte, geht es fast schon stereotyp um den rechtsgleichen Zugang zu Leistungen oder Einrichtungen oder um die willkürliche Verweigerung desselben. ${ }^{954}$ In keinem dieser Urteile wird die Anwendung anderer Grundrechte aber ausdrücklich ausgeschlossen. Und so hielt das Bundesgericht in einem Urteil von 2013 die Bindung an sämtliche Grundrechte ausdrücklich fest: «Wer staatliche Aufgaben wahrnimmt und diese gegebenenfalls mit Nebenaktivitäten finanziert, ist nicht nur an das Willkürverbot und den Grundsatz der Rechtsgleichheit gebunden, sondern muss generell auch dem besonderen ideellen Gehalt der Freiheitsrechte Rechnung tragen» ${ }^{955}$.

In darauffolgenden Urteilen prüfte das Bundesgericht in solchen Konstellationen - ohne dies für speziell erklärungsbedürftig zu halten - auch Eingriffe in andere Schutzbereiche: die geschlechterspezifische Gleichbehandlung956, die persönliche Freiheit ${ }^{957}$, den Schutz der Privatsphäre ${ }^{958}$ und die Vereinigungsfreiheit ${ }^{959}$. Sofern das Bundesgericht also die Gelegenheit erhält, sich über die Anwendbarkeit anderer Grundrechte zu äussern, erklärt es den privatisiert auftretenden Staat auch daran selbstverständlich gebunden.

\section{c. Ergebnis: Eingeschränkte Geltung nur auf ausdrückliche Anordnung des Gesetzgebers hin}

Damit kann festgehalten werden, dass die Verfassung und die Gerichtspraxis keinen Anhaltspunkt geben, die Grundrechtsbindung generell auf gewisse Schutzbereiche einzuschränken aus Rücksicht auf privatisierte Organisations- und Handlungsformen. Wenn «der Gesetzgeber diese Art der Aufgabenbesorgung in der Absicht gewählt hat, dem Gemeinwesen grössere Bewegungsfreiheit zu verschaffen» ${ }^{960}$, steht es ihm frei, dieser grösseren Freiheit mit ausdrücklichem Dispens von der Einhaltung gewisser Grundrechte Nachachtung zu verschaffen. Dafür ist allerdings eine entsprechende gesetzliche Anordnung zu fordern - eine Einschränkung auf dem Auslegungsweg

954 BGE143 I 37 E. 7.1 S. 44; 140 I 201 E. 6.5.1 S. 210; 127 I 84 E. 4c S. 89-91; 103 Ia 544 E. 6 e S. 555. Unter dem besonderen Gleichbehandlungsgebot im Rahmen der Wirtschaftsfreiheit geprüft in BGE 141 V 557 E. 4 S. 565 f., E. 7.2 S. 569 f.; 138 I 289 E. 2.3 S. 292. Mangels Grundrechtsbindung kein Schutzbereich geprüft, aber ähnliche Konstellation im grundlegenden Post-Urteil BGE 129 III 35.

955 BGE 139 I 306 E. 3.2.3 S.311; fast gleichlautend schon BGE 138 I 274 E. 2.2.2 S. 283.

956 BGE 140 I 201 E. 6.4.2 S. $209 \mathrm{f}$.

957 BGE 140 I 2 E.10.3 S.30 f.

958 BGE 140 I 2 E.10.5.1 S. 32 (ohne eingehende Prüfung).

959 BGE 140 I 201 E. 6.5.2 S.210 f.

960 TSCHANNEN, Staatsrecht, §7 Rz. 50. 
ist unzulässig.961 Da die verfassungsrechtlichen Pflichten an der Aufgabe hängen, können sie durch die Wahl der Erfüllungsmodi nicht automatisch als reduziert angesehen werden. ${ }^{962}$

Ein Zurückdrängen der verfassungsrechtlichen Anforderungen an das staatliche Handeln - wie hier des Umfangs der einzuhaltenden Grundrechte - lässt sich nicht mit den Beweggründen einer Privatisierung begründen. Die an das staatliche Handeln angelegten Bindungen schmälern sich nicht schon deshalb, weil der ausgelagerte und sich privatrechtlicher Instrumente bedienende Aufgabenträger ein Bedürfnis nach unternehmerischen Freiräumen hat. Die Verfassung stellt dieselben Anforderungen für sämtliches Staatshandeln auf. Sollen diese Anforderungen herabgesetzt werden, muss dies der Gesetzgeber ausdrücklich anordnen. Da dem Gesetzgeber diese Möglichkeit aber grundsätzlich offensteht, schafft die Grundrechtsbindung einen besonderen Spielraum für die Erfüllungsprivatisierung.

\section{Insbesondere bei den privatisierten Organisationsformen}

\section{a. Herabgesetzte Forderung einer «protection juridique minimum»}

Im Bestreben danach, den Staat durch die Auslagerung von Staatsaufgaben auf privatisierte Träger nicht aus der Grundrechtsbindung zu entlassen, scheint das Bundesgericht den Weg zu einem herabgesetzten Schutzniveau zu öffnen. Bezogen auf die Anforderung von Art. 35 Abs. 2 BV führte es aus:

«Afin que cette exigence [...] soit effective, l'Etat se doit d'assurer une certaine surveillance voire un certain contrôle sur les activités déléguées [...], ainsi que de garantir une protection juridique minimum aux personnes susceptibles d'être touchées dans leurs droits fondamentaux par les actes du délégataire de tâches publiques, du moins pour les atteintes potentielles d'une certaine gravité» 963 .

Anstatt sämtliche rechtsstaatlichen Anforderungen - insbesondere die Grundrechte in ihrem gesamten Bestand - als Massstab anzulegen, wird eine blosse «protection juridique minimum»964 akzeptiert. Wie soeben in 1.c dargelegt, darf der Umfang der Grundrechtsbindung nicht alleine auf dem Auslegungsweg eingeschränkt werden; dies kann nur der Gesetzgeber vorsehen.

961 So auch HÄSLER, Geltung der Grundrechte, S.127.

962 So aber TSCHANNEN, Systeme, Rz.150. Ähnlich, aber weniger deutlich auch TSCHANNEN, Staatsrecht, \$7 Rz. 57.

963 BGE 133 I 49 E.3.2 S. 56. Darauf Bezug nehmend auch HäFELIN/MÜLLER/UHLMANN, Allgemeines Verwaltungsrecht, Rz.1854.

964 BGE 133 I 49 E.3.2 S. 56; dazu auch HÄFELIN/MÜLLER/UHLMANN, Allgemeines Verwaltungsrecht, S. 406 (Rz. 1854). 
Doch das Bundesgericht scheint hier zumindest sich selbst die Möglichkeit zu öffnen, bei privatisierten Organisationsformen einen grundsätzlichen Spielraum zu gewähren und eine weniger intensive Grundrechtsgeltung anzunehmen. Ob das Bundesgericht künftig die Rechtsprechung in diesem Sinn präzisieren wird, muss sich erst zeigen. Es ist auch möglich, dass es die Grundrechte insgesamt als Mindestrechtsschutz meint und somit bereits in corpore als nicht zu unterschreitendes Minimum schützen wird.

\section{b. Unterschiedliche Aufsicht über privatisierte Aufgabenträger als Spielraum bei der Rechtsgleichheit}

Selbst wenn die "protection minimum» privatisierte Aufgabenträger von der Gewährleistung gewisser Freiheitsrechte befreien sollte, an die Rechtsgleichheit bleiben sie auf jeden Fall gebunden (siehe vorne 1.a). Diesem Grundrecht wohnt aber ein bedeutender Spielraum inne, den privatisierte Organisationsformen ausnützen können: Rechtsgleiche Behandlung kann nur gegenüber derselben Behörde eingefordert werden. Es betrifft die Rechtsgleichheit nicht, wenn die Gewerbepolizei einer Restaurateurin eine Nachtbetriebsbewilligung erteilt für ein Gartenrestaurant, das Bauamt ihr aber die Bewilligung für eine Überdachung des Innenhofs verweigert.

Im Detail ist umstritten, was im Sinne von Art. 8 Abs. $1 \mathrm{BV}$ noch dieselbe Behörde ist (siehe vorne \$3/II.6.a.bb). Es gibt Lehrmeinungen, die darunter alle Verwaltungseinheiten fassen wollen, die derselben Aufsichtsbehörde unterstehen. ${ }^{965}$ Verwaltungsträger, die nach anderen Rechtsgrundlagen einer eigenen Aufsicht unterstehen, dürften jedenfalls nicht darunter fallen. ${ }^{966}$ Darin liegt der besondere Spielraum für privatisierte Organisationsformen. Ihre Beaufsichtigung wird spezialgesetzlich geregelt; die Behördenpraxis anderer Einheiten liegt also ausserhalb der vergleichbaren Situationen, die bei einer Gleichbehandlungsprüfung zu berücksichtigen sind. Privatisierte Aufgabenträger sind dadurch freier, ihre eigene Praxis zu prägen, und müssen nur in ihrem eigenen Handeln konsistent sein.

\section{c. Grundrechtsträgerschaft ausserhalb des staatlichen Aufgabenbereichs beigemischten Organisationen}

Privatisierte Aufgabenträger, die neben staatlichen Aufgaben auch privatwirtschaftlich tätig sind, profitieren von einem Spielraum bezüglich Grund-

965 Darstellung dieser Lehrmeinungen bei MüLLER, Kommentar aBV, Art. 4 Rz. 39. Frage offengelassen in Urteil 2P.283/2001 vom 25. Februar 2002, E. 5.1.1; tendenziell wohl auch BGE 138 I 321 E. 5.3.7 S.330. Ferner HÄFELIN/MÜLLER/UHLMANN, Allgemeines Verwaltungsrecht, Rz. 588.

966 So ist auch BGE 138 I 321 E. 5.3.7 S.330 zu verstehen. 
rechtsbindung, der genau genommen gar keiner ist. Ausserhalb der staatlichen Aufgaben sind sie funktionell private Akteure und können sich auch ihrerseits auf den Schutz der Grundrechte berufen. ${ }^{967}$ Dies entspringt dem - noch geltenden - funktionellen Ansatz der Grundrechtsbindung, wurde aber bezüglich öffentlich beherrschter Unternehmen noch offengelassen (dazu vorne $\$ 5 /$ V.1.a). ${ }^{968}$ Dieser Spielraum betrifft also von vornherein nicht die staatliche Aufgabenerfüllung; diesbezüglich entstehen keine Lockerungen durch die Konstruktion von Akteuren, die neben staatlichen Aufgaben auch privatwirtschaftliche Tätigkeiten wahrnehmen.

Der Spielraum entsteht, weil staatlichen Akteuren die Betätigung im originär privaten Bereich erlaubt wird. Dann nämlich ist das fragliche Handeln nicht mehr staatliche Beschränkung privater Freiheiten, sondern bloss wettbewerbliches Handeln. Oder wie es das Bundesgericht im Glarnersach-Urteil ausdrückte:

«Die individualrechtliche Komponente der Wirtschaftsfreiheit (Art. 27 BV) gibt hingegen dem Einzelnen keinen Schutz vor Konkurrenz. Tritt ein staatliches Unternehmen mit gleichen Rechten und Pflichten wie ein privater Unternehmer und im Wettbewerb zu diesem auf, so entsteht den Privaten bloss ein weiterer Konkurrent, was keine Einschränkung der individualrechtlichen Wirtschaftsfreiheit darstellt» 969 .

\section{Insbesondere bei den privatisierten Handlungsformen}

Mit privatisierten Handlungsformen verzichtet der Staat auf einen Teil seines Machtvorsprungs, wenn er statt zwingender Mittel auf Gegenseitigkeit und Konsens setzt. Auf der anderen Seite bedeutet dies, dass die Verwaltungsadressaten freiwillig Rechtsbeziehungen mit dem Staat eingehen, statt dass er ihnen gegenüber lediglich Rechte und Pflichten einseitig anordnet. Diese Freiwilligkeit soll die Akzeptanz der staatlichen Vorkehrungen fördern (siehe vorne die Kennzeichen \$4/III.3.d und e).

Die gesteigerte Wirkungserwartung, die sich aus dieser Akzeptanz ergibt, hängt mitunter daran, dass Private nicht alle Rechtsansprüche geltend machen, die ihnen zustehen. Bezüglich der Grundrechte eröffnet dies die Diskussion über die Möglichkeit des Grundrechtsverzichts. Auch wenn es nach vorherrschender Lehrmeinung nicht möglich ist, von vornherein auf den Grundrechtsschutz zu verzichten, so können sich Private wenigstens

967 BAUMANN, Wettbewerbsverzerrungen, Rz.128.

968 BGE 142 II 369 E. 6.3.2 S.387.

969 BGE 138 I 378 E. 6.2.2 S. 386. 
entscheiden, ihre grundrechtlichen Ansprüche nicht durchzusetzen. ${ }^{970}$ Diese Möglichkeit besteht unabhängig von den eingesetzten Handlungsformen. Je mehr Mitwirkung aber ein Instrument erlaubt und je eher Verwaltungsadressaten der getroffenen Lösung zustimmen müssen, desto wahrscheinlicher dürfte ein solcher Verzicht auf die Durchsetzung von Grundrechtsansprüchen werden. 


\section{Zwischenfazit: Erfüllungsprivatisierung nutzt Einzelfallregelungen und Freiwilligkeit}

\begin{tabular}{|c|c|c|c|c|}
\hline \multicolumn{5}{|c|}{ Spielräume aus den Anforderungen: Übersichtsdarstellung der rasterartigen Prüfung in $§ 6$} \\
\hline & & \multicolumn{3}{|c|}{ Privatisierung der ... (\$4) } \\
\hline & & Organisationsformen & Handlungsformen & Finanzierungsformen \\
\hline \multicolumn{2}{|c|}{ Allgemein } & \multicolumn{3}{|c|}{$\begin{array}{l}\text { Spielraum: Keine Pflicht zu hoheitlichen Formen } \\
\text { Spielraum: Keine Pflicht zu übersichtlicher Verwaltungsorganisation }\end{array}$} \\
\hline \multirow{7}{*}{ 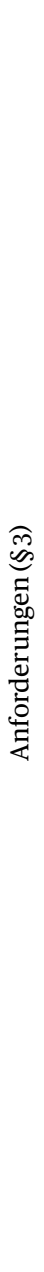 } & $\begin{array}{l}\text { Gesetzmässigkeit } \\
\text { Art. } 5 \text { und } 36 \text {, } \\
\text { jeweils Abs. } 1 \text { BV }\end{array}$ & $\begin{array}{l}\text { Spielraum: Einzelfall- } \\
\text { regelungen zulässig } \\
\text { Spielraum: Auch } \\
\text { Verbandsaufsicht prüft } \\
\text { Rechtsverletzungen } \\
\text { Spielraum: Spezialitäts- } \\
\text { prinzip lässt Konkreti- } \\
\text { sierung ausserhalb des } \\
\text { Gesetzes zu }\end{array}$ & \begin{tabular}{|l|} 
Spielraum: Einwilligung \\
bzw. Freiwilligkeit \\
setzen Anforderungen \\
an Normdichte herab
\end{tabular} & $\begin{array}{l}\text { Spielraum: Geringe } \\
\text { Normdichte kompen- } \\
\text { siert durch Äquivalenz- } \\
\text { prinzip und Marktpreise } \\
\text { Spielraum: Aufgeweich- } \\
\text { ter Verursacherbegriff } \\
\text { erweitert das } \\
\text { Äquivalenzprinzip }\end{array}$ \\
\hline & $\begin{array}{l}\text { Öffentliches } \\
\text { Interesse } \\
\text { Art. } 5 \text { und } 36 \text {, } \\
\text { jeweils Abs. } 2 \text { BV }\end{array}$ & $\begin{array}{l}\text { Spielraum: Renditeziele } \\
\text { auch als (einzige) öffent- } \\
\text { liche Interessen zulässig }\end{array}$ & $\begin{array}{l}\text { Spielraum: Handlungs- } \\
\text { formen dürfen private } \\
\text { Interessen fördern }\end{array}$ & $\begin{array}{l}\text { Spielraum: Verhalten } \\
\text { darf mit Gebühren } \\
\text { gesteuert werden }\end{array}$ \\
\hline & $\begin{array}{l}\text { Verhältnis- } \\
\text { mässigkeit }\end{array}$ & \multicolumn{3}{|c|}{$\begin{array}{l}\text { Allgemeiner Spielraum: Gerichtliche Zurückhaltung schafft } \\
\text { Abwägungsspielräume }\end{array}$} \\
\hline & $\begin{array}{l}\text { Art. } 5 \text { Abs. } 2 \text {, } \\
\text { Art. } 36 \text { Abs. } 3 \text { BV }\end{array}$ & - & \begin{tabular}{|l} 
Spielraum: Einbezug \\
von Fachwissen fördert \\
private Interessen \\
Spielraum: Einver- \\
nehmliche Instrumente \\
sind eher zumutbar
\end{tabular} & $\begin{array}{l}\text { Spielraum: Äquivalenz- } \\
\text { prinzip kompensiert } \\
\text { offene Normen } \\
\text { Spielraum: Abhängig- } \\
\text { keit von Gebühren- } \\
\text { zahlern fördert private } \\
\text { Interessen }\end{array}$ \\
\hline & $\begin{array}{l}\text { Treu und Glauben } \\
\text { Art. } 5 \text { Abs. } 3 \text {, } \\
\text { Art.9 BV }\end{array}$ & - & $\begin{array}{l}\text { Spielraum: Unverbind- } \\
\text { liche Instrumente sind } \\
\text { i.d.R. keine Vertrauens- } \\
\text { grundlage }\end{array}$ & - \\
\hline & $\begin{array}{l}\text { Grundrechte } \\
\text { Art. } 35 \text { Abs. 2, } \\
\text { Art. } 7-34 \text { BV }\end{array}$ & \multicolumn{3}{|c|}{$\begin{array}{l}\text { Allgemeiner Spielraum: Grösserer Ermessensspielraum } \\
\text { Allgemeiner Spielraum: Gesetzgeber kann einzelne Ansprüche von } \\
\text { Grundrechtsrechtsbindung ausnehmen }\end{array}$} \\
\hline & & $\begin{array}{l}\text { Spielraum: Blosse } \\
\text { "protection juridique } \\
\text { minimum» } \\
\text { Spielraum: Rechts- } \\
\text { gleichheit nur gegen- } \\
\text { über derselben Behörde }\end{array}$ & $\begin{array}{l}\text { Spielraum: Verzicht auf } \\
\text { Geltendmachung von } \\
\text { Grundrechtsansprüchen } \\
\text { dank freiwilliger } \\
\text { Instrumente }\end{array}$ & - \\
\hline
\end{tabular}


Die Erfüllungsprivatisierung soll den Staat flexibler, effizienter und günstiger machen. Um diese Erwartung zu erfüllen, muss die privatisierte Erfüllung staatlicher Aufgaben von besonderen Lockerungen innerhalb der verfassungsrechtlichen Anforderungen profitieren können. Dieser Paragraf hat einige solcher Lockerungen feststellen können, die nicht allgemein aus den Anforderungen hervorgehen, sondern durch Erfüllungsprivatisierungen erst entstehen.

Ein grundlegendes Kennzeichen der Erfüllungsprivatisierung zu einem ebenso grundlegenden Spielraum: Gesetzgeberische Speziallösungen und Einzelfallregelungen verringern die Aufsichtsintensität und schaffen Ermessensspielräume. Ausserdem leiten sich zwar alle Pflichten der Aufgabenträger von ihren Aufgabenbereichen ab, doch erlaubt das Spezialitätsprinzip gerade, dass die Details dieser Aufgaben ausserhalb des Gesetzes, etwa in Leistungsvereinbarungen, definiert werden.

Was im $§ 5$ als zusätzlicher Normsetzungsaufwand zu bemängeln war, erscheint hier in der Gestalt von massgeschneiderten Sonderlösungen, die Spielräume entstehen lassen.

Ein weiterer Spielraum ist nur der Tendenz nach festzustellen und hängt massgeblich vom konkreten Fall ab: Privatisierte Instrumente setzen auf freiwillige Kooperation Privater, was diesen als Einwilligung in die staatlichen Freiheitsbeschränkungen ausgelegt werden kann. Dies kann bis hin zu einem Verzicht auf die Geltendmachung von Rechtsansprüchen gehen. Öffentliches Recht bleibt aber zwingendes Recht, die blosse Mitwirkung der Machtunterworfenen lässt den Rechtsschutz nicht untergehen. Der Spielraum, der hier entsteht, ist eher faktischer denn rechtlicher Natur: Bürgerinnen und Bürger könnten ihre Ansprüche zwar (in den meisten Fällen) weiterhin gerichtlich durchsetzen; der Staat gewinnt aber an Freiheit, weil er darauf vertrauen kann, dass sie ebendies nicht tun werden.

Die Erfüllungsprivatisierung befreit den Staat nicht von seinen verfassungsrechtlichen Bindungen und lockert diese nur vereinzelt. Einzig das Erfordernis der genügenden Normdichte, ein ohnehin dehnbares Prinzip, erfährt systematische Lockerungen. Wenn der Staat einvernehmliche Instrumente und verursachergerechte Finanzierungen einsetzt, darf er sich auf unbestimmtere gesetzliche Grundlagen abstützen. Leicht vereinfachend ausgedrückt: Je freier die Aufgabenerfüllung sein soll, desto offener darf das Gesetz sein. 


\section{§7 Ergebnisse}

\section{Die Erfüllungsprivatisierung im Kontext eines funktionellen Staatsbegriffs}

Die Verfassung grenzt die staatliche von der privaten Sphäre ab, indem sie dem Staat Pflichten auferlegt, die für Private nicht gelten. Diese Pflichten sind die verfassungsrechtlichen Anforderungen an staatliches Handeln: Gesetzmässigkeit, öffentliches Interesse, Verhältnismässigkeit, Teilgehalte von Treu und Glauben, Grundrechtsbindung und Staatshaftung. Weil diese Anforderungen nur den Staat treffen, muss die Verfassung einen Staatsbegriff prägen. Dieser ist funktionell, wie in den $§ \S 2$ und 3 hergeleitet wurde; er hängt am Begriff der Staatsaufgabe. Staatlich ist jedes Handeln, das auf die Erfüllung staatlicher Aufgaben gerichtet ist. Eine staatliche Aufgabe ist dabei jeder Tätigkeitsbereich, der einem Akteur durch Verfassung oder Gesetz verpflichtend zur Erfüllung übertragen ist.

Nach der hier vertretenen Auffassung kennt der Staatsbegriff der Verfassung keine Abstufungen: Es gibt kein mehr oder weniger staatliches Handeln. Jede Tätigkeit, die als Aufgabenerfüllung anzusehen ist, ist staatlich. Auch die Anforderungen kennen keine Abstufungen bezüglich Umfang oder Intensität: Es gelten für jedes Staatshandeln dieselben Anforderungen. Allerdings darf die Bindungsintensität dieser Anforderungen von vornherein nicht überschätzt werden: Insbesondere das Bestimmtheitsgebot (als Teil der Gesetzmässigkeit), das öffentliche Interesse und das Äquivalenzprinzip (als abgabenspezifische Ausprägung der Verhältnismässigkeit) legen vergleichsweise lockere Fesseln an das Staatshandeln. Ihre Voraussetzungen sind dehnbar und daher leicht einzuhalten.

Unter diesen Vorzeichen können Privatisierungen entweder bei den staatlichen Aufgaben oder bei deren Erfüllung ansetzen. Werden staatliche Aufgaben privatisiert (Aufgabenprivatisierung), sind die verfassungsrechtlichen Konsequenzen einfach zu ziehen: Der betreffende Tätigkeitsbereich ist nicht mehr staatlich und unterliegt nicht mehr den verfassungsrechtlichen Anforderungen. Schwieriger liegt dies bei allen Vorhaben, die lediglich die Erfüllung der staatlichen Aufgaben privatisieren, die staatliche Aufgabenverantwortung aber unberührt lassen (Erfüllungsprivatisierung). 
Die Erfüllungsprivatisierung setzt bei den verschiedenen Aspekten der Aufgabenerfüllung an. Es sind organisatorische, instrumentelle und finanzielle Erfüllungsaspekte zu unterscheiden. \$4 legt dar, was die Privatisierung in diesen drei Hinsichten kennzeichnet. Privatisierte Organisationsformen bedeuten grössere Bewegungsfreiheiten für die Aufgabenträger und eine Verlagerung der Steuerung und Leitung hin zu einer punktuellen und nachträglichen Kontrolle. Privatisierte Handlungsformen bedeuten einen Verzicht auf verbindliche, einseitige Anordnungen und die Hoffnung auf kooperationsbereite und dankbare Verwaltungsadressaten. Privatisierte Finanzierungsformen letztlich bedeuten, dass Staatsaufgaben zur wirtschaftlichen Verhandlungsmasse werden.

\section{Aufgabenfremde Ziele und Anreize der Erfüllungsprivatisierung}

Werden bloss die Erfüllungsaspekte privatisiert, betrifft dies die Staatlichkeit der zugewiesenen Aufgaben nicht; dies ist mit der Aufgabenzuweisung per Verfassung und Gesetz abschliessend bestimmt. Die Erfüllungsprivatisierung kann aber die Aufgabenerfüllung mit aufgabenfremden Überlegungen beladen.

Dies geschieht etwa, indem der Staat auf gegenseitige, nicht verbindliche Instrumente setzt, um Rechte und Pflichten zu regeln, statt diese einseitig anzuordnen. So wird die Akzeptanz und Kooperation Privater auf dieselbe Ebene gehoben wie die verbindliche Durchsetzung gesetzlicher Vorgaben. Oder wenn Regelungsbereiche als zu kompliziert und technisch für staatliche Behörden angesehen werden und daher die Normsetzung an Fachexperten ausgelagert wird - so treten die Bedürfnisse der betroffenen Adressaten in der Gestalt von Sondergruppeninteressen neben die öffentlichen Ziele, die mit der Aufgabe verfolgt werden. Eine ähnliche Aufladung der Aufgabenerfüllung um sachfremde Aspekte zeigt sich insbesondere auch bei den privatisierten Finanzierungsformen: Sie erheben die Mittelbeschaffung für die Erfüllung der staatlichen Aufgaben auf dieselbe Stufe wie die Aufgaben selber. Der Gesetzgeber könnte abschliessend festlegen, welche Leistungen zwingend zu erbringen sind, und die erforderliche Finanzierung dafür sicherstellen. Stattdessen werden Aufgabenträger dazu angehalten, sich selber zu finanzieren und jeden Bestandteil ihrer Tätigkeit als abrechnungsfähige Leistung auszuweisen. Und wenn Trägern staatlicher Aufgaben erlaubt wird, sich nebenbei (oder gar hauptsächlich) mit privatwirtschaftlichen Leistungen am Markt zu betätigen, werden unternehmerische Überlegungen ebenso wichtig wie zielgerichtetes Umsetzen gesetzlicher Vorgaben. 
Ob die Erfüllungsprivatisierung die staatliche Aufgabenerfüllung effizienter und günstiger macht, muss im konkreten Fall geprüft werden - auf jeden Fall verlängert sie den kürzestmöglichen Weg von der zugewiesenen Aufgabe zu deren Erfüllung. Wie wirksam und wirtschaftlich die privatisierte Aufgabenerfüllung in einem konkreten Bereich ist, könnten nur ökonomische Methoden eruieren; die Rechtswissenschaft kann dazu nur Vermutungen aufstellen.

\section{Einzelfallregelungen als Mehraufwand und Garant für Freiräume zugleich}

Erfüllungsprivatisierung heisst, neue Formen einzusetzen. Die Eigenschaften und Rechtsfolgen dieser Formen und Instrumente müssen erst definiert werden. Die nichtprivatisierte Aufgabenerfüllung auf Bundesebene beruht - nur unwesentlich vereinfacht - auf drei Gesetzen: dem RVOG für das Organisatorische, dem VwVG für das Instrumentelle und dem FHG für das Finanzielle. Jede privatisierte Aufgabenerfüllung bedingt ihr eigenes Gesetz: Die spezialgesetzliche Aktiengesellschaft will errichtet, der öffentlichrechtliche Vertrag an die Hand gegeben und die Aufsichtsabgabe vorgesehen werden. Dies fordert einerseits das Gesetzmässigkeitsprinzip und ist zweitens Ausdruck der Notwendigkeit, die Einhaltung der verfassungsrechtlichen Anforderungen sicherzustellen.

Die Erfüllungsprivatisierung verursacht somit in jedem Fall einen Mehraufwand. Dies ist aber nur die eine Seite: Die Einzelfallregelung, das Erfordernis einer spezialgesetzlichen Sonderlösung, bringt dem Aufgabenträger auch einmalige Freiheiten. Es gelten eben nur gerade die Voraussetzungen und Rechtsfolgen, die das Spezialgesetz vorsieht. Und spezialgesetzlich eingerichtete Autonomiebereiche sind zu beachten - unabhängig davon, wie gut sie sich mit der Zweckrichtung auf die vorgegebene Aufgabe vertragen.

\section{Einzelne Probleme bei der privatisierten Erfüllung staatlicher Aufgaben}

Diese Arbeit misst die verschiedenen Erscheinungen der Erfüllungsprivatisierung (\$4) an den verfassungsrechtlichen Anforderungen an staatliches Handeln (\$3). Privatisierte Organisations-, Handlungs- und Finanzierungsformen führen zu einer Reihe von Problemen und Spannungen. Von Problemen 
spricht diese Arbeit, wie eingangs bei § 5 dargelegt, wenn sich eine bestimmte Privatisierung mit einer verfassungsrechtlichen Anforderung schlechterdings nicht verträgt. Spannungen liegen hingegen vor, wenn eine Privatisierung lediglich in eine andere Zielrichtung weist als die Anforderung, mit ihr aber noch zu vereinbaren ist.

Eine umfassende Darstellung der Probleme und Spannungen findet sich im Zwischenfazit bei \$5/VII. An dieser Stelle sollen nur einige nennenswerte Probleme hervorgehoben werden:

- Problem aus der Gesetzmässigkeit bei privatisierten Handlungsformen: Ausgelagerte Rechtsetzung - sprich von Fachexperten geschriebene Normen - ist tendenziell schwerer auffindbar und kann sprachlich schwer verständlich sein. Dies verträgt sich schlecht mit dem Erfordernis der Normdichte (vorne \$5/I.2.d).

- Problem aus der Verhältnismässigkeit bei privatisierten Handlungsformen: Unverbindliche Handlungsinstrumente sind weniger zwecktauglich und zielgenau, weil ihre Wirkung von indirekten Anreizen abhängt (vorne \$5/III.2.b). Dadurch können beispielsweise informelle Absprachen, Empfehlungen und Warnungen ungeeignet sein, um die angestrebten öffentlichen Interessen zu erreichen.

- Problem aus Treu und Glauben bei den privatisierten Handlungsformen: Bei einvernehmlich ausgehandelten Instrumenten, die nachträglich rechtswidrig werden, dürfte das Vertrauen in ihre Beständigkeit das entgegenstehende Interesse an einer gesetzeskonformen Anpassung regelmässig überwiegen (vorne \$5/IV.2.b).

- Problem aus der Grundrechtsbindung bei privatisierten Organisationsformen: Die Aufsicht gegenüber privatisierten Aufgabenträgern erfolgt oft nur noch punktuell und nachträglich. Das erschwert einen wirksamen Grundrechtsschutz (vorne \$5/V.1.c).

- Problem aus der Grundrechtsbindung bei privatisierten Finanzierungsformen: Die Verursacherfinanzierung kann Träger von Grundrechten davon abhalten, ihre Ansprüche auszuüben. Kostentragepflichten für polizeiliche Einsätze beispielsweise schrecken davon ab, sich auf die Meinungsäusserungs- oder die Versammlungsfreiheit zu berufen (vorne \$5/V.3.b).

- Querschnittsproblem: Privatisierte Organisations-, Handlungs- und Finanzierugsformen lassen die Erfüllung staatlicher Aufgaben wie privates Handeln erscheinen - was für die Durchsetzung aller verfassungsrechtlichen Anforderungen ein Problem ist. Damit sich Verwaltungsadressaten überhaupt auf ihre verfassungsrechtlichen Ansprüche berufen, müssen sie wissen, dass sie es mit dem Staat zu tun haben (vorne $\$ 5 / V I .1$ ). 
Der Gesetzgeber könnte die verfassungsrechtlichen Anforderungen herabsetzen, wenn er die Aufgabenerfüllung in privatisierte Formen giesst. Die erwähnten Probleme entstehen oft dadurch, dass er dies nicht tut und dann nur unzureichende Vorkehrungen trifft, um die Einhaltung der weitergeltenden Anforderungen sicherzustellen.

\section{Einzelne Spielräume bei der privatisierten Erfüllung staatlicher Aufgaben}

Die verfassungsrechtlichen Anforderungen $(\$ 3)$ können für die verschiedenen Erscheinungen der Erfüllungsprivatisierung (\$4) nicht nur Probleme bedeuten, sie können umgekehrt auch Spielräume bieten. Darin liegt die Hoffnung begründet, Privatisierungen brächten einen kleineren, schlankeren und günstigeren Staat. Wie am Anfang von $\$ 6$ erörtert, können solche Spielräume ausserhalb oder innerhalb der Anforderungen bestehen: also entweder dort, wo gerade keine Anforderungen greifen, oder dort, wo die Anforderungen gelockert sind. Diese Lockerungen wiederum können sich allgemein aus den Anforderungen ergeben oder erst besonders bei privatisierten Organisations-, Handlungs- und Finanzierungsformen auftreten. Vor allem diese besonderen Lockerungen innerhalb der Anforderungen können die Erwartung von weniger Staat einlösen.

Eine umfassende Darstellung der Spielräume findet sich im Zwischenfazit bei \$6/VII. An dieser Stelle sollen nur einige nennenswerte Spielräume hervorgehoben werden:

- Allgemeiner Spielraum: Die Verfassung verpflichtet den Staat nicht darauf, sein Organigramm möglichst übersichtlich zu halten (vorne \$6/I). Das Rechtssatzerfordernis von Art. 164 Abs. 1 Bst. g BV, die Koordinationspflicht von Art. 180 Abs. 1 Satz 2 BV und das Öffentlichkeitsgesetz verpflichten die Verwaltung nur auf Transparenz, nicht auf Nachvollziehbarkeit.

- Spielraum bei der Gesetzmässigkeit für privatisierte Organisationsformen: Wenn einem staatlichen Akteur privatwirtschaftliche Tätigkeit erlaubt wird, wandelt sich das Gesetzmässigkeitsprinzip zu einem Spezialitätsprinzip. Die spezialgesetzliche Aufgabenübertragung muss nur noch den Sachbereich umschreiben und braucht nicht mehr gleich detailliert zu sein (vorne \$6/II.1.c).

- Spielraum bei der Gesetzmässigkeit für privatisierte Handlungsformen: Auch einvernehmliche Instrumente setzen die Anforderungen an die 
Bestimmtheit von Normen herab. Wenn Verwaltungsadressaten einer Pflicht zustimmen oder eine Rechtseinschränkung freiwillig auf sich nehmen, reicht eine Kompetenznorm als gesetzliche Grundlage (vorne \$6/II.2). Dieser Spielraum besteht auch bei der Grundrechtsbindung, indem Private auf die Durchsetzung ihrer grundrechtlichen Ansprüche verzichten können (vorne \$6/VI.3).

- Spielraum bei der Gesetzmässigkeit für privatisierte Finanzierungsformen: Kostenanlastungsabgaben und insbesondere Aufsichtsabgaben weiten das Verursacherprinzip aus. Dies verhilft dem Äquivalenzprinzip zu einer breiteren Anwendung, was wiederum ungenügend bestimmte gesetzliche Grundlagen kompensieren kann (vorne \$6/II.3.a)

- Spielräume bei der Verhältnismässigkeit für privatisierte Handlungsformen: Konsensuale Instrumente und ausgelagerte Rechtsetzung beziehen die Sichtweise Privater stärker in die Aufgabenerfüllung ein. Dies liegt im Sinn der Verhältnismässigkeitsprüfung und kann insbesondere die Zumutbarkeit von staatlichen Eingriffen garantieren (vorne \$6/IV.2).

- Allgemeiner Spielraum bei der Grundrechtsbindung: Auch der privatisiert handelnde Staat ist an die Grundrechte gebunden. Es stünde dem Gesetzgeber aber grundsätzlich offen, die privatisierte Aufgabenerfüllung ausdrücklich von der Bindung an gewisse Schutzbereiche zu befreien (vorne \$6/VI.1). Nach der hier vertretenen Meinung kann dies aber nicht schon auf dem Weg der Rechtsanwendung geschehen.

- Spielraum bei der Grundrechtsbindung für privatisierte Organisationsformen: Privatisierte Aufgabenträger gelten aufgrund ihres je eigenen Aufsichtsregimes nicht als dieselbe Behörde im Sinn der Rechtsgleichheit. Im Unterschied zur Zentralverwaltung sind sie also weniger stark an das Verhalten anderer Verwaltungseinheiten gebunden (vorne \$6/VI.2.b).

Diese Spielräume entstehen meist dann, wenn das Spezialgesetz den Aufgabenträgern Entscheidungsspielräume gewährt und wenn Verwaltungsadressaten auf die Durchsetzung ihrer verfassungsrechtlichen Ansprüche verzichten. Die freiwillige Kooperation Privater lockert insbesondere die Anforderungen der Gesetzmässigkeit und der Grundrechtsbindung. 


\section{Empfehlungen}

So weit der Rückblick auf die gewonnenen Erkenntnisse. Diese Arbeit soll aber nicht mit abstrakten Problemschilderungen enden. Vielmehr gibt sie dem Gesetzgeber Mittel an die Hand, um die verfassungsrechtlich problematischen Folgen der Erfüllungsprivatisierung zu minimieren.

Abschliessend werden daher die Probleme und Spannungen, wie sie in \$5 herausgearbeitet wurden, zu einem Fragekatalog aufbereitet (1). Diese Fragen können im Sinn einer Checkliste durchgegangen werden, um zu beurteilen, ob ein Privatisierungsvorhaben die auftretenden Probleme bedenkt und adressiert. Anschliessend folgt - in Rückbesinnung auf das funktionelle Staatsverständnis der Verfassung - die allgemeine Empfehlung, die zugewiesenen Aufgaben im Gesetz selbst möglichst präzise zu beschreiben (2). Danach werden die wichtigsten Erkenntnisse bezüglich jedes Erfüllungsaspekts zu einer Forderung verdichtet (3-5).

\section{Probleme und Spannungen als Checkliste für Privatisierungsvorhaben}

Bei der Privatisierung der Organisationsformen entstehen Probleme vor allem aus zwei Gründen: Erstens, weil die Aufsicht spezialgesetzlich geregelt werden muss, und zweitens, wenn privatwirtschaftliche und staatliche Tätigkeit in derselben Organisation vermischt werden. Bevor Aufgabenträger aus der Zentralverwaltung herausgelöst werden, sind folgende Fragen zu beantworten:

- Umschreibt das Spezialgesetz die übertragenen Aufgaben präzis? Insbesondere: Ist die Aufgabenzuweisung bestimmt genug, dass ohne grossen Aufwand erkennbar ist, welche Tätigkeiten an die Grundrechte gebunden sind?

- Macht das Spezialgesetz, das den Aufgabenträger errichtet oder ihm Aufgaben übertragt, deutlich, auf welche Ziele die Aufgabenerfüllung auszurichten ist?

- Falls einem Aufgabenträger Renditeziele vorgegeben werden: Können diese die zweckgebundene Ausrichtung auf die Aufgabenerfüllung gefährden?

- Ist die spezialgesetzlich eingerichtete Aufsicht genügend intensiv, um die Einhaltung der verfassungsmässigen und gesetzlichen Vorgaben zu garantieren?

- Bleibt der Akteur als Träger staatlicher Aufgaben erkennbar?

- Überwiegen die Gründe, staatliche und privatwirtschaftliche Tätigkeiten in derselben Organisation zu vereinen, die allfälligen Marktverzerrungen? 
Mit privatisierten Handlungsformen handelt der Staat tendenziell weniger verbindlich und verlässt sich mehr auf die Mitwirkung Privater. Bevor Verträge, informelle Instrumente oder ausgelagerte und überwachte Rechtsetzung und Rechtsanwendung für die Aufgabenerfüllung eingesetzt werden, stellen sich folgende Fragen:

- Besteht die Notwendigkeit, ein neues Instrument zu schaffen, oder kann ein Instrument mit gefestigter Dogmatik eingesetzt werden? Kann zum Beispiel eine Rechtsverordnung ein Selbstregulierungsreglement ersetzen?

- Ist sichergestellt, dass der Aufgabenträger hoheitliche Instrumente anwenden kann, wenn er Rechte und Pflichten verbindlich regeln muss?

- Sind insbesondere Verträge nur in Bereichen vorgesehen, in denen eine nachträgliche Anpassung an eine veränderte Rechtslage nicht notwendig werden wird?

- Stellt das Spezialgesetz mittels Aufgabenzuweisung und Aufsichtsmitteln sicher, dass Branchenvertreter und Fachexperten auf öffentliche Interessen verpflichtet bleiben, soweit sie in die Aufgabenerfüllung einbezogen werden?

- Führt der Verweis auf das Zivilprozessrecht zu Rechtsschutzdefiziten, die nicht bewusst gewählt sind?

- Bleibt das Verwaltungshandeln als staatliches Handeln erkennbar?

Bei der Privatisierung der Finanzierungsformen schliesslich werden Aufgabenträger mit ihrer eigenen Mittelbeschaffung beauftragt und es ziehen wirtschaftliche Faktoren in die Aufgabenerfüllung ein. Bevor die Steuerfinanzierung zugunsten anderer Finanzierungsformen verdrängt wird, sind folgende Fragen zu beantworten?

- Werden wirtschaftliche Anreize gesetzt, die mit gesetzlichen Leistungspflichten oder Rechtsansprüchen in Konflikt geraten? Insbesondere: Schrecken Kostenfolgen von der Geltendmachung von grundrechtlichen Ansprüchen $\mathrm{ab}$ ?

- Werden wirtschaftliche Anreize gesetzt, die den Aufgabenträger zu zusätzlichem Aufwand anleiten, der für die Aufgabenerfüllung nicht nötig ist?

- Wird ein Aufgabenträger derart abhängig von seinen Gebührenzahlern, dass er deren Interessen über die öffentlichen Interessen zu stellen droht?

- Abgesehen von der politischen Machbarkeit, besteht ein sachlicher Grund dafür, anstelle von Steuern Kostenanlastungsabgaben einzurichten? Und ist bei diesen Abgaben sichergestellt, dass die Abgabepflichtigen in möglichst engem Bezug zum Aufgabenbereich stehen? 


\section{Detaillierte Aufgabenzuweisung im Gesetz}

Die Verfassung bindet jedes Handeln, das der Erfüllung staatlicher Aufgaben dient, an besondere Anforderungen. Diese Arbeit hat das funktionelle Staatsverständnis der Verfassung zur Genüge betont und leitet daraus eine übergreifende Empfehlung ab: Das Gesetz soll möglichst präzis festlegen, welche Tätigkeiten einem Akteur zur verbindlichen Erfüllung übertragen werden. Je präziser die Aufgabe definiert ist, desto klarer sind die Ziele und Zwecke, an denen die staatliche Tätigkeit zu messen ist. Je genauer das Gesetz vorgibt, welche Eingriffe vorzunehmen und welche Leistungen zu erbringen sind, desto einfacher wird die Kontrolle der staatlichen Tätigkeit. Damit die Pflichterfüllung überprüft werden kann, müssen die Pflichten klar erkennbar sein. Und nur wenn der Aufgabenbereich bestimmt ist, besteht Gewissheit für eine möglichst schlanke und effiziente Ausrichtung auf diese Tätigkeit. Wenn der Aufgabenbereich zu einer Verhandlungssache wird (Stichwort Leistungsvereinbarung) und die Mittelbeschaffung die Tätigkeit vorbestimmt, werden Aufgabenträger ihre Zuständigkeitsbereiche ausweiten. 971

Die verfassungsrechtlichen Anforderungen müssen möglichst treffgenau auf dasjenige Handeln angelegt werden können, das als staatliche Aufgabe mit Erfüllungspflicht vorgegeben ist. Das Gesetz sollte daher nachvollziehbar und beständig definieren, welche Tätigkeiten in welchem Umfang in welcher Qualität verlangt werden.

\section{Insbesondere bezüglich der Organisationsformen}

Was die organisatorischen Erfüllungsaspekte betrifft, liegt die wichtigste Empfehlung auf der Hand: Vermische nicht, was nicht zusammengehört! In aller Regel geht es zulasten von Privatpersonen, wenn staatliche Aufgabenbereiche mit privatwirtschaftlicher Tätigkeit verbunden werden. Die unternehmerischen Freiheiten mögen dem ehemaligen Monopolisten erlauben, neue Geldquellen zu erschliessen, sie werden die staatliche Aufgabenerfüllung aber nicht effizienter und zielgerichteter machen.

Die verfassungsrechtlichen Anforderungen werden zum toten Buchstaben, wenn sie nicht angerufen werden. Der Verfassung wäre also gedient, wenn Aufgabenträger ein Bewusstsein dafür haben, Staat zu sein, und wenn Verwaltungsadressaten wissen, wann sie es mit dem Staat zu tun haben. Die saubere Trennung von staatlichen Aufgaben und anderen Tätigkeiten erleichtert diese Erkennbarkeit.

971 Vgl. zu diesem Spannungsverhältnis auch BAUMANN, Wettbewerbsverzerrungen, Rz.131. 


\section{Insbesondere bezüglich der Handlungsformen}

Handlungsformen sind ein Mittel zum Zweck. Verwaltungsträger erfüllen damit ihre vorgegebenen Aufgaben. Um Spannungen und Probleme zu vermeiden, empfiehlt sich Folgendes: Der Gesetzgeber muss die Ausgangslage analysieren und überlegen, wie er das Verhalten seiner Bürgerinnen und Bürger beeinflussen will - und dies dann auf möglichst direkte Weise tun.

Diese Empfehlung drückt zweierlei aus. Erstens ist zu fordern, dass Verwaltungsträger das nötige Fachwissen selber aufbauen und erhalten, um die ihnen übertragenen Aufgaben selber zu erfüllen. Wenn der staatliche Akteur nicht in der Lage ist, über die geeigneten Instrumente zu entscheiden, liegt die Aufgabe am falschen Ort. Und zweitens soll sich der Staat darauf zurückbesinnen, dass Staatshandeln Machtausübung ist. Was mit freiwilligen Absprachen und informellen Vorgaben geregelt werden kann, muss nicht zwingend in staatlicher Verantwortung liegen. Der Staat sollte selbstbewusst verfügen und Recht setzen, wo verbindliche Anordnungen angebracht sind, und beim Rest hinterfragen, ob die betreffende Aufgabe bei ihm am richtigen Ort ist.

Zu einer ähnlichen Schlussfolgerung kommt etwa auch eine Untersuchung der ausgelagerten Normsetzung im Stromversorgungsrecht. Die «festgestellten Steuerungsmisserfolge [seien] nur zum Teil auf staatliche Wissensdefizite zurückzuführen». ${ }^{972} \mathrm{Mehr}$ privatisierte Rechtsetzung sei also nicht die Lösung.

\section{Insbesondere bezüglich der Finanzierungsformen}

Eine möglichst verursachergerechte Finanzierung der staatlichen Tätigkeit ist nicht zu bemängeln, doch sie darf der hauptsächlichen Aufgabe der Verwaltung nicht im Weg stehen: Staatliche Aufgabenträger sollen in erster Linie ihre Aufgaben erfüllen können. Die Mittelbeschaffung muss daher der Aufgabenerfüllung vorgelagert sein. Die Empfehlung bezüglich der finanziellen Erfüllungsaspekte lautet daher: Was nicht nachträglich und einzelfallweise mit Gebühren beglichen werden kann, gehört steuerfinanziert.

Anstatt das Verursacherprinzip zu überstrapazieren und jeden Aspekt der Verwaltungstätigkeit in verrechenbare Leistungen zu giessen, kann die Aufgabenerfüllung wieder fokussiert werden, indem zumindest die Strukturfinanzierung von Verwaltungsträgern über Steuermittel bestellt wird. ${ }^{973}$

972 WALTHER, Kooperative Steuerungsinstrumente, S.222.

973 Vgl. in diese Richtung auch BIAGGINI, Kommentar BV, Art. 94 Rz. 6a a.E. 


\section{Schlusswort}

Mit einem verfassungsdogmatischen Blick erscheinen viele Privatisierungen wenig zielführend. Sie verkomplizieren die staatliche Aufgabenerfüllung und laden sie mit aufgabenfremden Motiven auf. Dabei könnte es auch einfacher sein: Welche Ziele muss der Staat befördern? Welche Verwaltungseinheiten braucht er dafür? Welche Pflichten muss er Privaten auferlegen und welche Rechte ihnen gewähren? Und wie viel Steuereinkommen braucht er, um das alles zu bewältigen? Punkt.

Diese Arbeit plädiert nicht gegen Privatisierungen. Sie will aber die Erwartungen mässigen, die damit verbunden sind. Was den Staat ausmacht, sind die Aufgaben, die er erfüllen muss. Schraubt man nun an den Erfüllungsformen, wird man immer mehr Komplexität und Ungewissheit erlangen, aber nicht sicher mehr Effizienz und Bürgernähe. Dies will wohlüberlegt sein. 

Dieses Werk ist erschienen in der Reihe sui generis, herausgegeben von Daniel Hürlimann und Marc Thommen.

1. Auflage 29. Mai 2020

(C) 2020 Dr. iur. Dominik Elser

Dieses Werk wurde unter einer Creative Commons Lizenz als Open Access veröffentlicht, die bei Weiterverwendung nur die Nennung des Urhebers erfordert (CC BY 4.0 - https://creativecommons.org/licenses/by/4.0).

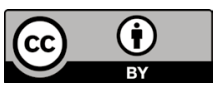

Die Druckvorstufe dieser Publikation wurde vom Schweizerischen Nationalfonds zur Förderung der wissenschaftlichen Forschung unterstützt.

ISBN: 978-3-907297-09-4

DOI: $10.38107 / 009$

Korrektorat: Christoph Meyer

Gestaltung: Müller+Hess, Basel

Druck: Ebner \& Spiegel, Ulm 
sui generis ist ein Verein, der sich der Förderung des freien Zugangs zu juristischer Literatur, Gerichtsurteilen, Behördenentscheidungen und Gesetzesmaterialien verschrieben hat. Unter dem Label suigeneris erscheint seit 2014 eine juristische Open-Access-Fachzeitschrift. 2019 erfolgte die Gründung des sui generis Verlags.

sui generis Buchreihe herausgegeben von Daniel Hürlimann und Marc Thommen

In dieser Reihe werden juristische Dissertationen und Habilitationen sowie Lehrbücher und Fachpublikationen einem breiten Publikum zugänglich gemacht. Die Bücher dieser Reihe erscheinen als gedruckte Werke und online. Die digitale Version ist weltweit kostenlos zugänglich (Open Access). Die Urheberrechte verbleiben bei den AutorInnen; die Werke werden unter einer Creative-Commons-Lizenz veröffentlicht.

Bisher in der sui generis Reihe erschienen:

001 - Monika Simmler: Normstabilisierung und Schuldvorwurf

002 - Marc Thommen: Introduction to Swiss Law

003 - Silvio Hänsenberger:

Die zivilrechtliche Haftung für autonome Drohnen unter Einbezug von Zulassungs- und Betriebsvorschriften

004 - Mais A.M. Qandeel:

Enforcing Human Rights of Palestinians in the Occupied Territory

005 - Moritz Oehen:

Der Strafkläger im Strafbefehls- und im abgekürzten Verfahren

006 - Jens Lehne:

Crisis at the WTO: Is the Blocking of Appointments to the WTO

Appellate Body by the United States Legally Justified?

007 - Lorenz Garland: Waffengleichheit im Vorverfahren

008 - Christoph Urwyler:

Die Praxis der bedingten Entlassung aus dem Strafvollzug

009 - Dominik Elser: Die privatisierte Erfüllung staatlicher Aufgaben

010 - David Henseler:

Datenschutz bei drohnengestützter Datenbearbeitung durch Private 



\section{9}
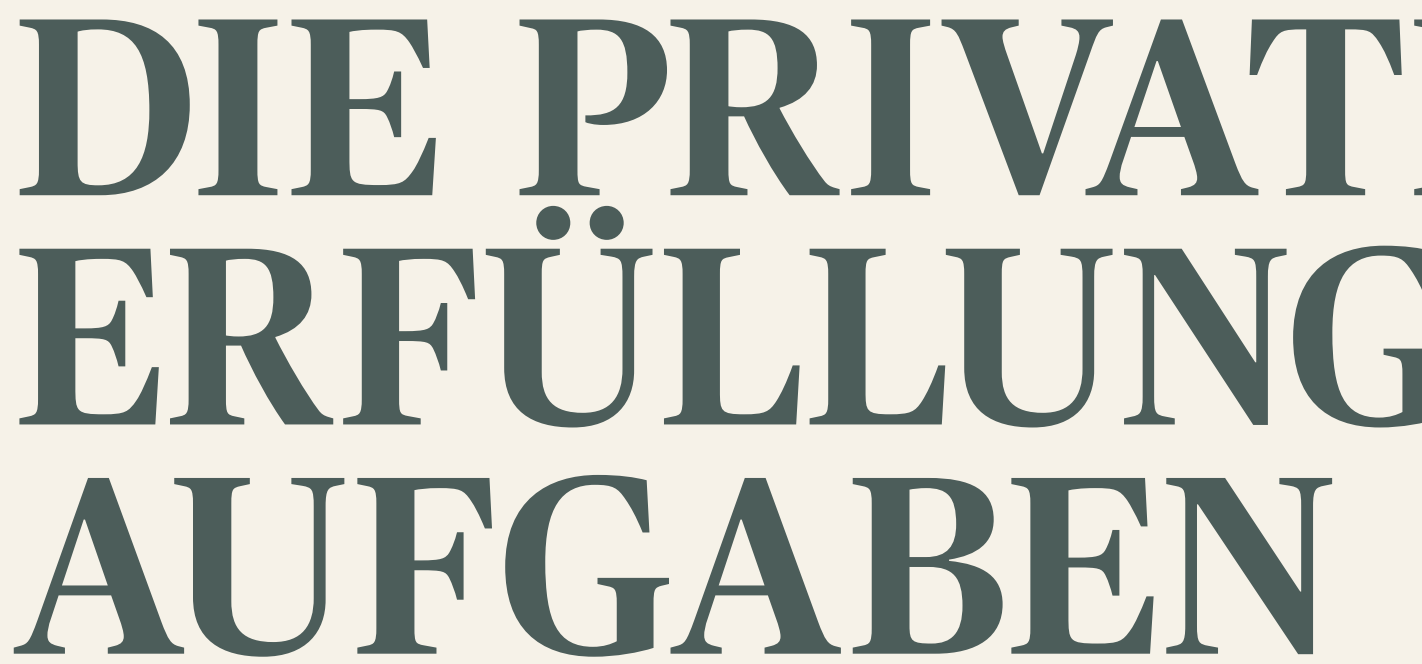

Der Staat ist zu gross, zu langsam, zu teuer und zu weit von seinen Bürgerinnen und Bürgern entfernt. Diesen Eindruck vermitteln die zahlreichen Privatisierungsvorhaben, die den Staat reformieren möchten. Können Privatisierungen die Erwartung von weniger Staat einlösen, oder sind umgekehrt die Befürchtungen berechtigt, wonach damit die rechtsstaatlichen und demokratischen Vorgaben zurückgedrängt werden?

Was den Staat ausmacht, sind die Aufgaben, die er erfüllen muss. Wer nur die Art und Weise der Aufgabenerfüllung verändert, wird den Staat nicht verkleinern, verursacht aber oft zusätzlichen Kontrollaufwand. Zudem können aufgabenfremde Motive in die staatliche Aufgabenerfüllung einfliessen und die Grenzen des Staates schwieriger zu ziehen sein. Auf der anderen Seite stehen grössere Flexibilität, Erfüllungsweisen, die für den Einzelfall passen, und die erhöhte Akzeptanz seitens der Adressatinnen und Adressaten von staatlichem Handeln.

Dieses Buch arbeitet mit Blick auf das schweizerische Verfassungsrecht heraus, wo Probleme und Spielräume auftreten, wenn die staatlichen Aufgaben privatisiert erfüllt werden.

suigeneris

ISBN 978-3-907297-09-4

DOI $10.38107 / 009$ 